
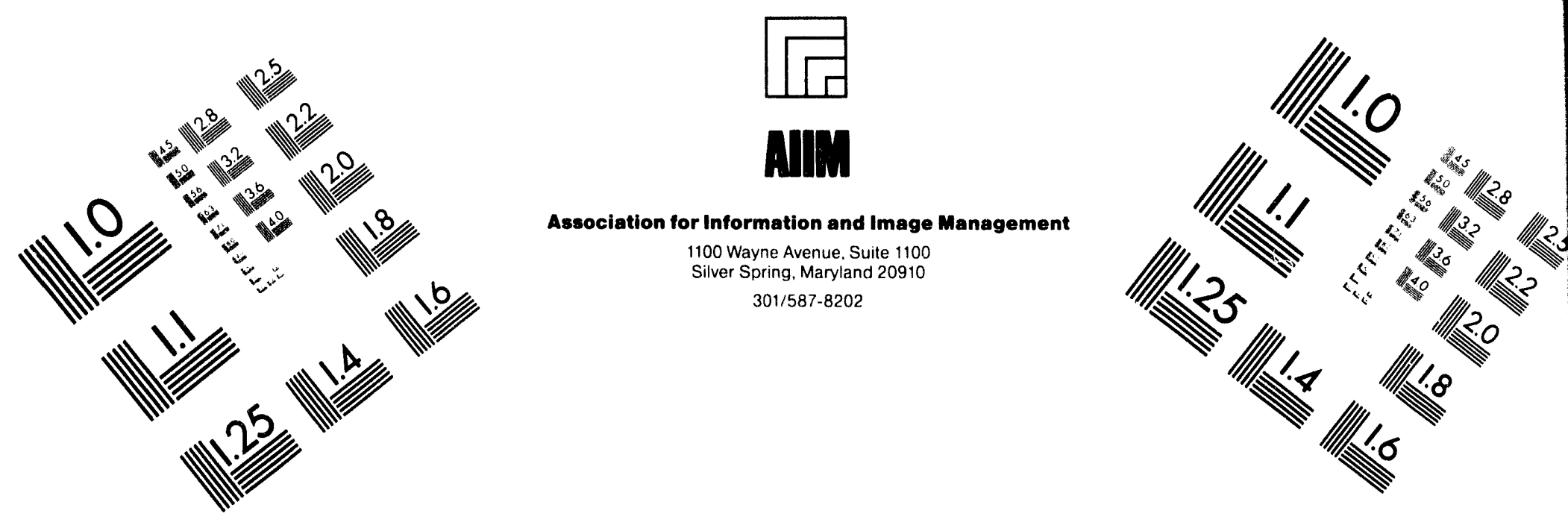

\title{
Centimeter
}

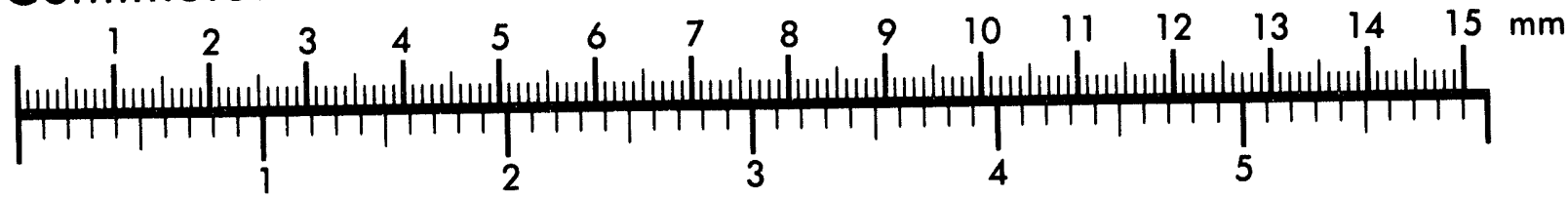

Inches
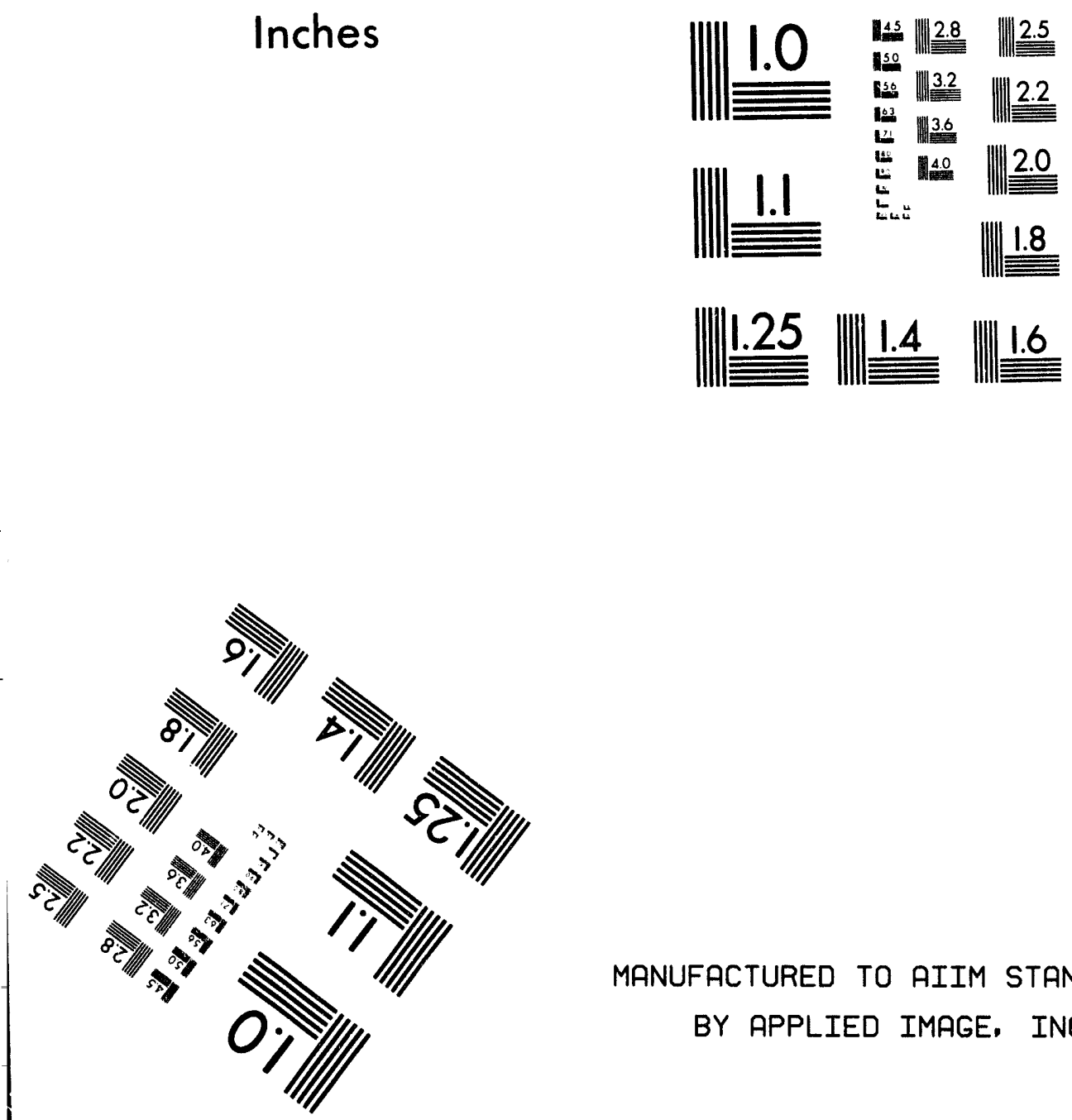

MANUFACTURED TO AIIM STANDARDS

BY APPLIED IMAGE, INC.

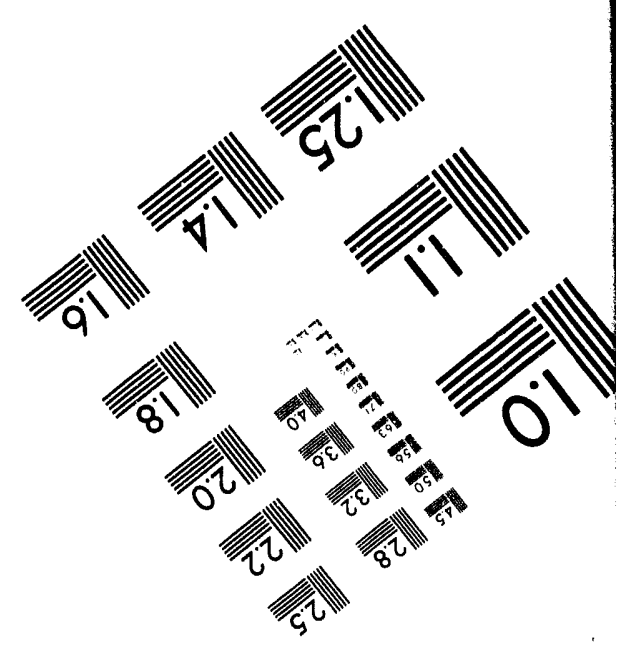



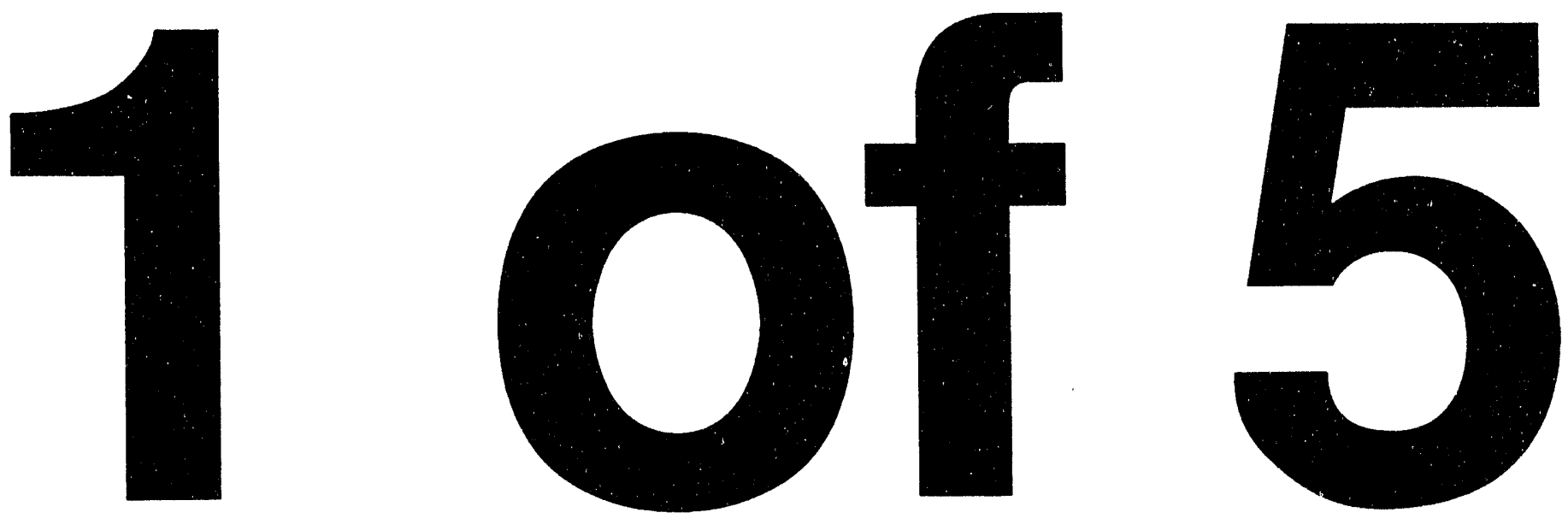
PFC/RR-94-4

\title{
A Passively-Safe Fusion Reactor Blanket with Helium Coolant and Steel Structure
}

\author{
Kenneth Mitchell Crosswait
}

April, 1994

\author{
Plasma Fusion Center \\ Massachusetts Institute of Technology \\ Cambridge, MA 02139 USA
}

This work was performed under appointment to the Magnetic Fusion Energy Technology Fellowship program which is administered for the U.S. Department of Energy by the Oak Ridge Institute for Science and Education. Reproduction, translation, publication, use and disposal, in whole or in part by or for the United States Government is permitted.

By acceptance of this article, the publisher and/or recipient acknowledges the U.S. Government's right to retain a non-exclusive, royalty-free license in and to any copyright covering this paper.

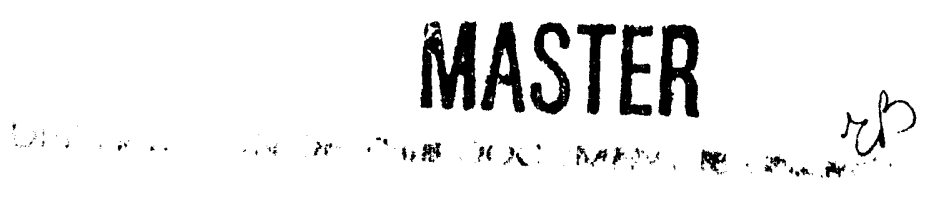




\section{A Passively-Safe Fusion Reactor Blanket with Helium Coolant and Steel Structure}

by

\section{Kenneth Mitchell Crosswait}

B.S. in Applied and Engineering Physics, Cornell University

(1984)

Submitted to the Department of Nuclear Engineering in Partial Fulfillment of the Requirements for the Degree of

Doctor of Philosophy in

Nuclear Engineering

at the

Massachusetts Institute of Technology

April 1994

(C) Kenneth Mitchell Crosswait 1994

$\frac{\text { Signature of Author }}{\text { Department of Nuclear Engineering }}$

Certified by

John E. Meyer

Professor, Department of Nuclear Engineering Thesis Supervisor

Accepted by

Allan F. Henry

Chairnan, Department Committee on Graduate Students 
This work was performed under appointment to the Magnetic Fusion Energy Technology Fellowship program which is administered for the U.S.

Department of Energy by the Oak Ridge Institute for Science and Education.

The author hereby grants to MIT permission to reproduce and to distribute publicly copies of this thesis document in whole or in part. 
A Passively-Safe Fusion Reactor Blanket with Helium Coolant and Steel Structure

by

Kenneth Mitchell Crosswait

Submitted to the Department of Nuclear Engineering, MIT, on April 7, 1994 in partial fulfillment of the requirements for the degree of Doctor of

Philosophy in Nuclear Engineering.

\begin{abstract}
Helium is attractive for use as a fusion blanket coolant for a number of reasons. It is neutronically and chemically inert, nonmagnetic, and will not change phase during any off-normal or accident condition. A significant disadvantage of helium, however, is its low density and volumetric heat capacity. This disadvantage manifests itself most clearly during undercooling accident conditions such as a loss of coolant accident (LOCA) or a loss of flow accident (LOFA).
\end{abstract}

This thesis describes a new helium-cooled tritium breeding blanket concept which performs significantly better during such accidents than current designs. The proposed blanket uses reduced-activation ferritic steel as a structural material and is designed for neutron wall loads exceeding $4 \mathrm{MW} / \mathrm{m}^{2}$. The proposed geometry is based on the nested-shell concept developed by Wong, ${ }^{1}$ but some novel features are used to reduce the severity of the first wall temperature excursion. These features include the following: (1) A "beryllium-joint" concept is introduced, which allows solid beryllium slabs to be used as a thermal conduction path from the first wall to the cooler portions of the blanket. The joint concept allows for significant swelling of the beryllium (10 percent or more) without developing large stresses in the blanket structure. (2) Natural circulation of the coolant in the water-cooled shield is used to maintain shield temperatures below 100 degrees $\mathrm{C}$, thus maintaining a heat sink close to the blanket during the accident. This ensures the long-term passive safety of the blanket.

By using jet-pumps in the shield water system, bypass valves around the shield water coolant pumps are not necessary, hence natural circulation of the water can be assured. The peak first wall temperature during a complete LOFA is less than 700 degrees C. Therefore, the blanket can withstand such an accident without any damage even if the helium coolant system remains pressurized.

The thesis discusses two different options for a naturally circulating shield cooling system. The first option uses a single coolant loop between the shield and the air-cooled

${ }^{1}$ C.P.C. Wong, et.al., "Blanket Design for the ARIES-I Tokamak Reactor," Proc. IEEE 13th Symp. on Fusion Eng., 1990, p. 1035. 
heat exchanger. The second option uses two coolant loops, together with an intermediate heat exchanger that transfers heat from one coolant loop to the other. Cost comparisons are made for these two options, and the radioactivity hazards for the single loop system are explored. It is shown that the cost for a naturally circulating shield water system is small compared to the cost of the reactor blanket and shield complex, and that the radioactivity hazards for a single loop system can be mitigated. Because of the low cost of a naturally circulation cooled shield coolant system, and the potential passive safety benefits it provides, the author considers that designers of future fusion machines with potentially large afterheat generation rates, such as ITER and DEMO, should consider incorporating such a "safe" shield cooling system.

Thesis Supervisor: Dr. John E. Meyer Title: Professor of Nuclear Engineering 


\section{Acknowledgments}

It is not possible, in this short space, to thank everyone who has helped me complete this thesis and made my stay at MIT both enjoyable and worthwhile. The following acknowledgments include but a small portion of the people from whom I learned invaluable knowledge, both technical and otherwise.

Firstly, I would like to thank Professor John Meyer, my thesis advisor, teacher, and friend. He not only helped to ensure that the technical foundation of my thesis was strong, but through his advice and companionship greatly added to the enjoyment of my MIT experience. My thanks also to Mrs. Meyer for hosting wonderful gatherings in Lexington. I am grateful to Dr. Daniel Cohn for introducing me to MIT and welcoming me to his group. Dr. Cohn and the other merrbers of his group, notably Dr. David Rhee, Paul Thomas (Mr. Magnet), and Janet Anderson provided me with technical, administrative, and "extra-curricular" support without which I would have suffered greatly.

Many thanks to the wonderful people at the Oak Ridge Institute for Science and Education for the awesome help and cooperation I received while on my fellowship. I would particularly like to thank Sandra Johnson, whose email I hope will continue to brighten my days even after I leave MIT. Thanks also to Gene Nardella at DOE for providing me with advice, wisdom, contacts, and most importantly, money.

There are many members of the MIT/Plasma Fusion Center faculty and staff to whom I am indebted. Professor Kazimi provided a pleasant and useful technical forum each week in the Fusion Technology meeting. Paul Rivenberg and Pat Stewart are superb administrators and great friends. Professor Jeff Freidberg is quite simply the best teacher I have ever had. And the PFC library staff, Kathy and Jason, not only provided the best librarian service in the universe, but a welcome haven of irreverence as well.

I never would have made it through MIT had it not been for the friendships I made here. My thanks to Dr. Tom and Sue Hsu, Chris and Eva Kurz, Peter Biegelbauer and Karin Zambra, John and Deyann Urbahn, Jim Reardon, Matt Fulton and "the CPO staff," among many others. Thanks to Dr. Mathias Koch especially for helping me understand the subtleties of ONEDANT and REAC, and for ensuring my optimism was properly tempered.

The support of my family has helped me though another milestone in life, as it has thorough all of the previous milestones. Special thanks to my parents and my brother Glenn for all of the encouragement and love.

No words can describe how mich my wife, Cheryl, has meant to me for the past three years. Her love has made the triumphs infinitely more sweet, and the failures infinitely more bearable. I only wish I had met her earlier. Thank you, my best sweetie, for everything. 


\section{Contents}

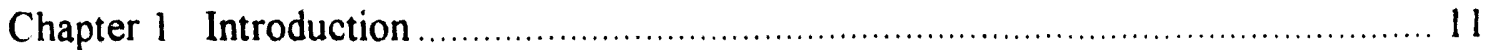

Chapter 2 Overall Fusion Reactor Configuration......................................... 15

Chapter 3 Neutronics, Activation and Heat Transfer Codes ................................ 19

3.1. The TWODANT Neutronics Code .............................................. 19

3.1.1 The TRANSX Interfacing Code .......................................... 23

3.1.2 MATXS Cross-Section Libraries .......................................... 23

3.1.3 Neutronics Model Geometry................................................ 24

3.2. The REAC Activation Code ..................................................... 31

3.3 The HEATING Heat Transfer Code .................................................. 33

Chapter 4 Past Blanket Design Concepts ................................................. 35

4.1 Pressurized Module Designs ................................................ 35

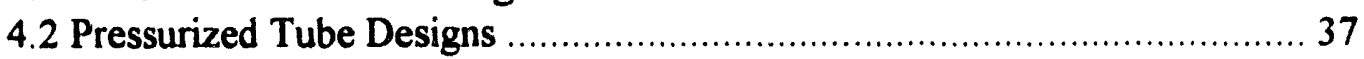

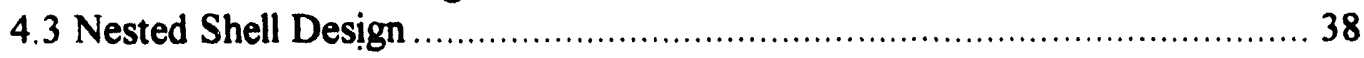

4.4 Helium-Particulate Designs ........................................................ 40

4.5 Breeder, Neutron Multiplier, and Structural Materials ........................ 41

4.5.1 Breeder Materials .......................................................... 41

4.5.2 Neutron Multipliers ....................................................... 48

4.5.3 Pebble Bed Design Concept....................................... 52

4.5.4 Structural Materials ...................................................... 54

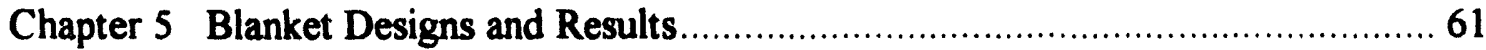

5.1 Blanket Design Constraints .........................................................6. 62

5.2 Blanket Design 1 -- Nested Shell with Mixed Pebble Bed ......................... 66

5.2.1 Lithium Oxide and Beryllium Mixed Bed: Neutronics ............... 68

5.2.2 Lithium Oxide and Beryllium Mixed Bed: Thermo-

Hydraulics............................................................................ 77

5.2.2.1 Pebble Bed Conductivity ..................................... 77

5.2.2.2 Mixed Bed Region Thicknesses............................... 80

5.2.2.3 Structural Shell Thicknesses................................... 83

5.2.2.4 Cooling of the First Wall ....................................... 87

5.2.2.5 Cooling of the Mixed Bed Regions........................... 90

5.2.6.6 Required Pumping Power for Blanket and Helium

Loop ..................................................................... 93

5.2.3 Blanket Design 1: Accident Analysis................................... 95

5.2.3.1 Simplified Analyses of Separate LOCA and LOFA....... 96

5.2.3.2 Afterheat Calculations........................................... 100

5.2.3.3 One-D Model for Accident Analysis.......................... 103

5.2.3.4 The Shield Model................................................. 106

5.2.3.5 Results of the No-Flow LOFA Analysis.................... 107

5.2.3.6 Failure Analysis for the First Wall........................... 110

5.3 Blanket Design 2 -- Nested Shell with Beryllium Joints .......................... 128

5.3.1 Blanket Design 2: Neutronics ........................................... 133

5.3.2 Blanket Design 2: Beryllium Joint Design .......................... 136 
5.3.3 Blanket Design 2: Thermo-Hydraulics ................................... 150

5.3.4 Blanket Design 2: Accident Analysis...................................... 154

5.3.4.1 Immediate Plasma Shut-Off..................................... 155

5.3.4.2 Plasma Continuation ............................................. 158

5.3.5 Blanket Design 2: Sensitivity Analysis ................................... 160

5.3.5.1 Effect of Gap Conductance ...................................... 162

5.3.5.2 Effect of Beryllium Conductivity ............................... 167

Chapter 6 Design of Shield Water System........................................................ 199

6.1 Shield Water System Heat Loads ...................................................... 202

6.1.1 Normal Operation............................................................. 202

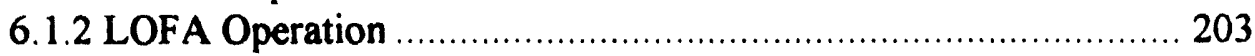

6.2 Configuration of Single-Loop Shield Water System................................. 205

6.3 The $\varepsilon$-NTU Method for Heat Exchanger Design ..................................... 207

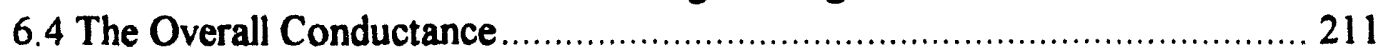

6.4.1 Heat Transfer Coefficient - Primary Side ................................ 213

6.4.2 Heat Transfer Coefficient - Secondary Side ……..................... 213

6.5 Heat Exchanger Pressure Drops ........................................................ 221

6.5.1 Pressure Drop - Primary Side ………...................................... 221

6.5.2 Pressure Drop - Secondary Side ……………….................... 222

6.6 Cooling Tower/Auxiliary Heat Exchanger Sizing for Single Loop

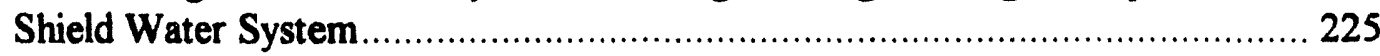

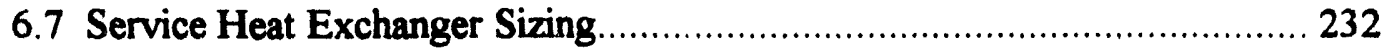

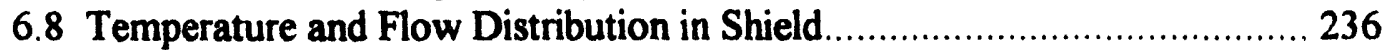

6.9 Shield Water System Pumping Power.................................................. 240

6.10 Activity in Single Loop Shield Water System Coolant ........................... 242

6.10.1 Long-Lived SWS Coolant Activity ..................................... 242

6.10.1.1 Corrosion Product Activity .................................... 244

6.10.1.2. Argon-41 Activity ............................................... 270

6.10.2 Short-Term N-16 Activity ................................................. 272

6.10.2.1 Model for N-16 Activity in the SWS ....................... 274

6.10.2.2 Biological Dose Rate from AHE ............................ 277

6.10.2.3 Shielding of SWS Piping ........................................ 281

6.11 The Two-Loop Shield Water System Option......................................... 284

6.11.1 Two Loop SWS Components Size and Cost .......................... 284

6.11.2 Cost Comparison between Single and Two Loop SWS ….......... 295

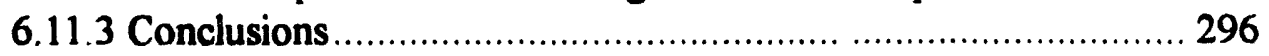

Chapter 7 Conclusions and Recommendations for Future Work ................................ 298

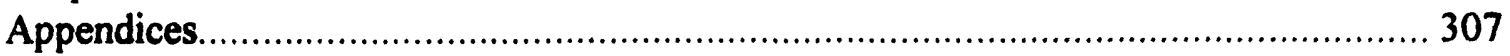

Appendix 1 Geometrical Correction Factor Details ….............................................. 307

Appendix 2 Sizing of Shell Coolant Channels ................................................... 309

Appendix 3 Determination of Bed Thicknesses for Nested Shell Blanket.................... 317

Appendix 4 Calculation of Tritium Inventory in Blankets....................................... 323

Appendix 5 Thermal Stresses in Slabs............................................................... 333

Appendix 6 Cooling Tower/Auxiliary Heat Exchanger Sizing for the Shield

Water System. 
Appendix 7 Shield Water System Pumping Power Calculations

Appendix 8 Cost Data for Shield Water System Components

Appendix 9 Composition and Properties of HT/MT-9 Steels

Appendix 10 MATHCAD Code for Calculating Pebble Bed

Conductivity.

Appendix 11 Effect of Shield Water System Design on a

Vanadium/Lithium/Lithium Blanket Design 


\section{Chapter 1 Introduction}

"The heave'ns declare the glory' of God:

and the firmament sheweth his handiwork." (Ps. 19, v. 1)

"Fusion power, the energy source of the sun and stars, may well be mankind's ultimate energy source." (from the cover jacket of The Man-Made Sun, by T. A. Heppenheimer)

The pursuit of practical, peaceful fusion energy production has involved a considerable amount of international scientific and engineering effort since the 1950's. By the early 1990 's, although ruuch progress has been made toward understanding how to confine and control a magnetically-confined fusion plasma, a practical fusion reactor is still probably decades in the future. Like fission reactors, fusion reactors will burn no fossil fuels, and hence will release no carbon dioxide into the atmosphere. However, fusion offers potential safety benefits as compared to fission. These potential safety benefits are a strong justification for continuing an aggressive fusion research program, despite the long-term nature of the effort.

Some of the safety advantages of fusion have been examined extensively in past studies. 1

2 These advantages include the following: (1) Fusion reactors cannot sustain runaway reactions, since the amount of fuel inside the reactor at any given time would only operate the reactor for a few seconds; (2) The radioactive waste from fusion reactors will decay much faster than fission reactor waste, will have lower initial levels of radioactivity and will be orders of magnitude less hazardous; (3) The worst-case accidents that could occur in a fusion reactor should represent a much lower potential

'J. P. Holdren, et.al., "Report of the Senior Committee on Environmental, Safety, and Economic Aspects of Magnetic Fusion Energy," Lawrence Livermore National Laboratory Report \#UCRL-53766 dated September 25, 1989.

${ }^{2}$ U.S. Congress, Office of Technology Assessment, Starpower: The U.S. and the International Quest for Fusion Energy, OTA-E-338 (Washington, DC: U.S. Government Printing Office, October 1987). 
hazard to the public than worst-case fission reactor accidents, chiefly because a fusion reactor will contain a lower radioactive inventory, and will have less stored energy which could be used to release radioactivity in the event of an accident; and (4) Fusion reactors should require simpler post-shutdown or emergency cooling systems than fission reactors, if such systems are needed at all.

Many fusion safety studies have focused on the worst-case accident scenarios for fusion reactors, in an effort to determine reactor designs which would be least likely to harm the public, even in the event of a very unlikely, very severe, accident. An example of such an accident is one in which the large-scale structure of the reactor plant is disturbed, perhaps as a result of an extremely severe earthquake, or deliberate sabotage. Such studies have led to the determination that use of silicon carbide ( $\mathrm{SiC}$ ), or vanadium alloy as structural materials, coupled with the use of helium or molten salt (FLiBe) coolants, would offer the greatest safety advantages, since these materials and coolants have low activation characteristics, and have low potential for exothermic reactions with air or water. The problem with these materials and coolants is that with the exception of helium, none have been used before in any large-scale engineering application, let alone a nuclear application. Hence, much development work is required before these options can be used.

The focus by the fusion safety community on the very improbable, worst-case accident, while laudable in its own right, has perhaps drawn attention away from the less severe, but more probable accidents which would not endanger the public, but could severely damage the fusion reactor itself. As the accident at Three Mile Island showed, an accident which results in an insignificant radioactive release to the public can have disastrous consequences to the plant itself. Fusion reactors have the potential to exhibit passive operational safety to relatively more probable accidents such as a loss-of-site- 
power. Passive operational safety is defined here as the ability to sustain an accident, without reliance on active safety systems, and without damage to the reactor. After such an accident, the reactor could be operated again normally.

As will be seen, the focus of the work presented herein will be on passive operational safety for more probable accidents. A blanket concept will be developed which exhibits passive operational safety for accidents such as a Loss of Site Power (LOSP) [also known as a Station Blackout], and which uses helium coolant and low activation ferritic steel structure. Because they are in common use in engineering and nuclear applications currently, helium and ferritic steel should be easier to develop for fusion applications than more exotic choices such as $\mathrm{SiC}$, vanadium alloy, and $\mathrm{FLiBe}$, and should involve less risk to the fusion reactor builder. Although low activation ferritic steel has higher activation levels than $\mathrm{SiC}$ or vanadium alloy, it has much lower long-term activity than a normal steel.

Many helium/steel blanket designs have been developed in the past (see Chapter 4). However, generally speaking these designs do not exhibit operational passive safety. The basic reason for this is that helium is a poor thermal conductor and has low heat capacity. Therefore, if the helium circulators fail (as they would during a LOSP), the helium becomes essentially useless for heat removal, and the afterheat from the steel overheats the blanket structure, causing structural failure. The result of the present work is a helium/steel blanket design that overcomes this major disadvantage.

The basic thesis layout is as follows. The first four thesis chapters provide relevant background information, including a description of the overall fusion reactor configuration, and the basic functions of the reactor blanket and shield (Chapter 2), a 
description of the neutronics, activation, and heat transfer codes used herein (Chapter 3), and a review of past fusion reactor blanket design concepts (Chapter 4)

The heart of the thesis is Chapter 5, which covers the description and analysis of two new blanket and shield conceptual designs which use helium coolant and a ferritic steel structure (Blanket Designs 1 and 2). Blanket Design 1 is a modification of a previously developed design, and is used as a starting point to establish $i$ e required overall blanket and shield dimensions. Analysis of Blanket Design 1, however, shows that it does not meet the goal of passive operational safety. Blanket Design 2 modifies Blanket Design 1 by incorporating a novel feature, "beryllium joints," to help it meet the operational passive safety goal.

The iong-term passive operational safety of Blanket Design 2 depends on having the shield act as a heat sink for the blanket. This is provided by the design of a Shield Water System (SWS) with natural circulation capability. Chapter 6 describes options for such a naturally circulating SWS. Chapter 6 also explores how a naturally circulating SWS could potentially benefit blanket designs other than the ones developed in this work. The thesis closes with a chapter providing overall conclusions, as well as recommendations for future work (Chapter 7). 


\section{Chapter 2 Overall Fusion Reactor Configuration}

Currently, the most advanced magnetic fusion confinement device is the tokamak.

Hence, most studies, including the present study, assume that the tokamak will be the reactor of choice for future fusion power reactors. This assumption is advantageous, since the tokamak configuration has been studied extensively, and hence the operational requirements and characteristics for a tokamak fusion power reactor are better known than for the less-advanced designs, such as the stellerator. The overall arrangement of a typical tokamak power reactor design is shown in Figure 2.1.

The hollow center of the torus is where the fusion plasma is confined. The plasma is surrounded by the blanket, which in turn is surrounded by the shield. Both the blanket and shield are segmented to allow for easier installation and replacement. In the STARFIRE reactor design, the blanket and shield are both cooled by water; hence the water coolant inlet and outlet pipes indicated in the figure. Outside the shield are the toroidal field (TF) and equilibrium field (EF) superconducting magnets, which generate the proper magnetic field configuration to confine the fusion plasma. 


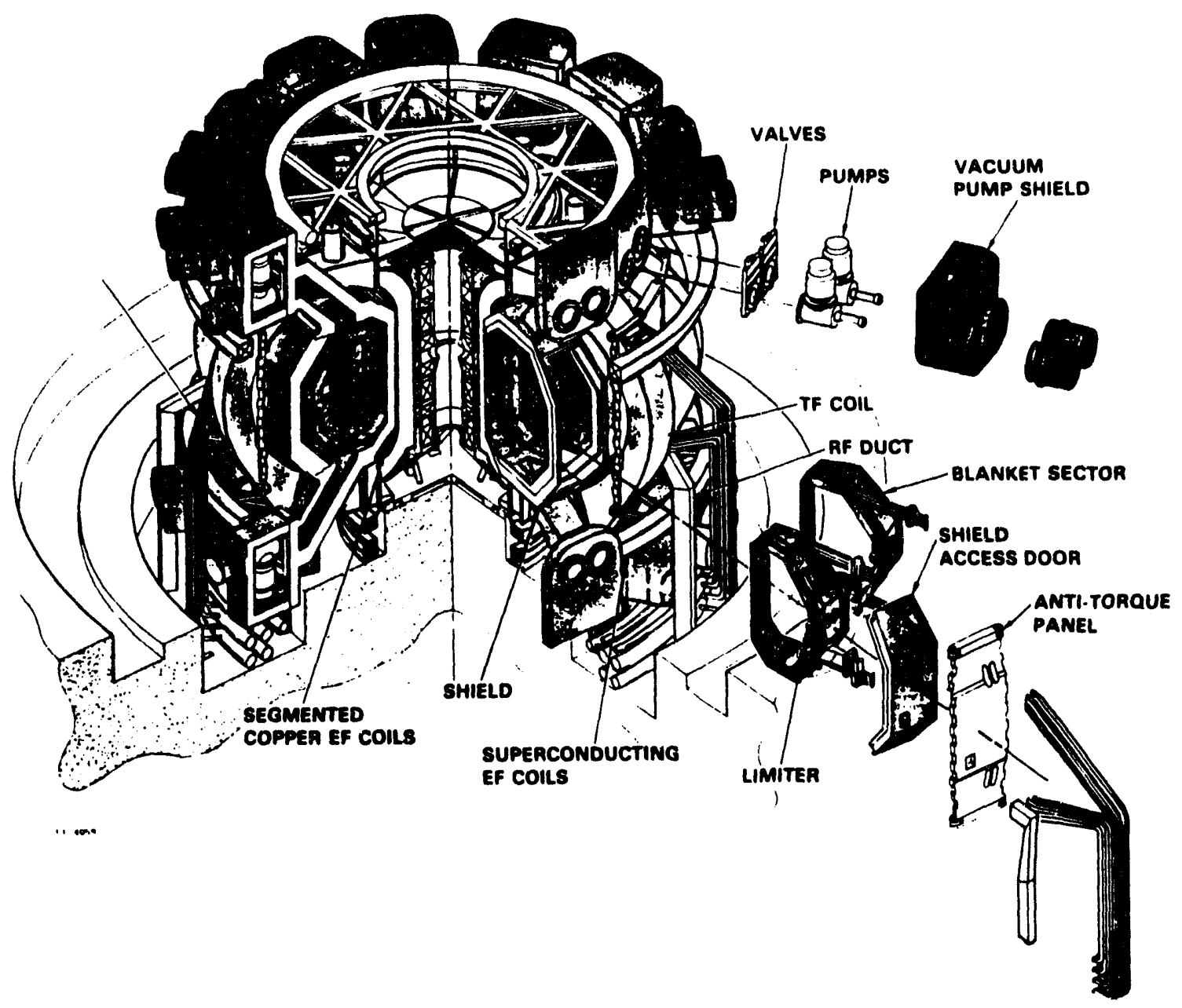

Near-term fusion power reactors will probably fuse deuterium and tritium to generate power, simply because this reaction has the largest fusion cross-section of the available reaction types, and has been the most studied. This reaction is shown schematically as follows:

$$
{ }_{1}^{2} \mathrm{D}+{ }_{1}^{3} \mathrm{~T} \Rightarrow{ }_{2}^{4} \mathrm{He}(3.52 \mathrm{MeV})+{ }_{0}^{1} \mathrm{n}(14.1 \mathrm{MeV}) \text {. }
$$

The products of the D-T fusion reaction are an alpha particle of $3.52 \mathrm{MeV}$ energy, and a neutron of $14.1 \mathrm{MeV}$ energy. Two important issues related to this reaction are, firstly,

${ }^{3}$ C. C. Baker, et.al., "STARFIRE - A Commercial Tokamak Fusion Power Plant Study," Argonne National Laboratory Report \#ANL/FPP-80-1, 1980, Volume 1, Chapter 2, Figure 2.2. 
that tritium is not available naturally, and secondly, that the neutron carries most of the fusion reaction's energy.

The two main purposes of the blanket portion of a fusion reactor are: (1) To convert the kinetic energy of the neutrons resulting from the D-T reaction into thermal energy which can be used to boil water for the reactor's steam plant; ${ }^{*}$ and (2) To manufacture tritium for use in the D-T reaction. Tritium is "bred" in a fusion blanket by the fusion neutrons reacting with lithium in the blanket, forming tritium. The main purpose of the shield is to further attenuate the neutron flux coming from the plasma in order to protect the superconducting magnets from radiation damage and from an excessive neutron heat load.

\footnotetext{
\# Note that since the neutrons are uncharged, they are not confined in the tokamak's magnetic field with the main fusion plasma. Hence, all neutrons generated in the D-T reaction immediately travel to, impact on and penetrate into the blanket, where most of their kinetic energy is converted to thermal energy as they slow down.
} 


\section{Chapter 3 Neutronics, Activation and Heat Transfer Codes}

The most important characteristic of any fusion blanket and shield design is its response to irradiation by the $14 \mathrm{MeV}$ fusion neutrons. In order to calculate this response, a number of different codes must be utilized. These codes, used in conjunction with each other, will provide such information as the neutron flux as function of position within the blanket, the operational and post-shutdown volumetric heating of different blanket regions, and the tritium breeding ratio of the blanket. Chapter 3 provides a brief description of the codes used for the present analysis.

\subsection{The TWODANT Neutronics Code}

The starting point for the determination of the blanket response to neutron irradiation is, of course, the neution flux as a function of position during reactor operation. The neutron fluence impacting on the plasma-facing wall of the blanket, the "neutron wall load," varies with position; this issue in discussed further in Section 6.1. Once the neutron wall load (energy, direction and current) for a particular position is known, determining the position-dependent neutron flux within the blanket is a standard reactor physics problem which is discussed in a number of elementary textbooks, for example, see Henry. ${ }^{1}$ It basically involves solving the time-independent inhomogeneous Boltzmann transport equation. This equation, in one spatial dimension, can be written as follows:

\footnotetext{
'A. Henry, Nuclear-Reactor Analysis, The MIT Press, Cambridge, MA, 1982.
} 


$$
\begin{aligned}
& \nabla \cdot \underline{\Omega} \psi(r, E \underline{\Omega})+\sigma(r, E) \psi(r, E, \underline{\Omega}) \\
& \quad=\iint d E^{\prime} d \Omega^{\prime} \sigma_{s}\left(r, E^{\prime} \rightarrow E, \underline{\Omega} \underline{\Omega}\right) \psi\left(r, E^{\prime}, \underline{\Omega}\right) \\
& +\frac{1}{4 \pi} \iint d E^{\prime} d \Omega \Omega^{\prime} \chi\left(r, E^{\prime} \rightarrow E\right) \vee \sigma_{i}\left(r, E^{\prime}\right) \psi\left(r, E^{\prime} \cdot \underline{\Omega}\right) \\
& +S(r, E, \underline{\Omega})
\end{aligned}
$$

where $\psi(r, E, \underline{\Omega})$ is the neutron flux defined such that $\psi(r, E, \underline{\Omega}) d E d l^{\prime} d \Omega$ is the flux in the energy range $d E$ about $E$, in the volume element $d V$ about $r$, with a direction of motion in the solid angle element $d \Omega$ about $\underline{\Omega} . S(r, E, \underline{\Omega})$ is the source rate of neutrons, and is similarly defined. $\sigma$ is the total macroscopic cross section, and $\sigma_{s}$ is the macroscopic probability of scattering from energy $E^{\prime}$ to $E$ through an angle $\underline{\Omega} \cdot \underline{\Omega^{\prime}}$, and the macroscopic fission cross section is $\sigma_{f}$. The fission term is used for reactions such as $(n, 2 n)$. The number of particles emitted isotopically $(1 / 4 \pi)$ per fission is $v$, and the fraction of these particles appearing in energy $d E^{\prime}$ about $E^{\prime}$ is $\chi\left(r, E^{\prime} \rightarrow E\right)$.

The Boltzmann equation above is applicable to photons as well as neutrons, provided the appropriate photon cross-sections are incorporated. Since neutron interactions with matter produce a substantial amount of gamma radiation, some of which will in turn generate additional neutrons, TWODANT as well as most other neutronics codes are capable of treating neutrons and gammas as a coupled set.

A number of neutronics codes exist which solve the Boltzmann equation, such as ANISN $^{2}$, TRISM $^{3}$, ONEDANT $^{4}$, and TWODANT ${ }^{3}$. Previous fusion reactor studies at

\footnotetext{
${ }^{2}$ W. Engle, Jr., "A User's Manual for ANISN: A One-Dimensional Discrete Ordinates Transport Code with Anisotropic Scattering," Oak Ridge Gaseous Diffusion Plant Computing Technology Center report K-1693, 1967.

${ }^{3}$ J. Davidson, et.al., "TRISM, A Two-Dimensional Finite-Element Discrete-Ordinates Transport Code," Los Alamos National Laboratory draft report, 1986.
} 
the MIT Plasma Fusion Center have used the ONEDANT code, which is a onedimensional discrete-ordinates neutron transport $\operatorname{code}^{6}{ }^{7}$. The TWODANT code is similar to ONEDANT, but allows 2-dimensional calculations to be performed TWODANT was used for the present study.

To solve the Boltzmann equation, TWODANT represents the macroscopic scattering cross-section $\sigma_{s}$ as a finite Legendre polynomial expansion. The order of this expansion is specified by the user, but the cross-section file to be used by TWODANT must have cross-section data to the required order (see the subsection below on TRANSX). The order of the Legendre expansion used is typically specified as $P_{0}$ for a zeroth-order expansion, $\mathrm{P}_{1}$ for a first-order expansion, etc. The order used for the present calculations is $P_{3}$.

The energy and solid angle variables are also discretized by TWODANT. The number of neutron energy groups and gamma energy groups can be specified by the user, again subject to the constraint that the cross-section library must have appropriate crosssections. For the present work, 30 neutron energy groups and 12 gamma groups were used. The order of the discretization of the solid angle variable is typically specified in the form $\mathrm{S}_{\mathrm{N}}$, where $\mathrm{N}$ is the number of quadrature directions is plane geometry. Order $S_{12}$ was used for the present work.

${ }^{4} R$. O'Dell, F. Brinkley, Jr., and D. Marr, "User's Manual for ONEDANT: A Code Package for One Dimensional, Diffusion-Accelerated, Neutral-Particle Transport, "Los Alamos National Laboratory report LA-9184-M, February, 1982.

${ }^{5}$ R. Alcouffe, F. Brinkley, Jr., D. Mart and R. O'Dell. "User's Guide for TWODANT: A Code Package for Two-Dimensional Diffusion-Accelerated. Neutral-Particle Transport," Los Alamos National Laboratory report LA-10049-M, Revision 1, 1984.

6J. Massidda and M. Kazimi, "Thermal Design Considerations for Passive Safety of Fusion Reactors", MIT Plasma Fusion Center Report PFC/RR-87-18, October 1987.

${ }^{7} \mathrm{M}$. Koch, "A Comparison of Radioactive Waste from First Generation Fusion Reactors and Fast Fission Reactors with Actinide Recycling," MIT Plasma Fusion Center report PFC/RR-91-9. April 1991. 
In principle it is simple to run TWODANT. The user supplies the appropriate geometry for the problem, which includes the material compositions of the various physical regions, together with the region boundaries. The code must also have an appropriate cross-section data file, which it combines with the geometrical information to calculate the appropriate position-dependent macroscopic cross-sections in the Boltzmann equation. Finally, the user supplies the neutron source term (which, for a fusion blanket, is the neutron flux from the plasma). Specifying the geometry, cross-section data, and source flux is sufficient to solve the Boltzmann equation, and is all TWODANT needs to provide the neutron flux distribution as a function of position within the blanket.

The geometry and source term data are easy for the user to generate and supply to TWODANT. The cross-section information, however, requires a bit more work. This is because standard cross-section data files are not in a format which is most suitable for use in a neutron transport code such as TWODANT. Additionally, TWODANT does not automatically provide such information as tritium breeding; in order to get such output, an appropriate cross-section must be generated for tritium breeding, and added to the cross-section file to be used by TWODANT. Fortunately, there is another code, called TRANSX, ${ }^{8}$ the purpose of which is to convert standard cross-section files into the format used by TWODANT and other neutronics codes. TRANSX also makes it possible to generate information such as tritium breeding during a TWODANT run. The following subsection briefly describes the TRANSX code.

${ }^{8}$ R. E. MacFarlane, "TRANSX 2: A Code for Interfacing MATXS Cross-Section Libraries to Nuclear Transport Codes," Los Alamos National Laboratory, May 20, 1992. 


\subsubsection{The TRANSX Interfacing Code}

TRANSX was developed as an interface between the neutronics codes such as TWODANT and the standard cross-section libraries. Cross-section libraries generally are not in a format which is suitable for direct use in a neutronics code. This is because the data in the cross-section library is typically ordered by material, and the neutronics codes work much more efficiently if the data is ordered by energy group. The crosssection libraries are obviously much easier to maintain if they are "material-ordered". The neutronics codes, however, generally solve the Boltzmann equation by sweeping down from energy group 1 (the highest energy group) to the lower energy groups. Hence, "group-ordering" of the cross-section data results in much faster code operation. The main purpose of TRANSX is to provide this conversion of the cross-section library to group-ordered form. TRANSX also allows the user to specify desired quantities such as the Legendre order of the group-ordered cross-section table. In this fashion, the group-ordered cross-section table is tailored to fit the code and the particular problem of interest.

Another purpose of TRANSX is to generate appropriate cross-sections for calculations of tritium breeding and other so-called "response-function edits". These response edit cross-sections are then placed in the group-ordered cross-section library for use by the TWODANT code.

\subsubsection{MATXS Cross-Section Libraries}

The MATXS format is a standard for material-ordered cross-libraries. ${ }^{9}$ One of the nice features of this format is that all information is identified using lists of Hollerith names, and usually the names are relatively self-explanatory. For example, entries NF and NG in

${ }^{9}$ For more information of the MATXS file structure, see Chapter IV of R. E. MacFarlane, "TRANSX 2: A Code for Interfacing MATXS Cross-Section Libraries to Nuclear Transport Codes," Los Alamos National Laboratory, May 20, 1992. 
the MATXS format correspond to the fission and $(n, \gamma)$ cross-sections. A variety of MATXS libraries exist, some tailored for fusion applications, some more useful for fastreactors. There are also MATXS libraries suitable for light-water reactor and shielding problems. The MATXS5 library was used for the present work. This library, which is appropriate for fusion applications, has 30 neutron energy groups and 12 gamma groups (42 total energy groups) and contains data for 100 materials. The Legendre order for scattering is $P_{4}$ (TRANSX is used to convert the MATXS5 library into a $P_{3}$ groupordered library called XSLIB for use in the present work).

\subsubsection{Neutronics Model Geometry}

A fusion reactor blanket has a complex shape, which makes it difficult to model accurately using anything less than a fully 3-dimensional geometry. To use the TWODANT neutronics code, however, it is necessary to approximate the true 3dimensional toroidal geometry using only two dimensions.

Of all the possible methods for approximating a toroidal shape in 2-dimensions, the straight cylinder approximation is perhaps the most commonly used. In this method, the tokamak major radius is allowed to increase to infinity. This creates a 2-dimensional configuration which has the advantage of preserving the poloidal geometry. Since parameters such as the neutron wall loading and blanket thickness depend strongly on poloidal angle, the straight cylinder approximation is appropriate for use in a blanket model. Two basic errors are introduced by the cylinder model. The first is an error in neutron fluxes within the blanket, and the second is an error in neutron wall load at the blanket first wall. Both types of error depend on the aspect ratio (a/R) of the tokamak, with a high aspect ratio resulting in a greater error. 
The general nature of the flux error within the blanket can be analyzed as follows Because of curvature around the central toroidal "hole", the neutron flux impacting on the inner portion of the blanket (that portion surrounding the ohmic transformer) will tend to be concentrated slightly by geometry as the neutrons penetrate toward the center of the tokamak. Thus, the neutron fluxes calculated by the TWODANT code using the cylinder model will be less than those actually seen by the inner portion of the blanket. Conversely, the calculated neutron flux impacting on the outer portion of the blanket will actually be diluted by geometry as the neutrons travei outward.

\section{Figure 3.1. Top View of Torus}

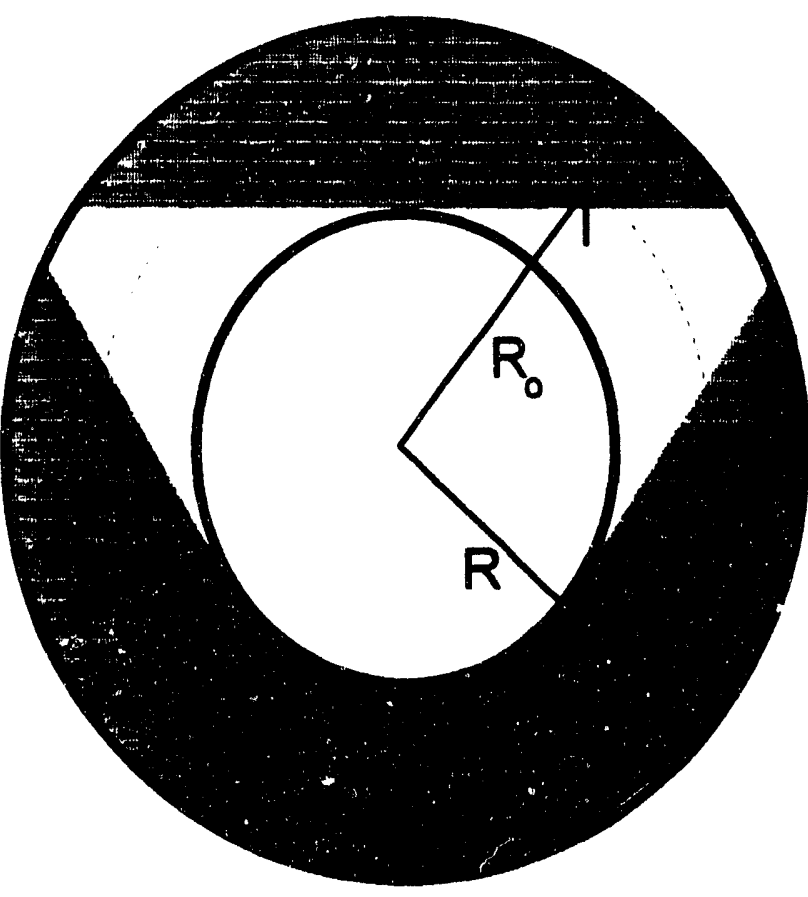

The magnitude of the error in neutron flux caused by this effect is easy to calculate using simple geometric arguments, and is equal to $R_{0} / R$, where $R_{0}$ is the major radius of the torus, and $\mathrm{R}$ is the horizontal distance from the vertical centerline of the torus (see Figure 3.1.)

The neutron wall load error can be described as follows. A uniform plasma confined in a toroidal geometry will not generate a

uniform neutron wall load. A point on the outer portion of the torus wall (point $A$ in Figure 3.1) has a line-of-sight to a greater volume of plasma than does a point on the inner portion of the torus wall (point B). This is illustrated in Figure 3.1 using the shaded areas around points A and B. "Seeing" a larger volume of plasma means receiving a higher neutron flux. Thus, the neutron wall load is higher for the outer 
portion of the blanket than for the inner portion. When the torus is approximated by a cylinder, this asymmetry vanishes, introducing an error in the neutron wall load. In addition, the plasma in a tokamak is generally not uniform, and can have large variations in density and temperature as one moves from one poloidal position to another. The non-uniformity of the plasma can result in large variations of neutron wall load depending on the poloidal angle. Figure 3.2 shows a typical example of neutron wall load as a function of poloidal angle for a tokamak reactor. Generally, one obtains neutron wall load profiles such as this from detailed modeling of the plasma properties as a function of position. Note that for this conceptual design study, no attempt is made to obtain the proper velocity distribution for the neutron s impacting on the wall - the neutrons are assumed to be isotropic in velocity space.

\section{Figure 3.2. Typical Neutron Wall Load Variation ${ }^{10}$}

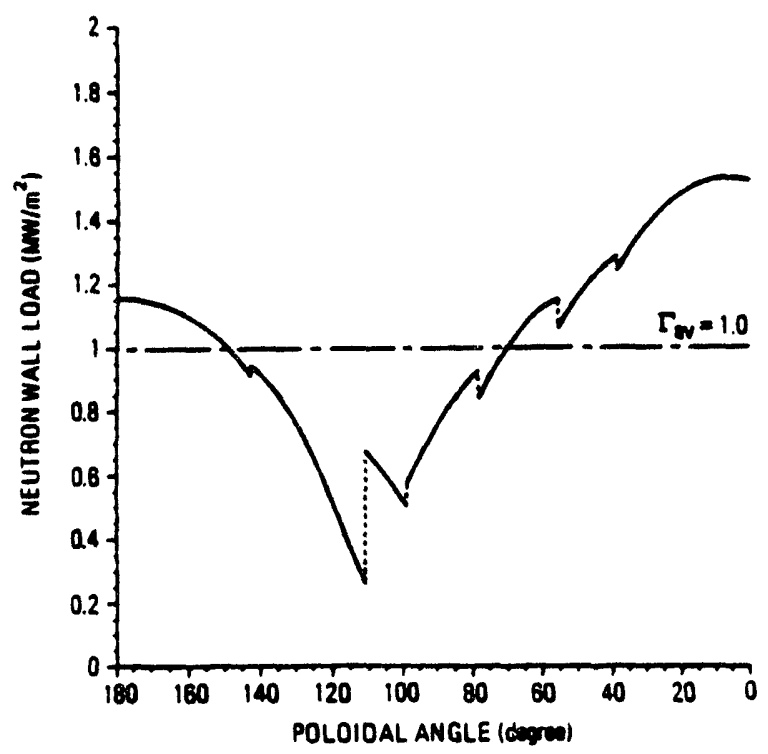

described in more detail in Section 5.1.
To account for both the

$1 / R$ geometry effect, and the poloidal variation in neutron wall load, the following procedures are used. First, the nonuniform wall loading is averaged, prior to being used as an input to the TWODANT code. This averaging procedure is

\footnotetext{
${ }^{10}$ from ITER Documentation Series. No. 3, IAEA, Vienna. 1989, p. 308.
} 
Second, the TWODANT neutron wall load input is modified to account for the geometry factor. For example, let us assume that a tokamak with a major radius $\mathrm{R}_{\mathrm{v}}=6 \mathrm{~m}$ has a neutron wall load of $1 \mathrm{MW} / \mathrm{m}^{2}$ at a position along the first wall corresponding to a distance $\mathrm{R}=7 \mathrm{~m}$ from the torus vertical centerline (a position in the outboard blanket wall). The neutron wall load at this position to be used in TWODANT should then be $R / R_{0}=7 / 6=1.17 \mathrm{MW} / \mathrm{m}^{2}$. Note that the wall load used for outboard blanket section positions is greater than the actual wall load. This is because the straight cylinder approximation concentrates the volume elements in the outboard section.

Finally, the TWODANT neutron flux output data is corrected using the inverse of the geometry factor used for the wall load, that is, $R_{0} / R$. See Appendix 1 for further details on how the $1 / R$ geometry factor was accounted for calculationally. It should be noted that tritium breeding ratio information, which is also provided by TWODANT, need not be corrected for in this fashion. This is because the tritium breeding ratio depends on the total number of neutrons entering the blanket, not on the neutron flux. In other words, any change in tritium breeding density in the blanket due to transformation from two to three dimensions is exactly offset by the change in the corresponding volume element.

A fusion blanket and shield is usually designed with an inboard section and an outboard section. The inboard section is designed to be as thin as possible, consonant with tritium breeding and shielding requirements, since the thickness of the inboard section has a direct impact on the overall size of the machine. The outboard section does not control the size of the machine as much, and hence is usually designed to be thicker than the inboard section. Figure 3.3 shows the vertical centerline of the tokamak, including a typical configuration of a reactor blanket/shield. 


\section{Figure 3.3. Typical Blanket/Shield Configuration}

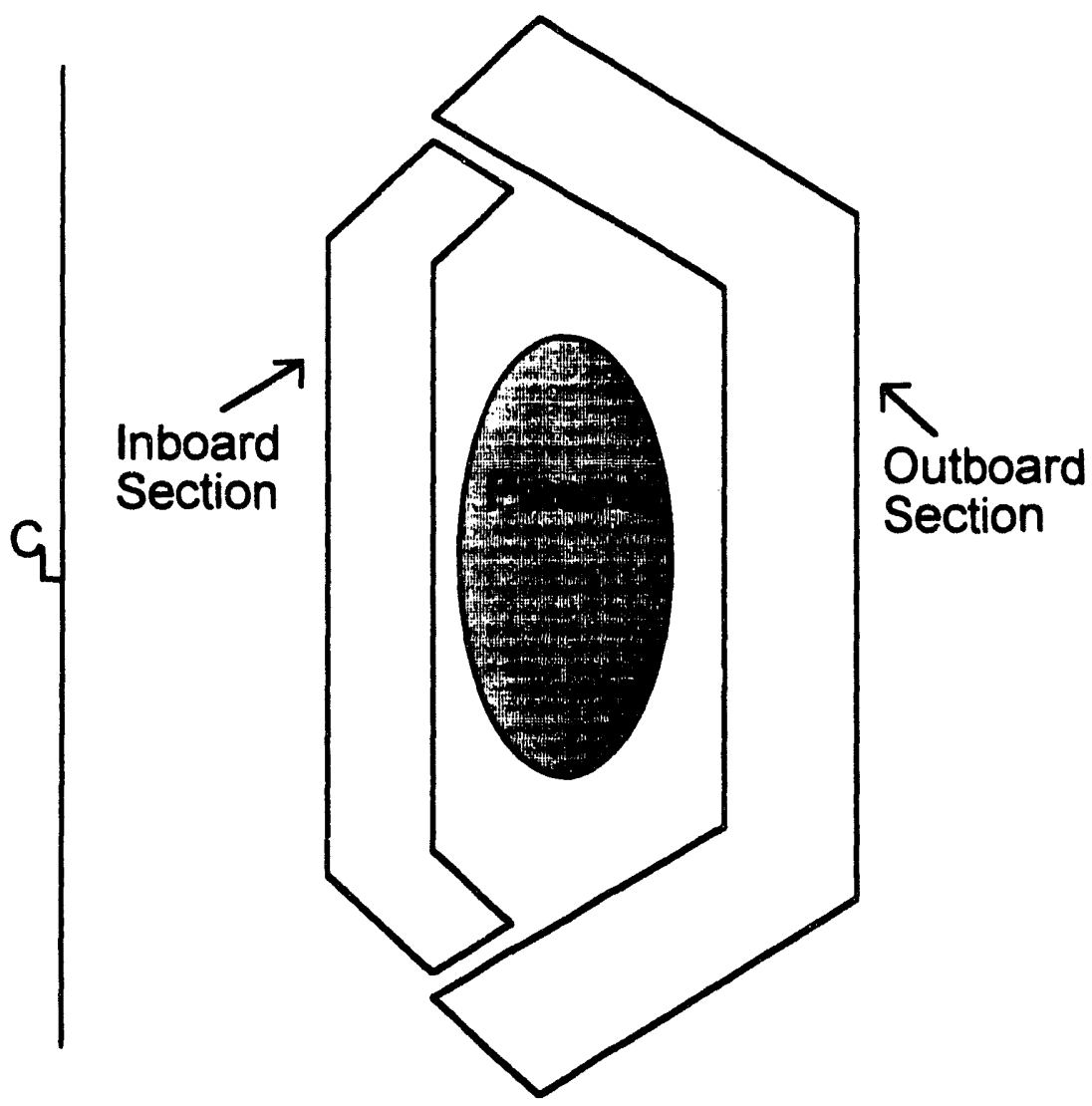

To model the blanket configuration shown above using TWODANT, we make a vertical slice through the center of the plasma, separating the problem into two parts, one part corresponding roughly to the inboard blanket/shield section, and the other part corresponding to the remaining outboard section (see Figure 3.4.) 


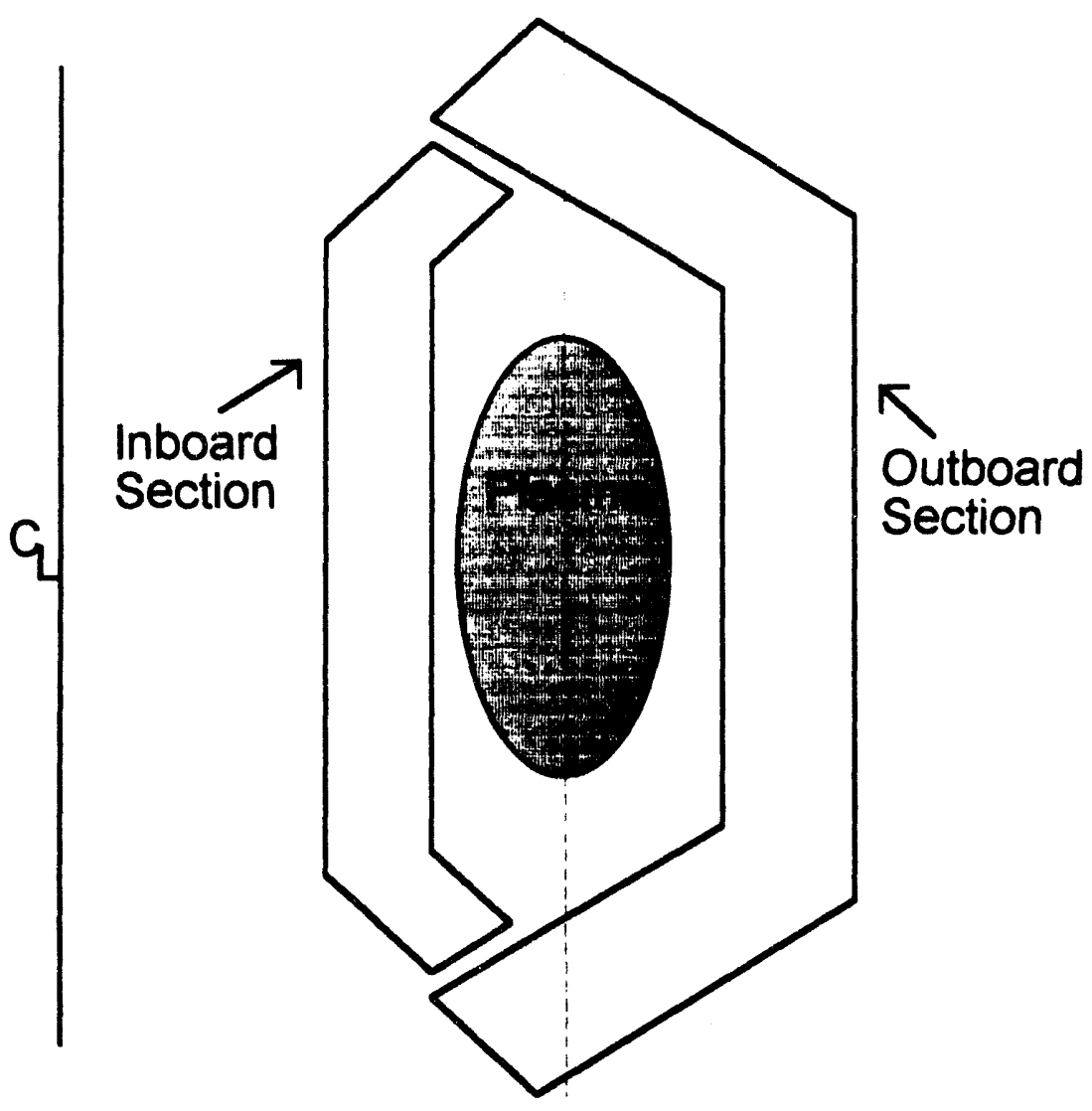

For simplicity, the outlines of the blanket/shield sections are approximated as semicircles, and the plasma is modeled as an isotropic semi-circular source, normalized to the appropriate wall loading. Figure 3.5 shows the simplified model used as TWODANT input.

It should be noted that the inboard and outboard sections of this model are run separately, not together. That is, the TWODANT model of the inboard section does not see the outboard section at all, there is simply a vacuum there. Similarly, the model of the outboard section does not see the inboard section. The significance of this is that any 
neutrons which are reflected back after hitting the outboard or inboard sections of the model cannot be reabsorbed into the other section, since it is not present in the model. Since in a real machine the reflected neutrons would in fact be able to be absorbed into Figure 3.5. Inboard and Outboard TWODANT Models

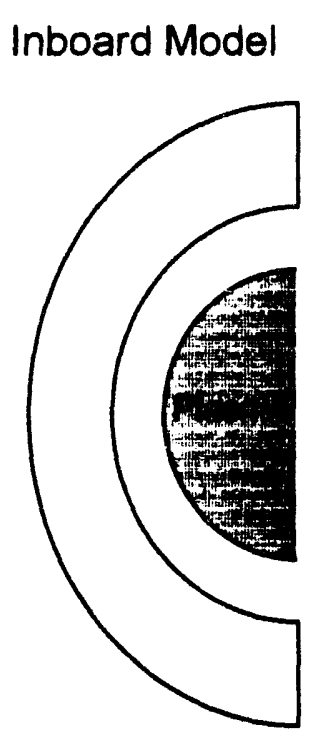

Outboard Model

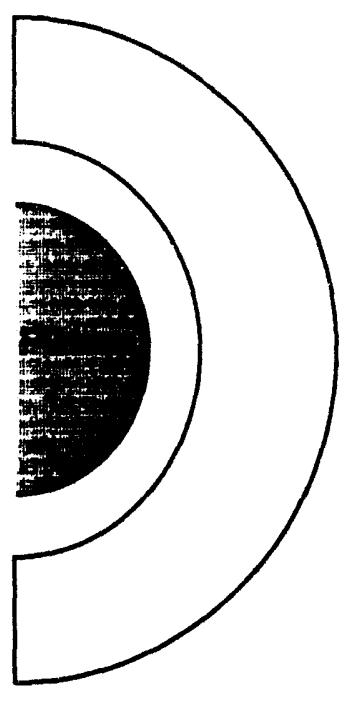

the other blanket/shield section (if they did not escape though a vacuum port or into the divertor), modeling the inboard and outboard sections separately is conservative with respect to tritium breeding considerations. However, the type of blankets analyzed in this work (helium-cooled, solid breeder blankets) do not have a significant amount of neutron reflection. TWODANT was used to calculate tritium breeding and volumetric heating rates for the case where the b'anket sections are modeled separately, and the case where they are modeled together. Because of the low amount of reflection, the heating and tritium breeding values were less than $1 \%$ larger for the case where both sections were modeled together. 
Based on recent comparisons between one-dimensional and three-dimensional neutronics calculations, modeling the plasma as isotropic is probably somewhat unconservative for tritium breeding, but yields conservative values for the neutron flux near the first wall. This means that afterheat values near the first wall, which are critical to the thermohydraulics calculations central to the present work, will also be conservative ${ }^{11}$ The lack of conservatism in the tritium breeding ratio can be accounted for by increasing the minimum tritium breeding ratio required for the blanket design.

\subsection{The REAC Activation Code}

The previous subsections described the TWODANT code and its supporting code TRANSX. The primary output of TWODANT is the neutron flux as a function of position in the blanket. In order to calculate volumetric heating rates during reactor operation and after shutdown, it is necessary to calculate how the neutron flux activates isotopes in the blanket. This is the purpose of the REAC code. ${ }^{12}$ REAC is a code which calculates the change in composition of materials in a neutron radiation field and also the activation of the irradiated materials. The transmutation of a set of nuclides exposed to a neutron flux can be expressed with the following equation:

$$
\begin{aligned}
\frac{d N_{i}(t)}{d t}= & \Phi(t) \cdot \sum_{j} N_{j}(t) \sigma_{j \rightarrow i}+\sum_{k} \lambda_{k \rightarrow 1} N_{k}(t) \\
& -\Phi(t) N_{i}(t) \cdot \sum_{l} \sigma_{t \rightarrow i}-N_{i}(t) \sum_{m} \lambda_{t \rightarrow m}
\end{aligned}
$$

where $N_{i}(t)$ is the number density of nuclide i at time $\mathrm{t}, \Phi(t)$ is the total neutron flux, $\sigma_{J \rightarrow 1}$ is the spectrum averaged cross-section for changing nuclide $\mathrm{j}$ into nuclide $\mathrm{i}$, and

\footnotetext{
${ }^{11} \mathrm{U}$. Fischer. "Qualification of Neutronic Blanket and Shielding Calculations in a One-Dimensional Approach to a Tokamak Reactor," Fusion Technology, Vol. 22, September 1992, p. 251.

${ }^{12}$ F. M. Mann. "REAC*2: Users Manual and Code Description." Westinghouse Hanford Company Report \#WHC-EP-0282. dated December 1989.
} 
$\lambda_{k \rightarrow,}$ is the decay constant for nuclide $\mathrm{k}$ changing into nuclide I. REAC3 is the code version used for this work

To use REAC3, the user must supply the appropriate material composition and neutron flux for the problem in question. Unlike TWODANT, which gives position-dependent output, REAC3 is setup to provide composition and activation results on a point by point basis. Therefore, if the decay heat rate for the entire blanket is desired, a number of different REAC3 runs must be performed to obtain the decay heat rate at different points on the blanket. Then, some sort of interpolation routine must be used to estimate the decay heat rate between these known points. This interpolation routine is described in Section 6.2.3.2., Afterheat Calculations.

REAC3 contains its own cross-section and decay data, which are used together with the user's material composition input to obtain the $\sigma$ 's and $\lambda$ 's of Equation 3.1. The crosssection library holds over 6,000 reactions covering over 325 isotopes, and uses a 63 energy group structure. The decay data library contains data for over 1,250 nuclides, which are the nuclides which can be reached via any of the reactions in the cross-section library.

The 63 energy group structure of REAC3's cross-section library is unfortunately incompatible with the 42 energy group structure of the TWODANT neutronics code. Thus, in order to use the neutron flux output file from TWODANT, this file must be converted from a 42 group structure to a 63 group structure. A code called FLXWRT was developed by Massidda ${ }^{13}$ to perform this conversion for ONEDANT flux files. This

13J. Massidda and M. Kazimi, "Thermat Design Considerations for Passive Safety of Fusion Reactors", MIT Plasma Fusion Center Report PFC/RR-87-18, October 1987. 
code was modified for the present work to perform the conversion on TWODANT flux files

\subsection{The HEATING Heat Transfer Code}

Once the decay heat, or afterheat, as a function of position in the blanket is known, the temperatures as a function of time and position within the blanket are determined using the HEATING heat transfer code. There are numerous versions of this code presently available. For this work, a modified version of HEATING3 is used ${ }^{14}$ Originally developed at Oak Ridge National Laboratory for use on an IBM 360 computer, some minor format modifications were made to the code by T. E. Hechanova and myself to allow it to run on an IBM PC as well as on the CRAY-2 supercomputers at the National Energy Research Supercomputer Center at Lawrence Livermore National Laboratory.

Once the geometry, volumetric heat generation rates, and boundary conditions are known, the HEATING code is capable of solving the 3-dimensional heat conduction equation, namely:

$$
\rho c_{p} \frac{\partial \mathrm{T}}{\partial t}=\nabla \cdot \mathrm{k} \nabla \mathrm{T}+\mathrm{q}^{\prime \prime \prime},
$$

where $\rho$ is the material density, $c_{p}$ is the specific heat, $\mathrm{k}$ is the thermal conductivity, and q"' is volumetric heat generation. HEATING is also capable of solving 1- or 2-

dimensional problems. For the present work, a 1-dimensional model of the blanket is used (see Section 5.2.3.3). HEATING accounts for temperature-dependent material properties, position- and time-dependent heat deposition rates, and a variety of boundary condition types, including surface-to-surface radiation., and constant heat flux boundary conditions.

\footnotetext{
${ }^{14}$ W. D. Turner M. Siman-Tov, "HEATING3 - An IBM 360 Heat Conduction Program," Oak Ridge National Laboratory Report \#ORNL-TM-3208, February, 1971.
} 


$$
\text { - }
$$




\section{Chapter 4 Past Blanket Design Concepts}

Over the last decade or so, there have been a number of different helium-cooled fusion blanket design concepts proposed. Virtually all of these concepts use a solid breeder material, and some use beryllium as a neutron multiplier. Although these past designs have obtained adequate tritium breeding and exhibited temperatures within relevant operational limits for normal operation, they have not generally been designed to withstand an accident such as a LOCA or LOFA in a passive manner. However, some configurations seem to have more potential to survive such an accident than others. Chapter 4 briefly reviews the basic helium-cooled blanket configurations which have been developed, and discusses characteristics which would impact the blanket's ability to withstand a LOCA or LOFA. This chapter also discusses candidate tritium breeding and neutron multiplier materials applicable to helium-cooled blankets.

\subsection{Pressurized Module Designs}

One of the earliest designs uses pressurized modules or canisters ,hich wrap poloidally around the inside of the reactor.' These modules are roughly one-third of a meter in the toroidal direction and half a meter long radially and contain the breeder, which is mounted inside the modules in the form of plates. The helium flows first through a thin channel along the first wall of the module to cool it, then into the breeder region. See Figure 4.1.

In order to ensure flow distribution to the regions between breeder plates, a large gap is needed between the first wall and the breeder plates. This gap creates a significant thermal barrier to heat transfer from the hot first wall to the rest of the blanket and makes

\footnotetext{
$\mathrm{I}_{\mathrm{M}}$. Huggenberger and K. Schultz, "Helium-Cooled Solid Breeder Blanket Design for a Tokamak Fusion Reactor," Nuclear Technology/Fusion Vol. 4, November 1983, p. 456.
} 
Figure 4.1. Pressurized Module Design

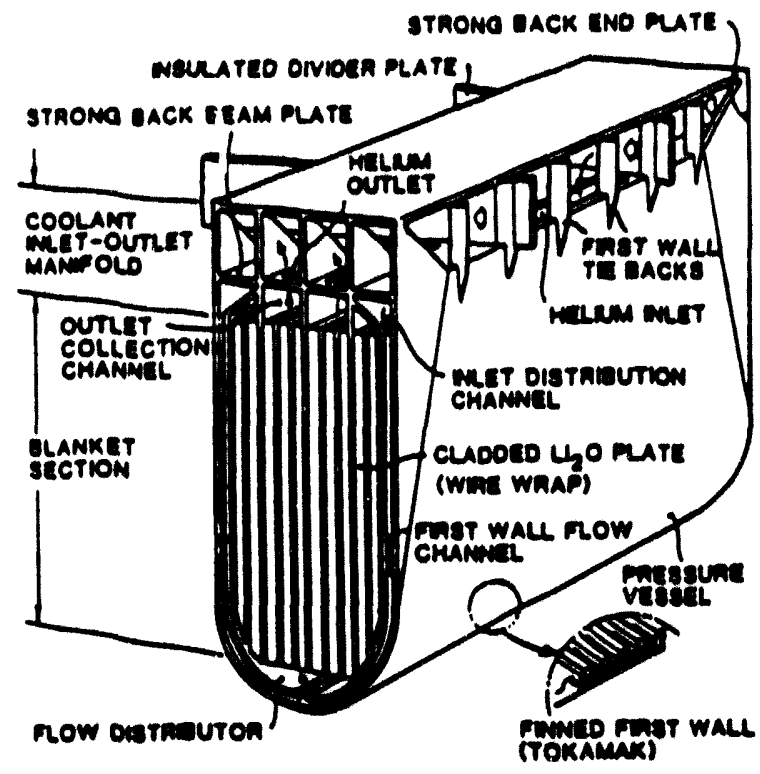

sustaining a LOCA or LOFA difficult. This design has in fact been analyzed for these casualties, and unacceptably high temperatures were shown to occur at the first wall. ${ }^{2}$

More recently, various other canister-type designs have been proposed, 456789 and although they differ from the original concept in detail, all have the characteristic of

2J. E. Massidda and M. S. Kazimi, "Thermal Design Considerations for Passive Safety of Fusion Reactors," DOE/D-01570-3, PFC/RR-87-18, October 1987.

${ }^{3}$ C.P.C. Wong, et. al., "Helium-Cooled Blanket Designs," Fusion Technology, Vol. 8, 1985, p. 114.

${ }^{4}$ L. Anzidei, et. al., "Il Mantello - A Helium Cooled Solid Breeder Blanket for NET," Proc. 14th Symp. on Fusion Technology, Avignon, 1986, p. 1299.

'E. Proust, et. al., "Progress in Fusion Reactors Blanket Analysis and Evaluation at CEA," Proc. 14th Symp. on Fusion Technology, Avignon, 1986, p. 1261.

${ }^{6} \mathrm{M}$. Abdou, et. al., "A Helium-Cooled Solid Breeder Concept for the Tritium-Producing Blanket of the International Thermonuclear Experimental Reactor," Fusion Technologv. Vol. 15, March 1989, p. 166.

${ }^{7}$ C. Baker, "U. S. ITER Shield and Blanket Design Activities," Fusion Technology, Vol. 15, March 1989, p. 849. 
relatively poor thermal conductivity from the first wall to the rest of the blanket. Although a simplified analysis of the ITER pressurized module design has been performed which indicated that structural integrity may be preserved during a LOCA, this analysis was performed using a neutron wall loading of just over $1 \mathrm{MW} / \mathrm{m}^{2}$, which is relevant for ITEK but much too low for an economical power reactor. This analysis also assumed a minimal flow of helium purge gas during the accident, which would require an active safety system. ${ }^{10}$

\subsection{Pressurized Tube Designs}

Another common design configuration consists of an array of tubes carrying pressurized helium which is embedded in the breeder/multiplier matrix. 111213 In these designs, the helium tubes are arranged more densely nearer the first wall to account for the higher heat load there (see Figure 4.2.) The LOCA/LOFA performance of such designs depends critically on the composition and configuration of the matrix. If the matrix is a good conductor of heat, this should help cool the first wall during an accident by allowing heat to flow from the first wall into the cooler interior regions of the blanket. Since the matrix is bound to have a much higher thermal conductivity than helium, the pressurized tube designs seem to offer more potential than the module designs for surviving such an

8. Raffray, et. al., "Helium-Cooled Solid Breeder Blanket for ITER," Fusion Technology, Vol. 15, March 1989, p. 858.

${ }^{9}$ M. Chazalon, et. al., "Next European Torus In-Vessel Components," Fusion Technology, Vol. 14, March 1988, p. 82.

${ }^{10} \mathrm{Z}$. Gorbis, et. al., "LOCA Study for a Helium-Cooled Solid Breeder Design for ITER." Fusion Technology, Vol. 15, March 1989, p. 821.

"M. Ferrari and G. Simbolotti, "Thermal and Stress Analysis of the Solid B.O.T. Blanket for NET," Proc. Isth Symp. on Fusion Technology, Avignon, 1986, p. 1231.

${ }^{12}$ M. Dalle Donne, et. al., "Pebble Bed Canister: A Ceramic Breeder Blanket with Helium Cooling for NET," Proc. 14th Symp. on Fusion Technology, Avignon, 1986, p. 423.

${ }^{13}$ A. Cardella, et. al., "Design and Thermohydraulic Optimization of a Solid Ceramic Breeder Blanket for NET." Proc. 14th Symp. on Fusion Technology, Avignon, 1986, p. 1291. 
casualty. In addition, proper orientation of the helium coolant tubes can allow enhanced heat transfer via natural circulation.

Figure 4.2. Pressurized Tube Design ${ }^{14}$

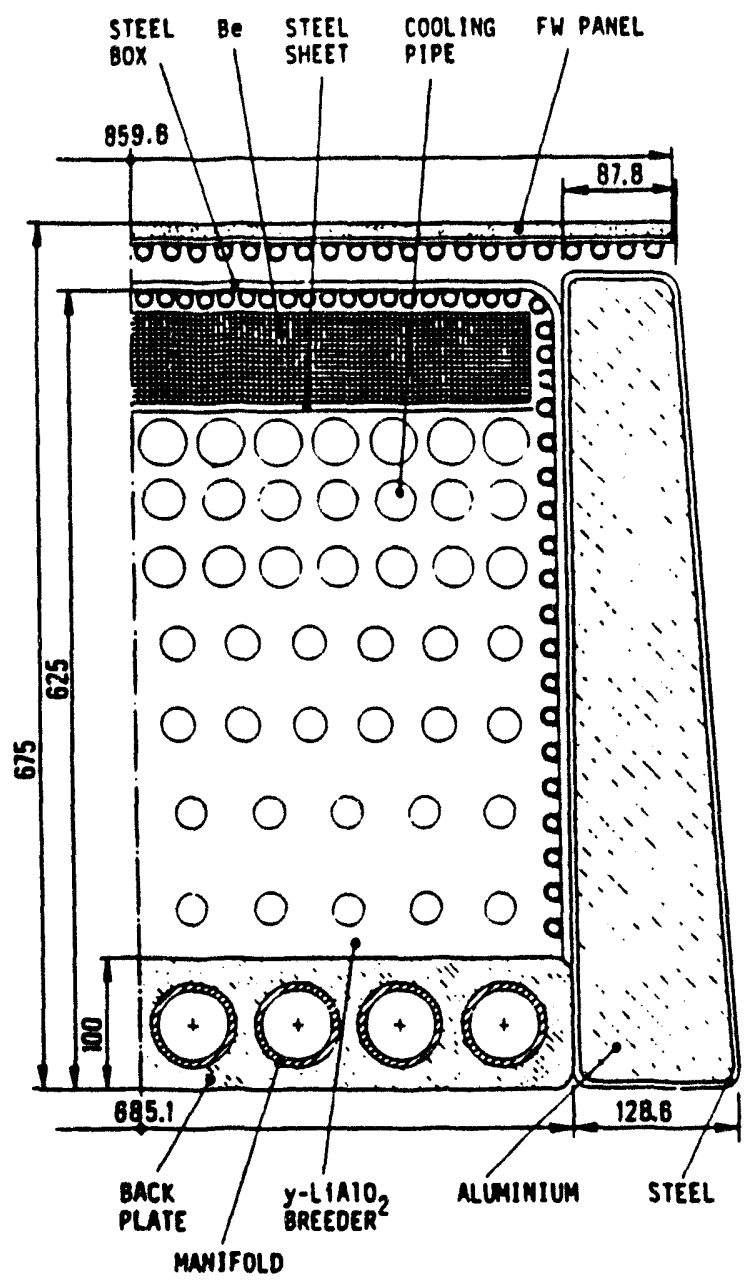

\subsection{Nested Shell Design}

This design concept is the newest of the concepts discussed here, and was used in the ARIES-I design ${ }^{15} 16$. In this design, pressurized helium is circulated in channels which are

${ }^{14}$ M. Ferrari and G. Simbolotti, "Thermal and Stress Analysis of the Solid B.O.T. Blanket for NET," Proc. 14th Symp. on Fusion Technology, Avignon, 1986, p. 1232.

${ }^{15}$ F. Najmabadi, et. al., "The ARIES-I Tokamak Reactor Study, Final Report," UCLA-PPG-1323, 1991. 
part of U-shaped shells. These shells contain the breeder/multiplier matrix (see Figure 4.3, which shows a nested shell blanket module).

\section{Figure 4.3 Nested Shell Design ${ }^{17}$}

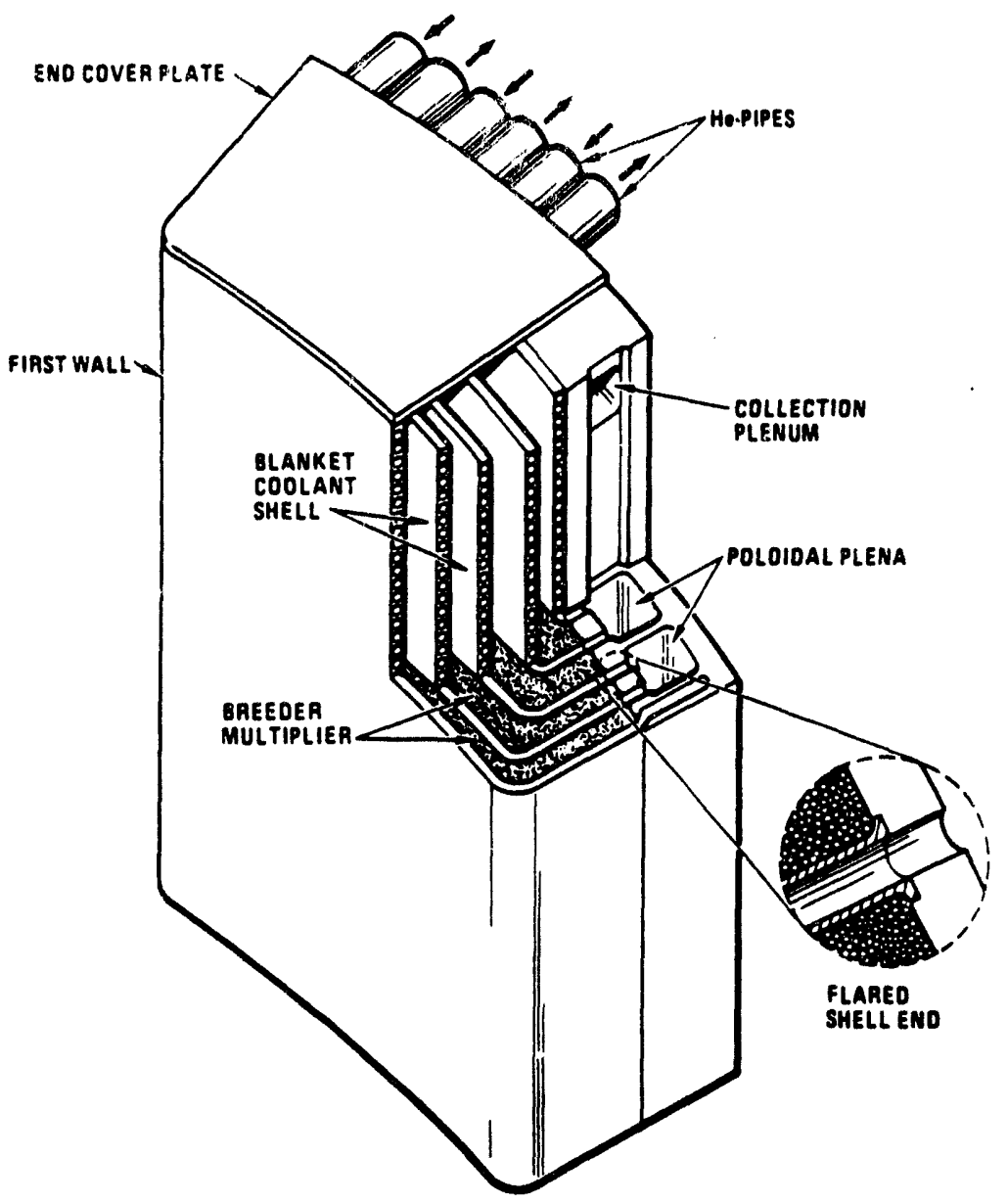

This configuration has the same advantages that the pressurized tube designs have with respect to LOFA's and LOCA's, in that the matrix provides a conduction path from the first wall into the cooler regions of the blanket. Proper orientation of the coolant channels can similarly allow for some natural circulation. In addition to the potential for good

${ }^{16}$ C. P. C. Wong, et. al., "Blanket Design for the ARIES-I Tokamak Reactor," Proc. IEEE 13th Symp. on Fusion Eng., Knoxville, TN, 1990, p. 1035.

${ }^{17}$ F. Najmabadi, "The ARIES-I Tokamak Reactor Study, Final Report," Volume II, University of California at Los Angeles Report \#UCLA-PPG-1323, 1991, p. 8-7. 
thermal characteristics during a LOFA or LOCA, the nested shell design has some other advantages over the other concepts. These include relatively simpler design, a smaller number of welded joints, and a smooth plasma facing surface.

\subsection{Helium-Particulate Designs}

The final configuration to be discussed enhances the thermal performance of the helium coolant by adding particulates to the helium flow stream. These flowing particulates may be lithium compounds, in which case they perform the tritium breeding function as well as augmenting the cooling effectiveness of the helium. 1819 Alternatively, the particulates may be compounds which do not contain lithium, in which case they increase the thermal effectiveness of the helium coolant, which lowers the required helium pumping power and increases the reactor's thermal efficiency ${ }^{20}$.

Although the concept of using particulates in the coolant of a gas-cooled reactor is not a new idea (such designs were proposed in the 1960's for fission reactors), no fission reactor has ever been operated with such a coolant. Hence, many uncertainties on the performance of such a design remain. Additionally, the thermal benefits of such a suspended coolant would be lost in the event of a LOCA or LOFA. For these reasons this concept is not considered to have advantages over the concepts discussed previously.

\footnotetext{
${ }^{18} \mathrm{C}$. Wong, et. al., "A Li-Particulate Blanket Concept for ITER," Fusion Technology Vol. 15, March 1989, p. 871.

${ }^{19}$ Y. Gohar, et.al., "Helium-Cooled Lithium Compound Suspension Blanket Concept for ITER," Fusion Technology, Vol. 15, March 1989, p. 876.

${ }^{20}$ S. Mori, et. al., "Preliminary Design of a Solid Particulate Cooled Blanket for the Steady State Tokamak Reactor (SSTR)," Fusion Technology Vol. 21, May 1992, p. 1744.
} 


\subsection{Breeder, Neutron Multiplier, and Structural Materials}

Many issues are involved with the selection of the breeder and neutron multiplier materials in a fusion blanket, as well as with the form in which the materials are used (e.g. slabs, pebble bed, etc.) Any workable fusion blanket design must account for the following considerations: (1) the allowable operating temperature ranges for the materials; (2) irradiation effects such as swelling and helium production; (3) tritium transport characteristics; (4) chemical compatibility; and (5) possibly complex thermal conductivity characteristics, especially with pebble bed designs.

These considerations, among others, must be kept in mind while striving to obtain the best possible tritium breeding, energy multiplication, and shielding performance in the least amount of space. Additionally, for the helium-cooled blanket designs of concern here, a primary consideration is the performance of the design during a LOCA or LOFA.

Generally speaking this means that the thermal conductivity of the breeder and neutron multiplier materials should be made as high as possible, to facilitate conduction of heat from the first wall toward the cooler sections of the blanket. The following discussion will describe the relevant characteristics of the breeder and multiplier materials currently of interest for helium-cooled blanket designs. Following discussion of breeder and multiplier options, the structural materials of interest in a helium-cooled blanket are reviewed.

\subsubsection{Breeder Materials}

In addition to adequate tritium breeding performance, the breeder material used in a fusion blanket should satisfy the following necessary criteria:21 (1) Low tritium retention, to minimize associated safety problems; (2) Chemical compatibility with structural and neutron multiplier materials, and with the tritium purge gas, which is used to remove

${ }^{21}$ S. Mori. et. al., "Preliminary Design of a Solid Particulate Cooled Blanket for the Steady State Tokamak Reactor (SSTR)," Fusion Technology Vol. 21, May 1992, p. 1744. 
tritium from the breeder; (3) Chemical compatibility with the blanket coolant in case of a coolant leak; (4) Stability at high neutron fluences and high temperatures; and (5) Industrial fabricability at reasonable cost. For helium-cooled blankets, the materials which satisfy the above requirements best are lithium-bearing ceramics.

\section{Table 4.1. Breeder Properties at 500 degrees $\mathrm{C}^{22}{ }^{23}$}

\begin{tabular}{|c|c|c|c|c|c|}
\hline Breeder Matl: & $\mathrm{Li}_{2} \mathrm{O}^{*}$ & $\mathrm{Li}_{4} \mathrm{SiO}_{4}{ }^{* *}$ & $\mathrm{LiAlO}_{2}{ }^{*}$ & $\mathrm{Li}_{2} \mathrm{ZrO}_{3}{ }^{*}$ & $\mathrm{Li}_{2} \mathrm{TiO}_{3}{ }^{* * *}$ \\
\hline Li atom density: & $750 \mathrm{~kg} / \mathrm{m}^{3}$ & $530 \mathrm{~kg} / \mathrm{m}^{3}$ & $220 \mathrm{~kg} / \mathrm{m}^{3}$ & $300 \mathrm{~kg} / \mathrm{m}^{3}$ & $360 \mathrm{~kg} / \mathrm{m}^{3}$ \\
\hline Thermal Cond: & $4.0 \mathrm{~W} / \mathrm{m}-\mathrm{K}$ & $1.4 \mathrm{~W} / \mathrm{m}-\mathrm{K}$ & $2.8 \mathrm{~W} / \mathrm{m}-\mathrm{K}$ & $1.4 \mathrm{~W} / \mathrm{m}-\mathrm{K}$ & $1.9 \mathrm{~W} / \mathrm{m}-\mathrm{K}$ \\
\hline $\begin{array}{l}\text { General Upper } \\
\text { Temp. Limit : }\end{array}$ & $850^{\circ} \mathrm{C}$ & $660^{\circ} \mathrm{C}$ & $970^{\circ} \mathrm{C}$ & $970^{\circ} \mathrm{C}$ & $920^{\circ} \mathrm{C}$ \\
\hline $\begin{array}{l}\text { Creep Upper } \\
\text { Temp. Limit : }\end{array}$ & $650^{\circ} \mathrm{C}$ & $750^{\circ} \mathrm{C}$ & $1000^{\circ} \mathrm{C}$ & N/A & N/A \\
\hline $\begin{array}{l}\text { Temp. for } 1 \text { day } \\
T \text { residence : }\end{array}$ & $325^{\circ} \mathrm{C} \#$ & $380^{\circ} \mathrm{C}$ & $430^{\circ} \mathrm{C}$ & $310^{\circ} \mathrm{C}$ & $\left(380^{\circ} \mathrm{C} ?\right)$ \\
\hline
\end{tabular}

Table 4.1 shows the basic physical properties of various candidate breeder materials, as well as some relevant temperatures. The materials in this table are among the beststudied, best-performing solid breeders, and all except lithium titanate have been used in recent fusion reactor design studies. It should be noted, however, that substantial uncertainties remain with respect to the properties of these materials, especially after exposure to high levels of neutron irradiation. Note also that some entries in the table

\footnotetext{
${ }^{22}$ M. A. Abdou, et. al., "Modeling, Analysis and Experiments for Fusion Nuclear Technology," PPG-1021 or UCLA-ENG-86-44 or FNT-17, January 1987.

${ }^{23}$ E. Proust. et.al., "Solid Breeder Blanket Design and Tritium Breeding," Fusion Engineering and Design 16,1991, p. 73.
} 
remain blank due to lack of data. In order to facilitate future blanket design efforts, more testing of ceramic breeder materials is clearly warranted

A purge gas flows through the breeder region of the blanket to carry away the tritium generated in the breeder. It is important that the tritium generated in the bulk of the breeder material be able to escape into this purge gas flow. To facilitate this, the breeder material is fabricated with a porosity of up to 20 percent. The data shown in the table is for materials which have a porosity within that range.

The 1 day tritium residence time temperature shown in the table is important because at temperatures below this, tritium will be retained by the breeder material for an unacceptably long period of time. This results in an excessive tritium inventory in the blanket. To allow for sufficient tritium release, the breeder should be operated at temperatures above this value.

A number of factors influence the choice of the upper temperature limits shown in the table. Basically, the upper temperature limit is the temperature above which the breeder would experience a loss of integrity, or be reduced in its ability to release tritium to the tritium purge stream. Possible causes of such breeder degradation are 24 : (1) vapor phase mass transport, which could close the pores of the breeder and prevent tritium release; (2) sintering, which would also close the breeder pores; (3) phase changes, which could adversely affect the melting point and other physical breeder properties; and (4) creep and deformation which could cause changes in breeder geometry.

\footnotetext{
${ }^{24}$ M. A. Abdou, et. al., Modeling, Analysis and Experiments for Fusion Nuclear Technology (FNT Progress Report: Modeling and FINESSE), University of California at Los Angeles report UCLA-ENG86-44, January 1987.
} 
The first three items in the above list must be avoided in any breeder design. However the fourth item, creep, may not be an important concern depending on the geometry of the breeder material. For example, in the pebble bed design to be described below, creep is not a significant concern, since the gross geometry of the pebble bed would be largely unaffected if the individual pebbles experienced some creep. Thus, two upper temperature limits are shown in Table 4.1. The general, higher limit corresponds to mass transport, sintering, and phase change issues which affect all breeder designs. The lower limit is a creep limit which may or may not be relevant, depending on the particular breeder geometry.

The creep rate temperature limit shown in the table is the highest temperature the breeder can sustain without experiencing a creep rate in excess of $10^{-6} \mathrm{~mm} / \mathrm{mm}-\mathrm{hr}$ at a stress of $10 \mathrm{MPa}$. By operating at temperatures below this point, it can be assumed that the breeder will not experience deformation during operation as a result of thermal creep. ${ }^{25}$ The following subsections describe the individual breeder materials in more detail, and explain the basis for the general upper temperature limit in Table 4.1.

\section{Lithium Oxide}

Lithium oxide $\left(\mathrm{Li}_{2} \mathrm{O}\right)$ is one of the most important breeder candidates, because it has a significantly higher $\mathrm{Li}$ atom density than the other breeders. This makes it the only breeder material that can conceivably breed enough tritium to forego the use of a neutron multiplier ${ }^{26}$, though most blanket designs incorporating lithium oxide use some multiplier material as well. Lithium oxide generates virtually no afterheat, which makes it attractive from a safety point of view; it makes passive survival of an accident easier. Because of the

${ }^{25}$ M. Chazalon, et. al., "Next European Torus In-Vessel Components," Fusion Technology, Vol. 14, March 1988, p. 82.

${ }^{26} \mathrm{U}$. Fischer, "Qualification of Neutronic Blanket and Shielding Calculations in a One-Dimensional Approach to a Tokamak Reactor," Fusion Technology, Vol. 22. September 1992, p. 251. 
excellent breeding performance of lithium oxide, the material has been rather extensively studied, and probably more is known about this breeder than is known about the other candidate breeders. The thermal conductivity of lithium oxide is also somewhat higher than that of the other breeders; this can help to mitigate the consequences of a LOFA or LOCA. The general upper temperature limit for lithium oxide is determined by sintering concerns. The sintering limit is set at $0.66 \cdot T_{\text {melt }}$, where $T_{\text {melt }}$ is the melting point of lithium oxide, based on fission reactor experience. ${ }^{27}$

A major issue of concern for lithium oxide is its chemical compatibility with the neutron multiplier beryllium. There is a high chemical potential for reaction between these materials, which may be inhibited by the formation of a thin beryllium oxide layer ${ }^{28}$ Inreactor experiments are being conducted at present to determine whether this will be a problem. One way to prevent this from being an issue is to separate the lithium oxide from the beryllium using a mechanical divider plate; however, this complicates the blanket design and can potentially lower the multiplier's effectiveness. Another potential concern for lithium oxide is the generation and migration of LiOT from hot-to-cold regions of the blanket. How significant a concern this is requires further high-temperature, in-situ tritium extraction tests.

\section{Lithium Orthosilicate}

Like lithium oxide, lithium orthosilicate $\left(\mathrm{Li}_{4} \mathrm{SiO}_{4}\right)$ generates negligible amounts of afterheat. However, its $\mathrm{Li}$ atom density and thermal conductivity are significantly lower than those of lithium oxide. More importantly, however, at 665 degrees Celsius lithium

\footnotetext{
${ }^{27}$ M. A. Abdou, et. al., Modeling, Analysis and Experiments for Fusion Nuciear Technology (FNT Progress Report: Modeling and FINESSE), University of California at Los Angeles report UCLA-ENG86-44, January 1987.

${ }^{28}$ D. Smith, et.al, "ITER Blanket, Shield and Material Data Base," ITER Documentation Series, No. 29, International Atomic Energy Agency, Vienna, 1991.
} 
orthosilicate can undergo a phase change which might cause undesirable displacement of breeder material during operation. In addition, under irradiation, lithium orthosilicate can form a low-melting point eutectic which could increase tritium inventory and compromise breeder integrity. Hence, the general upper temperature limit for lithium orthosilicate is chosen to be 660 degrees, to reduce the potential for these phase changes. This relatively low temperature, coupled with the relatively high tritium residence temperature limit, makes the temperature window for lithium orthosilicate quite small.

\section{Lithium Aluminate}

Lithium aluminate $\left(\mathrm{LiAlO}_{2}\right)$ has the lowest $\mathrm{Li}$ atom density of the breeders considered here, which makes it more difficult to design a compact blanket with an adequate tritium breeding ratio. The aluminum in this breeder generates substantial amounts of afterheat, making lithium aluminate less attractive from an accident/safety standpoint. It has good thermal conductivity, however, and is highly resistant to creep. It has the highest tritium residence temperature limit, but also has a high creep temperature limit and general temperature limit (governed by the $0.66 \cdot T_{\text {melt }}$ sintering value). Thus, its temperature window is substantial.

\section{Lithium Zirconate}

Because zirconium generates a very high level of afterheat, use of lithium zirconate $\left(\mathrm{Li}_{2} \mathrm{ZrO}_{3}\right)$ causes difficult safety problems. In the ARIES-I reactor study which used this breeder material, the zirconium was assumed to have been isotopically tailored to reduce its afterheat levels. ${ }^{29}$ However, this is an expensive process, and does not entirely eliminate the problem. The thermal conductivity of lithium zirconate is also low; this adds to the effect of the high afterheat to make a LOFA or LOCA more severe.

${ }^{29}$ F. Najmabadi, et. al., "The ARIES-I Tokamak Reactor Study, Final Report," UCLA-PPG-1323, 1991. 
On the positive side, lithium zirconate has the lowest tritium residence temperature limit, coupled with a high general temperature limit, giving it a very large temperature window No creep data is currently available for lithium zirconate.

\section{Lithium Titanate}

The data on lithium titanate $\left(\mathrm{Li}_{2} \mathrm{TiO}_{3}\right)$ are a bit more sparse than that of the four other breeders discussed herein. However, it is a breeder material which merits consideration. It has a significantly higher $\mathrm{Li}$ atom concentration and generates somewhat less afterheat than does lithium aluminate. Moreover, it does not have the phase change problems of lithium orthosilicate, and has a somewhat higher thermal conductivity than the zirconate and orthosilicate.

Because of a lack of data, the tritium residence temperature limit was estimated for lithium titanate. The tritium residence temperature limit was assumed to be the same as lithium orthosilicate, based on similar tritium diffusion characteristics and case-specific tritium inventory calculations. ${ }^{30}$ No data is currently available on creep characteristics.

\section{Lithium-6 Enrichment}

Natural lithium is composed of 92.5 percent $\mathrm{Li}-7$ and 7.5 percent $\mathrm{Li}-6$. Both of these lithium isotopes can generate tritium when bombarded by a neutron. The applicable equations follow:

$$
\mathrm{Li}-6+\mathrm{n}(\text { thermal) } \Rightarrow \mathrm{He}-4+\mathrm{T} \text { (exothermic) }
$$

\footnotetext{
${ }^{30}$ M. A. Abdou, et. al., Modeling, Analysis and Experiments for Fusion Nuclear Technology (FNT Progress Report: Modeling and FINESSE), University of California at Los Angeles report UCLA-ENG86-44, January 1987.
} 


$$
\mathrm{Li}-7+\mathrm{n}(\text { fast }) \Rightarrow \mathrm{He}-4+\mathrm{T}+\mathrm{n}(\text { endothermic })
$$

For the neutron energy distribution seen in a fusion blanket, the Li-6 reaction has a substantially higher cross-section. Hence, generally speaking, increasing the Li-6 content in the breeder material by isotopic tailoring will lead to higher tritium production. However, the FINESSE study ${ }^{31}$ showed that for most breeder materials in a typical blanket configuration, the increase in tritium breeding saturates at a Li-6 enrichment of 60 percent or so. A notable exception is lithium oxide, for which the tritium breeding ratio peaks at about 20 percent enrichment and then declines for higher enrichment values.

The value of Li-6 enrichment chosen for a particular blanket design may not necessarily be the optimum value for tritium breeding. As the Li- 6 content is increased, the operational volumetric heat generated by the breeder increases as well. This can lead to unacceptably high temperatures in the breeder region, a 1 perhaps undesirably high lithium burnup rates. It should also be noted that the lithium enrichment need not remain constant throughout the blanket. For example, the Li- 6 enrichment could be minimized toward the front of the blanket to minimize the volumetric heat generated in this region of high neutron flux, and maximized toward the back of the blanket where the flux is lower.

\subsubsection{Neutron Multipliers}

As mentioned above, all solid breeder materials of interest, with the possible exception of lithium oxide, require a neutron multiplier to achieve the required tritium breeding performance. Of the possible multiplier choices, beryllium is the clear winner. Beryllium has a substantial $(n, 2 n)$ cross section with a low reaction threshold of $1.85 \mathrm{MeV}$, thus allowing secondary neutrons emitted in the $(n, 2 n)$ reaction to induce a second similar

${ }^{31}$ lbid. 
reaction. ${ }^{32}$ The neutron multiplication performance of beryllium is further enhanced by its low neutron absorption cross section. Beryllium can be utilized in a blanket in two different forms: pure metallic beryllium and beryllium oxide. The following paragraphs discuss these two options.

\section{Pure Bervllium}

Pure beryllium has a melting point of 1280 degrees $C$ (very much higher than its nearest competitor, lead), and has relatively high strength. ${ }^{33}$ Beryllium has an extremely high thermal conductivity, and generates no afterheat. These characteristics are very important in helping to mitigate the effects of a LOCA/LOFA. Pure beryllium has a very high tritium diffusion coefficient. This, coupled with the fact that tritium generation in beryllium is minimal, helps to minimize the tritium inventory in pure beryllium.

Pure beryllium also has some drawbacks from a design point of view. As discussed in the subsection on lithium oxide, there is a serious chemical compatibility concern for lithium oxide/beryllium systems. In addition, there are questions regarding the performance of beryllium under neutron irradiation. Finally, beryllium oxide is toxic, and since it is possible to oxidize pure beryllium during fabrication or during a reactor accident involving high temperatures and air ingress, beryllium is a potential safety hazard. This safety hazard is exacerbated by the potential for rapid beryllium oxidation (a beryllium fire), if high enough temperatures are reached during the accident, and if enough beryllium is exposed to air.

\footnotetext{
${ }^{32}$ E. Proust, et.al., "Solid Breeder Blanket Design and Tritium Breeding," Fusion Engineering and Design 16, 1991, p. 73.

${ }^{33}$ R.C. Weast, Ed., CRC Handbook of Chemistry and Phusics, 58th Ed., CRC Press. Inc., 1978.
} 
The most important irradiation effect for beryllium is its potential for swelling at high temperatures. The beryllium near the first wall of a power reactor will have a helium generation rate of about 20,000 appm per full power year. ${ }^{34}$ There is currently no swelling data for beryllium at these high helium levels. Because of the lack of data, and the difficulty of predicting swelling rates for such a case, the estimates of beryllium swelling vary widely. At 10,000 appm, for example, estimates of beryllium swelling range from 1.5-5 percent for $T<300$ degrees $C, 2-8$ percent for $T<400$ degrees $C$, and 3.5-14 percent for $500<\mathrm{T}<700$ degrees $C .{ }^{35}$ These are very large uncertainty ranges, and make design analysis difficult. There are efforts underway to more fully characterize the swelling performance of beryllium, as well as to determine whether beryllium can be fabricated with smaller grains, lower impurities, reduced oxides, or deliberate anisotropies in order to reduce radiation-induced swelling, with target swelling values of less than 4-5 percent for fusion-relevant fluences. ${ }^{36}$

Beryllium is fabricated using one of two basic methods, powder metallurgy (PM) and ingot metallurgy (IM). In the powder metallurgy method, beryllium powder is pressed and sintered together to form a solid. The PM method allows the fabricator to control the amount of porosity in the formed solid; values of porosity up to about 20 percent can be attained using this method. ${ }^{37}$ The ingot metallurgy process involves melting down flakes of beryllium to form ingots. The resulting material is 100 percent dense, and has superior

\footnotetext{
${ }^{34}$ F. Najmabadi, et. al., "The ARIES-I Tokamak Reactor Study, Final Report," UCLA-PPG-1323, 1991.

${ }^{35}$ D. Smith, et.al, "ITER Blanket, Shield and Material Data Base," ITER Documentation Series, No. 29, International Atomic Energy Agency, Vienna, 1991.

${ }^{36}$ G.R. Longhurst, Summary for the Beryllium Technology Workshop,, Idaho National Engineering Laboratory Report \#EGG-FSP-10017, December 1991.

${ }^{37}$ G.R. Longhurst, Idaho National Engineering Laboratory, personal communication, April, 1993.
} 
weldability. IM beryllium also has significantly greater thermal conductivity than PM beryllium. ${ }^{38}$

\section{Bervllium Oxide}

Beryllium oxide has been identified as a potential alternative to pure beryllium. It is not as good a neutron multiplier as pure beryllium, but there is much less of a chemical compatibility concern between lithium oxide and beryllium oxide than between lithium oxide and pure beryllium. There may be less of a radiation-induced swelling concern for beryllium oxide, and it has a very high melting point. It has almost as high a thermal conductivity as pure beryllium.

As mentioned above, beryllium oxide is toxic, although perhaps not to all people; there is evidence that only a small fraction of the public is susceptible to beryllosis. ${ }^{39}$ Furthermore, since manufacture and use of pure beryllium would already necessitate that precautions be taken against possible ingestion, perhaps the precautions necessary for beryllium oxide would not be substantially more difficult. Finally, beryllium oxide is far less of a chemical hazard than beryllium. In an air-ingress accident, pure beryllium has the potential to burn, releasing large amounts of energy and possibly damaging the blanket. Beryllium oxide has far less chemical potential energy, hence would represent much less of a safety concern with respect to this kind of accident.

A potential safety problem with beryllium oxide is its potential to retain tritium. Based on a limited and somewhat uncertain database, the tritium diffusion coefficient for beryllium oxide is very small. Because there is a low rate of tritium generation in beryllium oxide, a

\footnotetext{
${ }^{38}$ D.R. Floyd, Appendix J of the Beryllium Technology Workshop, G.R. Longhurst, Chairman. EGG-FSP10017. December 1991.

${ }^{39}$ F. Najmabadi, et. al., "The ARIES-I Tokamak Reactor Study, Final Report," UCLA-PPG-1323, 1991.
} 
small diffusion coefficient could lead to undesirably large tritium inventories in the beryllium oxide.

\subsubsection{Pebble Bed Design Concept}

The upper and lower temperature limits discussed above for the breeder materials constrain the design of a fusion blanket. To take advantage of the good thermal efficiency potential of helium-cooled blankets, it is desirable to have as large as possible difference between the helium coolant inlet and outlet temperatures. A reasonable temperature range for the coolant might be from 250 degrees $C$ to 450 degrees $C$. Given such a large coolant temperature range, it is a significant design problem to ensure that the breeder and multiplier materials remain within their operating temperature limits.

Early blanket designs generally used slabs or relatively large pellets of breeder and multiplier materials, usually with a cladding surrounding them. The thermal conductivity of these slabs and pellets was driven mostly by the thermal conductivity of the pure material itself (although some control in the thermal conductivity is provided by the choice of material porosity; some porosity is necessary to allow tritium release). Temperature control of the breeder and multiplier was attained by suitable routing of the helium coolant flow. Note that in the pressurized module blanket designs discussed above, the cold inlet helium flow is directed first along the first wall channel, where is heated. This heated helium then flows by the breeder slabs, thus keeping them above their lower temperature limit.

More recently, however, the idea of using a "pebble-bed" or "sphere-pac" configuration for the breeder and/or multiplier material has been developed. In this concept, the breeder or multiplier material is formed into small (diameter $\approx 1 \mathrm{~mm}$ ) spherical pebbles, and 
inserted into the appropriate blanket region. By varying the pebble sizes and gas pressure of the bed, the thermal conductivity of the bed can be varied. ${ }^{40}+1$ This allows the blanket designer much greater flexibility in choosing blanket configurations.

The pebble bed concept allows the breeder to be mixed uniformly with the multiplier, which is the optimum configuration for tritium breeding. ${ }^{42}$ Additionally, using pure beryllium in the form of pebbles may allow the helium which causes swelling to escape, resulting in much lower radiation-induced swelling. ${ }^{43}$

A design constraint which must be recognized when designing a pebble bed blanket is the maximum allowable packing fraction of the pebbles in the bed. Generally, it is desirable to have a high pebble packing fraction (that is, the fraction of volume in the bed taken up by the pebbles). This maximizes the tritium breeding and shielding effectiveness of the blanket. However, there is a limit to how tightly a bed can be packed. This limit depends on the geometry of the bed, with bed width and pebble diameter(s) being the lengths of interest. With single-sized pebbles, a packing fraction of 60 volume percent is attainable, provided the pebble diameter is no greater than one-fifth the bed width, and preferably less than one-tenth. 4445

\footnotetext{
${ }^{40}$ M.S. Tillack, et.al., "Experimental Study of the Effective Thermal Conductivity of a Packed Bed as a Temperature Control Mechanism for ITER Ceramic Breeder Blanket Designs," Proc. IEEE 13th Symp. on Fusion Eng., Knoxville, TN, 1990, p. 70.

${ }^{41}$ Z.R. Gorbis, et.al., "Thermal Resistance Gaps for Solid Breeder Blankets Using Packed Beds," Fusion Technology, Vol. 15, March, 1989, p. 695.

${ }^{42}$ U. Fischer, "Optimal Use of Beryllium for Fusion Reactor Blankets," Fusion Technology, Vol. 13, January, 1988, p. 143.

${ }^{43}$ F. Najmabadi, et. al., "The ARIES-I Tokamak Reactor Study, Final Report," UCLA-PPG-1323, 1991.

${ }^{44}$ P. Gierszewski and J. Sullivan, "Ceramic Sphere-pac Breeder Design for Fusion Blankets," \{lem Fusion Engineering and Design\}, Vol. 17, 1991, p. 95.

${ }^{45}$ R. McGeary, "Mechanical Packing of Spherical Particles," \{lem J. Amer. Cer. Soc\}, Vol. 44(10), 1961, p. 513.
} 
A higher packing fraction (up to 80 volume percent) can be attained using two different pebble sizes, with the smaller pebble diameter no greater than one-seventh the diameter of the larger pebble. Even higher packing fractions can be attained using a third pebble size, with a diameter again less than one-seventh the previous diameter. However, three-sized pebble beds have been shown using mathematical models to have characteristically excessive purge flow pressure drops (because the smallest sphere size eliminates too much bed porosity). Hence, three-sized pebble beds will not be considered further.

It is important to be able to determine the thermal conductivity of a pebble bed breeder with some precision; after all, control of this parameter is one of the main reasons to use a pebble bed in the first place. Unfortunately, calculation of the thermal conductivity of a pebble bed is difficult, especially since appropriate data for fusion-relevant materials is lacking. Fundamenski and Gierszewski recently did a comparison between various pebble bed conductivity relations and available data on fusion breeder materials to determine the relation that seems to give the best thermal conductivity estimate for a packed bed. ${ }^{46}$ This relation is discussed further in Section 5.2.2.1, Pebble Bed Conductivity, wherein a pebble bed design is developed for Blanket Design 1.

\subsubsection{Structural Materials}

The following subsection describes some of the main candidate structural materials for use in helium-cooled fusion reactor blankets. Some of the main characteristics necessary for a helium-cooled fusion blanket structural material to have are: (1) resistance to a high fluence of $14 \mathrm{MeV}$ neutrons, sufficient to allow the first wall to last for roughly 5 years at a wall loading of 3-5 MW/ $\mathrm{m}^{2}$; (2) high strength at high temperatures (up to 550

\footnotetext{
${ }^{46}$ W.R. Fundamenski and P.J. Gierszewski, "Comparison of Correlations for Heat Transfer in Sphere-Pac Beds," Canadian Fusion Fuels Project Report \#CFFTP G-9181, August, 1991.
} 
degrees $\mathrm{C}$ ), to allow for a high helium outlet temperature, and high helium coolant pressure; (3) compatibility with helium and the trace impurities that may be present (such as hydrogen, water, and carbon monoxide); ${ }^{47}$ (4) compatibility with the chosen breeder and multiplier materials; (5) fabricability into large-scale, complex geometries; and (6) reasonable cost.

In addition to these required characteristics, it is very desirable that a fusion blanket material have low levels of induced radioactivity and afterheat production, both in the short term (to enhance the safety of the blanket during an accident), and in the long term (to reduce the problems associated with disposal or recycle of the blanket structure after use). While not strictly required for blanket operation, having "low-activation" characteristics can vastly improve a material's safety and environmental ranking. Since the presumed safety and environmental benefits of fusion are two of the main reasons it is being pursued as an energy source, having low-activation characteristics should perhaps also be considered among the "required" characteristics of a structural material for a fusion reactor blanket.

The following paragraphs discuss the structural material options which have attracted the most attention for use in helium-cooled fusion blankets. All of the materials discussed below appear to have adequate chemical compatibility with helium coolant (and its impurities), and with the candidate breeder and multiplier materials. However, as will be seen, each of the structural materials discussed has one or more problems which could prohibit its use in a fusion blanket. Ongoing research by the international fusion community continues for all of these materials to solve these outstanding problems.

\footnotetext{
${ }^{47}$ For a discussion of typical helium impurities in an HTGR, see G. Melese and R. Katz, Thermal and Flow Design of Helium-Cooled Reactors, American Nuclear Society, 1984, p.193.
} 


\section{Ferritic Steels}

Ferritic, or martensitic steels constitute a class of stainless steels characterized by having up to 27 percent chromium as an alloying agent. ${ }^{48}$ Since ferritic steels are ferromagnetic, prior to the early 1980's concerns that these steels would adversely effect the magnetic configuration of a fusion reactor eliminated them from consideration as fusion blanket materials. However, design studies in the early 1980's indicated that the magnetic saturation of the ferritic steels would permit acceptable reactor operation. ${ }^{49} 50$

Ferritic steels have had widespread use in engineering applications in the past, hence are generally considered to be useful for relatively near-term fusion reactor applications. Ferritic steels have higher thermal stress factor ${ }^{\#}$ than austenitic stainless steels, thus reducing the thermal stresses induced in the first wall region of a fusion reactor blanket. The operating temperature limit for these steels is about 500-550 degrees $C .^{51}$

Significant progress has been made toward the development of ferritic steels which have a more rapid decay of activity than conventional steels. Programs to develop reducedactivation ferritic steels, begun in the mid-1980's by the international fusion community, have determined that replacing molybdenum (a typical alloying element in

${ }^{48}$ T. Baumeister, et.al., Mark's Standard Handbook for Mechanical Engineers, Eighth Ed., McGraw-Hill, 1978, p. 6-36.

${ }^{49} \mathrm{~T}$. Lechtenberg, et.al., in "Ferritic Alloys for Use in Nuclear Energy Technologies," Proc. Top. Conf. Snowbird. UT, 1983, Metallurgical Society and American Institute of Mining, Metallurgical and Petroleum Engineers, New York, 1984, p. 179.

${ }^{50}$ H. Attaya, et.al., ibid., p. 169.

\# The thermal stress factor is defined as $k \sigma(1-v) / E \alpha$, where $k$ is thermal conductivity, $\sigma$ is failure stress, $v$ is Poisson's ratio, $E$ is Young's Modulus, and $\alpha$ is the thermal expansion coefficient.

51D.L. Smith, et.al., "Reduced Activation Structural Materials Development for DEMO Fusion Reactor Applications," Argonne National Laboratory Report \#ANL/ER/CP--76020, September, 1992. 
ferritic/martensitic steels) by vanadium and/or tungsten significantly reduces the mediumto-long term activation level of the steel. ${ }^{52}$

A significant amount of data has been obtained on the performance of ferritic steels under neutron irradiation, including some of the reduced-activation ferritic steel options. ${ }^{53}$ Although the radiation-induced swelling for ferritic steels appears to be acceptably low, there is a significant shift in the ductile-to-brittle transition temperature (DBTT) for these steels during irradiation. The shift in DBTT appears to be the major issue of concern regarding the feasibility of these steels for use in fusion blankets.

\section{Austenitic Stainless Steels}

Austenitic stainless steels have both nickel and chromium as alloying agents. Like ferritic steels, austenitic stainless steels have been used extensively in past engineering applications, including nuclear applications. Because of the large industrial capability in existence for fabrication and weldment for these steels, plus the large experience base, austenitic stainless steels are leading contenders for the fusion experimental machines currently being designed (such as ITER and DEMO).$^{54}$

Unlike ferritic steels, austenitic steels have relatively poor thermal performance. The lower thermal stress factor of these steels leads to higher first wall stresses; this is particularly a problem for pulsed fusion reactor concepts. A larger problem for conventional austenitic stainless steels is the unacceptably large swelling which occurs

\footnotetext{
52R.L. Klueh. et.al., "Ferritic/Martensitic Steels: Promises and Problems," in Fusion Reactor Materials Semiannual Progress Report for Period Ending March 31, 1992, U.S. Dept. of Energy Report \#DOE/ER0313/12.

${ }^{53}$ R.L. Klueh, "Developing Steels for Service in Fusion Reactors," Journal of Materials, April, 1992, p. 20.

${ }^{54}$ E.E. Bloom. "Structural Materials for Fusion Reactors," Nuclear Fusion, Vol. 30, No. 9, 1990, p. 1879.
} 
even at modest doses of neutron irradiation. For example, cold-worked CRES316 irradiated at a neutron dose equivalent to 1 year of operation at a neutron wall load of 5 $\mathrm{MW} / \mathrm{m}^{2}$ exhibits 6 percent volume swelling. ${ }^{55}$ This level of swelling would make this steel clearly unacceptable for use in a fusion power reactor application.

This high level of swelling, and the concomitant loss of ductility, can be reduced by proper alloying techniques. Advanced austenitic stainless steels, such as PCA (prime candidate alloy), have been developed which have much lower swelling values than conventional austenitic stainless steels. For these advanced alloys, up to 3 years of operation at a neutron wall load of $5 \mathrm{MW} / \mathrm{m}^{2}$ should be achievable at temperatures up to 550 degrees C. .56

Nickel is an important alloying element for most austenitic stainless steels of interest to fusion, since it stabilizes the face-centered-cubic austenitic structure. Unfortunately, nickel contributes significantly to the high long-term activity of these steels. Manganese, which has significantly less long-term activity than nickel, also can be used to stabilize the crystal structure of the steel; however, it is not as effective as nickel. Development of reduced-activation, manganese-stabilized austenitic stainless steels is in the early stages, but it appears that alloys can probably be developed with properties similar to other conventional alloys. However, the reduced-activation austenitic stainless steel alloys studied to date do not have as low long-term activity levels as reduced-activation ferritic steels. 57585960

55 Ibid.

${ }^{56}$ Ibid.

${ }^{57}$ M. Zucchetti, M. Zublena, "A Study on the Prospects for Development of a New Low Activity Austenitic Stainless Steel for Fusion Applications," Fusion Technology, 1988, p. 991.

${ }^{58} \mathrm{~A}$. Khursheed, et.al., "Activation of Materials for the Fusion Reactor First Wall," Fusion Technology, 1988. p. 971. 


\section{Silicon Carbide}

From a safety and environmental standpoint, silicon carbide ( $\mathrm{SiC}$ ) is perhaps the most attractive fusion blanket structural material yet identified. A recent study has indicated that the very low short-term activation characteristics of $\mathrm{SiC}$, coupled with its high melting point, would probably make an undercooling accident such as a LOCA or LOFA easy to sustain passively without damage, regardless of the detailed design of the blanket ${ }^{61}$ The low long-term activation of $\mathrm{SiC}$ would also greatly reduce radioactive waste disposal concerns. ${ }^{62}$ In addition to these significant safety and environmental virtues, $\mathrm{SiC}$ also has a high thermal stress factor, making it a potentially good first wall material.

Despite its substantial positive attributes, a number of significant problems with $\mathrm{SiC}$ diminish its potential worth as a blanket structural material, at least for the near future. Since $\mathrm{SiC}$ is a ceramic, it is inherently brittle; hence use of monolithic $\mathrm{SiC}$ is inadvisable for a structure such as a fusion blanket. SiC fiber composites, however, offer the potential to behave in a more ductile-like fashion when stressed. SiC/SiC composite technology, however, is still in its infancy, and major issues, such as the hermeticity of the composite, remain currently unresolved.

The irradiation data base for SiC is very small. Much more information on how highenergy neutrons at high fluences affect the physical and mechanical properties of $\mathrm{SiC}$ is necessary before it can be used for blanket structure. Methods of fabrication and joining

${ }^{59} \mathrm{G}$. Piatti, et.al., "Development of Low Activation Cr-Mn Austenitic Steels for Fusion Reactor Applications," Fusion Technology, 1988, p. 983.

${ }^{60}$ E.E. Bloom, "Structural Materials for Fusion Reactors," Nuclear Fusion, Vol. 30, No. 9, 1990, p. 1879.

${ }^{61}$ F. Najmabadi, et.al., "The ARIES-I Tokamak Reactor Study, Final Report," UCLA-PPG-1323, 1991.

62 J.P. Holdren, et.al., "Report of the Senior Committee on Environment, Safety, and Economic Aspects of Magnetic Fusion Energy," Lawrence Berkeley Laboratory Report \#UCRL-53766, September 25, 1989. 
of $\mathrm{SiC}$ to itself, and to other materials, need to be developed in order to be able to build a large, complex engineering structure such as a fusion blanket. Finally, SiC's compatibility with tritium breeding materials still needs to be verified. ${ }^{63}$

The current problems with SiC may simply be due to the fact that it has never been developed as a structural material for large-scale engineering applications. Current work to resolve the main problems and uncertainties with $\mathrm{SiC}$ is underway within the international fusion program and elsewhere; this work may in time result in making SiC composites prime candidates for fusion blanket structures. However, currently SiC is well behind steels on the road to development of a structural material for fusion blankets. For this reason, $\mathrm{SiC}$ will not be considered further as a blanket structural material in the present work.

${ }^{63}$ D.L. Smith, et.al., "Reduced Activation Structural Materials Development for DEMO fusion reactor Applications," Argonne National Laboratory Report \#ANL/ER/CP--76020, September 1992. 


\section{Chapter 5 Blanket Designs and Results}

The previous chapters have provided an introduction and some background information on previous helium-cooled blanket designs as well as on breeder, multiplier and structural material options. This chapter, which forms the heart of the present work, develops the designs for two new helium-cooled blanket concepts, and analyzes the new designs to determine their performance during undercooling accidents.

This chapter is divided into three main sections. Section 5.1 describes the design constraints that are applied to both blanket designs, to ensure each design meets the minimum requirements a D-T fusion reactor blanket must have. Section 5.2 describes the first new blanket design (Blanket Design 1), which is similar in configuration to the nested shell-type, pebble bed blanket design discussed in Chapter 4. Although features of Blanket Design 1 such as the breeder and multiplier materials, and the breeder/multiplier ratio were optimized to provide the best performance during an undercooling accident, analysis of this design showed that it would not survive a NoFlow LOFA, the worst-case type of undercooling accident.

Section 5.3, the third main section of Chapter 5, describes Blanket Design 2. Although Blanket Design 2 is neutronically identical to Blanket Design 1, it uses a unique new configuration of beryllium multiplier and breeder material to give it even better thermal performance during undercooling accidents than Blanket Design 1. By replacing Blanket Design 1's pebble bed breeder/multiplier region with "beryllium-joints," the performance of the blanket during an accident is substantially improved, without degrading any of its other performance characteristics. As will be shown, Blanket Design 2 easily survives the worst-case undercooling accident. Hence Blanket Design 2 represents fulfillment of a primary goal of the present work: the development of a helium-cooled blanket with 
metal structure which can passively sustain a worst-case undercooling accident without damage.

\subsection{Blanket Design Constraints}

As has been discussed previously, there are many functions that a fusion reactor blanket must perform. Foremost among these required functions are the following: (1) the blanket must breed adequate tritium; (2) the blanket must provide (in conjunction with the shield) adequate neutron shielding; and (3) the blanket operational lifetime must be sufficient. All of these requirements must be met within a reasonable size envelope. The following paragraphs discuss these design constraints in detail.

\section{Design Constraint 1: Tritium Breeding Ratio}

Perhaps the most critical blanket function is the requirement for adequate tritium breeding, for if D-T fusion is to be a viable, widespread power source, it must provide for its own tritium needs. Thus, the overall tritium breeding ratio (TBR) must be greater than or equal to one for the whole tritium fuel cycle for the reactor to be self-sufficient. To account for losses due to the radioactive decay of tritium while in storage onsite, as well as tritium that will be needed for the startup of next generation reactors, the blanket must produce between 1.01 and 1.02 tritons per triton consumed in the plasma. ${ }^{1}$

The following scoping study on blanket designs uses a two-dimensional model for the blanket; this model is described is Chapter 3. The TBR which is calculated using a oneor two-dimensional model usually overestimates the actual, three-dimensional breeding ratio. Such a simplified model does not account properly for neutrons which are lost via streaming through pumping and heating ducts and diagnostic ports. In addition,

'F. Carré, et.al., "Analysis of the Tritium Requirements for a Power Reactor, Fusion Technology, Vol. 4, 1983, p. 805. 
simplified models which are not fully three-D do not properly account for neutrons which impact the divertor rather than the blanket. To account for the overestimation of the TBR in such simplified models, the TBR as calculated in the model must be substantially greater than the required 1.01 to 1.02 value. Although the present study uses a two-dimensional model, this model is relatively simplified, and does not account for losses through vacuum ports and the divertor. Hence, for conservatism, this study will adopt a correction factor which is appropriate for a one-D model.

Various studies have assumed different one-D TBR correction factors. In a recent comparison between one- and three-D neutronics calculations, the one-D TBR overestimation for a solid-breeder blanket is calculated to be 34 percent. ${ }^{2}$ This is more conservative than the one-D TBR of 1.21 used in the ARIES-1 study, ${ }^{3}$ and is consistent with the one-D TBRs calculated for the Blanket Comparison and Selection Study (BCSS) blanket designs. ${ }^{4}$ For the blanket designs developed herein, a design constraint will be that the TBR must be at least 1.35 .

\section{Design Constraint 2: Magnet Radiation Limit}

The primary purpose of the shield is to ensure (in conjunction with the blanket) that the superconducting magnets of the reactor are sufficiently protected from high-energy neutrons to last the life of the reactor. The shield also enhances the TBR of the blanket by acting as a neutron reflector. However, an investigation using TWODANT showed

\footnotetext{
${ }^{2} \mathrm{U}$. Fischer, "Qualification of Neutronic Blanket and Shielding Calculations in a One-Dimensional Approach to a Tokamak Reactor," Fusion Technology, Vol. 22, September 1992, p. 251.

${ }^{3}$ F. Najmabadi, et. al., "The ARIES-I Tokamak Renctor Study, Final Report, " UCLA-PPG-1323, 1991.

4J. E. Massidda and M. S. Kazimi, "Thermal Design Considerations for Passive Safety of Fusion Reactors," DOE/ID-01570-3, FFC/RR-87-18, October 1987.
} 
that for cases of interest here, the enhancement of TBR is minimal. Hence, the primary concern is protection of the magnets.

The fluence limit used for the ARIES-I reactor design magnets is also used herein. The ARIES-I magnets use $\mathrm{Nb}_{3} \mathrm{Sn}$ as the superconductor, and the allowable fast neutron fluence is $1.0 \times 10^{2.3} \mathrm{n} / \mathrm{m}^{2}$. This fluence was chosen to ensure that the $\mathrm{Nb}_{3} \mathrm{Sn}$ superconductor performs adequately over the life of the machine. This fluence should also be low enough to ensure that the polyamide insulator typically used for fusion reactor magnets will last the machine lifetime. As will be seen, the requirement to keep the neutron fluence seen by the magnets below this value will control the thickness of the inboard blanket/shield.

\section{Design Constraint 3: Neutron Wall Loading}

The lifetime of a fusion blanket is determined chiefly be the maximum neutron fluence sustainable by the blanket's first wall. To determine the operational lifetime, the neutron flux at the first wall must be known. The neutron flux at the first wall must also be specified in order to size the inboard blanket/shield to protect the magnets, and to determine the activation of the blanket structure. Because of the toroidal shape of the reactor and plasma, the neutron wall load varies as one travels along the first wall in the poloidal direction. This is discussed in Chapter 3. The neutron wall load peaks at a poloidal angle of 0 degrees (along the outboard circumference), and is smallest at poloidal angles of roughly 90 and 270 degrees (the top and bottom). Calculations performed for a reactor of representative dimensions $(R=5.8 \mathrm{~m}, \mathrm{a}=1.6 \mathrm{~m})$ have shown that the peak neutron wall load is 1.5 times the average wall load, and the minimum wall load is 0.4 times the average. These calculations also show that the average wall load

5ITER Documentation Series, No. 3, IAEA, Vienna, 1989, p. 308. 
seen by the outboard blanket is 1.2 times the overall average, and the average inboard blanket wall load is 0.82 times the overall average.

Historically, an average neutron wall load of about $5 \mathrm{MW} / \mathrm{m}^{2}$ has been used for blanket studies; the BCSS study assumed this value. ${ }^{6}$ More recent reactor studies have resulted in average neutron wall loads which are somewhat below that value, however. For example, the ARIES-I study has an average neutron wall load of only $2.2 \mathrm{MW} / \mathrm{m}^{2}$. For the present scoping study, the peak neutron wall load is assumed to be $5 \mathrm{MW} / \mathrm{m}^{2}$. This assumption, coupled with the variations in neutron wall load discussed above for a typical reactor, result in the neutron wall load values adopted for this scoping study (see Table 5.1.)

Table 5.1. Neutron Wall Loads

Overall Average: $3.3 \mathrm{MW} / \mathrm{m}^{2}$
Outboard Blanket Average:
$4.0 \mathrm{MW} / \mathrm{m}^{2}$
Inboard Blanket Average:
$2.7 \mathrm{MW} / \mathrm{m}^{2}$
Peak Load (Outboard):
$5.0 \mathrm{MW} / \mathrm{m}^{2}$
Peak Load (Inboard):
$3.4 \mathrm{MW} / \mathrm{m}^{2}$
Minimum Load:
$1.3 \mathrm{MW} / \mathrm{m}^{2}$

The outboard and inboard average wall loads are used to determine the blanket TBR and to determine the avsaze activation and afterheat values in the blanket structure, as discussed in Appendix 1. The inboard peak load is used to determine the maximum neutron load to the magnets, and hence the required inboard shield thickness.

${ }^{6}$ D.L. Smith, et.al., Blanket Comparison and Selection Study (BCSS) - Final Report, Argonne National Laboratory report \#ANL/FPP-84-1, September 1984. 


\subsection{Blanket Design 1 - Nested Shell with Mixed Pebble Bed}

Of the types of blankets described above in the section on past blanket design concepts, one of the more promising designs from the point of view of surviving a LOCA or LOFA accident is the nested-shell type. This blanket has a good thermal conduction path from the first wall back to the cooler interior portion of the blanket. As discussed in Chapter 4 , this blanket design also has the advantages of a relatively simple design, a minimum of critical welded joints, and a smooth plasma-facing surface. The nested shell design is therefore chosen for Blanket Design 1, the first blanket to be developed in this work.

The benefits of a pebble bed blanket arrangement have been discussed in previous sections. To summarize, a pebble bed allows the designer some control over the thermal conductivity of the blanket, which is advantageous when attempting to maintain the breeder material within its operating temperature window. Using beryllium in a pebble bed configuration may also help mitigate the effects of radiation-induced swelling of beryllium. However, the reduction in thermal conductivity characteristic of a pebble bed has a negative influence on the LOCA/LOFA accident. Blanket Design 1 will use a pebble bed for its breeder zone, but the thermal conductivity of the pebble bed will be increased as much as possible, by maximizing the amount of high-conductivity beryllium in the bed, and maximizing the size of the pebbles in the bed. In this way the best possible LOFA/LOCA performance will be obtained, consistent with obtaining an adequate tritium breeding ratio (TBR).

As discussed previously, mixing the breeder with the multiplier generally results in a better TBR than if the same materials (at the same densities) were placed separately in the blanket. Such mixing also increases the thermal conductivity of the bed, which helps with respect to the LOCA/OFA. In order to maximize the thermal conductivity as 
much as possible for the mixed bed design, the breeder with the highest thermal conductivity, namely lithium oxide, is chosen for Blanket Design 1. As discussed in Chapter 4, lithium oxide has the highest lithium atom density of the candidate breeders, which helps to minimize the thickness of the blanket; this also helps the blanket perform better during a LOCA/LOFA, by reducing the amount of afterheat-producing structure. There are chemical compatibility concerns for the lithium oxide/beryllium combination (see Chapter 4). For the purposes of this study, it is assumed that these concerns are manageable.

It has been shown ${ }^{7}$ that for homogeneous mixtures of breeder and multiplier, the breeding ratio is nearly insensitive to the amount of $\mathrm{Li}-6$ enrichment in the breeder. This is especially true for lithium oxide and beryllium. Hence, for this case, it is assumed for simplicity that the lithium in the breeder has a natural abundance ( 7.5 percent) of Li-6.

The structural material chosen for Blanket Design 1 is a reduced-activation ferritic steel. As discussed in Chapter 4 , the ferritic steels are a relatively near-term blanket structural material option, and much work has been done to develop reduced activation versions of these steels. The specific type of reduced-activation steel used for Blanket Design 1 was proposed by Fetter, ${ }^{8}$ and used in a previous blanket safety study. 9 It is a modification of the steel kno wn as Sandvik HT-9, with most of the Ni and Mo alloying elements replaced by tungsten. The modified HT-9 is called MT-9 in this study, and the compositions of both HT-9 and MT-9, are given in Appendix 9.

\footnotetext{
${ }^{7}$ M. A. Abdou, et. al., Modeling, Analysis and Experiments for Fusion Nuclear Technology (FNT Progress Report: Modeling and FINESSE), University of California at Los Angeles report UCLA-ENG86-44, January 1987.
}

8S. Fetter, "The Radiological Hazards of Magnetic Fusion Reactors," Fusion Technology, Vol. 11, March 1987, p. 400.

9J. E. Massidda and M. S. Kazimi, "Thermal Design Considerations for Passive Safety of Fusion Reactors," DOE/ID-01570-3, PFC/RR-87-18, October 1987. 


\subsubsection{Lithium Oxide and Bervllium Mixed Bed: Neutronics}

To begin the neutronics analysis of this design, which will ensure that the TBR goal of 1.35 is met, the proper ratio of beryllium to lithium oxide in the bed must be determined. It has been noted that to design a blanket for optimum tritium breeding, it may be necessary to vary the ratio of multiplier-to-breeder as one moves from the front of the blanket to the rear. For the present scoping-type study this was not done. Even assuming a uniform multiplier-to-breeder ratio, to determine the precisely optimum ratio at this initial stage of design is impossible, because none of the required blanket details, such as blanket thickness and structural material volume fraction are known yet.

To get an estimate of the optimum ratio, a one-D model is developed in which the ratio of beryllium-to-lithium oxide is varied, with other aspects of the model, such as blanket and shield depth, remaining constant. The model incorporates a $10 \mathrm{~mm}$ thick first wall region, followed by a $43 \mathrm{~cm}$ thick blanket region, and finally a $65 \mathrm{~cm}$ thick shield. Hence, the total blanket and shield thickness is $109 \mathrm{~cm}$. The first wall, blanket, and shield regions are assumed to be volumetrically uniform. The blanket and shield thicknesses chosen for the model are similar to the thicknesses used for the outboard helium/solid breeder reference blanket in the BCSS study. ${ }^{10}$ The helium coolant plenum located at the rear of the BCSS blanket region is neglected in this model, for simplicity. Figure 5.1 shows the geometry of the model.

\footnotetext{
${ }^{10}$ D.L. Smith, et.al., Blanket Comparison and Selection Study (BCSS) - Final Report, Argonne National Laboratory report \#ANL/FPP-84-1, September 1984.
} 
Figure 5.1. Multiplier Ratio Optimization Model

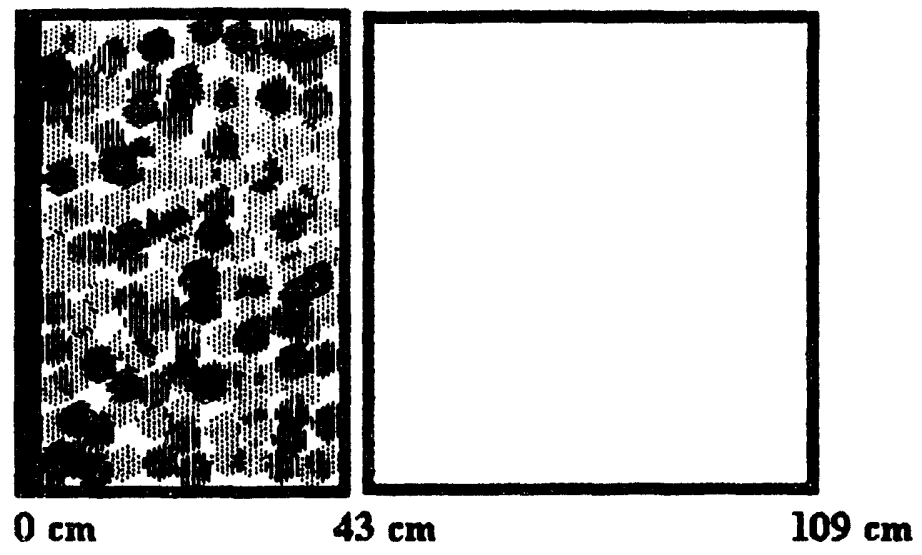

The first wall of this model is composed of MT-9 and helium, with the volume fraction of MT-9 equal to 0.28 . This volume fraction would result from using $5 \mathrm{~mm}$ radius tubes with a $0.71 \mathrm{~mm}$ wall thickness L- Mixed Bed Blanket Region for the first wall. The shield is $\square$ - Shield - First Wall (1 cm thick) composed of water and MT-9 with MT-9 having a volume fraction of 0.90 , which is the composition of the BCSS shield. The blanket region is composed of MT-9 structure, and the mixed bed of lithium oxide and beryllium. The volume fraction of MT-9 structure is fixed at 0.11 , a reasonable value for the nested shell configuration. (It should be noted that a previous neutronics study of a helium-cooled solid breeder blanket indicated that the optimum beryllium/breeder ratio depends on the volume fraction of structural material in the blanket. " Hence, it is important to ensure that the structural volume fraction used in the optimization is representative.)

The volume fractions of beryllium and lithium oxide in the breeder region depend on two factors: (1) the beryllium/lithium oxide ratio in the pebble bed; and (2) the ratio of helium to breeder/multiplier in the bed. The first factor is the one which is to be varied in the analysis. The second factor depends on how tightly the pebble bed can be packed. For a two-sized pebble bed, a maximum packing fraction of 0.80 can be attained (see

11Y. Watanabe, et.al., "Neutronics Performance of a Helium-Cooled Solid Breeder Blanket and Shield for ITER," Fusion Technology, Vol. 15, March 1989, p. 475. 
Section 5.2.2.1.) Hence, this is the packing fraction which is assumed for the mixed bed. It is assumed for simplicity here that the pebbles in the bed are 100 percent dense. Given the ratio of beryllium to lithium oxide in the mixed bed, we now have enough information to determine the volume fractions of beryllium and lithium oxide in the overall blanket region. Table 5.2 summarizes the compositions of the resulting models which were examined. 
Table 5.2. Volume Fractions in Ratio Optimization Model Regions

\begin{tabular}{|c|c|c|c|}
\hline Be:Li20 Ratio & First Wall Region & Blanket Region & $\frac{\text { Shield }}{\text { Region }}$ \\
\hline $10: 1$ & $\begin{array}{l}0.28 \text { MT-9/ } \\
0.72 \text { Helium } \\
\end{array}$ & $\begin{array}{l}0.436 \mathrm{Be} / 0.046 \mathrm{Li} 20 / \\
0.41 \mathrm{He} / 0.11 \mathrm{MT}-9\end{array}$ & $\begin{array}{l}0.90 \text { MT }-9 / \\
\text { 0.10 Water }\end{array}$ \\
\hline 8:1 & $\begin{array}{l}0.28 \mathrm{MT}-9 / \\
0.72 \text { Helium }\end{array}$ & $\begin{array}{l}0.427 \mathrm{Be} / 0.053 \mathrm{Li} 20 / \\
0.41 \mathrm{He} / 0.11 \mathrm{MT}-9\end{array}$ & $\begin{array}{l}\text { 0.90 MT-9/ } \\
\text { 0.10 Water }\end{array}$ \\
\hline $6: 1$ & $\begin{array}{l}0.28 \text { MT-9/ } \\
0.72 \text { Helium } \\
\end{array}$ & $\begin{array}{l}0.411 \mathrm{Be} / 0.069 \mathrm{Li} 20 / \\
0.41 \mathrm{He} / 0.11 \mathrm{MT}-9\end{array}$ & $\begin{array}{l}0.90 \text { MT-9/ } \\
\text { 0.10 Water }\end{array}$ \\
\hline $4: 1$ & $\begin{array}{l}0.28 \text { MT-9/ } \\
0.72 \text { Helium } \\
\end{array}$ & $\begin{array}{l}0.384 \mathrm{Be} / 0.096 \mathrm{Li20} / \\
0.41 \mathrm{He} / 0.11 \mathrm{MT}-9\end{array}$ & $\begin{array}{l}0.90 \text { MT }-9 / \\
0.10 \text { Water }\end{array}$ \\
\hline $3: 1$ & $\begin{array}{l}\text { 0.28 MT-9/ } \\
\text { 0.72 Helium }\end{array}$ & $\begin{array}{l}0.360 \mathrm{Be} / 0.120 \mathrm{Li} 20 / \\
0.41 \mathrm{He} / 0.11 \mathrm{MT}-9\end{array}$ & $\begin{array}{l}0.90 \text { MT-9/ } \\
0.10 \text { Water }\end{array}$ \\
\hline 2:1 & $\begin{array}{l}0.28 \text { MT-9/ } \\
0.72 \text { Helium }\end{array}$ & $\begin{array}{l}0.320 \mathrm{Be} / 0.160 \mathrm{Li} 20 / \\
0.41 \mathrm{He} / 0.11 \mathrm{MT}-9\end{array}$ & $\begin{array}{l}0.90 \text { MT }-9 / \\
0.10 \text { Water }\end{array}$ \\
\hline
\end{tabular}

The dimensions shown in Figure 5.1, together with the six sets of volumetric composition data shown in Table 5.2 are now used as input to the TWODANT neutronics code. The results of these six TWODANT runs are shown in Figure 5.2.

Figure 5.2. TBR as a Function of $\mathrm{Be} / \mathrm{Li2O}$ Volumetric Ratio

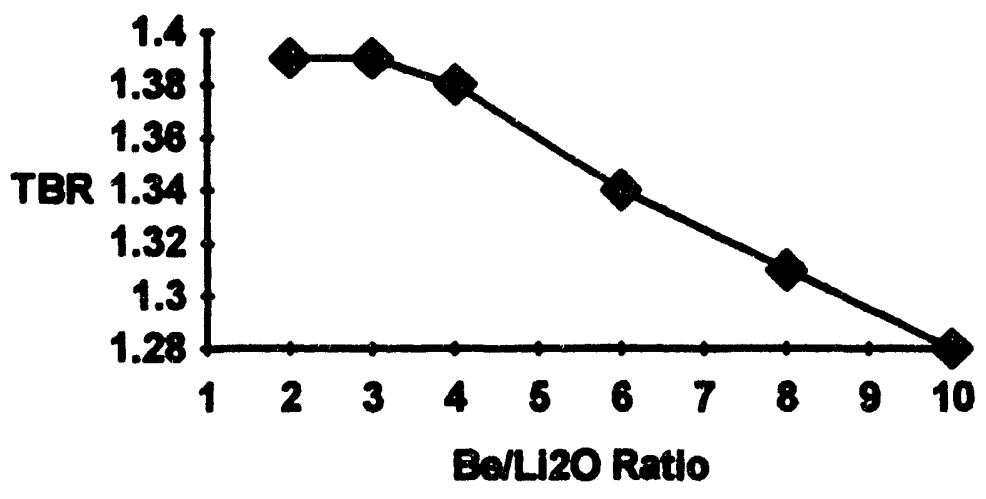

One can see from this figure that the TBR reaches a maximum of 1.39 as the beryllium to lithium oxide ratio becomes less than 3:1. 
Since beryllium is an excellent conductor of heat, it makes sense to maximize the amount of it in the pebble bed, consistent with having a good TBR; this will help the blanket's performance during a LOCA/LOFA. Hence, a beryllium to lithium oxide volumetric ratio of $3: 1$ is adopted for the mixed bed.

Now that the optimum beryllium to lithium oxide ratio has been established, the required inboard and outboard blanket and shield thicknesses can be determined. These thicknesses are driven by the requirement to have a TBR of 1.35 for the blanket, and by the shielding required to protect the magnetic field coils throughout the reactor lifetime. Because of space constraints, the inboard blanket/shield complex is designed to be thinner than the outboard blanket/shield complex. This makes sense from a neutronics point of view as well, since because of the toroidal geometry of a tokamak, the average neutron wall load is higher outboard blanket than inboard. The inboard and outboard blanket and shield of our design need to be thick enough, when the variation in neutron wall load is accounted for, so that the overall TBR is sufficient and the magnets are sufficiently protected.

To determine the proper inboard and outboard blanket and thicknesses, a neutronics model is created which includes the helium plenum region at the back of the blanket. Inclusion of the plenum region does not significantly affect the TBR, but does make the model more realistic. 
Figure 5.3. Model with Plenum Region
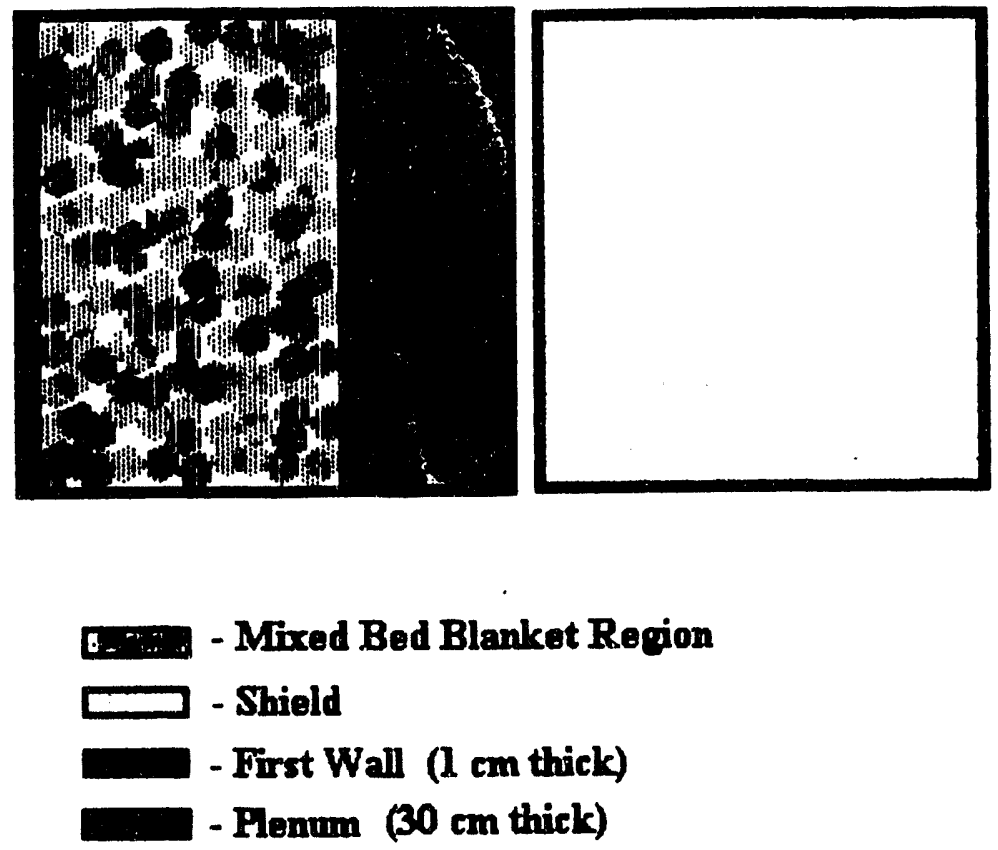

Figure 5.3 shows the model with the plenum region added. As shown in the figure, the plenum region thickness is fixed at $30 \mathrm{~cm}$, which is the thickness of the plenum region for the ARIES-I reactor nested shell plenum. ${ }^{12}$ Also like the ARIES-I plenum, the model's plenum is assumed to have a structural material volume fraction of 0.75 . For the present model the structural material is MT-9.

As discussed above, the beryllium to lithium oxide volumetric ratio is fixed at $3: 1$ for the mixed bed. In addition, for subsequent analysis the pebbles in the bed are assumed to be 90 percent dense. The pebbles need to have some porosity to allow the tritium generated inside them to escape. Table 5.3 below summarizes the composition of this model.

\footnotetext{
12F. Najmabadi, et. al., "The ARIES-I Tokamak Reactor Study, Final Report," UCLA-PPG-1323, 1991.
} 
Table 5.3. Volume Fractions in Model with Plenum Region

\begin{tabular}{|l|l|l|l|l|}
\hline$\frac{\text { Be:Li20 }}{\text { Ratio }}$ & $\frac{\text { First Wall }}{\text { Region }}$ & Blanket Region & $\frac{\text { Plenum }}{\text { Region }}$ & $\frac{\text { Shield }}{\text { Region }}$ \\
\hline $3: 1$ & $\begin{array}{l}0.28 \text { MT-9/ } \\
0.72 \text { Helium }\end{array}$ & $\begin{array}{l}\text { 0.360 Be/ 0.120 } \\
\text { Li2O/ 0.41 He/ } \\
0.11 \text { MT-9 }\end{array}$ & $\begin{array}{l}\text { 0.75 MT-9/ } \\
\text { 0.25 Helium }\end{array}$ & $\begin{array}{l}\text { 0.90 MT-9/ } \\
\text { 0.10 Water }\end{array}$ \\
\hline
\end{tabular}

TWODANT is again used to investigate the dependence of TBR on the blanket region thickness. With the shield region thickness fixed at $65 \mathrm{~cm}$, the blanket thickness is varied from $44 \mathrm{~cm}$ down to $24 \mathrm{~cm}$. Figure 5.4 shows the results. It can be seen that a blanket thickness of $40 \mathrm{~cm}$ will achieve a TBR of 1.35 .

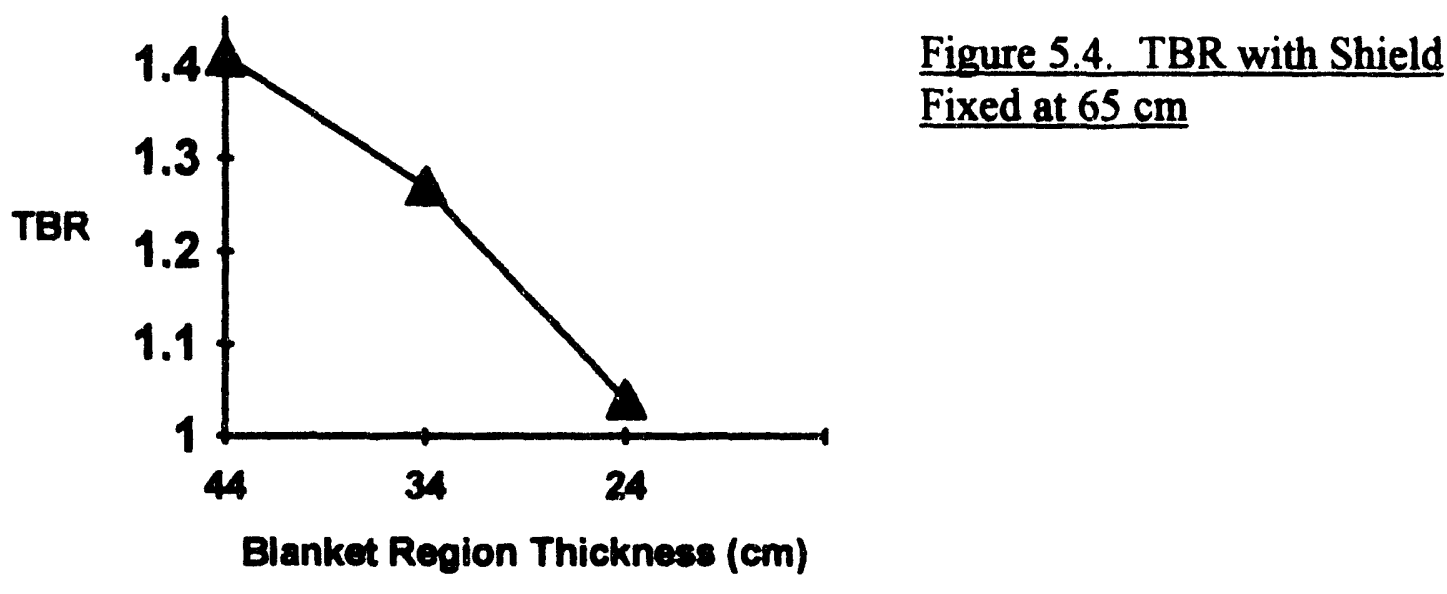

As mentioned previously, varying the shield thickness does not seem to affect TBR much, provided the shield is kept reasonably thick (cases down to $25 \mathrm{~cm}$ were investigated.) Therefore, the size of the inboard shield is determined solely by the requirement that the magnet's lifetime fluence limit is not exceeded. Using the inboard peak neutron wall load of $3.4 \mathrm{MW} / \mathrm{m}^{2}$ (see Table 5.1) and holding the blanket thickness constant at $44 \mathrm{~cm}$, obtains the results shown in Figure 5.5 . 
Figure 5.5. Lifetime Fluence to Magnet (Blanket

Thickness $=44 \mathrm{~cm}$ )

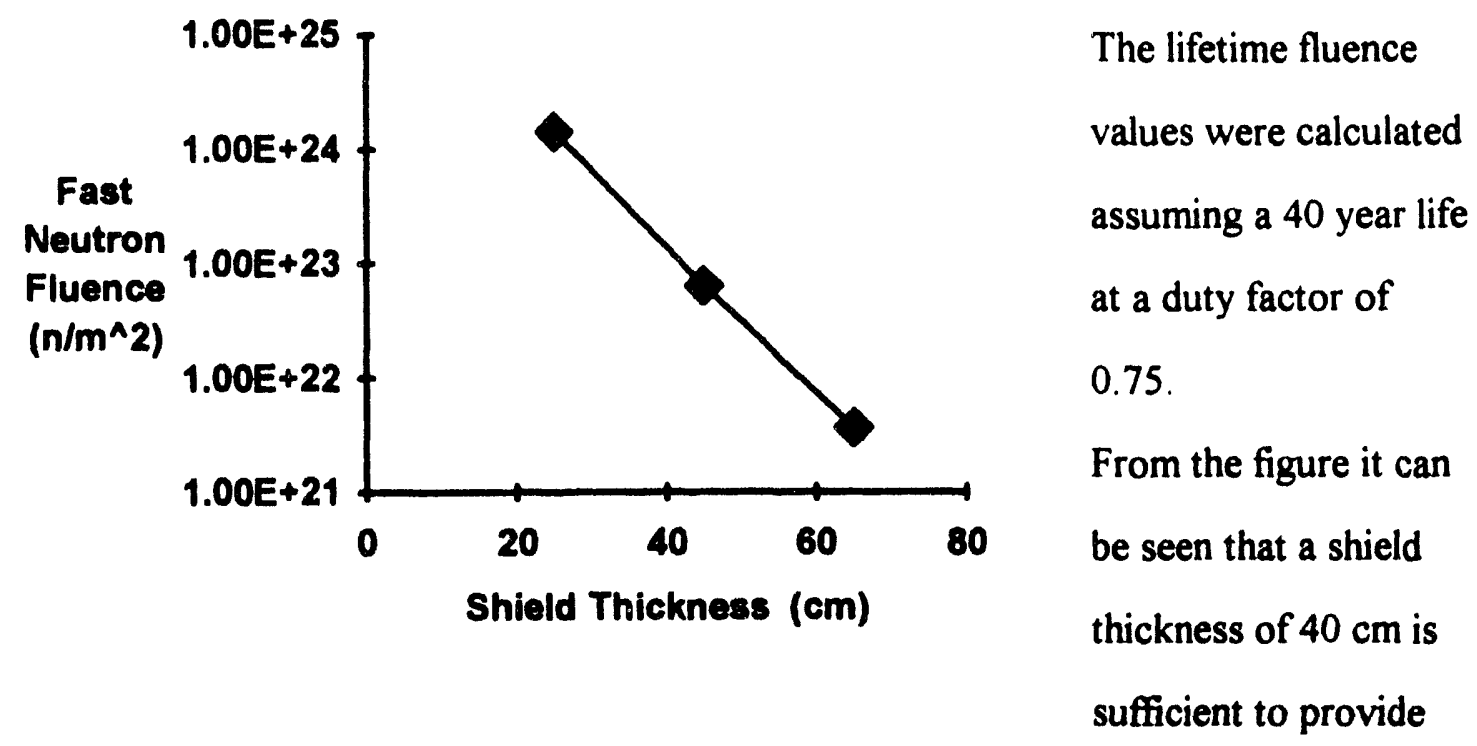

adequate shielding to keep the lifetime fast neutron fluence of the magnet below $1.0 \times 10^{23} \mathrm{n} / \mathrm{m}^{2}$, the fluence limit specified in Section 5.1 . Since only $40 \mathrm{~cm}$ of blanket is necessary for breeding, and since the shield is more than twice as effective as is the blanket is for attenuating fast neutrons, rather than using $44 \mathrm{~cm}$ of blanket and $40 \mathrm{~cm}$ of shield we will use $40 \mathrm{~cm}$ of blanket and $42 \mathrm{~cm}$ of shield. These blanket and shield thicknesses, plus $30 \mathrm{~cm}$ for the plenum, gives a total inboard blanket/shield thickness of $1.12 \mathrm{~m}$, which is reasonable based on past fusion reactor studies. (It is actually quite a bit thinner than the inboard blanket/shield thickness adopted for ARIES-I of $1.4 \mathrm{~m}$. This is due to the fact that the combination of MT-9 and water serves as a more effective shield than the silicon carbide/boron carbide combination used for ARIES-I.)

It should be noted that the fluence values shown in Figure 5.5 are somewhat higher than would actually be seen in a real reactor with this shield thickness, since the TWODANT calculations neglected the effect of the magnet structure which surrounds the magnet, and the cryostat which in turn surrounds the magnet structure. Both of these would add shielding "for free," thereby reducing the fluence seen by the magnet superconductor and 
insulator. However, the shielding effect of these two items is small, and neglecting them is conservative.

The thickness of the outboard shield is usually chosen based on what available space exists in the outboard region. The amount of space is governed for the most part by the toroidal field coil geometry. For the present work, 1 meter is assumed to be available for the outboard shield

Estimates of the required blanket/shield thicknesses for Blanket Design 1 have now been found. These estimates will be confirmed when the blanket thermal-hydraulic design and the corresponding blanket design details are known. Table 5.4 summarizes the dimensions of the inboard and outboard shields and blanket.

Table 5.4. Estimated Blanket/Shield Thicknesses for Blanket Design 1

\begin{tabular}{|c|l|l|}
\hline \multicolumn{2}{|c|}{ Inboard Section (Total Thickness $=1.12 \mathrm{~m}$ ) } \\
\hline Blanket Region & Plenum Repion & Shield Reqion \\
\hline 0.40 meters & 0.30 meters & 0.42 meters \\
\hline Outboard Section (Total Thickness $=1.7 \mathrm{~m})$ \\
\hline Blanket Rerion & Plenum Repion & Shield Region \\
\hline 0.40 meters & 0.30 meters & 1.0 meters \\
\hline
\end{tabular}




\subsubsection{Lithium Oxide and Beryllium Mixed Bed: Thermo-Hydraulics}

Now that estimates of the overall blanket and shield dimensions have been made, the detailed design of Blanket Design I can be completed. The basic dimensions left to be determined are the thickness of the mixed bed breeder regions, and the dimensions of the MT-9 structural shells. Before the thermo-hydraulic analysis is performed, the helium inlet and outlet temperatures must be specified.

The helium inlet temperature is specified as 250 degrees $\mathbf{C}$, consistent with previous a previous helium-cooled reactor design. ${ }^{13}$ The lower temperature limit ensures good steam quality on the secondary side of the steam generator. The upper temperature limit is set, ultimately, by the structural material temperature limit. Since ferritic steels in fusion applications are expected to be acceptable up to 550 degrees C (see Chapter 4), 450 degrees $C$ is chosen as the coolant outlet temperature." This gives 100 degrees of margin between the bulk coolant outlet temperature and the maximum allowable first wall temperature.

\subsubsection{Pebble Bed Conductivity ${ }^{14}$}

A critical issue in the determination of the mixed bed region thicknesses, and in the accident analysis of the blanket, is the conductivity of the mixed pebble bed regions of the blanket. Determining the conductivity of a heterogeneous mixture of solid pebbles and helium gas is difficult, especially when the pebbles are of two different materials. A

\footnotetext{
${ }^{13} \mathrm{M}$. Huggenberger and K. Schultz, "Helium-Cooled Solid Breeder Blanket Design for a Tokamak Reactor," Nuclear Technology/Fusion, Vol. 4, 1983, p. 456.
}

\# The average coolant outlet temperature is determined using the average wall loading for the outboard blanket of $4 \mathrm{MW}$ per square meter. For portions of the blanket receiving different wall loads, orificing and/or slightly different channel diameters would be necessary to ensure an outlet temperature of $\mathbf{4 5 0}$ degrees $\mathrm{C}$.

${ }^{14}$ Much of the following discussion on pebble bed characteristics comes from P. Gierszewski and J. Sullivan, "Ceramic Sphere-pac Breeder Design," Fusion Engineering and Design, Vol. 17, 1991, P. 95. 
number of correlations have been proposed to date, and recently these were compared with experimental data to determine which correlation works best for pebble beds of the type used in fusion applications. ${ }^{15}$ The correlation found to be most accurate is a modified version of the Schlunder, Zehner and Bauer (SZB) model. ${ }^{16} 17$ The details of the modified SZB model are given in the references. This model was found to be accurate to within $20 \%$ for a range of bed conductivities (experimental data was given for bed conductivities from 1 to $8 \mathrm{~W} / \mathrm{m} \cdot \mathrm{K}$ ).

In Section 5.2.1, the selected volumetric ratio of lithium oxide to beryllium in the mixed bed is $3: 1$. Hence, the mixed bed pebble sizes and volume fractions should be designed such that this ratio is achieved, while obtaining the highest thermal conductivity possible (to increase conduction in the blanket and help mitigate the effect of a LOCA/LOFA). To maximize the bed conductivity, the size of the beryllium pebbles (which have a very high conductivity) needs to be increased to the maximum possible. This maximum size is limited by packing considerations. For single sized spheres, the maximum packing fraction that can be achieved is about $60 \%$. This maximum packing fraction can only be achieved if the pebble diameter is less than or equal to roughly $1 / 5$ the bed thickness. Hence, the beryllium pebble diameter is limited by this constraint. The smallest bed thickness of Blanket Design 1 is about $15 \mathrm{~mm}$ (as will be shown in Section 5.2.2.2); this limits the maximum beryllium pebble diameter to $3 \mathrm{~mm}$.

\footnotetext{
${ }^{15}$ W.R Fundamenski and P. Gierszewski, "Comparison of Correlations for Heat Transfer in Sphere-Pac Beds," Canadian Fusion Fuels Technology Project report G-9181, August 1991.

${ }^{16}$ E. Schlunder, "Particle Heat Transfer," Proc. 7th Intl. Heat Transfer Conf., Munich, Vol. 1, May 1982, p. 195.

${ }^{17} \mathrm{M}$. Dalle-Donne and G. Sordon, "Heat Transfer in Pebble Beds for Fusion Blankets," Fusion Technology, Vol .17, 1990, p. 597.
} 
In between the beryllium spheres can now be placed smaller spheres of lithium oxide. If the smaller sphere's diameter is less than $1 / 7$ that of the larger spheres (say, $0.4 \mathrm{~mm}$ ), the total bed packing fraction can be increased to about $80 \%$, with the lithium oxide sphere packing fraction being equal to $20 \%$. It should be noted that lithium oxide pebbles as small as $0.3 \mathrm{~mm}$ have been fabricated, ${ }^{18}$ hence the beryllium and lithium oxide pebble sizes proposed for this bed should be easily fabricable. If both the lithium oxide and beryllium spheres have a porosity of $10 \%$, a volume fraction ratio for beryllium to lithium oxide of $3: 1$ is obtained, which is what is desired. The helium purge gas pressure, which is necessary to calculate the bed conductivity properly, is set at 1.5 bar (a typical value for fusion blanket designs.) Table 5.5 summarizes the characteristics of the pebble bed for Blanket Design 1.

\section{Table 5.5 Blanket Desion 1 Pebble Bed Parameters}

\section{Beryllium/Lithium Oxide}

Volumetric Ratio: 3:1

Beryllium Pebble Diameter: $3 \mathrm{~mm}$

Lithium Oxide Pebble Diameter: $0.4 \mathrm{~mm}$

Bed Packing Fraction: $\mathbf{0 . 8 0}$

Pebble Porosity: 0.10

Helium Purge Gas Pressure: 1.5 bar

The thermal conductivity of the pebble bed described in the above table is calculated using a modified version of the SZB model, using the code shown in Appendix 10." The results are shown in Figure 5.6.

${ }^{18}$ T.Terai. et.al., "Modeling of Tritium Recovery from CTR Solid Breeder," Fusion Engineering and Design, Vol. 8, 1989, p. 349.

\# The calculation of bed conductivity using the modified SZB model was greatly facilitated by P. Gierszewski, of the Canadian Fusion Fuels Technology Project, who graciously provided the author with 


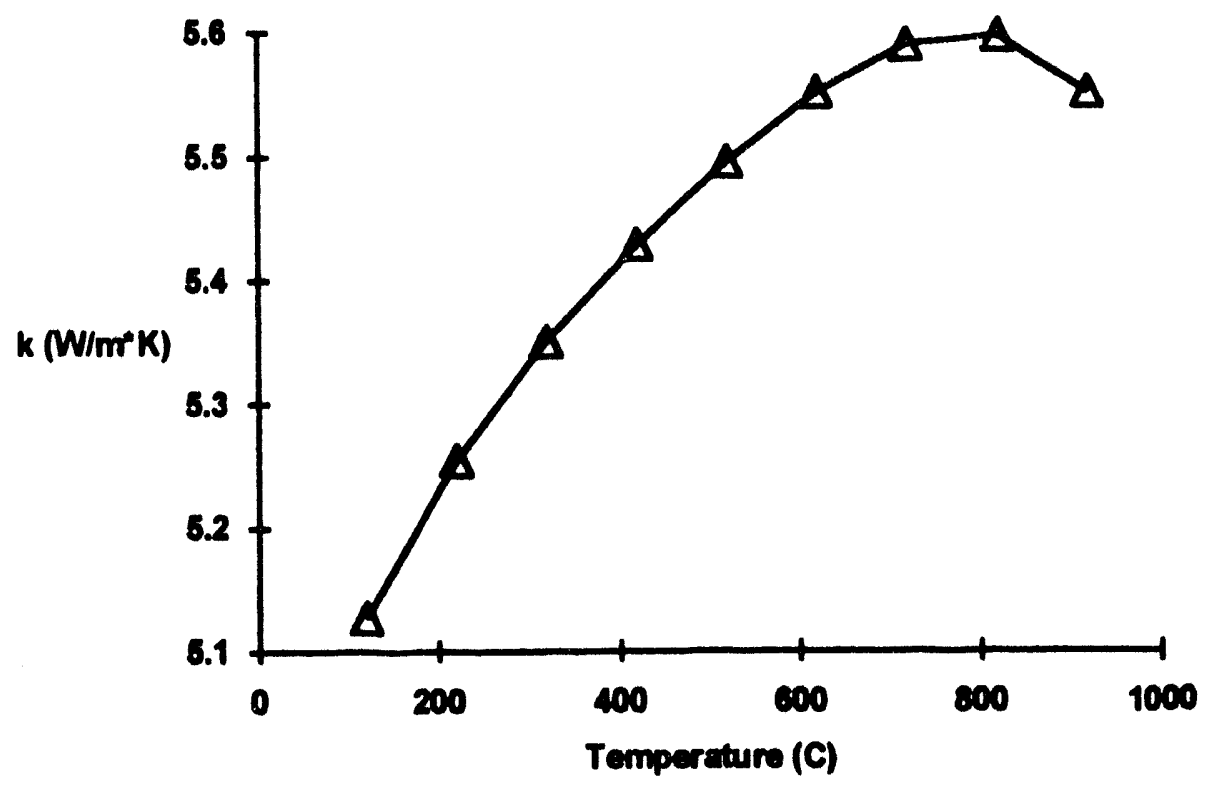

As can be seen in the figure, the bed conductivity stays between 5.1 and $5.6 \mathrm{~W} / \mathrm{m} \cdot \mathrm{K}$ over a wide temperature range. This is quite a low conductivity, despite the efforts made to increase the conductivity of the bed region. This low conductivity is the worst characteristic of pebble beds with respect to performance during a LOCA/LOFA.

\subsubsection{Mixed Bed Region Thicknesses}

To determine the thicknesses of the mixed pebble bed regions between the structural shells, it is necessary to know the volumetric heat generated in the mixed bed regions during reactor operation, and the conductivity of the mixed bed regions, which was discussed above. The bed thickness need to be made such that the peak temperature at the center of the bed does not exceed the upper temperature limit for lithium oxide, which is 800 degrees $C$ (Chapter 4). The amount of lithium oxide that operates below Appendix 10. 
the one-day tritium residence temperature of 325 degrees $\mathrm{C}$ should also be minimized. In addition, the MT-9 structural temperature limit of 550 degrees $C$ must not be exceeded.

The combination of these requirements means that the mixed bed region thickness should be as thick as possible, subject to the constraints on the maximum structural and breeder temperature limits. Making the breeder regions as thick as possible also minimizes the amount of structural material necessary in the blanket, thus minimizing the afterheat and mitigating the consequences of a LOCA/LOFA. As it turns out, for the breeder regions closest to the first wall, the structural temperature limit governs. For the regions further back, the breeder peak temperature is most limiting.

The volumetric heating in the mixed bed regions decreases exponentially as one moves from the front of the blanket to the rear. Thus, designing the mixed bed regions to be thicker as one goes toward the back of the blanket will ensure that the maximum and minimum temperatures in each region are similar.

In calculating the temperatures in the mixed bed regions (and the structural shells), it is assumed that the heat transfer coefficient into the helium at the channel wall is 1,800 $\mathrm{W} / \mathrm{K} \cdot \mathrm{m}^{2}$, a typical value used in the ARIES-I design. ${ }^{19}$ This value results in a reasonable pressure drop (and hence helium pumping power) for the blanket. The required pumping power and pressure drop will be calculated later. It was also assumed that the structural shell thicknesses are all $10 \mathrm{~mm}$. The actual channel/shell dimensions depend on the thickness of the mixed bed regions, as will be seen, but $10 \mathrm{~mm}$ is a reasonable value to start with. The type of model used for these calculations is shown in

19F. Najmabadi, et.al., "The ARIES-I Tokamak Reactor Study, Final Report," UCLA-PPG-1323, 1991. 
Figure 5.7, and the resultant bed thicknesses are shown in Table 5.6. For further details on how the bed thicknesses are determined, see Appendix 3.

Figure 5.7. Model for Mixed Bed Thicknesses
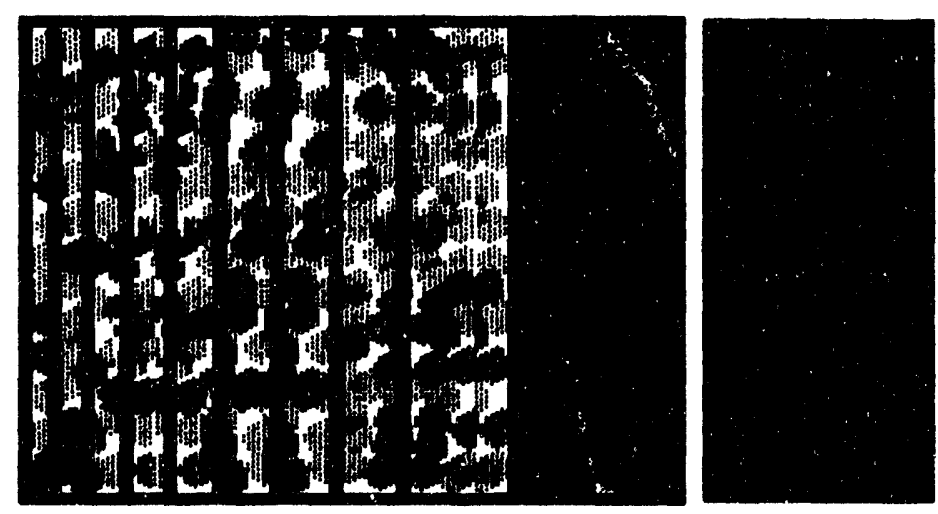

L.... - Mirod Bod

- Strield

- Structural Shells (1 cm thick)

- Plenum 
Table 5.6. Blanket Design 1 Bed Region Thicknesses

\begin{tabular}{|c|c|c|}
\hline \multicolumn{2}{|c|}{ Number of Bed Regions: } & 13 \\
\hline \multicolumn{3}{|c|}{ Bed Region Thicknesses } \\
\hline Region Number & \multicolumn{2}{|c|}{ Thickness (mm) } \\
\hline 1 & \multicolumn{2}{|c|}{12} \\
\hline 2 & \multicolumn{2}{|c|}{14} \\
\hline 3 & \multicolumn{2}{|c|}{15} \\
\hline 4 & \multicolumn{2}{|c|}{17} \\
\hline 5 & \multicolumn{2}{|c|}{19} \\
\hline 6 & \multicolumn{2}{|c|}{21} \\
\hline 7 & \multicolumn{2}{|c|}{24} \\
\hline 8 & \multicolumn{2}{|c|}{28} \\
\hline 9 & \multicolumn{2}{|c|}{32} \\
\hline 10 & \multicolumn{2}{|c|}{36} \\
\hline 11 & \multicolumn{2}{|c|}{40} \\
\hline 12 & \multicolumn{2}{|c|}{40} \\
\hline 13 & \multicolumn{2}{|c|}{43} \\
\hline
\end{tabular}

\subsubsection{Structural Shell Thicknesses}

The dimensions of the MT-9 shells are determined by the size of the coolant passages inside the shells. The thickness of each of the shells will be varied according to how much heat flows into the particular shell's coolant during normal operation. The shell next to the first wall has the highest heat load, and each successive shell toward the rear of the blanket has a smaller heat load. These smaller heat loads necessitate a lower mass flow rate through each shell to maintain the desired coolant inlet/outlet temperature 
difference of 200 degrees C. However, the velocity of the coolant must not be allowed to drop significantly as one lowers the mass flow rate, since this will also lower the surface heat transfer coefficient $h$, which is fixed at $1,800 \mathrm{~W} / \mathrm{K} \cdot \mathrm{m}^{2}$ in the analysis above for the mixed bed regions. To allow the mass flow rate to decrease but keep $\mathrm{h}$ relatively constant, the diameter of the channels must also decrease as one travels deeper into the blanket. This section will present the calculations which determine the appropriate coolant channel diameters and corresponding shell thicknesses.

To determine the appropriate size range for the channels, it is desirable to maximize the potential for cooling by natural circulation of helium coolant in the tubes. To get an estimate as to what size would maximize natural circulation cooling, a Loss of Flow Accident (LOFA) in a simplified coolant flow loop is modeled as shown in Figure 5.8. As shown in this figure, the flow loop includes a single coolant channel, inlet and outlet plena, the steam generator and hot and cold leg piping.

\section{Figure 5.8. Simplified Flow Loop for Coolant Channel Sizing}

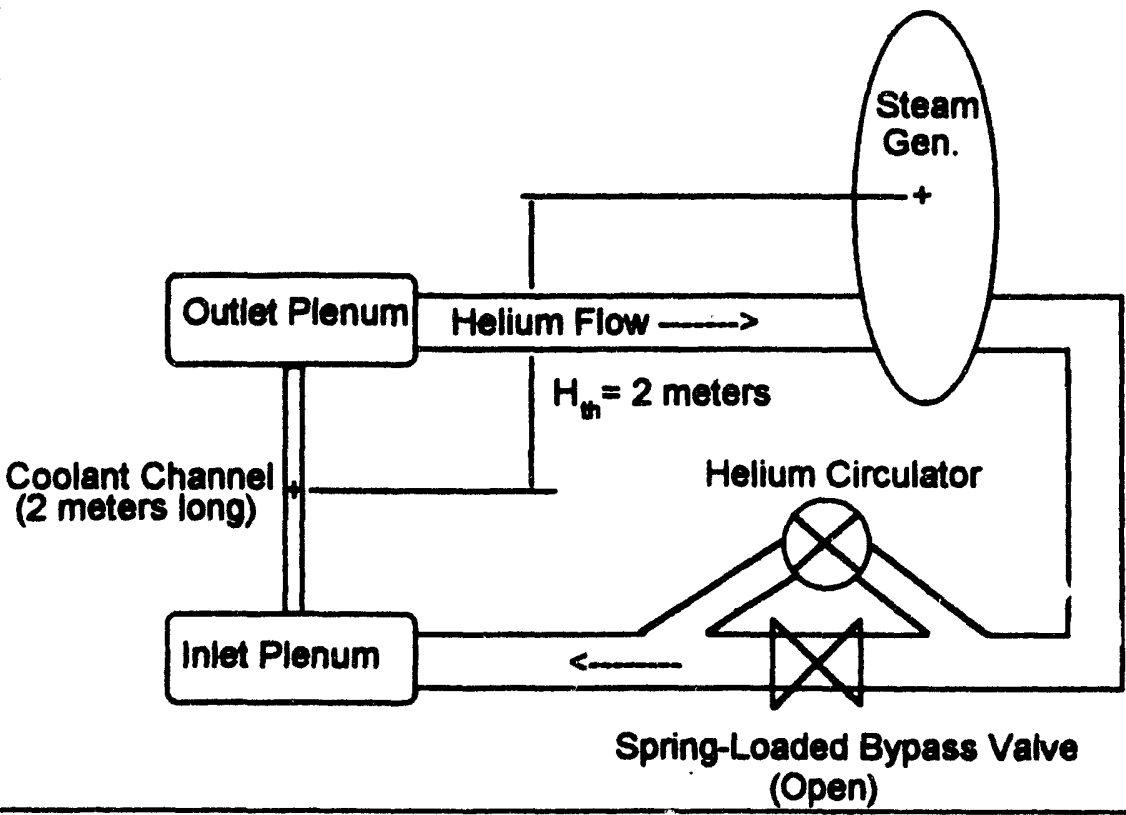


As can be seen, the helium circulator is assumed to be equipped with a bypass valve which will open automatically upon loss of circulator head (it could be a spring loaded flapper valve held shut during normal operation by the circulator's differential pressure, and opening via spring and/or gravity force when the differential pressure drops below a certain point.) Also shown is a vertical, 2 meter long coolant channel. This channel length is appropriate for a typical first wall tube for the nested shell-type blanket design. Since the steam generator is assumed to be located above the coolant outlet plenum, $\mathrm{H}_{\mathrm{w}}$ $=2$ meters represents the vertical distance available for development of a natural circulation driving head. This driving head is developed by decay heat from the coolant passage walls causing a change in temperature between the coolant channel inlet and outlet.

It should be noted that although this model does not depend on having the coolant channels in the reactor oriented vertically; in fact, the channels in the nested shell design are horizontal. The 2 meters of natural circulation driving head is developed by ensuring that the steam generator is located at least 2 meters above the top of the top if the reactor.

The coolant channel inlet temperature is assumed to be constant at 250 degrees $C$ (the steam generator is assumed to have a passive secondary water circulation system capable of maintaining this helium coolant outlet temperature). The helium coolant outlet temperature, and hence the maximum coolant channel wall temperature, varies depending on the assumed diameter of the coolant channel.

As the coolant channel diameter is increased (the channel is assumed to be cylindrical), the pressure drop in the channel goes down, which tends to help the natural circulation 
flow, thereby lowering the maximum wall temperature. However, the wall thickness must increase to contain the helium pressure (cases from 2 to $10 \mathrm{MPa}$ are examined.) Higher pressure increases the maximum wall temperature, because a thicker wall deposits more heat into the channel, increasing the coolant outlet temperature. In addition, a thicker wall has a higher associated temperature increase across the wall thickness. Figure 5.9 shows the results of this analysis. The analysis is performed for MT-9 coolant channels, and the afterheat is assumed to be the maximum value for the first wall tubes, which occurs at shutdown. The plasma is assumed to be extinguished at the onset of the LOFA, and the afterheat calculations assume 3 full-power-years of operation at a neutron wall load of $4 \mathrm{MW} / \mathrm{m}^{2}$ (see Section 5.2.3.2 for a discussion of MT-9 afterheat, and see Appendix 2 for further details on the analysis.)

Figure 5.9. Maximum First Wall Temperatures During Natural Circulation

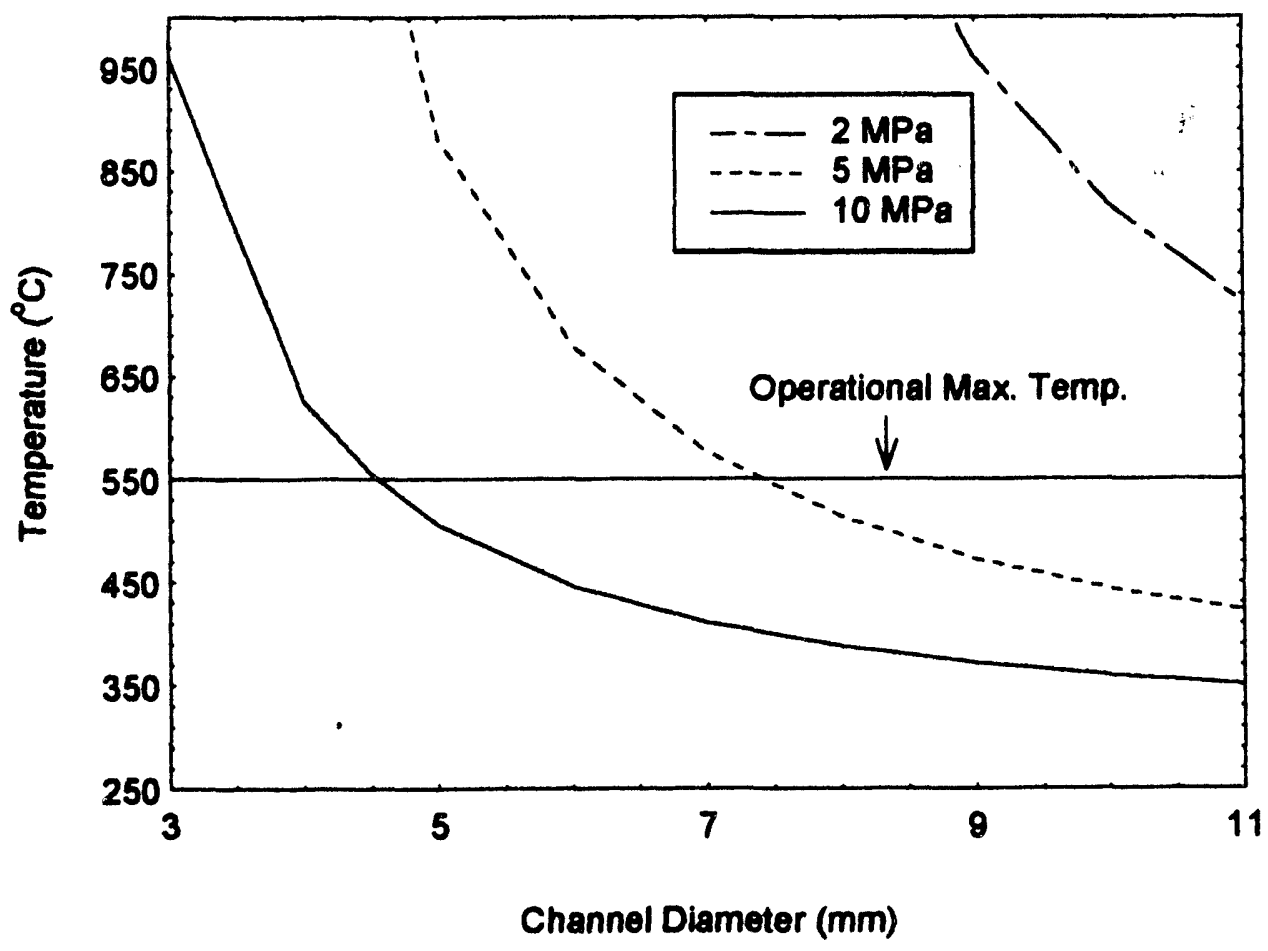


As can be seen in the figure, natural circulation can keep the maximum temperature of the coolant channel comparable to or even well below the maximum allowable operating temperature of 550 degrees $\mathrm{C}$, as long as the coolant pressure and channel diameter are chosen appropriately. The figure shows a range of helium coolant pressures; a pressure of $2 \mathrm{MPa}$ is clearly too low to obtain satisfactory cooling by natural circulation for any reasonable channel diameter. $10 \mathrm{MPa}$ is adopted as the helium pressure for subsequent analyses. For this pressure, channel diameters above $4 \mathrm{~mm}$ will give sufficient natural circulation performance. The specific channel diameters are determined in the discussion that follows.

\subsubsection{Cooling of the First Wall}

The structural shell which forms the first wall has a significantly higher heat load than the other shells in the blanket. An erosion layer of $2 \mathrm{~mm}$ thick MT-9 is also required on the plasma side of the first wall, to account for wall erosion during operation. ${ }^{20}$ This additional thickness increases the temperature drop across the first wall. The shell forming the first wall also experiences an extremely non-uniform heat load, since the plasma heat comes only from the plasma side. A non-uniform heat load of this magnitude has been shown analytically and using numerical models to significantly reduce the effective surface heat transfer coefficient h of the helium coolant. ${ }^{21}$ For

\footnotetext{
${ }^{20}$ This thickness value is based on values used in past studies, including the NET Pebble Bed Canister (M. Dalle Donne, et.al, "Pebble Bed Canister: A Ceramic Breeder Blanket with Helium Cooling for NET," Proc. 14th Symp. on Fussion Technology, 1986, p. 423.) which has a $2 \mathrm{~mm}$ steel erosion layer, and the SSTR (S. Mori, et.al., "Blanket and Divertor Design for the Steady State Tokamak Reactor," Second International Symposium on Fusion Nuclear Technology, 1991, p. 55.) which has a $2.5 \mathrm{~mm}$ steel layer. If this thickness should prove insufficient, the $2 \mathrm{~mm}$ of steel could be used as a base to attach ceramic tiles. The addition of ceramic tiles would not change the heat load to the first wall, hence would not affect this analysis.
}

${ }^{21}$ see, for example, W.C. Reynolds, "Turbulent Heat Transfer in a Circular Tube with Variable Circumferential Heat Flux," Int. J. Heat and Mass Transfer, Vol. 6, 1963, p. 445, and M. Z. Hasan, 
conditions similar to the case of interest here, the $h$ calculated for the non-uniform heat load is $37 \%$ lower than the corresponding $h$ for a uniform heat load. ${ }^{22}$

Figure 5.10. Pumping Power for Different First Wall Channels (10 MPa Coolant Pressure)

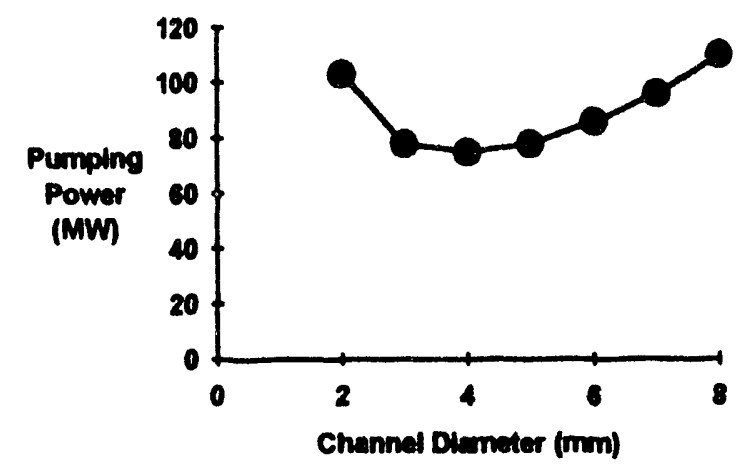

All of these factors mean that in order to maintain the maximum temperature of the first wall below the required value of 550 degrees C, the coolant channel for the first wall requires a very high coolant velocity to maintain $\mathrm{h}$ sufficiently high. The high velocity results in

a high mass flow rate and presstie drop through the channel. Since pumping power is proportional to the product of the mass flow rate and the pressure drop, the first wall channel requires a substantial pumping power to maintain the required flow through it. Because this pumping power is so high, the first wall cooling channel diameter is chosen to minimize the pumping power required to cool the first wall. Assuming a blanket module width of 2 meters (meaning that the length of first wall channel exposed to the plasma is 2 meters), and a first wall surface heat deposition of $1 \mathrm{MW} / \mathrm{m}^{2}$, Figure 5.10 shows the pumping power necessary to cool the entire first wall of the reactor (of major radius 6 meters and minor radius 1.5 meters) as a function of coolant channel diameter (assuming circular channels, and a helium circulator efficiency of 0.90 ). This figure was generated by determining the lowest coolant channel velocity which would result in a maximum first wall temperature of 550 degrees $C$. Appendix 2 contains details on the

"Effects of Nonuniform Surface Heat Flux and Uniform Volumetric Heating on Blanket Design for Fusion Reactors," Fusion Technology, Vol 16, 1989, p. 44.

22this value is recommended in the ARIES-I reactor study, which uses helium coolant at $10 \mathrm{MPa}$, and has first wall conditions similar to those of interest here (F. Najmabadi, et.al., "The ARIES-I Tokamak Reactor Study, Final Report," UCLA-PPG-1323, 1991, p. 8-98.) 
generation of this figure. A first wall channel diameter of $4 \mathrm{~mm}$ is clearly optimal, requiring a pumping power of $75 \mathrm{MW}$; this diameter also gives acceptable natural circulation results. Table 5.7 shows the resulting data for the first wall channel. ${ }^{\#}$

Table 5.7. First Wall Channel Data

\begin{tabular}{|c|c|c|c|}
\hline$\frac{\text { Channel Diameter }}{(\mathrm{mm})}$ & $\frac{\text { Channel Pressure }}{\text { Drop }(\mathrm{Pa})}$ & $\frac{\text { Channel } h}{\left(W / m^{2} \cdot K\right)}$ & $\frac{\text { Channel Mass Flow }}{\text { Rate }(\mathrm{kg} / \mathrm{s})}$ \\
\hline 4 & 460,000 & 6500 & 0.061 \\
\hline
\end{tabular}

Figure 5.11. First Wall Shell Arrangement

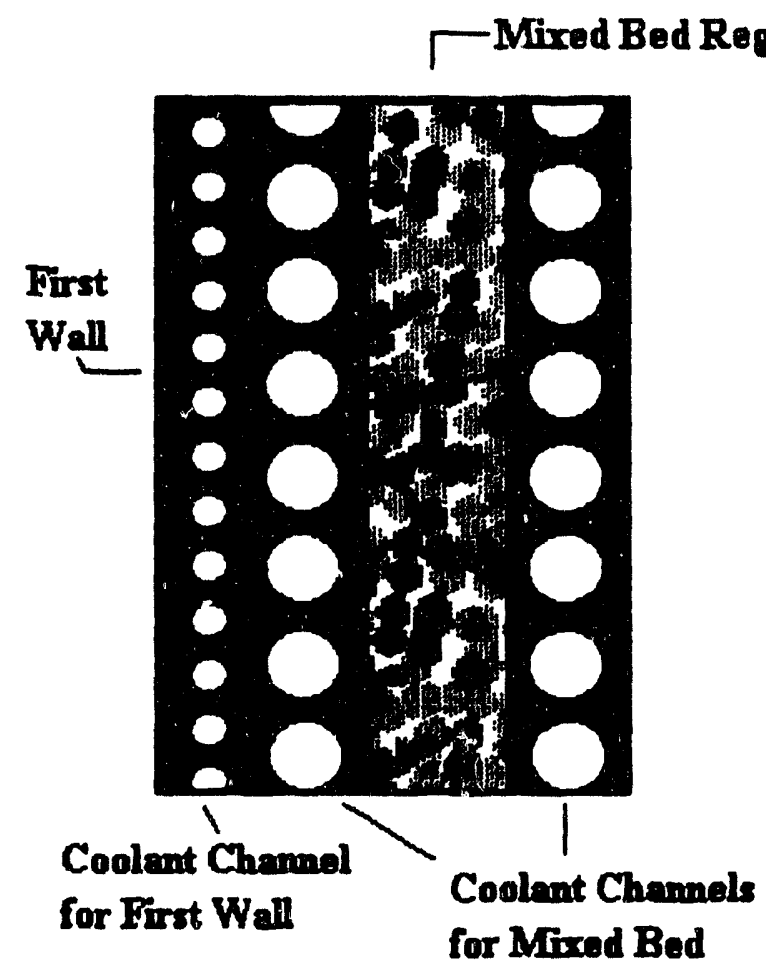

The high mass flow rates necessary in the first wall coolant channel to keep the maximum first wall temperature below 550 degrees result in a coolant outlet temperature well below the 450 degree $C$ value desired for thermal efficiency. It is not necessary or desirable, therefore, to have such high mass flow rates in the other blanket coolant channels. In addition, having a mass flow rate so high requires a

\footnotetext{
\# Note that $4 \mathrm{~mm}$ is a relatively small diameter from a fabrication stancopint. Concerns with the required fabrication tolerances might well make a larger channel diameter desirable, despite a the resulting increase in required pumping power. To address such fabrication issues in detail is beyond the scope of the present work.
} 
substantial pumping power, as shown above. If each blanket shell needed such a high pumping power to sustain the flow of coolant inside, the total pumping power required for the blanket would be unacceptably high.

Blanket Design 1 has two structural shells with interior coolant channels just behind the first wall, as shown in Figure 5.11. The first shell's coolant has a very high mass flow rate (see Table 5.7), and thus this shell will have a high pressure drop and pumping power. Nearly all of the heat from the first wall will be removed by the coolant in this shell. Directly behind this first wall shell is another shell which will remove the heat from the front side of the first mixed bed breeder region. This shell's coolant, and the coolant of all the other shells, has a much lower flowrate. The outlet temperatures for these shells are much higher, and the pressure drop and pumping power required for these shells are much lower. It would have been possible, of course, to have only one shell which cooled both the first wall and the front half of the first mixed bed region. However, the added heat load of the mixed bed region increases the pumping power required for this shell substantially, so this option was not pursued.

Since the pressure drop of the first wall coolant channel is much greater than any of the other channels, it is necessary to have a separate, higher pressure helium circulator to supply coolant to the first wall channels. This adds to the system complexity, but also allows the first wall region to have two, independent sources of coolant flow. If the circulator supplying coolant to the first wall coolant channels failed, the first wall region would still have some cooling as long as the lower pressure circulator supplying coolant to the channel just behind the first wall coolant channel continued to operate. 


\subsubsection{Cooling of the Mixed Bed Regions}

The cooling channels behind the first wall are designed to remove the heat from the mixed bed regions, heating the coolant from the inlet temperature of 250 degrees $\mathrm{C}$ to the desired outlet temperature of 450 degrees $C$ in the process. Since the thicknesses of the mixed bed regions (discussed in Section 5.2.2.3 above) are based on the surface heat transfer coefficient to the helium being $1,800 \mathrm{~W} / \mathrm{K}-\mathrm{m}^{2}$, the velocity in the channels must be high enough to produce this heat transfer coefficient. Despite the fact that the bed regions get thicker, the amount of heat flowing into each channel decreases as one goes towards the back of the blanket (both because the volumetric heating decreases and because the channels are getting shorter). Hence, the mass flow rate needed to obtain the desired inlet/outlet temperature difference becomes smaller. To keep the channel velocities high enough, the channel diameter must decrease correspondingly as one goes toward the back of the blanket. Using the mixed bed thicknesses in Table 5.6, the channel diameters required to maintain the required $\mathrm{h}$ and the inlet/outlet temperature difference of 200 degrees are shown in Table 5.8. The mass flow rates in these channels vary from $0.007 \mathrm{~kg} / \mathrm{s}$ per channel in the channels near the front of the blanket to 0.002 $\mathrm{kg} / \mathrm{s}$ per channel in the rearmost channel. 
Table 5.8. Required Structural Shell Channel Diameters (excepting the first wall channel)

\begin{tabular}{|c|c|c|c|}
\hline Channel $^{*}$ & $\frac{\text { Channel Diameter }}{(\mathrm{mm})}$ & $\begin{array}{c}\text { Channel Pressure } \\
\text { Drop }(\mathrm{Pa})\end{array}$ & $\begin{array}{c}\text { Channel h } \\
\left(\mathrm{W} / \mathrm{m}^{2} \cdot \mathrm{K}\right)\end{array}$ \\
\hline $1^{* *}$ & 9 & 6210 & 1850 \\
\hline 2 & 9 & 6210 & 1850 \\
\hline 3 & 9 & 6000 & 1850 \\
\hline 4 & 9 & 5600 & 1830 \\
\hline 6 & 9 & 5360 & 1820 \\
\hline 7 & 8 & 6900 & 2000 \\
\hline 8 & 8 & 6250 & 1970 \\
\hline 9 & 8 & 5720 & 1940 \\
\hline 10 & 7 & 4920 & 1870 \\
\hline 11 & 6 & 5560 & 1970 \\
\hline 12 & 5 & 5710 & 2000 \\
\hline 13 & 5 & 4450 & 1780 \\
\hline $14^{* *}$ & 5 & 1300 & $1100^{* * *}$ \\
\hline
\end{tabular}

* starting from the channel directly behind the first wall channel.

** these channels have an outlet temperature below 450 degrees $C$ because they have a breeder region only on one side.

*** a lower $h$ is acceptable for these channels without exceeding either the maximum breeder or maximum structural temperature limits, because of the lower volumetric heating at the rear of the blanket. Because of the low pressure drop in these channels, flow restrictors will probably be necessary to balance the flow between channels.

Table 5.9 shows relevant blanket temperatures which result from the above thermalhydraulic design of Blanket Design 1. The minimum temperatures occur in the vicinity of the coolant inlet, and the maximum temperatures occur near the coolant outlet. The maximum temperatures are below the upper temperature limits for the structural and breeder materials, and the lower temperature bound of the lithium oxide is only slightly 
below the desired lower limit of 325 degrees. This means that some of the breeder around the helium inlet region will be slightly below the temperature required to have a tritium residence time of $I$ day or less. Thus the blanket will have a somewhat higher tritium inventory in the breeder. However, most of the tritium in Blanket Design 1 will be in the beryllium. As is shown in Appendix 4, the tritium inventory in the beryllium is conservatively estimated to be about $3 \mathrm{~kg}$, compared to a few tens of grams in the breeder.

\section{Table 5.9. Blanket Design 1 Operating Temperatures (deg. C)}

\begin{tabular}{|l|l|}
\hline Helium Inlet Temperature: & 250 \\
\hline Helium Outlet Temperature: & 450 \\
\hline Maximum MT-9 Temperature: & 550 \\
\hline Maximum $\mathrm{Li}_{2}$ O/Be Temperature: & 785 \\
\hline Minimum $\mathrm{Li}_{2}$ O/Be Temperature: & 309 \\
\hline
\end{tabular}

The final design of the Blanket Design 1 was described in the above paragraphs. A TWODANT nu was performed on the final design to confirm the blanket tritium breeding ratio. This run indicated that the blanket has a TBR in excess of 1.40. This TBR exceeds the required value of 1.35 .

\subsubsection{Required Punping Power for Blonket and Helium Loop}

As discussed above, the first wall cooling channels have a much greater mass flow rate and associated pressure drop than do the mixed bed channels. As was seen, the pumping power necessary to supply cooling to the first wall channels is $75 \mathrm{MW}$. By comparison, the pumping power required for all the other channels combined is negligible. In 
addition to the pumping power required to move the coolant through the blanket channels, power is also required to move coolant through the plenum region, the steam generator, and the rest of the helium loop piping. Table 5.10 shows estimates of the pressure drops associated with these items.

\section{Table 5.10. Pressure Drops of Associated Components and Piping ( $\mathrm{Pa})$}

\begin{tabular}{|c|c|c|}
\hline Plenum Region $^{*}$ & Steam Generator & $\frac{\text { Piping to/from Steam }}{\frac{\text { Generator and }}{\text { Circulator }}}$ \\
\hline 18,310 & 30,000 & 36,900 \\
\hline
\end{tabular}

*based on the ARIES-I reactor plenum pressure drop, including pressure drops from turns, contraction and expansion (F. Najmabadi, "The ARIES-I Tokamak Reactor Study, Final Report," UCLA-PPG-1323, 1991, p. 8-81.)

** see M. Huggenberger and K. Schultz, "Helium-Cooled Solid Breeder Blanket Design for a Tokamak Reactor, Nuclear Technology/Fusion, Vol. 4, 1983, p.456.

The total mass flow rate for the entire blanket (assuming a reactor with a major radius of 6 meters and a minor radius of 1.5 meters) is about $2730 \mathrm{~kg} / \mathrm{s}$. Using this value, and the pressure drops of the above table (which total $85,210 \mathrm{~Pa}$ ), the pumping power to circulate coolant through the plenum regions, the steam generator and the irlet and cutlet piping can be determined. Assuming a helium circulator efficiency of 0.90 , a pumping power of $28 \mathrm{MW}$ is obtained. Adding this to the $75 \mathrm{MW}$ associated with the first wall yields a total pumping power of $103 \mathrm{MW}$ for the entire first wall and blanket system. Although this value is large, it is reasonable for a helium-cooled fusion reactor design. ${ }^{23}$

${ }^{23} \mathrm{sec}$, for example, G. Melese and R. Katz, Thermal and Flow Design of Helium-Cooled Reactors, American Nuclear Society, La Grange, Il, p. 369. 

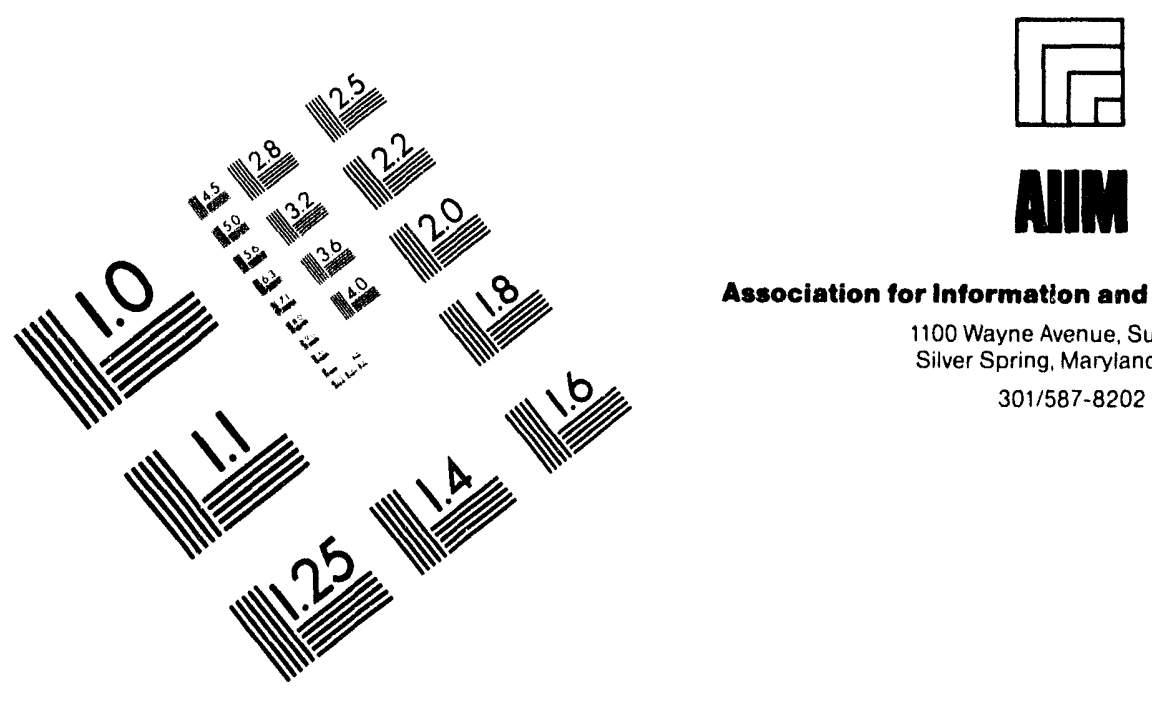

Association for Information and Image Management

1100 Wayne Avenue, Suite 1100

Silver Spring, Maryland 20910

301/587-8202

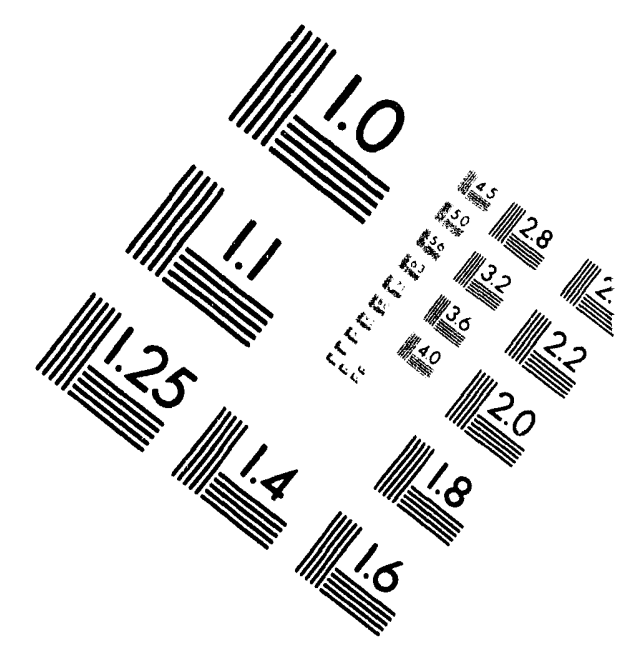

\section{Centimeter}

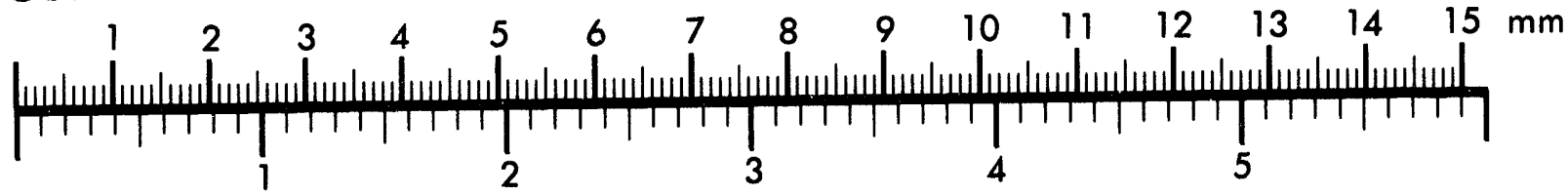
Inches
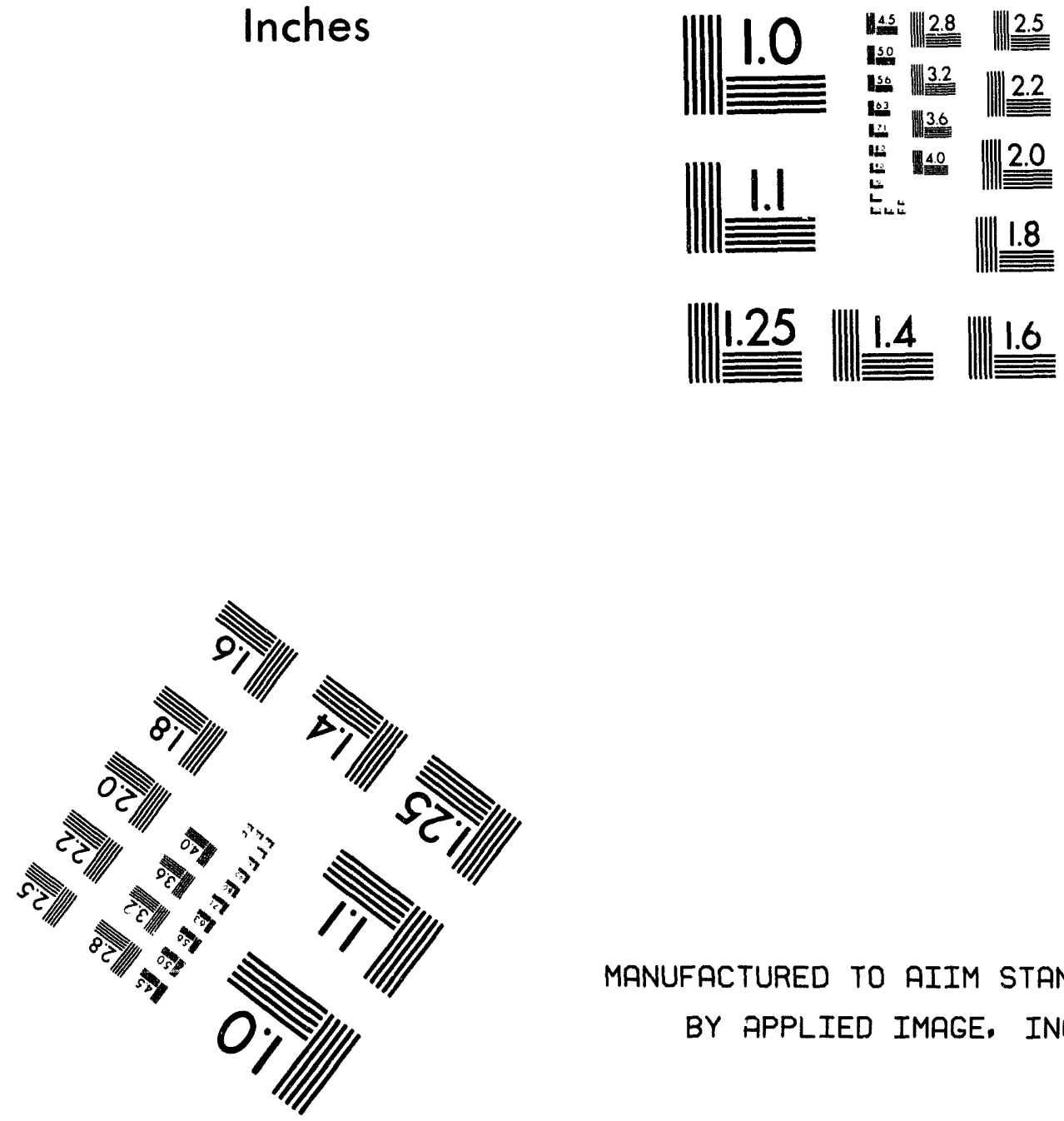

MANUFACTURED TO AIIM STANDARDS

BY APPLIED IMAGE, INC.

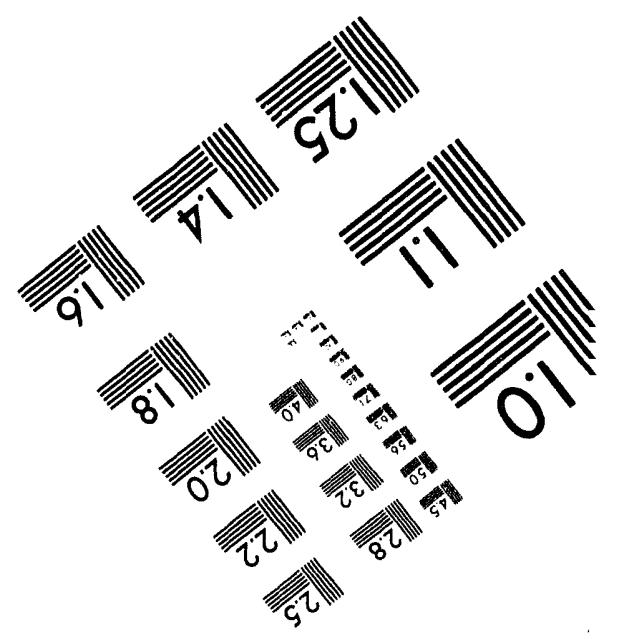



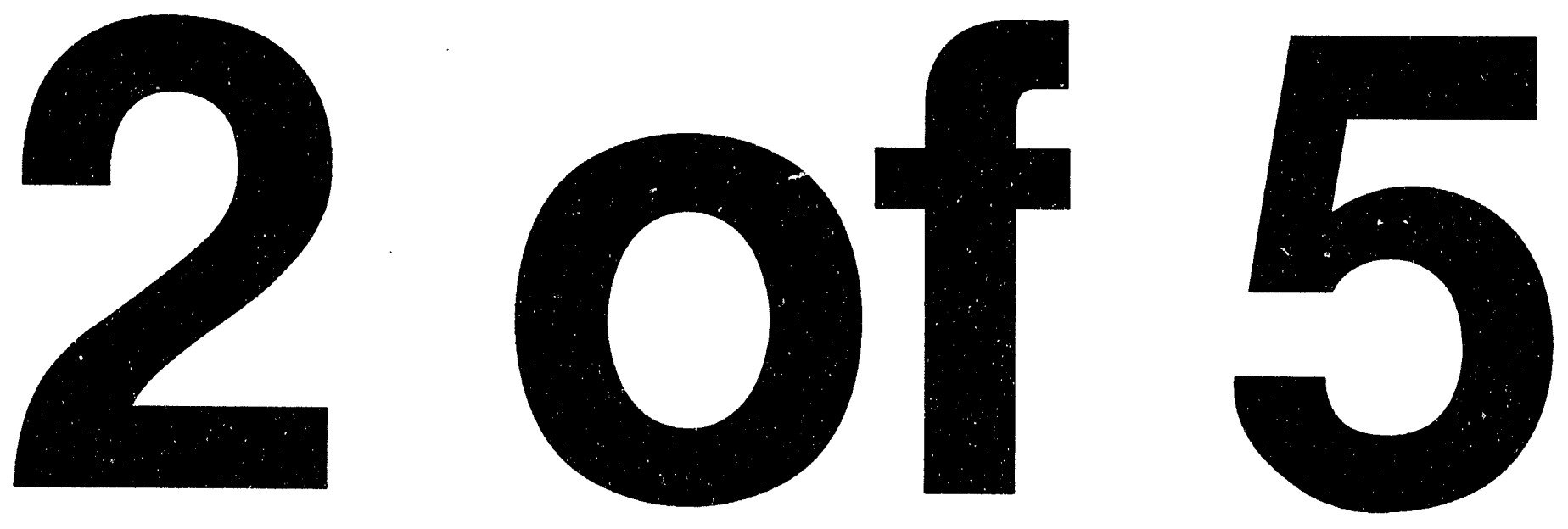


\subsubsection{Blanket Design 1: Accident Analysis}

The accidents of interest here involve loss of cooling to the blanket regions. The loss may be due to loss of helium coolant (a LOCA - Loss of Coolant Accident) due to a rupture in a coolant pipe or channel, or it may be due to a loss of coolant flow. The most severe loss of flow accident (LOFA) is the stoppage of all helium circulators at once, perhaps due to a loss of site power. The following paragraphs discuss the analysis of the LOFA and LOCA. The accident analysis in this section will be done for the outboard blanket region. This is becruse, as was shown in the Section 5.1, the outboard region has a higher average neutron wall load, hence it has more severe afterheating and thus reaches higher temperatures than would be reached by the inboard blanket region.

An important input to the blanket accident analysis is the behavior of the plasma after the accident. The temperature response of the blanket, especially the first wall, to the accident depends on how soon after the accident the plasma is extinguished.

Unfortunately, currently there is not much information from which to postulate what the plasma response following a particular accident might be. For a LOFA which occurs due to a loss of site power, it is reasonable to assume that the plasma control system would also be disabled, resulting in a rapid extinguishing of the plasma, probably via a disruption. The helium circulators do not immediately stop upon loss of power. Rather, there is a "coastdown" period during which the circulators slow down and coolant flow gradually lessens. Based on the above assumptions, for the purposes of the LOFA accident, the plasma can be considered to be shut off instantly. An "instant plasma shutoff" model has been used previously for the analysis of fusion blanket LOFAs. ${ }^{24}$ For conservatism in the LOFA analysis, no credit is taken for heat removal due to the circulator coastdown.

\footnotetext{
${ }^{24}$ See J. Massidda and M. Kazimi, "Thermal Design Considerations for Passive Safety of Fusion Reactors," MIT Plasma Fusion Center Report PFC/RR-87-18, October 1987.
} 
For a LOCA, it will take on the order of tens of seconds to fully depressurize the coolant system, even assuming a large helium header completely fails. ${ }^{25}$ In a recent study, it was assumed that the plasma could be shutdown within 5 seconds upon detection of an accident. ${ }^{26}$ Hence, assuming that the plasma control system shuts off the plasma within a few seconds of detection of loss of coolant pressure, the plasma will be shut off long before the coolant system is fully depressurized. Note that there are a number of ways to detect such a loss of pressure, such as a simple pressure detector in the coolant system, or an overpressure detector in the reactor building. Hence, as with the LOFA case, for the purpose of the LOCA analysis the plasma can be considered to be shut off instantly. For conservatism in the analysis, no credit is taken for heat removed by the escaping coolant.

\subsubsection{Simplified Analyses of Separate LOCA and LOFA}

Assuming there is some capability for natural circulation of helium coolant in the blanket, provided by an adequate driving head and by bypass valves around the helium circulators, a substantial amount of heat can be removed by the naturally circulating coolant as long as the coolant remains pressurized. Referring back to Figure 5.9, it can be seen that as long as helium pressure is maintained at $10 \mathrm{MPa}$, the maximum first wall temperature remains below 550 degrees $C$ for a first wall coolant channel diameter of 4 $\mathrm{mm}$, which is the diameter adopted for Blanket Design 1. It is clear, then, that natural circulation is completely adequate to prevent the first wall from failing, or even from

\footnotetext{
${ }^{25}$ based on design-basis accident analyses of High-Temperature Gas-Cooled (HTGR) fission reactor designs, for example, see G. Melese and R. Katz, Thermal and Flow Design of Helium-Cooled Reactors, American Nuclear Society, La Grange Park, IL, p. 243. This reference states that for a 3000 MWth HTGR design-basis LOCA, it takes over 100 seconds for the coolant system to fully depressurize.

26J. P. Holdren, et.al., "Report of the Senior Committee on Environmental, Safety, and Economic Aspects of Magnetic Fusion Energy (ESECOM)," Lawrence Livermore National Laboratory Report \#UCRL-53766 dated September 25, 1989.
} 
exceeding normal operating temperatures, provided that there is a modest driving head ( 2 meters was assumed in Figure 5.9) and the normal helium coolant pressure is maintained. The capability of natural circulation to reduce the maximum temperature of the first wall decreases markedly as helium pressure is decreased. It is reasonable to assume that the coolant does remain pressurized following a LOFA, however, since the most likely cause of a complete LOFA, namely a loss of site power, would probably not also result in a break in the helium coolant containment.

During a LOCA, the helium pressure rapidly decreases to atmospheric pressure, greatly reducing the density of coolant in the blanket, and consequently, the coolant's ability to remove heat efficiently. Since a LOCA presumes a break in the helium coolant containment, it is conceivable that air gets sucked into the coolant system as a result. of the LOCA, especially if the break occurs directly upstream of a helium circulator. Since air has a specific heat almost five times lower than helium, an air ingress will further degrade the ability of the coolant to remove heat from the blanket. However, presuming that the helium circulators continue to function following the LOCA, the depressurized coolant will continue to circulate in the blanket, and heat will be removed.

To see the effect of a LOCA with and without air ingress, simplified analyses are performed to determine the maximum first wall temperature during a LOCA. The first analysis assumes that no air enters the helium loop during the LOCA (perhaps because the leak is relatively small). The second analysis assumes ALL of the helium in the coolant loop is replaced by air. For both analyses, the helium circulators are assumed to continue operating. Since the operating head-flow curve as a function of pressure for the helium circulators depends on their detailed design, for these analyses it will be

"During the LOFA analysis in the present work, it is asssumed that the coolant pressure remains at the design operational pressure ( $10 \mathrm{MPa})$. It is assumed that relief valves maintain the coolant pressure at roughly operational pressure, even if the coolant expands during the accident. 
assumed that the volumetric flow rate through the circulator is the same before and after the LOCA. In other words, the depressurization of the coolant has no effect on the volumetric flow through the helium circulator, or thorough the coolant channels. As was done for the natural circulation analysis in Section 5.2.2.3, all of the afterheat from the first wall channels is assumed to be deposited in the coolant (either helium or air). The results of these simplified LOCA analyses are shown in Table 5.11.

\section{Table 5.11. Simplified LOCA Analysis Results}

\begin{tabular}{|c|c|}
\hline Coolant Type in Coolant Loop & Maximum First Wall Temperature \\
\hline Helium & 460 degrees C \\
\hline Air & 1260 degrees C \\
\hline
\end{tabular}

As shown in the table above, with helium in the loop, the maximum temperature for the first wall during a LOCA with continued helium circulator operation is below the maximum normal operating temperature for the first wall. This is because the reduction in heat removal capability caused by depressurization is more than balanced by the reduction in heat load caused by the plasma being extinguished. However, if air is assumed to displace the helium in the loop, the maximum first wall temperature rises to 1260 degrees $\mathrm{C}$. This maximum temperature occurs at the outlet of the first wall channel, and is much larger than the temperature seen with helium coolant because of the dramatic difference in specific heat between air and helium. As discussed in Section 5.2.3.6, 1260 degrees $C$ is well above the temperature at which the MT-9 structure would be expected to fail, even under very low stresses. Hence, this simplified analysis indicates that for a LOCA which involves a substantial air ingress, there is a concern for structural failure. However, as will be shown in the detailed accident analysis later in Section 5.2, which accounts for thermal conduction within the blanket, the simple 
analysis method used above significantly overestimates the temperature in the first wall during a LOCA with air ingress.

From the simple analyses above, it is clear that for a LOCA without air ingress or a normal LOFA, Blanket Design 1 will not be damaged. For a LOCA with air ingress, there is a potential concern for structural damage, based on the simplified, conservative analysis performed so far. The accidents analyzed so far involve a single failure (e.g. loss of power to the pumps, or a coolant line break). We turn now to a more severe, and less likely scenario, a LOFA without natural circulation. In this worst-case type of accident, it is assumed the helium circulators fail but there is no natural circulation of coolant. This is quite unlikely, but conceivable, and involves a second failure (for example, perhaps a bypass valve around a circulator fails to open upon loss of flow, preventing the blanket region served by that circulator from obtaining natural circulation of coolant). This is the worst type of undercooling transient the blanket can undergo, since the capability of the coolant to remove heat is eliminated by the flow blockage, but the pressure stress in the coolant channels from the high pressure coolant still exists. Showing that a blanket design can survive an accident which is so severe greatly enhances the desirability of such a design from the safety point of view.

The No-Flow LOFA, as we will call this accident, proceeds as follows. Upon initiation of the LOFA, the helium circulators coastdown as usual, but following coastdown the helium coolant in the blanket ceases to move. The No-Flow LOFA thereby eliminates the ability of the coolant system to remove heat. Hence, the only method by which heat can be removed is via conduction through the blanket, and then by radiation across the vacuum gap to the cooler shield. To analyze this accident properly requires that a timedependent heat transfer model be developed for the entire blanket. This model is now developed. 


\subsubsection{Afterheat Calculations}

The first step in analyzing a blanket design for the No-Flow LOFA involves determination of the afterheat as a function of position in the blanket. To do this, the neutron flux data for a particular position in the blanket are obtained from the TWODANT code and fed into the REAC3 activation code. REAC3 outputs the volumetric heating as a function of time for a particular position in the blanket. It is impractical to run the REAC3 code for every point in the blanket. Instead, REAC3 runs are performed for three positions in the blanket: the first wall, the middle of the blanket, and near the back of the blanket. This yields volumetric afterheat values as a function of time for three positions in the blanket, as shown in Figures 5.12 and 5.13. In generating these figures, a wall load of $4 \mathrm{MW} / \mathrm{m}^{2}$ is assumed. This corresponds to the average outboard blanket wall load (see Section 5.1). This wall load is applied for three full power years (corresponding to five years at a duty factor of 0.60 ).

\section{Figure 5.12. Afterheat of $100 \%$ MT -9 in Blanket Design 1}

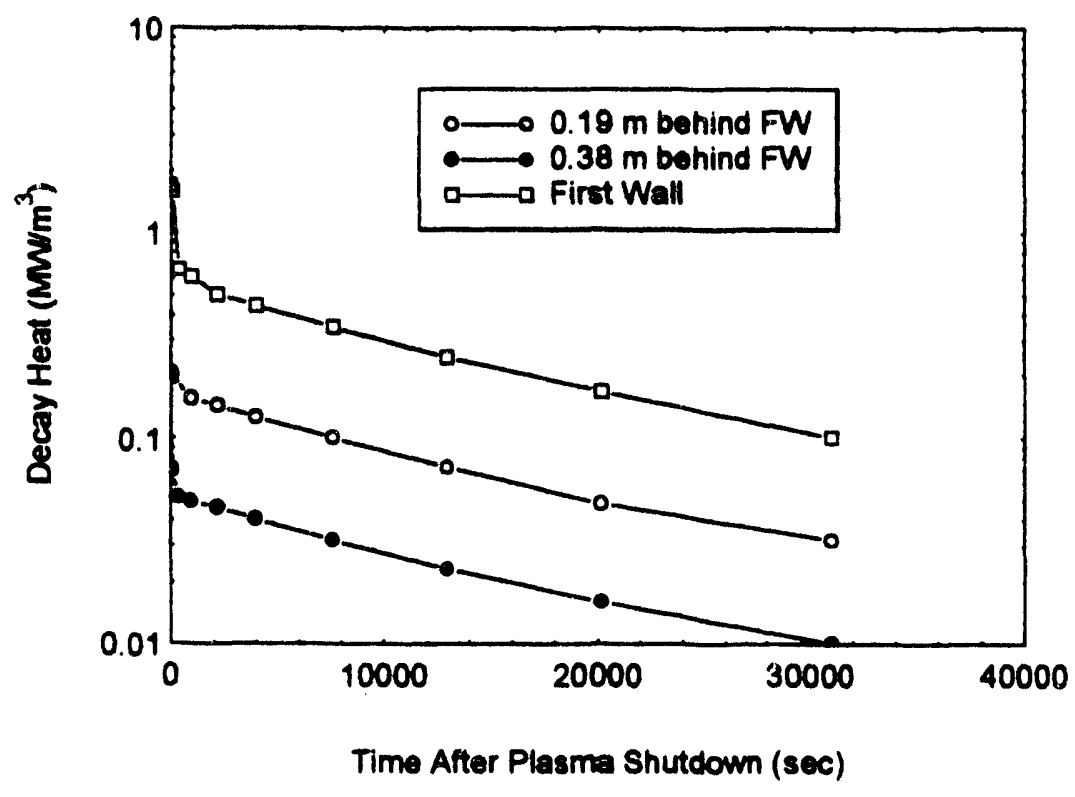




\section{Figure 5.13. Afterheat of Breeder Regions in Blanket Design 1}

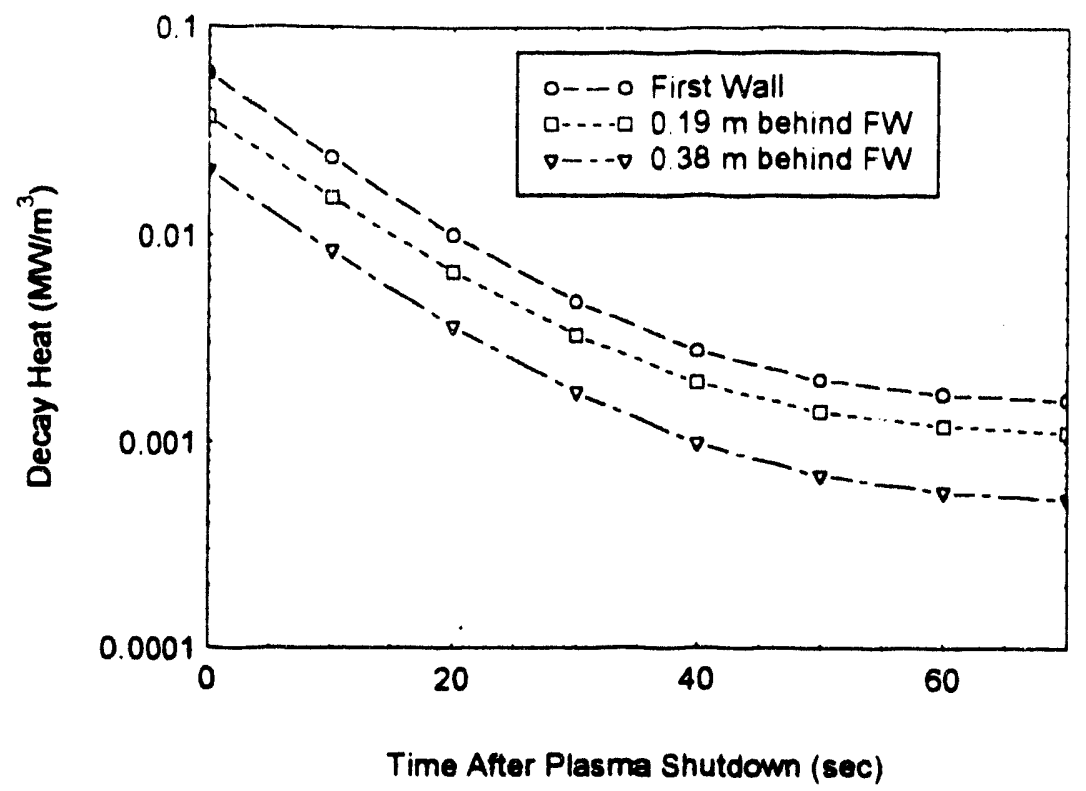

In a blanket with MT-9 structural material, lithium oxide breeder and beryllium multiplier, the predominant producer of afterheat is the MT-9 strusture, as can be seen by comparing Figures 5.12 and 5.13. Beryllium produces very little afterheat; roughly $10^{-3} \mathrm{MW} / \mathrm{m}^{3}$ for locations near the first wall for the first few hours after shutdown. By comparison, MT-9 produces between 0.1 and $1.0 \mathrm{MW} / \mathrm{m}^{3}$ for locations near the first wall during the same period. Lithium oxide produces afterheat mainly via the decay of two isotopes produced during irradiation: tritium and N-16. Of these, N-16 contributes the most initially, but has a very short halflife ( 7 seconds). The results in Figure 5.13 show the effect of the $\mathrm{N}-16$ decay. After $\mathrm{N}-16$ decays, the tritium decay dominates, but at a level far below the afterheat level for the MT-9. Note that the results shown in Figure 5.13 assume all of the tritium generated in the blanket remains in the blanket. Since the purge flow is in fact removing the majority of the tritium generated, Figure 5.13 represents a very conservative curve for breeder afterheat. 
To obtain the afterheat for regions of the blanket other than those analyzed using REAC3, it is assumed that the afterheat drops exponentially as one goes deeper into the blanket. This is not strictly true, however, especially for MT-9. As can be seen in Figure 5.12, the afterheat curve for MT-9 for the first wall has a more substantial initial peak, and decays away more rapidly with time than it does in the interior regions of the blanket. This is because the higher energy neutron flux near the front of the blanket generates more higher energy, short-lived isotopes in the MT-9. These isotopes decay away rapidly, however, and the curves for the three regions become similar in shape a few hundred seconds after shutdown.

To conservatively account for this fact, the afterheat-vs.-time curves for regions in the front half of the blanket (from 0 to $19 \mathrm{~cm}$ from the first wall) are assumed to have a shape similar to that of the top curve in Figure 5.12. For regions further back, the afterheat curve shapes are assumed to be similar to the those of the bottom two curves in Figure 5.12. 


\subsubsection{One-D Model for Accident Analysis}

The model used for the accident analysis is based on the nested shell blanket design discussed in the sections above. The finite difference heat transfer code HEATING3 is used to calculate the temperature distribution a function of time in a one-dimensional model of the blanket. The one-D model is based on taking a slice through the center section of the blanket module. Such a model, though computationally simple and requiring only a tnodest amount of computing time (less than a half-hour on a CRAY-2/8 machine for the transients of interest here), does not account for "end-effects" at the ends of the module. Thus, the temperature versus time plots calculated herein should be considered estimates. To obtain truly accurate results, a full, 3-D model, requiring much more computing time and effort, would have to be used.

Figure 5.14 shows the 1-D model geometry. In this figure are shown the various regions in the model, beginning with the first wall at the top of the figure, and ending with the shield at the bottom of the figure. The region thicknesses are shown, as well as the region compositions, by volume fraction. Note that the scale of the figure is approximate. 
Figure 5.14. One-Dimensional Model for Accident Analysis

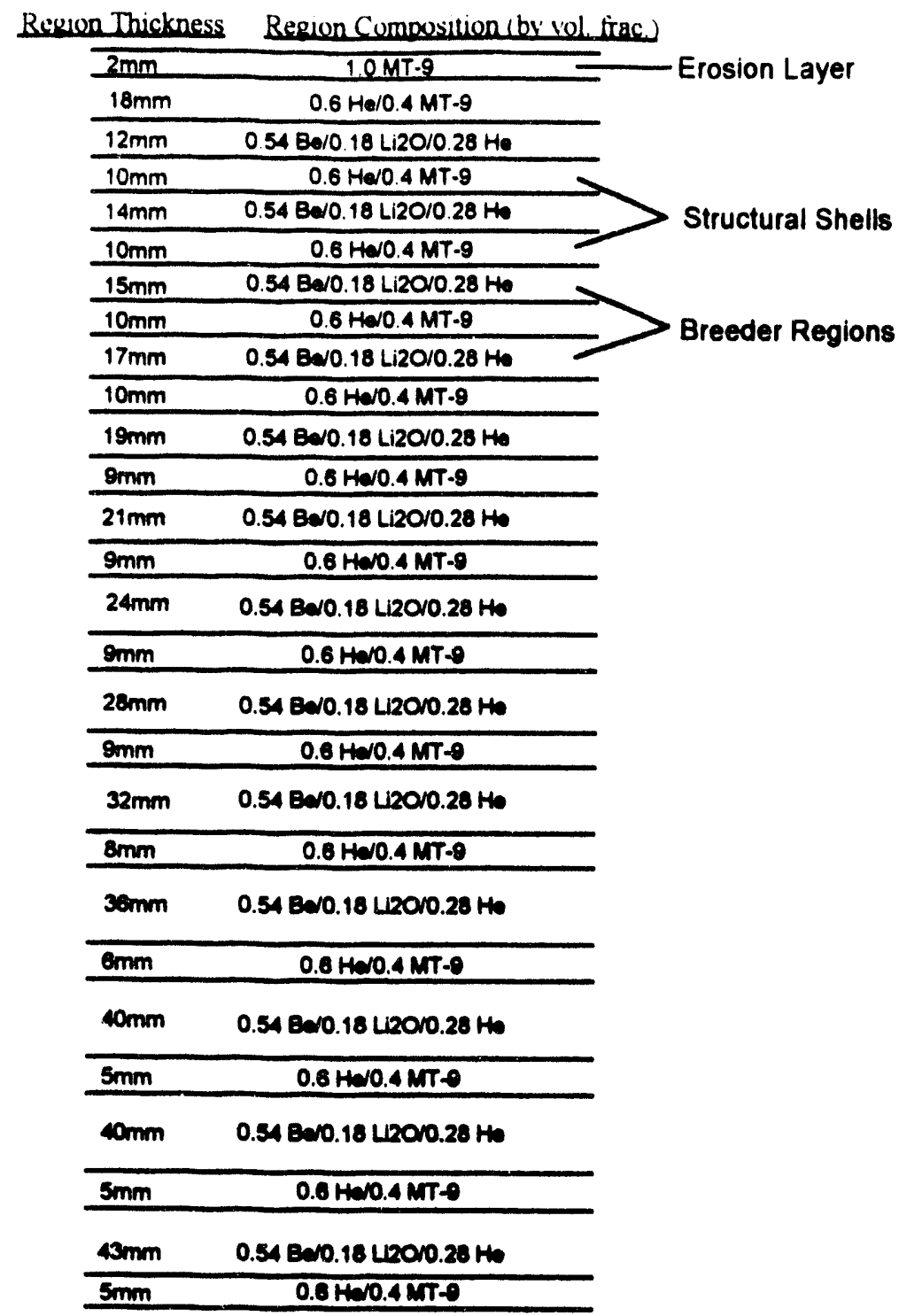

$300 \mathrm{~mm} \quad 0.25$ Har $0.75 \mathrm{MT} \theta \quad$ Plenum

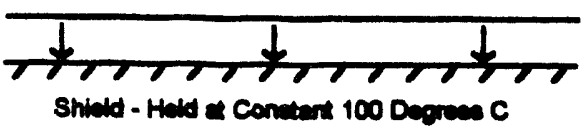


The bed thicknesses of Figure 5.14 correspond to the thicknesses that were shown in Table 5.6. The channel thicknesses of Figure 5.14 are larger than the channel diameters shown in Tables 5.7 and 5.8, because the channel wall thickness has been accounted for. ${ }^{27}$

The volume fractions in the mixed bed regions assume a 3:1 beryllium to lithium oxide ratio, a pebble porosity of 0.10 ; and a total pebble packing fraction of 0.80 , as discussed

\section{Figure 5.15. Geometry of a Channel}

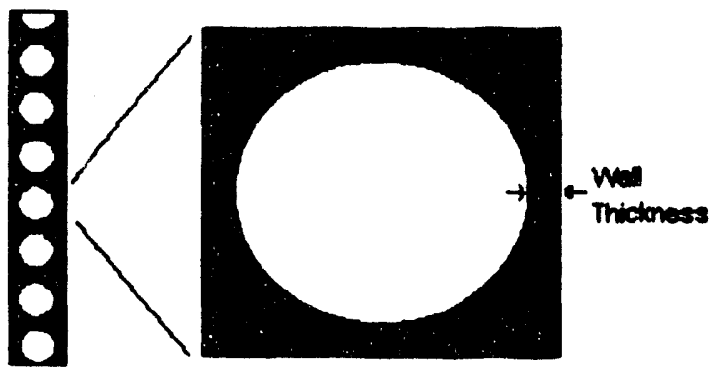

in previous sections. The volume fraction in the shell regions are based on a shell geometry as shown in Figure 5.15. Âs shown in this figure, the channel wall thickness is defined as the thinnest portion of the channel wall.

The arrows pointing at the shield region in Figure 5.14 signify cooling by radiation across the vacuum region between the back of the blanket and the front of the shield. The emissivity of the MT-9 on either side of the vacuum region is assumed to be 0.7 for this analysis. ${ }^{28}$ The rate of radiative heat transfer between parallel surfaces at temperatures $T_{h}$ and $T_{c}$ and emissivity $\varepsilon$ is as follows: 29

\footnotetext{
${ }^{27}$ The wail thicknesses were chosen as the largest of two values: the minimum thickness required to contain the assumed $10 \mathrm{MPa}$ helium coolant pressure $(t=\mathrm{Pr} / \mathrm{S}$, where $\mathrm{P}=10 \mathrm{MPa}$, $\mathrm{r}=$ the channel radius, and $S$ is the stress corresponding to $1 \%$ creep after 3 years of continuous operation at 500 degrees $C$ ), and the minimum thickness allowable to ensure structural ruggedness. The requirement for ruggedness was set, somewhat arbitrarily, at a value equal to $\mathrm{r} / 7$. For the channel thicknesees of concern here, the wall thickness is always governed by the ruggechness requirement, and the wall thickness between channels is twice the thickness required for ruggedness. Hence the coolant channels are all quite overdesigned for the helium pressure.
}

${ }^{28}$ The emissivity of oxidized HT/MT-9 is expected range from 0.7 to 0.8 for temperatures between 0 and 900 degrees C, based on data from other, similar metals (see The Materials Handbook for Fusion Energy 


$$
\mathrm{Q}=\frac{\varepsilon \sigma\left(T_{h}^{4}-T_{c}^{+}\right)}{2-\varepsilon}
$$

where $\sigma$ is the Stefan-Boltzmann constant $\left(5.67 \times 10^{-8} \mathrm{~W} / \mathrm{m}^{2} \cdot \mathrm{K}^{4}\right)$. Using an emissivity of 0.7 results in $\mathrm{Q}=0.5 \sigma\left(T_{h}^{+}-T_{c}^{4}\right)$. This formula is used in the HEATING3 code. The shield is assumed to be held at a constant temperature of 100 degrees $C$ by natural circulation of the shield water. The following section discusses the modeling of the shield region.

\subsubsection{The Sinield Model}

The shield is insulated from the blanket by a vacuum break, generates only a small amount of heat during operation (as compared to the blanket). Because the shield generates such a small amount of power during reactor operation, the heat generated by the shield is not used for electrical power generation. Hence, there is no need to generate steam from the shield water coolant system, and it can be designed to operate with depressurized water below 100 degrees $C$. The exact operating temperature of the shield depends on the design of the shield cooling system, which is discussed in Chapter 6.

For the purposes of the present analysis of the No-Flow LOFA, the shield temperature is an important input because the shield is the only heat sink available to the blanket. Past analyses of blanket casualties, notably the comprehensive blanket accident survey performed by Massidda, ${ }^{30}$ have assumed that the shield water remains stagnant during

Systems, USDOE Report wDOE/TC-10122, McDonnell Douglas Astronautics Co., Initial Issue April 30,1980 ). In the present analysis, 0.7 is adopted for conservatism.

${ }^{29}$ From T. Baumeister, oditor, Mark's Standard Handbook for Mechanical Engineers 8th Ed., McGrawHill, New York, 1978, p. 19-36.

30J. Massidda and M. Kazimi, "Thermal Design Considerations for Passive Safety of Fusion Reactors," MIT Plasma Fusion Center Report PFC/RR-87-18, October 1987. 
the blanket accident. This assumption, while conservative, tends to make the first wall of the blanket experience high temperatures for very long periods of time in the event of a blanket LOCA of LOFA.

The present study determines, in Chapter 6, whether natural circulation of the shield water can minimize the temperature of the shield enough to help reduce the length and/or magnitude of the first wall temperature excursion following a blanket accident such as a LOCA or LOFA. It should be noted that the shield water system can be designed in such a way as to guaramtee natural circulation of the shield water in the event of a LOFA in the shield water system, without relying on bypass valves (which could always stick shut). Whereas the helium circulators serving the blanket require bypass valves to permit natural circulation during a LOFA, the shield water system, since it is a water system requiring low flow rates and negligible pumping power, can be designed with jet pumps located outside the main shield water flow stream. The jet pumps, if they failed (as they would if the blanket LOFA was caused by a loss of site power), would thereby not create resistance to the natural circulation of the shield water. As is shown in Chapter 6, the natural circulation of water in the shield system will, with proper system design, maintain the shield temperature below 100 degrees $C$ for the duration of a No-Flow LOFA. Hence, this is the shield boundary temperature used in Figure 5.14.

\subsubsection{Results of the No-Flow LOFA Analysis}

All of the required information is at hand now to perform the No-Flow LOFA thermal analysis on Blanket Design 1, the nested shell design with MT-9 structure and a mixed bed of lithium oxide and beryllium. The results of this analysis are shown in Figures 5.16 and 5.17 . 
Figure 5.16 shows the temperature response of the first wall after the accident. It peaks at 772 degrees $C$ about 4 hours (15,400 seconds) after the onset of the accident and steadily decreases thereafter, approaching the normal operating temperature of 550 degrees $\mathrm{C}$ at the 30 hour (108,000 seconds) point.

\section{Figure 5.16. Temperature of First Wall of Blanket Design 1 After} No-Flow LOFA

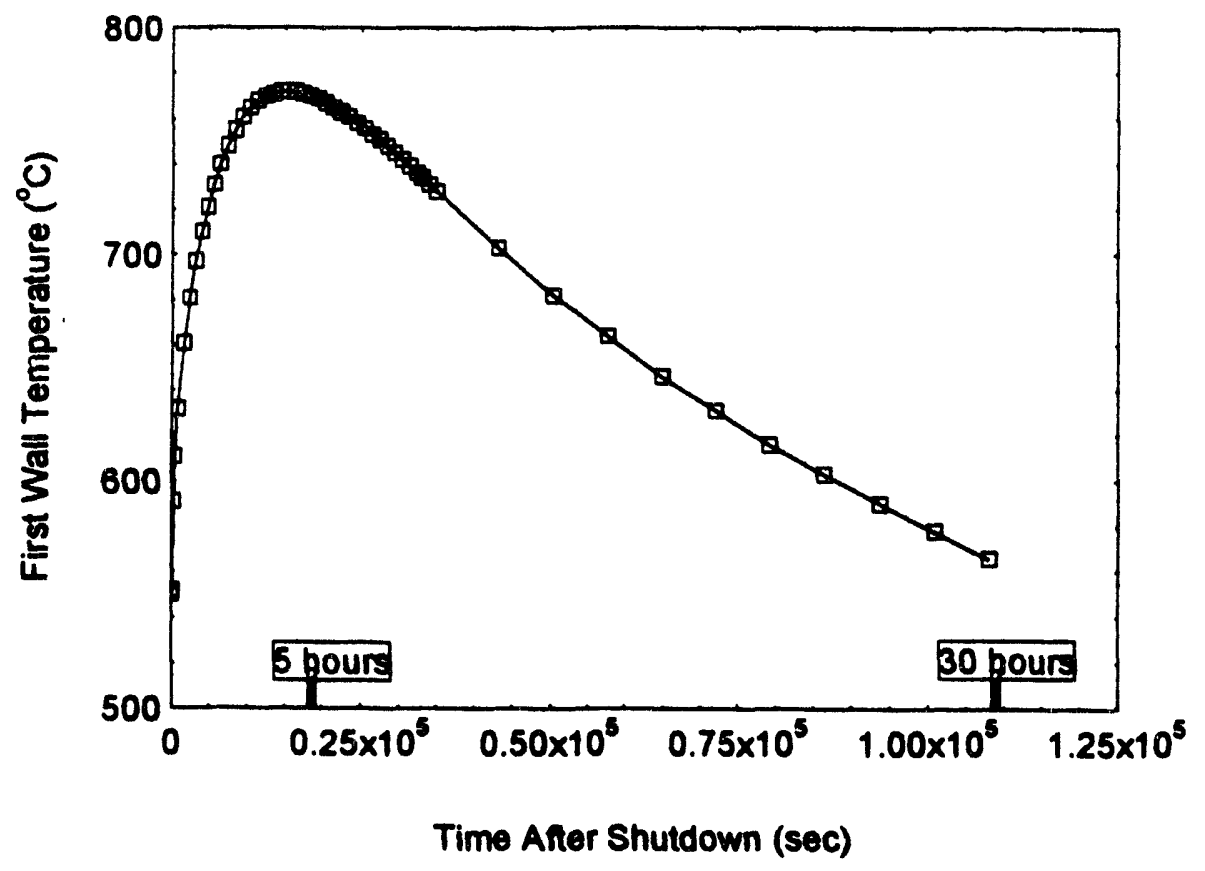

Figure 5.17 shows plots of temperature versus distance into the blanket at five different times: at shutdown, one minute after shutdown, 7.6 hours after shutdown, 13 hours after shutdown, and 32 hours after shutdown. The "at shutdown" curve shows the normal operating temperature distribution in the blanket at the hottest point in the shell, the helium coolant outlet side. This maximum temperature points in this curve are the central portions of the 13 mixed bed regions. The minimum temperatures correspond to the coolant channels. The flat portion of the temperature curve at the back of the blanket is the plenum region. This region is where the inlet and outlet manifolds for the 
helium are, and the normal operating temperature chosen for this region is 350 degrees $\mathrm{C}$, which is the average between the helium inlet temperature of 250 degrees and the outlet temperature of 450 degrees. The curve at one minute after the accident shows fewer temperature peaks, since the mixed bed regions have stopped generating heat and the temperature is equilibrating through them. The curve for 7.6 hours after shutdown shows a first wall temperature of 750 degrees $C$ (which occurs after the maximum first

\section{Figure 5.17. Temperatures In Blanket Design 1 After No-Flow LOFA}

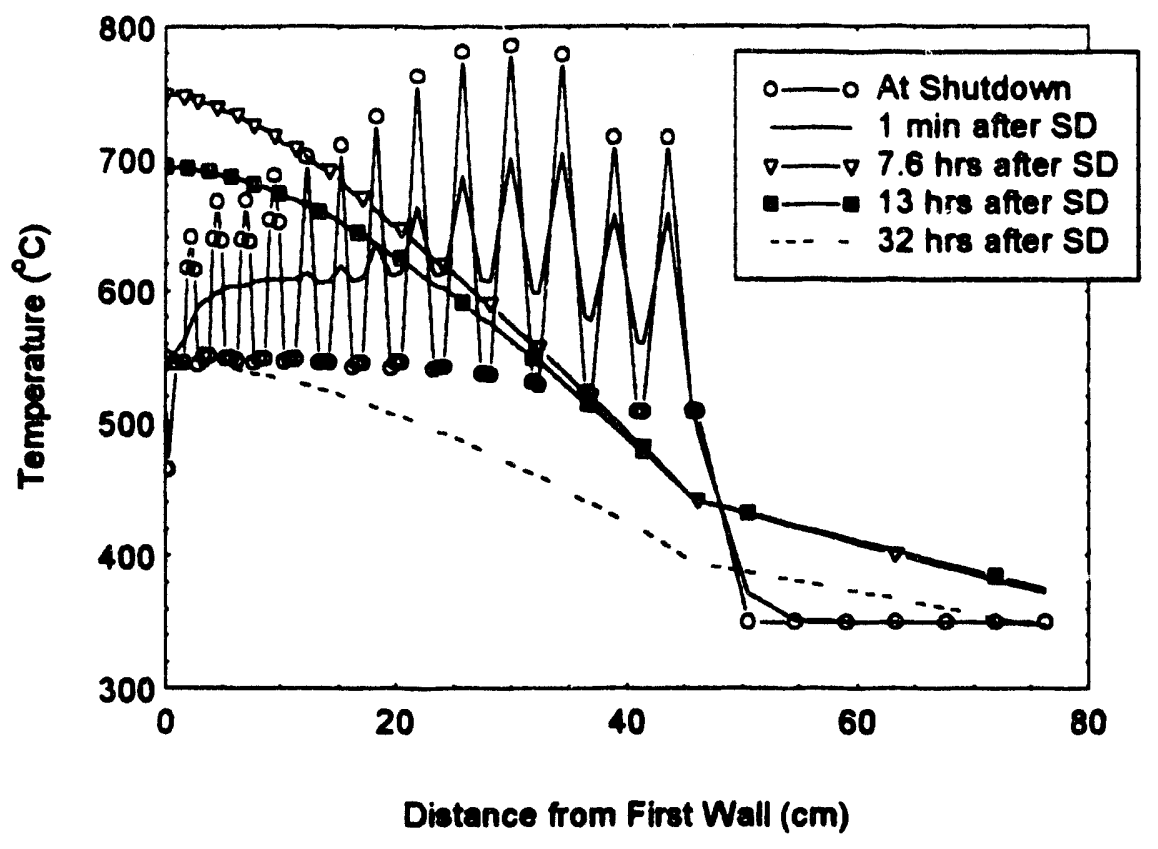

wall temperature of 772 degrees C). By this time, the temperature peaks in the mixed bed regions have all equilibrated away. The temperature at the back of the blanket has increased to almost $\mathbf{4 0 0}$ degrees, and is radiating to the shield, which is assumed to be at 100 degrees C. By 13 hours after shutdown, the afterheat in the blanket has substantially dropped, and the first wall temperature has also, although the back portion of the blanket remains at its peak temperatures. By 32 hours after shutdown, the entire blanket is beginning to cool off from the peak temperatures reached during the accident. 


\subsubsection{Failure Analysis for the First Wall}

Now that the temperature history for the first wall is known, a determination needs to be made of whether the first wall will sustain damage as a result of the accident. For the temperatures seen here, which are well below the melting point for MT-9, the two possible modes of failure are classified as acute structural failure, which occurs when the material exceeds its ultimate tensile strength, and creep rupture, which occurs when the material undergoes extensive high-temperature creep. The following paragraphs discusses these two failure modes further.

\section{Acute Structural Failure}

For a material to fail acutely, the stress level must exceed the material's ultimate tensile strength (UTS). The UTS for a material is temperature-dependent, and generally goes Figure 5.18. Ultimate Tensile Strength of HT/MT-9 down with temperature. Steel ${ }^{31}$

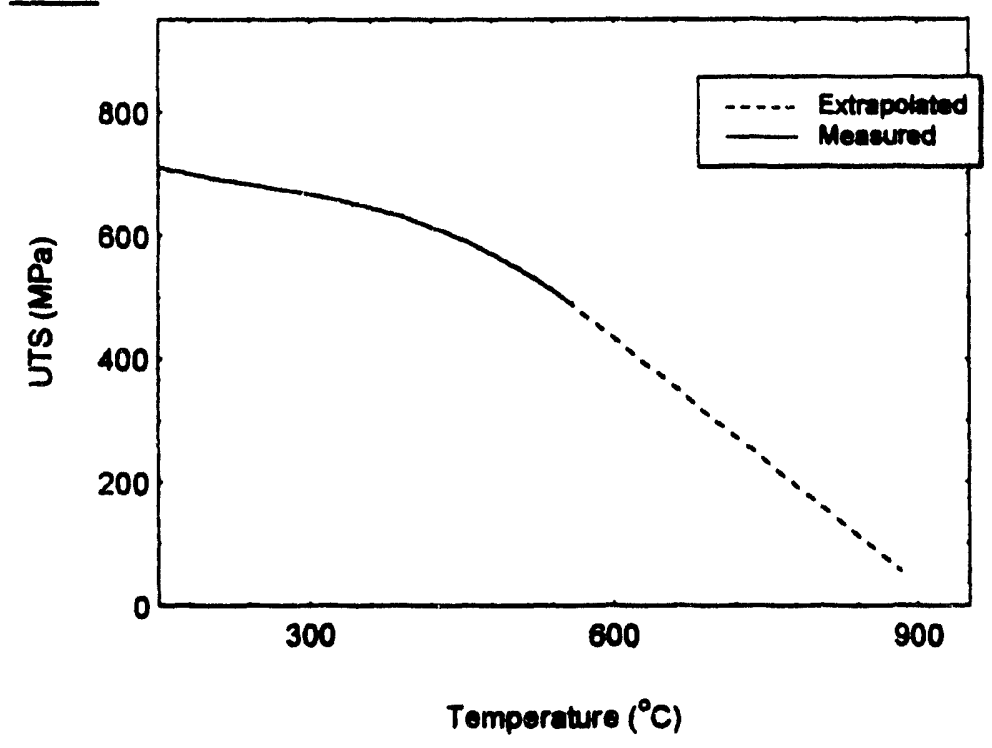

For MT-9/HT-9, the available data base for this material extends only up to 550 degrees $C$. Hence, values for the UTS (and for most other properties) are only available up to this temperature. Since the temperature of the first

wall exceeds 550 degrees $C$ in the accident, an extrapolation must be done from the available data base. For this extrapolation, it is assumed that the slope of the UTS vs.

\footnotetext{
${ }^{31}$ See the Materials Handbook for Fusion Energy Systems, USDOE Report \#DOE/TIC-10122, McDonnell Douglas Astronautics Co., Initial Issue April 30, 1980.
} 
temperature curve is constant for temperatures higher than 550 degrees $\mathrm{C}$. Figure 5.18 shows the UTS as a function of temperature for MT-9.

\section{Stress Rupture}

When materials are subjected to load at high temperatures, they experience a gradual flow, called creep, even for stresses that are significantly lower than the material's yield stress. Figure 5.19 shows a typical creep versus time curve for a metal at elevated temperature. $^{32}$

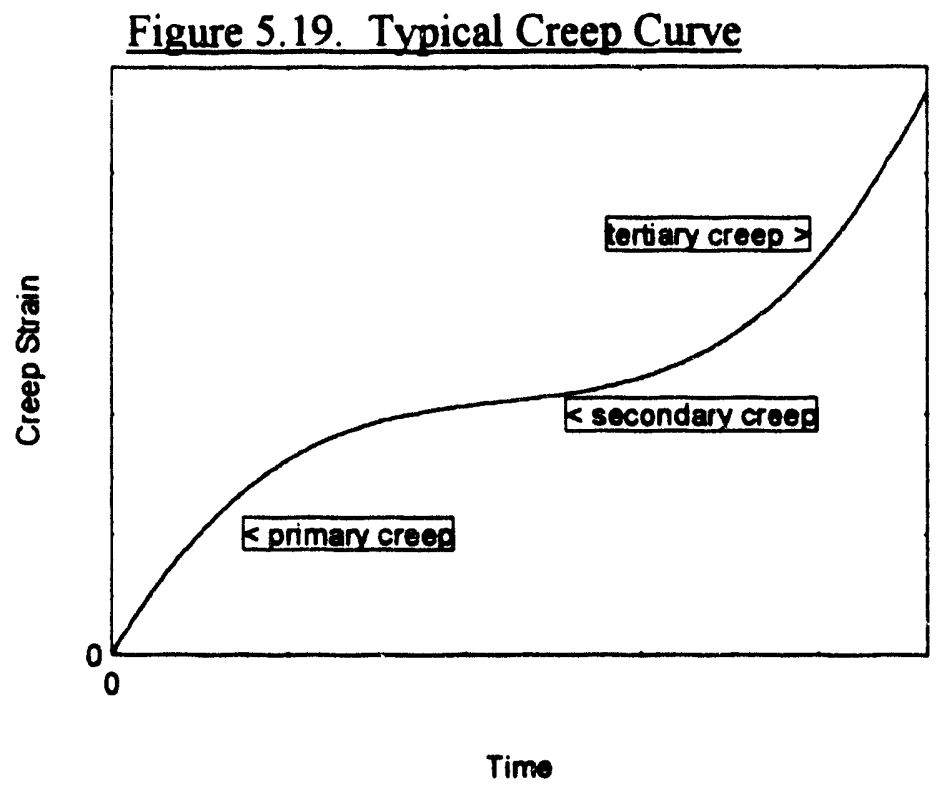

This figure shows the three regions which typify a creep curve. The first region, the region of primary creep, has a slope which is decreasing. This signifies that in this region the strain rate is decreasing with time. The secondary creep region exhibits a constant strain rate, and the tertiary creep region has an increasing strain rate. If the material enters the tertiary creep region, the increasing strain rate causes large deformations in the metal which eventually causes the metal to fail. This mode of failure is called stress rupture.

${ }^{32}$ T. Baumeister, et.al. Mark's Standard Handbook for Mechanical Engineers, Eighth Edition, McGrawHill, New York, 1979. 
Testing can be performed on a metal to determine how long it takes for it to undergo stress rupture at a given temperature and stress level. When such data is plotted, the resulting curves are known as stress rupture strength curves. The appropriate curves for HT/MT-9 are shown in Figure 5.20. The curves in this figure indicate the time it takes for MT-9 to experience stress rupture for a particular temperature and stress level. The curves represent a conservative lower bound for the rupture strength for HT/MT -9 , and were derived based on ASME guidelines. ${ }^{33}$ However, as with the UTS curve, extrapolation has to be done for temperatures greater than 550 degrees $\mathrm{C}$. This is done using the Larson-Miller parameter, which is used to predict creep behavior at a temperature for which there is no test data using test data at a different temperature. See Appendix 9 for a discussion of the Larson-Miller parameter, and for details on how the stress rupture strength curves are generated. The appropriate stress values to enter on the $y$-axis of this figure are the Von Mises equivalent stress values, which are defined in Equation 5.10 below.

\footnotetext{
${ }^{33}$ The ASME guidelines for generating conservative stress rupture curves are elucidated in P. M. Brister, "Code Design Criteria in the U.S.A. Evaluation of Strength Properties," in the handbook 1977 Design Criteria of Boilers and Pressure Vessels, The American Society of Mechanical Engineers, 1977, p. 35. The data used for generating the curves is from the Materials Handbook for Fusion Energy Systems, USDOE Report HDOE/TIC-10122, McDonnell Douglas Astronautics Co., Initial Issue April 30, 1980. See Appendix 9 for details on generation of the creep rupture curves.
} 
Figure 5.20. HT/MT-9 Stress Rupture Strength

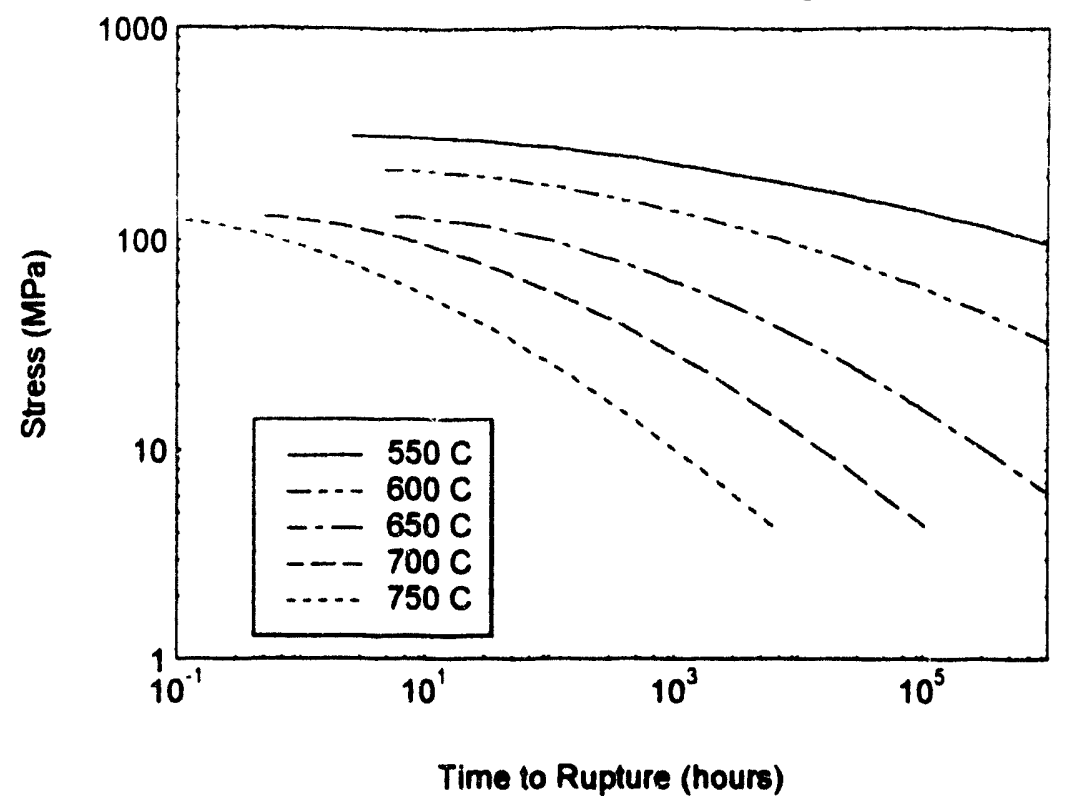

First Wall Stresses

A necessary input to the failure analysis for the first wall is the stress state seen by the first wall during the accident. The stresses in the first wall region during an accident can be classified into two categories: thermal and pressure stresses. Pressure stresses are caused by the $10 \mathrm{MPa}$ helium coolant inside the first wall channels; these stresses remain constant throughout the accident. Thermal stresses are caused by the temperature gradient which exists in the first wall during reactor operation (when the plasma is on), but which vanishes when the plasma is shut off during the accident. The temperature gradient which exists during reactor operation will, at the beginning of blanket life, cause initial thermal stresses in the first wall. These stresses will gradually lessen as the reactor operates, however, via creep of the first wall metal. By the end of the blanket lifetime (corresponding to about 3 years of full power operation), creep will presumably have eliminated the thermal stresses which existed during normal operation. However, when the plasma is shut off, and the first wall temperature distribution becomes essentially 
uniform, thermal stresses reappear in the first wall. These stresses are of the same magnitude as the thermal stresjes which occurred at the beginning of life, but are opposite in sign.

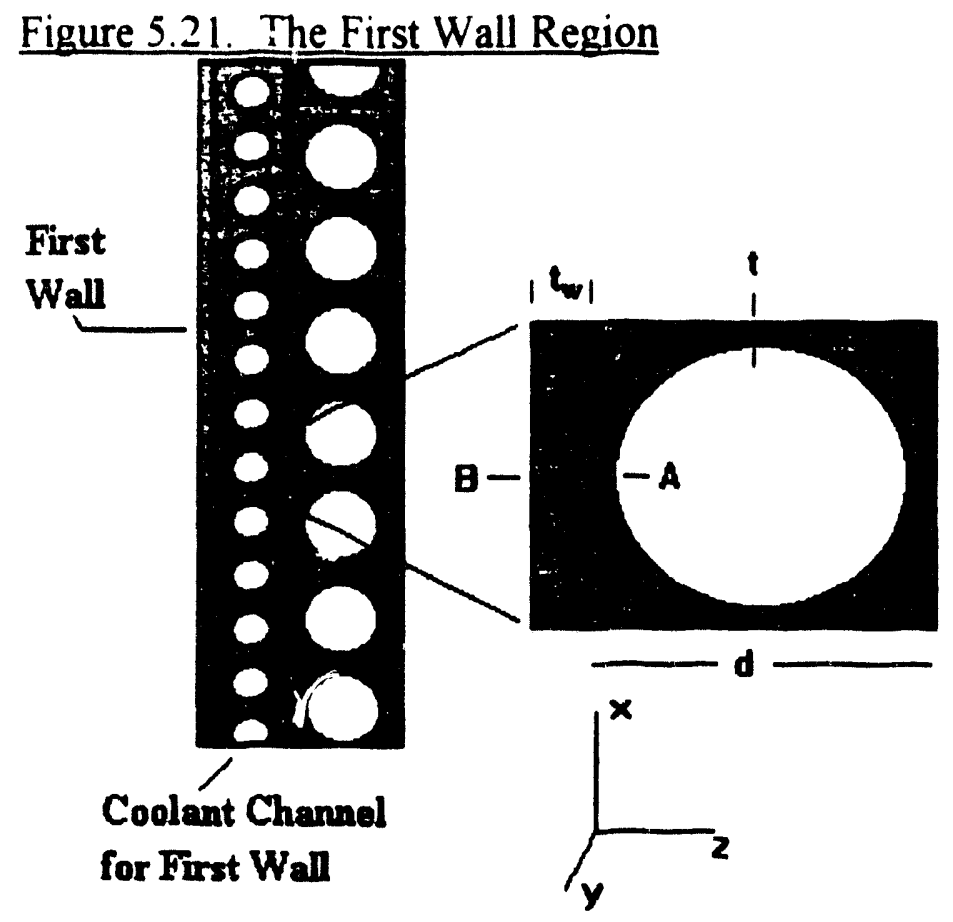

The geometry of the first wall region, shown in Figure 5.21, is somewhat complex. This means that the stress field in the region will also be relatively complex. To accurately calculate the stresses in the first wall during the accident would require detailed timedependent analysis of the region, using for example

finite-element techniques. This type of analysis is beyond the scope of the present work. A first-order estimate of the stresses, however, is possible using some simplifying approximations. These first-order estimates can provide an indication of how close the first wall region comes to structural damage during the accident. An estimate of the stresses at point A of Figure 5.21 is necessary. This is the point which is expected to be most limiting, as the following discussion demonstrates.

The thermal stresses experienced by the first wall at shutdown are examined first. It is assumed that before shutdown, the reactor has operated for a sufficiently long period that there are no thermal stresses in the first wall when the plasma is on. When the plasma shuts off, the plasma side of the first wall (point B in Figure 5.21) cools, creating a tensile thermal stress at point $\mathbf{B}$ and compressive stress at point $\mathbf{A}$ It is the tensile 
stress that is of concern, since tensile stress causes positive creep strain, and it is positive creep strain which ultimately causes failure ${ }^{34}$ The magnitude of thermal stress at points $A$ and $B$ can be estimated as follows. During operation, the front side of the slab (at point $B$ ) is exposed to high temperature $T_{\max }$, while the back side (at point $A$ ) is exposed to a lower temperature $T_{\min }$. These temperatures are determined based on the plasma surface heat load, the thickness and thermal conductivity of the first wall, and the temperature and surface heat transfer coefficient of the coolant. At plasma shutdown, the magnitude and sign of thermal strains at points $\mathbf{A}$ and $\mathbf{B}$ for such a slab is given by

$$
\varepsilon_{\text {Bxth }}=\varepsilon_{B y t h}=-\varepsilon_{A x t h}=-\varepsilon_{A y t h}=\frac{1}{2}\left(T_{\max }-T_{\min }\right) \alpha
$$

where $\alpha$ is the coefficient of thermal expansion for the metal. There are no thermal strains in the $\mathrm{z}$ direction.

To estimate the thermal stresses in the first wall, it is assumed that the first wall is constrained to have no deflection in the $z$ direction, but is allowed to expand at the edges. In this case, the stresses for a thin plate are given by: ${ }^{35}$

$$
\left.\sigma_{B x+h}\right|_{t=0}=\left.\sigma_{\text {Byoth }}\right|_{t=0}=-\left.o_{\text {Axth }}\right|_{t=0}=-\left.o_{\text {Ayth }}\right|_{t=0}=\frac{\left(T_{\max }-T_{\min }\right) \alpha E}{2(1-v)} .
$$

There are no thermal stresses in the $\mathrm{z}$ direction. Equation 5.2 gives the magnitude of thermal stress at the beginning of the accident $(t=0)$. However, as discussed above, the initial thermal stress induces creep which acts to reduce the thermal stress $\varepsilon_{a}$ as the accident proceeds. (The pressure stress, since it is a primary stress, and neglecting geometry changes, remains constant with time.)

\footnotetext{
${ }^{34}$ In Appendix T of ASME Code Case N-47-28 for Class 1 Components in Elevated Temperature Service, the deformation and strain limits are associated with positive values of strain.

${ }^{35}$ B. A. Boley and J. H. Weiner, Theory of Thermal Stresses, John Wiley and Sons, 1960, p. 405.
} 
For Blanket Design I, the first wall thickness (including the erosion layer) is $2.6 \mathrm{~mm}$, and the peak surface heat load seen by the outer blanket is $1.0 \mathrm{MW} / \mathrm{m}^{2}$ (corresponding to a neutron wall load of $5 \mathrm{MW} / \mathrm{m}^{2}$ ). This yields a temperature difference of 95 degrees $\mathrm{C}$ across the first wall, which corresponds to an initial thermal strain of less than 0.1 percent. According to the ASME Code, local strain magnitudes of less than 5 percent are considered acceptable. ${ }^{36}$ Since the maximum value of thermal strain is more than an order of magnitude below this, thermal strains are neglected in the following failure analysis for Blanket Design 1.

The pressure stresses seen by the first wall region are now examined. Point $A$, on the inside of the coolant channel, experiences the highest pressure stress caused by the coolant inside the channel. To estimate the pressure stress at point A, it is conservatively assumed that the first wall erosion layer (of thickness $t_{w}$ in Figure 5.21) does not help to contain the coolant pressure. The wall thickness $t$ in Figure 5.21 is equal to $r / 7$, where $r$ is the coolant channel radius. Use of this formula ensures that the wall thickness is greater than that necessary to contain the pressure during normal operation, and ensures that the wall thickness does not become excessively thin. An excessively thin wall thickness would result in the channel lacking adequate structural ruggedness.

Since the wall thickness is greater than $r / 10$, these channels can not be approximated as thin cylinders. Hence, for the stresses in the radial and azimuthal directions (the $x$ and $z$ directions of Figure 5.21), the pressure stresses for a thick cylinder are used, namely:37

\footnotetext{
36This criterion is stated in the Appendix $T$ of ASME Code Case N-47-28.

${ }^{37}$ From Mark's Standard Handbook for Mechanical Engineers, T. Baumeister, et.al., eds., McGraw-Hill, New York, 1978.
} 


$$
\begin{aligned}
& \sigma_{\text {Axp }}=\frac{P\left(r_{\text {in }}\right)^{2}}{\left(r_{\text {out }}{ }^{2}-r_{\text {in }}{ }^{2}\right)}\left(1+\left(\frac{r_{\text {out }}}{r_{\text {in }}}\right)^{2}\right) \\
& \sigma_{\text {A: } p}=\frac{P\left(r_{\text {in }}\right)^{2}}{\left({r_{\text {out }}}^{2}-r_{\text {in }}{ }^{2}\right)}\left(1-\left(\frac{r_{\text {out }}}{r_{\text {in }}}\right)^{2}\right)
\end{aligned}
$$

where $\mathrm{P}$ is the coolant pressure, $\mathrm{r}_{\mathrm{im}}$ is the inner radius of the channel, and $\mathrm{r}_{\text {out }}$ is the effective outer radius of the channel, defined as $r_{i=}+t$. For point $B$, there is no $z$-direction pressure stress (since there is no external pressure), and the $\mathrm{x}$-direction pressure stress is

$$
\sigma_{\text {Bxp }}=\frac{2 P\left(r_{\text {in }}\right)^{2}}{\left(r_{\text {out }}{ }^{2}-r_{\text {in }}{ }^{2}\right)}
$$

The longitudinal (y direction) stresses at points $\mathrm{A}$ and $\mathrm{B}$ are given simply by a force balance in the $y$ direction. Thus, we obtain

$$
\sigma_{A y p}=\sigma_{B y p}=P \frac{A_{c h}}{A_{s r}}=P \frac{\pi r_{i n}{ }^{2}}{d^{2}-\pi r_{i n}{ }^{2}}
$$

where $A_{m}$ is the channel cross-sectional area, $A_{*}$ is the cross-sectional area of structure associated with the channel, and $d$ is shown in Figure 5.21.

\section{Creep Rupture Analysis Methods}

The ASME code specifies in detail how to design components for high-temperature service in Code Case $\mathrm{N}-47$. The analysis methods to be used herein are not extensive nor detailed enough to demonstrate total compliance with Code Case N-47; this type of exhaustive analysis is not justified for the present conceptual design study. However, the analysis methods to be used are based on the requirements given in Code Case N-47. Hence, the results of the analysis should give a good indication of the likelihood the 
blanket will fail via high-temperature creep rupture during the accident. Two different analysis methods based on different databases for HT/MT-9 are used. Only if both analysis methods indicate the blanket does not fail will the blanket be considered to have survived the accident undamaged. Use of two methods in this fashion will serve both to demonstrate different analysis methods, and hopefully help offset the fact that substantial extrapolations in the existing database are necessary to perform the high temperature analysis for HT/MT-9.

\section{Rupture Fraction Method}

The first method of creep rupture analysis is called the rupture fraction method. This method makes use of the stress rupture curves for the material to be analyzed. The stress rupture strength curves in Figure 5.20 are only applicable when the stress level and temperature are constant for the entire time period. To analyze the more relevant case where the temperature and/or stress level varies with time, a method specified by the ASME Code is adapted for use here. ${ }^{38}$ This method involves calculation of the rupture fraction, $\mathrm{f}$, associated with a particular temperature-stress transient. The rupture fraction is a measure of how close the material is to stress rupture, with a rupture fraction equal to 1.0 signifying that stress rupture has occurred, and rupture fractions below 1.0 indicating no stress rupture.

To calculate the rupture fraction, the temperature-stress combination must be known as a function of time for the transient of interest. The transient is broken up into many small increments of time $\Delta t$, with the temperature and stress assumed to be constant over the time increment. The time to stress rupture can then be determined for the time

\footnotetext{
${ }^{38}$ Specifically, ASME Code Case N-47-28 for Class 1 Components in Elevated Temperature Service, 1988. The method used was also used in J. Massidda and M. Kazimi, "Thermal Design Considerations for Passive Safety of Fusion Reactors," MIT Plasma Fusion Center Report PFC/RR-87-18, October 1987.
} 
increment, using the stress rupture curves for the material. The rupture fraction for the time increment $i$, then, is simply

$$
f_{1}=\frac{\Delta t_{1}}{\left(t_{r}\right)_{1}}
$$

where $\left(t_{r}\right)_{1}$ is the time-to-rupture associated with the stress and temperature during the time interval $\Delta t_{1}$. The rupture fraction associated with the whole transient is given by

$$
f=\sum_{i} \frac{\Delta t_{1}}{\left(t_{r}\right)_{1}}
$$

\section{Direct Calculation of Creep Magnitude}

The second method of analysis involves a direct estimation of the magnitude of creep sustained by the first wall during the accident. ASME Code Case $\mathrm{N}-47$ stipulates that the total through-wall creep magnitude (in each principle direction) should not exceed 1 percent for high temperature components (for positive values of creep). Hence, if an estimate of the through-wall creep magnitude which occurs in the first wall during the accident can be obtained, we can determine how close the first wall comes to violating the ASME criterion. Since the creep correlations for a material generally come from a different database than the rupture strength data, calculating the creep magnitude serves as an independent check on the rupture fraction results.

To determine how much creep strain is induced by the pressure stresses as the temperature changes in the first wall region, a correlation for the creep of HT/MT-9 steel as a function of temperature and stress is needed. Such a correlation is generally given in one of two ways. Either the creep itself is given as a function of time for a given temperature and stress condition, or the creep rate is given as a function of stress and temperature. When the creep itself is given, the entire form of the creep curve shown in 
Figure 5.19 can be reproduced for a given stress level and temperature, with primary, secondary and tertiary creep regions. When the creep rate is given, it is presumed to be constant in time. Hence the creep rate correlations focus on the secondary, constant creep-rate region, neglecting the primary and tertiary regions.

\section{Form of the Creep Strain Relations ${ }^{39}$}

The following discussion discusses the form of creep strain correlations. Creep equations are generally of the form

$$
\varepsilon_{e q}=f\left(\sigma_{e q}, T, t\right)
$$

where $T$ is the temperature, $t$ is time, $\varepsilon_{\infty q}$ is the magnitude of the creep, and $\sigma_{e q}$ is the Von Mises equivalent stress, defined as follows:

$$
\sigma_{\infty q}=\left\{\frac{1}{2}\left[\left(\sigma_{x}-\sigma_{y}\right)^{2}+\left(\sigma_{y}-\sigma_{z}\right)^{2}+\left(\sigma_{x}-\sigma_{z}\right)^{2}\right]\right\}^{\frac{1}{2}}
$$

The above equation is valid when no shear stresses exist on planes normal to the coordinate axes, as in the simplified case being analyzed here.

Creep rate equations are generally of the form

$$
\dot{\varepsilon}_{\infty q}=f\left(\sigma_{\infty q}, T\right) \text {, }
$$

with the creep magnitude determined by multiplying $\dot{\varepsilon}_{\infty q}$ by an appropriate time increment.

The creep magnitude, $\varepsilon_{a q}$, is relateci to the creep strains in the principal directions as follows (again, for the case of no shear stresses):

${ }^{39}$ This subsection is based on notes for MIT Course 22.314, Fall 1991. 


$$
\varepsilon_{1}=\frac{\varepsilon_{e q}}{\sigma_{e q}}\left(\sigma_{1}-\frac{1}{2}\left(\sigma_{j}+\sigma_{k}\right)\right)
$$

where $\varepsilon_{1}$ is the creep rate in the ith direction, and $j$ and $k$ denote the other principal directions. Note that for an isotropic stress distribution, the creep strains become zero, as would be expected.

If a creep strain rate relation such as Equation 5.12 is to be used, an iterative approach is taken to determine the creep strain as a function of time. Since the pressure stress values are known and constant, and the temperature as a function of time is known, Equation 5.12 can be used to provide the creep strain rate for a short amount of time $\Delta t$ following time $t$. The new values of strain are thus:

$$
\left.\varepsilon_{i}\right|_{s+\Delta s}=\varepsilon_{i} l_{t}+\Delta t \cdot \dot{\varepsilon}_{i}\left(\sigma_{\infty q}, \prod_{t}\right)
$$

where the values on the right-hand side of the equation above are evaluated at time $t$, as indicated. The initial strain for the transient is assumed to be zero.

\section{Available Creep Strain Correlations}

For MT/HT-9 steel, creep strain and strain rate correlations exist which can be used to determine the creep as a function of time for a given stress and temperature condition. Unfortunately, as with the rupture time curves shown in Figure 5.20, these relations are based on data for a limited temperature range. The creep strain correlation is based on empirical data fitting to existing creep data for HT-9, and gives the creep as a function of time, temperature and stress, as follows: 40

$$
\varepsilon_{\propto q}=\theta_{1} \cdot t^{\theta_{2}}
$$

\footnotetext{
${ }^{40} \mathrm{G}$. Lewis and C.-C. Chuang, "Analyzing Thermal Creep Strain of a Tokamak First-Wall Steet," Journal of Materials, December, 1990, p. 22.
} 
where $\mathrm{t}$ is time in hours, $\theta_{1}=-A(T+\sigma)^{2}+B(T+\sigma)+C$, and $\theta_{2}=D(T+\sigma)+E$

Here, $A=7.491 \mathrm{E}-8, \mathrm{~B}=1.612 \mathrm{E}-4, \mathrm{C}=-8.494 \mathrm{E}-2, \mathrm{D}=-3.028 \mathrm{E}-4$, and $\mathrm{E}=6.681 \mathrm{E}-1$

$\mathrm{T}$ is in $\mathrm{K}$ and $\sigma$ is in $\mathrm{MPa}$. This correlation is based on data in the 500-600 degree $\mathrm{C}$ range.

The strain rate correlation is based on empirically fitting a phenomenologically-based model, and is written as follows: ${ }^{41}$

$$
\dot{\varepsilon}_{e q}=\frac{B}{k T}\left(\sigma-\sigma_{o}\right) \exp \left(-Q^{*} / k T\right),
$$

where $\mathrm{B}=7.385 \mathrm{e}-3, \mathrm{Q}^{*}=1.23 \mathrm{eV}, \mathrm{k}=8.6207 \mathrm{e}-5 \mathrm{eV} / \mathrm{K}$, and $\sigma_{o}=\mathrm{aT}+\mathrm{C} \mathrm{ksi}$, where $\mathrm{a}=$ -0.2185 and $C=198.1783$. The units of $\dot{\varepsilon}_{e q}$ are $(1 / \mathrm{hr}), T$ is in $\mathrm{K}$, and $\sigma$ is in ksi. As with the creep strain equation, this equation is based on data in the temperature range 500-600 degrees $C$.

\section{Comparison of Creep Strain Limits}

The rupture fraction, creep strain, and creep rate relations all suffer from a lack of data above 600-650 degrees C. Therefore, it is prudent to take a look at the extrapolated behavior of the three relations for temperatures above 650 degrees $\mathrm{C}$. To compare the relations, each of the relations are used to calculate the time to failure of a first wall channel as a function of temperature assuming it is pressurized to $10 \mathrm{MPa}$. For the rupture time relation, failure is of course considered to occur at the rupture time. For the creep strain and creep rate correlations, failure is considered to occur at $1 \%$ creep strain. The Von-Mises stress caused by pressure in the first wall channels is $75.9 \mathrm{MPa}$ at $10 \mathrm{MPa}$ helium pressure. Figure 5.21 shows the results of the comparison.

\footnotetext{
${ }^{41}$ R.J. Amodeo and N.M. Ghoniem, "Developmem of Design Equations for Ferritic Alloys in Fusion Reactors," Nuclear Engineering and Design/Fusion, Volume 2, 1985, p. 97.
} 


\section{Figure 5.22. Comparison of Creep Limit Relations}

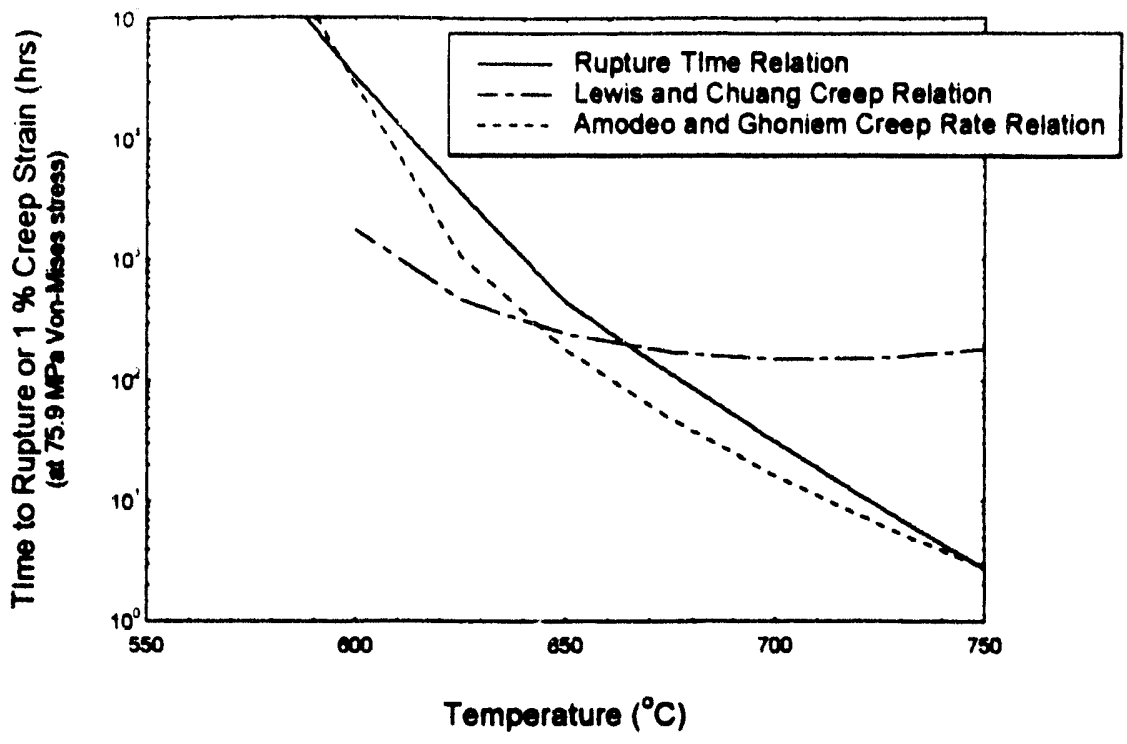

As can be seen in this

figure, the Amodeo

and Ghoniem creep

rate and the rupture

time relations behave

rather as one would

expect. That is, the

time to $1 \%$ strain or

rupture gets smaller as

the temperature increases and the rate of creep gets larger.

The Lewis and Chuang creep relation, however, indicates that the time to rupture increases with temperature at higher temperatures. This is a completely unphysical result which effectively means that the above extrapolation of the Lewis and Chuang relation above 600 degrees $C$ is inappropriate. Therefore, for the failure analysis of the present work, only the rupture time and the Amodeo and Ghoniem relations will be used. The blanket design will be considered to have survived the transient if the rupture fraction is less than 1.0 and the total creep is less than $1 \%$ at the end of the transient, based on the Amodeo and Ghoniem creep relation.

\section{Failure Analysis Rezults}

To evaluate whether the first wall is damaged by acute structural failure, the maximum Von Mises equivalent stress at point $B$ is evaluated. This maximum stress occurs at the onset of the casualty, before the thermal stress is relieved via creep, and at point B the thermal and pressure stresses are both positive. Hence they combine in the case of a NoFlow LOFA to make a larger positive stress. For an operational plasma heat load of 
$0.8 \mathrm{MW} / \mathrm{m}^{2}$ (corresponding to an average neutron wall load of $4 \mathrm{MW} / \mathrm{m}^{2}$ ), an erosion layer of $2 \mathrm{~mm}$, and a channel wall thickness of $0.6 \mathrm{~mm}$ (corresponding to the first wall channel diameter of $8 \mathrm{~mm}$ ) the Von Mises equivalent stress at the onset of a No-Flow LOFA is $197 \mathrm{MPa}$. For a LOCA, the pressure stress does not exist; the thermal Von Mises stress alone at point $B$ at the onset of the accident is $150 \mathrm{MPa}$. Creep of the first wall region will work to decrease the thermal stress as the accident proceeds.

Acute structural failure is predicted to occur if the Von Mises equivalent stress at point B exceeds the UTS for MT/HT-9. Although structural failure may not result in a loss of coolant channel integrity (for example, a small crack at point B which did not go through the wall may not compromise blanket performance), for the purposes of this study, any structural damage will be considered to be unacceptable.

Figure 5.18 shows the temperature dependence of the UTS for MT/HT-9. For the NoFlow LOFA, the maximum Von Mises stress, as we have seen, is $197 \mathrm{MPa}$. Figure 5.18 indicates that the UTS remains higher than $197 \mathrm{MPa}$ for temperatures below 780 degrees C. Since the temperature of the first wall reaches a maximum of 772 degrees $C$ during the No-Flow LOFA, acute structural failure should not occur.

A simplified analysis of a LOCA and a LOCA with air ingress was performed in Section 5.2.3.1, which neglected any conduction from the first wall to the back of the blanket. Now that the effect of such conduction has been accounted for, it can be seen that the maximum temperature of 1260 degrees $\mathrm{C}$ determined in Section 5.2.3.1 for a LOCA with air ingress (see Table 5.11) was much too high. The results of the No-Flow LOFA analysis indicate that conduction within the blanket will limit the first wall temperature to 772 degrees $\mathbf{C}$ even without any flow within the helium channels. Hence, clearly, for a 
LOCA with air ingress, the maximum temperature would be even less than 772 degrees $C$, since the air circulating within the channels would augment the conductive cooling Since the maximum Von Mises stress in the first wall during a LOCA is only $150 \mathrm{MPa}$ (due to thermal effects), clearly there should be no acute structural failure as a result of a LOCA with air ingress. Since there are no pressure stresses during a LOCA, there is no creep rupture concern. Hence, contrary to the results of the simplified analysis of Section 5.2.3.1, Blanket Design 1 should be able to withstand both a LOCA and a LOCA with air ingress with no damage. Furthermore, it is clear that the conductive cooling of the first wall is sufficient to keep the temperature below the temperature required for acute failure. Hence, Blanket Design 1 should withstand even a LOCA which occurs in tandem with a failure of the helium circulators.

Using the creep-rupture analysis methods discussed above, the rupture fraction and creep strain accumulated for the No-Flow LOFA in Blanket Design 1 can be calculated. The rupture fraction and total creep strains at the end of the transient are shown in Table 5.12 .

\section{Table 5.12. Blanket Design 1 Failure Analysis Results}

\begin{tabular}{|c|c|}
\hline Rupture Fraction: & 4.9 (failure) \\
\hline Total $\varepsilon$ in $x$-directio & $4.1 \%$ (failure) \\
\hline
\end{tabular}

As can be seen, the rupture fraction significantly exceeds 1.0 for the No-Flow LOFA, indicative of failure. Additionally, the creep strain in the x-direction (refer to Figure 5.21) exceeds $1 \%$, also indicative of failure. The creep strains in the $y$ - and $z$-directions are negative, hence they are not a concern from a structural failure standpoint. 
One possible method to improve Blanket Design 1 would be to reduce the Von-Mises pressure stress seen by the first wall coolant channels by making the walls thicker, or by reducing the coolant design pressure. To see how big the change would have to be to allow Blanket Design 1 to survive the No-Flow LOFA, the rupture fraction is calculated for a variety of coolant pressures, assuming the temperature vs. time profile of Figure 5.16. Figure 5.23 shows the results (note that the creep strain is not plotted here because the Von-Mises stress values drop far below the stress range wherein the creep correlation is valid, and the creep results become spurious).

\section{Figure 5.23. Rupture Fraction vs. Coolant Pressure} for Blanket Design 1

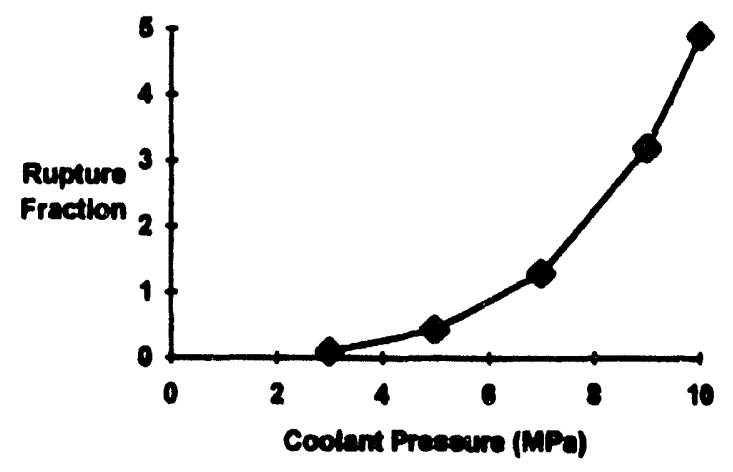

As can be seen in this figure, for coolant pressure above about $6 \mathrm{MPa}$, the rupture fraction rapidly exceeds 1.0 for the accident. Lowering the coolant pressure by 40 percent would have a serious impact on the required

pumping power for the first wall, which would decrease the overall blanket power conversion efficiency to undesirably low values.

Other methods to reduce the stress in the first wall, such as decreasing the coolant diameter or increasing the channel thickness would also significantly increase the required pumping power. Increasing the wall thickness increases the temperature change across the wall, which thereby requires a higher heat transfer coefficient to the helium in order to prevent the onboard side of the first wall from exceeding the maximum 
allowable operating temperature. This in turn requires a higher helium flow velocity, which translates into much greater pumping power necessary to maintain that velocity Increasing the wall thickness also increases the amount of afterheat released into the blanket during the casualty, making it more severe.

Since the pumping power is already relatively high for the first wall channels in this design, increasing the required pumping power by a large amount would result in an undesirable blanket design. Hence it can be concluded that although Blanket Design 1 survives a LOCA, a LOCA with air ingress, and even a LOCA in tandem with helium circulator failure, it is not a suitable blanket design for survival of the worst-case undercooling accident, a No-Flow LOFA. 


\subsection{Blanket Design 2 - Nested Shell with Beryllium Joints}

Blanket Design 1 fails to survive the worst-case No-Flow LOFA, despite efforts to maximize the conductivity of the pebble bed region and minimize the overall blanket thickness. Hence, a new, more radical blanket design concept will be investigated in an effort to find a design which can survive the No-Flow LOFA. Lithium oxide and beryllium perform well from a neutronics point of view, and so they will continue to be used as breeder and multiplier materials. Additionally, we desire to stay with MT-9 as a structural material, and with the nested shell blanket concept. Rather than changing materials or basic blanket design, then, we look toward modifying the breeder region to significantly increase its thermal conductivity.

Based on the analysis on Blanket Design 1, it appears that the pebble bed breeder region cannot be made conductive enough to allow the nested shell blanket design to survive the No-Flow LOFA. It will be recalled that the beryllium volume fraction and pebble size were maximized in Blanket Design 1 to increase the pebble bed region's conductivity to the highest achievable value. Hence, the next blanket design concept to be studied (Blanket Design 2) will use solid beryllium, rather than pebbles of beryllium, in the breeder regions.

It is desirable to use solid beryllium as a conductor to help heat flow from the first wall region toward the cooler back of the blanket (and ultimately to the shield), but the use of solid beryllium slabs is problematic because of beryllium's potential for severe swelling under high-energy neutron irradiation. In Blanket Design 2, a concept is proposed which allows the use of solid beryllium for thermal conduction while allowing for significant swelling of the beryllium. 
The key to this concept is the use of "beryllium joints," rather than solid beryllium slabs, for thermal conduction through the blanket. This joint concept takes advantage of thermal conduction through solid beryllium and yet provides for swelling of the beryllium without producing stresses in the blanket structure. A beryllium joint is composed of two slabs of beryllium placed next to each other in thermal contact, but not welded or brazed together. Thus, the two slabs are free to move relative to each other. Space is left between the slab and the other shell to accommodate swelling in the slab in the direction perpendicular to the shells. Figure 5.24 shows the beryllium joint configuration. The lithium oxide beds are separated from the beryllium slabs by dimpled metal divider plates, and the lithium bed is packed loosely to allow for swelling of the beryllium in the direction parallel to the shells.

\section{Figure 5.24. Beryllium Joint Concept}

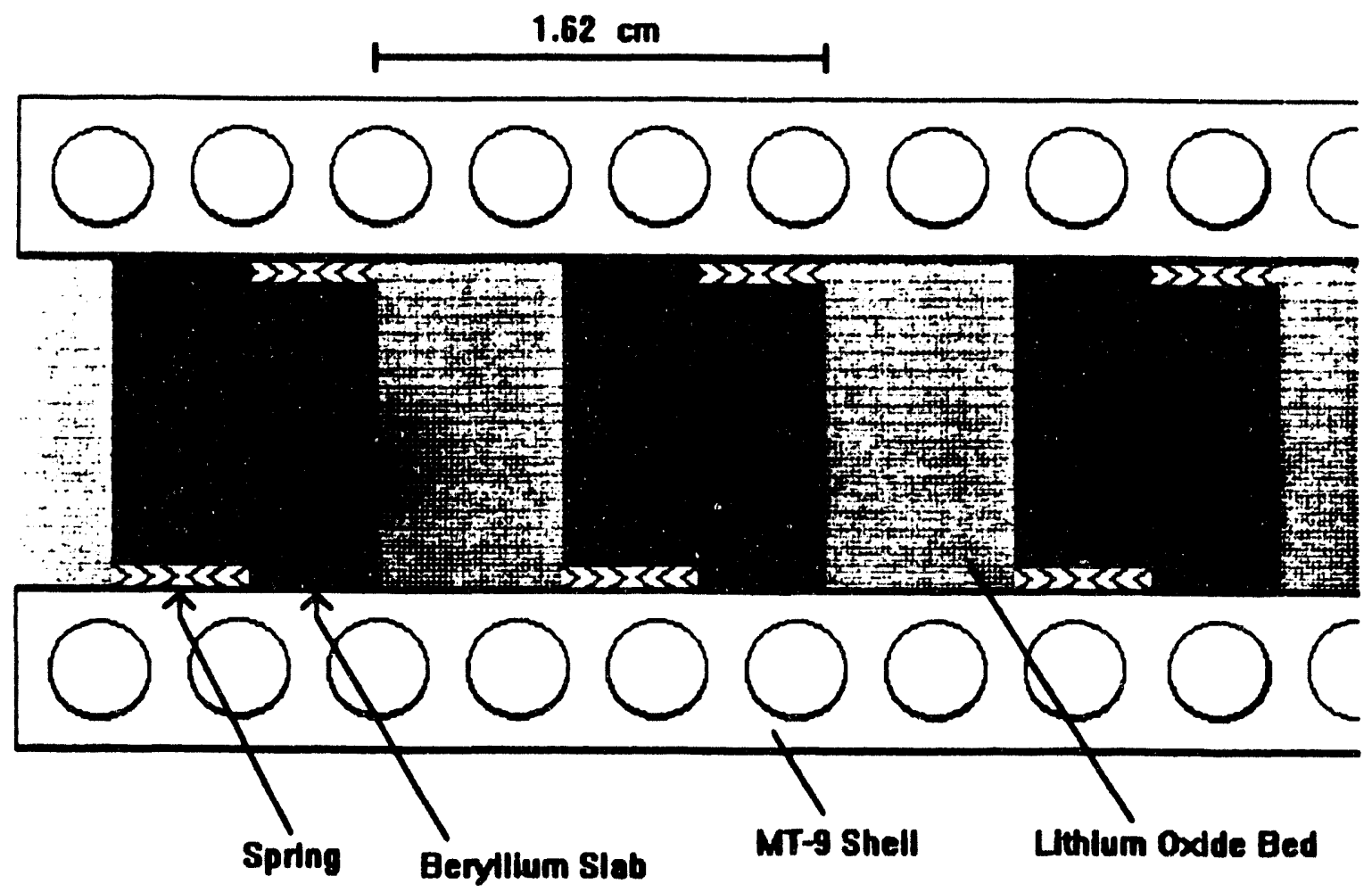


It is obviously important to ensure that the beryllium slabs remain in contact with the MT-9 shells and with each other, to ensure a flow path for heat through the beryllium is maintained. It would be very difficult to ensure this if the beryllium slabs were attached to the MT-9 shells permanently, via brazing or welding. The tolerances required to fabricate the blanket if this were done would be quite difficult to achieve, since each beryllium slab would have to be perfectly lined up with the one opposite it. Additionally, even if the blanket could be fabricated to the required tolerances, the slightest strain (due, for example, to thermal effects as the blanket heats up to normal operating temperatures) could result in a gap appearing between the slabs, and the resultant large decrease in thermal conductivity.

Rather than attach the slabs permanently, then, the beryllium slabs are held next to the MT-9 shells via springs located at the ends of the slabs, which cause the slabs to be pressed against the MT-9. These springs do not need to be complicated (see Figure 5.25 for an example of what the spring might look like), and ensure that the beryllium slabs always remain in contact with the MT-9 shells, barring extreme deformation of the shell geometry (which would not be expected). To keep the slabs in contact with each other, the thin metal plates dividing the lithium oxide beds from the beryllium slabs are equipped with dimples, which maintain contact pressure between the slabs (refer to Figure 5.25). The divider plates also provide tension to keep the MT-9 shells on either side of the breeder regions from separating due to the beryllium slab spring forces. The "tensioner" wire shown in Figure $\mathbf{5 . 2 5}$ helps to maintain lateral contact pressure between the slabs, and helps in fabrication of the blanket. During fabrication, the beryllium joints would be assembled as shown in Figure 5.25 prior to nesting together the blanket shells. The shells would then be nested together with the joints installed between them, and the metal divider plates would be electron beam welded to the adjacent structural shell. 
To further ensure thermal contact between the beryllium slabs and the MT-9 and between the two slabs in the event some misalignment does occur at the contact regions, porous copper inserts are used between contact surfaces (see Figure 5.25). This porous copper is fabricated by sintering copper powder together with a built-in porosity. Such porous copper is used in fuel filters and in porous bearings. Copper metal has a high thermal conductivity, and a low yield strength, especially at high temperatures. The high conductivity ensures good heat transfer in the gap regions, and the low yield strength ensures the copper will conform to misalignments of the slabs. Adding porosity to the copper reduces the thermal conductivity, but also makes the copper more compliant, helping to ensure good conductivity even with low contact pressures.

The proposed beryllium joint design has the benefit of separating the lithium oxide from the beryllium, eliminating the possibility of chemical interactions between the two materials. There is serious concern for the possibility of such an interaction between lithium oxide and beryllium (see Chapter 4), hence, physical separation of these materials is indeed an attractive feature. 


\section{Figure 5.25. Beryllium Joint Details}

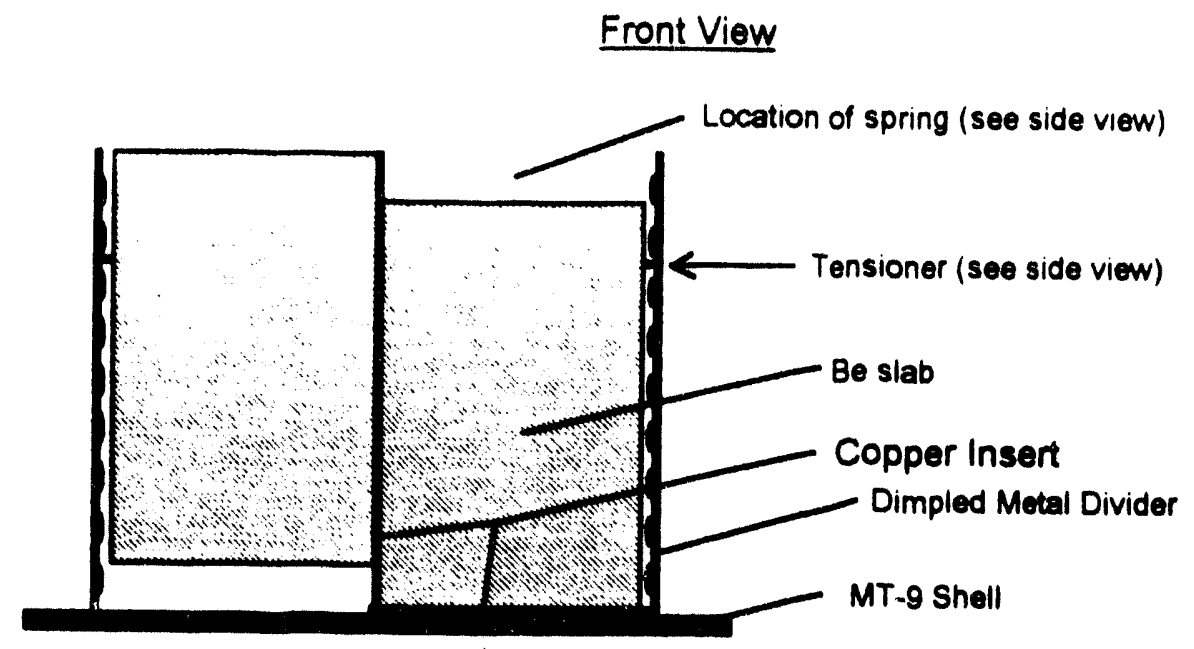

Side View (showing spring)

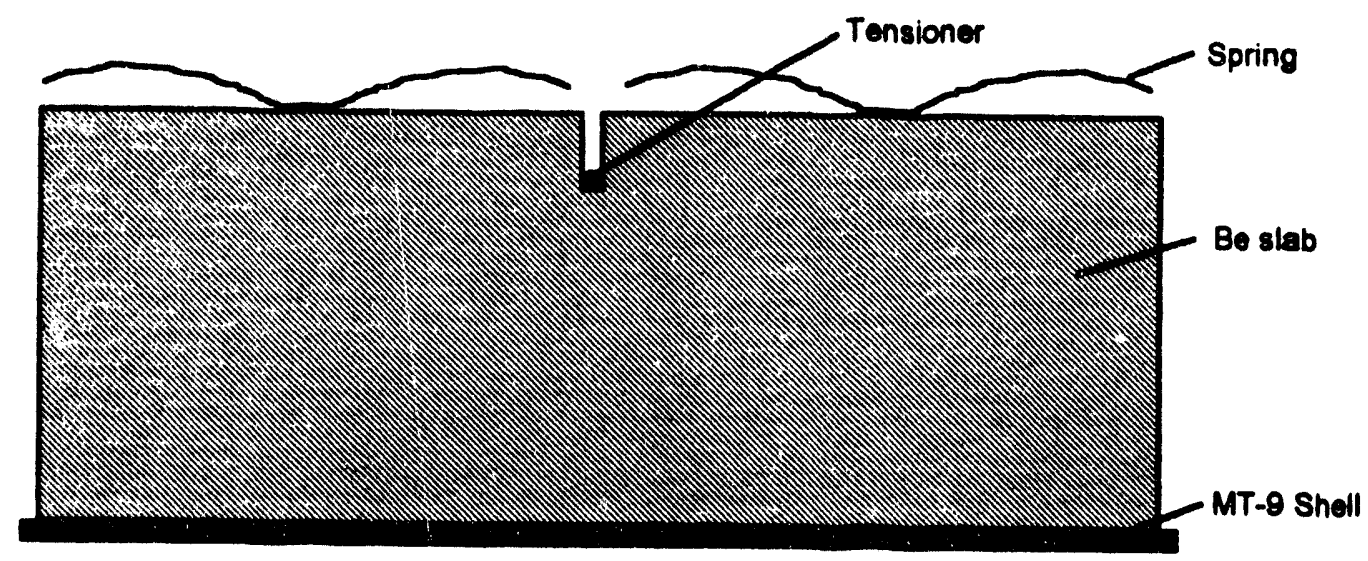

The beryllium joint design proposed here can be designed to accommodate a large range of swelling values, by making the gaps at the ends of the beryllium appropriately large, and by sufficiently under-packing the lithium oxide beds. In the analysis which follows, 10 percent beryllium swelling is somewhat arbitrarily taken as the design value for the blanket. This value is above the 4-5 percent swelling range targeted by materials experts as a goal for beryllium used for fusion applications, but is below the possible value that could be seen at higher operating temperatures (see the paragraph on beryllium in 
Chapter 4 for more information on beryllium swelling behavior). Higher or lower values could have been chosen and designed for as well, with lower swelling values resulting in smaller gap sizes and somewhat better thermal and neutronics performance, and higher values resulting in somewhat worse performance.

\subsubsection{Blanket Design 2: Neutronics}

The neutronics analysis of Blanket Design 1 resulted in an optimum beryllium-to-lithium oxide ratio, as well as the required thickness of the blanket for adequate tritium breeding. Blanket Design 2 uses the same materials as Blanket Design 1, but in a different configuration. The configuration of Blanket Design 2 is significantly less homogenous than that of Blanket Design 1, since the beryllium and lithium oxide in the blanket are in separate regions, rather than in a mixed pebble bed. This non-homogeneity makes the blanket much more complex to analyze from a neutronics point of view, and to start from the beginning and analyze Blanket Design 2 as Blanket Design 1 was analyzed would require a significant amount of computational time and effort.

Fortunately, the results of Blanket Design 1 can be used for Blanket Design 2 as well, provided the length scale of the separate lithium oxide and beryllium regions in Blanket Design 2 are small enough. The effect of inhomogeneity on breeding performance in breeding blankets has been investigated by Fischer ${ }^{42}$ He showed that since the mean free path of a neutron is rather long in a typical fusion breeder blanket ( 3 to $6 \mathrm{~cm}$ ), as long as the length scale of the heterogeneous regions is kept small enough (of order $1 \mathrm{~cm}$ or less), the tritium breeding performance of the heterogeneous blanket is the same as that of a homogeneous blanket. Therefore, the neutronics and tritium breeding performance will be the same for the two blanket designs if the following design features

\footnotetext{
42U. Fischer, "Optimal Use of Beryllium for Fusion Reactor Blankets," Fusion Technology, Vol. 13, 1988, p. 143.
} 
are incorporated in Blanket Design 2: (1) keep the widths of the beryllium joint region and the lithium oxide bed regions of Figure 5.24 less than or equal to $1 \mathrm{~cm}$; and (2) maintain a similar average volume fraction of lithium oxide and beryllium for Blanket Design 2 as existed for Blanket Design 1.

The first of the two requirements stated above is accomplished by simply requiring that the beryllium joint regions (which are thicker than the adjoining lithium oxide bed regions) be $1 \mathrm{~cm}$ thick (note the scale of Figure 5.24). The second requirement is satisfied by picking the appropriate region thickness, packing fraction, and pebble porosity for the lithium oxide bed regions. The beryllium slabs are chosen to be 100 percent dense, to maximize their conductivity. The lithium oxide pebble bed packing fraction is chosen to be 54 percent. This is 10 percent less than the maximum singlesized packing fraction of about 60 percent, allowing for 10 percent swelling in the beryllium slabs on either side of the bed. If the lithium oxide bed region is $0.62 \mathrm{~cm}$ thick, the resulting average volume fractions for the breeder region are shown in Table 5.13. Table 5.13 also shows the volume fractions in the mixed bed breeder region for Blanket Design 1, for comparison.

\section{Table 5.13. Volume Fractions in Breeder Regions}

\begin{tabular}{|l|c|c|}
\hline & Berrllium & Lithium Oxide \\
\hline Blanket Design 1 & 0.54 & 0.18 \\
\hline Blanket Design 2 & 0.55 & 0.19 \\
\hline
\end{tabular}

As can be seen in this table, Blanket Design 2 has slightly higher beryllium and lithium oxide volume fractions than Blanket Design 1. It also has a slightly lower beryllium-tolithium oxide ratio. Referring back to Figure 5.2, however, it can be seen that a slightly lower beryllium-to-lithium oxide ratio will not hurt the tritium breeding ratio. Hence, 
because Blanket Design 2 has higher volume fractions, there should be a slight increase in the tritium breeding ratio for Blanket Design 2, assuming the shell spacing and the number of shells are kept the same as Blanket Design 1. Since the tritium breeding ratio of Blanket Design I was more than sufficient, the analysis to follow will assume that Blanket Design 2 has the same blanket thickness and shell spacing as Blanket Design 1, with the only change being the configuration of the breeder regions. 


\subsubsection{Blanket Design 2: Beryllium Joint Design}

The following section discusses the design parameters for the beryllium joints, including the appropriate porosity range to use for the copper inserts and the required magnitude of the contact pressure to ensure the inserts comply with misalignments of the beryllium slabs. The following paragraphs also discuss the thermal performance of the beryllium joint design and Blanket Design 2.

It is recognized that the effectiveness of the design of the springs, dimpled divider, and porous copper arrangement for the beryllium joint would have to be confirmed by actual construction and testing of the joint, which is beyond the scope of the present work. However, the design discussed here is proposed as a feasible method to ensure that thermal contact between the beryllium slabs and the MT-9 shell, as well as between the slabs themselves, is maintained during operation of the blanket.

To model the beryllium joints in a one-dimensional thermal-hydraulics code for the NoFlow LOFA analysis, the effective thermal conductivity of the joints between coolant channels as a function of temperature must be found. This effective one-dimensional conductivity can then be used in the HEATING code to calculate the heat flow through the joints toward the back of the blanket during the accident.

The first necessary piece of information is the conductivity of the beryllium slabs in the joint. There are two basic processes for producing beryllium slabs, the ingot metallurgy (IM) process and the powder metallurgy (PM) process (see Chapter 4). 100 percent dense beryllium produced via the $\mathbf{I M}$ process has a significantly higher conductivity than beryllium produced by powder metallurgy. Hence, this type of beryllium is used for the 
beryllium slabs to enhance the conductivity of the joint. Figure 5.26 shows the conductivity of IM beryllium.

Figure 5.26. Conductivity of $\mathrm{MM}$ Beryllium $(100 \text { percent dense })^{43}$

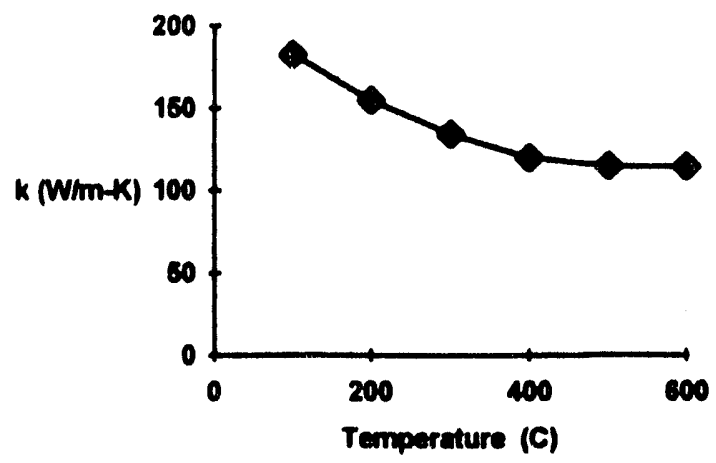

As this figure shows, the conductivity of IM beryllium is above $100 \mathrm{~W} / \mathrm{m} \cdot \mathrm{K}$ for temperatures up to 600 degrees $C$, and approaches 200 at lower temperatures. (For temperatures above 600 degrees $C$, conductivity data are unavailable; the conductivity is considered to remain constant at the 600 degree

C value for these temperatures.)

The conductivity of the lithium oxide bed is the next necessary ingredient. This depends on the size of the lithium oxide pebbles in the bed. As discussed in the section on neutronics above, the bed packing is such that the pebbles take up 54 percent of the bed (which allows for swelling of the beryllium). A pebble diameter of $0.88 \mathrm{~mm}$ is chosen, which is well below $1 / 5$ of the bed width of $0.64 \mathrm{~cm}$, ensuring good bed packing (refer to Section 5.2.2.1). The thermal conductivity for the bed is about $1.3 \mathrm{~W} / \mathrm{m} \cdot \mathrm{K}$ for the temperature range 220 to 820 degrees C. This conductivity is used in the following NoFlow LOFA analysis.

\footnotetext{
${ }^{43}$ From D.R. Floyd, Thermophysical Properties of a New Ingot Metallurgy Beryllium Proctuct Line, presented to the Beryllium Technology Workshop at the Fifth International Conference on Fusion Reactor Materials, Idaho National Engineering Laboratory Report \#EGG-FSP-10017 dated December 1991.
} 
A critical element in the beryllium joint concept is the thermal conductance for the contact regions between the slabs and the MT -9 shells and between two slabs. The conductance of such a region is sensitive to a number of factors, including the nature of the surfaces in contact, the characteristics of the porous copper in the gap, the pressure applied to the contact region, and whether or not there is a bond between the surfaces.

The porous copper between the regions helps to maintain thermal conductivity even if there is misalignment between surfaces. The reason copper is chosen as the material for the inserts in the beryllium joints is that it has a relatively low yield stress. This makes it more compliant, and hence less contact pressure is required to ensure good thermal contact in the gap regions. It is assumed that good compliance, and hence good thermal contact, occurs in the gap regions if the contact pressure is equal to or greater to the yield strength of the porous copper. A lower porosity is desirable since it results in higher thermal conductance. However, the copper becomes more compressible at higher

Figure 5.27. 100 Percent Dense Copper Yield Streingth 44

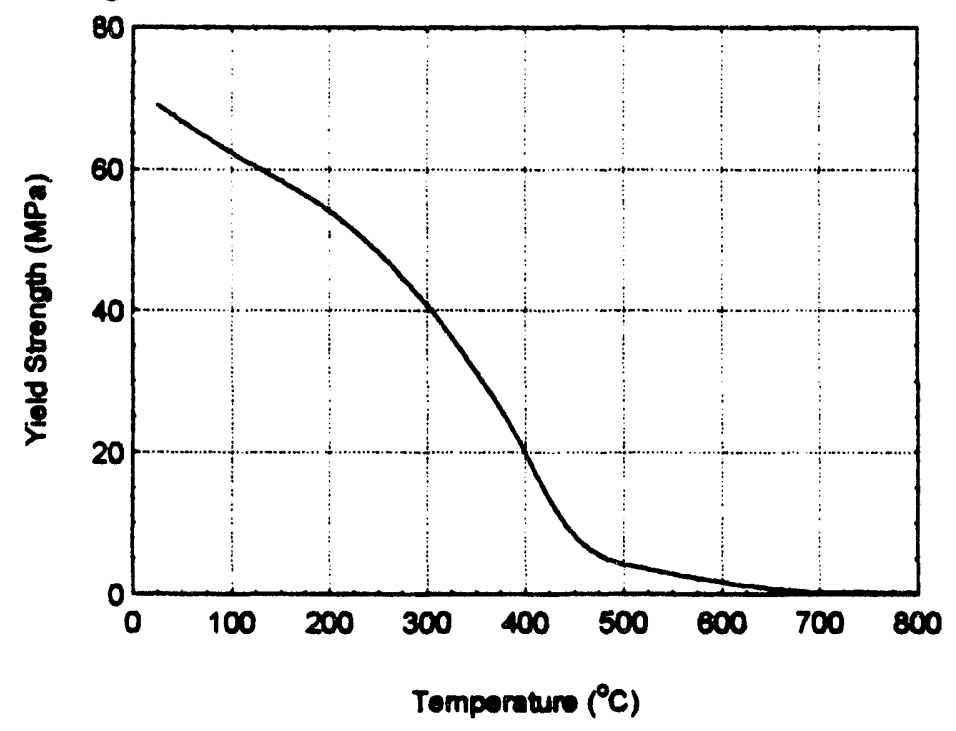

porosities, therefore more

able to accommodate misalignments between the surfaces.

The yield strength of 100 percent dense copper is strongly temperaturedependent. Figure 5.27 shows the estimated yield

\footnotetext{
${ }^{44}$ For OSOSO temper, oxygen-free (OF) copper. Curve derived by scaling the yield strength vs. temperature curve of 1180 temper OF copper (which has a room temperature yield strength of $415 \mathrm{MPa}$ ) to the room temperature yield strength of OSO50 temper OF copper. Source, W. H. Cubberly, et al. eds., Metals Handbook, Ninth Edition, Vol. 2, American Society for Metals, 1979, pp. 275-277.
} 
strength curve for oxygen-free copper. As shown, the yield strength drops rapidly as temperature is increased. As it turns out, the temperature in all of the beryllium joints reaches 500 degrees $\mathrm{C}$ or above quickly after the No-Flow LOFA begins (refer ahead to Figure 5.38). Hence, the yield strength for the copper matrix of the gap inserts rapidly decreases to $5 \mathrm{MPa}$ or less after the onset of the LOFA.

The yield strength for a porous material with a matrix material which yields plastically has been shown to be determined by the following relation:45

$$
\sigma_{p t}^{*}=C \sigma_{y}(1-P)^{1.9} \text {, }
$$

where $\sigma_{p l}^{*}$ is the yield, or plastic collapse, strength of the porous material, $\sigma_{y}$ is the yield strength of the matrix material, $\mathrm{P}$ is the porosity, and $\mathrm{C}$ is a constant which varies from 1.0 to 0.3 . $\mathrm{C}$ is 0.3 for porosities greater than 0.7 , and approaches 1.0 as porosity decreases to 0 . For the following analysis, it is assumed that $\mathrm{C}=1.0$ for all porosities.

Figure 5.28. Copper Insert Yield Strength at 500 degrees $\mathrm{C}$ This yields a

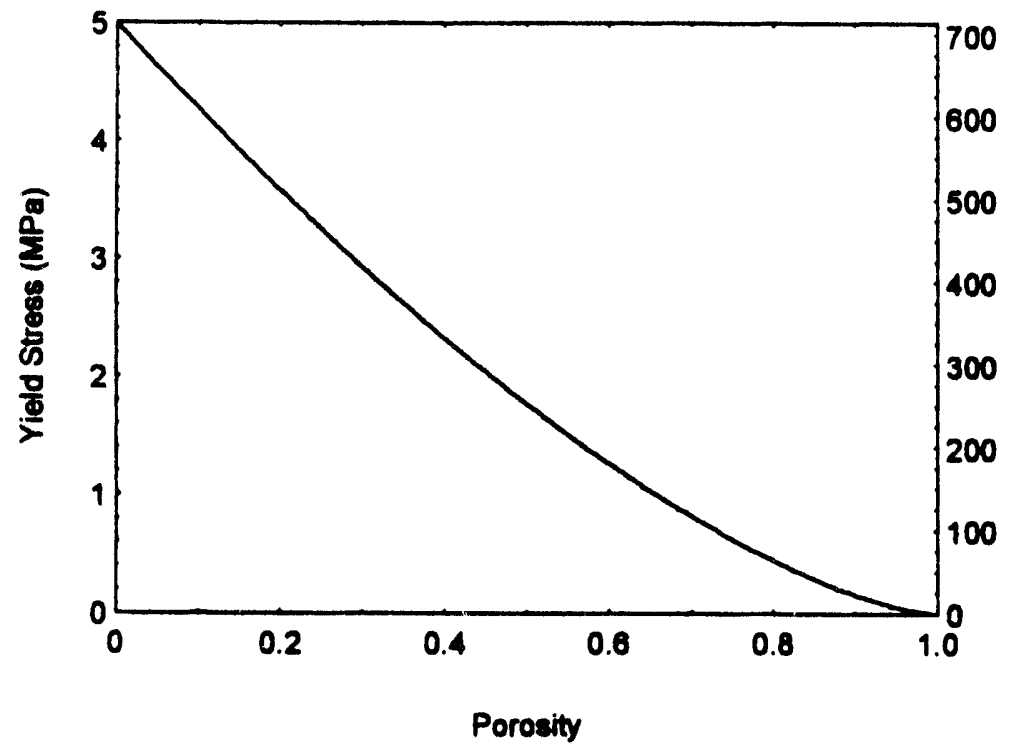
conservatively high value for the required

stress to obtain yielding of the copper inserts. Assuming the copper matrix yield strength is $5 \mathrm{MPa}$, Figure 5.28 shows the stress required to cause yielding as a

\footnotetext{
45S. K. Maiti, et. al., "Deformation and Energy Absorption Diagrams for Cellular Solids," Acta metall., Vol. 32, No. 11, 1984, p. 1963.
} 
function of porosity in the copper inserts. For porosities greater than 0.3 , the yield strength is below $3 \mathrm{MPa}(430 \mathrm{psi})$. This is quite a modest required stress, and is the magnitude of the pressure required to be imposed on the beryllium slabs by the beryllium joint springs and dimpled dividers to ensure good thermal contact in the gap regions This pressure is resisted by the dimpled metal dividers between the lithium oxide beds and the beryllium slabs (refer to Figure 5.25).

At this point the effective conductance of the gap regions containing the inserts can be determined. A schematic of the Be/Be gap region is shown in Figure 5.29. As shown in the figure, the gap region is modeled as a slab of porous copper of thickness $t$ surrounded by spaces of thickness $\delta$. These spaces are filled with helium purge gas at 1 atmosphere. The width of the gap regions is governed by the surface roughness of the porous copper and the beryllium slabs.

The gap region between the Figure 5.29. Schematic of Be/Be Gap Region beryllium slabs and the MT-9 coolant shells is similar to that shown in Figure 5.29, except that one side of the gap is MT-9 material Additionally, it is desirable to braze the copper directly to the MT-9 during assembly, so that only the beryllium side of the MT-9/Be

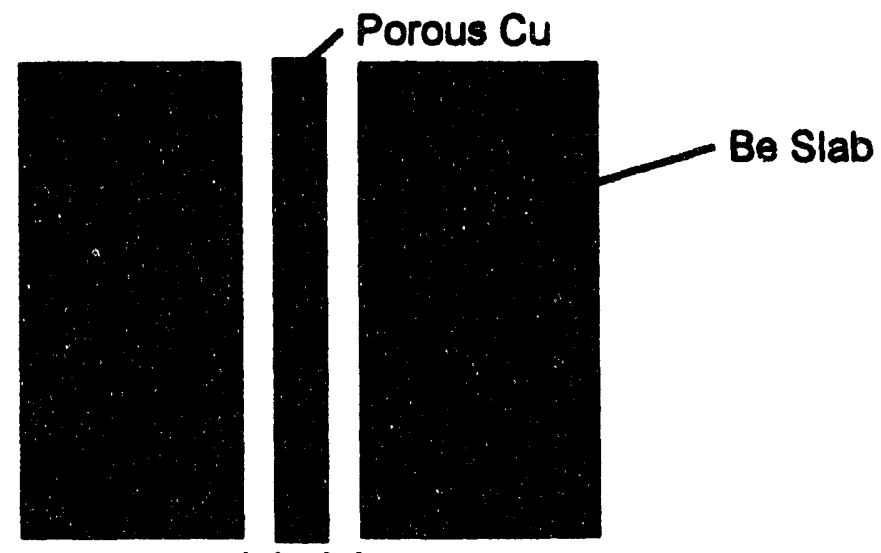
$|\delta|+|\delta|$ gap has a space $\delta$. 
To calculate the conductance of the gap regions, we focus on the $\mathrm{Be} / \mathrm{Be}$ gap shown in the figure. Since the MT-9/Be gap has a space $\delta$ only on one side of the copper, it has a higher conductance that the $\mathrm{Be} / \mathrm{Be}$ gap. However, the conductance calculated for the $\mathrm{Be} / \mathrm{Be}$ gap is used in all of the gap regions for conservatism.

The conductance of the gap region is calculated by calculating the series conductances of the porous copper and the two spaces, as follows:

$$
\mathrm{h}_{\text {sep }}=\frac{1}{\frac{1}{\mathrm{~h}_{\mathrm{cv}}}+\frac{2}{\mathrm{~h}_{\delta}}},
$$

where $h_{c u}$ is the conductance of the porous copper, and $h_{\delta}$ is the conductance of the space $\delta . h_{c o}$ can be calculated with the following relation:

$$
h_{\mathrm{cos}_{0}}=\frac{k_{\mathrm{ce}}}{\mathrm{t}} \text {, }
$$

where $k_{\mathrm{cu}}$ is the conductivity of the porous copper. The conductivity of copper with porosity $\mathbf{P}$ is given by the following formula:

$$
k_{c u}=k_{c a 0} \frac{1-P}{1+11 \cdot P^{2}}
$$

where $k_{c \text { co }}$ is the conductivity of 100 percent dense copper. ${ }^{46}$ Using the value of $k_{c a}$ as calculated by Equation 5.19 is conservative, since any compression of the copper insert in service tends to reduce the porosity, and hence increase the conductivity of the insert.

Calculation of the conductance of the spaces $\delta$ is somewhat more involved. In general, conductance across a gap is given by the following equation: ${ }^{47}$

\footnotetext{
${ }^{46}$ Correlation for copper presented by M. C. Billone at the Beryllium Technology Workshop in Clearwater Beach, FL, Idaho National Engineering Laboratory report EGG-FSP-10017, 1991.
} 


$$
h_{s}=h_{\text {i.oper }}+h_{\text {sontect }}
$$

where $h_{5,0 p e s}$ is the conductance which would exist with no contact pressure, and $h_{\text {conuct }}$ is the added conductance caused by contact pressure. Neglecting radiation effects, which are negligible for cases of interest here, $h_{\delta, \text { ppen }}$ is given as follows

$$
\mathrm{h}_{\text {o. opes }}=\frac{\mathrm{k}_{\mathrm{gua}}}{\delta_{\text {eff }}}
$$

where $k_{g m}$ is the conductivity of the cover gas, and $\delta_{\text {ef }}$ is the effective gap width, defined as:

$$
\delta_{\text {eff }}=\delta+\delta_{\text {jmpl }}+\delta_{\text {jump2 }}
$$

where the $\delta_{j}$ 's are correction terms which account for mean free path effects near the gas-solid surface caused by the small number of gas molecules present near the surface. The subscripts 1 and 2 correspond to the two surfaces bounding the space $\delta$, namely the copper and beryllium surfaces. For conservatism, negligible contact pressure is assumed in the gap regions. In this case, the gap conductance to be dominated by the $h_{8,0 p e n}$ term of Equation 5.22 .

$\delta_{\mathrm{j} m}$ can be calculated by the following formula:

$$
\delta_{j-m}=\left\{\frac{2-a}{a} \cdot \frac{2}{\gamma+1} \cdot \frac{k_{m}}{\mu C_{v}}\right\} \lambda
$$

where $\lambda$ is the gas mean free path, $C_{v}$ is the specific heat, $\mu$ is the viscosity, $\gamma$ is the ratio of specific heats, and $\mathrm{a}$ is called the accommodation coefficient. ${ }^{48} \mathrm{C}_{\mathrm{v}}, \mu, \mathrm{k}_{\mathrm{om}}$ and $\gamma$ for helium are obtained from Melese and Katz, ${ }^{49}$ with $\mu$ and $\mathbf{k}_{\mathbf{m}}$ being temperature

${ }^{47}$ The following discussion of gap conductances is from N. Todreas and M. Karimi, Nuclear Systems I, Thermal Hydraulic Fundamentals, Hemisphere, New York, 1990.

${ }^{48}$ E. H. Kennard, Kinetic Theory of Gases, Mc-Graw Hill, 1938, p. 314.

${ }^{49} \mathrm{G}$. Melese and R. Katz, Thermal and Flow Design of Helium-Cooled Reactors, American Nuclear SI : : rty, La Grange Park, II, 1984. 
dependent. The mean free path $\lambda$ depends on the pressure, temperature and type of gas; for helium at atmospheric pressure at a temperature of 600 degrees $C, \lambda$ is about 0.2 $\mu \mathrm{m} .{ }^{\nexists}$ The accommodation coefficient (a) is a measure of how gas molecules' energy is adjusted upon bouncing off the surface. The numerical value of the accommodation coefficient is never greater that 1 , but varies widely depending on the surface characteristics, gas type, and temperature, and is somewhat difficult to measure experimentally. However, Song and Yovanovich ${ }^{50}$ have provided an empirical correlation based on numerous experimental measurements. The accommodation coefficient is very sensitive to surface conditions; the Song and Yovanovich correlation is based on data from "engineering" surfaces, that is, surfaces which have not been specially cleaned. Hence, the correlation is particularly applicable for use in the present work. The correlation is presented below:

$$
a=\exp \left[C_{0} \frac{i}{273}\right]\left(\frac{M_{s}}{C_{1}+M_{3}}\right)+\left\{1-\exp \left[C_{0} \frac{T}{273}\right]\right\}\left\{\frac{2.4 \omega}{(1+\omega)^{2}}\right\},
$$

where $T$ is the temperature in degrees $C, M_{3}$ is the molecular weight of the gas, and $\omega$ is equal to $M_{s} / M_{s}$, where $M_{s}$ is the molecular weight of the surface material. $C_{0}$ equals -0.57 and $C_{1}$ equals 6.8 . The accommodation coefficient, as can be seen, depends on temperature and the masses of the surface material and the cover gas. Since it is known that beryllium develops a surface coating of beryllium oxide in the presence of air, the molecular weight for the beryllium surface is assumed to be that for BeO, not Be. This results in a higher jump distance, hence is conservative.

\footnotetext{
\# $\lambda=1 /(1.4140 \mathrm{n})$, where $\sigma$ is the molecular collision cross section and $\mathrm{n}$ is the number density.

30S. Song and M. M. Yovanovich, "Correlation of Thermal Accommodation Coefficient for 'Engineering' Surfaces," in Fundamentals of Conduction and Recent Developments in Contact Resistance, M. Imber, et. al., eds., The American Society of Mechanical Engineers, 1987, p. 107.
} 
We now are in a position to calculate the conductance of the gap region given the thickness of the copper insert ( $t)$, the porosity of the copper (P), the temperature of the gap region, and the width of the spaces $(\delta)$. To calculate $\delta$, the following relation is used, derived from a detailed geometric analysis of conforming rough surfaces: ${ }^{51}$

$$
\delta=1.184 \sigma[-\ln (3.312 \mathrm{P} / \mathrm{H})]^{0.447},
$$

where $P$ is the contact pressure, $\mathrm{H}$ is the hardness of the softer surface (in this case, copper), and $\sigma$ is the effective rms surface roughness, defined as:

$$
\sigma^{2}=\sigma_{1}^{2}+\sigma_{2}^{2}
$$

where $\sigma_{1}, \sigma_{2}$ are the rms roughnesses of the two contacting surfaces. The hardness $H$ for copper at room temperature is $50 \mathrm{MPa}^{32}$ and if a very low contact pressure of 5000 $\mathrm{Pa}$ (less than $1 \mathrm{psi}$ ) is assumed, then we obtain:

$$
\delta=4.26 \sigma \text {, }
$$

which is used for conservatism to calculate $\delta$. The surface roughness for engineering surfaces may vary from about $50 \mu \mathrm{m}$ for rough, flame-cut or sand-cast surfaces to about $0.01 \mu \mathrm{m}$ for surfaces which are specially polished. ${ }^{53}$ A variety of techniques exist for obtaining highly polished surfaces, but as would be expected, highly polished surfaces cost more to fabricate.

Since surface roughness has a strong effect on the overall gap conductance, it is initially assumed that the copper and beryllium surfaces have an ms roughness of $0.03 \mu \mathrm{m}$, which results in an effective rms roughness $(\sigma)$ of $0.05 \mu \mathrm{m}$. These roughness values are quite small, and will be relaxed to larger values in Section 5.3.5 as part of optimization

\footnotetext{
"1M. M. Yovanovich, "Thermal Contact Correlations," in Spacecraft Radiative Transfer and Temperature Control, T. E. Horton, ed, Vol. 83 of Progress in Astronautics and Aeronautics, M. Summerfield, od, 1982, p. 83.
}

${ }^{32}$ Ibid

${ }^{33}$ T. Baumeister, et. al., eds. Marks' Standard Handbook for Mechanical Engineers, Eighth Edition, McGraw-Hill, 1978, p. 13-79. 
of the gap parameters. The copper insert thickness ( $t$ ) must be small to maximize the gap conductance, but must also be fabricable. A value of $0.5 \mathrm{~mm}$ is initially assumed for the copper insert thickness. The porosity (P) of the copper is initially assumed to be 0.3 . Note that in Section 5.3.5 optimum values for all of these gap parameters are determined.

Figure 5.30 shows the gap conductance as a function of temperature for the chosen gap parameters. As can be seen, the gap conductance increases with temperature, mostly because the conductivity of helium increases. Figure 5.31 shows the effect of temperature on helium conductivity, with the conductivity of air for comparison.

\section{Figure 5.30. Gap Conductance vs. Temperature}

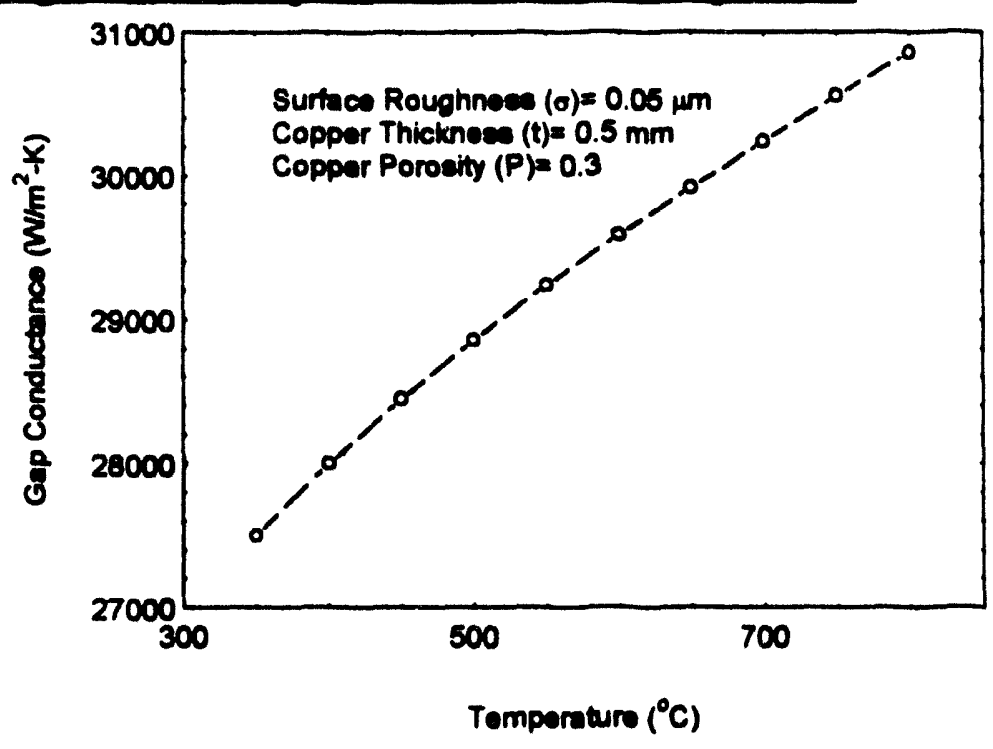


Figure 5.31. Air and Helium Conductivities

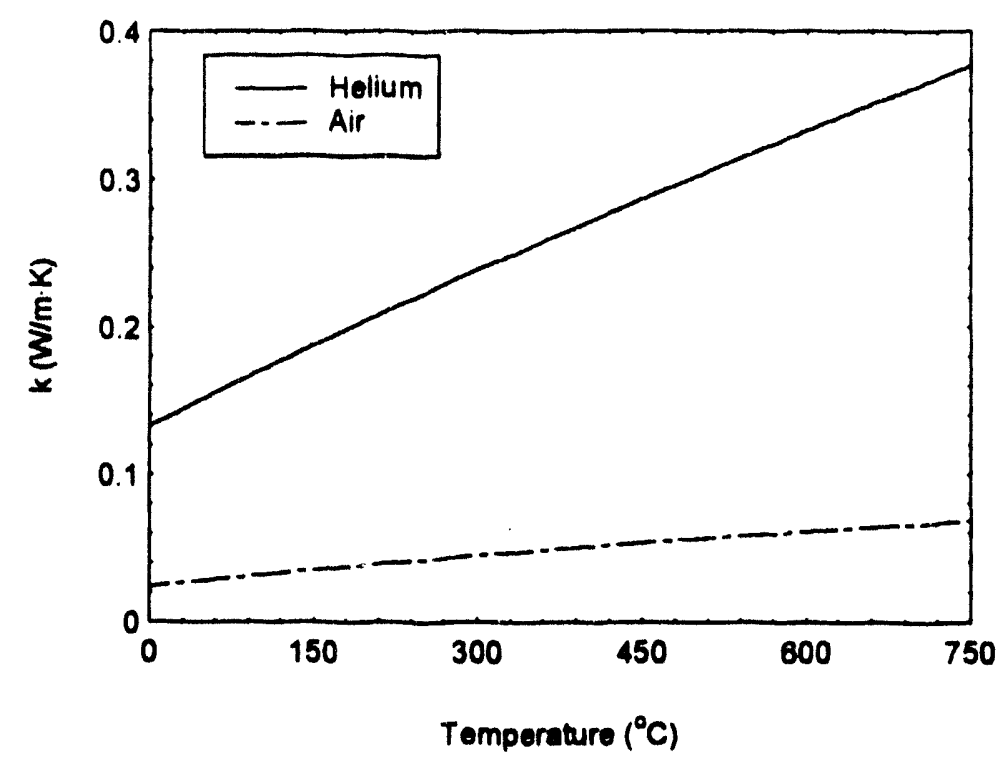

One-Dimensional Thermal Modeling of Beryllium Joint

To incorporate the beryllium joints into a one-dimensional thermal-hydraulics code, the effective one-dimensional conductivity of the joints must be known. This varies as a function of temperature (since both the conductivity of beryllium and the conductance of the gap regions varies with temperature), and as a function of joint thickness. To determine appropriate one-dimensional conductivity values for the joints, a number of two-dimensional HEATING3 runs is performed to evaluate the temperature distribution through the joints for a given value of heat flow through the joints. The average temperature drop through the joints is then calculated, and this average temperature drop is used to determine the effective conductivity of the joint, via the relation: 


$$
k_{\text {edf }}=\frac{q^{\prime \prime} \cdot t}{\Delta T_{\text {ung }}},
$$

where $k_{\text {eff }}$ is the effective conductivity of the joint, $q^{\prime \prime}$ is the specified heat load flowing through the joint, $t$ is the joint thickness (the distance between the adjacent cooling channels), and $\Delta T_{\text {avg }}$ is the average temperature drop across the joint. The twodimensional model used for determination of $k_{\text {edf }}$ is shown in Figure 5.32. Note that $q^{\prime \prime}$ in this figure is the amount of heat flowing into the joint during the No-Flow LOFA.

\section{Figure 5.32. Model for Beryllium Joint Effective Conductivity}

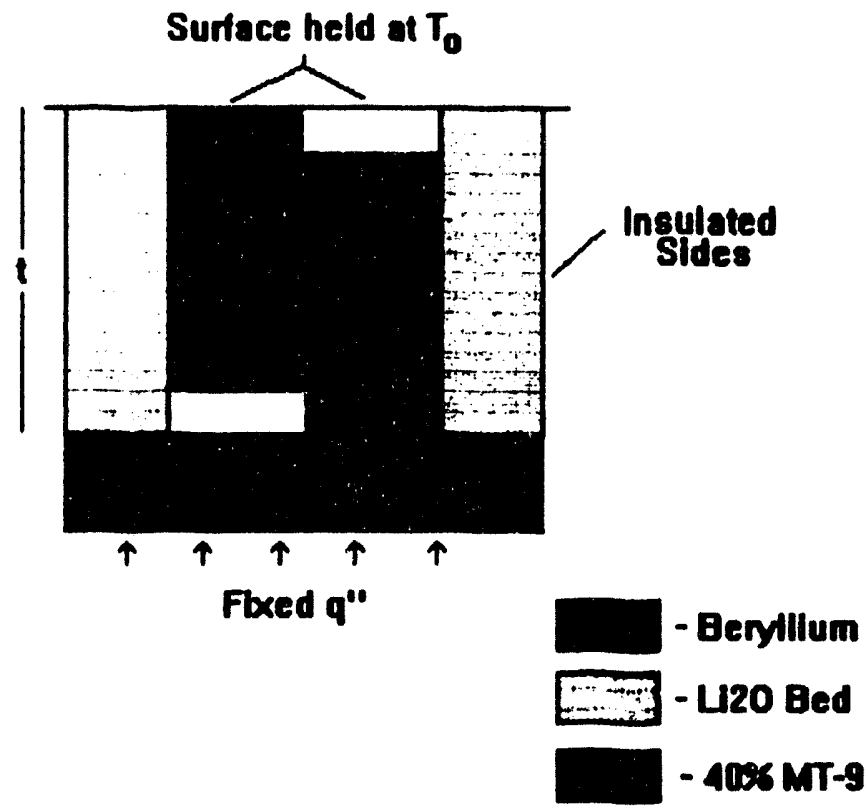

The value of $q^{\prime \prime}$ depends on the time after the onset of the accident, and on where the joint is in the blanket. The joints further back in the blanket have higher heat flow through them at any particular time during the accident, because the heat from all channels closer to the first wall must ultimately flow through the rear-most joints in order to get to the shield. The thickness of the $40 \%$ MT-9 in Figure 5.32 is $9 \mathrm{~mm}$ (a 
typical channel thickness for channels near the front of the blanket.) The beryllium slabs are each $5 \mathrm{~mm}$ thick, and the lithium oxide bed regions are $3.1 \mathrm{~mm}$ thick (half the thickness of the actual beds; the lithium oxide bed midplanes are the planes of symmetry for the joints, and are modeled as insulated boundaries.)

Figure 5.33. Results from 2-D Model of Be Joint

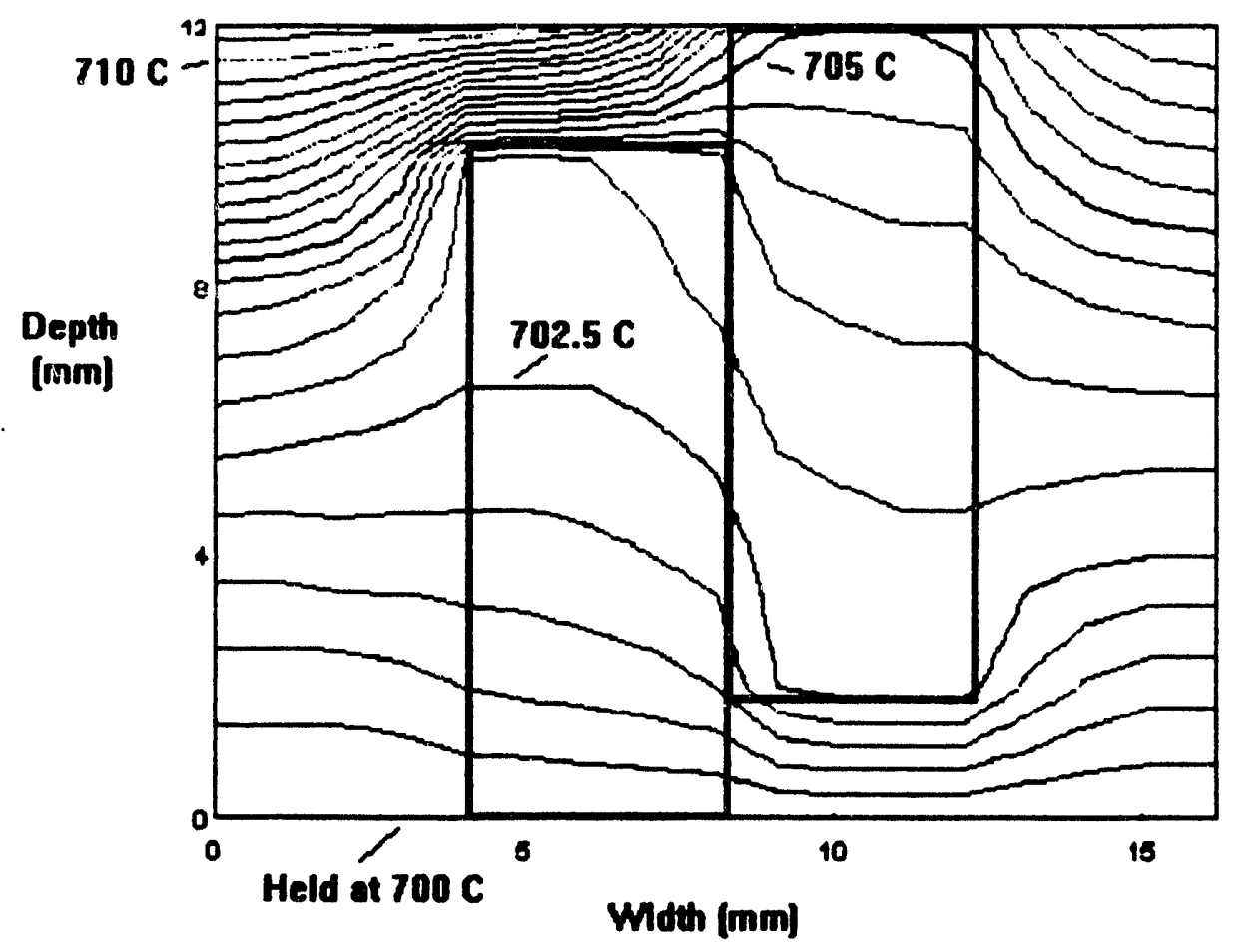

Figure 5.33 shows the temperature distribution of a beryllium joint $12 \mathrm{~mm}$ thick, with a $q^{\prime \prime}$ value of $7200 \mathrm{~W} / \mathrm{m}^{2}$ (note that the orientation of Figure 5.33 is 180 degrees opposite the orientation of Figure 5.32). $12 \mathrm{~mm}$ is the thickness of the first breeder region, and $7200 \mathrm{~W} / \mathrm{m}^{2}$ is a reasonable value for the heat load which would be expected to flow through this channel roughly an hour after shutdown. This heat load comes from the afterheat of the first wall erosion layer, and the two coolant channels behind this layer. 
A possible concern with using the average temperature drop across the joint in our calculation of the effective conductivity is the fact that the temperature of the MT-9 in contact with the joint is not uniform. Hence, using the average temperature drop across the joint as a basis may not be conservative. Figure 5.33 can be used to estimate how large this nonuniformity of temperature is, and whether it is a concern. Looking at the temperatures along the top portion of Figure 5.33, which is where the MT-9 coolant channel connects to the hot side of the breeder region, we note a temperature difference of about 10 degrees $C$ between the hottest and coolest portions of the MT-9. This means that the hottest portion of the first wall is a maximum of about 5 degrees hotter than the average temperature of the first wall. This temperature difference is small, and is therefore neglected.

Figure 5.34. Effective Conductivity of Be Joints

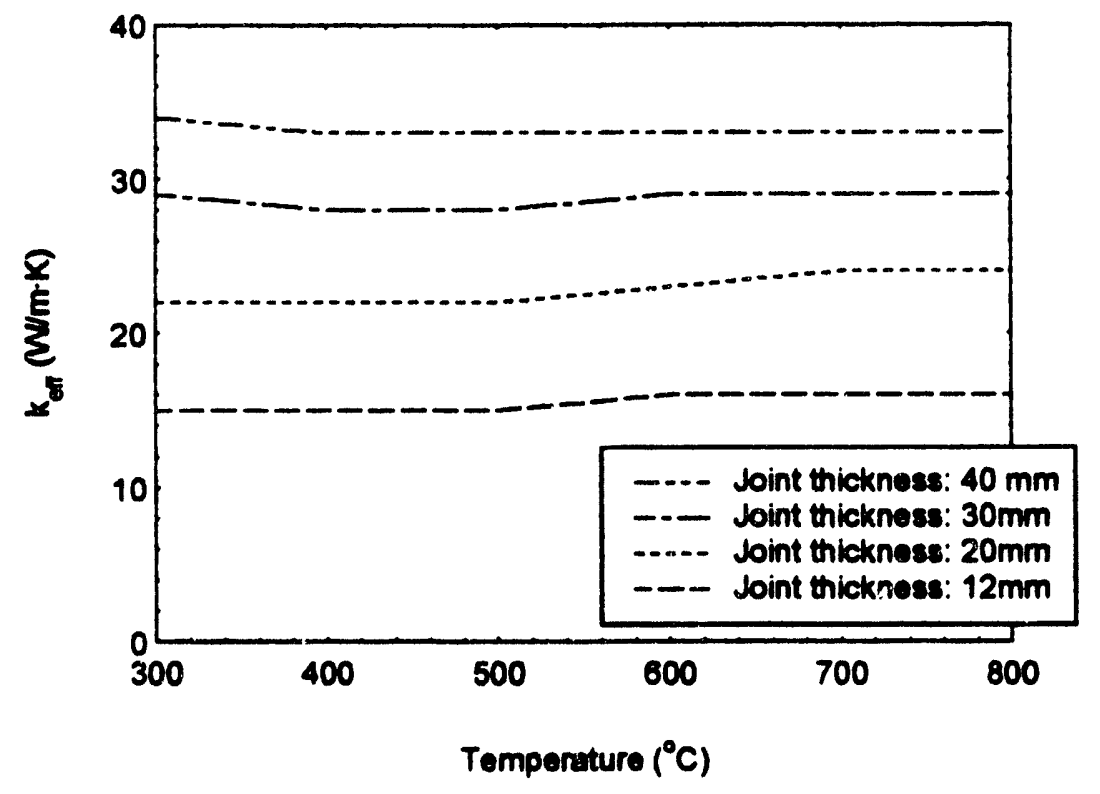

Figure 5.34 shows the effective conductivity of the beryllium joints based on the analysis just described. Note that for a given joint thickness, there is very little conductivity 
variation with temperature. This can be explained by noting that the conductivity of the beryllium slabs decreases with temperature (see Figure 5.26), but the gap conductance increases with temperature, as the conductivity of helium rises (see Figure 5.31). These two effects tend to cancel making the temperature dependence on the effective joint conductivity negligible.

On the other hand, Figure 5.34 shows that there is a strong dependence of the effective conductivity on the joint thickness. This is easily explained by noting that the heat which flows through the joint basically follows a path which takes it through one of the beryllium slabs where it contacts the MT-9 channel, across the gap between the beryllium slabs, and out through the other slab's contact region into the other MT-9 channel. This flow path can easily be discerned by noting the direction of the isothermal lines of Figure 5.33. Since most of the heat has to flow through the gap between the beryllium slabs, the longer the beryllium joint is, the lower the resistance to heat flow, since the contact area in the gap region gets larger. Hence the effective conductivity rises along with the joint thickness.

\subsubsection{Blanket Desien 2: Thermo-Hydraulics}

Because the average volume fractions of beryllium and lithium oxide in the breeder region of Blanket Design 2 are so close to those of the mixed bed section of Blanket Design 1, the average heat load from the breeder is also similar. Hence, the overall shell sizing, spacing and helium flow rate calculations performed for Blanket Design 1 (in Section 5.2.2) are generally applicable for Blanket Design 2 as well. The heterogeneous nature of the breeder region of Blanket Design 2, however, makes the normal operating temperature distribution of this region more complicated than for Blanket Design 1. The HEATING3 code is used to determine the two-dimensional operational temperature 
distribution, and thereby the maximum and minimum temperatures for the MT -9 , lithium oxide and beryllium materials.

To ensure that the maximum temperatures in Blanket Design 2 are acceptable, HEATING3 is run for two limiting cases, which are now described. Referring back to Figure 5.17, note that during normal operation the first nine breeder regions of Blanket Design 1 (which are identified by the temperature peaks in the "at shutdown" curve of Figure 5.17) are bordered by coolant shells which are at the maximum operational MT-9 temperature of 550 degrees. To ensure that the MT-9 structure of Blanket Design 2 does not exceed 550 degrees $C$, then, the temperature distribution of one of the first nine breeder regions in the blanket must be evaluated using HEATING3. The first breeder region is chosen, and this is the first limiting case.

For the second limiting case, note that the tenth breeder region in the at shutdown curve of Figure 5.17 has the highest peak breeder temperature of 780 degrees C. To determine the highest lithium oxide and beryllium temperatures for Blanket Design 2, the temperature distribution in the tenth breeder region is evaluated. This is the second limiting case. If the maximum temperatures for these two cases are acceptable, the temperatures in the other regions of Blanket Design 2 should be also.

Because the Blanket Design 1 breeder regions had a uniform pebble bed geometry, and the Blanket Design 2 breeder regions alternate between beryllium slabs and lithium oxide pebble beds, there are temperature peaks along the cooling channels of Blanket Design 2. Because of these peaks, it is expected that the surface heat transfer coefficient $(h)$ for the helium coolant will have to be modestly increased for the cooling channels in Blanket Design 2 to ensure the MT-9 shell material does not exceed its maximum allowable operational temperature of 550 degrees C. Using the HEATING3 code, it is found that 
increasing the coolant channel $h$ to $3000 \mathrm{~W} / \mathrm{m}^{2} \cdot K$ (from the values of between 1000 and $2000 \mathrm{~W} / \mathrm{m}^{2} \cdot K$ used in Blanket Design 1) ensures that the MT-9 shells stay below 550 degrees $\mathrm{C}$ for Blanket Design 2. The increase in $\mathrm{h}$ is accomplished by making the coolant channel diameters smaller for Blanket Design 2, while retaining the channel mass flowrates. This makes the blanket a bit thinner overall, which helps reduce the severity of the LOFA. However, the overall blanket thickness does not change by much, hence the cooling channels were conservatively left at the diameters used for Blanket Design 1 for the accident analysis. Increasing the $h$ in the Blanket Design 2 breeder region channels also increases the coolant velocity and channel pressure drops. However, the pumping power required is still small for these channels (about $5 \mathrm{MW}$ ) relative to the pumping power required for the first wall channels (121 MW).

Figure 5.35 shows the temperature distribution in the first breeder region of Blanket Design 2, and Figure 5.36 shows the temperatures for the tenth breeder region. The helium coolant $\mathrm{h}$ is assumed to be $3000 \mathrm{~W} / \mathrm{m}^{2} \cdot K$ for both of these figures.

Figure 5.35. Maximum Operating Temperatures for 1st Breeder Region of Blanket Design 2

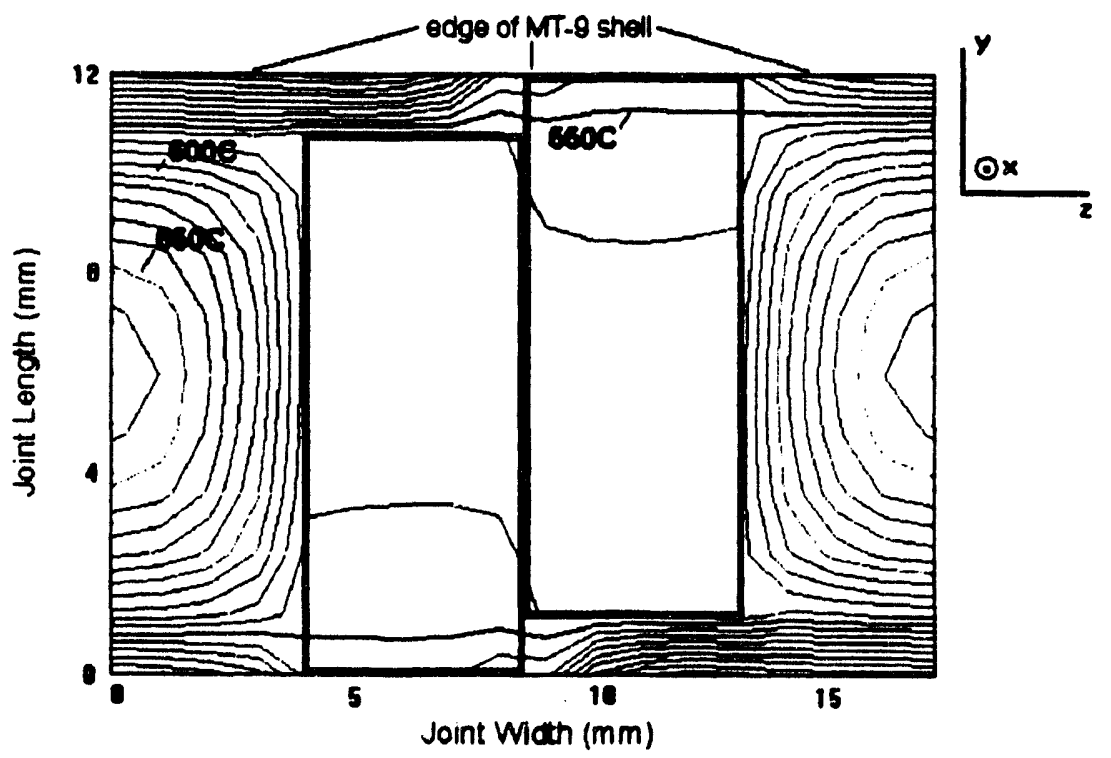


Figure 5.36. Maximum Operating Temperatures for 10th Breeder Region of Blanket Design 2

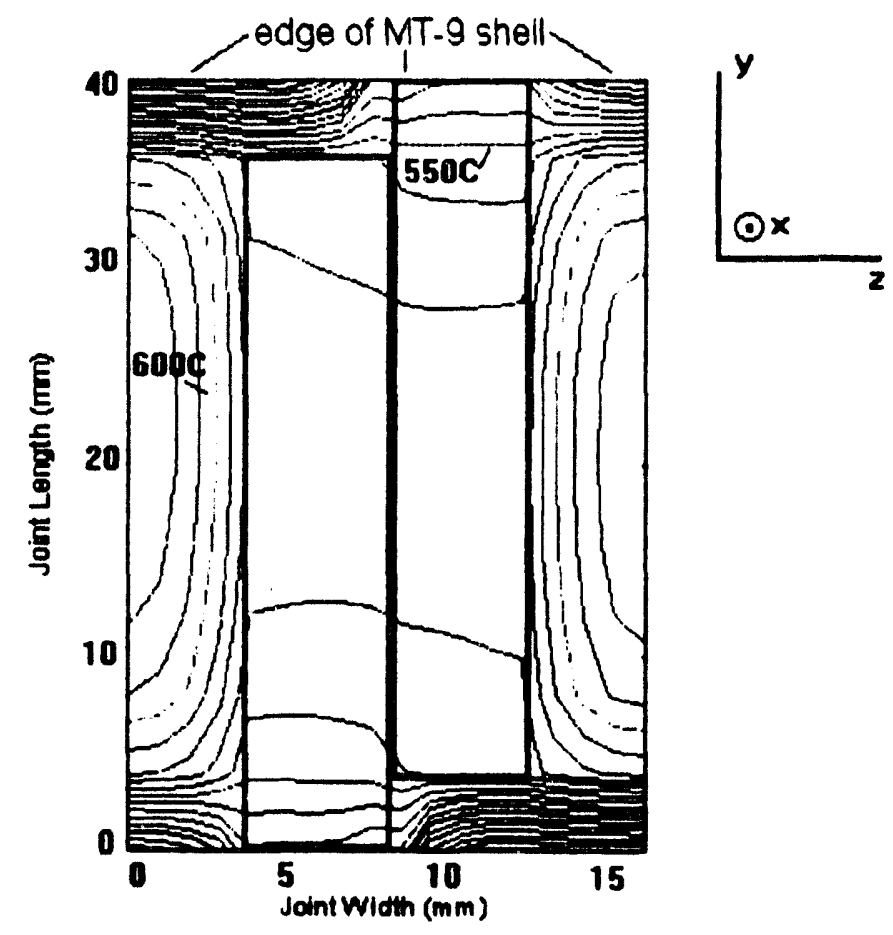

Both figures show the beryllium slabs in outline, and exhibit rotational symmetry about the center of the joint, as is expected. The isotherms in the figures are each 10 degrees $C$ apart. The figures show the large temperature drop which occurs in the gap between the beryllium slab and the edge of the MT-9 coolant shell, and one can clearly see how the heat flows into the beryllium slabs from the lithium oxide beds, and then into the MT-9 coolant shell through the areas where the beryllium contacts the MT-9. The peak temperature at the edge of the MT-9 shell occurs at those areas, and is below 550 degrees $\mathrm{C}$ for both cases. The peak temperature in the lithium oxide bed is about 660 
degrees $\mathrm{C}$ for both breeder regions. Table 5.14 summarizes the operational temperatures for Blanket Design 2. The tritium inventory in Blanket Design 2 is about $4 \mathrm{~kg}$, and as with Blanket Design 1, most of the tritium resides in the beryllium. See Appendix 4 for details in tritium inventory estimation.

\section{Table 5.14. Blanket Design 2 Operating Temperatures (deg. C)}

\begin{tabular}{|l|l|}
\hline Helium Inlet Temperature: & 250 \\
\hline Helium Outlet Temperature: & 450 \\
\hline Maximum MT-9 Temperature: & 550 \\
\hline Maximum Be Temperature: & 575 \\
\hline Maximum Li 20 Temperature: & 660 \\
\hline Minimum $\mathrm{Li}_{2} \mathrm{O}$ Temperature: & 309 \\
\hline
\end{tabular}

\subsubsection{Blanket Design 2: Accident Analysis}

Now that the one-dimensional conductivity of the beryllium joints was determined in section 5.3.2, these joints can be incorporated into the one-dimensional HEATING model for the blanket. Since the geometry and materials of Blanket Design 2 are the same as Blanket Design 1, the dimensions of the one-dimensional model shown in Figure 5.14 still apply. The normal operating temperatures for Blanket Design 2 are similar to those of Blanket Design 1, as was seen in the last section. 


\section{3.t.1 Immediate Plasma Shut-Off}

For the first analysis we assume the same type of No-Flow LOFA as was analyzed for Blanket Design 1 (see Section 5.2.3), that is, a No-Flow LOFA wherein the plasma shuts off immediately after the LOFA. The same afterheat values for the MT-9 channels are used here as were used in the analysis for Blanket Design 1, since the neutronics

\section{Figure 5.37. Temperature of First Wall of Blanket Design 2 After No-} Flow LOFA

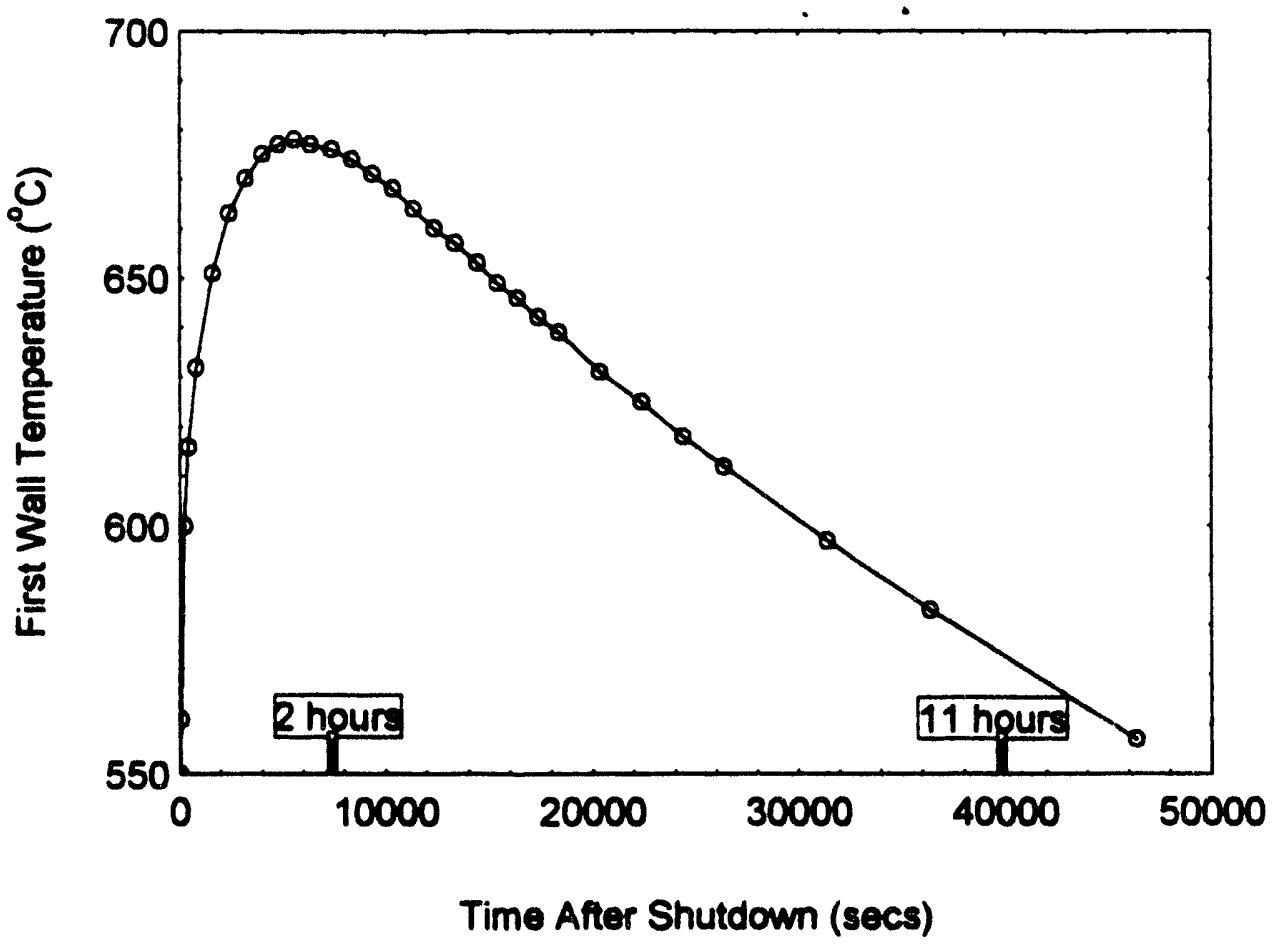

behavior is almost identical between Blanket Designs 1 and 2. The resulting first wall temperature vs. time graph for Blanket Design 2 is shown in Figure 5.37.

As can be seen, the peak first wall temperature is only 678 degrees $C$, well below the peak temperature of the first wall of Blanket Design 1 (772 degrees C). The time at the 
elevated temperature is also shorter than for Blanket Design 1. Both of these facts make Blanket Design 2 much more capable of sustaining the No-Flow LOFA.

Figure 5.38 shows the temperature distribution throughout the blanket at various times after shutdown for Blanket Design 2. As with Blanket Design 1, within the first few seconds the temperature distribution smoothes as conduction between adjacent blanket regions occurs. The time of the peak first wall temperature ( 1.5 hours after shutdown) is shown, and the last time plotted is 12.9 hours after shutdown, when the first wall cools almost back to its normal operating temperature of 550 degrees $\mathrm{C}$.

Figure 5.38. Temperatures in Blanket Design 2 After No-Flow LOFA

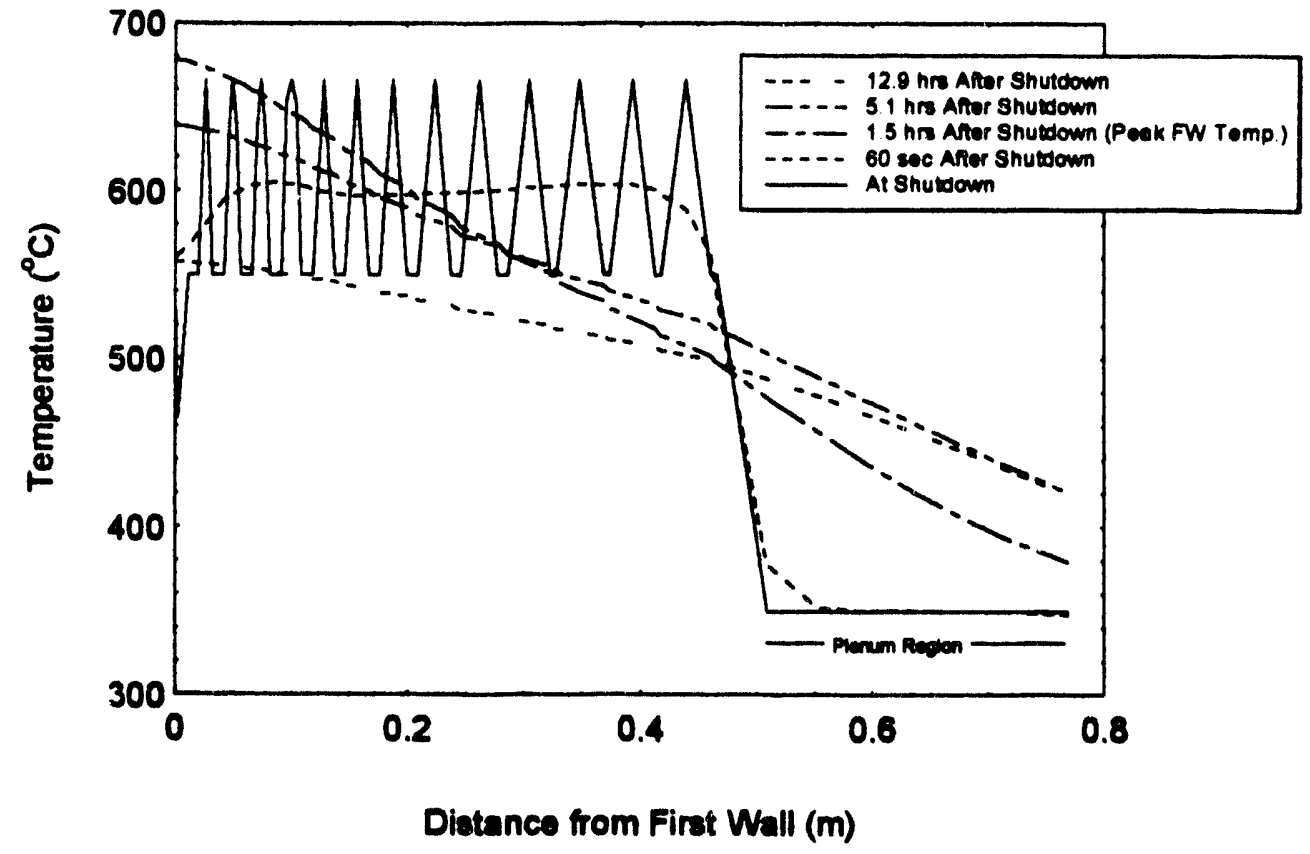

A failure analysis of the type previously described for Blanket Design 1 is now performed. The results are shown in Table 5.15. The rupture fraction is much less than 1.0 , and the maximum tensile creep strain (in the x-direction) is also much less than $1 \%$. Hence, Blanket Design 2 can easily sustain a No-Flow LOFA without structural damage, 
assuming the plasma shuts off immediately. In fact, since the values shown in Table 5.15 are so low, it is evident that this blanket design could survive quite a few such accidents without exceeding the creep or rupture time limits.

Table 5.15. Blanket Design 2 Failure Analysis Results

\begin{tabular}{|r|c|}
\hline Rupture Fraction: & 0.027 \\
\hline Total $\varepsilon$ in the x-direction: & $0.063 \%$ \\
\hline
\end{tabular}




\section{3.+2 Plasma Continuation}

We now turn to the No-Flow LOFA case wherein the plasma continues to burn following the onset of the LOFA. This is unlikely if the LOFA is assumed to occur due to Loss of Site Power, however, if an individual circulator pump failed, the plasma would continue to burn until an active or passive system shut it off. It is of interest to determine how long Blanket Design 2 can sustain a LOFA with the plasma on without structural failure, since this would be a measure of the required response time for a passive or active plasma shutdown system. Note that for this analysis, the effects of a plasma disruption, which could deposit additional energy onto the first wall, are neglected.

As discussed in the previous analysis of the LOFA with immediate plasma shut-off, the first wall may fail acutely or due to creep rupture. For the plasma continuation case, acute failure is much more of a concern because of the very high first wall temperatures which are reached as the plasma burns when there is no coolant flowing through the first wall coolant channels. When the plasma shuts off, thermal and pressure stresses exist in the first wall. Calculation of these stresses was performed in the Section 5.2.3.6.

The maximum Von-Mises equivalent stress in the first wall at plasma shutdown (assuming no plasma continuation, and combining the thermal and pressure stresses) was determined in Section 5.2.3.6 to be $197 \mathrm{MPa}$. Although the thermally induced portion of this stress would be expected to decrease during a plasma continuation via creep, this effect will be neglected for conservatism and simplicity. Assuming, then, that an equivalent stress of $197 \mathrm{MPa}$ exists in the first wall throughout the transient, acute structural failure will occur when the temperature of the first wall increases to the point where the ultimate tensile strength of MT-9 decreases to $197 \mathrm{MPa}$. Referring back to Figure 5.18, note that the temperature corresponding to $197 \mathrm{MPa}$ is 780 degrees $\mathrm{C}$. 
Therefore, a necessary condition for the first wall to sustain a plasma continuation NoFlow LOFA is that the first wall temperature remain below 780 degrees $C$

Figure 5.39 shows the maximum first wall temperature as a function of plasma

Figure 5.39. Maximum First Wall Temperature for Plasma Continuation Cases

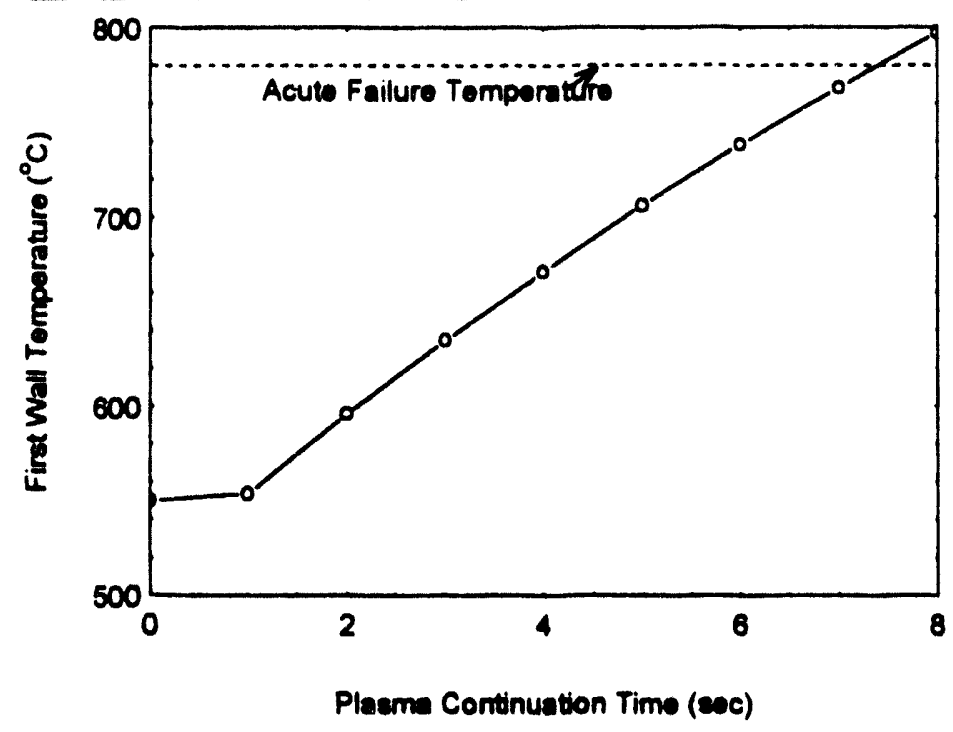

continuation time for a NoFlow LOFA. The first wall of Blanket Design 2 can sustain at least 7 seconds of full-power plasma operation without coolant before reaching the acute failure temperature of 780 degrees $\mathrm{C}$.

It has been shown that Blanket Design 2 can sustain 7 seconds of plasma continuation without acute structural failure. Performing the creep rupture calculation will determine if the first wall fails over the long term. The temperature of the first wall versus time for

\section{Figure 5.40. First Wall Temperature for 7} Second Plasma Continution

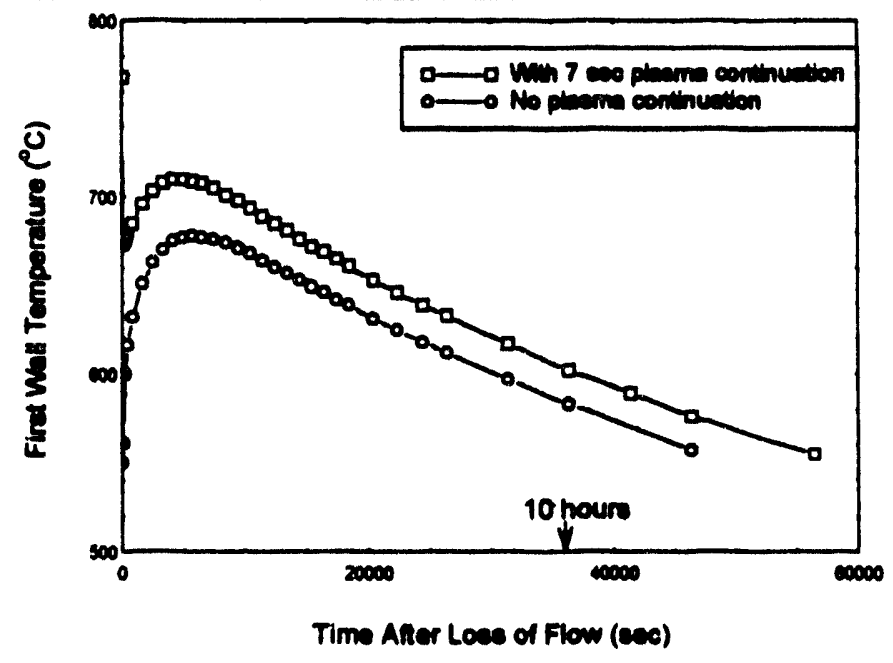

the 7 second plasma continuation case is shown in Figure 5.40. For comparison, the case previously analyzed with no plasma continuation (that is, immediate plasma shutoff) is also shown. 
The first thing to note about the plasma continuation case is that the first wall temperature rapidly decreases from its maximum when the plasma shuts off. This is because the short, 7 second burn of the plasma heats up only the region very close to the first wall. Then, following plasma shut down, the hot first wall region rapidly equilibrates via conduction with the much cooler regions just behind the first wall, rapidly reducing the temperature of the first wall below 700 degrees $C$.

The second thing to note is that the curve for the 7 second plasma continuation is, except for the first few seconds after the plasma shuts off, only about 25 degrees $\mathrm{C}$ hotter than the case with no plasma continuation. This modest temperature increase means the amount of creep will not be significantly larger for the 7 second plasma continuation case. The results of failure analysis for this case are shown in Table 5.16. As expected, the rupture fraction and creep values are well below those necessary to cause failure. Hence, Blanket Design 2 is capable of sustaining multiple No-Flow LOFAs with plasma continuation without damage, as long as the plasma shuts off within 7 seconds.

\section{Table 5.16. 7 Second Plasma Continuation Failure} Analysis Results for Blanket Design 2

\begin{tabular}{|r|c|}
\hline Rupture Fraction: & 0.14 \\
\hline Total 8 in the $x$-direction: & $0.3 \%$ \\
\hline
\end{tabular}

\subsubsection{Blanket Desion 2: Sensitivity Analusis}

The accident performance of Blanket Design 2 relies on the thermal conduction performance of the beryllium joints. This in turn relies on the conductance of the gap regions, and the conductivity of the beryllium slabs. It is difficult to predict precisely what the gap region conductances will be in service, since swelling of the beryllium and 
possible misalignment of the joint configuration may result in changes to the gap conductances over the blanket lifetime. Because of the difficulty of predicting precise values for the gap conductances, a conservative approach was taken in Section 5.32 for calculation of the conductances. To ensure conservative conductance values, contact pressure, which can dramatically improve the conductance of a gap region, was assumed to be less than $7 \mathrm{kPa}(1 \mathrm{psi})$.

In the gap conductance analysis assumptions had to be made regarding the surface roughness of the gap materials and the porosity and thickness of the copper inserts. It is difficult to determine without actual fabrication and testing of the joints (which is beyond the scope of the present work) whether these assumptions are all truly reasonable. For example, perhaps the assumed copper insert thickness of $0.5 \mathrm{~mm}$ is too small to be readily fabricated and installed in a large-scale blanket structure. Or perhaps obtaining a very smooth surface is cost-prohibitive. The values for these parameters are speculative, and perhaps may be overly optimistic.

In the accident analysis just performed, the conductivity of the beryllium slabs was assumed to be that of 100 percent dense beryllium. This assumption is somewhat optimistic, since during irradiation the beryllium swells from the generation of helium atoms in the beryllium grains. At higher swelling vahues, these helium atoms begin to congregate in voids, creating a porosity in the beryllium. This porosity can reduce the conductivity of the beryllium slabs. Additionally, there is the possibility of thermal stresses and/or the stresses caused by differential swelling values within the beryllium slab causing cracking in the slab, which would reduce the conductivity further.

The purpose of this section is to evaluate the effects of a decrease in gap conductance and beryllium slab conductivity on the performance of Blanket Design 2. The likelihood 
of cracking in the beryllium, and the possible effects of swelling on the slab conductivity are also discuessed. From this evaluation, it can be determined how robust the design is to adverse changes in some of the assumptions used in the previous analysis.

\subsubsection{Effect of Gap Conductance}

To evaluate the effect of lowering the conductance of the gap regions, a simplified version of the accident analysis of Section 5.3.4 is performed. Rather than allowing the gap conductance to vary with temperature, as was done previously, the gap conductance will be fixed at a chosen value. The beryllium slab conductivity will also be fixed at the low value of $114 \mathrm{~W} / \mathrm{m} \cdot \mathrm{K}$ for the present analysis. This will result in a conservative beryllium joint effective conductivity for a given gap conductance. Figure 5.41 shows the result of these assumptions.

Figure 5.41. Be Joint Effective Conductivity vs. Gap Conductance (k of Be fixed at $114 \mathrm{~W} / \mathrm{m}-\mathrm{K}$ )

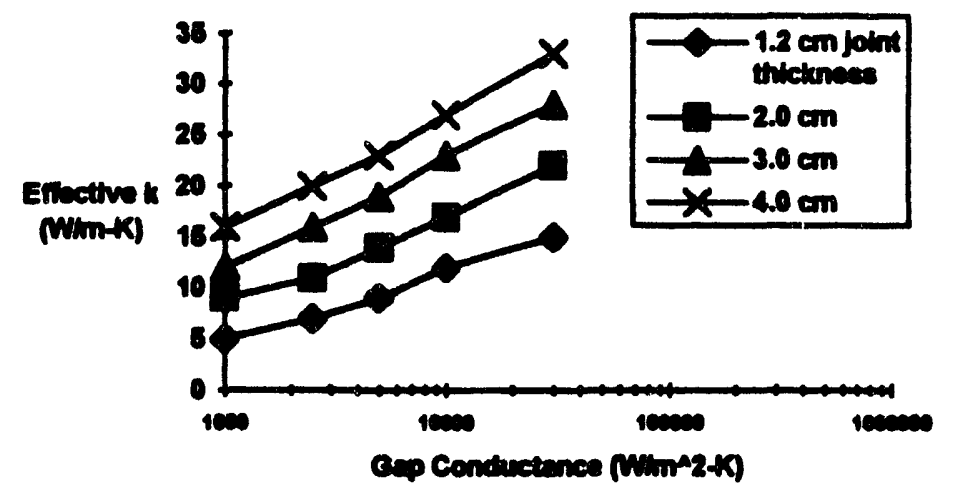

As shown in Figure 5.41, the effective conductivity of the beryllium joints is not very sensitive to the gap conductance. An order of magnitude reduction of the gap conductance results in a

drop of the effective conductivities by only a factor of two or so. This result is somewhat surprising, and indicates that a high gap conductance may not be required in order to have Blanket Design 2 survive a No-Flow LOFA.

Using the effective beryllium joint conductances shown in Figure 5.41, the No-Flow LOFA case with 7 second plasma continuation is run again for the different gap conductance values. The resulting temperature profiles are shown in Figure 5.42. The 
curve labeled "Initial Case" is the curve already presented in Section 5.3.4. For this curve, as will be recalled, the gap conductance was allowed to vary with temperature (from 23,400 to $39,200 \mathrm{~W} / \mathrm{m}^{2} \cdot \mathrm{K}$ ). The beryllium conductivity for this curve also varied with temperature.

Figure 5.42. First Wall Temperatures for Different Gap Conductivities (for 7 second plasma continuation)

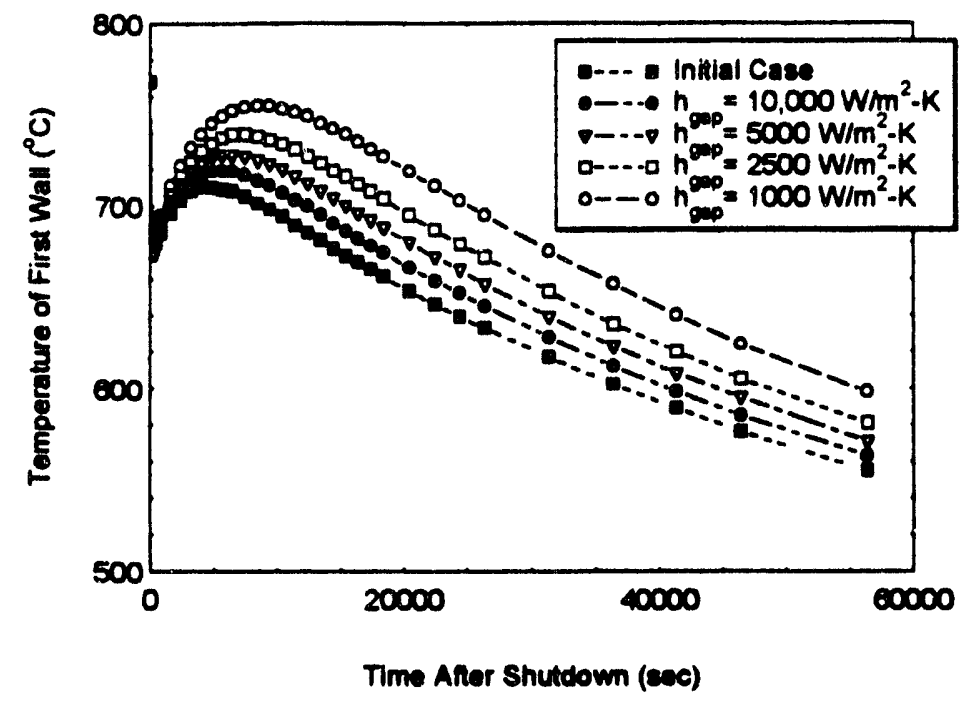

As expected, as the gap conductivities are lowered, the temperature profile shifts upward, resulting in a more severe transient.

Performing a creep faihure analysis on the plots shown in Figure 5.42 results in graphs of rupture fraction and total $\mathrm{x}$-direction creep strain, shown in Figure

\subsection{This figure shows that} both the total creep strain and the rupture fraction remain well below the failure limit until the gap conductance drops to below $2500 \mathrm{~W} / \mathrm{m}^{2} \cdot \mathrm{K}$, at which point further decreases in gap conductance result in a rapid increase if both creep strain
Figure 5.43. Blanket Design 2 Margin to Failure for 7 Second Plasma Continuation

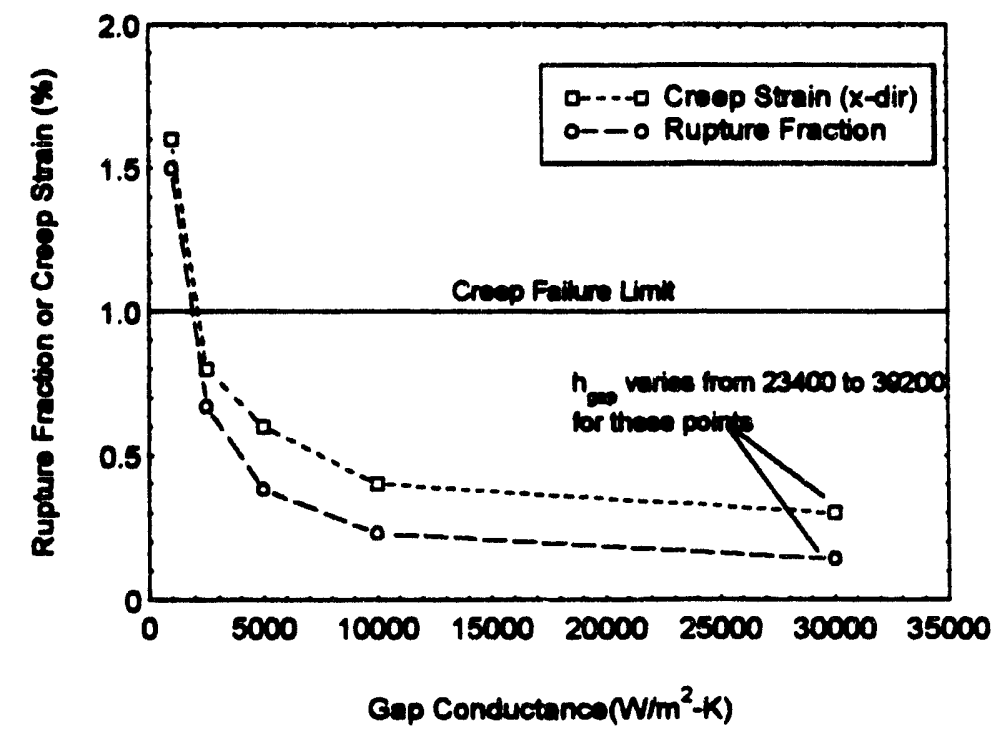


and rupture fraction to beyond the failure limit.

As was expected when the effective conductivity of the beryllium joints was evaluated earlier in this section, Blanket Design 2 can survive a No-Flow LOFA with 7 second plasma continuation even if the gap conductances are reduced by an order of magnitude from their original assumed values. Additionally, the effect of changes in gap conductance above a gap conductance value of $10000 \mathrm{~W} / \mathrm{m}^{2} \cdot \mathrm{K}$ is small, as indicated by the flat slope of Figure 5.43 in this region. This is encouraging, since the gap conductance is difficult to predict accurately.

Recall from Section 5.3.2 that the gap conductance depends on three design parameters, the thickness ( $(t)$ and porosity $(P)$ of the copper insert, and the surface roughness $(\sigma)$ of the gap surfaces. (The gap conductance also depends on temperature and contact pressure.) Recall also that the choices for $t, P$, and $\sigma$ were rather arbitrary, and may have been overly optimistic. Hence, it would be interesting to see how different combinations of $t, P$ and $\sigma$ influence the gap conductarces. Hence we can determine the allowable ranges of these parameters which still result in no blanket failure during the 7 second plasma continuation case.

Table 5.17 shows how much one can vary each of the parameters $t, P$ and $\sigma$ before the gap conductance decreases below $2500 \mathrm{~W} / \mathrm{m}^{2} \cdot \mathrm{K}$, which is the point below which the

\section{Table 5.17. Variation of Gap Design Parameters}

\begin{tabular}{|c|c|c|c|c|}
\hline & $t(\mathrm{~mm})$ & $\mathbf{P}$ & $\sigma(\mu \mathrm{m})$ & $n_{\infty}\left(W / m^{2}-K\right)$ \\
\hline Initial Case: & 0.5 & 0.3 & 0.03 & 30000 \\
\hline Maximum t Case: & 48 & 0.3 & 0.03 & 2500 \\
\hline Maximum P Case: & 0.5 & 0.96 & 0.03 & 2500 \\
\hline Maximum $\sigma$ Case: & 0.5 & 0.3 & $\underline{9}$ & 2500 \\
\hline
\end{tabular}


first wall is damaged according to Figure 5.43. The assumed gap temperature is 500 degrees $\mathrm{C}$. The underlined values are those varied.

Table 5.17 shows that each of the gap design parameters - the copper insert thickness, porosity and the surface roughness - can be greatly increased from the values assumed for the initial Blanket Design 2 case without causing the first wall to fail during the 7 second plasma continuation No-Flow LOFA. The maximum allowable copper insert thickness and porosity are absurdly large, at $48 \mathrm{~mm}$ and 0.96 respectively. Clearly, a real design would not come close to values which are that large. Hence, a real design would perform better, and would have more margin to failure. The maximum allowable surface roughness is $9 \mu \mathrm{m}$. This value is very rough, and can easily be attained via sawing or snagging processes, or even sand casting. ${ }^{54}$

Although lowering the gap conductance to $2500 \mathrm{~W} / \mathrm{m}^{2} \cdot \mathrm{K}$ would not result in blanket failure during a LOFA with 7 second plasma continuation, there is very little margin to failure for this case. On the other hand, Figure 5.43 indicates that reducing the gap conductance to $10,000 \mathrm{~W} / \mathrm{m}^{2} \cdot \mathrm{K}$ has little effect on the rupture fraction or total creep strain. Using $10,000 \mathrm{~W} / \mathrm{m}^{2} \cdot \mathrm{K}$ also allows some relaxation of the gap parameters $\sigma, t$ and $P$. Using somewhat larger $\sigma$ and $t$ parameters would make fabrication of the blanket easier. Using a higher $\mathbf{P}$ would allow the copper inserts to be more compliant. Table 5.18 shows a revised set of gap parameters which result in a gap conductance of 10,000 $\mathrm{W} / \mathrm{m}^{2} \cdot \mathbf{K}$.

34T. Baumeister, et. al., ods., Marks' Standard Handbook for Engineers, Eighth Edition, McGraw-Hill, 1978, p. $13-79$. 
Table 5.18. Revised Gap Parameters for Blanket Design 2

\begin{tabular}{|l|c|c|c|c|}
\hline & $\mathbf{t}(\mathrm{mm})$ & $\mathbf{P}$ & $\frac{\sigma(\mu \mathrm{m})}{\boldsymbol{h}_{\text {sep }}\left(W / \mathrm{m}^{2}-K\right)}$ \\
\hline Revised Values: & 2 & 0.5 & 1 & 10000 \\
\hline
\end{tabular}

As can be seen, the reduction in required gap conductance to $10,000 \mathrm{~W} / \mathrm{m}^{2} \cdot \mathrm{K}$, while not affecting the blanket design's margin to failure by much, allows the required surface roughness to be increased by almost 2 orders of magnitude. This roughness value is easily attained by a large variety of inexpensive processes, including sawing, planing, and casting. ss At the same time, the thickness can be increased by a factor of 4 , and the porosity can also be increased. The values used in Table 5.18 will be used in subsequent analysis for Blanket Design 2.

${ }^{55}$ Ibid., p. $13-79$. 


\subsubsection{Effect of Beryllium Conductivity}

The previous analyses for Blanket Design 2 were based on the beryllium slab conductivity varying from $134 \mathrm{~W} / \mathrm{m} \cdot \mathrm{K}$ to $114 \mathrm{~W} / \mathrm{m} \cdot \mathrm{K}$, depending on the temperature (see Figure 5.26). This temperature range is appropriate for 100 percent dense beryllium. However, there are two means by which the conductivity of the slabs could be reduced during reactor operation, cracking and swelling. This section discusses these processes, and determine an estimate of the maximum plausible conductivity reduction for the slabs. Then, the No-Flow LOFA case with 7 second plasma continuation is again run with the reduced slab conductivity to see the effect of the conductivity reduction on blanket performance.

\section{Bervllium Cracking}

The first process that could reduce the conductivity of the beryllium slabs is cracking of the slabs. Cracking could occur due to thermal stresses imposed on the slabs during operation or during accident conditions. Cracking could also occur due to differential swelling in different regions of the slab. (Residual stresses from fabrication are assumed to be negligible.) Such differential swelling can induce stresses in the slab, just as temperature differences induce thermal stresses. It is difficult to predict the effect of cracking on thermal conductivity, especially without knowing the details of the crack geometries. Fortunately, it appears unlikely that cracking will occur in the beryllium slabs of Blanket Design 2. The following paragraphs will describe analysis methods used to support this conclusion.

\section{Thermal Stressea}

The order of magnitude of the thermal stresses in a solid is generally governed by the maximum temperature difference $(\Delta \mathrm{T})$ seen in the solid. Because of the heat deposition in the beryllium and the lithium oxide which occurs during normal operation, the 
maximum $\Delta \mathrm{T}$ in the beryllium slabs occurs during normal operation (compare Figure 5.33 with Figure 5.35). As would be expected, the slabs in the thicker breeder regions have a higher $\Delta \mathrm{T}$ than the slabs in the thin regions (compare Figure 5.35 with Figure 5.36). Hence, the thicker breeder regions will have higher induced thermal stresses than the thinner regions.

However, as will be discussed later, the thinner breeder regions sustain much higher neutron fluences over the blanket lifetime, and hence see greater reduction in fracture toughness, or crack resistance. Therefore, in this analysis both the 10th breeder region behind the first wall, which is the region with the highest thermal stresses, and the breeder region just behind the first wall channels, which sees the highest neutron fluence, are examined.

Figure 5.44. Maximum Operating Temperatures for 1 st Breeder Region of Blanket Design 2

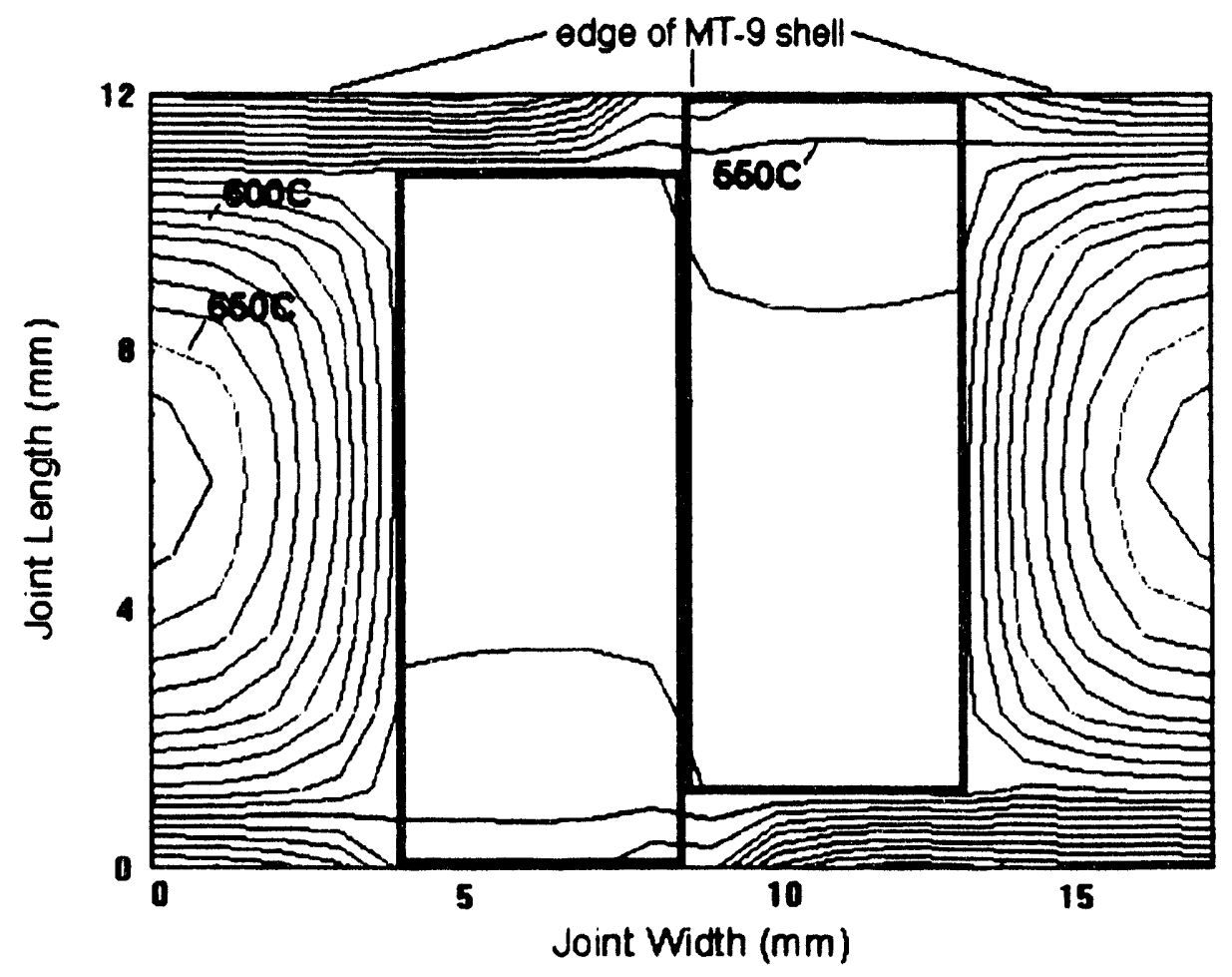


Figure 5.45. Maximum Operating Temperatures for 10th Breeder Region of Blanket Design 2

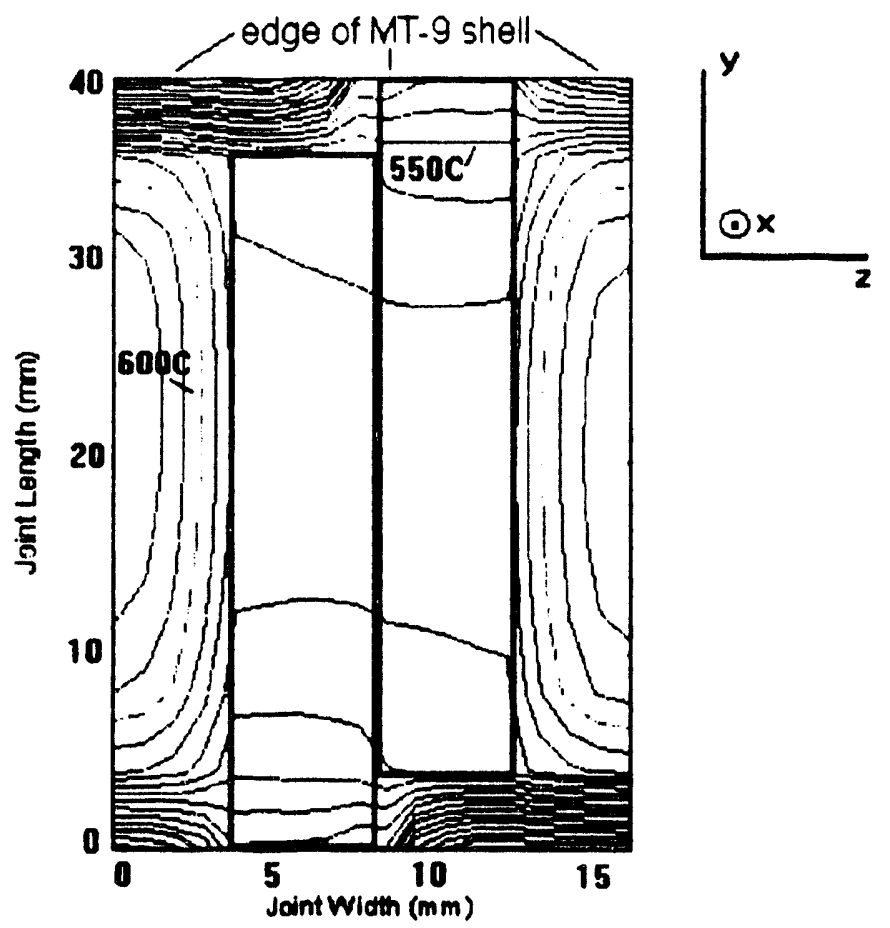

Figure 5.35 and Figure 5.36 are reproduced again here for convenience as Figures 5.44 and 5.45. As can be seen in these figures, the isotherms in the beryllium slabs are for the most part horizontal. Hence, the temperature distribution within the slabs can be reasonably approximated by assuming that the temperature varies only in the vertical direction of the figures. 
Figure 5.46. Temperature Distribution in Beryllium Slab in 1st Breeder Region

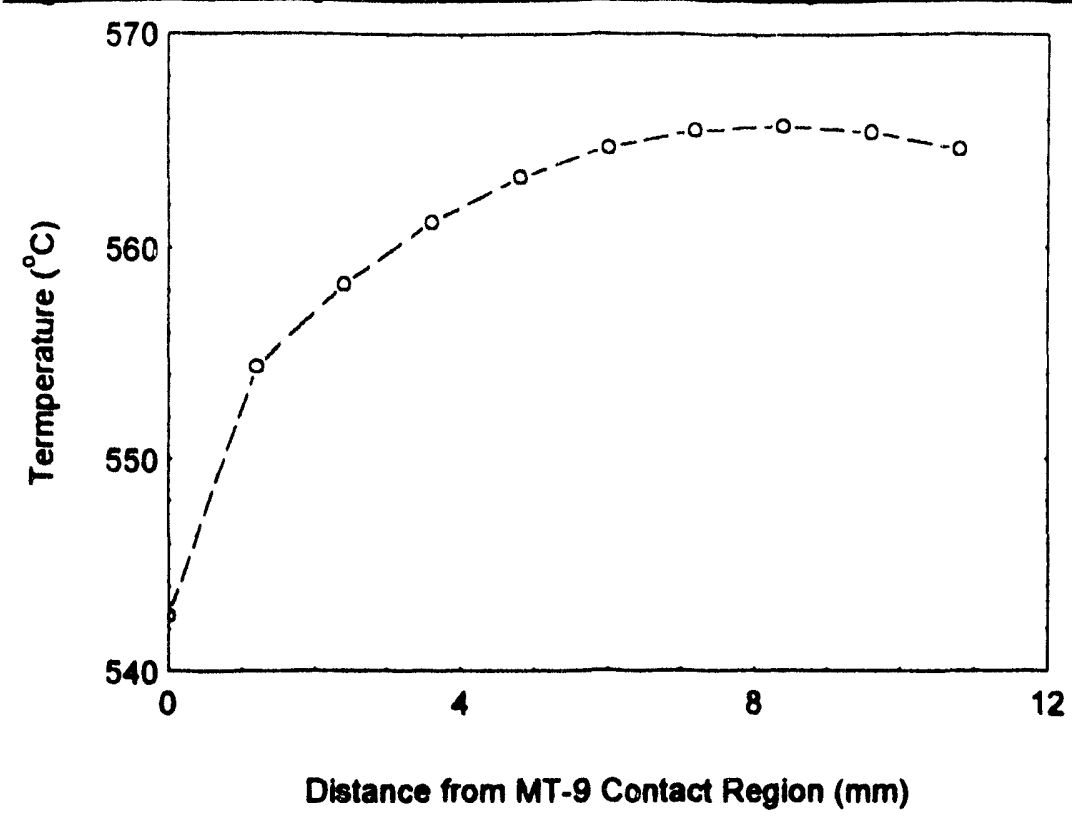

Figure 5.47. Temperature Distribution in Beryllium Slab in 10th Breeder Region

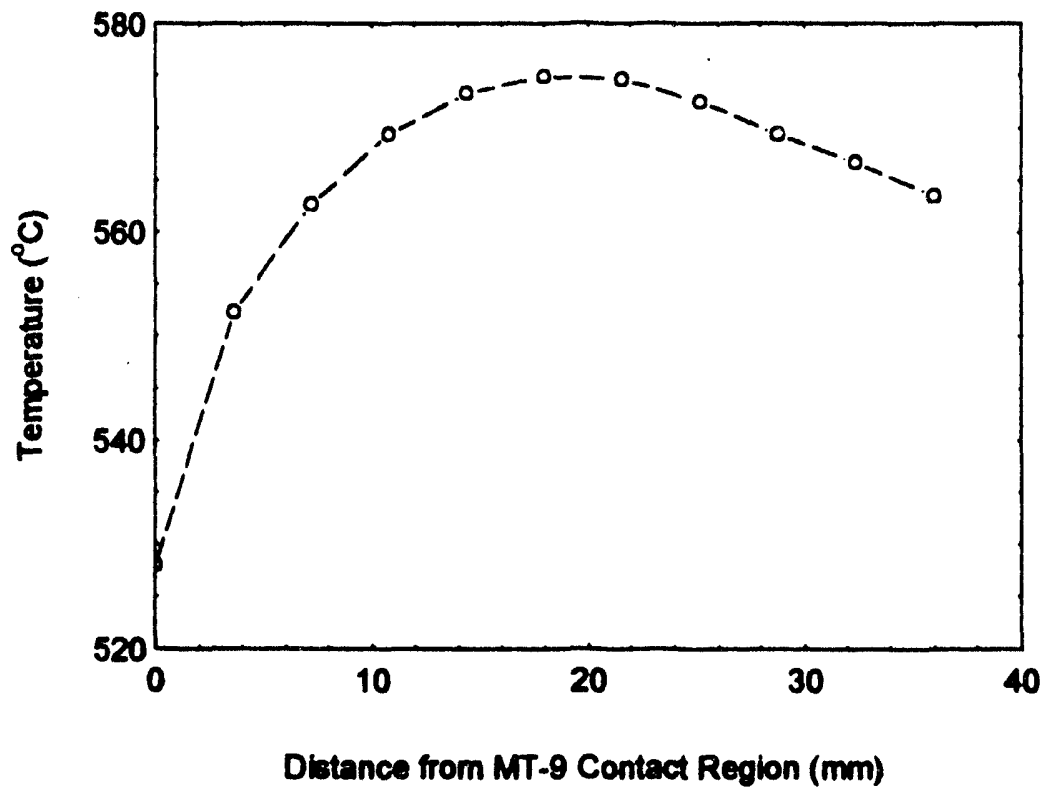

Plots of the temperature distribution in the vertical direction for the middle of the left beryllium slab of Figures 5.44 and 5.45 are shown in Figures 5.46 and 5.47. The thermal strains resulting from the temperature distributions of Figures 5.46 and 5.47 can to some 
extent be accommodated by slight deformations of the slabs (of order $0.1 \mathrm{~mm}$ for the 3.6 $\mathrm{cm}$ long slabs). However, because the temperature distribution is not linear, there will be some thermal strains which cannot be accommodated via deformation, and hence will result in thermal stresses within the slabs. Assuming no external loads, and assuming that the vertical direction is defined as the $\mathrm{y}$ direction, and the $\mathrm{x}$ direction is the direction out of the plane of Figures 5.44 and 5.45, the thermal stress in the slabs is in the $\mathrm{x}$ direction, and is given by: 56

$$
\begin{gathered}
\sigma_{x x}(y)=-\alpha \operatorname{ET}(y)+\frac{P_{\mathrm{T}}}{A}+\frac{M_{T:} y}{I_{z}}, \text { where } \\
P_{T}=\int_{A} \alpha \operatorname{ET}(y) d A, \text { and } \\
M_{\mathrm{Tz}}=\int_{A} \alpha y \operatorname{ET}(y) d A .
\end{gathered}
$$

Where $y$ is the distance from the center of the slab, $\alpha$ is the thermal expansion coefficient, $E$ is Young's Modulus, and $A$ is the cross sectional area of the slab in the $y-z$ plane. The thermal expansion coefficient $\alpha$ for beryllium is about $1.6 \times 10^{-3}\left({ }^{\circ} \mathrm{C}^{-1}\right)$ from 400 to 600 degrees C..$^{57}$ Young's Modulus for beryllium decreases linearly with temperature for temperatures above room temperature, such that at 650 degrees $C$ it is only about 88 percent of the value at room temperature. ${ }^{38}$ The value of Young's Modulus can vary depending on the purity and method of fabrication for beryllium; however, these effects are usually not large. Young's Modulus for a typical sample of beryllium in shown in Figure 5.48 .

\footnotetext{
${ }^{56}$ See Appendix 5 for derivation.

57D. Smith, et. al., ITER Blanket, Shield and Material Data Base, ITER Documentation Series, No. 29, International Atomic Energy Agency, Vienna, 1991.

58D. W. White, Jr. and J. E. Burke, eds., The Metal Beryllium, U.S. Atomic Energy Commission, 1954, p. 316.
} 
Figure 5.48. Young's Modulus for Beryllium

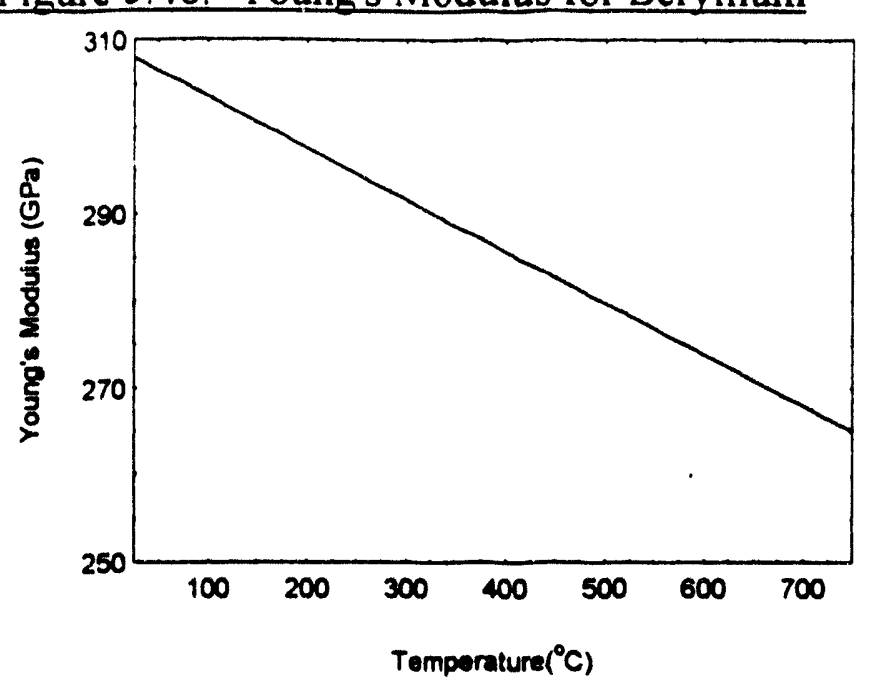

Using the temperature

distributions shown in Figures

5.46 and 5.47, and the Young's

Modulus curve of Figure 5.48, the thermal stresses in the 1st and 10th region beryllium slabs can be calculated. The results are shown in Figures 5.49 and 5.50 .

Figure 5.49. Thermal Stresses in 10th Region Beryllium Slab

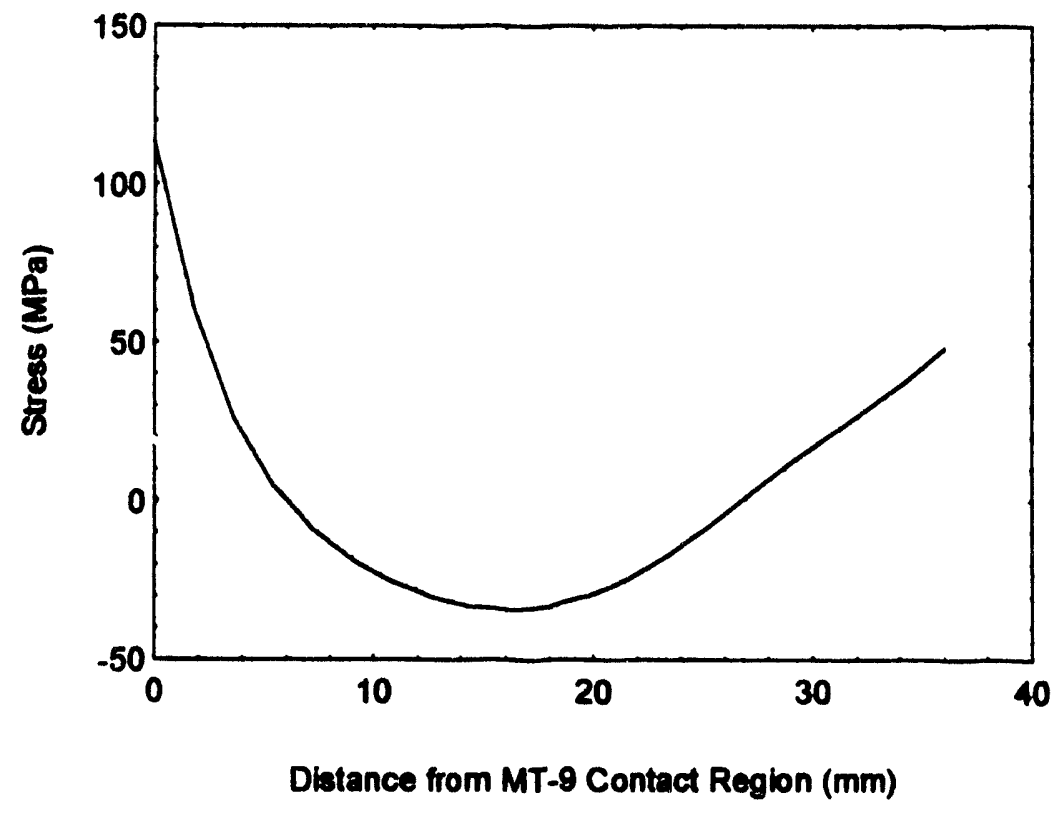


Figure 5.50. Thermal Stresses in 1st Region Beryllium Slab

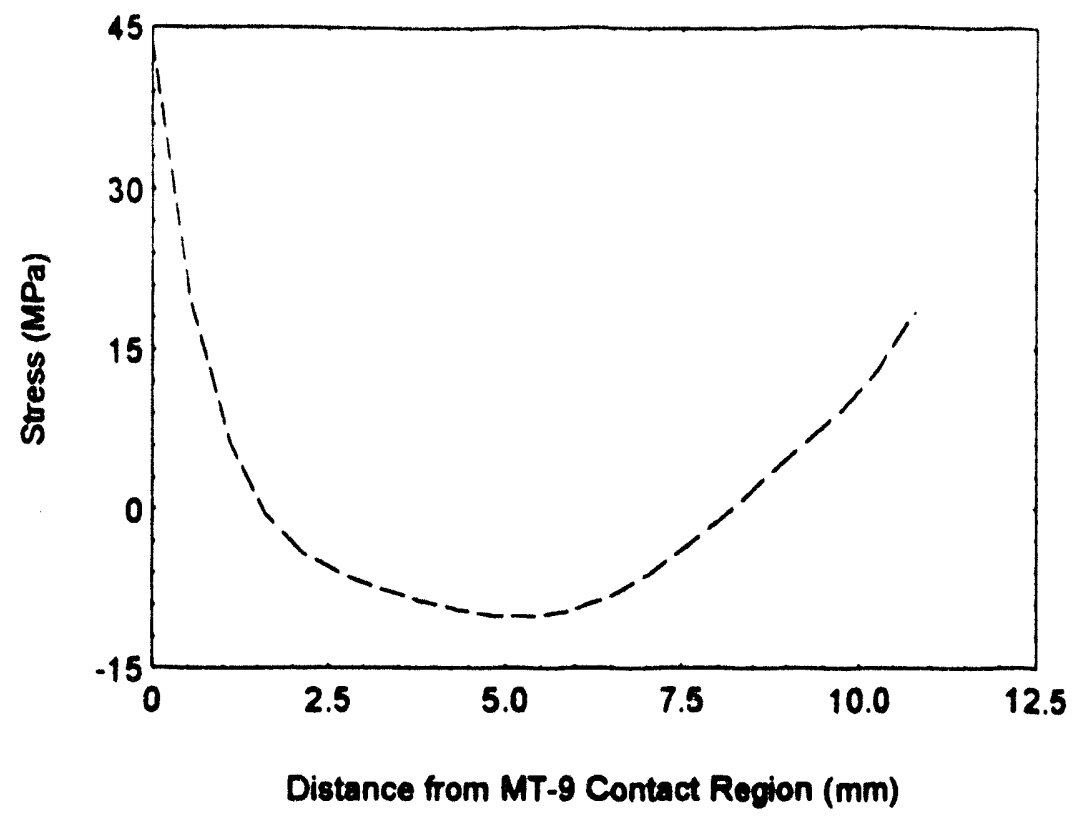

The thermal stress is positive (tensile) at the edges of the slabs, where the slabs are coolest, and negative (compressive) at the hotter inboard areas. The maximum stress is tensile, and occurs at the contact region with the MT-9 coolant shell. As expected, the thicker slab of the 10th breeder region has higher thermal stresses; the magnitude of the maximum stress for this slab is $113 \mathrm{MPa}$. For the 1 st breeder region slab, the maximum stress is only $43 \mathrm{MPa}$. As a first test to determine if such thermal stresses will cause cracking, the thermal stresses are compared to the ultimate tensile strength of the slabs. This is a conservative approach, since thermal stresses are secondary stresses, and hence ductile yielding within the slab would limit the actual magnitude of the stresses to the yield stress of the slab.

The ultimate tensile strength (UTS) of unirradiated beryllium as a function of temperature is shown in Figure 5.51. At the highest temperature of the slabs, $\mathbf{5 7 5}$ degrees $\mathrm{C}$, the UTS is $145 \mathrm{MPa}$, and the UTS will increase with irradiation. Hence, the maximum thermal stress in the slabs (113 $\mathrm{MPa}$ ) remains below the minimum UTS. This 
indicates that for a slab with no

internal flaws, thermal stresses

should not cause the slab to

crack.

The analysis presented above showed that an unflawed slab would not be expected to crack due to thermal stresses.

However, there is a possibility Figure 5.51. Beryllium Ultimate Tensile Strength ${ }^{59}$

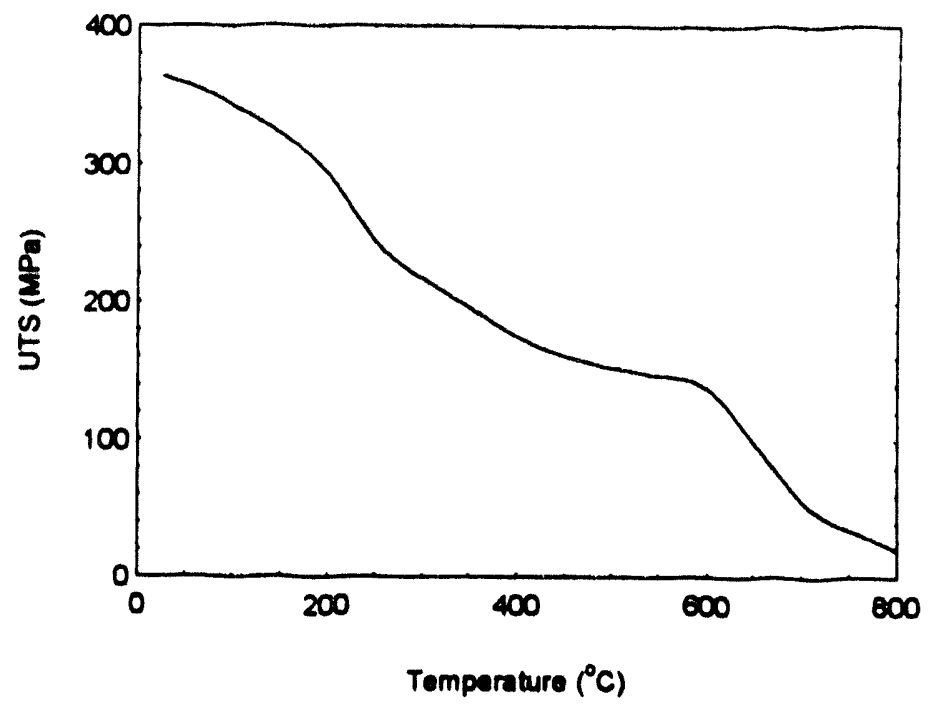

that fracture of the slab could

occur even at stress levels well below the UTS. Fracture could occur if there were a flaw in the beryllium slab of a sufficient size to initiate crack growth at the maximum thermal stress value seen in the slab. To test for the likelihood of such a fracture, a simple fracture mechanics analysis is performed. 60

Decades of study of fracture behavior have demonstrated that the magnitude of the applied stress which causes failure is related to the size and orientation of the crack or flaw within the structure. The larger the crack or flaw, the smaller the required failure stress. Through geometric analysis of simple cracks, coupled with materials testing, a simple rule has been developed to ensure against fracture. The rule is based on the

39 R. E. Taylor, A. al. , "Thermophysical Properties of Be Metal, A Report to Manufacturing Sciences Corp.," Report No. TPRL 1071, Thermophysical Properties Research Laboratory, Schenl of Mechanical Engineering, Purdue University, presemed by D. R. Floyd at the Beryllium Technology Workshop, Clearwater Beach, FL, November 20, 1991, Idaho National Engineering Laboratory Report No. EGGFSP-10017, G. R Longhurst, ed.

${ }^{60}$ The following discussion on frecture mechanics is adopled from J. A. Collins, Failure of Materials in Mechanical Design: Analysis, Prediction, Prevention, John Wiley and Sons, 1981, pp. 49-67 and from T. Baumeister, et. ai., ods., Mark's Standard Handbook for Mechanical Engineers, Eighth Edition, McGraw-Hill, 1978, pp. 5-7 to 5.9. 
principle that fracture is not expected to occur unless the work required for an increment of crack extension is exceeded by the elastic-strain energy released at the crack tip Based on this principle, fracture is not expected to occur in a material if the following relation is satisfied:

$$
\mathrm{K}_{\mathrm{c}} \geq \mathrm{C} \sigma \sqrt{\pi \mathrm{a}},
$$

where $\mathrm{K}_{\mathrm{c}}$ is called the critical stress intensity, and is a property of the material and the state of strain. $\sigma$ is an applied stress, $a$ is the crack length, and $C$ is a constant which depends on the crack geometry. For a given material, the critical stress intensity $\mathrm{K}_{\text {。 }}$ decreases to a lower limiting value as the state of strain approaches a plane strain condition. This lower limit defines a basic material property, known as the plane strain fracture toughness, or $\mathrm{K}_{\mathrm{k}}$. Thus, if the exact state of strain in the material is unknown, Equation 5.33 can be modified to obtain the following criterion:

$$
\mathrm{K}_{\mathrm{lc}} \geq \mathrm{C} \sigma \sqrt{\pi \mathrm{a}} .
$$

As long as Equation 5.34 is satisfied, fracture is not expected, no matter what the strain state. The constant $\mathrm{C}$ can vary from about 0.3 to 1.122 , for a simple crack or crackshaped flaw, depending on where the crack is located and what the stress distribution is. For conservatism is this analysis, $\mathrm{C}$ is assumed to be at the high end of its range, namely 1.122 .

If $\mathrm{K}_{\mathrm{lc}}$ is known for the material, and the stress state, Equation 5.34 can be solved to give the critical crack length above which fracture is expected. Denoting this critical crack length as $a_{\alpha}$, we have:

$$
a_{\alpha}=\frac{1}{\pi}\left(\frac{K_{\mathrm{l}}}{C \sigma}\right)^{2}
$$


Very little information exists on the value of $\mathrm{K}_{\mathrm{lc}}$ for beryllium, especially at high temperatures and after/during neutron irradiation. However, there are some data on the effect of temperature and irradiation on parameters such as the yield strength and the ductility of beryllium. Since these parameters can be related to $K_{l c}$, a qualitative estimate of the effects of irradiation and temperature on the fracture toughness of beryllium can be made.

There are a number of standard test methods used to calculate $\mathrm{K}_{\mathrm{lc}}$ for a material (for example, ASME tests E-399 or E-813). However, these test methods are expensive, time consuming, and require substantial quantities of material. Because of this, a method has been developed to relate $K_{l c}$ to quantities which are obtained though simple, inexpensive tensile tests. In the critical strain model, failure is assumed to occur when a critical fracture strain $\left(\varepsilon_{f}^{*}\right)$ is locally exceeded over some microstructurally significant characteristic distance $\left(l_{o}^{*}\right)$ ahead of the crack tip. Using this model, fracture toughness can be expressed in the form: ${ }^{61}$

$$
\mathrm{K}_{\mathrm{bc}}=\text { constant } \sqrt{\varepsilon_{f}^{*} \cdot l_{o}^{*} \cdot \sigma_{y} \cdot \mathrm{E}},
$$

where $\sigma_{y}$ is the material yield stress, and $\mathrm{E}$ is Young's Modulus.

Since the critical fracture strain $\varepsilon_{f}^{*}$ is difficult to determine experimentally, it has been proposed 62 to substitute the fracture strain, or ductility, $\varepsilon_{f}$ from a simple, $u$ : jaxial tensile test for the critical fracture strain in Equation 5.36. Although there is no theoretical basis for such a substitution, the critical fracture strain has been shown to be

\footnotetext{
${ }^{61}$ R O. Ritchie, et. al., "Critical Fracture Stress and Fracture Strain Models for the Prodiction of Lower and Upper Shelf Toughness in Nuclear Pressure Vessel Steels," Metallurgical Transactions A, Vol. 10A, October, 1979, p. 1557.

62F. M. Haggag and R. K. Nanstad, "Estimating Fracture Toughness Using Tension of Ball Indentation Tests and a Modified Critical Strain Model," in Innovative Approaches to Irradiation Damage and Fracture Analysis, D. L. Marrioth, et. al., eds., PVP Vol. 170, The American Society of Mechanical Engineers, 1989, p. 41.
} 
proportional to the ductility in tensile tests. Furthermore, tests for low strength steels indicate that at room temperature, $\varepsilon_{,}^{*} \equiv \varepsilon,{ }^{63} l_{o}^{*}$ is a distance related to the material microstructure, and is usually obtained empirically. The constant of Equation 5.36 is 3.00 for steels, but may be different for different materials.

Substituting $\varepsilon_{f}$ for $\varepsilon_{f}^{*}$ in Equation 5.36 yields: ${ }^{64}$

$$
\mathrm{K}_{\mathrm{Ic}}=\operatorname{constant} \sqrt{\varepsilon_{f} \cdot l_{o}^{*} \cdot \sigma_{y} \cdot \mathrm{E}} .
$$

In this equation, the yield stress $\sigma_{y}$ and ductility $\varepsilon_{f}$ can both be measured using a simple tensile test. Such tensile test data are available for beryllium at high temperatures, and for irradiated samples. The value of $\mathrm{E}$ has also been measured as a function of temperature for beryllium. If $E$ and $l_{o}^{*}$ are assumed to be constant functions of irradiation, and furthermore that $l_{o}^{*}$ remains constant with temperature, then knowledge of how $E$ changes with temperature, and how $\sigma_{y}$ and $\varepsilon_{f}$ change with both temperature and irradiation will give information on how $\mathrm{K}_{\mathrm{Lc}}$ could be expected to change. ${ }^{65}$ Specifically, with these assumptions we can write:

$$
K_{k}(T, \phi)=C \cdot \sqrt{\varepsilon_{f}(T, \phi) \cdot \sigma_{y}(T, \phi) \cdot E(T)}
$$

where $T$ is temperature and $\phi$ is a measure of irradiation damage.

\footnotetext{
${ }^{63}$ R. K. Pandy and S. Banejoe, "Strain Induced Fracture in Low Strength Steels," Engineering Fracture Mechanics, Vol. 10, p. 817.
}

${ }^{64}$ Note that in Hageng and Nantad, Equation 6 was written with $K_{f_{0}}$, rather that $K_{b o}$, on the left hand

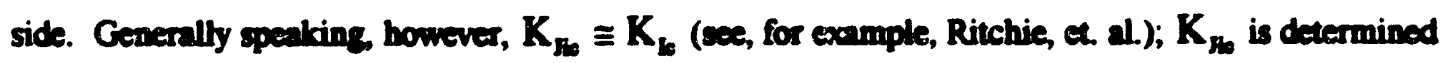
by firs determining the value of the so-called J-integral, and then relating it to $\mathbf{K}_{\mathrm{k}}$. For information on J-integral methods, see for exomple D. Brodk, Elementary Engineering Fracture Mechatics, Sijthof and Noordhofi, Aphen and den Rijn, The Netherlands, 1978.

${ }^{65}$ Since $I_{0}^{0}$ depends on the moterial microanceure, it may in fact change as a reault of irradimion. For beryllium, however, the efiects of irmdimion and high temperanre on $f_{0}^{b}$ are unknown, so herein they are asoumed to te small compared to the changes in cuctility and yield strese. 
Since the fracture toughness and other room temperature properties of beryllium are presumably known, it would now appear to be a simple matter to use tensile test data for irradiated beryllium samples, and data on how the properties of beryllium change with temperature, to determine via Equation 5.38 how the fracture toughness changes with temperature and irradiation. There are a number of complexities which arise when attempting to do this, however. First of all, depending on the detailed fabrication process and microstructure for the beryllium, and such variables as the level of beryllium oxide present in the metal, its properties can vary significantly. For example, room temperature tests of different samples of unirradiated, $100 \%$ dense beryllium have resulted in yield strength values from 175-245 $\mathrm{MPa}$, and fracture strain values from 3-30 percent. ${ }^{66}$ With differences which are that large, one must be very careful when trying to generalize the properties of beryllium, and especially when comparing data from tests on different beryllium samples.

Based on a short survey of available literature on beryllium, it appears that typical yield strength and fracture strain values are closer to the lower bounds of the ranges shown above. 67 The room temperature fracture strain, yield stress and fracture toughness of unirradiated IM beryllium manufactured by Material Sciences Corporation is shown in Table 5.19. This type of beryllium is the same as that assumed for the LOFA analysis of Blanket Design 2, and has properties which are "typical" (i.e. close to the lower bounds

\footnotetext{
${ }^{66}$ Compare data in (1) J. M. Becton, et. al., "Mochanical Properties of Irradisted Beryllium," Journal of Nuclear Materials, Vol. 195, 1992, p. 102; (2) J. M. Beeston, et. al., "Comparison of Compression Properties and Swelling of Beryllium Irratiated at Various Temperatures," Journal of Nuclear Materials, Vol. 122-123, 1984, p. 802; and (3) R. E. Taylor, et. al. , Thermophysical Properties of Be Metal, A Report to Manufecturing Sciences Corp.," Report No. TPRL 1071, Thermophysical Properties Research Laboratory, School of Mochanical Engineering, Purcue University, presented by D. R. Floyd at the Beryllium Technology Workshop, Clearwater Beach, FL, November 20, 1991, Idaho National Engineering Laboratory Report No. EGG-FSP-10017, G. R Longhurst, ed

${ }^{67}$ See, for example, 1) D. W. White, Jr. and J. E. Burke, eds., The Metal Beryllium, U.S. Atomic Energy Commission, 1954; 2) R. E. Taylor, et. al.; 3) J. M. Beeston, et. al., 1992; and 4) D. S. Gelles and H. L. Heinisch, "Neutron Damage in Beryllium," Joumal of Nuclear Materials, Vol. 191-194, 1992, p. 194.
} 
of the yield strength and fracture strain ranges discussed above) for room temperature beryllium.

Table 5.19. Properties of Unirradiated, $100 \%$ Dense, IM Beryllium at RT ${ }^{68}$

\begin{tabular}{|c|c|}
\hline Fracture Toughness $\left(\mathrm{MPa} \cdot \mathrm{m}^{05}\right)$ & 41 \\
\hline Tensile Yield Stress $(\mathrm{MPa})$ & 175 \\
\hline Tensile Fracture Strain $(\%)$ & 4 \\
\hline Young's Modulus $(\mathrm{GPa})$ & 308 \\
\hline
\end{tabular}

Test data are available for high-temperature properties of irradiated beryllium. However, the room temperature fracture strain reported for the beryllium used in these tests was very high, at 30 percent. ${ }^{69}$ This is a very high value, and based on the survey discussed above, is not representative of typical beryllium samples. It is therefore not clear whether the test results of this study are relevant to more typical beryllium samples fabricated with standard procedures. Hence, the results of this particular study will not be used.

Since no other study to date has performed elevated temperature testing on irradiated beryllium, we have no choice but to evaluate the effects of high temperature and irradiation on fracture toughness separately, and, based on these evaluations, hopefully get an idea of what the combination of high temperature and irradiation may do to

\footnotetext{
${ }^{68}$ R. E. Taylor, a. al. "Thermophysical Properties of Be Metal, A Report to Manufacturing Sciences Corp.," Report No. TPRL 1071, Thermophysical Properties Research Laboratory, School of Mechanical Engineering, Purdue University, presented by D. R. Floyd at the Beryllium Technology Workshop, Clearwater Beach, FL, November 20, 1991, Idaho National Engineering Laboratory Report No. EGGFSP-10017, G. R. Longhurst, ed.

69J. M. Beeston, et. a.., "Comparison of Compression Properties and Swelling of Beryllium Irradiated at Various Temperatures," Journal of Nuclear Materials, Vol. 122-123, 1984, p. 802
} 
toughness. Figure 5.52 shows how the yield strength and fracture strain vary with temperature for unirradiated beryllium. The yield strength gradually decreases with Figure 5.52. Yield Strength and Fracture Strain of 100 temperature, but the $\%$ Dense, Unirradiated IM Beryllium

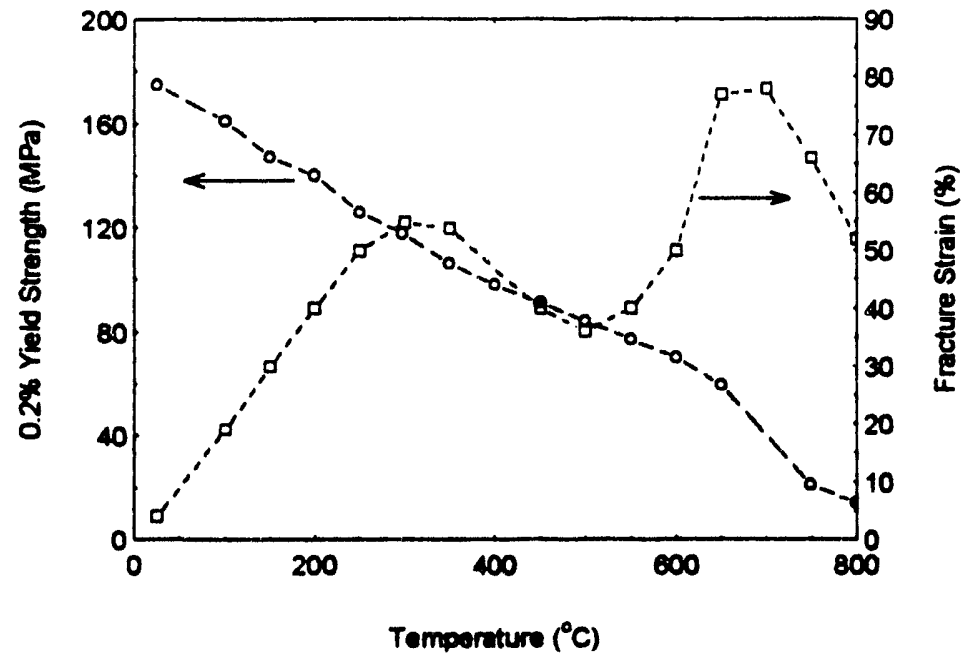

fracture strain increases substantially. Equation 5.38 indicates that the square root of the product of the yield strength, fracture strain and Young's Modulus is proportional to the fracture toughness.

Hence, we define a quantity called the toughness parameter, as follows:

$$
\text { Toughness Parameter }=\sqrt{\frac{\left(\varepsilon_{\mathrm{f}} \cdot \sigma_{y} \cdot \mathrm{E}\right)_{\tau}}{\left(\varepsilon_{\mathrm{f}} \cdot \sigma_{y} \cdot \mathrm{E}\right)_{R T}}}=\frac{\left.\mathrm{K}_{\mathrm{lc}}\right|_{T}}{\left.\mathrm{~K}_{\mathrm{lc}}\right|_{\mathrm{RT}}},
$$

where the denominator is evaluated at room temperature (RT) and the numerator is

\section{Figure 5.53. Beryllium Toughness Parameter versus} Temperature

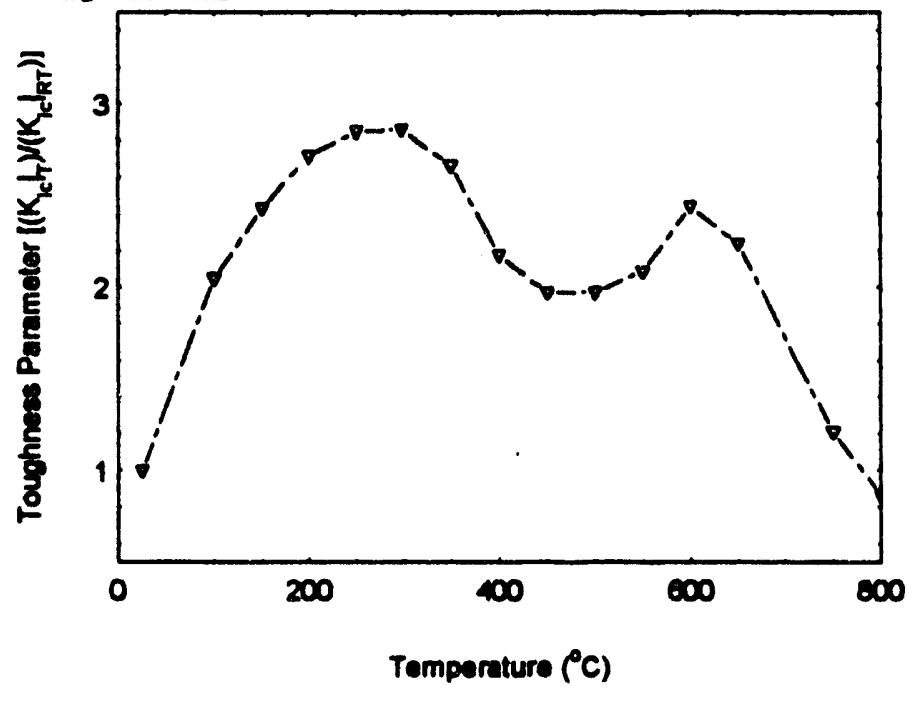

evaluated at temperature

T. The toughness

parameter is equal to the ratio of the fracture toughness at temperature $T$ to the room temperature fracture toughness.

Figure 5.53 shows a plot of the toughness parameter versus 
temperature, obtained from the data shown in Figures 5.48 and 5.52. For the operational temperature range for the beryllium in the blanket (280-575 degrees $C$ ), the toughness parameter lies between 2.0 and 2.9 .

Now we turn to the data on irradiated beryllium. The U.S. has initiated a beryllium irradiation study in support of ITER (the International Thermonuclear Experimental Reactor). As part of this study, the mechanical properties of unirradiated and irradiated beryllium are sought for various initial beryllium porosities. Although this study is not yet completed, some results are currently available.

Although fast neutron fluence is a usual measure for irradiation studies, it turns out that the amount of helium generated in the beryllium (usually measured in atomic parts-permillion, or appm) is a better measure for fusion-related beryllium studies. This is because helium generation results in voids being formed in the beryllium. It is these voids which drive the swelling behavior of the beryllium. Presumably, these voids are also largely responsible for the property changes in beryllium resulting from irradiation. Because most of the helium is produced from an $(n, 2 n)$ reaction with a threshold energy of 2.7 $\mathrm{MeV}$, the vastly different spectra between thermal, fast fission, and fusion reactors can result is great differences in the amount of helium produced for a given neutron fluence. ${ }^{70}$

Blanket Design 2 is to be operated for 3 effective-full-power-years at an average first wall load of $4 \mathrm{MW} / \mathrm{m}^{2}$, resulting in $12 \mathrm{MW} \cdot \mathrm{yr} / \mathrm{m}^{2}$ of fluence. From ITER calculations, it is known that the amount of helium generated in beryllium near the first wall is about 3000 appm/(MW/ $\left.\mathrm{m}^{2}\right)^{71}$ This information, plus data on the shape of the

70M. Billone, Argonne National Laboratory Fusion Power Program, personal communication, June, 1993. 
helium concentration curve as a function of distance in Blanket Design 2 (from the REAC3 and TWODANT codes), is used to generate Figure 5.54. Inspection of this figure reveals that the helium concentration in the beryllium slabs of Blanket Design 2 drops roughly exponentially as one moves from the first wall toward the rear of the blanket. This figure also indicates the locations of the 1 st breeder region, directly behind the first wall, and the 10th breeder region, $28.6 \mathrm{~cm}$ behind the first wall. In the $1 \mathrm{st}$ region, the helium concentration is rather large, at $36,000 \mathrm{appm}$. For the 10 th region, the helium concentration is only about 4500 appm.

Unfortunately, irradiation data obtained to date for beryllium properties are for samples with only about $800 \mathrm{appm}$ helium, well below the expected value for either breeder region. Since these are the only data available, we need to extrapolate rather far from the existing data base in order to get results which are meaningful for Blanket Design 2 at end-of-life. An additional unavoidable problem with the current data is that the irradiation temperature was much lower than the operational temperature for the

Figure 5.54. Helium Concentration in Blanket Design 2 Be Slabs at EOL

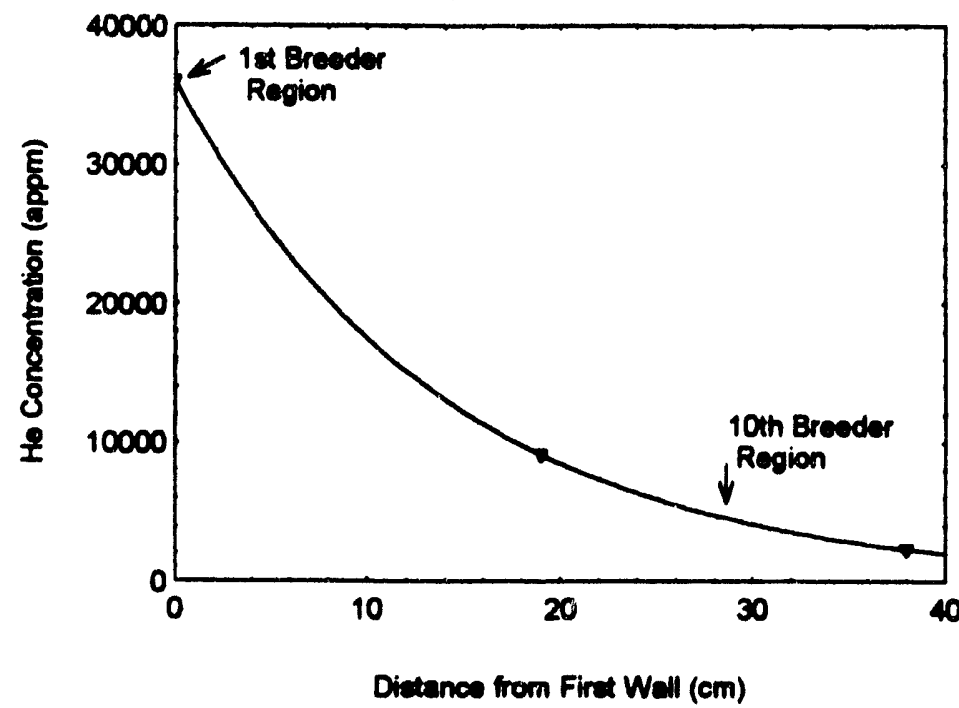

beryllium in Blanket

Design 2. Irradiation temperature may affect the magnitude of the changes in yield strength and fracture strain.

The irradiation test data currently available come from beryllium samples irradiated in the Advanced

IIIbid. 
Test Reactor (ATR) ${ }^{72}$ The samples were of 80 percent and 97 percent dense beryllium produced via powder metallurgy, and were irradiated with a fluence of $2.6 \times 10^{21} \mathrm{n} / \mathrm{cm}^{2}$ $(E>1 \mathrm{MeV})$. These samples had measured helium concentrations of 733 appm for the 80 percent dense samples, and 872 appm for the 97 percent dense samples. In the following analysis, it is assumed for simplicity that both types of sample were irradiated to 800 appm helium. Because $\mathrm{i}_{i}$ is easier to perform compression testing than tensile testing on irradiated beryllium, the yield strength and fracture strain were measured via compression, rather than tensile tests. The tests were performed at room temperature. For the purpose of this analysis, the change in compression properties after irradiation is assumed to be proportional to the change in tensile properties.

Table 5.20 shows the properties of the samples before and after irradiation.

\footnotetext{
${ }^{72}$ Information on the property data of irradiated beryllium comes from J. M. Becaton, et. al., "Mechanical Testing of Irradiated Beryllium," presented at the Beryllium Technology Workshop, G. L. Longhurst, chmn., Idaho Engineering Leboratory Report EGG-FSP-10017, December, 1991. There has been another recent irratiation test of $100 \%$ dense beryllium (D. S. Gelles and H. L. Heinisch, "Neutron Damage in Beryllium," Joumal of Nuclear Materials, Vol. 191-194, 1992, p. 194). In this teat, however, all of the irradiated beryllium samples exhibited zero cuctility during tensile testing. This result is contrary to other irradiation test recults on beryllium, and may have been che to a poor specimen fabrication technique.
} 
Table 5.20. Change in Beryllium Properties After Irradiation

\begin{tabular}{|c|c|c|}
\hline \multicolumn{3}{|c|}{ BEFORE IRRADIATION } \\
\hline Sample Density & $\frac{\text { Compression Yield }}{\text { Strength (MPa) }}$ & $\frac{\text { Compression Rupture }}{\text { Strain (\%) }}$ \\
\hline $97 \%$ & $216 \pm 7$ & 3.1 \\
\hline $80 \%$ & $140 \pm 3$ & 1.74 \\
\hline \multicolumn{3}{|c|}{ AFTER IRRADIATION } \\
\cline { 2 - 3 } TO 800 appm HELIUM \\
\hline Sample Density & $\frac{\text { Compression Yield }}{\text { Strength (MP) }}$ & $\frac{\text { Compression Rupture }}{\text { Strain (\%) }}$ \\
\hline $97 \%$ & $928 \pm 4.3$ & $0.64 \pm 0.04$ \\
\hline $80 \%$ & $611 \pm 58$ & $0.40 \pm 0.05$ \\
\hline
\end{tabular}

As can be seen, for both the 80 and 97 percent dense samples, the rupture strain decreases with irradiation, and the yield strength increases. These trends are characteristic of an irradiated material. There is approximately a four-fold decrease in the rupture strain values, and a four-fold increase in the yield strength values. Hence, these two effects tend to balance when the fracture toughness is calculated.

As was done before for temperature, a toughness parameter for irradiation can be defined as the ratio between the irradiated fracture toughness and the unirradiated fracture toughness. Using the data in Table 5.20, the toughness parameter corresponding to an 800 appm helium concentration is 0.94 for the 97 percent dense samples, and 1.00 for the 80 percent dense samples. Hence, the fracture toughness of the 97 percent dense samples is decreased by 6 percent, and the fracture toughness of the 80 percent dense samples stays the same. For the purpose of the present work, it is conservatively assumed that the results of the 97 percent dense beryllium samples are representative of the 100 percent dense beryllium used in Blanket Design 2. 
Extrapolating from 800 appm helium test results to the 36,000 appm level expected near the first wall of a reactor is obviously a questionable process. This process is made even more difficult because there are not enough usable irradiation data on beryllium at different helium levels to obtain information on the shape of the fracture toughnessversus-helium concentration curve. The assumption is therefore made, a priori, that for every 800 appm helium generated in the beryllium, the fracture toughness decreases by 6 percent, as it did for the first 800 appm helium generated in the 97 percent dense samples. This assumption is theoretically unfounded, and hence should be considered very speculative.

A toughness parameter for irradiation can be defined which is analogous to the toughness parameter defined above for temperature. Specifically, the toughness parameter for irradiation is defined as follows:

$$
\text { Irradiation Toughness Parameter }=\frac{\mathbf{K}_{\mathbf{k}} l_{0}}{\mathbf{K}_{\mathbf{k}} b},
$$

where $K_{k} l_{0}$ is the irradiated fracture toughness, and $K_{b_{0}} l_{0}$ is the unirradiated fracture toughness, both evaluated at the same temperature. Table 5.21 shows the toughness parameter for 800,6200 , and 50,000 appm helium, based on the assumptions discussed above.

\section{Table 5.21. Irradiation Toughness Parameters}

\begin{tabular}{|c|c|}
\hline Belium Level (apnm) & Toughneens Parnmeter \\
\hline 800 & 0.94 \\
\hline 4500 (10th brd. reg., EOL) & 0.71 \\
\hline 36,000 (1st brd. reg., EOL) & 0.06 \\
\hline
\end{tabular}

Now that the behavior of the fracture toughness of beryllium as a function of irradiation and of temperature has been separately explored, the results are now combined to obtain 
an estimate of the expected end-of-life toughness for the beryllium slabs in Blanket Design 2. The toughness parameter as a function of temperature was presented in Figure 5.53. If the minimum toughness parameter associated with the operational temperature range of the slabs (2.0) is multiplied by the end-of-life irradiation toughness parameters shown in Table 5.21 for the 1 st and 10 th breeder regions, a minimum end-oflife toughness parameter for each region is obtained which includes the effects of temperature and irradiation. If this toughness parameter is multiplied by the unirradiated, room temperature toughness of beryllium (shown in Table 5.19), an estimate of the minimum end-of life fracture toughness for the beryllium in each region is obtained. Table 5.22 shows the results.

Table 5.22. Fracture Toughness Estimate for Beryllium Slabs

\begin{tabular}{|c|c|c|c|c|}
\hline Breeder Region & $\frac{\text { Room Temp. Unirrad }}{\text { Fracture Toughness }}$ & $\begin{array}{c}\text { Temp } \\
\text { Toughness } \\
\text { Parameter }\end{array}$ & $\begin{array}{c}\text { Irrad } \\
\text { Toughness } \\
\text { Parameter }\end{array}$ & $\begin{array}{c}\text { Qperating Temp. } \\
\text { End-of-Life Fracture } \\
\text { Toughness }\end{array}$ \\
\hline 1 st & $41 \mathrm{MPa} \cdot \mathrm{m}^{05}$ & 2.0 & 0.06 & $4.9 \mathrm{MPa} \cdot \mathrm{m}^{05}$ \\
\hline $10 \mathrm{th}$ & $41 \mathrm{MPa} \cdot \mathrm{m}^{05}$ & 2.0 & 0.71 & $58 \mathrm{MPa} \cdot \mathrm{m}^{05}$ \\
\hline
\end{tabular}

As shown in Table 5.22, the end-of-life fracture toughness for the beryllium slabs in the 1st breeder region is greatly reduced due to the effects of irradiation, whereas the toughness for the 10th breeder region slabs is actually higher than the unirradiated room temperature value, because the increase in the toughness parameter caused by the elevated operating temperature outweighs the irradiation-induced decrease. These results can now be used in conjunction with the maximum thermal stresses seen in the slabs to determine the maximum allowable flaw size in the slabs to ensure against fracture. The maximum thermal stress actually seen in the slab may be lower than the $113 \mathrm{MPa}$ calculated above. Since thermal stresses are secondary stresses, the maximum stress actually seen in the slab is expected to be the yield strength. Although the yield 
strength for unirradiated beryllium at elevated temperatures is lower than $113 \mathrm{MPa}$ (see Figure 5.52), irradiation will tend to increase the yield strength, perhaps to above 113 $\mathrm{MPa}$. Hence, for conservatism, it is assumed the thermal stresses are not reduced by yielding

Solving for $a_{n}$ in Equation 5.35 using the end-of-life fracture toughnesses of Table 5.22 , together with the maximum thermal stresses of the slabs in each region, and assuming $C=1.122$ yields a maximum allowable flaw size of $6.7 \mathrm{~cm}$ for the 10 th breeder region and $3 \mathrm{~mm}$ for the $1 \mathrm{st}$ breeder region. The slabs in the 1 st breeder region show a smaller allowable crack size due to the detrimental effect of the irradiation on the fracture toughness of these slabs.

To avoid cracking in the slabs near the first wall, it would appear that non-destructive testing of these slabs may be warranted to ensure no flaw size greater than $3 \mathbf{m m}$ is present. Such testing should not be difficult, since the slabs have a simple geometry and relatively small cross sections. ${ }^{73}$ Since $3 \mathrm{~mm}$ is a rather large flaw size, it may be shown that beryllium slabs of the sizes needed for Blanket Design 2 can be easily fabricated with no flaws of this magnitude. If this turns out to be the case, such non-destructive testing may turn out to be unnecessary. For the larger slabs deeper in the blanket, it is clear that such testing is not warranted, since the maximum allowable flaw size is larger than the width of the slabs themselves. It can be concluded, based on the results of this section, that with proper fabrication and inspection of the slabs near the first wall, the beryllium slabs would not be expected to crack due to thermal stresses for the lifetime of Blanket Design 2.

\footnotetext{
${ }^{73}$ Note that $3 \mathrm{~mm}$ is much larger then the smallest flaw size $(0.4 \mathrm{~mm})$ used in referenoe blocks for ultrasonic calibrations. See T. Baumeinter, et.al., Mark's Standard Handbook for Mechanical Engineers, McGraw -Hill, 1978, p. 5-83.
} 
A final note of caution is in order concerning the previous analysis. A large amount of extrapolation was necessary to calculate the effect of irradiation on the end-of-life toughness. As previously mentioned, the shape of the fracture toughness-versus-helium concentration curve is unknown, and had to be assumed for this study. Hence, it is very important that further tensile test data be obtained for beryllium at higher irradiation levels and helium concentration levels. Furthermore, actual fracture toughness tests need to be performed on irradiated beryllium samples. Until such tests are performed, the conclusions reached herein, and in fact any conclusions on the fracture toughness of highly-irradiated beryllium in fusion reactors, must be considered to be very tentative. 


\section{Swelling Stresses}

Formation of helium in beryllium occurs under fast neutron irradiation due to the $(n, 2 n)$ reaction which has a threshold energy of $2.7 \mathrm{MeV}$. As has been discussed previously, the helium which forms causes swelling in the beryllium, to an extent which at present is not well established. For the purposes of design of the breeder regions of Blanket Design 2, it will be recalled that an end-of-life swelling value of 10 percent was assumed for all of the beryllium slabs, irrespective of their temperature or amount of helium generation. It is known, however, that the swelling is dependent on both the amount of helium produced and the temperature of the beryllium. Based on a substantial number of data points for 100 percent dense, hot-pressed beryllium with $\sim 25 \mu \mathrm{m}$ grain diameter, Billone developed a correlation describing helium-induced swelling in Be, which is as follows: ${ }^{74}$

$$
\frac{\Delta V}{V_{0}}=0.115\left(\frac{G_{\alpha}}{10^{3}}\right)\left[1+3.0 \times 10^{-3}\left(\frac{G_{\alpha}}{10^{3}}\right)^{0.5} T^{13} \exp \left(\frac{-3940}{T}\right)\right]
$$

where $\frac{\Delta V}{V_{0}}$ is the volumetric swelling in percent, $G_{\alpha}$ is the He content in appm, and $T$ is

Figure 5.55. Swelling in Beryllium Slabs

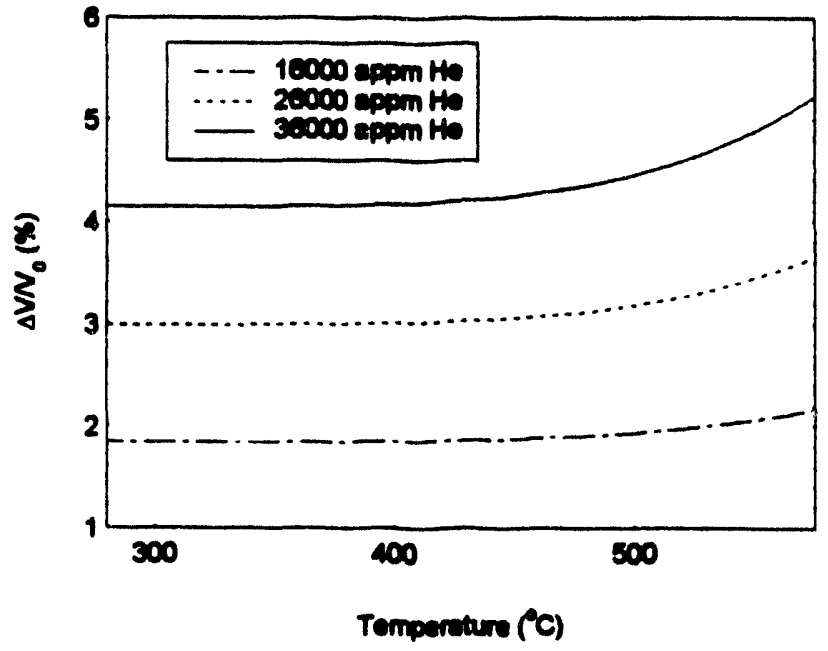

the temperature in $\mathbf{K}$.

Although the data base for this correlation exists only up to a

He content of 26,000 appm, the same correlation is assumed to hold up to the maximum end-of-life value for Blanket Design 2, which is 36,000 appm. Figure 5.55

${ }^{74}$ M. Billone, Argonne National Laboratory Fusion Power Program, personal communication, June, 1993. 

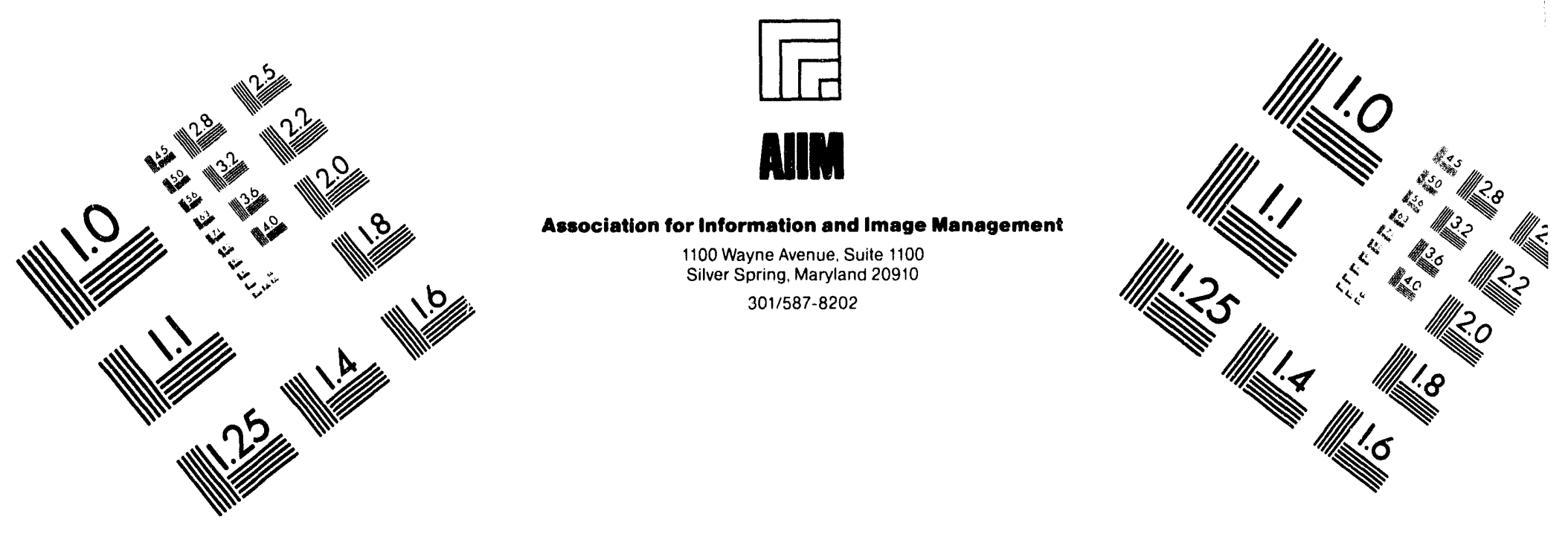

\section{Centimeter}

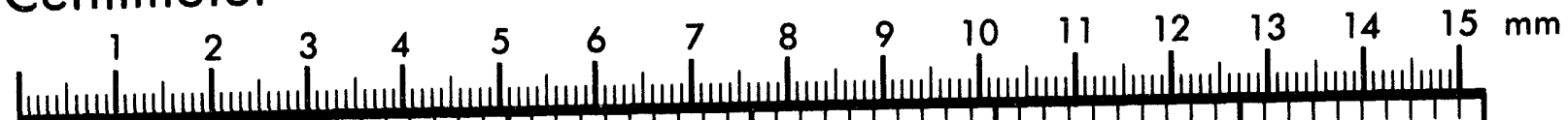
1нulum Inches
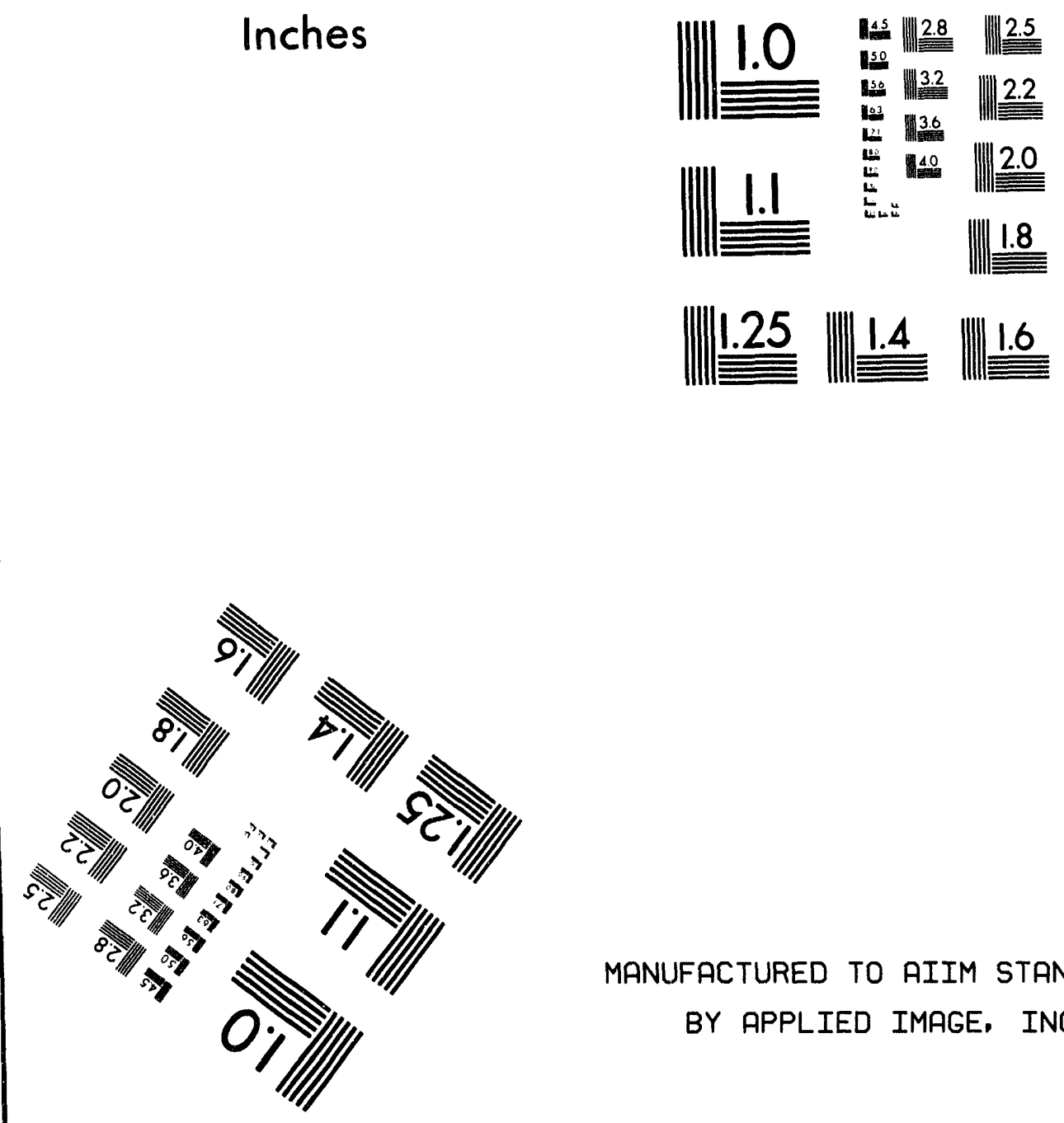

MANUFACTURED TO AIIM STANDARDS

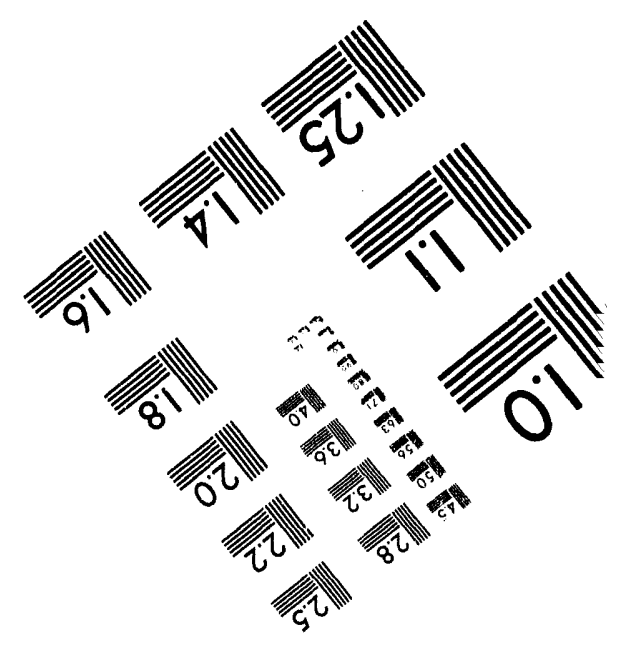



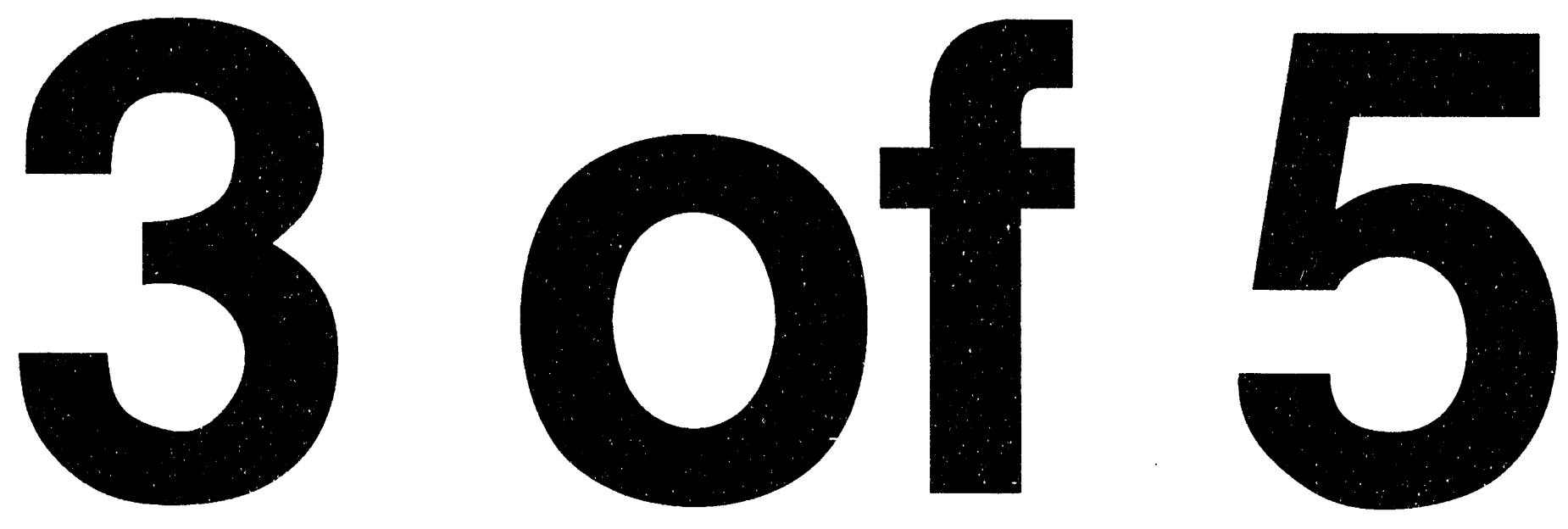
shows the swelling values as a function of temperature for three different helium concentrations. There is a significant difference in the amount of swelling at different temperatures, especially at high helium concentrations. ${ }^{75}$ This differential swelling can cause stresses in the beryllium slabs, since different portions of the slab are at different temperatures. For temperatures below about 450 degrees $C$, the swelling-versustemperature curve is almost flat, indicating that if the slabs operate below this temperature, the swelling is basically uniform in the slab, and hence no swelling-induced stresses appear. At higher temperatures, however, there is a marked slope in the curve, and therefore a potential for significant induced stresses.

The svelling-induced stresses, unlike the temperature stresses discussed previously, appear only gradually over the lifetime of the blanket. Hence, there is time for creep mechanisms to occur to relieve the stresses induced by swelling. The two creep mechanisms of interest for the beryllium slabs are high-iemperature and irradiation creep. Creep is facilitated by the motion of interstitials and vacancies in the metal lattice; for the case of high-temperature creep the mobility of interstitials and vacancies is induced by their increased thermal energy, and for the case of irradiation creep the mobility is induced by the energy imparted to the metal lattice by the incoming energetic neutrons.

For the beryllium slabs in an operating fusion blanket, these two creep mechanisms operate simultaneously, and both help to reduce the stresses induced by the differential swelling of the slabs. The rate of thermal creep for unirradiated beryllium is well known as a function of temperature and applied stress, as it is for most unirradiated metals. The

\footnotetext{
${ }^{75}$ Note that the maximum swelling value shown is less than the 10 percent assumed for design of Blanket Design 2, indicating some conservatism in design.
} 
rate of irradiation creep for beryllium is much less well known, due to a severe lack of data (there is only one data point for irradiation creep currently available for beryllium ${ }^{76}$ ).

Generally speaking, one expects irradiation to accelerate the rate of creep of a metal at a certain temperature and applied stress. ${ }^{77}$ This effect can be very dramatic (well over an order of magnitude for stainless steels in a fast neutron flux, ${ }^{78}$ and about an order of magnitude for zirconium ${ }^{79}$ ). For beryllium, a similar increase in the thermal creep rate due to irradiation is expected. However, irradiation also produces large amounts of helium in the beryllium. This helium forms voids in the metal, restricting the ability of the vacancies and interstitials to move. These voids reduce the out-of-pile fracture strain of the beryllium, as seen in the last section. The voids may also restrict the ability of the beryllium to creep. Hence, although irradiation should increase the creep rate of beryllium at low fluences, at high fluences the formation of helium voids may reduce the magnitude of this increase, or could even reduce the creep rate to a value below that of the unirradiated metal.

As indicated previously, there are not enough in-pile data on the creep behavior of beryllium to estimate the effect of either neutron flux or fluence on its high-temperature creep behavior. Hence, for simplicity, it is assumed that the beryllium creeps at a rate which corresponds to an unirradiated sample at the appropriate temperature. Hence the

\footnotetext{
${ }^{76}$ M. C. Billone, Argonne National Laboratory, private communication, July, 1993.

${ }^{77}$ D. R. Olander, Fundamental Aspects of Nuclear Reactor Fuel Elements, NTIS TID 26711-P1, 1976, p. 499.

${ }^{78}$ B. M. Ma, Nuclear Reactor Materials and Applications, Van Nostrand Reinhold Co., 1983, p. 110.

79D. G. Franklin, et. al., Creep of Zirconium Alloys in Nuclear Reactors, ASTM Special Technical Publication 815, American Society for the Testing of Materials, p. 60.
} 
irradiation enhancement of creep is conservatively neglected, and any reduction in creep that may conceivably occur at high helium concentrations is also neglected.

Figure 5.56. Creep of Beryllium ${ }^{80}$

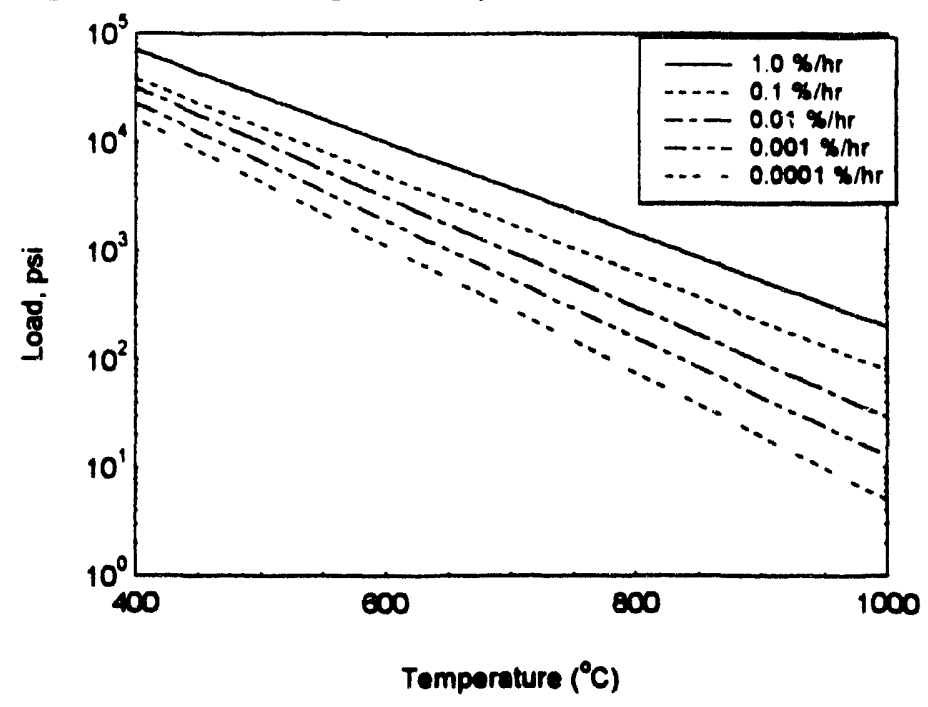

Figure 5.56 shows the unirradiated creep behavior of 100 percent dense beryllium. This figure shows the load required to cause a particular creep rate as a function of temperature. The creep rate data are now used in conjunction with Equation

5.41 to estimate the maximum stress induced in the beryilium slabs by swelling.

To find an upper-bound estimate for the swelling-induced stresses in the beryllium slabs, accounting for the effect of thermal creep, the following method is used. First, using Equation 5.41, the maximum swelling rate (swelling per unit He content) is determined. Second, Figure 5.56 is used to determine the minimum load required to obtain a creep rate corresponding to the maximum swelling rate at the appropriate temperature. For the present case, this minimum load is the maximum stress which would be experienced due to swelling if the beryllium slab were restrained at the edges. Since the slab is not restrained, this load represents a conservative estimate of the stress which would be seen in the slab. If this stress can be shown to be acceptable, then swelling of the slabs should not result in cracking of the slabs.

\footnotetext{
${ }^{80}$ Adapted from data in D. W. White and J. E. Burke, eds., The Metal Beryllium, U.S. Atomic Energy Commission, 1955, p. 322. The $0.0001 \%$ /hr curve obtained from extrapolation from other curves.
} 
Differentiating Equation 5.41 with respect to the helium concentration gives the following swelling rate relation:

$$
\frac{\partial}{\partial G_{\alpha}}\left(\frac{\Delta V}{V_{0}}\right)=1.15 \times 10^{-4}\left(1+1.423 \times 10^{-4}\left(G_{\alpha}\right)^{0.5} T^{1.9} \exp \left(\frac{-3940}{T}\right)\right)
$$

where the units of this expression are (\%/appm He). As discussed in the previous section on thermal stresses, the beryllium near the first wall of Blanket Design 2 is expected to generate about 12,000 appm He/full-power-year. This fact can be used in conjunction with the above equation to obtain the following swelling rate equation in more useful units:

(5.43) Swelling Rate $(\% / h r)=1.57 \times 10^{-4}\left(1+1.423 \times 10^{-4}\left(G_{\alpha}\right)^{0.5} T^{1.5} \exp \left(\frac{-3940}{T}\right)\right)$.

This swelling rate relation is maximized when the helium concentration $G_{\alpha}$ and temperature $\mathrm{T}$ are maximized.

There are two possible regimes which could result in the highest swelling-induced stresses. At high temperatures, the swelling rate is the largest, which tends to result in higher stress values. However, at low temperatures, the creep rate for a given value of stress is smaller, which again tends to result in higher stresses to induce the required creep rate. Hence, two cases are analyzed, a high temperature case (at 575 degrees $C$, the highest operational temperature for the beryllium in Blanket Design 2) and a low temperature case (450 degrees $\mathrm{C}$, the lowest temperature for which differential swelling is a concern based on Figure 5.55). For conservatism, the helium concentration for both cases is assumed to be the maximum seen in the first wall at end-of-life, or 36,000 appm. 
For the 575 degrees $\mathrm{C}$ case, a maximum volumetric swelling rate of $0.001 \% / \mathrm{hr}$ is obtained from Equation 5.43. To convert this volumetric swelling rate into a creep rate, note that if a cube with unit volume expands on all three sides by a small amount $\varepsilon$, then the change in volume of the cube is equal to $(1+\varepsilon)^{3}-1$. For small values of $\varepsilon$ this expression is approximately equal to $3 \varepsilon$. Hence, if $\varepsilon$ is paired with the creep rate associated with the volumetric swelling rate of $0.001 \% / \mathrm{hr}$, the creep rate corresponding to the maximum swelling rate is $\varepsilon=0.00033 \% / \mathrm{hr}$. From Figure 5.56 , it can be concluded that at 575 degrees $C$, the load required to obtain a creep rate of this magnitude is less that $14 \mathrm{MPa}$ (2000 psi). For the 450 degrees $\mathrm{C}$ case, a similar analysis results in a volumetric swelling rate of $0.0005 \% / \mathrm{hr}$, corresponding to a creep rate of $0.00017 \% / \mathrm{hr}$. At 450 degrees $\mathrm{C}$, the load required to obtain this creep rate is less than $70 \mathrm{MPa}$.

It can be concluded, then, that $70 \mathrm{MPa}$ is the maximum possible stress expected due to swelling in any of the beryllium slabs. This stress is significantly less that the stress levels induced by the thermal stresses discussed in the previous section. Hence, the thermal stresses are more limiting than the swelling-induced stresses, and if the slab can sustain the thermal stresses without fracture, it should also be able to withstand the swellinginduced stresses.

\section{Effece of Swelling Voids on Conductivity}

Althcugh it has just been demonstrated that swelling-induced stresses in the beryllium slabs should not cause the slabs to crack, the voids which form as part of the swelling process can reduce the conductivity of the slabs, even if cracking does not occur. It is assumed, as done during the design of Blanket Design 2, that all beryllium slabs experience 10 percent volumetric swelling at end-of-life. Furthermore, 10 percent of the 
beryllium slab is assumed to be composed of uniformly spaced helium bubbles at end-oflife. This is equivalent to assuming there is 0.10 porosity in the slabs at end-of-life

The reduction in thermal conductivity caused by this porosity is given as follows, based on data for beryllium at various temperatures: ${ }^{81}$

$$
k_{B e}(T)=k_{0}(T) \frac{1-P}{1+3.7 P^{2}},
$$

where $k_{B o}(T)$ is the conductivity with porosity $P$, and $k_{o}(T)$ is the conductivity of 100 percent dense beryllium at temperature $T$. For a porosity of 0.10 , this relation reduces the conductivity to 87 percent of its 100 percent dense value.

To see the effect of this reduction in conductivity, the LOFA case with 7 second plasma continuation is run again, reducing all of the beryllium conductivities to 87 percent of their original, 100 percent dense values. (Note that this analysis does not account for the fact that the beryllium slabs grow as they swell, increasing the thermal conductivity of the breeder regions - hence it is conservative). Figure 5.57 shows the first wall temperature as a function of time after shutdown for both the 0.10 porosity case and for the fully dense beryllium case. The assumed gap conductance for this figure is 10,000 $\mathrm{W} / \mathbf{m}^{2} \cdot \mathbf{K}$.

${ }^{81}$ M. Billone, "Beryllium in the ITER Blanket," presented at the Beryllium Technology Workshop, Clearwater F1, Idaho National Engineering Laboratory Report EGG-FSP-10017, December 1991. 
Figure 5.57. Effect of Be Porosity on First Wall Temperature Profile During NoFlow LOFA with $7 \mathrm{sec}$ Plasma Continuation $\left(\mathrm{h}_{\mathrm{gep}}=10,000 \mathrm{~W} / \mathrm{m}^{2} \cdot \mathrm{K}\right)$

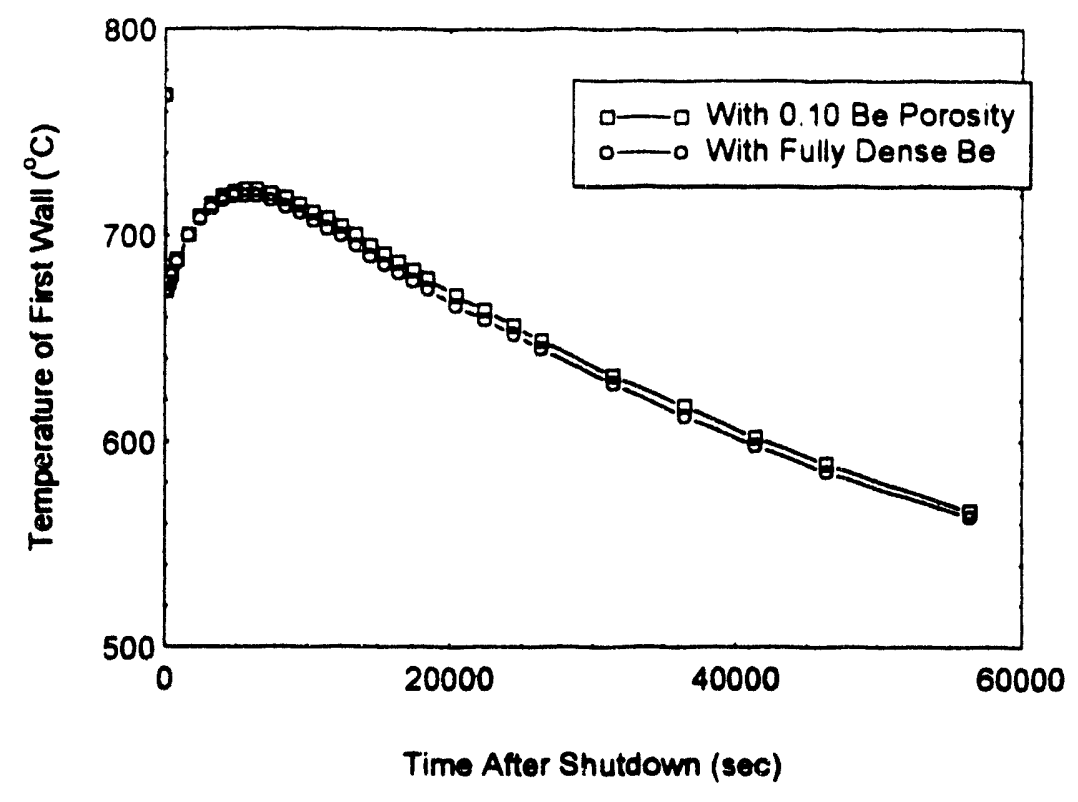

As can be seen, the reduction in conductivity caused by the porosity in the beryllium has very little effect on the temperature of the first wall during the LOFA. The resulting rupture fraction and $\mathbf{x}$ direction creep strain values are also barely effected, increasing from 0.40 to 0.44 and from 0.23 to 0.27 , respectively. There is clearly substantial margin to first wall failure, even assuming porous beryllium.

The small effect the reduction in conductivity in the beryllium has on the first wall temperatures in Figure 5.57 is perhaps somewhat surprising, but can be explained by noting that the gap conductance, which is also an important player in the effective conductivity of the breeder regions, remains unchanged as the conductivity of the beryllium is reduced. Additionally, the reduction in conductivity is not severe, so a drastic effect on the first wall temperatures should not be expected. 


\section{Conclusions}

This section analyzed the possible mechanisms which could reduced the conductivity of the beryllium slabs. As has been seen, cracking due to thermal stresses is not expected, nor is cracking due to swelling-induced stresses. This conclusion rests on some speculative assumptions regarding the fracture toughness of highly irradiated beryllium, which must be confirmed by fracture toughness testing on irradiated beryllium samples. The porosity in the beryllium caused by the end-of-life helium concentrations is expected to decrease the conductivity of the beryllium to 87 percent of its original value, but NoFlow LOFA analysis shows that the effect of this conductivity reduction on the first wall temperatures is very small. 


\section{Chapter 6 Design of Shield Water System}

The thermal analysis of the blanket designs in the previous chapters of this work assumed the shield was cooled with naturally-circulating water. More specifically, one of the boundary conditions for the No-Flow LOFA analyses was that the temperature at the front of the shield remains at 100 degrees $C$ for the duration of the transient. In Chapter 6 , the importance of this boundary condition is explored, and a conceptual design is developed for a shield water system (SWS) capable of keeping the front of the shield at or below 100 degrees for the entire accident via natural-circulation. The SWS to be developed in this chapter uses Blanket Design 2 as a reference blanket design.

To see how lack of a natural-circulation cooled shield affects the temperature response of Blanket Design 2, the No-Flow LOFA analysis (with no plasma continuation) is performed again, this time assuming that the water in the shield remains stagnant during the accident, rather than assuming that the front of the shield remains at 100 degrees $C$.

Figure 6.1. Blanket Design 2 Response to No-Flow LOFA with No Natural Circulation in Shield (Outboard Blanket)

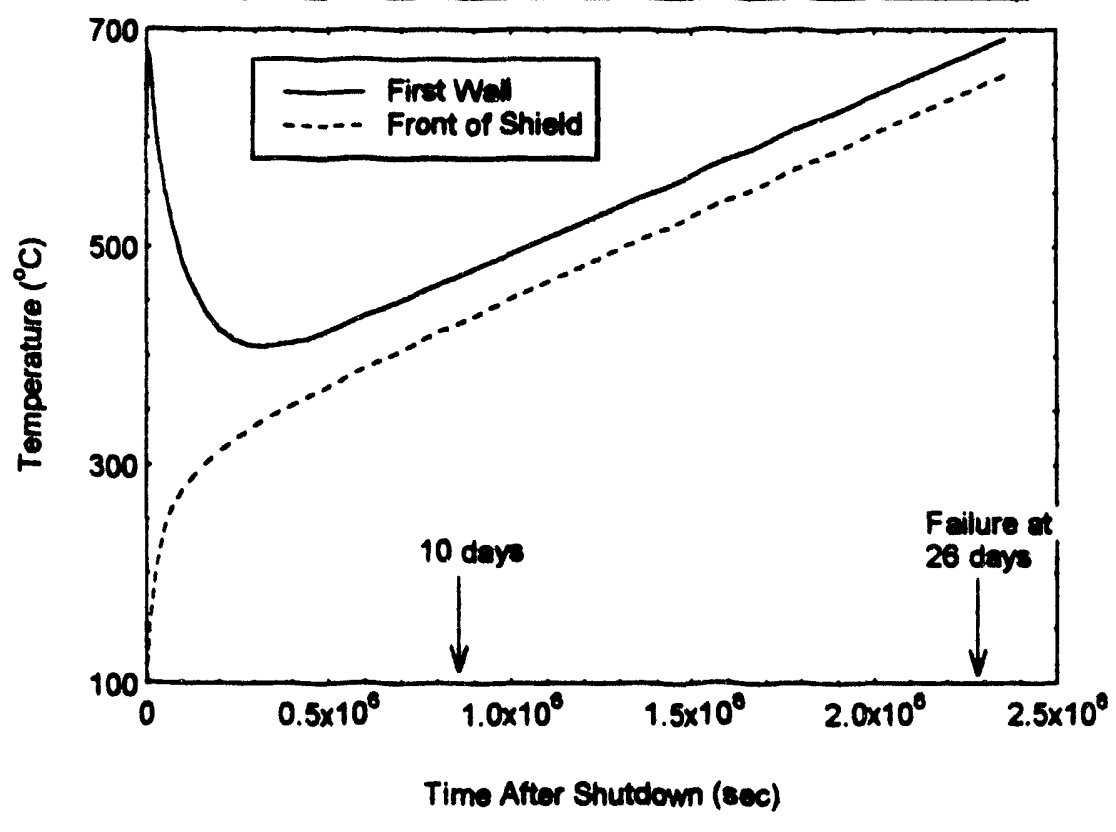

For this analysis, it is assumed that the shield is thermally isolated from the surroundings. Although this is obviously not strictly true, the thermal coupling between the shield and the surrounding 
structure is probably not good, hence such a conservative assumption may be quite reasonable until the shield heats up to high temperatures. Figure 6.1 shows the temperature response of the outboard blanket first wall and shield following a No-Flow LOFA, and Figure 6.2 shows the response of the inboard blanket configuration. The

Figure 6.2. Blanket Design 2 Response to No-Flow LOFA with No Natural Circulation in Shield (Inboard Blanket)

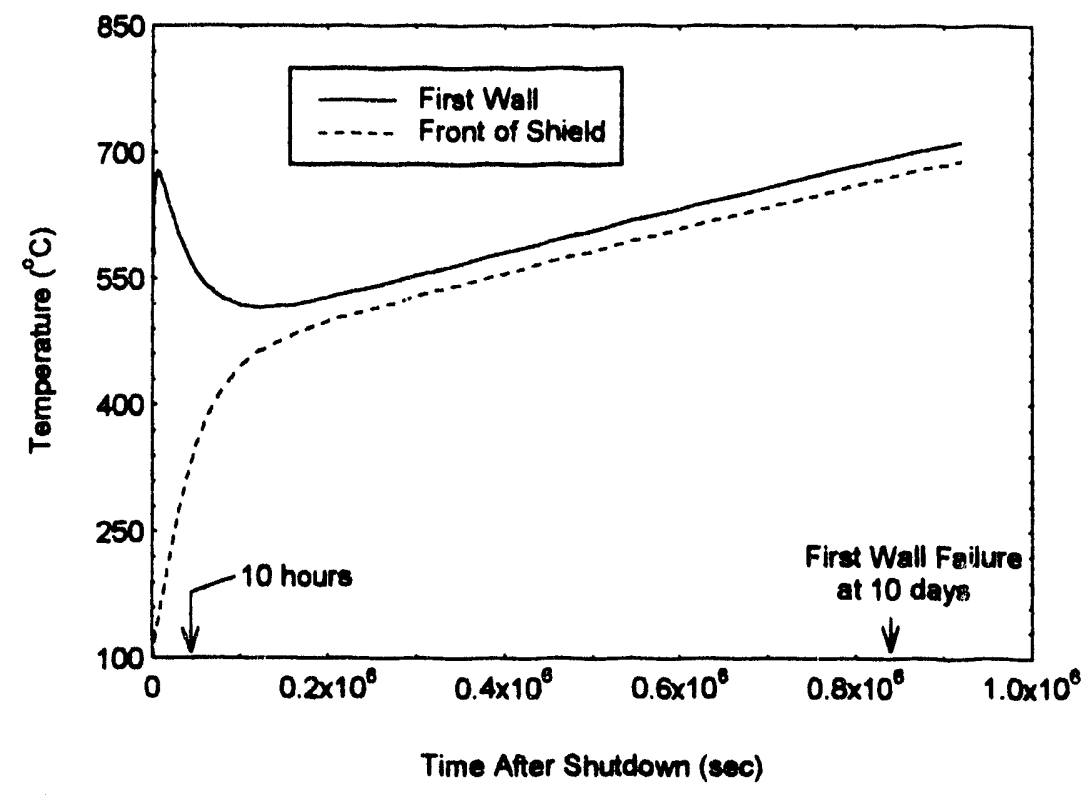

afterheat deposited in the shield region (used in generating these figures) is discussed in Section 6.1.2.

Note that in these figures, the time scale is much longer than for the

No-Flow LOFA curves displayed in Section 5.3. The characteristic heating up portion of the first wall curve at the beginning of the transient is hence not visible, although it is nearly identical to the heating up portion of the first wall curves of Section 5.3. Note, however, how the cooling trend of the first wall reverses, since the front of the shield is heating up, retarding heat flow from the blanket into the shield. This reversal happens much sooner for the inboard blanket and shield configuration than for the outboard configuration, since the inboard shield is much thinner than the outboard shield, reducing its total heat capacity. As the shield heats up, it is assumed that the water remains in the shield in a pressurized, liquid state. Hence effects such as vaporization and dryout of the shield water are neglected. 
As indicated on the figures, the outboard first wall fails due to creep rupture 26 days after the onset of the accident, whereas the inboard first wall fails 10 days after onset. Hence, although cooling of the shield is not necessary for the short.term safety of Blanket Design 2, it is necessary to ensure the long-term safety of the design.

The importance of a naturally-circulating SWS depends on the particular blanket design in question. In Appendix 11, a blanket design using vanadium as structural material and liquid lithium as coolant and breeder is analyzed. It is demonstrated therein that for such a blanket, having a shield water system capable of natural circulation can markedly change the short term accident performance of the blanket. Hence, having a naturalcirculation-cooled SWS is a potential benefit to any blanket design. The specific blanket design determines whether this benefit is seen in the short-term or long-term post accident performance of the blanket. 


\subsection{Shield Water System Heat Loads}

As a first step towards design of the SWS, determination of the heat loads on the system during the relevant modes of operation is necessary. The two modes of operation of interest here are normal operation, and the No-Flow LOFA situation. The head loads on the SWS originate in the shield itself (dominant during normal operation) as well as from thermal radiation from the blanket to shield (dominant during a LOFA). The following paragraphs characterize these two heat loads for both normal and LOFA operational modes.

\subsubsection{Normal Operation}

The dominant heat load on the SWS during normal operation is volumetric heating of the water and steel in the shield (recall that the shield is composed of 10 percent water arid

Figure 6.3. Operational Heating in the Outboard Shield

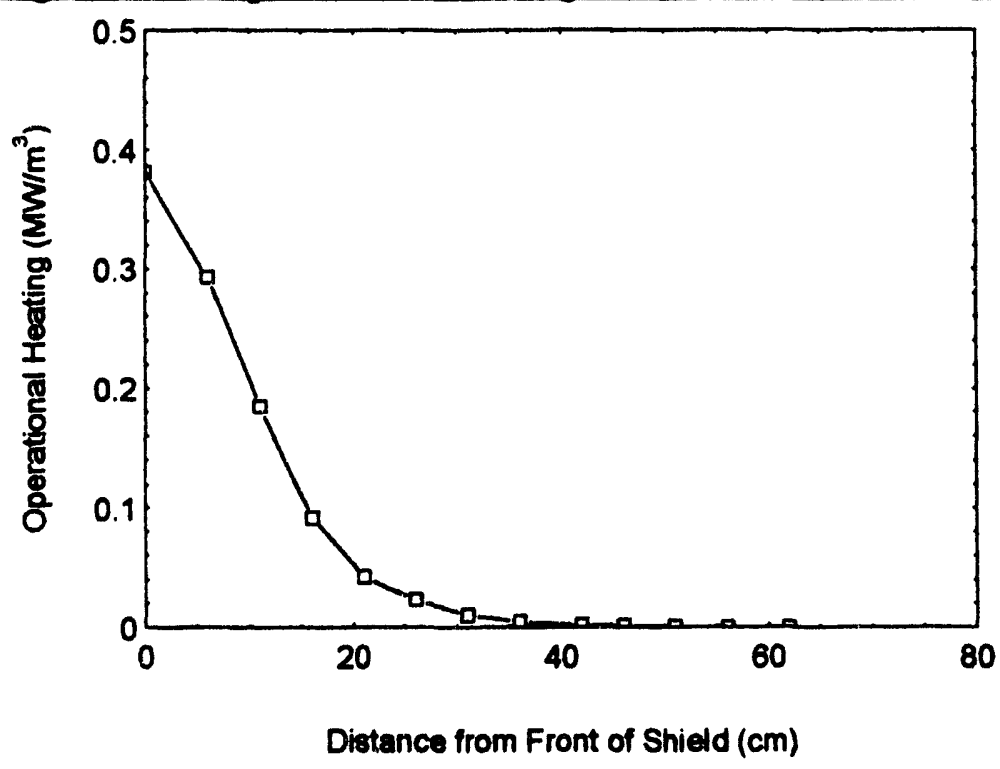

90 percent MT-9 steel)

The TWODANT neutronics code provides the heating rate from neutrons and gammas as a function of distance from the front of the shield. The results for the outboard shield are shown in Figure 6.3. The front of the shield generates about 0.4

$\mathrm{MW} / \mathrm{m}^{3}$, and this value drops rapidly with distance toward the back of the shield. For distances greater than about $40 \mathrm{~cm}$ behind the front, the operational heating becomes quite low. The results for the inboard shield are similar, except that the average neutron 
wall load for the inboard blanket and shield portion is only $2.7 \mathrm{MW} / \mathrm{m}^{2}$, rather than 4.0 $\mathrm{MW} / \mathrm{m}^{2}$ for the outboard portion. Hence, the operational heating is correspondingly less. Noting that the outboard shield is $1 \mathrm{~m}$ thick, and the inboard shield is $0.42 \mathrm{~m}$ thick, and assuming a tokamak major radius of $6 \mathrm{~m}$ and minor radius of $1.5 \mathrm{~m}$, the volumetric heating rate of the shield is integrated to obtain the total heat load on the SWS from the shield and obtain a value of about $25 \mathrm{MW}$.

The heat load from the blanket to the shield comes from thermal radiation across the vacuum gap between the blanket and shield. Since this gap is designed to prevent significant amounts of heat from flowing from the blanket into the shield (thereby being wasted), the heat load from the blanket is not significant during normal operation. Recall from Section 5.2.3 that the emissivity of the gap surfaces is assumed to be 0.7 . The back of the blanket region (the helium plenum) maintains an average temperature of $\mathbf{3 5 0}$ degrees $\mathbf{C}$ during normal operation. Conservatively assuming the shield operates at $\mathbf{5 0}$ degrees $C$ (the normal operating temperature of the shield is to be below 100 degrees $C$ ), the heat leaking from the blanket to the shield is only about $2 \mathrm{MW}$. Hence, the total heat load to the SWS during normal operation is about $27 \mathrm{MW}$.

\subsubsection{LOFA Operation}

During a LOFA, the heat load from the shield diminishes to the level determined by afterheat in the shield structure. Using the REAC3 afterheat code in conjunction with the TWODANT neutronics code, the afterheat level in the shield is plotted for three different distances from the front of the shield in Figure 6.4. As can be seen, the afterheat values are much lower than the operational heating values. The total integrated amount of afterheat in the shield immediately after shutdown is less than $1 \mathrm{MW}$. 
Figure 6.4. Afterheat in Outboard Shield

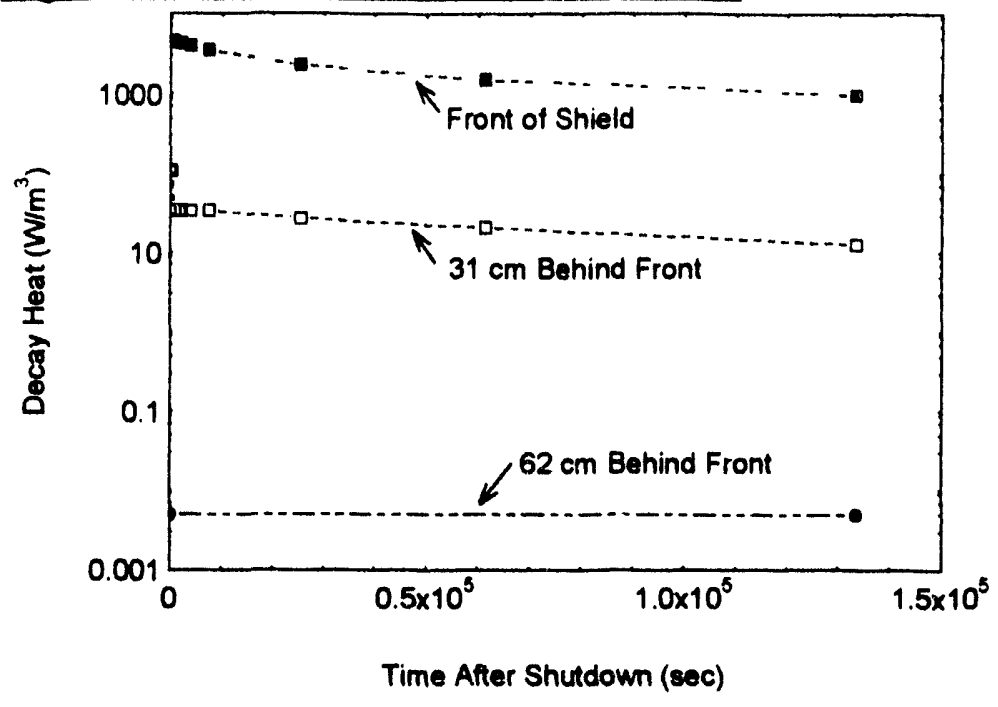

The heat flowing from the blanket to the shield, however, increases during the LOFA, since the back of the blanket heats up during the accident, resulting in more heat flow across the vacuum gap to the shield. The peak temperature of the back of Blanket Design 2

during the No-Flow LOFA is 420 degrees $\mathrm{C}$ assuming no plasma continuation (refer back to Figure 5.38), and 437 degrees $C$ for the 7 second plasma continuation case. Using 437 degrees $C$ as the temperature of the back of the blanket, and again assuming conservatively that the average temperature of the front of the shield is 50 degrees $\mathrm{C}$, the amount of heat flowing from the blanket to the shield is about $4 \mathrm{MW}$. The maximum heat load to the SWS from afterheat in the shield comes at shutdown, and the maximum heat load from the blanket comes many minutes after shutdown. However, for conservatism, the SWS is designed to accommodate both maximum heat loads simultaneously. Thus, the design heat load for the SWS during the LOFA is about 5 MW. Table 6.1 summarizes the SWS design heat loads discussed in the above paragraphs.

Table 6.1. Shield Water System Design Heat Loads

\begin{tabular}{|c|c|c|c|}
\hline $\begin{array}{c}\text { Operational } \\
\text { Mode }\end{array}$ & $\frac{\text { Heat Load from }}{\text { Shield (MW) }}$ & $\frac{\text { Heat Load from }}{\text { Blanket (MW) }}$ & $\frac{\text { Total Heat Load }}{\text { (MW) }}$ \\
\hline Normal Operation & 25 & 2 & 27 \\
\hline LOFA Operation & 1 & 4 & 5 \\
\hline
\end{tabular}




\subsection{Configuration of Single-Loop Shield Water System}

The SWS must be capable of passively removing, without the action of pumps or fans, both the heat deposited in the blanket and radiated to the shield and the heat generated in the shield itself during the LOFA. It must remove this heat while maintaining the temperature at the front of the shield below 100 degrees C. In addition, the SWS must be able to remove the normal operational heat load for the shield, using installed pumps and/or fans, if necessary.

Before design of the SWS can begin, the ultimate heat sink (UHS) for the system must be chosen. The UHS is the reservoir which accepts the heat rejected by SWS operation. Examples of possible UHS choices include a body of water such as a river or spray pond, or the atmosphere. For this study, the atmosphere is chosen as the UHS, for the following reasons: (1) Use of a cooling tower conveniently allows for natural circulation flow for the SWS coolant, because of the elevation of the tower's heat exchanger; (2) Use of a "dry" cooling tower prevents the release of any activation products which may exist in the SWS coolant (a spray pond could not use SWS water as a coolant without the possible release of these activation products into the atmosphere); and (3) Use of a naturally-occurring body of water such as a river presumes the existence of such a body of water at the reactor site.

Another choice which must be made in the design of the SWS is whether the cooling tower used for removal of the heat during a LOFA via natural circulation is also used to remove the normal operational heat load, or whether a separate heat exchanger is used instead. As can be seen in Table 6.1 above, the LOFA heat load is smaller than the normal operational heat load by over a factor of five. This large difference between the 
accident and normal operational heat loads makes it desirable to use a separate heat exchanger for the normal operational heat load, to minimize the size and expense of the cooling tower and tower heat exchanger. This conclusion is further justified later in this chapter.

The SWS coolant becomes radioactive during reactor operation, due to the neutron flux in the shield. Depending on the radioactive inventory in the coolant, a spill of coolant into the environment may be unacceptable. In addition, high levels of gamma radiation from decay of N-16 (created by an (n,p) reaction with 0-16 in the water) exist in the SWS coolant during operation. Both of these concerns could be alleviated using an intermediate cooling loop between the SWS coolant and the cooling tower heat exchanger. However, such an intermediate loop would complicate the system and decrease the system's reliability and thermal efficiency. Hence, it is worthwhile investigating an SWS which does not use ar: intermediate cooling loop. The issues of radioactivity and gamma radiation in the SWS coolant are addressed in later sections. Following investigation of a "single-loop" SWS with no intermediate heat exchanger, a "two-loop" SWS is examined.

\section{Figure 6.5. Single-Loop Shield Water System Diagram}

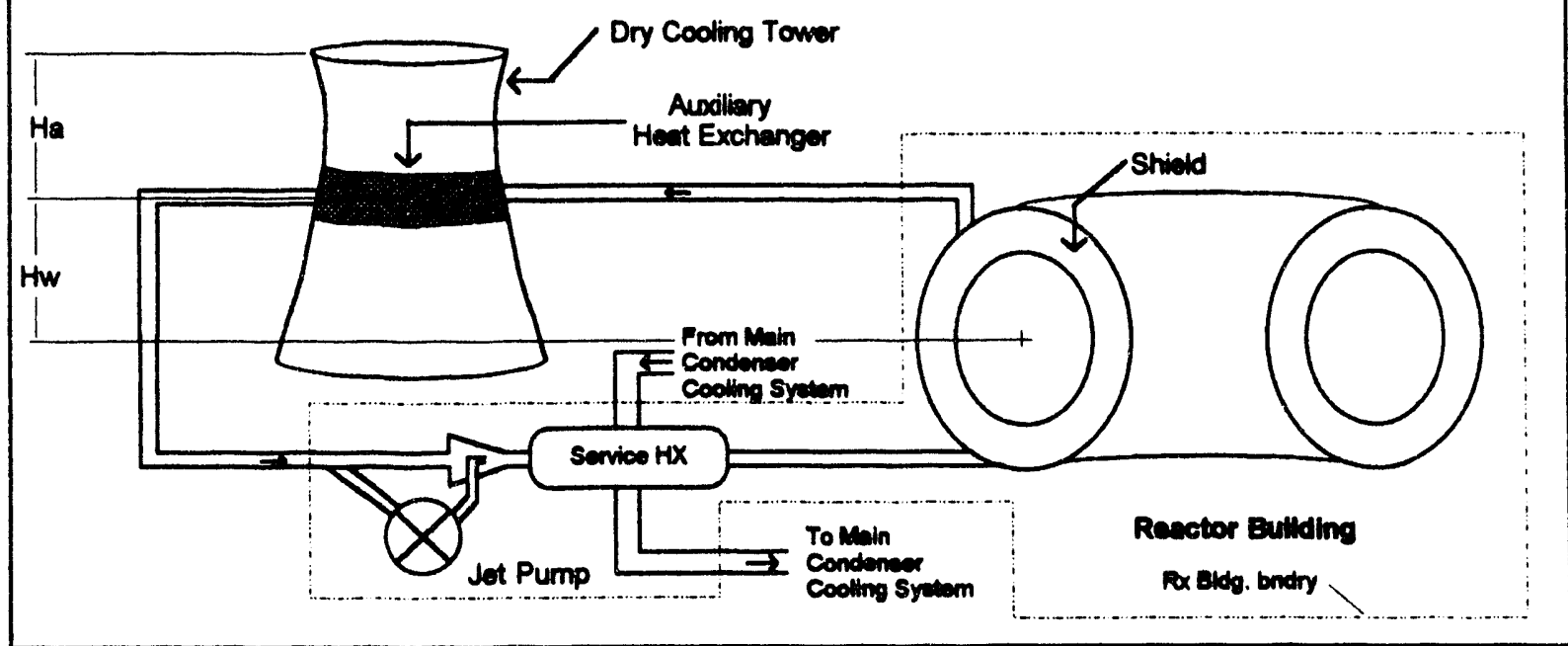


Figure 6.5 shows the arrangement of the "single-loop" SWS. The reactor shield is shown in this figure with a hot coolant leg connected to the top of the shield, and a cold leg connected to the bottom of the shield. The coolant flows through the hot leg to the Auxiliary Heat Exchanger in the cooling tower, then through the pump/pump-bypass lines, then through the Service Heat Exchanger, then back to the shield. As indicated in the figure, the Service Heat Exchanger is cooled by the same water system that cools the steam plant's Main Condenser. It is this heat exchanger which is used to remove the normal operation heat load, with the SWS jet pump providing the required driving head. The Auxiliary Heat Exchanger is designed to remove the LOFA heat load via natural circulation of the SWS coolant and the air in the cooling tower. Note that the SWS pump is a jet pump; if the pump fails, natural circulation flow can still occur through the system past the jet pump nozzle. Note also the reactor building boundary in the figure, indicating that the Auxiliary Heat Exchanger is located outside of the reactor building (of course), and hence outside of any barriers to radioactivity release. This issue is discussed at length later in this chapter.

\subsection{The $\varepsilon$-NTU Method for Heat Exchanger Design}

In order to size the Auxiliary Heat Exchanger and the associated cooling tower, as well as the Service Heat Exchanger, a method for heat exchanger sizing and design is needed. The method to be used herein is known as the $\varepsilon-\mathrm{NTU}$ method. The $\varepsilon-\mathrm{NTU}$ method differs in method (not end result) from the more familiar log-mean-temperaturedifference (LMTD) method, but is a more effective tool for the design of air-cooled heat exchangers such as the Auxiliary Heat Exchanger. ${ }^{1}$ The following description of the $\varepsilon-$ NTU method is adapted from a widely-referenced textbook on air-cooled heat exchanger design by Kays and London. ${ }^{2}$

\footnotetext{
'W. M. Kays and A. L. London, Compact Heat Exchangers, Mc-Graw Hill, 1984, p. 24.

${ }^{2}$ Ibid, Chapter 2.
} 
For a two-fluid heat exchanger, the input parameters which allow calculation of the thermal performance can be summarized as follows:

$$
\begin{aligned}
& \mathrm{U}=\text { overall conductance for heat transfer, in } \mathrm{W} / \mathrm{m}^{2} \cdot \mathrm{K} \text { of } \mathrm{A} \\
& A=\text { surface area on which } U \text { is based (usually the surface area on one or the } \\
& \text { other side of the heat exchanger tubes) in } \mathrm{m}^{2} \\
& \mathrm{~T}_{\text {pri, }}=\text { hot (inlet) temperature of primary fluid to be cooled (degrees } \mathrm{C} \text { ) } \\
& \mathrm{T}_{\text {pri.c }}=\text { cold (outlet) temperature of primary fluid (degrees } \mathrm{C} \text { ) } \\
& \mathrm{T}_{\text {sec, }, \mathrm{b}}=\text { hot (outlet) temperature of secondary fluid used to cool primary fluid } \\
& \text { (degrees C) } \\
& \mathrm{T}_{\mathrm{soc,c}}=\text { cold (inlet) temperature of secondary fluid (degrees } \mathrm{C} \text { ) } \\
& \mathrm{W}_{\mathrm{pri}},\left(\mathrm{W}_{\mathrm{soc}}\right)=\text { mass flow rate of primary (secondary) fluid }(\mathrm{kg} / \mathrm{sec} \text { ) } \\
& c_{p, p i},\left(c_{p, c o c}\right)=\text { specific heat at constant pressure for primary (secondary) fluid } \\
& (\mathrm{J} / \mathrm{kg} \cdot \mathrm{K}) \\
& \mathrm{C}_{\mathrm{pri}}=\mathrm{W}_{\mathrm{pri}} \cdot \mathrm{c}_{\mathrm{pri}}=\text { primary fluid "capacity rate" }(\mathrm{W} / \mathrm{K}) \\
& \mathrm{C}_{\text {soc }}=\mathrm{W}_{\mathrm{eco}} \cdot \mathrm{c}_{\mathrm{ecoc}}=\text { secondary fluid "capacity rate" }(\mathrm{W} / \mathrm{K})
\end{aligned}
$$

Flow arrangement $=$ counterflow, parallel flow, crossflow, both fluids mixed, one fluid mixed, etc.

Note the introduction of the quantity called "capacity rate", which, when multiplied by the appropriate inlet-to-outlet temperature difference, yields the rate of heat transfer from the primary coolant or to the secondary coolant. The flow arrangement depends on the type of heat exchanger used. For the Auxiliary Heat Exchanger in the present work, the flow arrangement is assumed to be crossflow, with the primary (SWS water) side unmixed, and the secondary (air) side mixed. The "unmixed" designation for the water side refers to the fact that the water runs inside tubes, and does not mix when traveling through the heat exchanger unit. The air runs past the outside of these tubes, and hence mixes as it passes through the unit. 
To begin to relate all of these parameters in order to facilitate sizing and design of a heat exchanger, the conservation of energy is used to derive the relation:

$$
q=C_{p r i}\left(T_{p r i, b}-T_{p r i, c}\right)=C_{s e c}\left(T_{s e c, b}-T_{\text {sec }, c}\right) \text {, }
$$

where $q$ is the heat exchanger's heat transfer rate. For an ideal counterflow heat exchanger of infinite heat transfer area, the maximum possible heat transfer rate is:

$$
\mathrm{q}_{\max }=\mathrm{C}_{\min }\left(\mathrm{T}_{\text {pri,b }}-\mathrm{T}_{\mathrm{soc}, \mathrm{c}}\right) \text {, }
$$

where $C_{\text {min }}$ is the smaller of $C_{p r i}$ and $C_{\text {sec }}$. The effectiveness of the heat exchanger, $\varepsilon$, is defined by the ratio of the actual heat transfer rate divided by the maximum possible heat transfer rate:

$$
\varepsilon \equiv \frac{q}{q_{\max }}
$$

The other definition which is integral to the $\varepsilon$-NTU method is the definition of the number of heat transfer units (NTU's). This quantity is defined as the heat transfer rate per unit temperature divided by the minimum heat capacity rate, or:

$$
\mathrm{NTU} \equiv \frac{\mathrm{UA}}{\mathrm{C}_{\min }} .
$$

Since the heat transfer area A scales with heat exchanger size, the number of heat transfer units is a measure of the physical size of the heat exchanger, for given $\mathrm{C}_{\min }$ and $U$. $C_{\min }$ and $U$ depend on heat exchanger geometry, and coolant flow rates.

It can be shown that, generally, the heat exchanger effectiveness $\varepsilon$ can be expressed as a function of the flow arrangement, $N T U$, and $C_{\min } / C_{\max }$. The quantity $C_{\min } / C_{\max }$ is called the capacity rate ratio $(C r)$, where $C_{\max }$ is the larger of $C_{p i}$ and $C_{m o}$. Specific equations for $\varepsilon$ can be derived given the flow arrangement of the heat exchanger. For a 
single pass, cross flow heat exchanger with one fluid mixed and one fluid unmixed, ${ }^{3}$ two different relationships exist for $\varepsilon{ }^{4}$ If the unmixed capacity rate is the maximum one $\left(C_{\max }=\right.$ unmixed, $C_{\min }=$ mixed $)$, then:

$$
\varepsilon=1-\exp \left[-\frac{1}{C r}(1-\exp (-N T U \cdot C r)]\right.
$$

For $\mathrm{C}_{\min }=$ unmixed, $\mathrm{C}_{\max }=$ mixed, we have:

$$
\varepsilon=\frac{1}{C r}\{1-\exp [-C r(1-\exp (-N T U)\rangle]\} .
$$

The effectiveness correlations of Equations 6.5 and 6.6 are plotted in Figure 6.6.

\section{Figure 6.6. Effectiveness vs. NTU Relations}

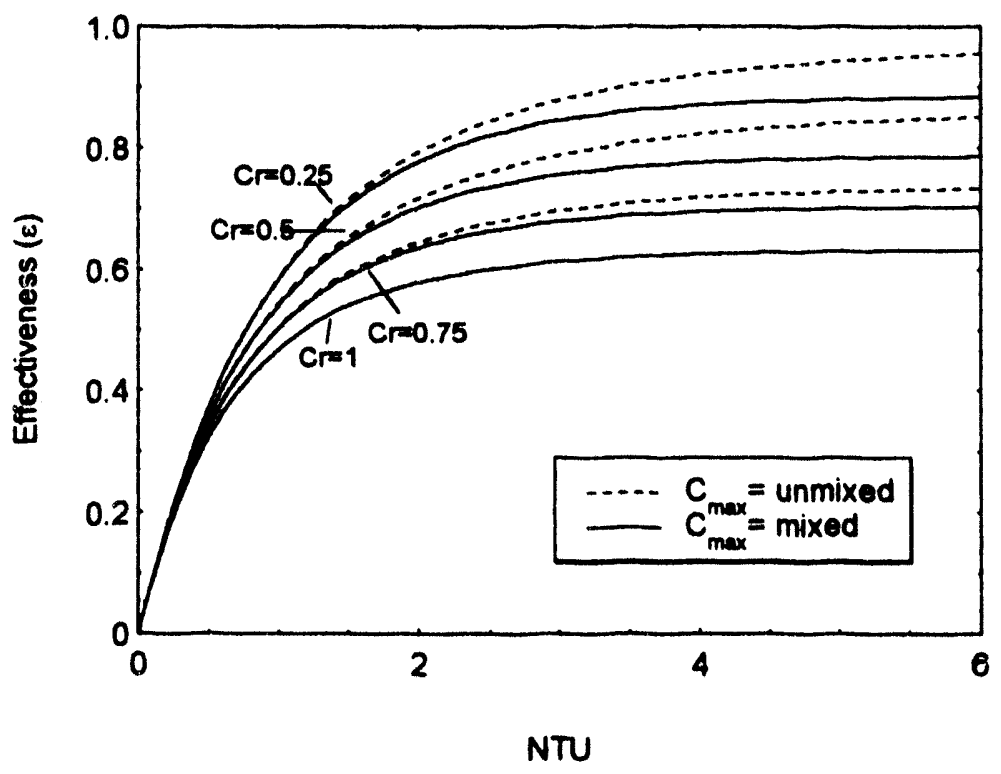

Figure 6.6 shows that the effectiveness increases with NTU, indicating that the larger the heat exchanger, the closer to ideal performance it has (as one would expect). Also shown is that the heat exchanger effectiveness is larger for a given NTU when $C_{\max }=$

\footnotetext{
${ }^{3}$ This is the type of heat exchanger which will be used for both the Auxiliary and Service Heat Exchangers.

${ }^{4}$ For a derivation of these relationships, see W. M. Kays and A. L. London, Compact Heat Exchangers, Mc-Graw Hill, 1984, Appendix C.
} 
unmixed. This indicates that a shell-and-tube heat exchanger with the secondary fluid on the shell side, should be designed so that the primary fluid capacity rate is larger than the secondary fluid capacity rate, or

$$
C_{p r i} \geq C_{s e c} \text {, or } W_{p r i} \cdot c_{p r i} \geq W_{s e c} \cdot c_{s e c}
$$

This relation limits the secondary mass flow rate once the primary mass flow rate is known. Furthermore, Equation 6.1 gives:

$$
T_{p r i, b}-T_{p r i, c} \leq T_{s e c, b}-T_{s e c, c}
$$

which limits the primary side temperature difference to be less than the secondary side temperature difference. ${ }^{3}$ Equations 6.7 and 6.8 are relations which significantly "bound" the heat exchanger design space to designs which have better efficiencies. These relations, in conjunction with Equation 6.5, describe the fundamental conclusions obtained using the $\varepsilon-\mathrm{NTU}$ design method.

\subsection{The Overall Conductance}

The overall conductance for heat transfer, $U$, is basically a series conductance for heat flow from the bulk of the primary fluid through the fluid film layer on the primary side of the heat exchanger tubes, then through the tubes themselves, and finally through the secondary fluid film layer. Hence, the equation for $U$ has three terms, each corresponding to a conductance through these three regions. The specific definition of $\mathrm{U}$ depends on which area $\mathrm{A}$ is chosen as a reference area. For the present work, the secondary side area (the air side area in the Auxiliary Heat Exchanger) is chosen as the reference area. The $U$ corresponding to the secondary side area (neglecting fouling) is hence:

\footnotetext{
${ }^{5}$ The mass flow rate and temperature difference relations were discussed in J.R. Tang, et.al., Conceptual Design of the Passive Light Water Cooled and Moderated Pressure Tube Reactor (PLPTR), MIT Nuclear Engineering Department Report MIT-ANP-TR-013, August 9, 1992.
} 


$$
\frac{1}{U_{\text {sec }}}=\frac{1}{\eta_{0, s e c} h_{s e c}}+\frac{t_{w}}{\left(A_{m} / A_{s e c}\right) k_{w}}+\frac{1}{\left(A_{p r i} / A_{s e c}\right) \eta_{n, p r i} h_{p r i}},
$$

where $t_{w}$ and $k_{w}$ are the tube wall thickness and conductivity, respectively.

$A_{p r i}$ and $A_{\text {sou }}$ are the primary and secondary heat transfer areas (including any fins,

$A_{m}$ is an effective mean heat transfer area for the tube wall (defined below),

$h_{p r i}$ and $h_{s e c}$ are the fluid film heat transfer coefficients, and

$\eta_{0, p r i}$ and $\eta_{0, \text { sec }}$ are effectiveness coefficients for the heat transfer areas, which account for inefficiencies if fins are used on either the primary or secondary side.

For cylindrical tubes, the effective mean heat transfer area for the tube wall is given by a relation for conduction through the walls of hollow cylinders, as follows: ${ }^{6}$

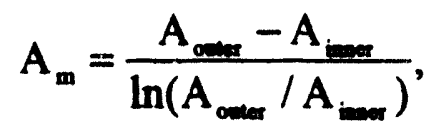

where $A_{\text {ower }}$ and $A_{\text {more }}$ correspond to the inside and outside areas of the tube wall (not including fins). The effectiveness coefficients $\eta_{0, p i}$ and $\eta_{0,000}$ are equal to unity if no fins are present. Since there are no fins inside the tubes for the heat exchangers of interest here, $\eta_{0, \text { mi }}=1$. If fins are present to increase heat transfer (as there are for the secondary (air) side of the Auxiliary Heat Exchanger), then we have:

$$
\eta_{0,000}=1-\frac{A_{\mathrm{f}, e 00}}{A_{200}}\left(1-\eta_{\mathrm{f}}\right),
$$

where $A_{f, \infty \infty}$ is the portion of the secondary side area associated with the fins, and $\eta_{\mathrm{f}}$ is the fin efficiency (for circumferential fins $=\tanh (\mathrm{ml}) / \mathrm{ml}$, where $l$ is the fin length, and $m=\left(4 h_{n o 0} / k_{f} D_{\text {oem }}\right)^{0 s}$, where $k_{f}$ and $D_{\text {omer }}$ are the conductivity of the fin and the outer diameter of the tube).

${ }^{6}$ T. Baumeister, et.al., eds., Mark's Standard Handbook for Mechanical Engineers, 8th Ed., McGrawHill, 1978, p. $4-60$. 


\subsubsection{Heat Transfer Coefficient - Primary Side}

For a shell-and-tube type heat exchanger, the fluid film heat transfer coefficients, $h_{p r i}$ and $h_{\text {sec }}$, are evaluated using different methods for the tube side and shell side fluids. For the flow inside the tubes (tube side), a simple, general relation (Dittus-Boelter) can be used for any tube size, because of the simplicity of the geometry. The Dittus-Boelter relation for a fluid being cooled is as follows:?

$$
\mathrm{Nu}_{\mathrm{pri}}=0.023 \operatorname{Re}_{\mathrm{pri}}^{0.8} \operatorname{Pr}_{\mathrm{pri}}^{0.3} \text {, }
$$

where $\mathrm{Nu}_{\mathrm{pri}}$ is the Nusselt number on the primary side (inside the tubes), $\operatorname{Re}_{\mathrm{pri}}$ is the Reynolds number, and $\operatorname{Pr}_{p i}$ is the Prandtl number. This relation is valid for $\operatorname{Re}_{p r i}>$ 10,000 , and $L_{\text {rubo }} / D_{\text {in }}>60$, conditions well satisfied for the present work. Recalling that the Nusselt number is defined as $h_{p r i} D_{i} / k_{p r i}$, where $D_{i n}$ is the inner tube diameter and $k_{p r i}$ is the conductivity of the primary water, $h_{p r i}$ can easily be solved for.

\subsubsection{Heat Transfer Coefficient - Secondary Side}

For flow outside the tubes, the geometry is much more complex, depending on parameters such as tube size and spacing, whether or not the tubes are finned, and overall heat exchanger tube arrangement. For such involved geometries, a single general relation does not suffice to determine the appropriate heat transfer coefficient, and more complex methods must be used, based heavily on empirical test data on heat exchangers with geometries and similar to the geometry being considered. The methods used are generally different depending on whether air or water is used as a secondary coolant. Hence, the methods used herein are different for the air-cooled Auxiliary Heat Exchanger and the water-cooled Service Heat Exchanger.

For air-cooled heat exchangers, it is necessary to use finned tubes to increase the heat transfer coefficient on the air side, since air has less favorable heat transfer properties

\footnotetext{
${ }^{7}$ N. E. Todreas and M.S. Kazimi, Nuclear Systems 1, Thermal Hydraulic Fundamentals, Hemisphere,
} 1990, p.443. 
than water. Because use of such fins drastically changes the heat transfer performance, and because there are so many possible ways to arrange such fins, it is generally necessary to test such a heat exchanger to determine its air-side heat transfer coefficient. Empirical relations are not useful because of the vast number of variables involved, such as fin size, shape, spacing, etc.

Fortunately, such testing has been performed on a large number of air-cooled heat exchanger designs, and the results of the tests have been compiled into a useful form by Kays and London. ${ }^{8}$ Each tested design has different fin and tube size and spacing. Choosing the best possible fin and tube configuration for the present work is difficult and time-consuming, and is not particularly warranted for a conceptual design. Hence, the fin and tube geometry from in a previous conceptual study by Tang is used. ${ }^{9}$ A cross section of the tube bundle region of the Auxiliary Heat Exchanger is shown in Figure 6.7 , indicating the fin and tube geometry. Table 6.2 shows the relevant parameter values for the Auxiliary Heat Exchanger.

\footnotetext{
${ }^{8}$ W. M. Kays and A. L. London, Compact Heat Exchangers, Mc-Graw Hill, 1984, Chapter 10.

9J.R. Tang, et.al., Conceptual Design of the Passive Light Water Cooled and Moderated Pressure Tube Reactor (PLPTR), MIT Department of Nuclear Engineering Report MIT-ANP-TR-013, August, 1992.
} 
Figure 6.7. Auxiliary Heat Exchanger Fin/Tube Geometry (not to scale)

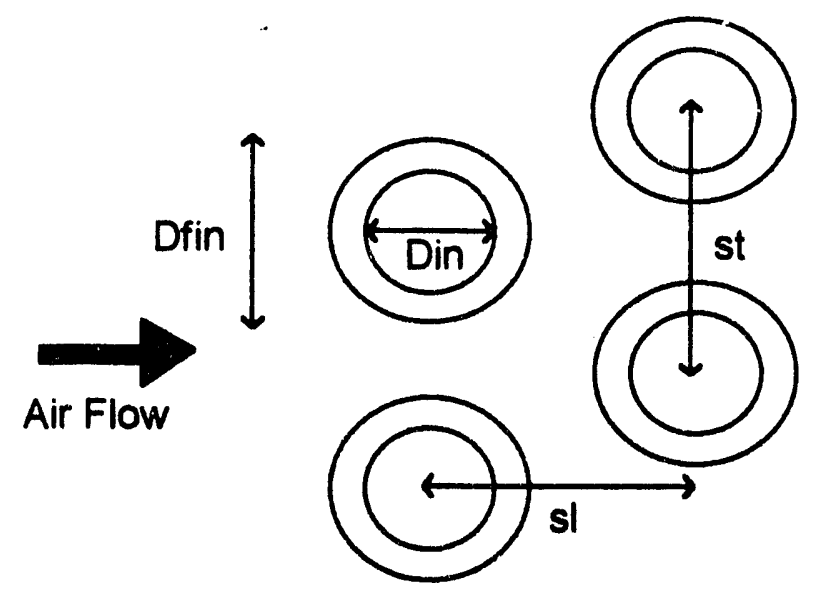

Table 6.2. Auxiliary Heat Exchanger Parameters ${ }^{10}$

\begin{tabular}{|c|c|}
\hline Longitudinal Tube Pitch (sl): & $52.4 \mathrm{~mm}$ \\
\hline Transverse Tube Pitch (st): & $78.2 \mathrm{~mm}$ \\
\hline Inner Tube Diameter (Din): & $23.4 \mathrm{~mm}$ \\
\hline Outer Tube Diameter (Dout): & $26.0 \mathrm{~mm}$ \\
\hline Fin Outer Diameter (Dfin): & $44.1 \mathrm{~mm}$ \\
\hline Fin Thickness (tf): & $0.30 \mathrm{~mm}$ \\
\hline Fins per Unit Tube Length (sf) & 346 per meter \\
\hline Fin Area/ Heat Transfer Area, $\mathbf{A}_{m \text { co }}$ (Farea): & 0.825 \\
\hline Air Flow Hydraulic Diameter (Dh): & $13.2 \mathrm{~mm}$ \\
\hline Free-Flow /Frontal Arsa $(\sigma)$ : & 0.642 \\
\hline Heat Transfer Area, $A_{e c c} / H X$ Volume $(\beta)$ : & 191 per $\mathrm{m}$ \\
\hline
\end{tabular}

${ }^{10}$ From Kays and London, Fig. 10-90, surface CF-8.8-1.0J/B. 
In addition to the basic geometry parameters, Table 6.2 also includes other important heat exchanger parameters, such as the ratio of the free-flow to frontal areas. The significance of these parameters is discussed shortly.

The test data for the heat transfer coefficient for the air-side of the Auxiliary Heat

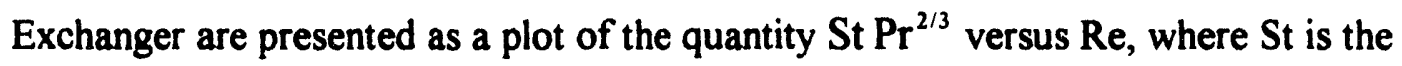
Stanton number. This data are shown in Figure 6.8, where Re in this figure is defined as follows:

$$
\operatorname{Re}=\frac{W_{\mathrm{air}} \cdot \mathrm{Dh}}{\mathrm{Aff} \cdot \mu_{\mathrm{air}}} .
$$

where $\mathrm{W}_{\text {air }}$ is the mass flow rate of air through the air-side, $A f f$ is the air-side free-flow area, and $\mathrm{Dh}$ is defined in the table above.

Figure 6.8. Heat Transfer Parameter for Air-Side of Auxiliary Heat Exchanger ${ }^{11}$

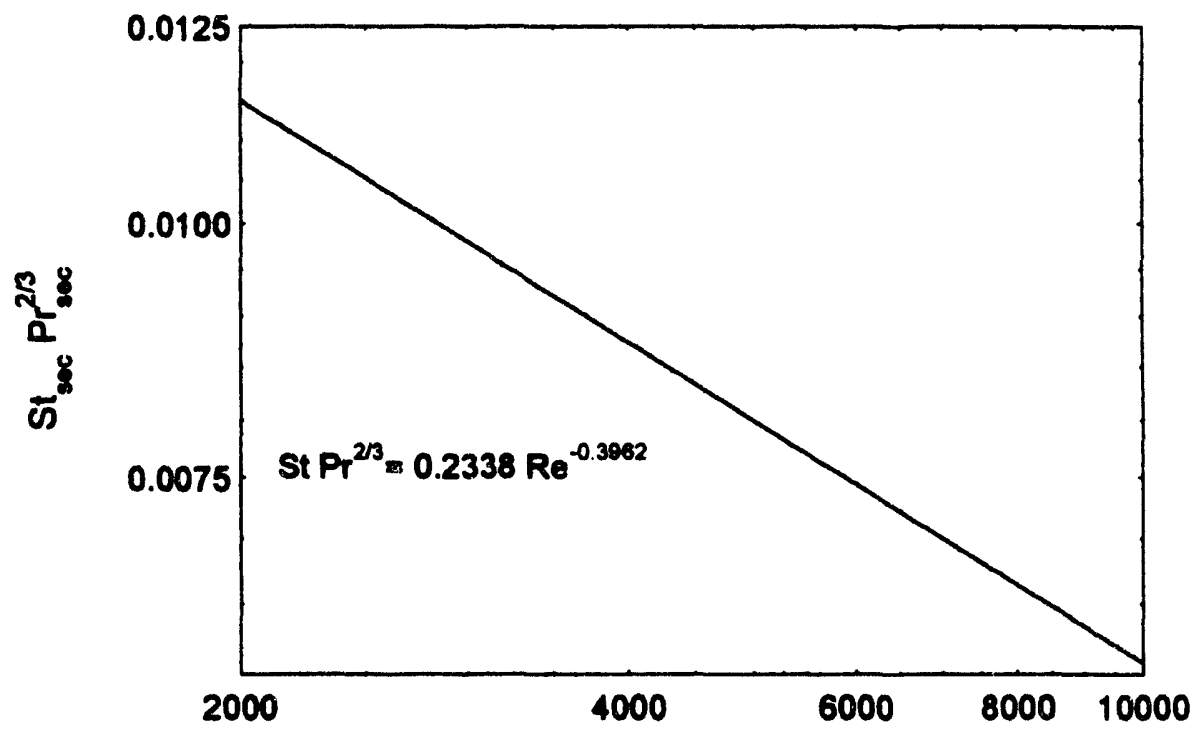

Re

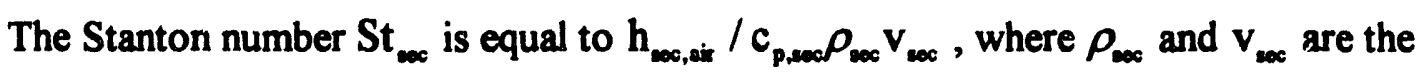
secondary (air) side density and velocity, respectively. Hence, Figure 6.8 directly yields

${ }^{11}$ W. M. Kays and A. L. London, Compact Heat Exchangers, Mc-Graw Hill, 1984, Fig. 10-90, surface CF-8.8-1.0J/B. 
the heat transfer coefficient for the air-side of the Auxiliary Heat Exchanger. Also

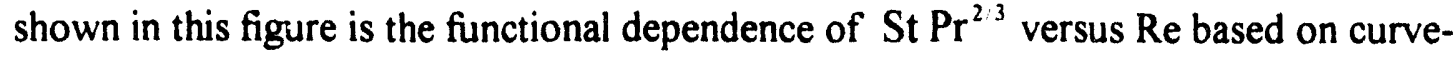
fitting the test data curve shown.

The Service Heat Exchanger uses water as a coolant on the secondary side. Generally, heat exchangers using water on both sides do not use finned tubes, since the heat transfer properties are not significantly improved by adopting them. Hence, the geometry is simplified as compared to the finned tube case, and there are fewer variables to consider for the design. The standard type of heat exchanger used when water is the coolant on both the primary and secondary sides is the "shell-and tube" type in a crossflow configuration. A relatively simple type of shell-and-tube heat exchanger is adopted as the configuration for the Service Heat Exchanger, one with a single internal baffle on the secondary (shell) side, and a "once-through" flow pattern for the primary (tube) fluid. Figure 6.9 shows this configuration. A baffle on the secondary side helps ensure uniform flow of the secondary fluid by reducing the cross-sectional area seen by the fluid and hence eliminating areas where the fluid flow could stagnate. For this type of heat exchanger, a somewhat general method of analysis has been developed which yields performance results without direct testing of the specific heat exchanger configuration. This method is called the "Delaware method." 12

\footnotetext{
${ }^{12}$ The description of the Delaware method is adopted from K.J. Bell, "Delaware Method for Shell Side Design," in Heat Exchangers, Thermal-Hydraulic Fundamentals and Design, S. Kakac, et.al., Eds., Hemisphere, 1981, p. 581.
} 


\section{Figure 6.9. Service Heat Exchanger Configuration}

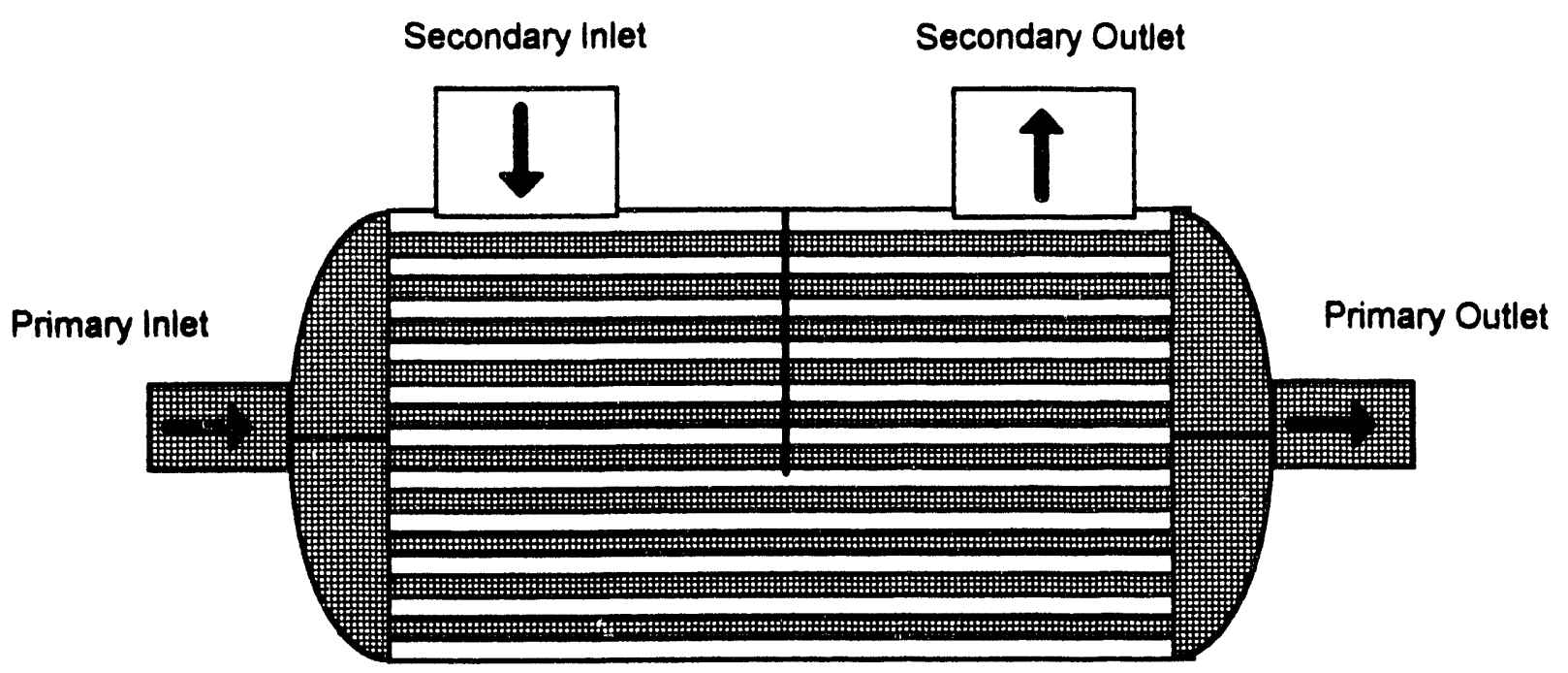

Determination of the heat transfer coefficient on the water-cooled secondary side of the Service Heat Exchanger using the Delaware method requires specification of the tube geometry. As with the Auxiliary Heat Exchanger, it would be possible to expend a considerable amount of effort trying to optimize the tube geometry in order to minimize the size of the Service Heat Exchanger. However, as was done for the Auxiliary Heat Exchanger, a representative tube geometry is adopted for the purposes of the present conceptual design. The tube geometry adopted for the Service Heat Exchanger is shown in Figure 6.10, and relevant parameter values are shown in Table 6.3. As previously mentioned, the Service Heat Exchanger tubes do not have fins. 


\section{Figure 6.10. Service Heat Exchanger Tube Geometry}

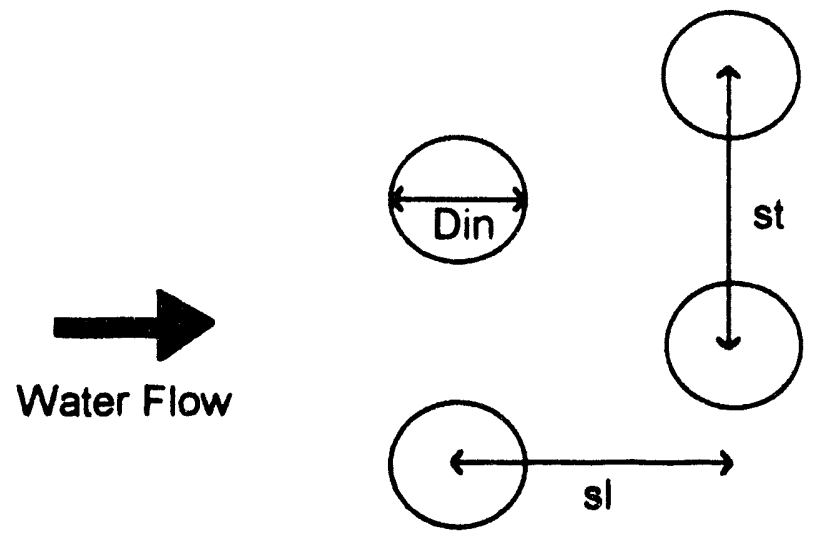

\section{Table 6.3. Service Heat Exchanger Parameters}

\begin{tabular}{|c|c|}
\hline Longitudinal Tube Pitch (sl): & $27.5 \mathrm{~mm}$ \\
\hline Transverse Tube Pitch (st): & $31.7 \mathrm{~mm}$ \\
\hline Inner Tube Diameter (Din): & $22.9 \mathrm{~mm}$ \\
\hline Outer Tube Diameter (Dout): & $25.40 \mathrm{~mm}$ \\
\hline
\end{tabular}

The heat transfer coefficient as calculated by the Delaware method is of the form:

$$
h_{\text {soc,wem }}=h_{\text {idal }} \cdot \text { (correction factors) }
$$

where $h_{\text {ideal }}$ is the ideal heat transfer coefficient for the specified tube bank geometry, and the correction factors account for effects such as tube bundle bypass flow. The Delaware method describes a number of rather involved methods for estimating the value of these correction factors given the detailed heat exchanger geometry. However, for the present work it is assumed that the combined effect of the correction factors is to reduce the ideal heat transfer coefficient by 40 percent, which is typical for a well designed shell-and-tube heat exchanger. ${ }^{13}$ Hence we have:

\footnotetext{
${ }^{13}$ K.J. Bell, "Delaware Method for Shell Side Design," in Heat Exchangers, Thermal-Hydraulic Fundamentals and Design, S. Kakac, et.al., Eds., Hemisphere, 1981, p. 585.
} 


$$
h_{\text {sei, water }}=0.6 h_{\text {ideal }} \text {. }
$$

To calculate $h_{\text {idest }}$, use is made of the " $j$-factor" method. In this formalism, $h_{\text {ideal }}$ is given by:

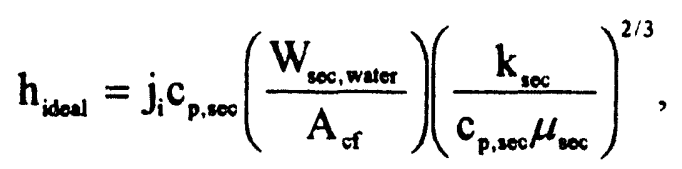

where $j_{i}$ is the $j$-factor (determined empirically for the particular tube bank geometry), $A_{o f}$ is the crossflow area near the heat exchanger centerline, and $k_{\text {esc }}$ and $\mu_{\text {sec }}$ are the secondary side water conductivity and viscosity, respectively. ${ }^{14}$ The other constants were defined at the beginning of this section. The value of $j_{i}$ for the tube bundle geometry of the Service Heat Exchanger is given in Figure 6.11, which shows the empirically-determined data curve together with a functional relationship determined by curve-fitting the data. In Figure 6.11, Re is defined as follows:

$$
\operatorname{Re}=\frac{W_{\text {coc, weer }} \cdot \text { Dout }}{\mu_{\text {esc }} \cdot A_{\text {cf }}} \text {. }
$$

\footnotetext{
${ }^{14}$ K.J. Bell, p. 606.
} 
Figure 6.11. Heat Transfer Parameter for Secondary Side of Service Heat Exchanger $^{15}$

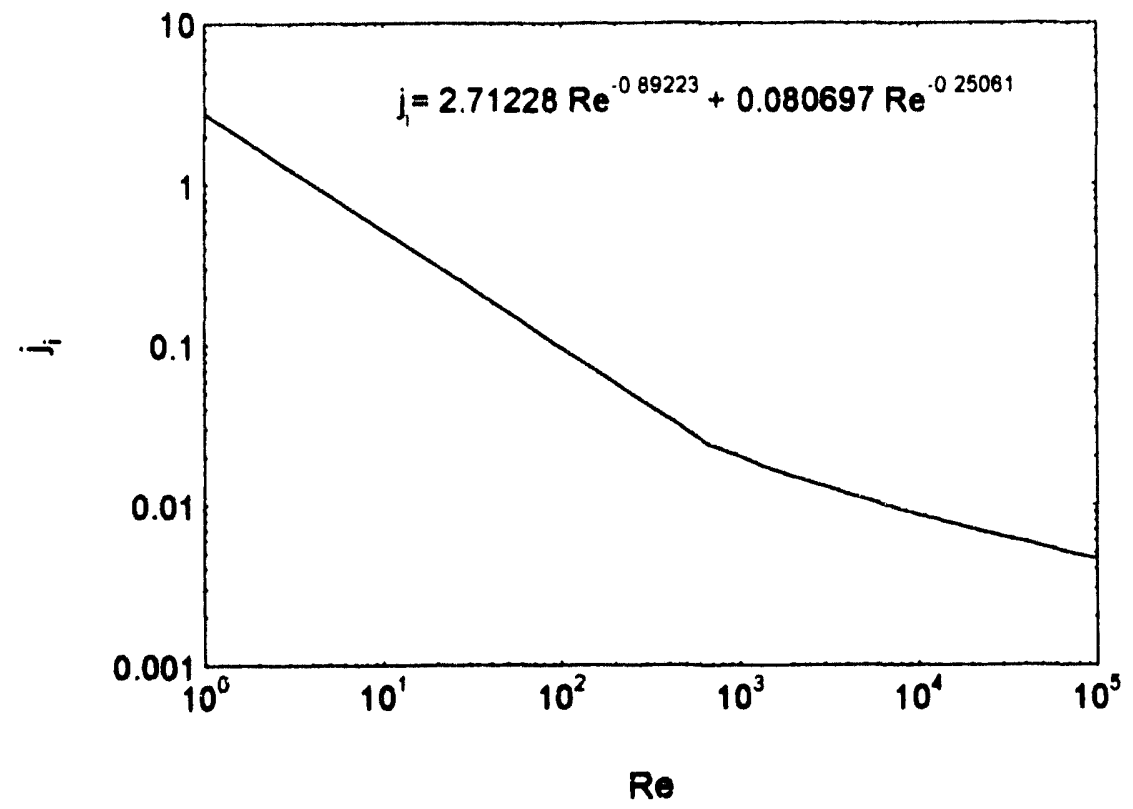

\subsection{Heat Exchanger Pressure Drops}

To determine the required pumping power and/or natural circulation driving head for a particular heat exchanger design, the pressure drop through both the primary and secondary sides of the heat exchanger must be determined. As was seen for the heat transfer coefficients, the calculational method used for the pressure drop is different depending on whether the fluid is inside or outside the tubes, and whether it is air or water.

\subsubsection{Pressure Drop - Primary Side}

As with the heat transfer coefficient, the pressure drop for the primary side can be determined using simple semi-analytical relations. The pressure drop for fluid flowing inside a tube is given by:

${ }^{15}$ K.J. Bell, Figure 14. 


$$
\Delta p_{p r i}=\frac{f_{p r i}}{2 \rho_{p r i}}\left(\frac{W_{\text {tube }}}{A_{\text {tube }}}\right)^{2}\left(\frac{L_{\text {rube }}}{D_{\text {tube }}}\right),
$$

where $f_{p r i}$ is the friction factor (defined below). ${ }^{16} \mathrm{~W}_{\text {mube }}$ and $A_{\text {tube }}$ are the mass flow rate in the tube and the tube flow area, respectively. $L_{\text {rube }}$ and $D_{\text {tube }}$ are the tube length and diameter, and $\rho_{\mathrm{pri}}$ is the primary water density. The friction factor for turbulent flow is given by: ${ }^{17}$

$$
\begin{aligned}
f_{p i}= & 0.316 \operatorname{Re}^{-0.25}(\text { for } \operatorname{Re}<30,000) \\
& 0.184 \operatorname{Re}^{-0.2} \quad(\text { for } 30,000<\operatorname{Re}<1,000,000)
\end{aligned}
$$

\subsubsection{Pressure Drop - Secondary Side}

For the air-cooled Auxiliary Heat Exchanger, the secondary side (air-side) pressure drop is composed of two main components: the friction pressure drop (similar in form to the friction pressure drop discussed above), and the flow acceieration pressure drop which is caused by acceleration of the air as it flows through the heat exchanger. The acceleration term has a component which depends on the ratio of the free-flow/frontal area ratio ( $\sigma$ in Table 6.2). This component gets small as the frontal area of the heat exchanger gets larger, indicating that the air is accelerated less as it enters the unit, hence has less corresponding pressure drop. The acceleration term also has a component which depends on the ratio of inlet/outlet air densities. This is because as the air heats up as it passes through the heat exchanger, the decrease in density causes the air the accelerate. Generally, the acceleration term accounts for entrance and exit effects, but these are small for a cooling tower, and are neglected herein.

The relation for the air-side pressure drop for the Auxiliary Heat Exchanger is given by:

${ }^{16}$ N. E. Todreas and M.S. Kazimi, Nuclear Systems 1, Thermal Hydraulic Fundamentals, Hemisphere, 1990 , p. 610.

${ }^{17}$ Ibid., p. 379. 


$$
\Delta \mathrm{p}_{\mathrm{sec} \text {.air }}=\frac{1}{2 \rho_{\mathrm{in}}}\left(\frac{\mathrm{W}_{\mathrm{sec} . \mathrm{arf}}}{\mathrm{Aff}}\right)^{2}\left[\left(1+\sigma^{2}\right)\left(\frac{\rho_{\mathrm{in}}}{\rho_{\mathrm{out}}}-1\right)+\mathrm{f}_{\mathrm{sec}} \frac{\mathrm{A}_{\mathrm{sec}}}{\operatorname{Aff}} \frac{\rho_{\mathrm{m}}}{\rho_{\mathrm{m}}}\right],
$$

where $\rho_{\text {in }}, \rho_{\text {out }}$, and $\rho_{\mathrm{m}}$ are the inlet, outlet, and average air densities, $\mathrm{W}_{\text {soc,air }}$ is the air mass flow rate, Aff is the air-side free flow area, $A_{s e c}$ is the total air side heat transfer area, and $\mathrm{f}_{\mathrm{soc}}$ is the air-side friction factor. As was the case for the air-side heat transfer parameter $\mathrm{St} \mathrm{Pr}^{2 / 3}, \mathrm{f}_{s o x}$ must be determined by testing the specific heat exchanger tube/fin geometry being considered. A plot of $\mathrm{f}_{\mathrm{soc}}$ for the Auxiliary Heat Exchanger is shown in Figure 6.12. The Reynolds number for this figure was defined in Equation 13.

\section{Figure 6.12. Pressure Drop Parameter for Air Side of Auxiliary Heat} Exchanger $^{18}$

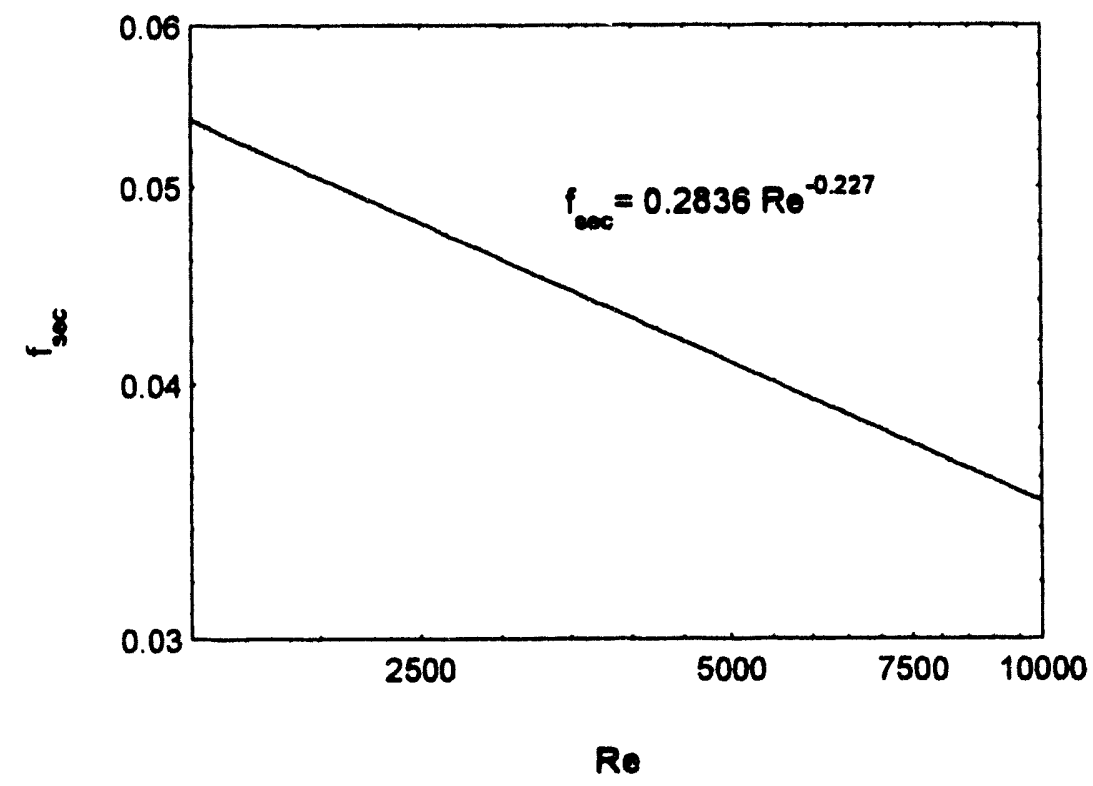

In addition to the friction pressure drop caused by passage through the Auxiliary Heat Exchanger, air passing through the cooling tower also experiences a friction pressure drop due to passage through the chimney of the tower. This chimney pressure drop

${ }^{18}$ W. M. Kays and A. L. London, Compact Heat Exchangers, Mc-Graw Hill, 1984, Fig. 10-90, surface CF-8.8-1.0J/B. 
(determined in a similar fashion as the friction pressure drop of water through tubes is determined) obviously depends on the diameter of the chimney, with smaller chimney diameters causing a higher chimney pressure drop, for a given air mass flow rate.

For the water-cooled secondary side of the Service Heat Exchanger, we return again to the Delaware method for shell side design. The full-blown Delaware method pressure drop correlation is rather complex, and requires knowledge of the design details of the heat exchanger which are not known at the conceptual design stage. Hence, a simplified relation is used to calculate the pressure drop. This relation neglects effects such as bypass flow around the tube bundle, hence it is conservative in that it yields higher values for pressure drop than the full Delaware correlation. The simplified pressure drop relation gives the pressure drop for an ideal crossflow section and is as follows:

$$
\Delta p_{\text {soc, water }}=\frac{2 f_{i} N_{c}}{\rho_{\text {reo }}}\left(\frac{W_{\text {sec, weser }}}{A_{c f}}\right)^{2},
$$

where $N_{c}$ is the number of tube rows crossed by the secondary flow, and $f_{i}$ is the friction factor, which is dependent on the tube geometry. Figure 6.13 shows $f_{i}$ for the Service Heat Exchanger geometry. The Reynolds number for this figure was defined in Equation 6.17. 
Figure 6.13. Pressure Drop Parameter for Secondary Side of Service Heat Exchanger

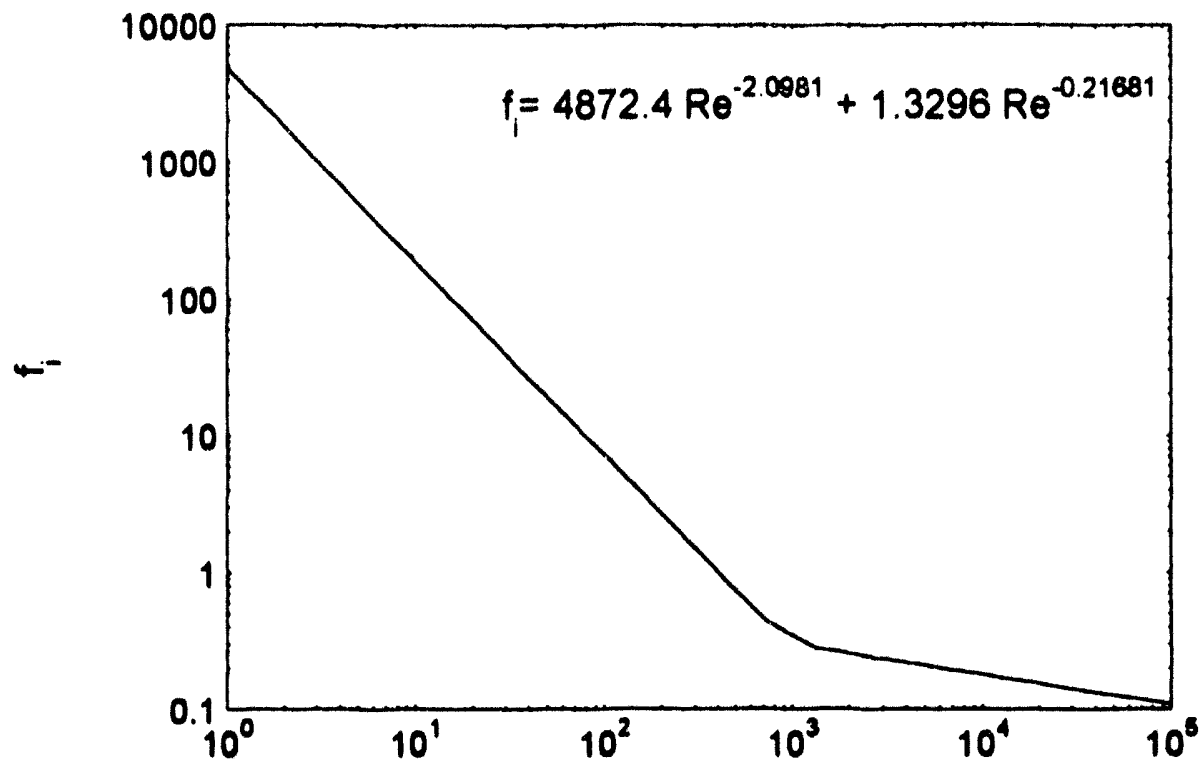

Re

\subsection{Cooling Tower/Auxiliany Heat Exchanger Sizing for Single Loop Shield Water System}

In the previous three sections, the basic elements of heat exchanger design were developed. In this section, the required sizes of the cooling tower and Auxiliary Heat Exchanger (AHE) are determined, using the methods previously outlined, with the added requirement that the natural circulation pressure drop of the system be equal to the frictional pressure drop. As a starting point for the sizing process, we need not only the fin/tube geometry, which was given in Figure 6.7 and Table 6.2 , but also the choice of fin/tube materials. A standard choice of material for low temperature, low pressure aircooled heat exchangers is aluminum, because of its high thermal conductivity. However, the protective corrosion film on aluminum breaks down above $60-70$ degrees $C,{ }^{19}$

19P. Cohen, Water Coolant Technology of Power Reactors, Gorden and Breach Science Publishers, New York, 1969, p. 309. 
making it unsuitable as a tube material for the present design, which operates at temperatures above 90 degrees $\mathrm{C}$. Hence, 304 stainless steel is chosen as a tube material, and aluminum is used only for the fins. Stainless steel has the additional benefit of allowing relatively high flow velocities in the heat exchanger tubes without concerns for tube erosion.

In a typical heat exchanger sizing problem, the inlet and outlet temperatures for both fluids are specified, and the required heat transfer rate for the heat exchanger is known. For the present design, the required heat transfer rate is the heat load under accident conditions, and is $5 \mathrm{MW}$, as discussed in Section 6.1.2. However, only two of the four relevant temperatures are specified, the AHE primary water inlet temperature (which must be below 100 degrees $C$, say 95 degrees $C$ ), and the air inlet temperature (assumed to be at 25 degrees $C$ ). The primary water outlet temperature during accident conditions, and the air outlet temperature are not specified initially. In addition, the allowable air- and water-side pressure drops (which are provided by natural circulation) are not specified either, but are functions of the cooling tower size and the assumed height of the AHE above the shield centerline.

In order to move forward in the heat exchanger sizing process, the number of unspecified variables must be reduced. The air outlet temperature can be fixed by noting that if the air exiting the cooling tower is too hot, it may be an environmental threat. Hence, the air outlet temperature is fixed at a value consistent with the outlet temperatures of other power plant dry cooling tower designs, namely 40 degrees $C .{ }^{20}$ The height of the center of the AHE is also fixed (somewhat arbitrarily) as 2 meters

\footnotetext{
${ }^{20}$ For examples of typical dry cooling tower design exit temperatures, see D.W. Kearney and B.E. Boyack, "Plume Behavior and Potential Environmental Effects of Large Dry Cooling Towers," in Dry and Wet/Dry Cooling Towers for Power Plants, RL. Wedd and R.E. Barry, Eds., The American Society of Mechanical Engineers, 1973, p. 35.
} 
above the center of the shield. Referring back to Figure 6.5 , Hw is therefore set at 2 meters.

The length and diameter of the piping which connects the shield to the AHE must be specified to determine the overall primary water-side pressure drop. The actual configuration of this piping, with the associated shield module inlet and outlet piping manifolds, is dependent on the detailed design of the shield and reactor plant. In addition, the length of the piping depends on detailed arrangements of shield water system components. Rather than attempting to specify such details here, the pressure drop behavior of the detailed piping arrangement is approximated by assuming the shield and AHE are connected by a single inlet and single outlet pipe of a specified length and diameter, with four long radius pipe bends in the loop. To account for pressure drops associated with pipe bends, tees, etc. which are not modeled directly, the inlet and outlet pipes are assumed to be relatively long, at 100 meters each.

The diameter of the inlet/outlet piping, as it turns out, is specified by the requirement to allow for decay time of N-16 activity. This issue is discussed in Section 6.10. To minimize the N-16 activity in the AHE to acceptable levels, the inlet/outlet pipe diameter have a diameter of 1.1 meters. Note that for a system which operates at atmospheric pressure, such a large pipe size is reasonable.

The final variable to be fixed is the primary water outlet temperature from the AHE under accident conditions. As it happens, with the other system parameters fixed as discussed above, the dimensions and cost of the AHE and cooling tower are not significantly affected by variations in the primary water outlet temperature. This temperature is therefore fixed at 80 degrees C. With the AHE outlet and inlet 
temperatures, and the heat load to the system all fixed, the system mass flow rate is also fixed by conservation of energy at $79 \mathrm{~kg} / \mathrm{sec}$.

A number of variables necessary to size the cooling tower and AHE have now been determined, and are shown in Table 6.4. The diameter of the cooling tubes in the shield (necessary to calculate the primary water side pressure drops) is specified as $22 \mathrm{~mm}$ in this table. As it turns out, the pressure drop of the shield is negligible compared to the pressure drop of the rest of the piping and components in the primary loop. This is demonstrated later, in Section 6.9.

Table 6.4. Shield Water System Design Parameters (Accident Conditions)

\begin{tabular}{|r|c|}
\hline Auxiliary Heat Exchanger (AHE) Heat Load : & $5 \mathrm{MW}$ \\
\hline Cooling Tower Air Inlet Temperature: & 25 degrees C \\
\hline Cooling Tower Air Outlet Temperature: & 40 degrees C \\
\hline Primary Water Outlet Temperature from AHE : & 80 degrees C \\
\hline Primary Water Inlet Temperature to AHE: & 95 degrees C \\
\hline System Mass Flow Rate: & 79 kg/sec \\
\hline Height of AHE above Shield Centertine (Hw): & 2 meters \\
\hline Shield-to-AHE Connecting Pipe Length (Inlet and Outlet): & 200 meters \\
\hline Shield-to-AHE Inlet and Outlet Pipe Diameter: & 1.1 meters \\
\hline Shield Cooling Tube Diameter (7400 tubes in shield): & 22 mm \\
\hline
\end{tabular}

The remaining variable necessary to specify the size of the of the cooling tower and AHE is the chimney height, or the height of the cooling tower above the heat exchanger ( $\mathrm{Ha}$ in Figure 6.5). The chimney height is varied in the following analysis to determine the optimum tower and heat exchanger size. 
In order to find the optimum cooling tower and Auxiliary Heat Exchanger sizes, the chimney height is now varied to see the effect of chimney height on the dimensions of the heat exchanger, and the cooling tower itself. The heat exchanger and cooling tower sizes change as the chimney height changes in order to keep the natural circulation pressure head of the system equal to the frictional pressure drop of the system. The details of the heat exchanger/cooling tower sizing procedure are not given in this section; the reader is referred to Appendix 6 for these details. The results of the heat exchanger sizing procedure detailed in Appendix 6 are plotted in Figure 6.14.

\section{Figure 6.14. Auxiliary Heat Exchanger Volume vs.} Chimney Height

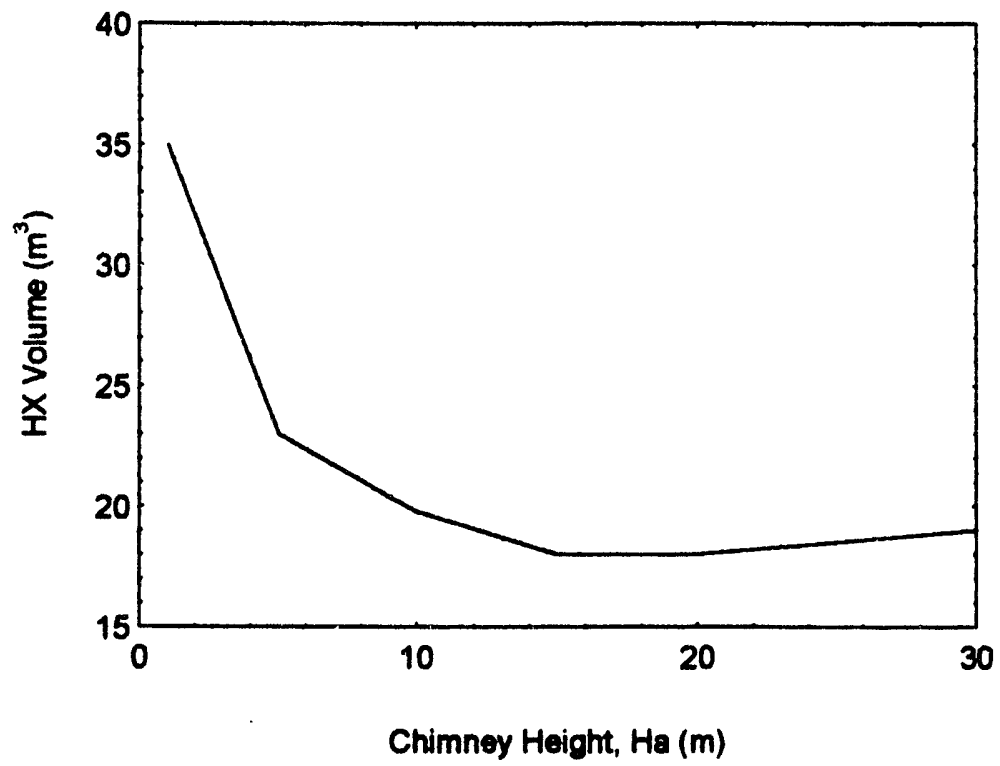

This figure shows that as the chimney height increases, the required heat exchanger size decreases, until a chimney height of about 15 meters, at which point the heat exchanger size begins to gradually increase again. The explanation for this curve

shape follows. Generally, a larger chimney height results in a greater allowable pressure drop across the heat exchanger. This in turn allows for a smaller flow area for the given (fixed) air mass flow rate, and hence a smaller heat exchanger. This explains the lefthand side of the figure, when heat exchanger size is decreasing with Ha. However, a smaller heat exchanger also means a smaller chimney diameter (see Figure 6.15 below). At heat exchanger volumes below 18 cubic meters, the chimney diameter becomes so 
small that the chimney pressure drop begins to dominate the air-side pressure drop. This reduces the rate of air flow for a given heat exchanger size, driving the exchanger size toward larger values again to allow the required air flow rate through heat exchanger/tower complex. A plot of relevant cooling tower parameters versus chimney height is given in Figure 6.15.

\section{Figure 6.15. Cooling Tower Parameters vs. Chimney Height}

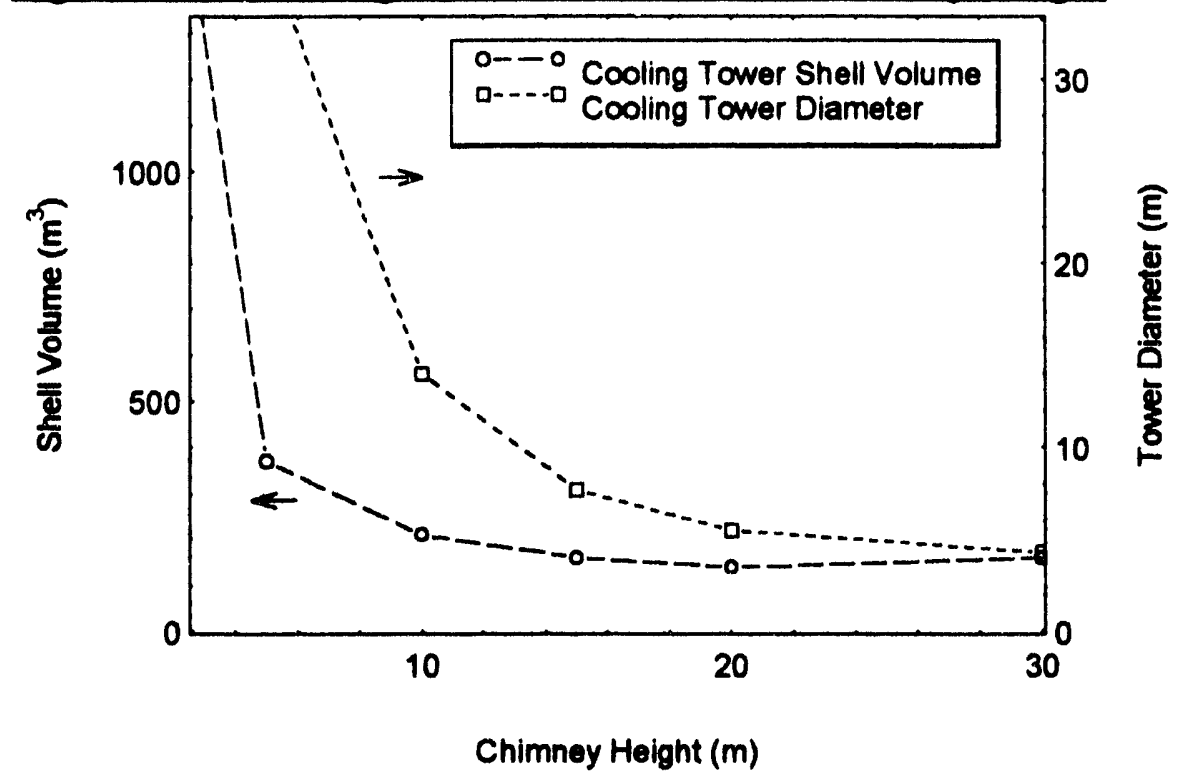

As shown in this figure, the cooling tower diameter drops with chimney height. As chimney height is increased, the cooling tower air gap increases, since the air gap is assumed to scale with the chimney height (see Appendix 6 for details on the configuration of the cooling tower/AHE complex). This allows more vertical room for the heat exchanger within the cooling tower, thereby allowing the tower diameter to decrease (since the heat exchanger must fit into the air gap region around the tower's diameter). The decrease in tower diameter tends to control the volume of the tower shell, despite the fact that the chimney height is increasing. Hence, the shell volume generally decreases with $\mathrm{Ha}$ until about $\mathrm{Ha}=20$ meters, at which point the shell volume begins to increase slowly because the diameter is not decreasing as fast as $\mathrm{Ha}$ is increasing. 
Since both the heat exchanger size and tower size decrease with increasing chimney height until about $\mathrm{Ha}=15$ meters, it would appear that this would be an optimum height for the tower. Table 6.5 shows the resulting Auxiliary Heat Exchanger/cooling tower parameters for this chimney height. This is the reference Auxiliary Heat Exchanger/cooling tower complex for the single coolant loop SWS.

Table 6.5. Reference Auxiliary Heat Exchanger/Cooling Tower Parameters (Single Loop SWS)

\begin{tabular}{|l|l|}
\hline Cooling Tower Chimney Height: & 15 meters \\
\hline Total Cooling Tower Height: & 21 meters \\
\hline Cooling Tower Diameter: & 7.7 meters \\
\hline Cooling Tower Shell Volume: & $160 \mathrm{cu}$. meters \\
\hline Heat Exchanger Volume: & $18 \mathrm{cu}$. meters \\
\hline
\end{tabular}




\subsection{Service Heat Exchanger Sizing}

We turn now to sizing of the Service Heat Exchanger (SHE), for use during normal operation. Figures 6.9 and 6.10 and Table 6.3 showed the basic configuration and geometry of the SHE. As with the Auxiliary Heat Exchanger, the tube material choice must be specified prior to sizing the SHE.

The tube material choice for the SHE is aluminum brass, a copper alloy with 20.5 percent zinc and 2 percent aluminum added as alloying materials. This is a standard, high conductivity material used in power plant heat exchangers, and is inexpensive relative to the 304 stainless steel used in the Auxiliary Heat Exchanger. However, unlike stainless steel, which can sustain high tube coolant velocities without erosion, aluminum brass has a tube velocity limit of about $7.0 \mathrm{ft} / \mathrm{sec}(2.13 \mathrm{~m} / \mathrm{sec}) .^{21}$ A tube velocity limit is acceptable for design of the SHE, since it is desirable to limit the pressure drop of this unit anyway, to facilitate natural circulation flow in the shield water system during accident conditions.

The normal operational heat load for the SHE has already been determined in a previous section of this chapter, and is $27 \mathrm{MW}$. To size the heat exchanger, the primary and secondary water inlet and outlet temperatures must also be specified. The secondary water used for cooling the SHE is assumed to come from the same water system which cools the main condenser in the steam plant for the reactor. Hence, it is logical to assume similar inlet and outlet temperatures for the secondary side of the SHE as would be reasonable for a main condenser cooling system.

\footnotetext{
${ }^{21}$ T. Baumeister, et.al., eds., Mark's Standard Handbook for Mechanical Engineers, 8th Ed., McGrawHill, 1978, p. 9-62.
} 
Since the water for cooling the main condenser (and the SHE) might come from a river or lake, the inlet temperature should be chosen to be at or above the maximum temperature for such natural bodies of water. For the U.S., this temperature is about 30 degrees $C ; 22$ hence 30 degrees $C$ is chosen as the design secondary water inlet temperature. The water temperature rise for condensers is typically about 10 degrees $\mathrm{C},{ }^{23}$ so the secondary coolant outlet temperature is specified as 40 degrees $\mathrm{C}$.

The primary Shield Water System coolant inlet temperature to the SHE is specified as 85 degrees $\mathrm{C}$, to ensure a large margin to 100 degrees $\mathrm{C}$ during normal operation. The primary coolant inlet temperature is then fixed by the results of the $\varepsilon-\mathrm{NTU}$ analysis discussed previously in this chapter. Recall that this analysis showed that for best heat exchanger efficiency, it is desirable to ensure that the following relation holds:

$$
\mathrm{T}_{\mathrm{pri,h}}-\mathrm{T}_{\mathrm{pri,c}} \leq \mathrm{T}_{\mathrm{sec,h}}-\mathrm{T}_{\mathrm{wec,c}} \text {. }
$$

Hence, the temperature drop across the SHE on the primary side must be at most equal to the temperature increase on the secondary side. To minimize the required flow rates, and the corresponding tube velocities, a primary side temperature drop of 9 degrees $\mathrm{C}$ is chosen, which is just slightly smaller than the secondary temperature rise. Table 6.6 shows the Shield Water System design parameters necessary to size the SHE.

\section{Table 6.6. Shield Water System Design Parameters (Normal Operation)}

\begin{tabular}{|l|l|}
\hline Service Heat Exchanger (SHE) Heat Load: & $27 \mathrm{MW}$ \\
\hline SHE Primary Water Inlet Temperature: & $\mathbf{8 5}$ degrees C \\
\hline SHE Primary Water Outlet Temperature: & 76 degrees C \\
\hline SHE Secondary Water Inlet Temperature: & 30 degrees C \\
\hline SHE Secondary Water Outlet Temperature: & 40 degrees C \\
\hline
\end{tabular}

\footnotetext{
${ }^{22}$ Ibid. p. 9-62.

${ }^{23}$ Ibid, Chapter 9.
} 
The parameters above determine the required mass flow rates on the primary and sides of the SHE. Given these, the dimensions of the cylindrically-shaped heat exchanger (namely the length and diameter) determine the flow velocity on both the inside and outside of the tubes. Specifying one dimension (diameter, for example) fixes the other dimension as well, since the heat exchanger has to have a certain surface area in order to transfer the required heat flow.

Figure 6.16. Service Heat Exchanger Length vs. Diameter

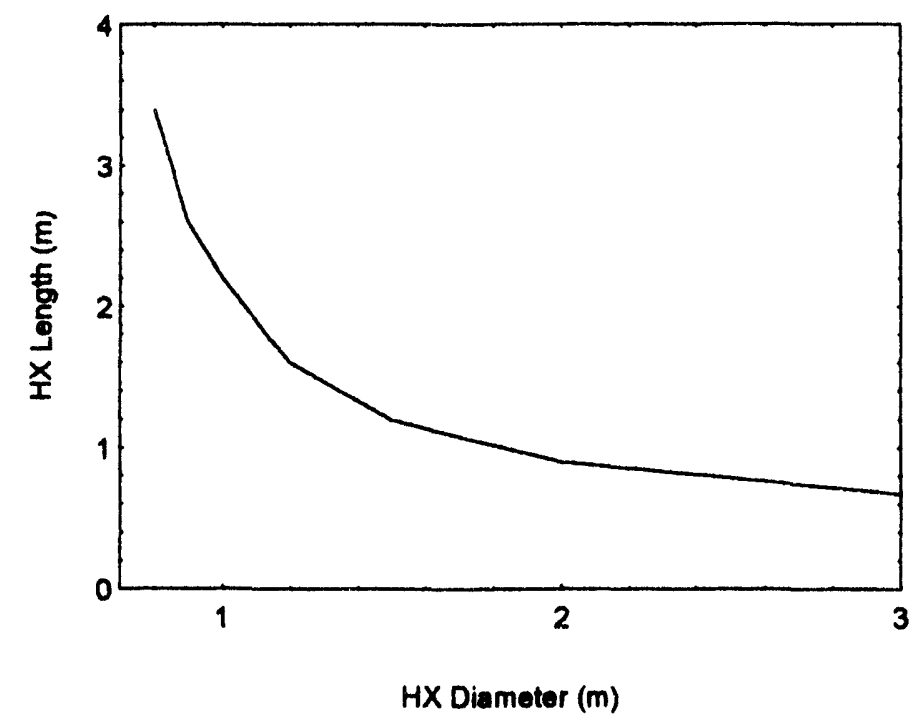

Using a procedure similar to the procedure described for sizing the Auxiliary Heat Exchanger (described in Appendix 6), and choosing the SHE diameter as a variable, a plot of SHE length versus diameter is generated, as shown in Figure 6.16. As the diameter is increased, the required heat exchanger length decreases, since the required surface area remains relatively constant as the diameter is changed. 


\section{Figure 6.17. Service Heat Exchanger Flow Velocities}

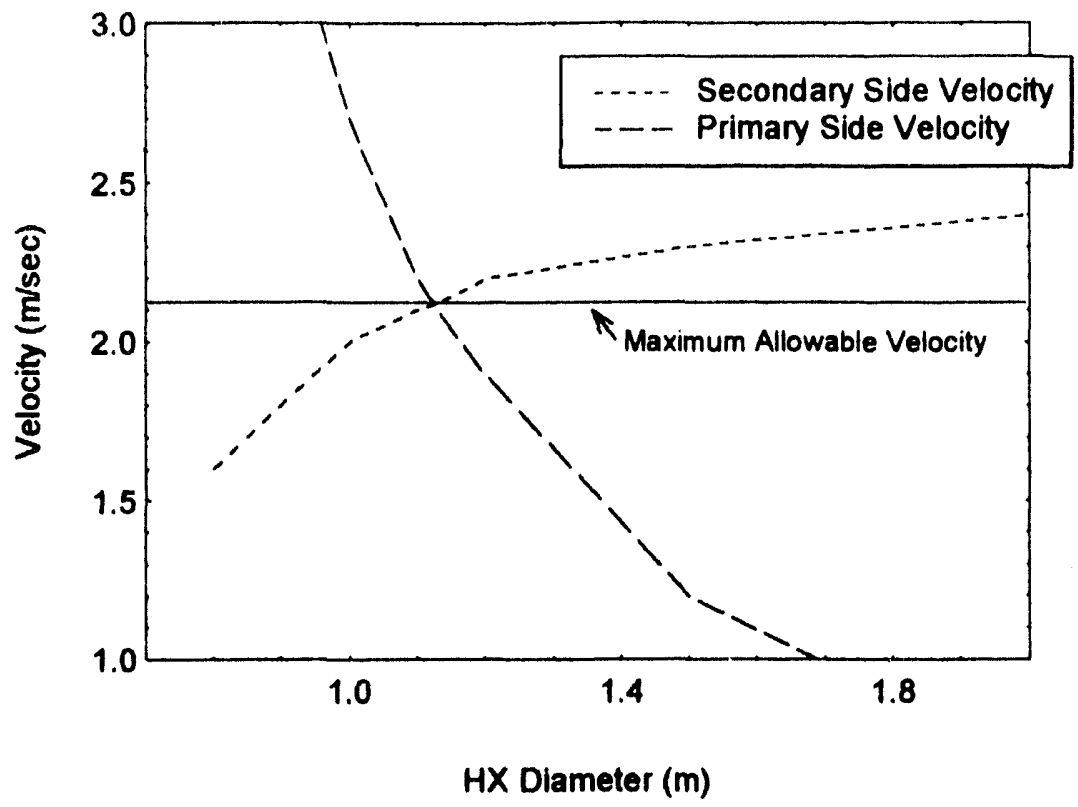

Given the results shown in Figure 6.16, it is straightforward to calculate the resulting flow velocities on the primary side (through the inside of the tubes) and the secondary side (on the outside of the tubes). The results are shown in Figure 6.17. As can be seen, for heat exchanger diameters below about 1.1 meters, the primary side velocity exceeds the maximum allowable velocity limit. This is because there is less primary side crosssectional flow area. For diameters above 1.1 meters, however, the secondary side velocity exceeds the allowable limit, since as the heat exchanger diameter increases, its required length decreases, reducing the secondary fluid's cross-sectional flow area. Hence, an SHE diameter of 1.1 meters is chosen for our design. The dimensions of the reference Service Heat Exchanger are thus shown in Table 6.7.

Table 6.7. Reference Service Heat Exchanger Dimensions

\begin{tabular}{|l|l|}
\hline Service Heat Exchanger Diameter: & 1.1 meters \\
\hline Service Heat Exchanger Length: & 1.8 meters \\
\hline
\end{tabular}




\subsection{Temperature and Flow Distribution in Shield}

The SWS must ensure that the temperature of the coolant water at the front of the shield does not reach 100 degrees during either normal operation, or during the LOFA.

However, the design input for the SWS is the average coolant water outlet temperature from the shield, not the maximum temperature within the shield. To relate the average coolant water outlet temperature to the maximum temperature seen at the front of the shield, an analysis of the temperature and flow distribution in the shield needs to be performed.

For normal operation with the driving head in the SWS provided by a pump, the average coolant outlet temperature from the shield is only 85 degrees $C$, as discussed in the previous section. With this large margin to 100 degrees $C$, there should be no concern with the coolant temperature in the front tubes exceeding this temperature. If necessary, the temperature distribution for coolant within the shield can be controlled using flow restrictors in the coolant pipes in the rear portions of the blanket. This forces more flow through the front sections of the shield to remove the higher heat load there, ensuring that the coolant outlet temperature in for each coolant tube remains below 100 degrees C.

For accident operation with no coolant pump, however, the driving head in each coolant tube is provided by heatup of coolant in each individual tube, which creates density changes in the tube and results in a natural circulation driving head. This driving head is different depending on how much heat is deposited in the tube. The tubes with the highest heat loads during the accident are the tubes at the front of the shield. Hence, the front tubes have greater natural circulation driving heads, higher flow velocities, and higher outlet temperatures than tubes further back in the shield. If the water in the front 
shield tubes can be shown to remain below 100 degrees $C$ during the accident, all of the water in the shield remains below this temperature.

Figure 6.18 illustrates the model used to perform the accident flow and temperature distribution analysis for the shield coolant tubes. This figure shows a simplified schematic of the entire SWS loop, including the shield, SHE and AHE. Also shown is the shield inlet and outlet plena temperatures, and the system mass flow rate, determined in Section 6.6. The first five rows of shield tubes behind the back of the blanket are also shown (the other tube rows are omitted for clarity). Finally, Figure 6.18 shows a cross section of the shield coolant tubes, indicating a "coolant tube unit cell", defined as a coolant tube and the surrounding associated structure.

\section{Figure 6.18. Shield Model}

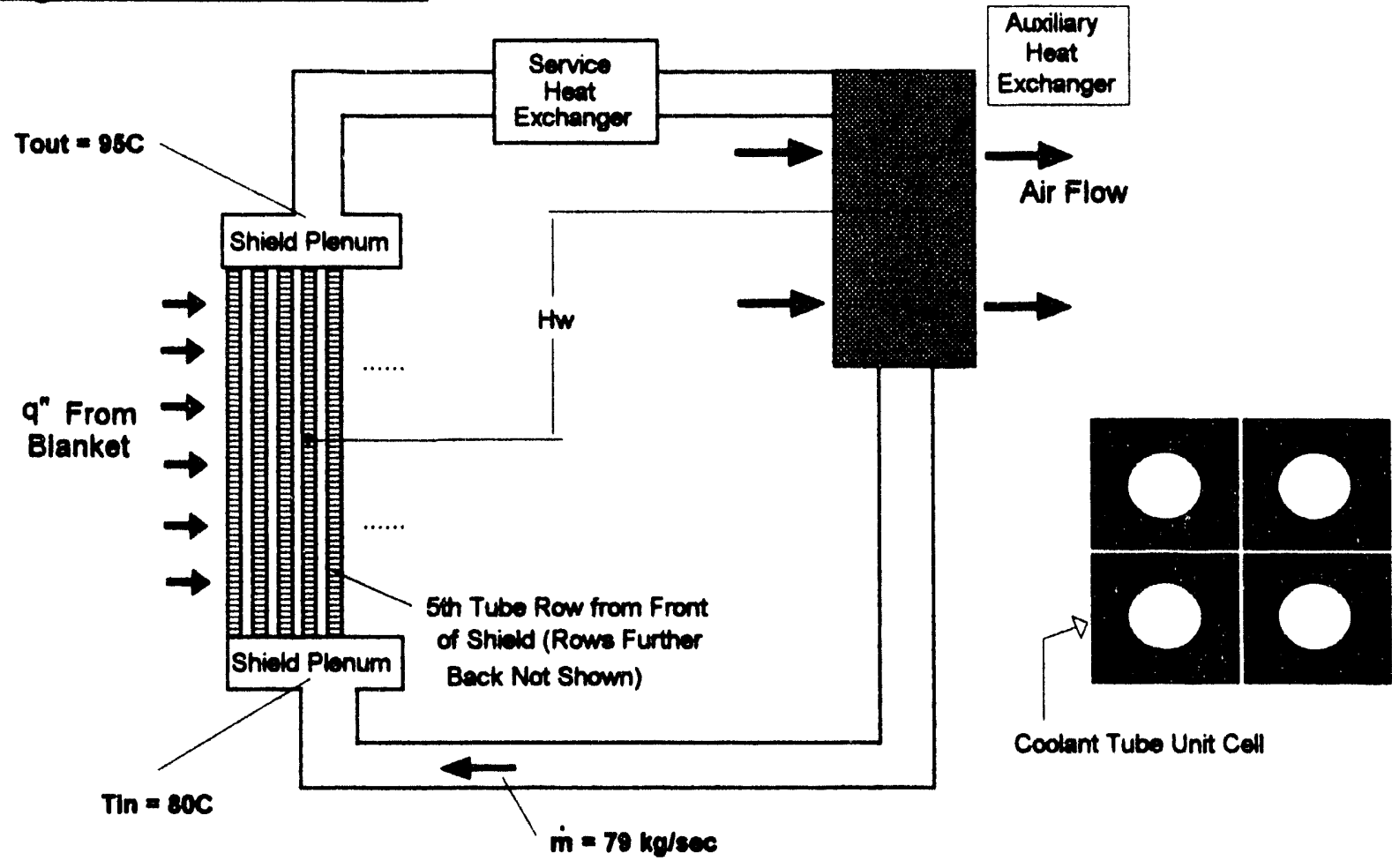


The model assumes that there is no conduction from the hotter front portion of the shield to the back of the shield. Rather, all of the afterheat generated in the structural part of the unit cell is assumed to be deposited in the coolant tube. Furthermore, all of the radiant heat load from the blanket is assumed to be deposited in the first row of shield tubes. The afterheat generation rate is assumed to be the peak generation rate, which occurs at shutdown. For conservatism, the peak radiant heat load from the blanket is used as well, even though the peak radiant heat load occurs well after shutdown. The no conduction assumption yields the most asymmetric temperature and flow distribution and the highest peak temperature in the front tubes, and hence is conservative. With the shield plena temperatures and the heat load in each shield tube specified, the channel pressure drop can be determined as a function of mass flux in each tube. The results for

Figure 6.19. Channel Pressure Drop vs. Mass Flux in four representative Shield Tubes

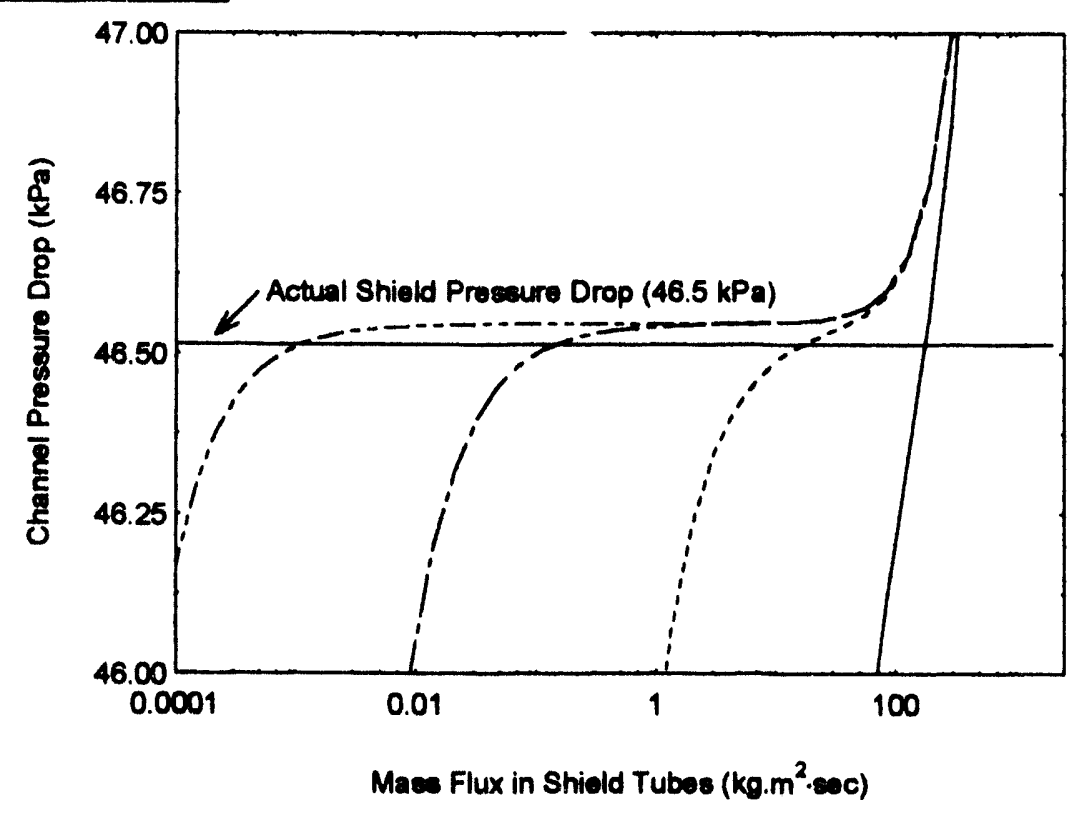

shield tubes are shown in Figure 6.19.

At lower mass fluxes, buoyancy pressure head is dominant over friction pressure drop, and the channel pressure drop decreases. Because of the much greater heat load in the front

shield tubes (caused by the radiant heat load from the blanket), buoyancy begins to dominate at much higher mass fluxes for these tubes than for tubes further back in the shield (note that the x-axis in Figure 6.19 is logarithmic.) The tube denoted as "Behind 
Front of Shield" represents the second row back in Figure 6.18. Because of the no conduction assumption, this row sees none of the radiant heat from the blanket, and consequently buoyancy dominates only for much lower mass fluxes as compared to the front shield tubes. For the other two representative tubes shown, the afterheat in the tube structure is lower, because the tubes are further back in the shield. Hence, buoyancy dominates at even lower mass fluxes for these tubes.

The pressure drop versus mass flux curves for each tube row in the shield can now be used to determine the actual operational pressure drop across the shield during the accident, and the operational mass fluxes in each shield tube. Note that during system operation, the overall pressure drop in each tube channel must be equal (neglecting any pressure drops in the plena). Also note that the total mass flow rate through all of the shield tube channels must equal $79 \mathrm{~kg} / \mathrm{sec}$, as shown in Figure 6.18. These two conditions can be used to find the actual shield pressure drop, and hence the actual mass flux in each shield tube during SWS operation.

The horizontal line in Figure 6.19 shows the correct shield pressure drop during accident operation, which is $46.5 \mathrm{kPa}$. When this is compared to the pressure head developed by the cold leg of the loop during natural circulation, it is found that the bouyancy generated in the shield tubes almost exactly balances the friction pressure drop of the tubes. Since the mass flux is known for each tube, the maximum tube outlet temperature can also be calculated. Most of the flow in the shield flows through the front tubes (as can be seen in Figure 6.19). The outlet temperature for these tubes is also the highest, but remains safely below 100 degrees, even with the conservative assumptions used in this model. Hence, the conclusion can be drawn that the SWS system, as designed in the above sections, keeps all of the water in the shield below 100 degrees during both normal and accident operation. 


\subsection{Shield Water System Pumping Power}

As has been mentioned previously in this study, it is necessary for the Shield Water System pump be a jet pump, with a nozzle inserted into the main shield water system piping which provides the pumping head required for normal operation, but with flow able to bypass the nozzle and pump during natural circulation operation following a loss of electrical power. A simple schematic of the jet pump configuration was shown in Figure 6.5 at the beginning of this chapter. One of the reasons a jet pump can be used in this system is that the system pumping power requirements are low enough that using an inefficient jet pump configuration does not result in excessive power consumption. To show this, the pumping power requirement for the Shield Water System's jet pump is determined.

Using conservation of momentum arguments, the overall efficiency of the jet pump can be shown to be only about 0.27 for the normal operational flow conditions of the system, assuming the jet pump nozzle takes up $1 / 3$ of the available flow area (see Appendix 7 for details on this calculation). The pressure drops for the system during normal operation, together with the required electrical pumping power are shown in Table 6.8. The required pumping power of $20 \mathrm{~kW}$ is small, despite the jet pump's inherent inefficiency. 
Table 6.8. Shield Water System Pressure Drop/Pumping Power Data (Normal Operation)

\begin{tabular}{|r|c|}
\hline Service Heat Exchanger Pressure Drop: & $3.6 \mathrm{kPa}$ \\
\hline Auxiliary Heat Exchanger Pressure Drop: & $3.1 \mathrm{kPa}$ \\
\hline Shield Pressure Drop: & $0.4 \mathrm{kPa}$ \\
\hline Piping Pressure Drop: & $0.5 \mathrm{kPa}$ \\
\hline Total Pressure Drop: & $7.6 \mathrm{kPa}$ \\
\hline Primary Water Mass Flow Rate: & $714 \mathrm{~kg} / \mathrm{sec}$ \\
\hline Jet Pump Efriciency: & 0.27 \\
\hline Required Electrical Pumping Power: & $20 \mathrm{~kW}$ \\
\hline
\end{tabular}




\subsection{Activity in Single Loop Shield Water System Coolant}

The single loop SWS has been designed without an intermediate loop between the SWS cooling water and atmospheric air in the Auxiliary Heat Exchanger. This raises the question of whether a leak in the Auxiliary Heat Exchanger could release unacceptable amounts of long-lived radioactivity to the environment. Since the coolant will emit gamma radiation due to short-lived $\mathrm{N}-16$ activity during operation, there is also a possibility that the SWS piping and Auxiliary Heat Exchanger could radiate a substantial amount of radiation, even if there is no leak.

In this section, the concentration of the most significant long-lived radionuclides in the SW coolant will be estimated, and it will be demonstrated that the coolant activity levels in the SWS is likely to be low enough so that a coolant leak should not be of concern. Following this calculation, the operational N-16 activity levels will be addressed, and a system design resulting in acceptable exposure levels presented.

\subsubsection{Long-Lived SWS Coolant Activity}

The possible sources of long-lived activity in the SWS coolant are: (1) activation of the coolant water itself, (2) activation of coolant water additives and impurities; and (3) release of activation products from the shield structure due to corrosion and solubility mechanisms. The most significant activation product for the ccolant water itself is $\mathrm{N}-16$, generated by an $(\mathrm{n}, \mathrm{p})$ reaction with $0-16$. However, the half-life of $\mathrm{N}-16$ is only 7.4 seconds, so it does not represent a long-term environmental hazard in the event of a leak. The $\mathrm{N}-16$ is a potential operational hazard, and will be addressed later.

Impurities in the coolant water come chiefly from dissolved gases from the air. Atmospheric air consists of 1 percent argon gas, which has a solubility of up to $5.6 \mathrm{cc}$ 
gas to $100 \mathrm{cc}$ water, depending on the water temperature. ${ }^{24}$ Of all the impurities which could dissolve into the coolant from the air, the argon is of most concern from an activation standpoint. ${ }^{25}$ This dissolved argon undergoes the reaction Ar-40 $+n \rightarrow \operatorname{Ar}-41$ in the neutron flux of the shield. Ar-4I has a 1.83 hour halflife, and so represents a potential environmental hazard for a few hours, in the event of a leak. We will see that the potential activity of $\mathrm{Ar}-41$ in the coolant warrants installing a deaeration system in the SWS loop to reduce the argon level to well below saturation.

Activation of coolant water additives depends, of course, on what additives are chosen. Pressurized Water fission Reactor (PWR) experience has shown that additives are necessary to getter oxygen at startup and to control coolant water $\mathrm{pH}$. Control of oxygen and $\mathrm{pH}$ are desirable to minimize the corrosion of the piping system. Hydrazine $\left(\mathrm{N}_{2} \mathrm{H}_{4}\right)$ is usually added to remove oxygen at startup. To control $\mathrm{pH}$, either lithium hydroxide $(\mathrm{LiOH})$, or ammonium hydroxide $\left(\mathrm{NH}_{4} \mathrm{OH}\right)$ is added. ${ }^{26}$ The significant difference between $\mathrm{LiOH}$ and $\mathrm{NH}_{4} \mathrm{OH}$ for the present work is that the lithium in the LiOH can result in the generation of tritium in the SWS coolant. Since it is desirable to minimize the generation of radioisotopes in the coolant as much as possible, we will adopt $\mathrm{NH}_{4} \mathrm{OH}$ as the additive to control pH in the SWS coolant. None of the constituents of $\mathrm{NH}_{4} \mathrm{OH}$ or $\mathrm{N}_{2} \mathrm{H}_{4}$ generate activation products as a result of neutron irradiation. ${ }^{27}$

${ }^{24}$ R. C. Weast, od, CRC Handbook of Chemistry and Physics, 58th Ed, CRC Press, 1977. ${ }^{25}$ P. Cohen, Water Coolant Technology of Power Reactors, Gorden and Breach Science Publishers, New York, 1969, p. 169.

${ }^{26}$ C. C. Baker, et. al., STARFIRE - A Commercial Tokamak Fusion Power Plant Study, Argonne National Laboratory Report ANL/FPP-80-1, 1980, Appendix G.

27The structural materials and operating conditions for a PWR are significantly differemt than for the SWS of the present work. Hence, it can not be said with complete confidence that the use of PWR-type coolant additives are the ideal choice for the SWS coolant. However, it is beyond the scope of the present study to perform a detailed study of this issue. It is sufficient to note that coolant additives should not be a major contributtor of coolant activity, provided the proper additives are chosen. 
The above paragraphs have shown that the main contributors to SWS coolant activity due to coolant and impurity elements are $\mathrm{N}-16$ and $\mathrm{Ar}-41$. These isotopes will be addressed in detail later in this chapter. Now, we turn to the effect of corrosion products which are released from the SWS structure into the coolant.

\subsubsection{Corrosion Product Activity}

The accurate calculation of the deposition, release, and transport of corrosion products is extremely complicated, and is an active area of research in the fission community. Extensive, detailed computer codes are generally used which model the individual processes involved, such as precipitation and dissolution of particulates, deposition of ions and particulates by crystal growth, adsorption, and ion exchange, and convection of ions and particulates into the bulk coolant. ${ }^{28}$

\section{Figure 6.20. Methods of Activity Transport}

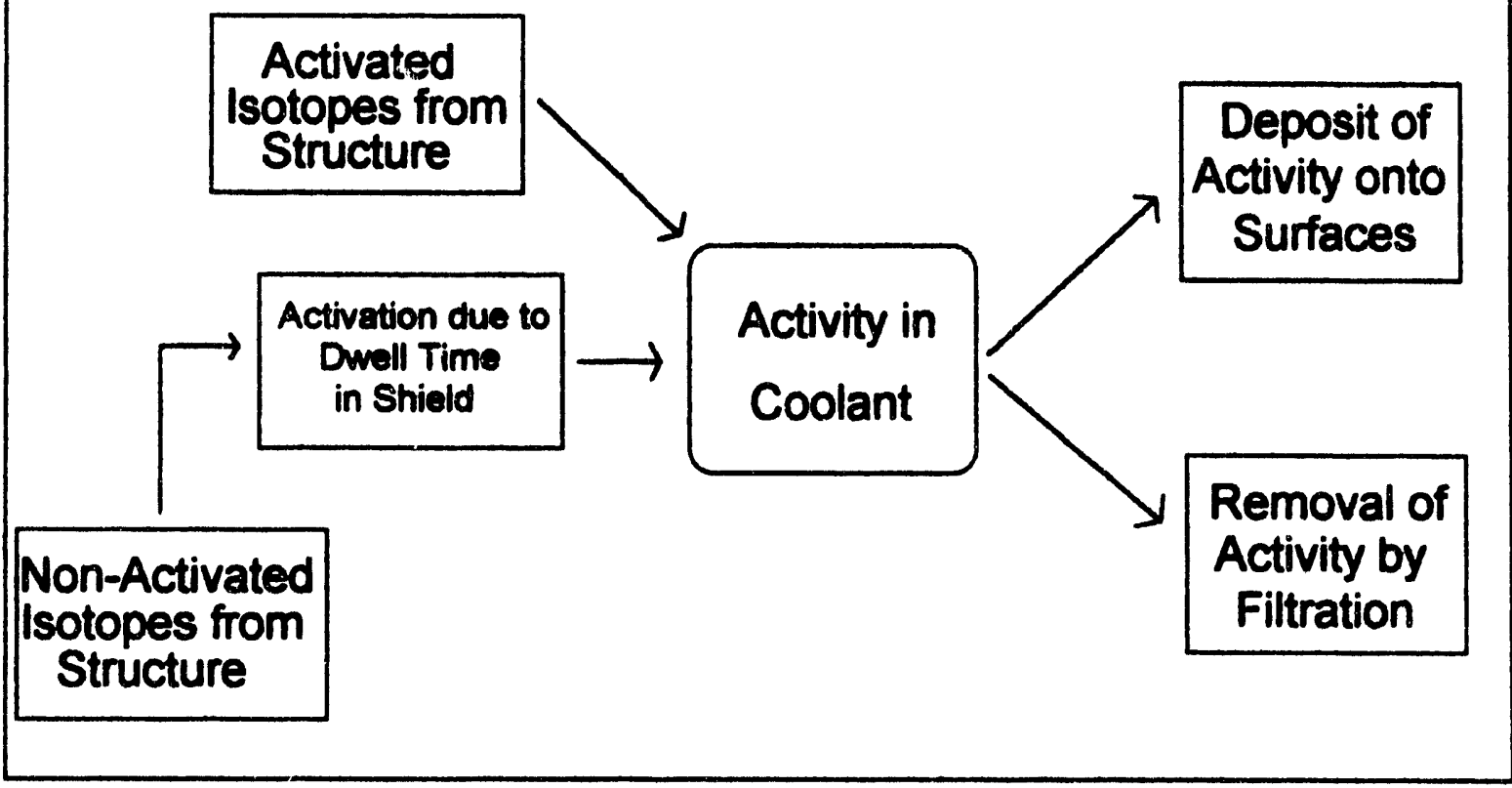

${ }^{28}$ See, for example, C. B. Lee, Modeling of Corrosion Product Transport in PWR Primary Coolant, MIT Nuclear Engineering Ph. D. Thesis, 1990. 
For the present conceptual design study, however, such detail is not warranted. Rather, a simpler model, which embodies the basic mechanisms responsible for corrosion product transport, is used instead. This model was developed as part of the STARFIRE reactor study to evaluate the level of corrosion products in the water-cooled blanket system for that reactor ${ }^{29}$ The current study modifies this model to adapt it to the lower temperature of SWS coolant loop.

Figure 6.20 shows the mechanisms for transport of activity to and from the SWS coolant. Activated corrosion products from the shield structure can be deposited directly to the coolant in either particulate or ionic form. Non-activated corrosion products can also be deposited to the coolant from other components in the SWS and become activated as it passes through the shield and sees the neutron flux there. Once in the coolant, activated products may be redeposited onto surfaces again, removing the products from circulation. Activation may also be removed from the coolant through the action of active filters installed in the system. Magnetic filters with 90 percent efficiency and a flow rate of $46 \mathrm{~kg} / \mathrm{sec}$ were available as of the early 1980's. ${ }^{30}$ In order to minimize the amount of activation products in the SWS coolant, it is assumed that two such filters are installed in the SWS system around the SWS pump, resulting in filtration of about 10 percent of total system flow.

\section{Activity Transport Model}

The activity transport model will now be described. Following this general description, a discussion of the necessary inputs to the model will be provided. The model performs two basic functions. Firstly, it calculates the generation and transport of both activated

${ }^{29}$ C. C. Baker, et. al., STARFIRE - A Commercial Tokamak Fusion Power Plant Study, Argonne National Laboratory Report ANL/FPP-80-1, 1980, Appendix G.

${ }^{30}$ Ibid. 
and non-activated corrosion products in the system. The resulting mass balance equations provide the concentration of corrosion products circulating in the coolant. Secondly, it calculates the amount of activity in the circulating corrosion products. We begin with a description of the corrosion product generation and transport portion of the model.

\section{Corrosion Product Generation and Transport}

The corrosion product release rate from structural surfaces is assumed to occur via two mechanisms: direct release of the solid corrosion product (crud) layer from the surface, and dissolution of the crud particles and ions into the coolant. The direct release mechanism is governed by a surface release rate term $R_{r}$, which is tied to the overall corrosion rate $R$. In the literature, these terms generally have units of $\left(\mathrm{mg} / \mathrm{dm}^{2} \cdot \mathrm{mo}\right)$, or milligrams per decimeter of surface area per month. The amount of radioisotopes released via the direct release mechanism depends on the amount of each isotope present in the crud at the time of the direct release.

The dissolution of crud particles and ions is modeled by release rate terms which indicate the fraction of the particular isotope in the wall crud layer which dissolves per unit time. The dissolution rate in PWRs is higher for in-reactor surfaces than for out-of-reactor surfaces; we will assume the same relationship for in-shield and out-of-shield surfaces. Additionally, the dissolution rate constants are assumed to be the same for each isotope. Hence, we have an in-shield dissolution rate term $r_{i}$ and an out-of-shield dissolution rate term $\mathbf{r}_{\mathbf{0}}$. These terms have units $(1 / \mathrm{sec})$.

The solubility of ions in the coolant, $C_{2}$, is important in determining the total amount of corrosion product ions in the coolant. The solubility (in ppm) is a function of temperature, $\mathrm{pH}$, and ion species. It is also generally a function of the structural 
material. However, as will be discussed later, lack of data for MT/HT-9 structure in appropriate coolant chemistries necessitates the use of data based on a different structural material. In addition to the soluble ions in the coolant, there also is a certain concentration of crud particulates $C_{c}$ which circulates in the coolant. The value of $C_{c}$ is determined by a mass balance, once the corrosion product generation and removal rates are known. The total concentration of corrosion products in the coolant is thus $C_{1}=C_{2}+C_{c}$.

The corrosion product deposition rate onto surfaces is governed by a soluble ion deposition rate term $\mathbf{k}_{\mathbf{a}}$, and a crud (or particulate) deposition rate term $\mathbf{k}_{\mathrm{c}}$. Both terms have units $\left(\mathrm{g} / \mathrm{m}^{2} \cdot \mathrm{sec}\right)$. The corrosion product is assumed to have the same ratio of metal species as does the structural material (in this case, MT-9). Furthermore, both the deposition and the release of individual chemical species in the corrosion product is assumed to occur in proportion to the abundance in the corrosion product (or, equivalently, the structural material).

The final inputs needed for the corrosion product transport portion of the model are the total volumetric flow rate for the system, $Q_{1}\left(\mathrm{~m}^{3} / \mathrm{sec}\right)$, the amount of coolant filtered by the corrosion product filters per unit time, $Q_{f}\left(\mathrm{~m}^{3} / \mathrm{sec}\right)$, and the total wetted surface area in the system, $\mathbf{A}\left(\mathrm{m}^{2}\right)$. A list of all of the inputs to this portion of the model, and the values for these parameters used in the STARFIRE blanket coolant system study, are shown in Table 6.9. 
Table 6.9. Corrosion Product Transport Inputs from STARFIRE Blanket Study ${ }^{31}$

\begin{tabular}{|c|c|}
\hline Corrosion Rate, R (mg / $\mathrm{dm}^{2} \cdot \mathrm{mo}$ ) & 3 \\
\hline Surface Release Rate, $R_{,}\left(\mathrm{mg} / \mathrm{dm}^{2} \cdot \mathrm{mo}\right)$ & 1 \\
\hline \multicolumn{2}{|l|}{ Dissolution Release Rates (1/sec) } \\
\hline In-blanket, $r_{i}$ & $1.8 \times 10^{-6}$ \\
\hline Out-of-blanket, $r_{0}$ & $10^{-7}$ \\
\hline \multicolumn{2}{|l|}{ Deposition Rates $\left(\mathrm{g} / \mathrm{m}^{2} \cdot \mathrm{sec}\right)$} \\
\hline For Soluble Ions, $\mathbf{k}_{\mathrm{a}}$ & 20 \\
\hline For Particulates (Crud), $k_{c}$ & 5 \\
\hline Total Ion Solubility, C (ppm) & 5 \\
\hline Total Coolant Flow Rate, $\mathrm{Q}_{\mathrm{t}}\left(\mathrm{m}^{3} / \mathrm{sec}\right)$ & 16.6 \\
\hline Effective Filter Flow Rate, $Q_{\mathrm{f}}\left(\mathrm{m}^{3} / \mathrm{sec}\right)$ & 0.17 \\
\hline Total Wetted Surface Area, A $\left(\mathrm{m}^{2}\right)$ & 60,000 \\
\hline In-Blanket Wetted Surface Area, $A_{i}\left(m^{2}\right)$ & 12,000 \\
\hline
\end{tabular}

The coolant system conditions for the STARFIRE reactor blanket are similar to the conditions in a PWR, but are very much different than for the SWS coolant under consideration in the present work. The most notable difference is that the STARFIRE blanket coolant operated at high temperature (300 degrees C), whereas the SWS operates at a maximum temperature of 85 degrees $C$. Hence, it will be necessary to find different values for the corrosion and release rates, deposition rates, and solubility which are more pertinent to the low temperature SWS system.

${ }^{31}$ Ibid. 
Determining the solubility at a lower temperature is straightforward, since solubility data exists over a wide temperature range. Solubility data exists based for both laboratory and PWR testing. ${ }^{33} 34$ The piping materials for PWRs (and for laboratory testing oriented towards PWRs) are generally nickel-based alloys such as Inconel or Incoloy, or austenitic stainless steels such as AISI 316 SS or 304 SS. Hence, the solubility data base concentrates on coolant in equilibrium with such materials. For the SWS shield, we have chosen to use MT-9, a ferritic stainless steel, as the structural material. The rest of the piping in the system is assumed to be austenitic stainless steel. The small amount of Table 6.10. Soluble Corrosion Products for PWR piping in the Service Heat Materials (93 degrees $\mathrm{C}, \mathrm{pH}=10$. Ammoniated) ${ }^{32}$

\begin{tabular}{|c|c|}
\hline Element & Solubility (opb) \\
\hline Iron & 1.1 \\
\hline Nickel & .4 \\
\hline Chromium & .1 \\
\hline Manganese & .7 \\
\hline Cobalt & .1 \\
\hline Total & 2.4 \\
\hline
\end{tabular}

Exchanger is aluminum brass; this is not expected to affect the results of this analysis significantly. For the purposes of this study we will use data based on PWR-type materials, since there is no relevant data for ferritic steels. The corrosion product oxides which have significant solubility in water for PWR-type materials are listed in Table 6.10, along with their solubilities at 93 degrees $C$ in water with $\mathrm{NH}_{4} \mathrm{OH}$ added to maintain the $\mathrm{pH}$ at 10 . Cobalt is not an alloying element in stainless steel; it is included in this list because Co-58 is generated via $(n, p)$ reactions with Ni-58.

\footnotetext{
${ }^{32}$ P. Cohen, Water Coolant Technology of Power Reactors, Gorden and Breach Science Publishers, New York, 1969, p. 51.

${ }^{33}$ Ibid.

${ }^{34}$ C. C. Baker, et. al., STARFIRE - A Commercial Tokamak Fusion Power Plant Study, Argonne National Laboratory Report ANL/FPP-80-1, 1980, Appendix E.
} 
In addition to the elements shown in Table 6.10, MT-9 has high concentrations of vanadium and tungsten as alloying elements. In the absence of data on the solubility of these elements for the assumed conditions of the SWS coolant, it will be conservatively assumed that the solubilities of both tungsten and vanadium are equal to the solubility of the most soluble element in the table, iron. This gives a total solubility of $\mathrm{C}_{\mathbf{s}}=2.4+$ $2(1.1)=4.6 \mathrm{ppb}$.

We turn now to development of new corrosion product deposition and release rate constants applicable to the low-temperature SWS. The mechanisms for the deposition and release of corrosion products are still not well known, despite much research. Hence it is difficult to develop a concrete theoretical basis with which to determine the temperature dependence of the relevant rate constants. Using empirical data, and a simple theoretically-based model, however, an estimate of the effect of the lower temperature on the rate constants of Table 6.9 can be determined.

By gathering corrosion data from different sources, the following relation for the surface corrosion release rate, $R_{r}$, has been developed: ${ }^{35}$

$$
R_{r}(\mathrm{~cm} / \mathrm{sec})=1.59 \times 10^{-14} \exp \left(0.0046 \cdot \mathrm{T}\left({ }^{\circ} \mathrm{K}\right)\right) .
$$

Using the maximum operational SWS coolant temperature of 85 degrees $C$, and converting to a mass loss rate (using the density of MT-9), this formula yields a new $R_{r}$ $=0.2 \mathrm{mg} / \mathrm{dm}^{2} \cdot \mathrm{mo}$, which is $1 / 5$ of the release rate used in the STARFIRE study.

The corrosion rate $\mathbf{R}$, which drives the corrosion release rate, is assumed to be 3 times the surface release rate, as it is in the STARFIRE case. Hence $\mathrm{R}=0.6 \mathrm{mg} / \mathrm{dm}^{2} \cdot \mathrm{mo}$.

${ }^{35}$ A.C. Klein, Activation Proctuct Transpont in Fusion Reactors, Nuclear Engincering PhD Thesis, University of Wisconsin, Madison, 1983, Appendix C. 
No empirical correlation as a function of temperature was found for the other rate constants of Table 6.9. Hence, we turn to a simple theoretical model to give an indication of the effect of temperature on these constants. Intuitively, one expects that as temperature drops, the processes by which the corrosion products diffuse to and from the solid surfaces will be slowed. There is evidence that the rate controlling step for corrosion product release is mass transfer across the boundary between the surface and the fluid ${ }^{36}$ If it is assumed the same is true for corrosion product deposition, then determining how temperature affects the mass transfer process will indicate how temperature affects the rate constants.

The well-known Dittus-Boelter correlation for heat transfer (for a cooled fluid) is as follows: ${ }^{37}$

$$
\mathrm{Nu}=0.023 \operatorname{Re}^{0.3} \mathrm{Pr}^{0.3},
$$

where $\mathrm{Nu}$ is the Nusselt number, Re is the Reynolds number, and $\mathrm{Pr}$ is the Prandtl number. An equivalent correlation for mass transfer is the following: ${ }^{38}$

$$
\mathrm{Sh}=0.023 \mathrm{Re}^{0.8} \mathrm{Sc}^{0.3} \text {, }
$$

where Sh is the Sherwood number, and Sc is the Schmidt number, defined below.

$$
\begin{aligned}
& \text { Sherwood Number, } S h=\frac{D_{b} h}{D_{\star}} \\
& \text { Schmidt Number, } S c=\frac{\mu}{\rho D_{\downarrow}} \text {, where }
\end{aligned}
$$

\footnotetext{
${ }^{36}$ See C.B. Lee, Modeling of Corrosion Product Transport in PWR Primary Coolant, Nuclear Engineering PhD Thesis, MIT, 1990, p.68.

${ }^{37}$ N.E. Todreas and M.S. Kazimi, Nuclear Systems I, Thermal Hydraulic Fundamentals, Hemisphere, 1990, p.443.

${ }^{38}$ For example, see C.B. Lee, Modeling of Corrosion Product Transport in PWR Primary Coolant, Nuclear Engincering PhD Thesis, MTT, 1990, p.56.
} 
$h=$ the mass transfer coefficient, which is proportional to the mass

transfer rate,

$D_{e}=$ the hydraulic diameter of the flow channel,

$\mu, \rho$ are the fluid viscosity and density, respectively, and

$D_{\omega b}=$ the diffusion coefficient.

Solving for the mass transfer coefficient, using the definition of the Reynolds number yields:

$$
\mathrm{h}=0.023\left(\frac{\rho}{\mu}\right)^{0.9} \frac{\mathrm{v}^{0.8}}{\mathrm{D}_{e}^{0.2}} \mathrm{D}_{\omega \mathrm{\omega}}^{0.7},
$$

where $v$ is the fluid velocity. The temperature dependence of the mass transfer coefficient $h$ is wholly contained in the diffusion coefficient $D_{\omega}$, and in the water properties $\mu$ and $\rho$. Noting that the mass transfer coefficient is proportional to the mass transfer rate, according to this simple model, the temperature dependence of the rate constants is as follows:

$$
\text { Rate Constants } \propto h \propto\left(\frac{\rho(T)}{\mu(T)}\right)^{0.9} D_{\omega}(T)^{0.7}
$$

The temperature dependence of the diffusion coefficients must now be determined. The mechanisms for diffusion of ions and particles are fundamentally different. For ions, the diffusion coefficient is determined using the Nernst-Einstein equation for an electrolyte in a dilute solution; for particles, the Stokes-Einstein relation for the mobility of a particle due to hydrodynamic friction is used. ${ }^{39}$ The diffusion coefficient for Fe+t ions has been determined as a function of temperature, and is plotted in Figure 6.21.

${ }^{39}$ loid, pp. 69.78. 
Figure 6.21 Diffusion Coefficient for $\mathrm{Fe}^{++}$Ions $^{\text {t11 }}$

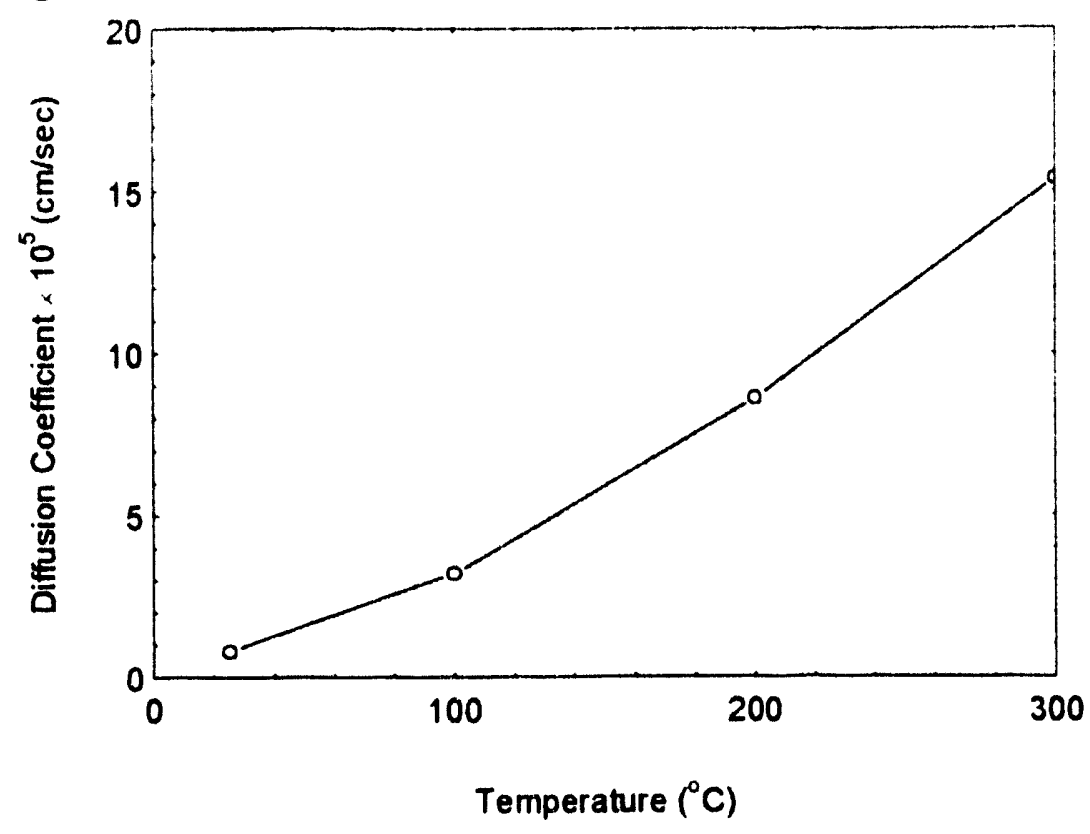

As intuitively expected. the diffusion coefficient increases with temperature, indicating that the mass transfer coefficient also increases with temperature. If it is assumed all the ion diffusion constants vary as the $\mathrm{Fe}^{++}$ diffusion constant

does, an estimate of the rate constant for ion deposition $\left(k_{\mathrm{s}}\right)$ in the SWS (at $85 \mathrm{C}$ ) can be obtained using the Nernst-Einstein equation below:

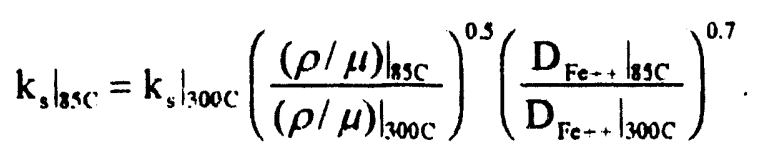

We thereby obtain a new $k_{\mathrm{s}}=3.0 \mathrm{~g} / \mathrm{m}^{2} \cdot \mathrm{sec}$, roughly $1 / 6$ of the STARFIRE value.

To calculate the effect of temperature on the diffusion coefficient for particles, the Stokes-Einstein relation for the mobility of a particle in a fluid is used. This relation is given below:

$$
D_{p}=\frac{k T}{4 \pi \mu r_{p}}
$$

where

$$
\begin{aligned}
& D_{p}=\text { the diffusion coefficient for a particle, } \\
& r_{p}=\text { the particle radius, } \\
& k=\text { Boltzmann's constant, }
\end{aligned}
$$

\footnotetext{
4)Ibid., p. 77, from the "literature values."
} 


$$
\begin{aligned}
& \mu=\text { the fluid viscosity, and } \\
& T=\text { temperature }(k)
\end{aligned}
$$

The above equation predicts the correct order of magnitude for particulate diftusion coefficients ${ }^{+1}$ Noting that the temperature dependence of the particulate diffusion coefficient is bound up in the ratio $T / \mu$, we can use Equation 6.28 again to write

$$
\begin{aligned}
& \left.k_{v}\right|_{8 s c}=\left.k_{v}\right|_{3000}\left(\frac{\left.(\rho / \mu)\right|_{396}}{\left.(\rho / \mu)\right|_{3000}}\right)^{109}\left(\frac{\left.D_{p}\right|_{896}}{\left.D_{p}\right|_{3000}}\right)^{0.7}
\end{aligned}
$$

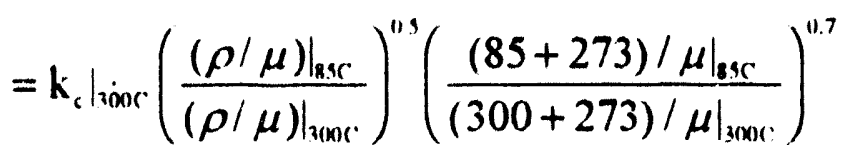

This yields a new value for $k_{c}$, the particulate, or crud, concentration of $0.4 \mathrm{~g} / \mathrm{m}^{2} \cdot \mathrm{sec}$, or about $1 / 10$ the STARFIRE value.

Revised values have been found for all of the rate constants of Table 6.9 except for the dissolution release rates $r_{i}$ and $r_{w}$. For these dissolution rates, no distinction is made between particulate and ion release rates. Hence it is not clear whether these release rates are governed by particulate or ion diffusion coefficients. For the present work, it will be conservatively assumed that ion diffusion governs these release rates. This results in higher release rates, because the ion diffusion rate dropped less percentage-wise than the particle diffusion rate between the STARFIRE and SWS cases. Using this assumption, we obtain:

$$
\left.r_{i, 0}\right|_{8 s C}=\left.r_{i, 0}\right|_{300 C} \frac{\left.k_{s}\right|_{8 s c}}{\left.k_{6}\right|_{300 C}} .
$$

\footnotetext{
"libid.. p. 78.
} 
Thus the revised values for these rate constants are: $r_{i}=2.7 \times 10^{7} \mathrm{sec}^{-1}$, and $r_{11}=1.5 \times 10^{-1} \sec ^{-1}$.

All of the rate constants in Table 6.9 have now been revised to values appropriate for use in the low-temperature Shield Water System. Assuming that the SWS has two magnetic corrosion product filters with an effective flow rate of $2 \cdot 46 \cdot 0.90=82.8 \mathrm{~kg} / \mathrm{sec}$ (as discussed at the beginning of this section), a new table similar to Table 6.9 can be generated for the SWS (see Table 6.11).

Table 6.11. Corrosion Product Transport Inputs for the SWS

\begin{tabular}{|c|c|}
\hline Corrosion Rate, $R\left(\mathrm{mg} / \mathrm{dm}^{2} \cdot \mathrm{mo}\right)$ & 0.6 \\
\hline Surface Release Rate, $R_{f}\left(\mathrm{mg} / \mathrm{dm}^{2} \cdot \mathrm{mo}\right)$ & 0.2 \\
\hline \multicolumn{2}{|l|}{ Dissolution Release Rates (1/sec) } \\
\hline In-blanket, $r_{i}$ & $2.7 \times 10^{-7}$ \\
\hline Out-of-blanket, $r_{0}$ & $1.5 \times 10^{-8}$ \\
\hline \multicolumn{2}{|l|}{ Deposition Rates $\left(\mathrm{g} / \mathrm{m}^{2} \cdot \mathrm{sec}\right)$} \\
\hline For Soluble Ions, $k_{3}$ & 3 \\
\hline For Particulates (Crud), $\mathbf{k}_{\text {。 }}$ & 0.4 \\
\hline Total Ion Solubility, $C_{s}$ (ppm) & 4.6 \\
\hline Total Wetted Surface Ar $A\left(\mathrm{~m}^{2}\right)$ & 5300 \\
\hline Surface Area in Neutron Flux, $A_{i}\left(m^{2}\right)$ & 1470 \\
\hline Total Coolant Flow Rate, $\mathrm{Q}_{\mathrm{t}}\left(\mathrm{m}^{3} / \mathrm{sec}\right)$ & 0.714 \\
\hline Efrective Filter Flow Rate, $\mathrm{Q}_{\mathrm{f}}\left(\mathrm{m}^{3} / \mathrm{sec}\right)$ & 0.083 \\
\hline
\end{tabular}




\section{Activity Calculations}

The method for determining the level of activity in the coolant is now discussed.

Referring back to Figure 6.20 , we note that activity can enter the coolant directly, from activated corrosion products in the shield structure, or by non-activated corrosion products from elsewhere in the system becoming activated as they pass through the neutron flux of the shield. Each of these mechanisms is now discussed in turn.

The activity of the corrosion products adhering to shield structure can be calculated by assuming that the radionuclides are all in steady state, with the decay rate matching the production rate. This assumption is conservative in that it maximizes the activity level, and is reasonable for most of the radionuclides of interest, assuming the reactor has been operating for a number of years. The decay rate for all of the atoms of a particular isotope in a given amount of MT-9 corrosion product is given by the product of the decay constant $\lambda(1 / \mathrm{sec})$ and the number of atoms of that isotope per $\mathrm{kg}$ of MT-9, $\beta$ (atoms $/ \mathrm{kg}$ ). ${ }^{42}$ Production of a given isotope occurs when a particular target nuclide is hit by a neutron. The production rate of a given isotope is hence given by the product of the number of target atoms per $\mathrm{kg}$ MT-9, $\mathrm{C}_{\mathbf{2}}$ (atoms $/ \mathrm{kg}$ ) and the transmutation rate for that isotope. The transmutation rate is given in turm by the product of the flux and target cross sections, $\sigma \phi(1 / \mathrm{sec})$. Setting the production rate equal to the decay rate thus yields:

$$
\lambda \beta=C_{-} \sigma \phi
$$

The TWODANT neutronics code and REAC3 activation code provide the values for the specific activity $(\lambda \beta)$ for various regions in the blanket. Knowing $C_{w}$ for the target

\footnotetext{
${ }^{42}$ We recall that the corrosion product is assumed to have the same composition of metal species as does the parent structural material.
} 
nuclides for MT-9 yields the appropriate values for the transmutation rate $(\sigma \phi)$ for the isotopes of interest. The isotopes contributing most to the activity in the MT-9 shield structure during operation are as follows: Cr-51, Mn-56, Fe-55, W-185, W-187, Mo-93, Mo-99 and Co-58. These isotopes contribute over 94 percent of the activity in the shield structure after 30 years of operation. Isotopes of molybdenum (which generate 4 percent of the shield activity) have generally not been found in the coolant of PWR reactors, despite their presence in the PWR piping structure. ${ }^{43}$ Therefore, we will assume that the molybdenum isotopes are not released to the SWS coolant, and will not consider them further.

The specific activity for the various isotopes drops off rapidly as a function of distance from the front of the shield. At $31 \mathrm{~cm}$ behind the front of the shield, the specific activity values drop by over an order of magnitude from the activity at the first wall. Hence, the contribution from the portion of the shield behind the $31 \mathrm{~cm}$ point is negligible.

Therefore, only corrosion products from the first $31 \mathrm{~cm}$ of the shield will be assumed to contribute to the activity in the SWS coolant. The specific activity values for the corrosion products in this section of the shield are conservatively assumed to be the average of the values at the front of the shield and the values at $31 \mathrm{~cm}$. Table 6.12 shows these average specific activity values for each radionuclide, along with the corresponding parent isotope density and transmutation rate values.

\footnotetext{
${ }^{43}$ C. C. Baker, et. al., STARFIRE - A Commencial Tokamak Fusion Power Plant Study, Argonne National Laboratory Report ANL/FPP-80-1, 1980, Appendix G.
} 
Table 6.12. Activity Parameters for SWS

\begin{tabular}{|c|c|c|c|c|c|}
\hline$\frac{\text { Radioisotope }}{\text { Name }}$ & $\begin{array}{c}\text { Decay } \\
\text { Constant } \\
(\lambda), 1 / \mathrm{sec}\end{array}$ & $\frac{\text { Specific Activity }}{(\lambda \beta), 1 / \mathrm{kg} \cdot \sec }$ & $\begin{array}{l}\frac{\text { Number }}{\text { Density in }} \\
\text { MT-9(B) } \\
\text { atoms/kg }\end{array}$ & $\begin{array}{l}\begin{array}{c}\text { Parent Nuclei } \\
\text { Isotope and } \\
\text { Number Density }\end{array} \\
\frac{\text { in MT-9 } 9}{\text { atoms/kg }}\end{array}$ & $\frac{\frac{\text { Transmutation }}{\text { Rates }(\sigma \phi)}}{1 / \mathrm{sec}}$ \\
\hline $\mathrm{Cr}-51$ & $2.9 e-7$ & $4.3 \mathrm{e} 11$ & $1.5 \mathrm{e} 18$ & $\mathrm{Cr}-50,5.8 \mathrm{e} 22$ & $7.4 e-12$ \\
\hline $\mathrm{Mn}-56$ & $7.5 e-5$ & $1.3 \mathrm{e} 12$ & $1.8 \mathrm{e} 16$ & $\mathrm{Mn}-55,6.0 \mathrm{e} 22$ & $2.2 \mathrm{e}-11$ \\
\hline $\mathrm{Fe}-55$ & $8.5 \mathrm{e}-9$ & $1.1 \mathrm{e} 12$ & $1.3 \mathrm{e} 20$ & $\mathrm{Fe}-54,5.4 \mathrm{e} 25$ & $2.0 \mathrm{e}-14$ \\
\hline W-185 & $1.1 \mathrm{e}-7$ & $2.4 \mathrm{e} 11$ & $2.2 \mathrm{e} 18$ & $\mathrm{~W}-184,3.4 \mathrm{e} 21$ & $6.9 e-11$ \\
\hline W-187 & $8.0 \mathrm{e}-6$ & $1.8 \mathrm{e} 12$ & $2.3 \mathrm{e} 17$ & $\mathrm{~W}-186,3.2 \mathrm{e} 21$ & $5.7 e-10$ \\
\hline Co-58 & $1.1 \mathrm{e}-7$ & $5.0 \mathrm{e} 09$ & $4.4 \mathrm{e} 16$ & $\mathrm{Ni}-58,3.5 \mathrm{e} 22$ & $1.4 \mathrm{e}-13$ \\
\hline
\end{tabular}

\section{Transport Equations}

Now that the required transport and activity inputs have been calculated for the SWS, the appropriate equations describing the activity transport in the system may be discussed. ${ }^{44}$ The equations which describe the activity/corrosion product transport are as follows: (1) the overall corrosion product mass balance; (2) the mass balance for the in-flux ${ }^{45}$ and out-of-flux surfaces; (3) the coolant balance for each radioisotope; (4) the in-flux surface balance for each radioisotope; and (5) the out-of-flux surface balance for each radioisotope. These equations are discussed in turn below.

\section{Overall Mass Balance for Corrosion Products}

Assuming all components in the system reach steady state, the net production of corrosion products must equal the net sink. The net production is given by the corrosion

\footnotetext{
44 The following methodology is adopted from C. C. Baker, et. al., STARFIRE - A Commercial Tokamak Fusion Power Plant Study, Argonne National Laboratory Report ANL/FPP-80-1, 1980, Appendix G.

${ }^{45}$ In the neutron flux (assumed to be the first $31 \mathrm{~cm}$ of the shield).
} 
rate constant $R$ times the total surface area $A$. The total sink rate is given by the filter effective flow rate $\mathrm{Q}_{\mathrm{f}}$ multiplied by the total coolant corrosion product concentration $\mathrm{C}_{1}=\mathrm{C}_{\mathrm{s}}+\mathrm{C}_{\mathrm{c}}$. Hence we have:

$$
R \cdot A=Q_{f}\left(C_{s}+C_{c}\right)
$$

Since all parameters are known in this equation except for $C_{c}$, we can solve for it as follows:

$$
C_{c}=\frac{R \cdot A}{Q_{f}}-C_{s} .
$$

Using the SWS parameters in Table 6.11, we obtain a negative value for $C_{c}$ in the above equation. This is of course unphysical, and it indicates that the filtration rate is higher than the corrosion production rate. This effectively means that the equilibrium value for $\mathrm{C}_{\mathrm{c}}$ will be very small, and that the total corrosion product concentration will be dominated by the solubility term $C_{1}$. We will assume, therefore, that $C_{1}=C_{1}$.

\section{Corrosion Product Mass Balance for Surfaces}

For the steady-state corrosion product mass balance of both the in-flux and out-of-flux surfaces, the addition of corrosion product by corrosion of the surfaces and deposition of corrosion product from the coolant onto the surfaces must balance the removal of corrosion by both surface release and dissolution release mechanisms. For the in-flux surfaces, this means the following equation must be satisfied:

$$
R+k_{i} C_{s}+k_{c} C_{c}=R_{r}+r_{i} W_{i},
$$

where $W_{i}$ is the corrosion product surface thickness on the in-flux surfaces, and the other parameters have been previously discussed and evaluated. Similarly, we can write for the out-of-flux surfaces:

$$
R+k_{s} C_{c}+k_{c} C_{c}=R_{r}+r_{0} W_{o},
$$


where $\mathrm{W}_{\mathrm{o}}$ is the corrosion product surface thickness on the out-of-flux surfaces. We can now solve for the corrosion product surface thicknesses, and recalling that $C_{\mathrm{s}} \cong 0$, we get:

$$
\mathrm{W}_{\mathrm{i}, \mathrm{o}}=\frac{\mathrm{R}-\mathrm{R}_{\mathrm{r}}+\mathrm{k}_{\mathrm{s}} \mathrm{C}_{\mathrm{s}}}{\mathrm{r}_{\mathrm{i}, \mathrm{o}}} .
$$

For the SWS, we obtain $\mathrm{W}_{\mathrm{i}}=0.1 \mathrm{~mm}$, and $\mathrm{W}_{\mathrm{o}}=1.7 \mathrm{~mm}$.

\section{Coolant Balance for Radioisotopes}

The equation for the balance of each radioisotope in the coolant is as follows:

The change in the total number of atoms of the radioisotope in the coolant per unit time $($ atoms $/ \mathrm{sec})=$

Term 1 Isotope production due to dwell time in shield

Term $2+$ Addition due to release of corrosion product surface

Term $3+$ Addition due to dissolution of corrosion product surface

Term 4 - Removal by deposition on surfaces

Term 5 - Removal by decay in coolant

Term 6 - Removal by filtration.

Each of these terms is now discussed, and their magnitudes determined.

Term 1, the isotope production due to dwell time, is given by the following relation:

$$
\text { Term } 1=U \cdot C_{m, \text { ino }} \cdot V \cdot(\sigma \phi)_{\text {ino }},
$$

where $\mathrm{C}_{\text {a,io }}$ is the concentration of the target isotope which generates the radioisotope of interest, $\mathrm{U}$ is the fraction of coolant in the neutron flux field, $\mathrm{V}$ is the total volume of coolant, and $(\sigma \phi)_{\text {io }}$ is the transmutation rate for isotope "iso" (see Table 6.12).

Term 2 , the addition due to the release of the corrosion product surface, is given by: 


$$
\text { Term } 2=\mathrm{R}_{r} \cdot \mathrm{A}_{\mathrm{i}} \cdot \boldsymbol{\beta}_{\mathrm{iso}},
$$

where $R_{t} \cdot A_{i}$ is the total release rate of the corrosion product surface for the irradiated portion of the system, and $\beta_{\text {ito }}$ is the density of isotope $i$ in the corrosion product. These terms are known from Tables 6.11 and 6.12.

Term 3, the addition due to dissolution of the corrosion product surfaces, is given by:

$$
\text { Term } 3=r_{i} A_{i} \omega_{i, \text { ivo }}+r_{0} A_{0} \omega_{\text {oimo }} \text {, }
$$

where the $r$ terms and $A_{i}$ are listed in Table 6.11, $A_{o}$ is equal to $A-A_{i}$ (the out-ofshield surface area), and $\omega_{i, \text { iso }}$ and $\omega_{\text {o,iso }}$ are the number of isotope atoms on the in-shield and out-of-shield surface corrosion product deposits per unit area, respectively. $\omega_{i, \text {,wo }}$ and $\omega_{\text {o.ieo }}$ are unknowns, and will be eliminated shortly using the in- and out-of-shield surface balance equations.

Term 4, removal by deposition on surfaces, is given by:

$$
\text { Term } 4=-\mathbf{k} \cdot \mathbf{A} \cdot \gamma_{\text {ivo }},
$$

where $\gamma_{\text {ino }}$ is the number of radioisotope atoms per volume of coolant (which is the variable to be solved for), and $\mathbf{k}$ is the corrosion product deposition rate per unit surface area (atoms $/ \mathrm{m}^{2} \cdot \mathrm{sec}$ ) divided by the total corrosion product concentration in the coolant (atoms $/ \mathrm{m}^{3}$ ). Since the concentration of corrosion products in the coolant and the deposition rate constants are known, we can determine $k$, which turns out to be about $10^{-6} \mathrm{~m} / \mathrm{sec}$.

Term 5, removal by decay in coolant, is given by:

$$
\text { Term } 5=-V \cdot \lambda_{\text {ioo }} \cdot \gamma_{\text {ino }} \text {, }
$$

where $\lambda_{\text {ino }}$ is the radioisotope's decay constant, and Term 6 , removal by filtration, is given by:

$$
\text { Term 6 }=-Q_{\mathrm{f}} \cdot \gamma_{\text {ino }} \text {. }
$$


Finally, then, the following equation is obtained for the balance of each radioisotope in the coolant. Here steady state is assumed, so that the concentration in the coolant is assumed to be constant:

$$
\begin{aligned}
& 0=U \cdot C_{s s, i s o} \cdot V \cdot(\sigma \phi)_{i \text { seo }}+R_{r} \cdot A \cdot \beta_{i s o}+\left(r_{i} A_{i} \omega_{i, i s o}+r_{0} A_{0} \omega_{0, \text { iso }}\right) \\
& -\mathrm{k} \cdot \mathrm{A} \cdot \gamma_{\mathrm{iso}}-\mathrm{V} \cdot \lambda_{\text {iso }} \cdot \gamma_{\text {iso }}-\mathrm{Q}_{\mathrm{f}} \cdot \gamma_{\text {iso }}
\end{aligned}
$$

\section{In-Flux Surface Balance}

The balance for each radioisotope for the wall surfaces in the neutron flux (assumed to be the surfaces in the first $31 \mathrm{~cm}$ from the front of the shield) is given by the following equation:

The change in the number of radioisotope atoms per unit area of in-flux surface

$$
\text { per unit time }=\frac{d \omega_{i, \text { ieo }}}{d t}\left(\text { atoms } / \mathrm{m}^{2} \cdot \sec \right)=
$$

Term la Production of radioisotopes in the surface deposit (for in-flux surfaces)

Term $2 \mathrm{a}+$ Addition due to activated corrosion products remaining on the surface

Term 3a + Deposition of radioisotopes from coolant

Term 4a - Removal by dissolution into coolant

Term 5a - Removal by decay.

Term la, the production in the surface deposit, is given by:

$$
\operatorname{Term} 1 \mathrm{a}=\mathrm{W}_{\mathrm{i}, \mathrm{ino}}(\sigma \phi)_{\mathrm{iso}},
$$

where $\mathrm{W}_{i, \text { ino }}$ is the number of target atoms per unit area of surface deposit $\left(=\mathrm{W}_{\mathrm{i}} \cdot \mathrm{C}_{\mathbf{w , i n o}}\right)$.

Both components of this term have previously been evaluated. Term $2 \mathrm{a}$, the addition from corrosion, is given by:

$$
\operatorname{Term} 2 \mathrm{a}=\left(\mathrm{R}-\mathrm{R}_{\mathrm{r}}\right) \boldsymbol{\beta}_{\mathrm{ieo}}
$$


The quantity $\left(R-R_{r}\right)$ is the amount of corrosion product which is not lost via surface release into the coolant. All quantities in Term 2a are known.

Term 3a, the deposition of radioisotopes from the coolant, is related to Term 4 from Equation 6.44 , but is defined per unit area. Similarly terms $4 a$ and $5 a$ correspond to terms 3 and 5 of Equation 6.44. The equations for these terms are as follows:

$$
\begin{aligned}
& \text { Term 3a }=k \cdot \gamma_{i s o}, \\
& \text { Term } 4 a=-r_{i} \cdot \omega_{i, \text { iso }}, \\
& \text { Term } 5 a=-\lambda_{\text {iso }} \cdot \omega_{i, \text { iso }} .
\end{aligned}
$$

Combining these terms, the mass balance for the in-flux surfaces in steady-state is:

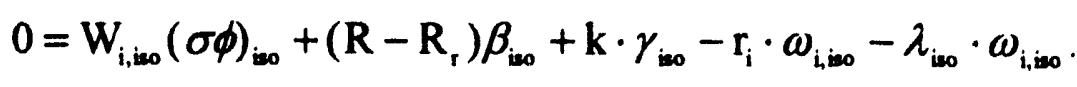

In this equation, all quantities are known except for $\gamma_{\text {iso }}$, which is the term we desire, and $\omega_{i, \text { iso }}$.

\section{Out-of-Flux Surface Balance}

For the out-of-flux surfaces, a similar mass balance equation can be formulated, except that there is no production of radioisotopes in the surface deposit, and no addition of radioisotopes due to corrosion of the out-of-flux surfaces. Hence, the first two terms of Equation 6.50 vanish, and we have:

$$
0=k \cdot \gamma_{\text {ino }}-r_{0} \cdot \omega_{0, i \infty 0}-\lambda_{\text {ino }} \cdot \omega_{0, i v 0} .
$$

$\omega_{0}$ represents an additional unknown. Rearranging the above two equations, and recalling that $\beta_{\text {ino }}=(\sigma \phi)_{i n o} \cdot C_{m, i \infty 0} / \lambda_{i n o}$, the unknowns $\omega_{i, i n o}$ and $\omega_{0, \text { ino }}$ can be solved for:

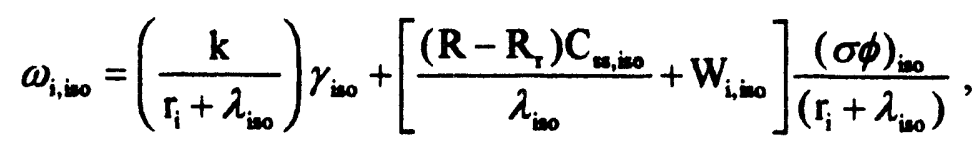

and 


$$
\omega_{0 . \text { iso }}=\left(\frac{\mathrm{k}}{\mathrm{r}_{\mathrm{o}}+\lambda_{\mathrm{iso}}}\right) \gamma_{\text {iso }} .
$$

If we now substitute Equations 6.52 and 6.53 into Equation 6.44 yields the relation for the concentration of each radioisotope in the SWS coolant, to wit:

$\gamma_{\text {soo }}\left(\right.$ atoms $\left./ \mathrm{m}^{3}\right)=$

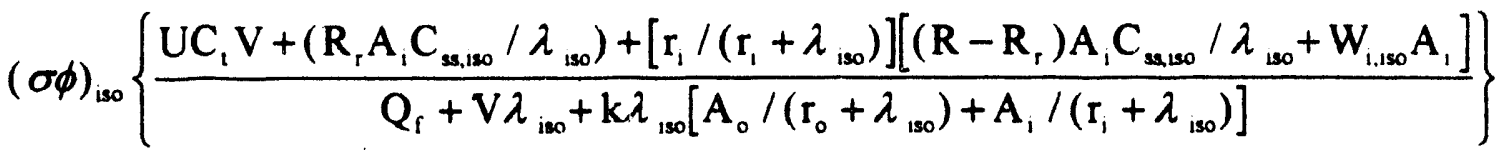

This equation can be simplified somewhat by noting that the production of radioisotopes in the coolant due to dwell time in the in-flux region is significantly less than the addition of isotopes to the coolant from the activated surface deposits. Mathematically, this means that the quantity $\mathrm{UC}_{\mathrm{t}} \mathrm{V}$ is substantially smaller that the other terms in the numerator of Equation 6.54, which is true for the radioisotopes of interest here. Further simplification can be achieved by noting that the decay of radioisotopes while in the coolant makes a negligible impact on the coolant concentrations, since:

$$
V \lambda_{\text {ino }} \ll<Q_{f}
$$

for all isotopes of interest. With these simplifications, and recalling that $R_{r}=R / 3$, we finally obtain:

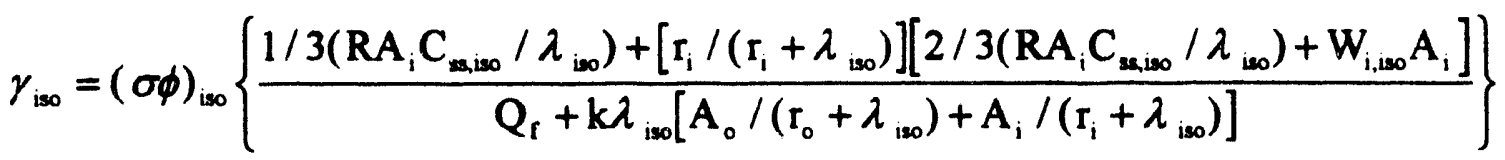

All of the terms on the left side of this equation are known. 
Multiplying the radioisotope concentrations in the coolant (obtained in the above equation) by their corresponding decay constants yields the activity for each radioisotope per unit volume of coolant. These values are shown in Table 6.13 , together with the maximum permissible concentration levels (MPC's) for each isotope. The MPC's are based on criteria for soluble radioisotopes released to unrestricted areas

\section{Table 6.13. Corrosion Product Activity Levels in SWS Coolant ${ }^{46}$}

\begin{tabular}{|c|c|c|}
\hline Radioisotope & Coolant Activity $\left(\mathrm{Ci} / \mathrm{m}^{3}\right)$ & MPC $\left(\mathrm{Ci} / \mathrm{m}^{3}\right)$ \\
\hline Cr-51 & $5.1 \mathrm{e}-6$ & $2.0 \mathrm{e}-3$ \\
\hline Fe-55 & $1.0 \mathrm{e}-5$ & $8.0 \mathrm{e}-4$ \\
\hline W-185 & $2.5 \mathrm{e}-6$ & $1.0 \mathrm{e}-4$ \\
\hline W-187 & $2.7 \mathrm{e}-5$ & $7.0 \mathrm{e}-5$ \\
\hline Co-58 & $5.3 \mathrm{e}-8$ & $1.0 \mathrm{e}-4$ \\
\hline Mn-56 & $2.0 \mathrm{e}-5$ & $1.0 \mathrm{e}-4$ \\
\hline
\end{tabular}

As shown in the above table, the activity of each corrosion product isotope is below the MPC level for unrestricted release of the SWS water.

In addition to ensuring that each isotope is below its MPC level, the total activity of all of the isotopes in the released water must be limited to allow for unrestricted release of the water. To limit the total activity, the following equation must be satisfied:

$$
\frac{\mathrm{C}_{\mathrm{A}}}{\mathrm{MPC}_{\mathrm{A}}}+\frac{\mathrm{C}_{\mathrm{B}}}{\mathrm{MPC}_{\mathrm{B}}}+\frac{\mathrm{C}_{\mathrm{C}}}{\mathrm{MPC}_{\mathrm{C}}}+\ldots \leq 1
$$

\footnotetext{
46MPC's from the Code of Federal Regulations - 10CFR20, Title 10, Part 20 - Standards for Protection Against Radiation (U.S. Government Printing Office, 1/1/1993). Values for soluble radionuclides used because the concentration of solubles is much greater than the concentration of insolubles in the SWS coolant.
} 
where $C_{A}, C_{B}$, etc. are the specific activities (or concentrations) of isotopes $A, B$, etc., and $M P C_{A}, M P C_{B}$, etc. are the corresponding maximum permissible concentrations. For the SWS, the summation on the left-hand-side of the above equation is dominated by contributions from $\mathrm{W}-187$ and $\mathrm{Mn}-56$, and is equal to 0.6 . Because this summation is less than 1, the SWS coolant water has low enough total activity to be released to an unrestricted area, based on the analysis presented here.

\section{Airborne Activity}

The results just discussed indicate that a release of coolant water from the SWS is not an environmental concern, as long as the radioisotopes remain waterborne. However, there is a possibility, in the event of a coolant leak in the Auxiliary Heat Exchanger, of these isotopes becoming airborne due to the flow of air through the cooling tower. Clearly, even in the event that all of the leaked water evaporated into the cooling tower airstream, not all of the radioisotopes would necessarily become entrained in the airstream. Since these isotopes are not volatile, a fraction of them, perhaps a large fraction, will probably remain on the heat exchanger surfaces after the water evaporates. Additionally, since the flow rate of air through the cooling tower is substantial, there will be a large dilution of the concentrations of any isotopes which become entrained in the airflow.

In order to determine the concentration of radioisotopes in the cooling tower air in the event of a leak in the Auxiliary Heat Exchanger, it is necessary to have an estimate of what fraction of the isotopes in the cooling water become entrained in the cooling tower air flow. This is a very difficult problem, depending not only on the coolant leak rate and air flow rate through the tower, but also on the location of the coolant leak, the inlet air conditions (temperature, humidity), the heat exchanger surface conditions, and the detailed air flow profiles in the vicinity of the leak. Such a detailed estimate is beyond 
the scope of the current conceptual design study. In lieu of such an estimate, then, a very conservative approach will be taken which will provide an upper bound to the airborne concentrations in the cooling tower air which could result from the largest conceivable leak rate into the tower air flow.

This conservative approach assumes the following:

(1) All radioisotopes present in the leaked coolant are immediately released to the air. No isotopes remain in the coolant, or plate out on surfaces.

(2) Any radioisotopes which enter the air flowing through the tower are uniformly mixed into the air as it exits the tower.

(3) The largest conceivable leak rate into the tower air flow results from a doubleended shear of a tube in the Auxiliary Heat Exchanger (AHE).

Note that although a double-ended shear of an AHE tube would not result in the largest conceivable leak rate from the system (since a double-ended shear of the main system piping would clearly result in a higher leak rate), the AHE tubes are the only portion of the system located in the air flow path for the cooling tower air. The flow rate through the ruptured tube is assumed (conservatively) to be driven by the total pressure head developed by the SWS pump, and the tube length through which the leak flows is assumed to be negligible.

To calculate the air flow rate through the tower during normal operation, it will be recalled that hot $(85 \mathrm{C})$ primary cooling water from the shield enters the Auxiliary Heat Exchanger/cooling tower complex before entering the Service Heat Exchanger (refer back to Figure 6.5). This hot water, flowing rapidly through the Auxiliary Heat Exchanger under the action of the jet pump, heats up air in the cooling tower and results in a substantial air flow rate through the tower. 
A thermal hydraulic analysis of the Auxiliary Heat Exchanger/cooling tower complex described earlier in this chapter has shown that the air flow rate through the tower during normal operation is $290 \mathrm{~m}^{3} / \mathrm{sec}$. This is somewhat less than the flow rate which occurs during accident operation, because the water in the Auxiliary Heat Exchanger is at a lower average temperature during normal operation. With the air flow rate known, the radioisotopic activities in the cooling tower air can be calculated. Table 6.14 shows the results. The MPC's in the table are again for soluble radionuclides.

Table 6.14. Corrosion Product Activity Levels in Cooling Tower Air ${ }^{77}$

\begin{tabular}{|c|c|c|}
\hline Radioisotope & Activity in Air $\left(\mathrm{Ci} / \mathrm{m}^{3}\right)$ & MPC in Air $\left(\mathrm{Ci} / \mathrm{m}^{3}\right)$ \\
\hline Cr-51 & $2.9 \mathrm{e}-11$ & $4.0 \mathrm{e}-7$ \\
\hline Fe-55 & $5.8 \mathrm{e}-11$ & $3.0 \mathrm{e}-8$ \\
\hline W-185 & $1.4 \mathrm{e}-11$ & $3.0 \mathrm{e}-8$ \\
\hline W-187 & $1.5 \mathrm{e}-10$ & $2.0 \mathrm{e}-8$ \\
\hline Co-58 & $3.0 \mathrm{e}-13$ & $3.0 \mathrm{e}-8$ \\
\hline Mn-56 & $1.1 \mathrm{e}-10$ & $3.0 \mathrm{e}-8$ \\
\hline
\end{tabular}

The table above shows that the specific activities for all of the corrosion products in the cooling tower air are more than 2 orders of magnitude lower than the corresponding MPC. Obviously, the total activity level would also satisfy an equation of the form of Equation 6.57. Hence, we can conclude that the corrosion product activity released into

\footnotetext{
${ }^{47}$ MPC's from the Code of Federal Regulations - 10CFR20, Title 10, Part 20 - Standards for Protection Against Radiation (U.S. Government Printing Office, 1/1/1993). Values for soluble radionuclides used because the concentration of solubles is much greater than the concentration of insolubles in the SWS coolant.
} 
the air flowing through the tower from a Auxiliary Heat Exchanger tube rupture will not exceed the values specified for release to unrestricted areas. 


\subsubsection{Argon-t1 Activity'}

As discussed previously in this chapter, argon is the most significant impurity in the SWS coolant from an activation standpoint. Argon gas is a constituent of the atmosphere, and has a significant solubility in water. Argon is composed of 99.6 percent $\mathrm{Ar}-40$, which converts to Ar-41 under neutron irradiation. Ar-41 in turn decays with a halflife of 1.83 hours, emitting both beta and gamma radiation. The halflife of argon is much longer than the SWS coolant loop transit time of about 4 minutes. Hence, the Ar-41 will not decay much during transport around the loop, and the Ar-41 activity level will be relatively constant around the loop at an equilibrium level determined by the neutron flux in the shield and the argon gas concentration in the coolant.

The federal Standards for Protection Against Radiation (10CFR20) specify no MPC in water for Ar-41, since it is a noble gas. Hence, the concern is airborne contamination resulting from a leak in the Auxiliary Heat Exchanger. We will calculate the airborne Ar41 activity levels in the air exiting the cooling tower, using methods described in the previous section. The assumptions used in this analysis are similar to those used in the previous section, namely:

(1) All Ar-41 present in the leaked coolant is immediately released to the cooling tower air.

(2) Any Ar-41 which enters the air flowing through the tower is uniformly mixed into the air as it exits the tower.

(3) The largest conceivable leak rate into the tower air flcw results from a double-ended shear of a tube in the Auxiliary Heat Exchanger (AHE).

We will first assume that the argon gas is present in the coolant at the saturation level (that is $5.6 \mathrm{cc}$ argon for every $100 \mathrm{cc}$ coolant, as discussed in Section 6.10.1). The argon activity level in the coolant and in the air exiting the cooling tower for this case are 
shown in Table 6.15. The Ar-41 coolant activity was calculated using the TWODANT and REAC3 codes.

Table 6.15. Ar-41 Activity Levels in Cooling Tower Air (Argon at Saturation) ${ }^{48}$

\begin{tabular}{|c|c|c|}
\hline$\frac{\text { Ar-41 Coolant Activity }}{\left(\mathrm{Ci} / \mathrm{m}^{3}\right)}$ & $\frac{\text { Activity in Air }}{\left(\mathrm{Ci} / \mathrm{m}^{3}\right)}$ & $\frac{\text { MPC in Air }}{\left(\mathrm{Ci} / \mathrm{m}^{3}\right)}$ \\
\hline 19 & $1.1 \mathrm{e}-4$ & $4.0 \mathrm{e}-8$ \\
\hline
\end{tabular}

As seen in the table, the argon activity in the cooling tower air is much higher than the MPC value when the argon is at saturation levels in the coolant water. Hence, a way must be found to reduce the Ar-41 activity in the SWS coolant.

The Ar-41 activity in the coolant depends only on the neutron flux in the shield and the concentration of argon in the coolant, as mentioned before. Since the neutron flux in the shield cannot be easily changed, the level of argon in the coolant must be reduced. $\mathbf{A}$ common way to reduce the concentration of gases in power plant coolants is to deaerate the coolant water. A brief discussion of deaeration is now provided. ${ }^{49}$

A typical deaerator reduces concentration of oxygen and most other dissolved gases to less than 0.005 cc/liter. ${ }^{50}$ Gases which form compounds with water, such as ammonia, may not be reduced to such levels. A deaerater functions by heating the water to saturation temperature, spraying it over trays to scrub the dissolved gases out, and sweeping the gases away by a supply of steam. The steam supply for the deaerator can

\footnotetext{
${ }^{48}$ The argon MPC is from 10 CFR 20 - The Code of Federal Regulations, Title 10, Part 20, Standards for Protection Against Radiation, U. S. Government Printing Office, January, 1993.

${ }^{49}$ The discussion of deacration is adapted from Chapter 9 of T. Baumeister, et.al., eds., Mark's Standard Handbook for Mechanical Engineers, Eighth Ed, McGraw-Hill, 1978.

s0Ibid., pp. 6-110 and 9-77.
} 
be obtained from the reactor's steam plant auxiliary steam supply. Tray-type deaerators are insensitive to water inlet temperature, and operate under wide load conditions. In addition to reducing the argon level in the SWS coolant, deaeration will also reduce corrosion of the SWS piping and equipment by reducing the oxygen level in the coolant. The deaerator can also act as a storage tank for SWS coolant.

With a deaerator installed in the SWS system, the argon level can be reduced to below $0.005 \mathrm{cc} / \mathrm{liter}$, which is over 10,000 times less than the saturation level. Assuming the argon level is $0.005 \mathrm{cc} / \mathrm{liter}$, we can recalculate the activity level in the cooling tower air. The results are shown in Table 6.16. As can be seen, the activity in the air is now below the MPC value. Hence, by deaerating the SWS coolant, the Ar-41 coolant activity is reduced to a level at which a Auxiliary Heat Exchanger tube rupture will not result in unacceptable Ar-41 activity in the air exiting the cooling tower.

\section{Table 6.16. Ar-41 Activity Levels in Cooling Tower Air (With Deaeration)}

\begin{tabular}{|c|c|c|}
\hline Ar-41 Coolant Activity & $\frac{\text { Activity in Air }}{\left(\mathrm{Ci} / \mathrm{m}^{3}\right)}$ & $\frac{\text { MPC in Air }}{\left(\mathrm{Ci} / \mathrm{m}^{3}\right)}$ \\
\hline$\left(\mathrm{Ci} / \mathrm{m}^{3}\right)$ & $9.6 \mathrm{e}-9$ & $4.0 \mathrm{e}-8$ \\
\hline $1.7 \mathrm{e}-3$ & &
\end{tabular}

\subsubsection{Short-Term N-16 Activity}

The radioisotopes considered in the previous sections of this chapter all have relatively long halflives. Hence, although these isotopes can be harmful to the environment in the event of a ieak, they will not cause significant occupational or public exposure doses as long as they remain in the coolant piping, since the long halflives result in relatively low activity levels. 
On the other side of the spectrum, $\mathrm{N}-16$ (which is generated by irradiation of $\mathrm{O}-16$ in the coolant) has a very short halflife of 7.3 seconds. Therefore, $\mathrm{N}-16$ is not a threat to the environment in the event of a leak. However, the activity of $\mathrm{N}-16$ during normal operation is very high, and the gamma rays which result from decay of $\mathrm{N}-16$ can shine through the SWS piping. This gamma radiation from the SWS piping and equipment is a potential operational hazard. The $\mathrm{N}-16$ gamma rays are an operational concern in watercooled fission reactors. Generally, a secondary shield encloses the reactor core and coolant piping to protect personnel against $\mathrm{N}-16$ radiation.

The $0-16(n, p) N-16$ reaction is a threshold reaction, requiring a neutron of a least $10 \mathrm{MeV}$ energy. ${ }^{51}$ Of the neutrons produced from thermal fission of U-235, less than 0.2 percent have energy above $10 \mathrm{MeV} .{ }^{32}$ The neutrons seen in a fusion reactor shield have a much harder spectrum, and the neutron flux at the front of a fusion reactor shield is similar to that of a fission reactor core. Therefore, the amount of $\mathrm{N}-16$ generated in the SWS coolant as it passes through the shield is much greater than the amount of $\mathrm{N}-16$ generated in fission reactor coolant.

To calculate the N-16 activity in the SWS coolant, we must account for the decay of N16 in the coolant piping as coolant flows through the unirradiated regions of the loop. The $\mathrm{N}-16$ activity level as the coolant exits the shield will be the highest level, and the activity drop as the coolant flows through the loop until it reaches the shield again. The $\mathrm{N}-16$ activity is not of concern for the coolant in the shield itself, since the fusion reactor, blanket, and shield will be inside a reactor compartment enclosed by secondary shielding.

\footnotetext{
${ }^{51}$ R. S. Amato, Shield Design and Radiation Analysis Course Notes, Bettis Reactor Engineering School, Bettis Atomic Power Laboratory, undated, p. 9-15.

32R. G. Jaeger, et.al., eds., Engineering Compendium on Radiation Shielding, Volume 1, SpringerVerlag, 1968, p. 69.
} 
Similarly, the SWS shield outlet piping can be shielded to protect personnel from the gamma radiation coming from coolant inside the pipe. However, the Auxiliary Heat Exchanger (AHE) would be very difficult to shield adequately to protect personnel and the environment, because of the configuration and large size of the AHE and cooling tower complex. Rather than shield the AHE, then, we will design the SWS piping system so that the radiation levels at the AHE are low enough to not require shielding. This can be done by ensuring that there is enough transit time between the shield outlet and the AHE inlet to provide adequate decay of the N-16 activity.

\subsubsection{Model for N-16 Activity in the SWS}

To determine the $\mathrm{N}-16$ activity level in the SWS as a function of position in the coolant loop, the basic flow pattern of the SWS must be modeled. Figure 6.22 shows a schematic of the SWS flow pattern.

\section{Figure 6.22. SWS Flow Pattern Schematic}

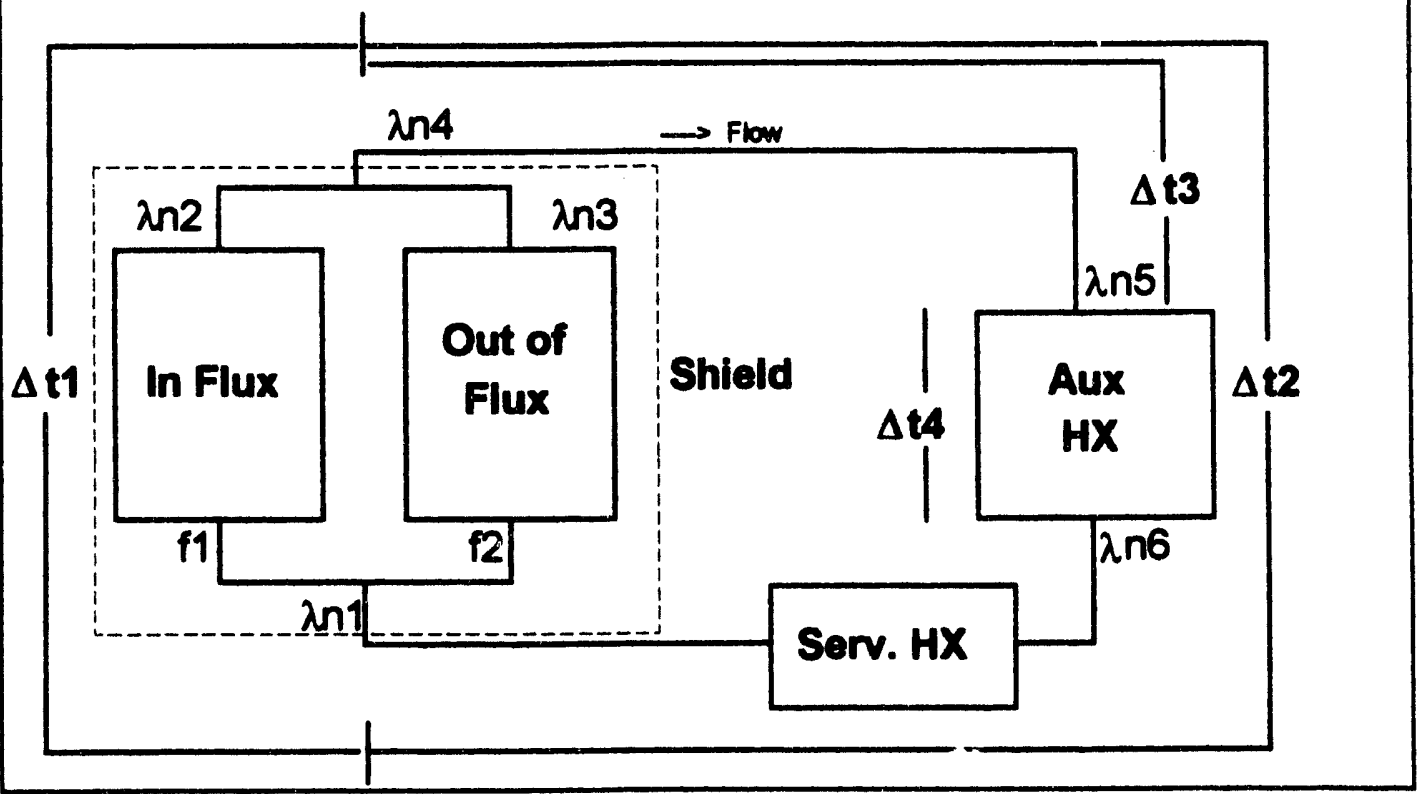


The flow schematic represents the shield as a dotted line, with the inboard third of the shield indicated as the "In-Flux" region, and the outboard two-thirds of the shield the "Out-of-Flux" region. The outboard two-thirds of the shield sees negligible neutron flux levels compared to the first third, as discussed in Section 6.10.1.1. A fraction (f1) of the coolant goes through the inboard third of the shield $(\mathrm{f} 1=1 / 3)$, and the rest of the coolant (f2) goes through the outboard two-thirds of the shield.

Coolant N-16 specific activity levels at various points in the system are indicated by $\lambda_{n} 1$ through $\lambda \mathrm{n} 6$. The $\lambda$ is the $\mathrm{N}-16$ decay constant, and $\mathrm{nl}$ through $\mathrm{n} 6$ are concentrations of $\mathrm{N}-16$ at the specified points. The highest activity level is $\lambda \mathrm{n} 2$, for coolant coming out of the inboard portion of the shield. The lowest activity level is $\lambda \mathrm{n} 1$, and the activity level for coolant entering the AHE in $\lambda \mathrm{n} 5$. Residence times for coolant in various portions of the system are indicated by $\Delta t \mathrm{l}$ through $\Delta \mathrm{t} 4$. The relationship between the activity levels and residence times is now derived. ${ }^{53}$

The equation governing the generation and decay of $\mathrm{N}-16$ activity is as follows:

$$
\frac{\mathrm{dn}}{\mathrm{dt}}=\mathrm{N} \sigma \phi-\lambda \mathrm{n},
$$

where $\mathrm{N}$ is the density of $0-16$ atoms in the coolant, and $\sigma \phi$ is the reaction rate in the neutron flux of the shield. The reaction rate used herein is the average value for the first third of the shield, as obtained by the TWODANT/REAC3 codes. Integrating the above equation allows us to solve for $\lambda \mathrm{n} 2$ in terms of $\lambda \mathrm{nl}$ :

$$
\lambda \mathrm{n} 2=\Lambda \sigma \phi\left(1-\mathrm{e}^{-\lambda \Delta t 1}\right)+(\lambda \mathrm{n} 1) \mathrm{e}^{-\lambda \Delta l 1} .
$$

The equation for $\lambda \mathrm{n} 3$ in terms of $\lambda_{\mathrm{n}} 1$ is similar, except there is no source term:

$$
\lambda \operatorname{nn}=(\lambda \ln 1) \mathrm{e}^{-\lambda \Delta 1} \text {. }
$$

\footnotetext{
${ }^{53}$ Derivation adapted from R. S. Amato, Shield Design and Radiation Analysis Course Notes, Bettis Reactor Engineering School, Bettis Atomic Power Laboratory, undated, Chapter 9.
} 
To determine $\lambda n 4$, we use a flow combining equation which depends on the flow fractions $\mathrm{fl}$ and $\mathrm{f} 2$, to wit:

$$
\lambda \mathrm{n} 4=\mathrm{f} 1 \cdot \lambda \mathrm{n} 2+\mathrm{f} 2 \cdot \lambda \mathrm{n} 3 .
$$

By analyzing the rest of the SWS flow loop, $\lambda \mathrm{n} 5$ and $\lambda \mathrm{n} 6$ can also be solved for. This results in six equations and six unknowns, and the various specific activity levels can be solved for in terms of No $\phi$, and $\Delta \mathrm{t} l$ through $\Delta \mathrm{t} 4$. The most important activity levels for the present analysis are the levels at the shield outlet $(\lambda n 4)$ and the AHE inlet $(\lambda n 5)$. $\lambda \mathrm{n} 4$ can be found based on the previously stated equations, plus the equation for $\lambda \mathrm{nl}$ :

$$
\lambda \mathrm{n} 1=\frac{\mathrm{N} \sigma \phi \cdot \mathrm{f} 1 \cdot e^{-\lambda \Delta t 2}\left(e^{-\lambda . \Delta t 1}-1\right)}{e^{-\lambda(\Delta t 1+\Delta t 2)}-1} .
$$

Once $\lambda \mathrm{n} 4$ is known, $\lambda \mathrm{n} 5$ can be found using the equation:

$$
\lambda \mathrm{n} 5=(\lambda n 4) e^{-\lambda \Delta t 3}
$$

Because the coolant flow rate has already been determined for the SWS $(0.714$ $\mathrm{m}^{2} / \mathrm{sec}$ ), as have the sizes of the Auxiliary and Service Heat Exchangers and the shield, the residence times through the shield $(\Delta t)$ and the AHE $(\Delta t 4)$ are determined, and are

Table 6.17. SWS Residence Times

\begin{tabular}{|r|c|}
\hline Shield $(\Delta t 1):$ & $28 \mathrm{sec}$ \\
\hline AHE $(\Delta t 4:)$ & $2.5 \mathrm{sec}$ \\
\hline
\end{tabular}
shown in Table 6.17. The residence time in the $\mathrm{AHE}$ is quite short (since the coolant flow path length of the unit is only a few meters). Hence, the $\mathrm{N}-16$ activity levels at the inlet and outlet of the AHE are similar.

The residence times $\Delta \mathrm{t} 2$ and $\Delta \mathrm{t} 3$ depend on the length and diameter of the piping connecting the Service and AHEs to the shield and to each other. The total length of the piping in this loop was previously determined to be 200 meters for the purposes of this study. However, the diameter has not yet been specified. The choice of pipe diameter 
strongly impacts the activity levels in the AHE, since a larger pipe diameter results in longer transit time from the shield to the AHE ${ }^{54}$ Table 6.18 shows the activity levels at the AHE inlet for different pipe diameters, as well as the residence times $\Delta \mathrm{t} 2$ and $\Delta \mathrm{t} 3$.

Table 6.18. N-16 Activity at AHE Inlet versus SWS Pipe Diameter

\begin{tabular}{|c|c|c|c|}
\hline Pipe Diameter $(\mathbf{m})$ & $\underline{\Delta} \mathbf{2}(\mathbf{s e c})$ & $\underline{\Delta} \mathbf{3}(\mathbf{s e c})$ & $\mathbf{N}-16$ Activity, $\lambda \mathbf{m} \mathbf{4}(\mathbf{C i} / \mathbf{c u} . \mathbf{m})$ \\
\hline 0.5 & 58 & 28 & 410 \\
\hline 1.0 & 220 & 110 & 0.16 \\
\hline 1.1 & 260 & 130 & 0.018 \\
\hline
\end{tabular}

\subsubsection{Biological Dose Rate from $A H E$}

In order to determine the minimum allowable pipe diameter, the $\mathrm{N}-16$ specific activity level in the coolant needs to be related to the biological dose rate of gamma radiation received in the vicinity of the AHE. Furthermore, the maximum allowable biological dose rate from the AHE must be specified. The following paragraphs discuss these issues.

Disintegration of an N-16 nucleus results in emission of a $6.1 \mathrm{MeV}$ gamma ray 55 percent of the time, and a $7.1 \mathrm{MeV}$ gamma ray 20 percent of the time. 55 Hence, the gamma yield is $0.55+0.20=0.75$. For simplicity in the following analysis, we will

\footnotetext{
${ }^{54}$ Rather than increasing the pipe diameter to increase the transit time to the AHE, a holding tank could be installed between the shield and the AHE. Coolant passing through the holding tank would be delayed while residing in the tank, thus reducing the activity level. However, by increasing the pipe diameter, the loop pressure drop is decreased, which is beneficial to the natural circulation operation of the SWS. As will be seen, the required pipe diameter is not excessively large. Hence, the holding tank option is not chosen.
}

${ }^{55}$ R. G. Jaeger, et.al., eds., Engineering Compendium on Radiation Shielding, Volume 1, SpringerVerlag. 1968, p. 92. 
assume that the emitted gamma ray energy is always $6.5 \mathrm{MeV}$. Thus, the gamma specific activity of the SWS coolant at the AHE inlet is given by:

$$
(\lambda \mathrm{n} 4)_{6.9 \text { MeV } y}=\lambda \mathrm{n} 4 \cdot(0.75) \text {. }
$$

To translate the gamma specific activity of the coolant in the AHE to a gamma flux in the vicinity of the AHE, the AHE is approximated as a cylindrical surface source with the diameter of the cooling tower and height equal to the AHE height in the cooling tower Figure 6.23 shows the assumed geometry.

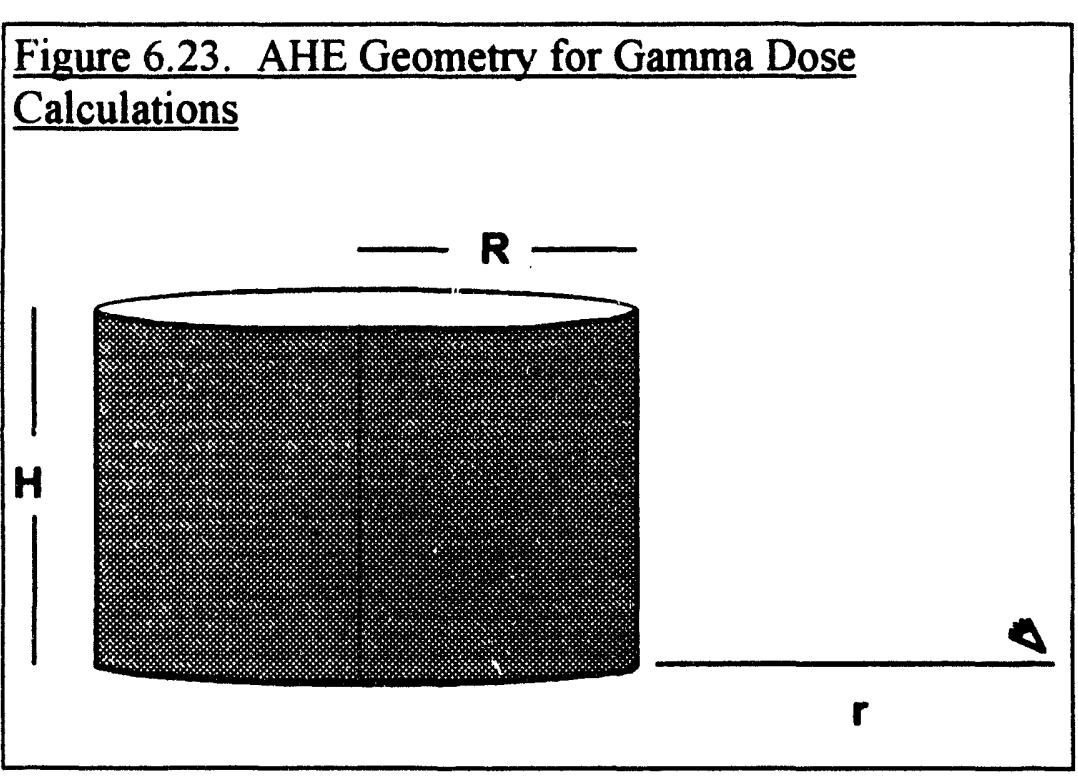

It will be recalled (Table 6.5) that the cooling tower radius $(\mathrm{R})$ is 3.85 meters, and the air gap height $(\mathrm{H})$ is 1.5 meters $(\mathrm{H}=$ $1 / 10$ the chimney height, from Appendix 6). The distance $r$ in the figure is the distance of the observer receiving the gamma ray flux from the bottom of the AHE. The observer is assumed to be level with the bottom of the AHE, which is conservative, since the bottom of the AHE is actually over 4 meters from the ground.

For a cylindrical surface source with the geometry shown in Figure 6.23, the gamma flux at the observer is given by the following equation:

$$
\phi\left(\mathrm{m}^{-2} \cdot \sec ^{-1}\right)=\frac{\mathrm{S} \cdot \mathrm{R}}{2(\mathrm{r}+\mathrm{R})} \mathrm{F}(\varphi, \mathrm{k}),
$$

where 


$$
\begin{gathered}
\varphi=\arctan \frac{\mathrm{H}}{\mathrm{r}-\mathrm{R}}, \\
\mathrm{k}=\frac{2 \sqrt{\mathrm{r} \cdot \mathrm{R}}}{\mathrm{r}+\mathrm{R}} \text {, and } \\
\mathrm{F}(\varphi, \mathrm{k})=\int_{0}^{\phi} \mathrm{e}^{-\mathrm{b} \cdot \sec \theta} \mathrm{d} \theta
\end{gathered}
$$

Here, $S$ is the surface source strength $\left(\mathrm{m}^{-2} \cdot \mathrm{sec}^{-1}\right)$ of the AHE. $S$ is evaluated by assuming all gamma emissions from the $\mathrm{AHE}$ come from a plane of height $\mathrm{H}$ and diameter $D$, taking into account the coolant activity at the AHE inlet, $(\lambda \mathrm{n} 4)_{65 \mathrm{MeV} \gamma}$, the fraction of coolant in the AHE volume, and the effective thickness of the AHE as installed in the cooling tower.

The gamma flux equation just described yields the number of $6.5 \mathrm{MeV}$ gamma rays crossing a unit surface area per unit time. To be useful in the present analysis, this gamma flux must now be converted into a biological dose rate, which is the rate at which energy is transferred to the human body. The standard unit for measure of biological dose is the rem or, radiation equivalent man. ${ }^{56}$ To convert from the gamma flux to the dose rate, a flux-to-dose-rate conversion factor is used, which depends on the energy of the gamma rays. For $6.5 \mathrm{MeV}$ gammas, the conversion factor is $6.93 \mathrm{e}-2$ $\mathrm{mrem} / \mathrm{hr}$ per $\gamma / \mathrm{cm}^{2} \cdot \sec .57$

Using this conversion factor, a table similar to Table 6.18 can be generated which shows the dose rate at different distances from the AHS for various SWS pipe diameters. Table

\footnotetext{
${ }^{56}$ The SI unit for biological dose is the sievert (Sv), which is equivalent to 100 rem for a particular type of absorbed radiation. However, the U.S. federal standard for radiation limits (10CFR20) continues to use rem. See R.A. Meyers, ed., Encyclopedia of Modern Physics, Academic Press, Inc., 1990, pp. 586587 for more information on radiation units.

57"Neutron and Gamma-Ray Flux-to-Dose-Rate Factors", American National Standard ANSI/ANS6.1.1-1977 (N666), American Nuclear Society, LaGrange Park. IL, March 17, 1977.
} 


\section{Table 6.19. Gamma Dose Rates versus SWS Pipe Diameter}

\begin{tabular}{|c|c|c|c|}
\hline$\frac{\text { Pipe Diameter }}{(\mathrm{m})}$ & $\begin{array}{c}\text { AHS Surface } \\
\frac{\text { Source Strength }}{\left(\mathrm{m}^{-2} \cdot \mathrm{sec}^{-1}\right)}\end{array}$ & $\begin{array}{c}\text { Dose Rate at AHS } \\
\frac{\text { Surface, } \mathbf{r}=\mathbf{0}}{(\mathrm{mrem} / \mathrm{hr})}\end{array}$ & $\begin{array}{c}\text { Dose Rate at } \mathbf{r}= \\
\frac{\mathbf{2} \text { meters }}{(\mathrm{mrem} / \text { week })}\end{array}$ \\
\hline 0.5 & $5.8 \mathrm{e} 11$ & $3.3 \mathrm{e} 4$ & $2.3 \mathrm{e} 6$ \\
\hline 1.0 & $2.2 \mathrm{e} 8$ & 12 & 870 \\
\hline 1.1 & $2.4 \mathrm{e} 7$ & 1.4 & 96 \\
\hline
\end{tabular}

6.19 shows the surface source strength of the geometry shown in Figure 6.23, and dose rates at the surface and 2 meters from the surface as functions of SWS pipe diameter. Note that the units for dose rate are different for each column.

The dose rate limits prescribed for unrestricted areas (that is, areas which do not have barriers to protect the public from radiation exposure) for members of the general public as follows: $100 \mathrm{mrem}$ for a person continuously occupying area for 7 days, and $2 \mathrm{mrem}$ for a person occupying area for a single hour. ${ }^{58}$ As can be seen in Table 6.19, a pipe diameter of 1.1 meters results in AHS dose rates which permits continuous occupation by members of the public at a distance of 2 meters from the AHS, since the dose rate there is less than $100 \mathrm{mrem} /$ week. Furthermore, this same pipe diameter results in dose rates below $2 \mathrm{mrem} / \mathrm{hr}$ at the surface of the AHS cylinder.

It can be concluded from the above analysis that a pipe diameter of 1.1 meters would result in dose rates from the AHS which would not be harmful either to the public or to the environment. This is therefore the pipe diameter chosen for the SWS. Although this

58 From 10 CFR 20 - The Code of Federal Regulations, Title 10, Part 20, Standards for Protection Against Radiation, U. S. Government Printing Office, January, 1993. 
is a large pipe diameter, the SWS is an unpressurized system. Hence, such a large pipe diameter would not be impractical.

\subsubsection{Shielding of SWS Piping}

Since the N-16 activity levels are very high at the shield outlet, regardless of SWS pipe diameter, the 1.1 meter diameter SWS piping near the shield outlet must be shielded to prevent excessive gamma exposures. To ensure that the required shielding thickness for this large piping is reasonable, the following discussion will determine the required thickness for the shield outlet piping.

From methods discussed above, the specific gamma ray activity in the coolant in the 1.1 meter pipe at the shield outlet can be determined. Given this value, we desire to know how much shielding (of what material) is necessary to limit the dose rate at the outer

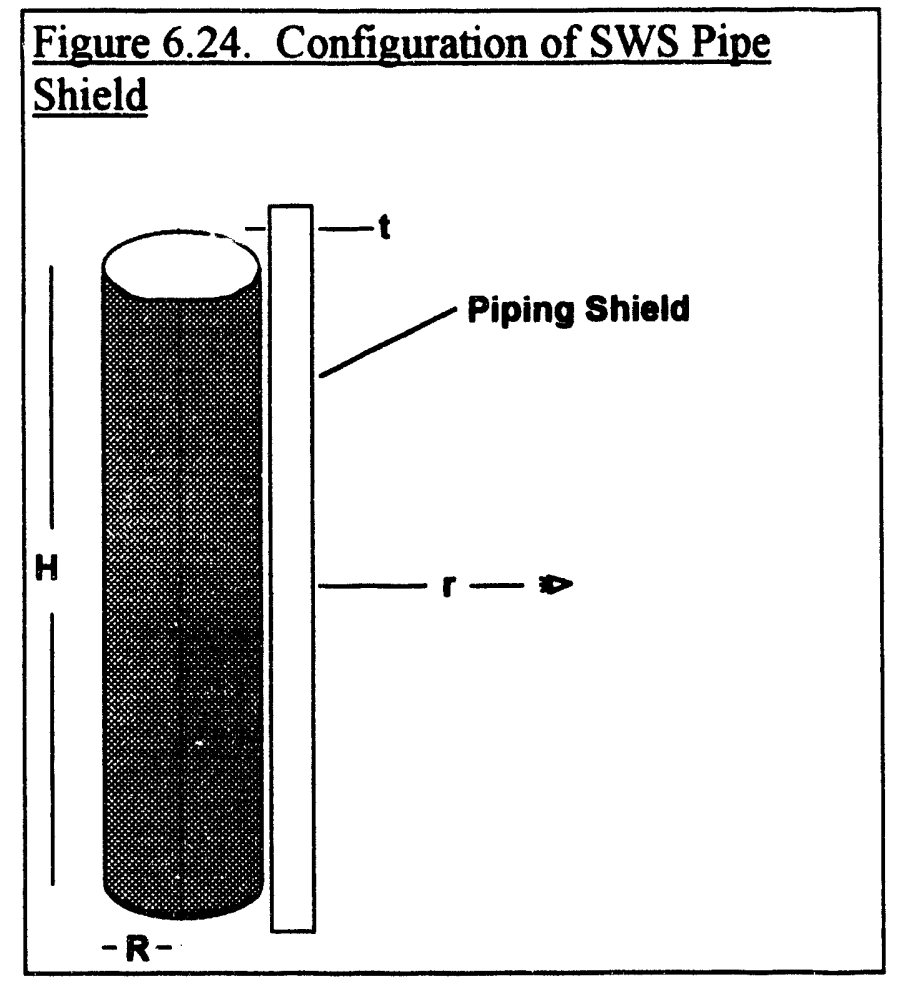

shield surface to below 2

$\mathrm{mrem} / \mathrm{hr}$. For a gamma ray

energy of about $6 \mathrm{MeV}$, gamma

ray attenuation in materials is

dominated by Compton scattering

and pair production. The

attenuation coefficients associated

with both of these processes

increase with the shield material's

atomic number. ${ }^{59}$ Therefore, the

higher the material's atomic

number, the better its gamma

shielding properties. Lead $(\mathrm{Z}=\mathbf{8 2})$ is therefore the material of choice for most gamma

${ }^{59}$ Lecture Notes for MIT Course 22.111, Nuclear Physics for Engineers, Spring 1991, Chapter 8. 
shielding applications, and will be used here. The shield is assumed to be a slab shield; the geometry of the shield configuration is shown in Figure 6.24 .

In calculating the required lead shield thickness, we must account for the fact that the water present in the pipe itself tends to self-shield some of the gamma rays being emitted from the center regions of the pipe. The appropriate gamma flux equation for a selfshielded, cylindrical source with a slab shield (as shown in Figure 6.24) is as follows: ${ }^{60}$

$$
\phi\left(\mathrm{m}^{-2} \cdot \sec ^{-1}\right)=\left[\frac{(\lambda \mathrm{n} 4)_{6,5 \mathrm{MeV} \gamma} \cdot \mathrm{R}}{2 \pi}\right] \cdot \mathrm{G}\left(\frac{\mathrm{H}}{\mathrm{R}}, \frac{\mathrm{r}}{\mathrm{R}}, \mu_{\mathrm{so}} \mathrm{R}, \mu_{\mathrm{sh}} \mathrm{t}\right) \cdot \mathrm{B}\left(\mu_{\mathrm{sh}} \mathrm{t}\right),
$$

where $\mathbf{G}$ is a function dependent on the geometry and the linear attenuation coefficients for gamma rays in the source $\left(\mu_{s o}\right)$ and the shield $\left(\mu_{s h}\right){ }^{61}$ B is called the buildup factor, and accounts for scattering in the shield reflecting gammas back toward the observation point, which tends to increase the gamma flux. Numerous approximations exist to analytically describe the value of the buildup factor. A common one is known as "Berger's Formula", and is as follows: 62

$$
\mathrm{B}\left(\mu_{s h} \mathrm{t}\right)=1+A \mu_{s h} \mathrm{t} \cdot \exp \left(\mathrm{B} \cdot \mu_{s h} \mathrm{t}\right) .
$$

Values of A, B and $\mu_{s h}$ for lead, as well as $\mu_{s o}$ for water are shown in Table 6.20.

The SWS coolant activity at the shield outlet, $(\lambda \mathrm{n} 4)_{65 \mathrm{MeV}_{\gamma}}$, is $1.8 \mathrm{e} 14$ per cubic meter per second. To shield a pipe of diameter 1.1 meters so that the shield surface dose rate is below $2 \mathrm{mrem} / \mathrm{hr}$, a lead shield thickness of less than 0.3 meters (or about one foot) is

\footnotetext{
${ }^{60}$ R. G. Jaeger, et.al., eds., Engineering Compendium on Radiation Shielding, Volume 1, SpringerVerlag, 1968, p. 382.

${ }^{61}$ Tables for the $G$ function are available in Jeager, et.al., pp. 377-381. These tables were utilized to evaluate $\mathrm{G}$ for this work.

${ }^{62}$ Jeager, et.al., p. 213-216.
} 
necessary. This required shield thickness is reasonable, and of course will become even smaller as one moves along the pipe and the $\mathrm{N}-16$ activity levels decrease

Table 6.20. Parameters for Pipe Shielding Calculations ${ }^{63}$

\begin{tabular}{|c|c|c|c|}
\hline \multicolumn{3}{|c|}{ Lead } & Water \\
\hline$\mu_{s h}(1 / \mathrm{m})$ & A & B & $\mu_{s o}(1 / \mathrm{m})$ \\
\hline 50.5 & 0.1344 & 0.1000 & 2.77 \\
\hline
\end{tabular}

${ }^{63}$ Values for $6 \mathrm{MeV}$ gammas, from Jeager, et.al., pp. 215 and 178. 


\subsection{The Two-Loop Shield Water System Option}

In the foregoing sections, the single-loop configuration of the SWS was discussed. It was claimed at the beginning of the section that a two-loop SWS configuration, although virtually eliminating the possibility of radioactive release or exposure to the environment, would be less efficient, hence more expensive. In this section, the two-loop SWS configuration will be analyzed. The components of the two-loop system will be sized, and the system cost estimated and compared to the estimated cost for the single-loop system.

\subsubsection{Two Loop SWS Components Size and Cost}

Figure 6.25 shows the overall configuration of the two loop shield water system. As shown in this figure, there is a primary loop which includes the Service Heat Exchanger (SHE), the shield, the jet pump, and one side of the Intermediate Heat Exchanger (IHE).

\section{Figure 6.25. Two-Loop Shield Water System Diagram}

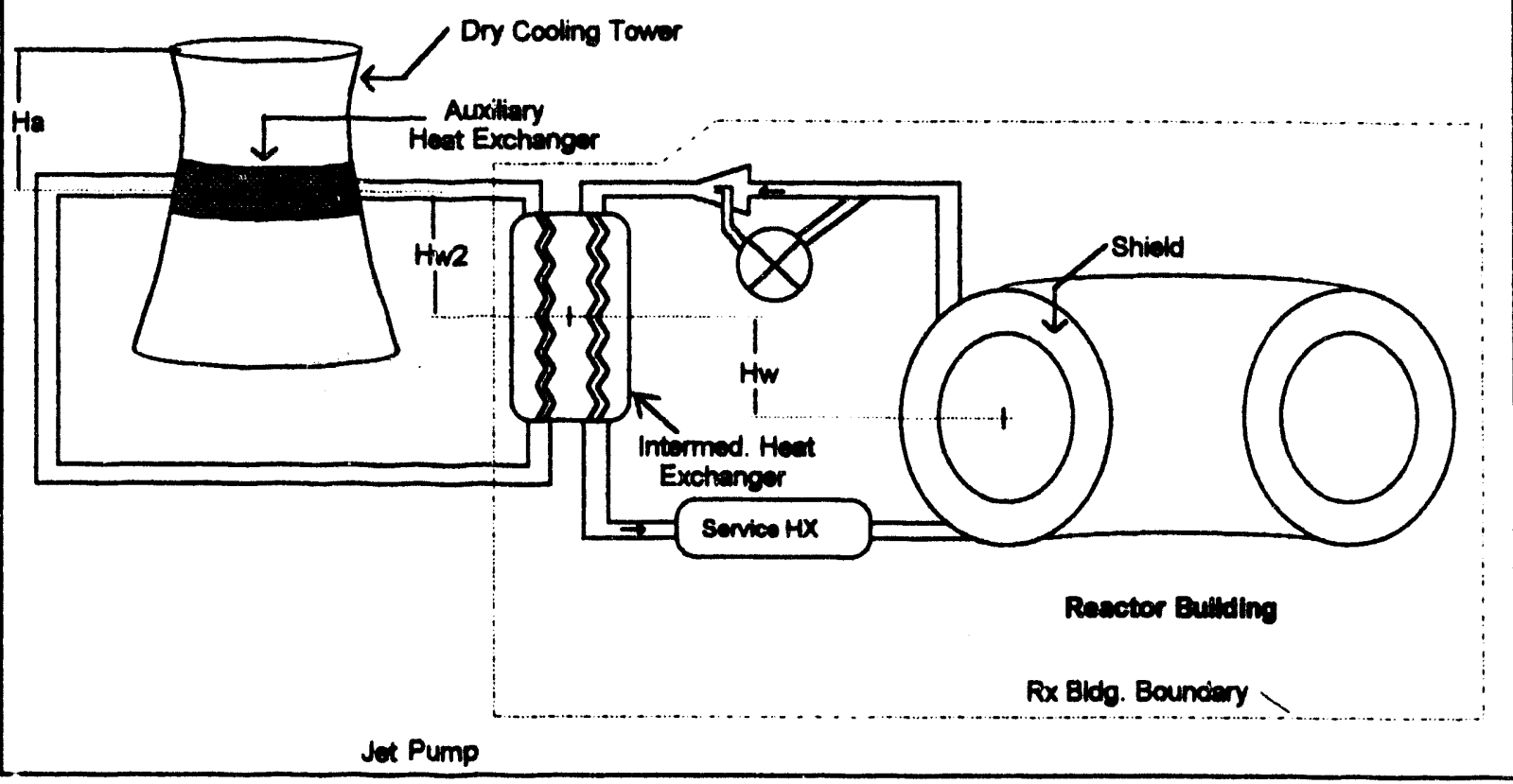

The primary loop is contaminated with radioactivity, but is fully enclosed within the 

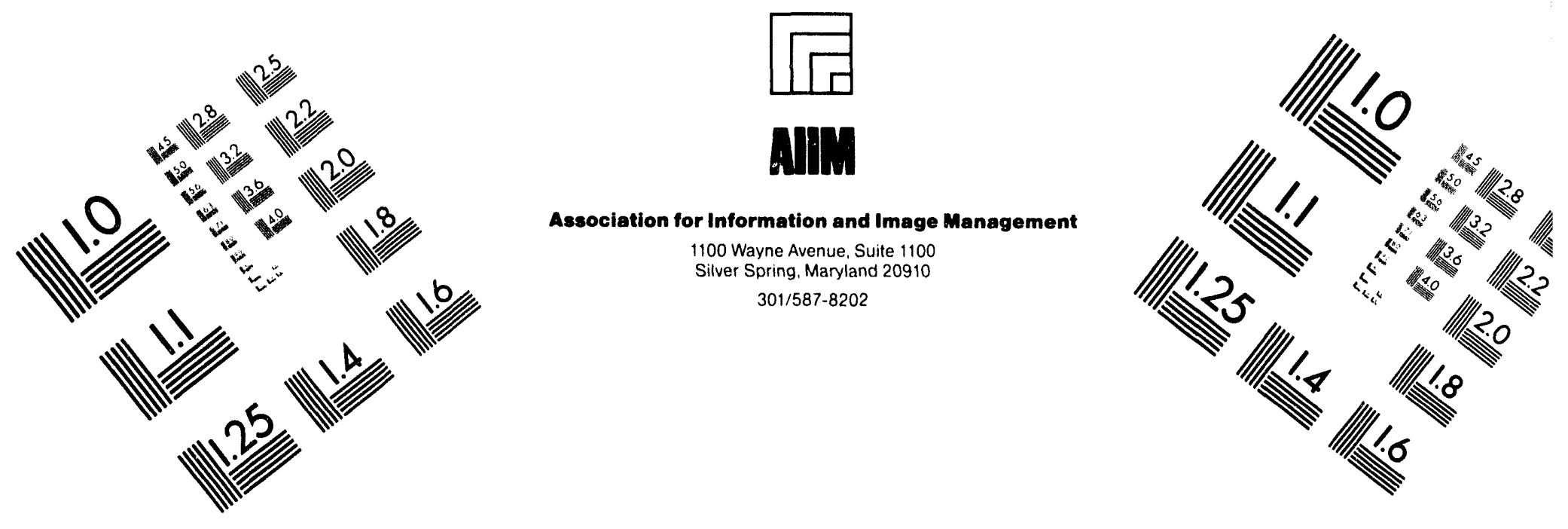

\section{Centimeter}

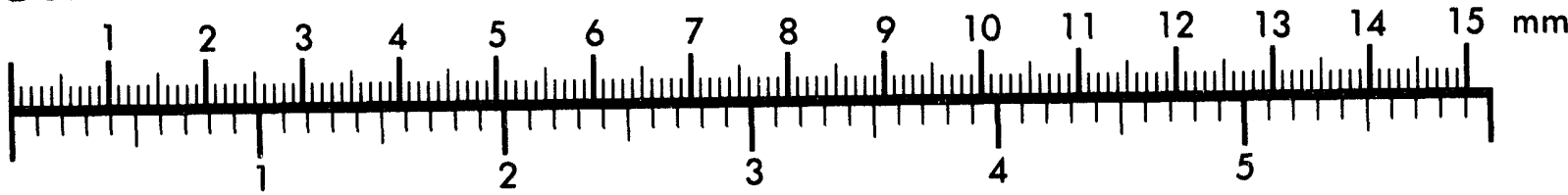
Inches
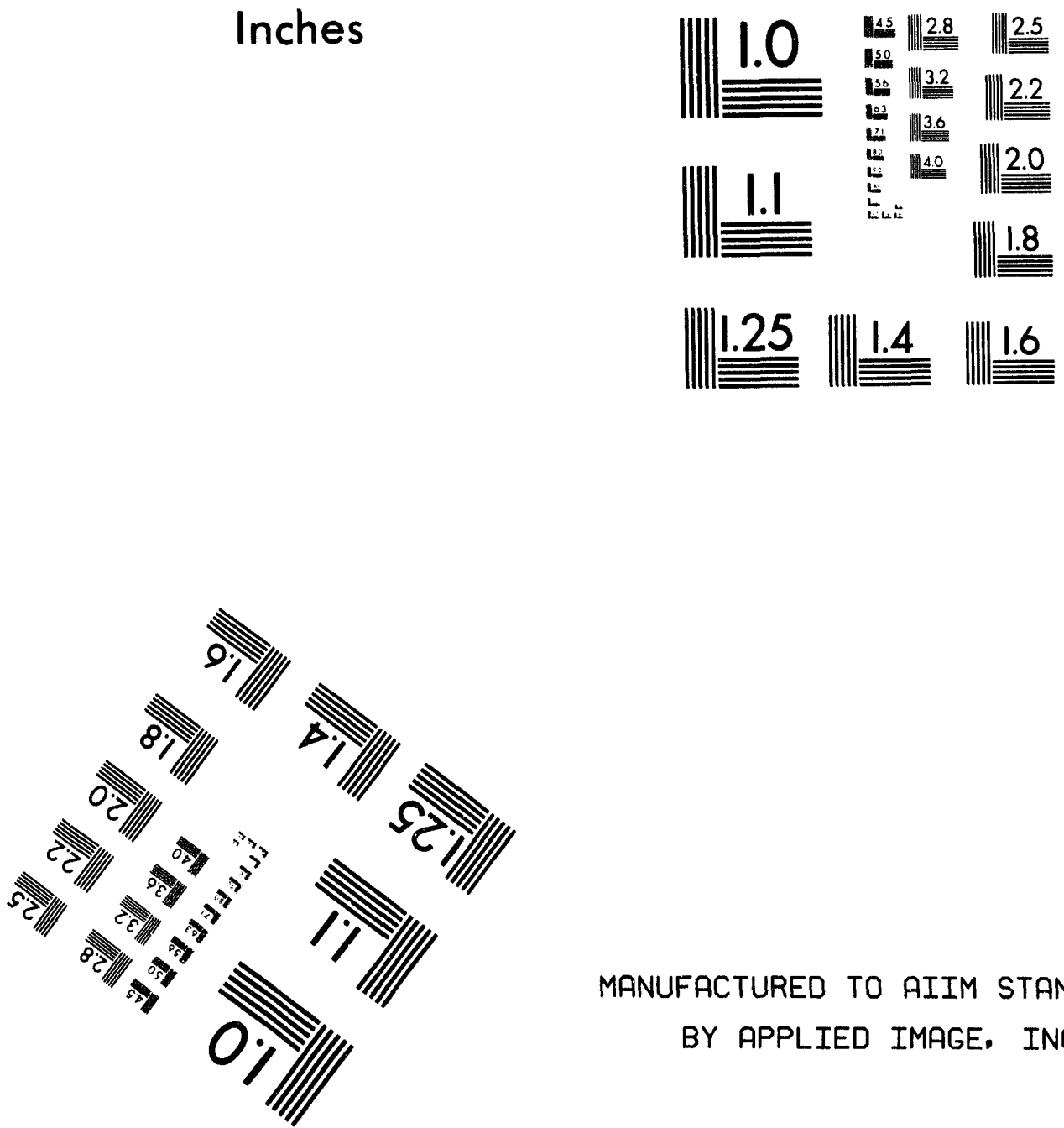

MANUFACTURED TO AIIM STANDARDS

BY APPLIED IMAGE. INC.

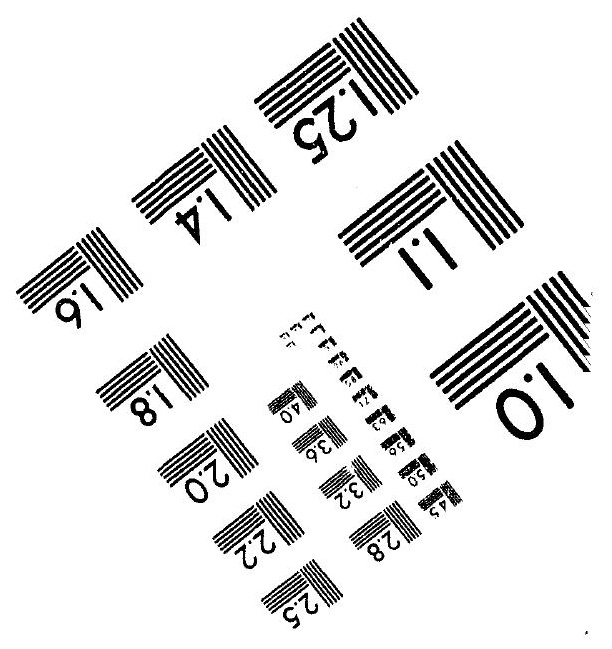



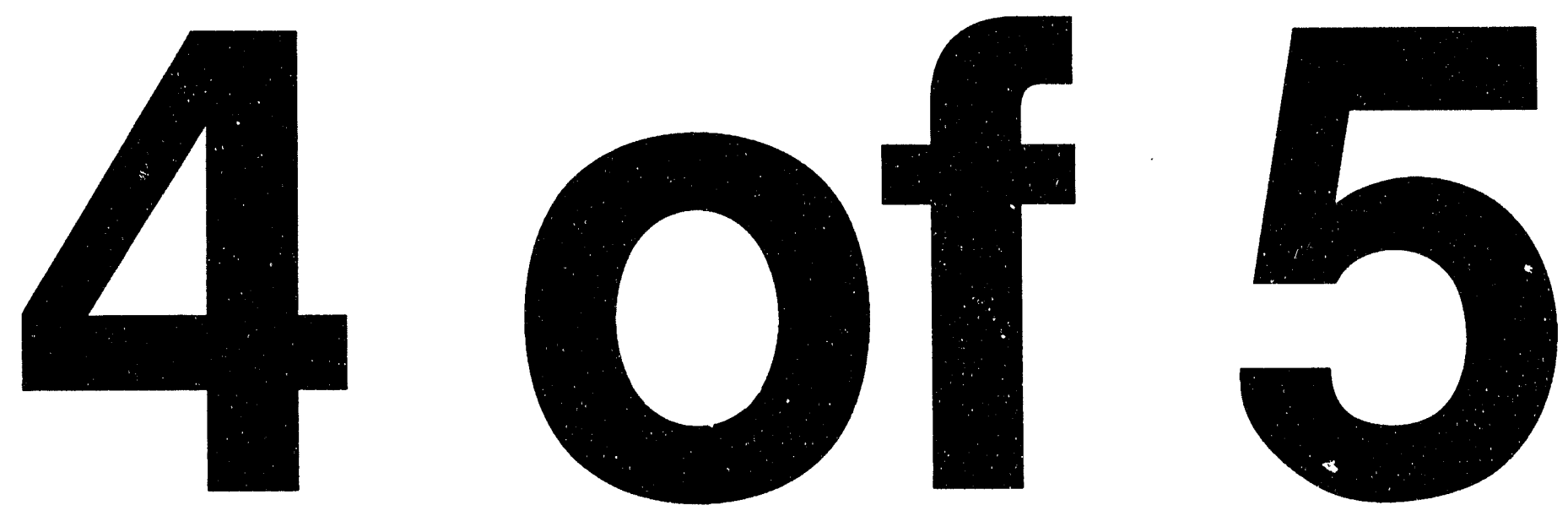
reactor building, thereby greatly reducing the chance of a radioactive release to the environment. The secondary loop includes the Auxiliary Heat Exchanger (AHE) and the other side of the IHE. This loop does not normally contain radioactivity, and for the most part runs outside of the reactor building.

During normal system operation, the primary loop water circulates in a forced-flow mode using the jet pump head. As with the single loop SWS, during normal system operation the SHE removes the heat from the primary loop using forced flow from the main condenser cooling system. During normal system operation, the secondary loop remains online, but plays virtually no part in removing herit from the primary luop. The size of the SHE required for the two loop system is similar to the size requiried for the single loop system.

If site power is lost, both the primary and secondary loops must naturally circulate in order to cool the shield. Hence, the IHE must be located above the shield by some amount (designated Hw in Figure 6.25), and the AHE must in turn be located above the IHE by some amount (Hw2), in order to provide natural circulation driving heads for the two loops. To determine appropriate values for $\mathrm{Hw}$ and $\mathrm{Hw} 2$, and to size the cooling tower, AHE and IHE for the two loop system, an analysis must be performed.

The basic tools used to perform the two loop analysis are the same as those used previously for the single loop analysis. Pressure drop and heat transfer coefficients for the AHE are the same as a function of Reynoids number, for example. The IHE is assumed to be a crossflow, shell-and-tube type heat exchanger. Hence the analytical methods used for the SHE are also applicable to the IHE. However, the two loop system is somewhat more complex than the single loop system, and has more parameters 
which must be specified. A list of the most significant parameters for the two loop system is shown in Table 6.21 .

Table 6.21. Two Loop SWS Parameters

\begin{tabular}{|l|l|l|}
\hline Primary Loop Piping: & \multicolumn{1}{l|}{ Length } & Diameter \\
\hline Secondary Loop Piping: & \multicolumn{1}{l}{ Length } & \multicolumn{1}{l|}{ Diameter } \\
\hline IHE Diameter & IHE Length & IHE, AHE Tube Configurations \\
\hline AHE Length & AHE Depth & AHE Width \\
\hline Primary Thot & Primary Tcold & Primary mass flow rate \\
\hline Secondary Thot & Secondary Tcold & Secondary mass flow rate \\
\hline Air Thot & Air Tcold & Air mass flow rate \\
\hline Air chimney height $(\mathrm{Ha})$ & Shield-IHE height $(\mathrm{Hw})$ & IHE-AHE height $(\mathrm{Hw} 2)$ \\
\hline
\end{tabular}

The above table indicates the large number of parameters that must be determined in order to specify the size and performance of the two loop SWS. To make the problem tractable, it is necessary to reduce the number of parameters which are to be varied in the analysis. In order to estimate the cost of the system (which is our primary goal in this section), we want to focus on the sizes of the major system components required for natural circulation operation, namely the cooling tower, the AHE and the IHE. For this first-order cost analysis, the cost of piping for the system will be neglected. (Note that the cost for piping for the two-loop and single loop systems will probably be similar hence, neglecting the piping cost is probably not significant when comparing the cost of the two systems.) 
First, the diameter and length of the system piping will be fixed. The diameter of the secondary and primary piping is fixed at 0.6 meters. This value was chosen after exploring a number of different sizes; it is the smallest diameter that does not overly constrain the rest of the system. Note that the diameter of the piping is not governed by $\mathrm{N}-16$ activation concerns, as it is for the single loop SWS. The secondary piping length is chosen is be 200 meters, to allow for a long run of piping to the AHE. This is the same value used for the single loop system piping length. The primary piping length is chosen to be 20 meters. As with the single loop analysis, pipe elbows, tees, and other flow restrictions are not explicitly modeled; rather, the piping lengths are chosen to be conservatively long, in part to account for such flow restrictions.

We will use the same AHE tube configuration as that used for the single loop analysis, and use the tube configuration previously used for the SHE for the IHE as well. The Tcold value for air will be chosen as 25 degrees $\mathrm{C}$, and the Thot value for air will be fixed at 40 degrees $C$. The Thot value for the primary side will be chosen as 95 degrees C. These temperature values were also used for the single loop analysis. The IHE diameter is fixed at 1 meter. This value ensures that the IHE diameter is of the same order as the inlet piping diameter. An IHE diameter of 1 meter also allows solutions to the heat transfer equations to be found for a wide range of other system parameters.

The air mass flow rate is fixed by the specification of the air inlet and outlet temperatures, plus the known required heat rejection rate of $5 \mathrm{MW}$ (same as for the single loop analysis). The cooling tower chimney height $(\mathrm{Ha})$ is fixed at $15 \mathrm{~m}$, the same value used in the final single loop cooling tower configuration (this choice will be further justified later). The secondary water mass flow rate is fixed by specifying a capacity rate ratio of 1.0 for the AHE. This is the same as the capacity rate ratio which characterized the final AHE size used in the single loop analysis, hence it represents an efficient choice 
for the temperature range, heat load, and mass flow rates associated with the SWS. The primary mass flow rate is also fixed by assuming a capacity rate ratio of 1.0 for the IHE as well. This is done primarily for simplicity. With the primary mass flow rate and primary side Thot specified, the primary side Tcold is determined as well. The fixed parameters discussed above are shown in Table 6.22.

\section{Table 6.22. Fixed Two Loop SWS Parameters}

\begin{tabular}{|c|c|c|}
\hline Primary Loop Piping: & Length $=20 \mathrm{~m}$ & Diameter $=0.6 \mathrm{~m}$ \\
\hline Secondary Loop Piping: & Length $=200 \mathrm{~m}$ & Diameter $=0.6 \mathrm{~m}$ \\
\hline IHE Diameter $=1 \mathrm{~m}$ & IHE Length & $\begin{array}{l}\text { IHE, AHE Tube } \\
\text { Configurations = Specified }\end{array}$ \\
\hline AHE Length & AHE Depth & AHE Width \\
\hline Primary Thot $=95 \mathrm{C}$ & Primary Tcold $=80 \mathrm{C}$ & $\begin{array}{l}\text { Primary mass flow rate }= \\
80 \mathrm{~kg} / \mathrm{sec}\end{array}$ \\
\hline Secondary Thot & Secondary Tcold & $\begin{array}{l}\text { Secondary mass flow rate } \\
=80 \mathrm{~kg} / \mathrm{sec}\end{array}$ \\
\hline Air Thot $=40 \mathrm{C}$ & Air Tcold $=25 \mathrm{C}$ & $\begin{array}{l}\text { Air mass flow rate }=330 \\
\mathrm{~kg} / \mathrm{sec}\end{array}$ \\
\hline $\begin{array}{l}\text { Air chimney height }(\mathrm{Ha}) \\
=15 \mathrm{~m}\end{array}$ & Shield-IHE height (Hw) & IHE-AHE height (Hw2) \\
\hline
\end{tabular}

With the parameters specified as shown above, the problem has been reduced to one having only 8 undetermined variables. The secondary cold and hot temperatures are not really independent, since the secondary mass flow rate and the required heat transfer rate have been fixed, hence the temperature drop on the secondary side has also been fixed.

As it turns out, if we choose the shield-IHE height $\mathrm{Hw}$ and the IHE-AHE height Hw2 as variables in a parametric study, the other parameters of Table 6.22 (and hence the 
desired heat exchanger sizes) are fixed by the other constraints of the problem, namely the need to balance the natural circulation pressure head with the frictional pressure drop of both water loops and the air flow path and the geometrical constraints on the heat exchangers. Details of the sizing procedure are provided in Appendix 6.

Because of the importance of minimizing the frictional pressure drops of both the primary and secondary loops of the two loop SWS (in order to minimize the required natural circulation driving heads and hence the component sizes and costs), the IHE is designed without baffles on the secondary side. This minimizes the secondary side pressure drop significantly. In order to ensure uniform crossflow through the IHE tube bundle, the IHE is assumed to have multiple inlet and outlet pipes serving the heat exchanger from a single inlet and a single outlet pipe. Figure 6.26 shows the IHE configuration.

\section{Figure 6.26. Intermediate Heat Exchanger Configuration}

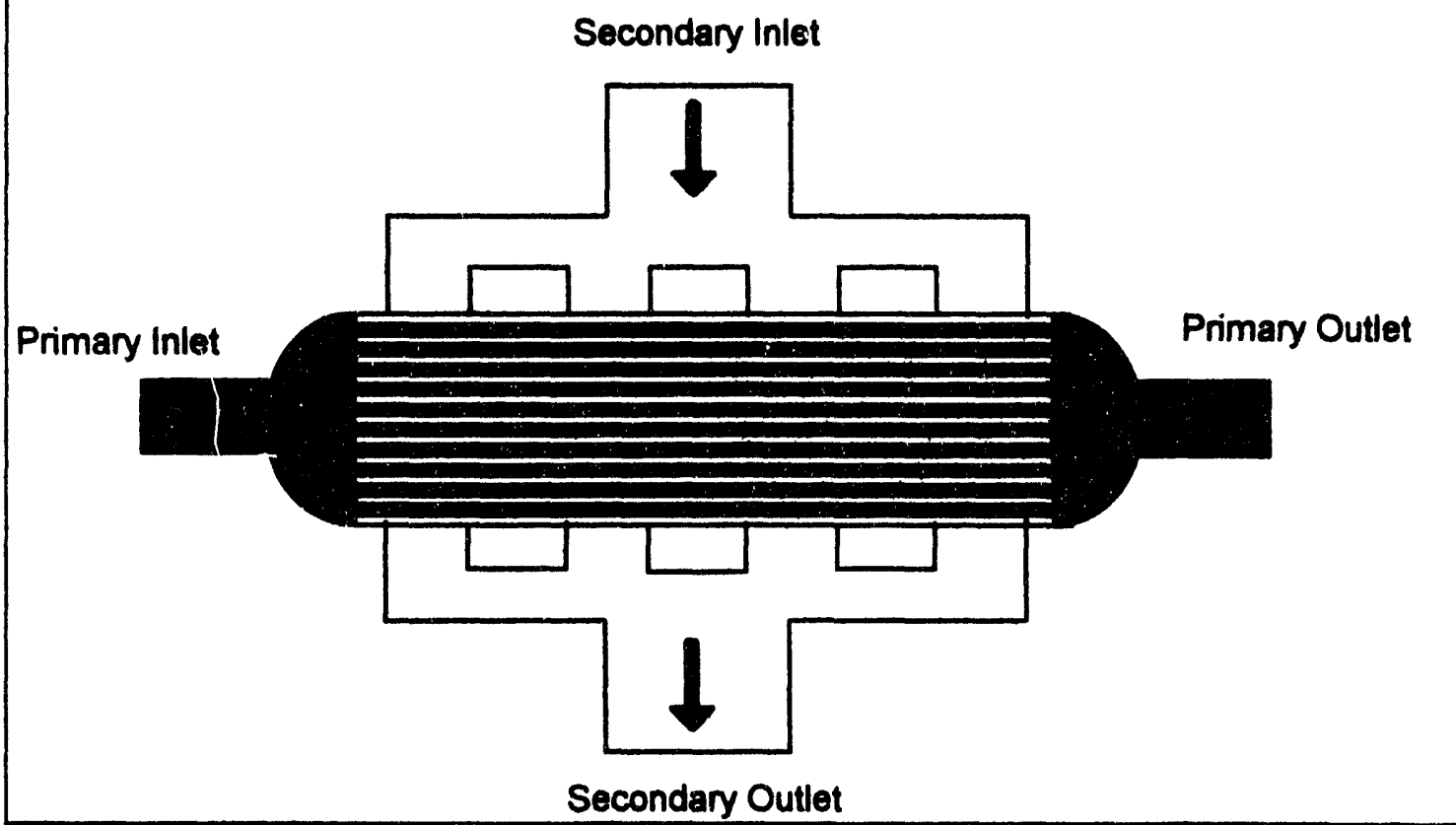


The tube material chosen for the IHE is the same as that chosen for the AHE tubes, stainless steel (CRES 304). This material is suitable for the IHE for the same reasons as it is suitable for the AHE, namely, a high tube velocity limit (for normal, forced flow operation, and compatibility with a pure water environment, which would be expected on both the primary and secondary sides of the two loop SWS.

We will now examine the results of the two loop SWS analysis, using Hw and Hw2 as parametric variables. Figure 6.27 shows how the AHE volume varies with Hw2 for two different values of $\mathrm{Hw}$. The AHE volume decreases with increasing $\mathrm{Hw} 2$ as well as with increasing $\mathrm{Hw}$, since the larger height differences permit more natural circulation driving head, hence the AHE can be made smaller, with a larger pressure drop.

\section{Figure 6.27. Auxiliary Heat Exchanger Volume}

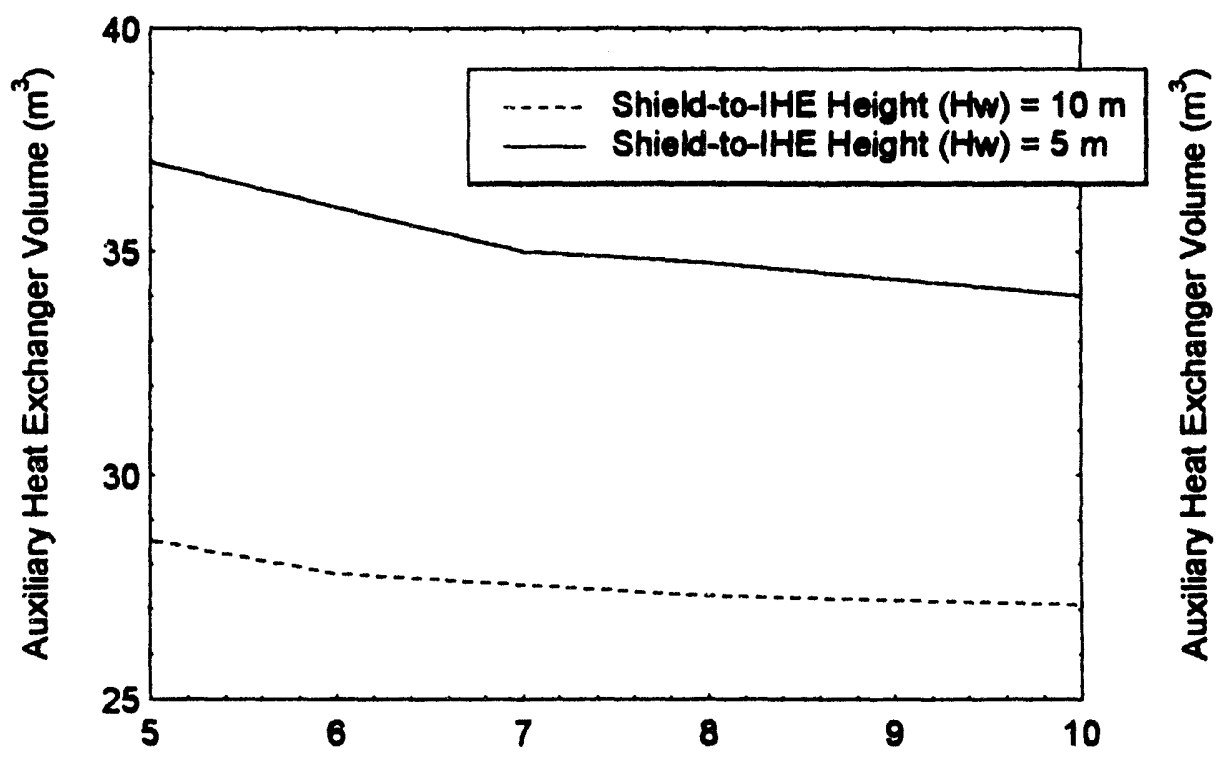

AHE-to-IHE Hoight (Hw2) (m) 
Figure 6.28 shows the volume of the cooling tower shell, as a function of the same $\mathrm{H}$ N and $\mathrm{Hw} 2$ values as were used for Figure 6.27.

\section{Figure 6.28. Cooling Tower Shell Volume}

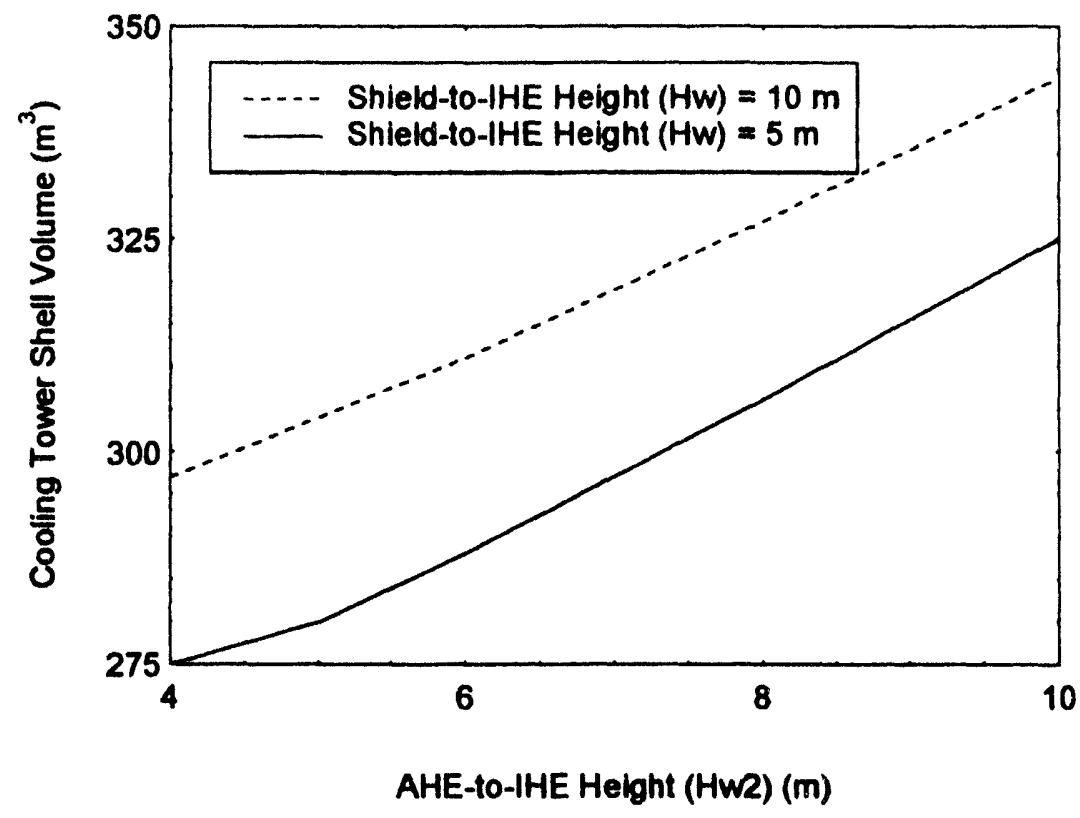

The cooling tower shell volume increases substantially with increasing $\mathrm{Hw}$ and $\mathrm{Hw} 2$, since the height of the cooling tower is a direct function of the required height differential between the shield, IHE and AHE.

Finally, Figure 6.29 shows the variation of the length of the IHE (which is proportional to the IHE volume since the IHE diameter is fixed) as a function of $\mathrm{Hw}$. The length of the IHE is not a function of Hw2 - with the prescribed set of fixed parameters shown in Table 2, the length of the IHE is determined solely by $\mathrm{Hw}$, the primary loop natural circulation height difference. 
Figure 6.29. Intermediate Heat Exchanger Length

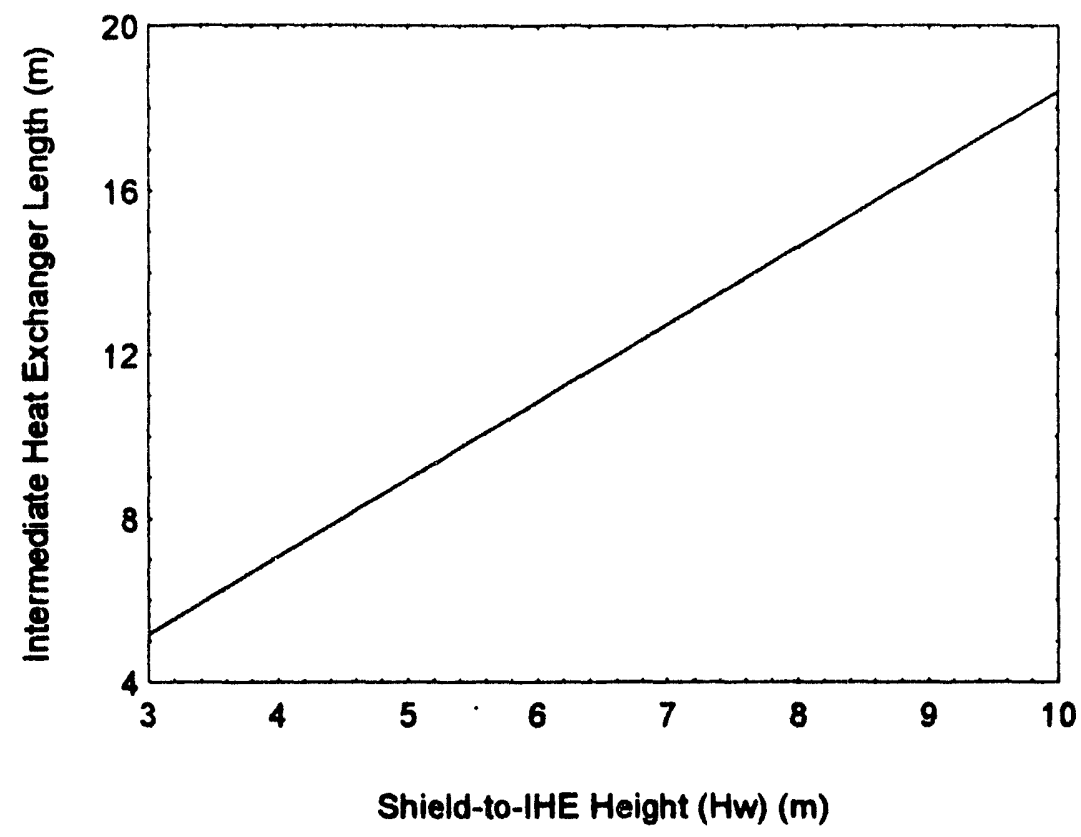

Figure 6.29 shows that the IHE length increases as $\mathrm{Hw}$ increases. As $\mathrm{Hw}$ increases, so does the natural circulation driving head in the primary SWS loop. This increased driving head permits a larger primary loop frictional pressure drop, and hence a longer IHE. The longer IHE in turn provides a larger heat transfer area, which permits a smaller average temperature difference between the primary and secondary fluids.

The previous three figures show the dependence of the size of the cooling tower, AHE and IHE on the heights $\mathrm{Hw}$ and Hw2. From these figures alone, it is not clear what the optimum values of $\mathrm{Hw}$ and $\mathrm{Hw} 2$ are to provide for the most efficient, least expensive two loop SWS system. To help determine the least expensive system, the overall cost of the cooling tower, AHE and IHE will be estimated and added together to provide a indication of the overall system cost. The cost estimate assumes that the AHE is composed of stainless steel (CRES 304) tubes with aluminum fins, that the IHE is composed of stainless steel tubes with a stainless steel shell, and that the cooling tower 
shell is composed of reinforced concrete. The details of the cost estimation method are provided in Appendix 8. The results of the cost calculations are shown in Figure 6.30.

Figure 6.30. Secondary Loop Components Cost vs. Hw and Hw2

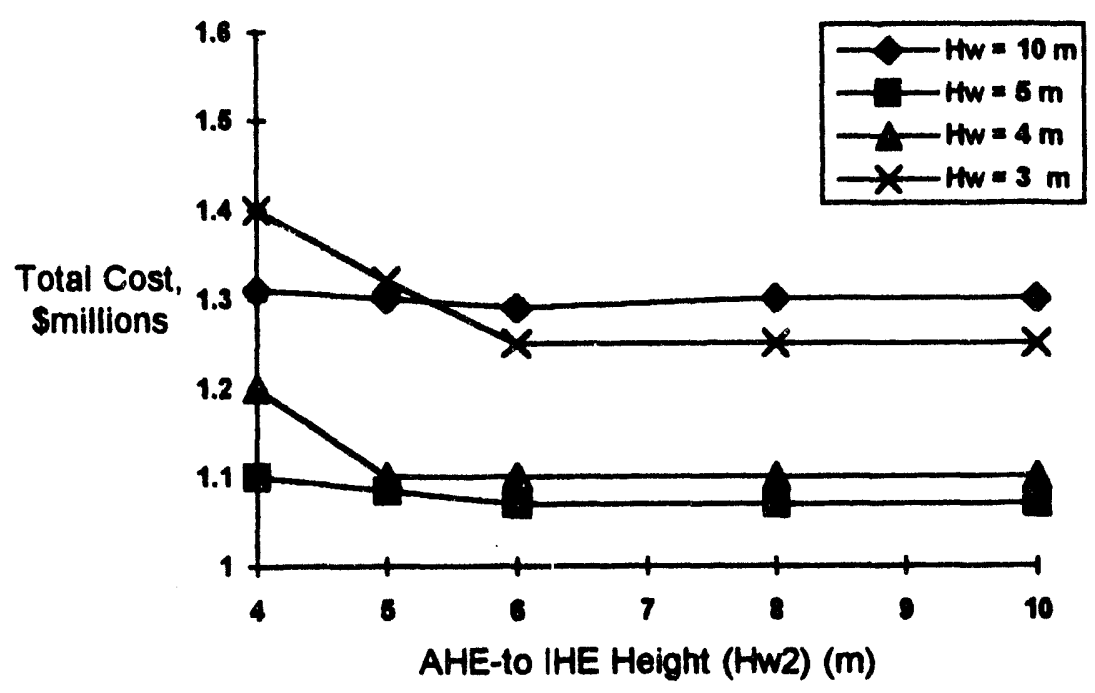

This figure shows, first of all, that the cost of the two loop SWS components do not substantially exceed about $\$ 1$ million, indicating that it is a relatively inexpensive system. ${ }^{1}$ Second of all, the figure shoes that varying $\mathrm{Hw}$ and $\mathrm{Hw} 2$ does not affect the cost of the system drastically. A minimum cost for the components of about $\$ 1.1$ million is given by choosing $\mathrm{Hw}$ in the range 4-5 meters, and Hw2 greater than 5 meters (note that as $\mathrm{Hw} 2$ increases, although the cost of the cooling tower increases along with $\mathrm{Hw} 2$, the cost of the AHE decreases, thus the total comronent cost in the figure remains constant).

Based on Figure 6.30, we will fix the height of the IHE above the shield (Hw), and the height of the AHE above the IHE (Hw2) at 5 meters each. With these parameters fixed, ${ }^{1}$ A typical fusion reactor blanket complex will cost hundreds of millions of dollars, by comparison. 
the effect of the cooling tower chimney height $(\mathrm{Ha})$ is now shown, to further justify the assumption to set $\mathrm{Ha}=15$ meters in Table 6.22. Figure 6.31 shows the effect of $\mathrm{Ha}$ on the secondary loop components cost, assuming $\mathrm{Hw}=\mathrm{Hw} 2=5$ meters, and other SWS parameters are as shown in Table 6.22.

Figure 6.31. Secondary Loop Components Cost vs. Ha

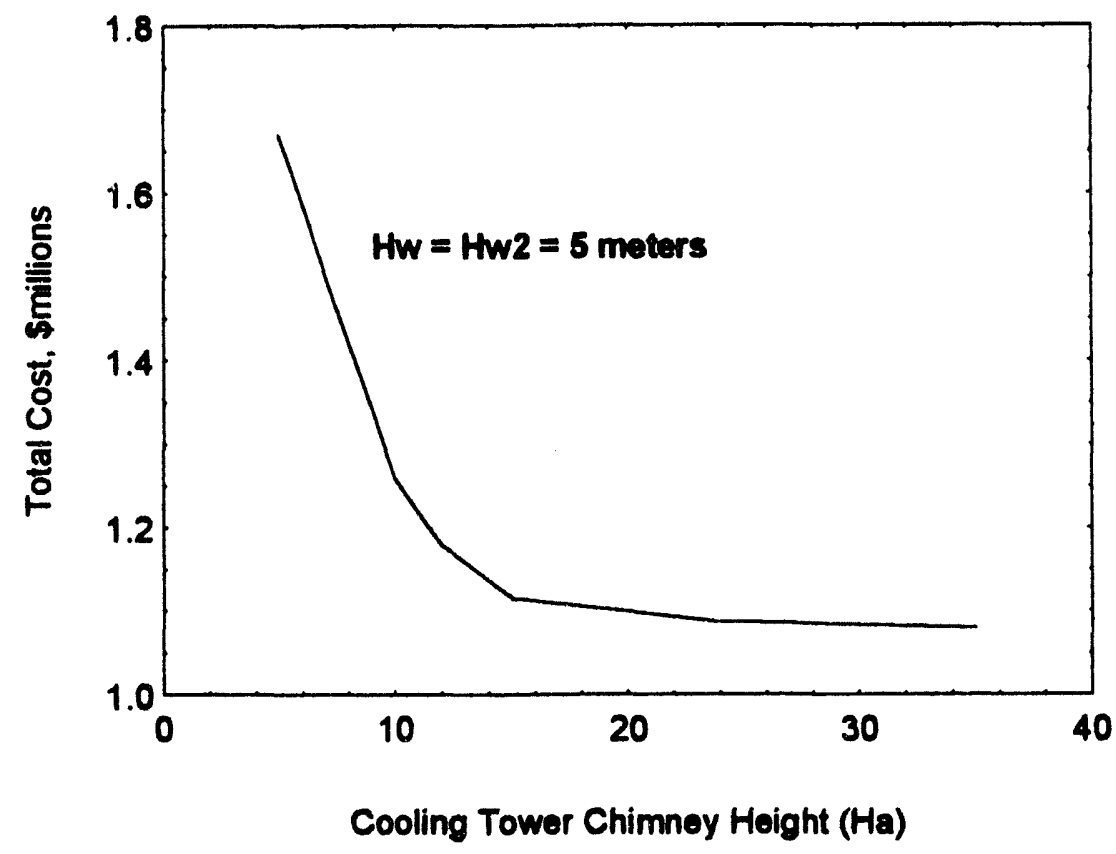

The total components cost decreases rapidly with increasing $\mathrm{Ha}$ until $\mathrm{Ha}$ is roughly 15 meters, at which point the rate of cost decrease slows substantially. Thus, ii is reasonable to pick $\mathrm{Ha}=15$ meters, to both minimize the cooling tower size and the components cost. 
The analysis in the two loop SWS discussed above yields the sizes shown in Table 6.23 for the components in the system. These values result from fixing $\mathrm{Hw}=\mathrm{Hw} 2=5$ meters, with the other SWS parameters as shown in Table 6.22 .

Table 6.23. Reference Component Parameters (Two Loop SWS)

\begin{tabular}{|l|l|}
\hline Total Cooling Tower Height: & 24 meters \\
\hline Cooling Tower Diameter: & 10 meters \\
\hline Cooling Tower Shell Volume: & $280 \mathrm{cu} . \mathrm{m}$. \\
\hline Auxiliary Heat Exchanger (AHE) Volume: & $38 \mathrm{cu} . \mathrm{m}$. \\
\hline Intermediate Heat Exchanger (IHE) Length: & 9 meters \\
\hline Intermediate Heat Exchanger (IHE) Volume: & $7 \mathrm{cu} . \mathrm{m}$. \\
\hline Total Secondary Components Cost: & $\mathbf{\$ 1 , 1 0 0 , 0 0 0}$ \\
\hline
\end{tabular}

\subsubsection{Cost Comparison between Single and Two Loop SWS}

The previous subsection estimated the minimum cost for the major components of the two loop SWS. To compare the cost of the single and two loop systems, a cost estimate of the single loop system needs to be performed. Since the single loop SWS does not have an Intermediate Heat Exchanger, the cost for the single loop system components is simply the cost of the cooling tower plus the cost of the Auxiliary Heat Exchanger. The minimum size for these components was determined in a previous subsection. The cost for the single loop components is estimated using the same methodology as used above for the two loop system, and the results are shown in Table 6.24 (the component sizes in Table 6.24 are reproduced from Table 6.5 for convenience). 


\section{Table 6.24. Reference Component Parameters (Single Loop SWS)}

\begin{tabular}{|l|l|}
\hline Cooling Tower Chimney Height: & 15 meters \\
\hline Total Cooling Tower Height: & 21 meters \\
\hline Cooling Tower Diameter: & 7.7 meters \\
\hline Cooling Tower Shell Volume: & $160 \mathrm{cu}$. meters \\
\hline Heat Exchanger Volume: & $18 \mathrm{cu}$. meters \\
\hline Total Components Coss: & $\$ 400,000$ \\
\hline
\end{tabular}

Comparing the component costs of Table 6.23 and Table 6.24, we see that the two loop system is roughly 3 times the cost of the single loop system. The increase in cost in the two loop system is due to two factors: (1) the additional cost of the IHE, and (2) the increased size of the AHE/cooling tower complex due to the lessened efficiency of the two loop system.

\subsubsection{Conclusions}

This section has been devoted to discussing the configuration, characteristics and cost of both the single loop and two loop Shield Water System. In this subsection, some general conclusions will be drawn based on the analyses presented in this section.

The single loop SWS has the potential to release radioactive coolant to the environment in the event of a leak, and there are concerns with the operational dose rates associated with N-16 gamma radiation. However, with proper system design (large pipe sizes and a deaeration system), the radioactivity concerns can probably be reduced to acceptable levels. The single loop system has the advantage of being simpler than the two loop system, and more efficient. In addition, the same, single loop which is used for cooling the shield during accident conditions is used for cooling during normal operation. Since 
the loop is used during normal operation, there is greater assurance that the loop will function as desired during accident conditions.

The two loop system virtually eliminates the conccrns over radioactive leakage and operational exposure, but is more complicated and less efficient than the single loop SWS. In addition, the secondary loop portion of the system is only used during accident conditions. Although it is online during normal reactor operation, it is idle. Idle fluid systems are by their nature harder to maintain in good operating condition than systems which are operated routinely. A specific problem in the case of the secondary loop of the two loop SWS is water chemistry/corrosion problems which could occur in the stagnant system due to the lack of circulation of coolant. Although this potential problem could be solved by installing a small jet pump in the secondary loop to maintain proper coolant circulation and chemistry, this would be a further complication to the two loop SWS.

Because the two loop SWS is less efficient than the single loop system, and because it requires an extra component (the Intermediate Heat Exchanger) the two loop system components are roughly 3 times as expensive as the single loop system. However, for both systems, the total component costs are equal to or less than about \$1 million. Considering that the cost of a typical fusion power reactor blanket system will cost in the hundreds of millions of dollars, the incremental cost of either the single or two loop SWS is very small. 


\section{Chapter 7 Conclusions and Recommendations for Future Work}

This chapter summarizes the main conclusions of this thesis, and provides recommendations for future work. One of the main results of this thesis is the conceptual design of Blanket Design 2, a helium-cooled fusion reactor blanket capable of passively surviving a worst-case undercooling accident with no damage. Included in the recommendations for future work are some of the steps which would be necessary to refine the results of the present conceptual design study in order to perform a more detailed design of Blanket Design 2.

\section{Conclusions}

The main conclusion of the present work is that it is possible to design a helium-cooled, solid breeder fusion reactor blanket which can survive a worst-case undercooling accident. This worst-case accident, dubbed a No-Flow LOFA, is characterized by a loss of helium coolant flow through the blanket, without loss of helium coolant pressure. The elevated temperatures of the first wall region caused by loss of coolant flow, coupled with the high stresses caused by the coolant pressure combine to make the No-Flow LOFA the most severe undercooling accident for a helium-cooled blanket. Blanket Design 2, using the novel "beryllium joint" concept, can survive such an accident easily.

Blanket Design 2 is based on the original nested shell blanket design developed by Wong (see Chapter 4). The original nested shell design used a mixed beryllium and lithium oxide pebble bed for the breeder regions. The first blanket design developed and analyzed herein (Blanket Design 1, described in Section 5.2) was also a nested shell design with a mixed pebble bed. The pebble bed region of Blanket Design 1 was optimized for survival of an undercooling accident by maximizing its thermal 
conductivity. This was done by: (1) maximizing the beryllium-to-lithium oxide ratio subject to retaining good breeding performance; and (2) maximizing the size of the beryllium pebbles subject to maintaining good bed packing performance. As is summarized in Table 7.1, Blanket Design 1 can survive all of the undercooling accidents analyzed except for the worst-case No-Flow LOFA.

\section{Table 7.1. Accident Performance of Blanket Designs 1 and 2}

\begin{tabular}{|c|c|c|c|}
\hline Blanket Type & Accident Trpe & $\begin{array}{l}\text { Survives } \\
\text { w/o Damare }\end{array}$ & Damaged \\
\hline \multirow{5}{*}{ Blanket Design 1} & LOCAt & $\mathbf{X}$ & \\
\hline & LOCA w/ air ingress & $\mathbf{X}$ & \\
\hline & $\begin{array}{l}\text { LOCA w/ helium } \\
\text { circulator failure }\end{array}$ & $\mathbf{X}$ & \\
\hline & LOFAt & $\mathbf{X}$ & \\
\hline & No-Flow LOFA & & $\mathbf{X}$ \\
\hline \multirow{5}{*}{ Blanket Design 2} & LOCAt & $\mathbf{X}$ & \\
\hline & LOCA w/ air ingress & $\mathbf{X}$ & \\
\hline & $\begin{array}{l}\text { LOCA w/ helium } \\
\text { circulator failure }\end{array}$ & $\mathbf{X}$ & \\
\hline & LOFAt & $\mathbf{X}$ & \\
\hline & No-Flow LOFA & $\mathbf{X}$ & \\
\hline
\end{tabular}

+ Assumes helium circulators continue operating, and there is no air ingress.

\$ Assumes natural circulation of pressurized helium coolant, with a 2 meter elevation difference between cool and hot coolant.

To design a helium-cooled blanket which can survive the No-Flow LOFA, development of the "beryllium-joint" concept was undertaken in Section 5.3. Incorporated in Blanket Design 2, the beryllium joint concept uses slabs of beryllium to conduct the heat away from the hot first wall region, minimizing its temperature during an accident. The 
beryllium joint concept also allows for significant ( 10 percent or more) beryllium swelling during blanket operation, without causing structural deformation or loss of thermal performance. Another factor which helps Blanket Design 2 survive a No-Flow LOFA is the fact that the coolant channel wall thickness was chosen based on ruggedness concerns (see Appendix 2). This resulted in overdesigning these channels with respect to the helium coolant pressure of $10 \mathrm{MPa}$. This overdesign lowers the stresses in the first wall channels, thus helping the first wall sustain the high temperatures of the accident without excessive creep. A summary of the design characteristics of Blanket Design 2 is shown in Table 7.2. As discussed in Section 5.3.4, Blanket Design 2 can survive a No-Flow LOFA with no damage even if the plasma remains on up to 7 seconds after the helium circulators cease to function.

Chapter 6 discussed the impact of Shield Water System (SWS) design on the accident response of Blanket Design 2. It was demonstrated that in order to ensure long-term passive safety for the blanket, a SWS capable of natural circulation in the event of pump failure is necessary. Analysis at the beginning of the chapter showed that the inboard blanket region of Blanket Design 2 may fail as early as 10 days after the onset of the NoFlow LOFA if there is no natural circulation in the shield. In addition, Appendix 11 showed that a SWS capable of natural circulation could eliminate the possibility of severe structural damage occurring in a vanadium/liquid lithium blanket within 9.2 hours after the onset of a LOCA with air ingress. 
Table 7.2. Blanket Design 2 Characteristics

Design Neutron Wall Load (Average):
$4 \mathrm{MW} / \mathrm{m}^{2} \mathrm{O}$
$5 \mathrm{MW} / \mathrm{m}^{2} \infty$
3 full-power-years

Design Neutron Wall Load (Peak):

Design Blanket Lifetime:

Blanket Thickness (Inboard and Outboard): $\quad 0.40 \mathrm{~m}$

Helium Plenum Thickness (Inboard and Outboard): $0.30 \mathrm{~m}$

Shield Thickness (Inboard):

$\underline{0.42 \mathrm{~m}}$

Total Inboard Thickness:

$1.12 \mathrm{~m}$

Blanket Module Width (Outboard): $\quad 1.7 \mathrm{~m}$

Helium Coolant Operating Pressure: $\quad 10 \mathrm{MPa}$

Helium Coolant Inlet Temperature: 250 degrees C

Helium Coolant Outlet Temperature: $\quad 450$ degrees $C$

Lithium Oxide Operational Temperature Range: $\quad 309-610$ degrees $\mathrm{C}^{++}$

Maximum MT-9 Operational Temperature: 550 degrees C

Maximum Beryllium Operational Temperature: $\quad 575$ degrees $\mathrm{C}$

Number of Coolant Shells: $\quad 15$

Inner Diameter of Coolant Shells: $\quad 4-9 \mathrm{~mm}^{*}$

Composition of Breeder Regions (by volume): $\quad 0.55 \mathrm{Be} / 0.19 \mathrm{Li} 2 \mathrm{O} / 0.26 \mathrm{He}$

Composition of Shield (by volume): $\quad 0.90 \mathrm{MT}-9 / 0.10$ water

One-Dimensional TBR: $\quad 1.4$

Tritium Inventory: $\quad \approx 4 \mathrm{~kg}$ in $\mathrm{Be} ; \approx 40 \mathrm{~g}$ in breeder

Maximum Fast Fluence to Magnets: $\quad 10^{23} \mathrm{n} / \mathrm{m}^{2 * *}$

Required Pumping Power:

Overall Power Conversion Efficiency:

$4.3 \%$ of total thermal power *** $0.35^{+}$

O Used for undercooling accident analysis calculations.

Do Used for shielding calculations.

* Depending on the location of the shell in the blanket.

** Assuming a 40 year lifetime at a cuty factor of 0.75 .

*** Assuming reactor total thermal power of $3500 \mathrm{MW}$. Separate helium circulators are necessary for the first wall coolant channels to avoid excessively high pumping power requirements.

+ Assumes a steam plant efficiency of 0.38 , a circulator efficiency of 0.9 , and accounts for helium circulator heating of coolant.

${ }^{++}$Except for very small region near helium inlet in which the temperature lies in the 290-300 degrees $C$ range. 
Chapter 6 provided conceptual designs and cost estimates for both a single-loop SWS and a two-loop SWS, and compared the advantages and disadvantages of the two options. The two-loop SWS has a primary coolant loop which circulates water between the shield and the Intermediate Heat Exchanger (IHE), and a secondary loop which circulates water between the IHE and the cooling tower. Table 7.3 summarizes the results of this comparison.

\section{Table 7.3 Comparison of Single- and Two-Loop SWS Options}

\section{Single-Loop Advantages:}

- Simpler and less-expensive design

- More efficient

- Easier to maintain

- Potentially more reliable

\section{Two-Loop Advantages:}

- No radioactivity in secondary loop

- No secondary loop shielding required

- No risk of radioactivity release to environment

The estimated cost of the components for the two-loop SWS is about 3 times more expensive than for the single-loop SWS (see Section 6.11). However, since the components for the two-loop SWS are estimated to cost only about $\$ 1$ million, the cost of either option will be very small compared to the overall cost of the reactor's blanket and shield system, which will cost hundreds of millions of dollars. Hence, the passive safety benefits of a natural circulation-cooled SWS can be achieved for a very reasonable price. In the author's opinion, the designers of future power reactors such as ITER and DEMO should consider incorporation of a natural circulation-cooled SWS into the reactor design, and, if possible, incorporate features into the blanket designs that permit rapid flow of heat from the first wall region toward the cooler portions of the blanket. 
During the development of the single-loop SWS, estimates were made of the radioactivity of corrosion products in the coolant to determine whether a coolant leak in the cooling tower/Auxiliary Heat Exchanger (AHE) complex would cause an unacceptable release of radioactivity into the environment. Assuming a worst-case leak in the AHE to be a double-ended rupture of an AHE tube, Section 6.10.1 showed that the radioactivity released to either the ground or to the air should not exceed the maximum permissible concentrations required by federal standards for release to unrestricted areas.

\section{Recommendations for Future Work}

The scope of the present work did not include the possible effects of a plasma disruption on the first wall temperature behavior. It is likely that the thermal effects of a disruption would be similar to the effects of the plasma continuation, but over a shorter timescale. It would be worthwhile to evaluate the possible impact of disruptions on the thermal behavior of Blanket Design 2 during a No-Flow LOFA. Furthermore, the effect of possible electromagnetic loads on the blanket structure during a disruption (if the plasma current is dumped into the blanket) should be addressed.

The present study simplified the geometry of the blanket to one-dimension during blanket analysis. Such simplification ignored the effects of the nested shell edges. During a detailed design of Blanket Design 2, the effect of the shell edges should be modeled using at least a two-dimensional, if not a three-dimensional treatment. This more detailed model should include the actual poloidal neutron wall distribution, which could not be modeled correctly in one-dimension. After development of the more detailed model, the model would be used to analyze both normal operating conditions (to confirm normal operating temperatures of breeder, multiplier, and structure) and accident conditions (to confirm that the blanket survives a No-Flow LOFA). 
During the sensitivity analysis performed on the beryllium joint concept in Section 5.3.5, it was tentatively concluded that stresses in the beryllium slabs caused by temperature and irradiation-induced swelling should not be enough to cause cracking of the beryllium slabs, and the consequent loss of thermal performance. However, it was recognized that a severe lack of high-neutron-fluence data on beryllium makes this conclusion speculative at best. High-energy neutron irradiation data at high fluences is sorely needed for beryllium to determine its swelling and fracture toughness performance

The scope of the present work did not include actual fabrication and test of the proposed beryllium joints. The next step in development of Blanket Design 2 would clearly involve such a "reality check." Some of the issues that could be explored during such testing are as follows: (1) confirmation of critical assumptions such as the gap conductances and effective thermal conductivity of the joints; (2) determination of the difficulty and cost of fabrication of large numbers of beryllium joints, which would be required to build a fusion reactor blanket; and (3) the effect of fabrication errors such as slab misalignment.

During failure analysis of Blanket Design 1 and 2, extrapolation of the data base for HT/MT-9 was necessary to estimate its high-temperature strength and creep properties. Prior to use of HT/MT-9 in an actual design, such high temperature performance data is needed. In addition, the effect of irradiation on the steel must be tested.

During development of both the single and two loop Shield Water Systems (SWS), it was assumed that only one cooling tower/Auxiliary Heat Exchanger complex is necessary to provide accident cooling to the shield. Further work could look into the desirability of adding redundancy to the SWS by, for example, using two cooling tower 
complexes instead of one. The possible effects of abnormal conditions in the cooling tower complex, such as ice on the heat exchange surfaces, could also be addressed. Such analysis was beyond the scope of the present work

To estimate the radioactivity in the single-loop SWS coolant, data from corrosion testing in high temperature water for typical PWR materials was coupled with temperaturedependent corrosion rate correlations to determine the expected corrosion rates in the low-temperature SWS system. To firmly establish the concentration of radionuclides which would be expected in the SWS coolant, low-temperature ( $\approx 85$ degrees $C$ ) corrosion testing is necessary for the materials expected to be used in the SWS. These materials include the ferritic steel used in the shield, the CRES 304 steel used for the inlet and outlet piping and heat exchanger tubes, as well as the aluminum brass tubing in the Service Heat Exchanger.

As was stated at the beginning of the chapter, most of the recommendations in this section suggest analyses which would have to be performed to advance Blanket Design 2 from the conceptual design stage presented in this thesis to the detailed design stage. Because of the conservative approaches adopted throughout the course of this conceptual design work, however, it is not expected that the main results of this thesis would change as a result of detailed design. 


\section{Appendix 1: Geometrical Correction Factor Details}

In the neutronics analysis used in the present work, the tokamak's geometry was approximated by allowing the major radius of the torus to go to infinity. As described in Chapter 3 , this results in an error in the calculated neutron flux results proportional to the distance from the centerline of the machine. Tn correct for this error, it is necessary to apply a correction factor to the TWODANT neutron flux results, prior to using them in the REAC3 activation code. This appendix describes in more detail how this correction factor is applied calculationally.

In Chapter 5, the neutron wall load used for calculating the tritium breeding ratio and the activation of the blanket is assumed to be uniform for a given inboard or outboard section, and equal to the average wall load for the appropriate section. The following discussion demonstrates how the neutron flux as a function of distance into the blanket is established for these runs.

Figure A1.1 shows a diagram of the outboard blanket section; the figure shows the tokamak vertical centerline, the tokamak major radius $\mathbf{R}_{0}$, the poloidal radius and angle of a point $P$ in the blanket $(r, \Theta)$, and the horizontal distance from the vertical centerline R. 


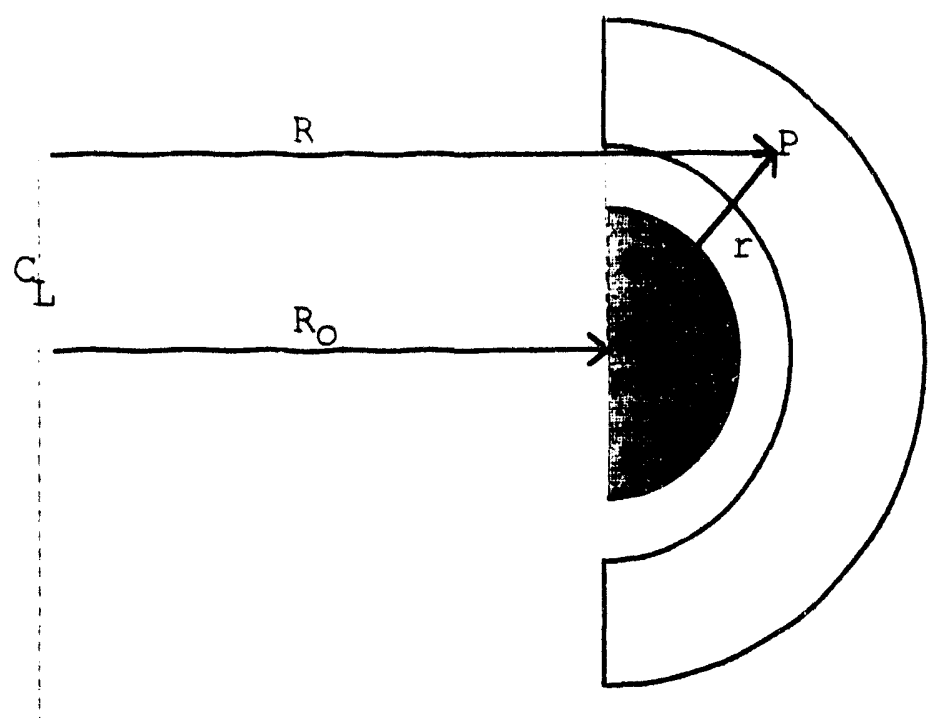

TWODANT gives the neutron flux at each distance $\mathrm{r}$ from the plasma center. Since the wall load is specified to be uniform, the neutron flux values that TWODANT outputs do not depend on poloidal angle $\Theta$. However, as can be seen in Figure A1.1, the distance from the centerline $R$ does depend on $\Theta$. By a simple integration of $\Theta$ from 0 to $\pi$, we determine that the average value of $R$ at a distance $r$ from the plasma center is given by $\langle R\rangle=R_{0}+\frac{2 r}{\pi}$ (for the outboard blanket). Hence, the average value of neutron flux at distance $r$, corrected for geometry, is the value given by TWODANT multiplied by the correction factor. It is these average values of neutron flux as a function of $r$ that are used as inputs to the REAC3 activation code. To calculate the neutron wall load of the outboard blanket, which is the input to TWODANT, the average poloidal neutron wall load (determined in Chapter 5) is multiplied by $\left\langle\left. R\right|_{0}\right\rangle / R_{0}$, where $\left\langle\left. R\right|_{0}\right\rangle=$ the value of $\langle R\rangle$ at the first wall $(r=a)$. The tokamak radius $R_{0}$ is chosen to be $6 \mathrm{~m}$, and the minor radius is chosen to be $1.5 \mathrm{~m}$ for the cases analyzed herein. 


\section{Appendix 2: Sizing of Shell Coolant Channels}

In the design of the nested shell blanket, an analysis of the effectiveness of natural circulation for various channel sizes is performed (Section 5.2.2.3), and pumping power is plotted as a function of first wall cooling channel diameter (Section 5.2.2.4.) This appendix describes the calculational model used for these analyses in greater detail

\section{Pumpine Power Calculation}

This calculation determines the minimum pumping power required to ensure that the first wall structural temperature does not exceed 550 degrees $C$. This maximum temperature occurs at the outlet side of the channel on the side exposed to the plasma.

As discussed at the beginning of the Chapter 5, the maximum neutron wall load for the blanket designs considered here is assumed to be $5 \mathrm{MW} / \mathrm{m}^{2}$. Since the alpha power generated in the fusion reactions is $1 / 4$ of the neutron power, if we assume the alpha power is uniformly distributed on the first wall, we obtain a maximum surface heat load of $\left(5 \mathrm{MW} / \mathrm{m}^{2}\right) / 4=1.25 \mathrm{MW} / \mathrm{m}^{2}$. However, a substantial amount of the alpha power is directed toward the divertor. This results in a reduced surface heat load to the first wall. For the ARIES-I reactor design, for example, the maximum surface heat load to the first wall is only $1 / 6$ of the maximum neutron wall load. ${ }^{1}$ Since it is beyond the scope of the present work to model the divertor heat load, the maximum surface heat load will be assumed to be 1/5 of the maximum neutron wall load (that is, midway between $1 / 4$ and $1 / 6$ ). This corresponds to a maximum surface heat load of $1 \mathrm{MW} / \mathrm{m}^{2}$. It is conservatively assumed for this calculation that this maximum heat load applies for the length of the channel considered.

\footnotetext{
1 F. Najmabadi, et.al., "The ARIES-I Tokamak Reactor Study, Final Report," UCLA-PPG-1323, 1991,p. 8-76.
} 
The helium inlet temperature is set at 250 degrees $\mathrm{C}$, and the helium pressure is $10 \mathrm{MPa}$. An erosion layer of $2 \mathrm{~mm}$ of MT-9 is assumed to exist on the first wall in addition to the channel wall thickness, which is chosen based on the minimum thickness allowable assuming the channel must contain the helium pressure. ${ }^{2}$

The maximum first wall temperature at the outlet of the channel is given by the following relation:

$$
\mathrm{T}_{\mathrm{Fw} \text {,max }}=\mathrm{T}_{\mathrm{bel,o}}+\mathrm{dT}_{\mathrm{film}}+\mathrm{dT}_{\mathrm{i}},
$$

where $\mathrm{T}_{\text {bel,o }}$ is the helium coolant outlet temperature (determined assuming all of the heat from plasma is deposited into the first wall channels),

$\mathrm{dT}_{\mathrm{fim}}$ is the temperature rise across the helium coolant film at the inside of the channel, and $d T_{1}$ is the temperature rise across the thickness of the erosion layer plus the channel thickness.

$\mathrm{dT}_{\text {film }}$ is given using the standard relation:

$$
\mathrm{dT}_{\mathrm{flm}}=\frac{\mathrm{q}^{\prime \prime}}{\mathrm{h}} \text {, where }
$$

$\mathrm{q}^{\prime \prime}$ is the heat flux across the helium film, and $\mathrm{h}$ is the convective heat transfer coefficient.

The convective heat transfer coefficient, in turn, defines the Nusselt number $\mathrm{Nu}$ :

$$
\mathrm{Nu} \equiv \frac{\mathrm{hD}}{\mathrm{k}},
$$

\footnotetext{
${ }^{2}$ based on the largest of the following two thickuresses: 1) the minimum thickness required to prevent creep strain from exceeding $1 \%$ at 500 degrees $C$ assuming 5 years of operation at a duty factor of 0.75 , and 2) the minimum thickness to ensure structural ruggedness (chosen to be $r / 7$, where $r$ is the channel radius). The creep data was obtained from The Materials Handbook for Fusion Energy Systems, USDOE Report \#DOE/TIC-10122, McDonnell Divglas Astronautics Co., Initial Issue April 30, 1980.
} 
where $\mathrm{D}$ is the channel inner diameter, and $\mathrm{k}$ is the helium thermal conductivity. The Nusselt number is usually determined by the Dittus-Boelter equation for heated fluids:

$$
\mathrm{Nu}=0.023 \mathrm{Re}^{0.8} \mathrm{Pr}^{0.4} \text {, }
$$

where $\operatorname{Re}$ is the Reynolds number ( $\rho$ VD $/ \mu$ ), and $\operatorname{Pr}$ is the Prandtl number, which can be taken as 0.7 for helium. However, for cases where the temperature gradient across the helium film is large (as is the case here), the heat transfer across the film is decreased; hence the Nusselt must be reduced. To account for this effect (and also to account for the effects of flow development at the inlet, which is not a large effect for the present case, the following correlation is used: ${ }^{3}$

$$
\mathrm{Nu}=0.021 \operatorname{Re}^{0.8} \operatorname{Pr}^{0.4}\left(\frac{\mathrm{T}_{\text {vall }}}{\mathrm{T}_{\text {bulk }}}\right)^{-0.9}\left[1+\left(\frac{\mathrm{z}}{\mathrm{D}}\right)^{-0.7}\right],
$$

where $z$ is the distance downstream from the inlet. This relation is valid for $z / D>5$, $15,000<\operatorname{Re}<600,000$, and $1<\mathrm{T}_{\text {wall }} / \mathrm{T}_{\text {balk }}<2.4$; conditions which are met in the present analysis.

Equations A2.3 and A2.5 can be used to determine $h$ for fully-developed flow in uniformly-heated round tubes. However, for the present case, the tube is actually very non-uniformly heated, since the intense plasma heat load comes only from one direction. To modify the $\mathrm{h}$ for the non-uniform heat flux, data reported in the ARIES-I reactor study, which also operated at $10 \mathrm{MPa}$ helium pressure with comparable channel diameters, were used. These data indicated that for turbulent helium coolant flows, $h$ is reduced by up to $37 \%$, thus increasing the temperature drop across the helium film by the same amount. Hence, in this calculation the $h$ is reduced by $37 \%$ from the value calculated by the equations above.

${ }^{3}$ D.M. McEligot, et.al., "Effect of Large Temperature Gradients on Convective Heat Transfer: The Downstream Region," J. Heat Transfer, Vol. 87, No. 1, February, 1965, p. 67. 
Equation A2.6 below, without the natural circulation terms on the left-hand side, and without the $\mathrm{K}_{\text {loop }}$ term, is used to determine the pressure drop in the straight lengths of the first wall coolant channel. The two channel bends at the corners of the blanket module are accounted for assuming $K_{\text {turn }}$ equals 0.5 , a reasonable value. ${ }^{4}$ The pumping power is determined by the following equation:

$$
\mathrm{W}_{\mathrm{p}}=\frac{1}{\varepsilon} \dot{\mathrm{m}} \frac{\mathrm{RT}}{\mathrm{P}} \Delta \mathrm{P}
$$

where:

$P$ is the helium pressure.

$\Delta P$ is the pressure drop in the channel

$\varepsilon$ is the helium circulator efficiency

$\dot{m}$ is the mass flow rate of helium through the channel

$R$ is the gas constant, and

$\mathrm{T}$ is the helium inlet temperature

Note that the mass flow rate $\dot{m}$ in Equation A2.6 is the total mass flow for the whole first wall, not just for one channel. (This is calculated by determining the number of channels necessary to cover the entire first wall area of a reactor of major radius 6 meters and minor radius 1.5 meters). The helium circulator efficiency is assumed to be 0.9 , as was done for ARIES-I. The pumping power goes up both as the mass flow rate and as the pressure drop does. To keep the temperature drop low enough across the helium film, the velocity of the coolant must kept relatively high. Hence, if one tries to decrease the pumping power by increasing the channel diameter, thereby decreasing the pressure drop in the channel, the mass flow rate increases, tending to keep pumping power high.

\footnotetext{
${ }^{4}$ The pressure drop at turn is given by $\left(K_{\text {tum }} \rho V^{\wedge} 2\right) / 2$. N.E. Todreas and M.S. Kazimi, Nuclear Systems I, Hemisphere Pub. Co., New York, 1990, p. 358, gives a $K_{\text {tum }}$ range of 0.35 to 0.9 for standard elbows. We have assumed a value in the middle of this range. Note that the pressure drop caused by the turns is small compared to the overall channel pressure drop.
} 
Similarly, if the mass flow rate is decreased to reduce the pumping power, the channel diameter must be decreased to maintain a sufficiently high velocity. An optimum can be found, as is seen in Section 5.2.2.4, around a channel diameter of $0.4 \mathrm{~cm}$.

\section{Natural Circulation Calculation}

Figure A2.1 shows the simplified flow loop used for the analysis. As described in the section on the blanket design, the figure reflects the following assumptions: (1) the first wall coolant channel is 2 meters long; (2) the steam generator is located above the top of the coolant channel, giving 2 meters for development of a thermal driving head; and (3) there is a helium circulator bypass valve which opens upon loss of circulator pump head.

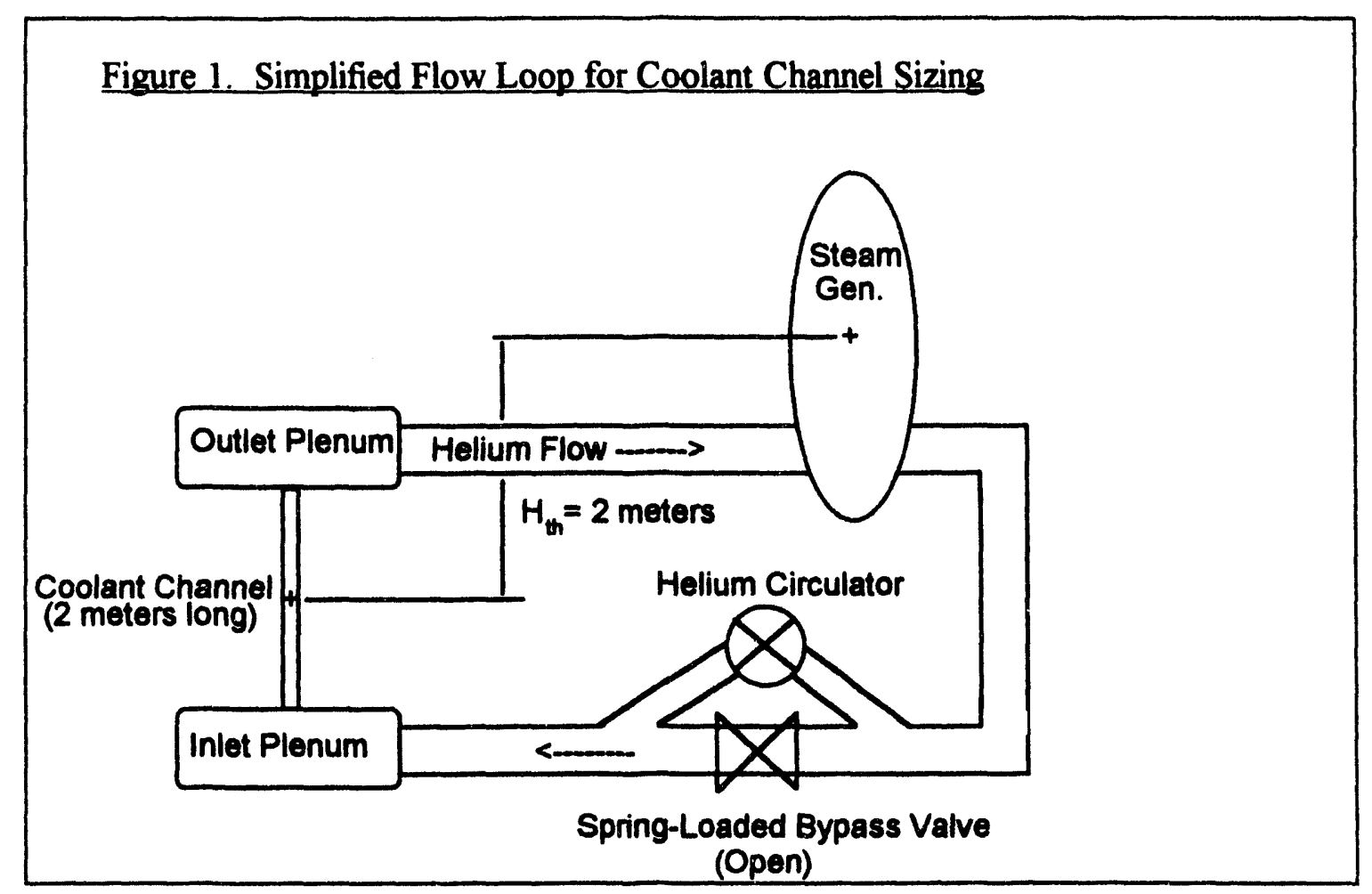

The equation for mass flow during natural circulation in the channel is given below: 


$$
\begin{aligned}
& g \mathrm{H}_{\mathrm{th}}\left(\rho_{\text {cold }}-\rho_{\text {hot }}\right)= \\
& \quad \dot{m}^{2}\left\{K_{\text {loop }}+\frac{R T_{\text {in }}}{2 P\left(A_{z}\right)^{2}}\left[\int_{z_{\text {in }}}^{:} \frac{f\left(z^{\prime}, \dot{m}\right) P_{f}}{A_{z}}\left(\frac{T_{b}\left(z^{\prime}\right)}{T_{\text {in }}}\right) d z^{\prime}+2\left(\frac{T_{\text {out }}}{T_{\text {in }}}-1\right)\right]\right\}
\end{aligned}
$$

where:

$H_{t h}$ is the vertical height available for natural circulation

$\rho$ is the helium density (in the cold leg or hot leg)

$\dot{m}$ is the mass flow rate of helium through the channel

$K_{\text {loop }}$ is the loop pressure drop coefficient

$A_{z}$ is the channel cross section

$P_{f}$ is the wetted perimeter of the channel

$f$ is the friction factor

$T$ is the helium temperature (at the inlet, outlet, or at point $z$ along the channel)

$P$ is the helium pressure.

The left side of the equation is the natural circulation driving head. The right side of the equation is the friction and expansion pressure drop. $K_{\text {loop }}$, the loop pressure drop coefficient, models the pressure drop through the steam generator, and inlet and outlet coolant piping. Consistent with previous analyses, 56 the loop pressure drop is assumed to simply scale with the mass flow rate through the system. The rest of the right hand side of the equation models the pressure drop within the heated first wall channel, and

5J.E. Massidda and M.S. Kazimi, Thermal Design Considerations for Passive Safety of Fusion Reactors, MIT Plasma Fusion Center Report \#PFC/RR-87-18 dated October, 1987.

${ }^{6}$ B. Malinovic, Natural Convection Characteristics of Liquid Metal Cooled Fusion Reactors, S.M. Thesis, Nuclear Engineering Department, Massachusetts Institute of Technology, 1986. 
includes both friction and expansion pressure drops. ${ }^{7}$ The friction factor $\mathrm{f}$ is given by the Koo correlation, which is valid for turbulent flow: ${ }^{8}$

$$
f=0.00140+0.125 \operatorname{Re}^{-0.32}
$$

This correlation is valid for $3000<\operatorname{Re}<3,000,000$

To solve Equation A2.7, the following steps are taken:

1) The helium pressure is chosen for the case to be run.

2) The channel diameter is chosen.

3) The channel wall thickness is chosen based on the minimum thickness allowable assuming the channel must contain the helium pressure, as discussed in the previous subsection.

4) The mass flow rate through the channel necessary to maintain the maximum first wall temperature at 550 degrees $\mathbf{C}$ during normal operation is calculated. This assumes a neutron wall loading of $5 \mathrm{MW} / \mathrm{m}^{2}$, and a first wall heat load of $1 \mathrm{MW} / \mathrm{m}^{2}$. This mass flow rate is the normal operating mass flow rate through the channel.

5) The decay heat deposited per unit length along the channel is conservatively assumed to be constant, and is calculated based on the volumetric decay heat being generated by the MT-9 first wall at shutdown ( $2 \mathrm{MW} / \mathrm{m}^{3}$.) Included in the decay heat are contributions from the first wall erosion layer (assumed to be $2 \mathrm{~mm}$ thick MT-9), and from the channel wall. The bulk temperature $T_{b}$ as a function of distance along the channel can then be related to the mass flow rate in the channel.

6) Using this relation between $T_{b}$ and the mass flow rate, Equation A2.7 can be solved iteratively to get the mass flow rate through the channel during natural circulation, and

\footnotetext{
${ }^{7}$ The correlation used for pressure drop in a heated channel of flowing helium was developed by Melese in G. Melese and R. Katz, Thermal and Flow Design of Helium-Cooled Reactors, American Nuclear Society, 1984.

8W.H. McAdams, Heat Transmission, 3rd Ed., McGraw-Hill Book Co., 1954.
} 
the consequent outlet temperature. ${ }^{9}$ For this calculation, the pressure drop through the portion of the loop not including the coolant channel is assumed to be $56 \mathrm{kPa}$ during normal operation, based on the ARIES-I nested shell blanket loop design value. ${ }^{10}$ For flow rates below the normal flow rate, the pressure drop is assumed to scale with the square of the mass flow rate, as discussed above.

7) The first wall temperature at the outlet during natural circulation is determined based on the helium coolant outlet temperature, plus the temperature rise from the bulk helium coolant to the first wall, as described by Equation A2.1. Note that no correction to $h$ based on non-uniform heat load need be made, since the plasma heat load is not present for this calculation.

${ }^{9}$ The MathCAD utility, by MathSoft Inc. is used to perform these calculations.

${ }^{10}$ F. Najmabadi, et.al., "The ARIES-I Tokamak Reactor Study, Final Report," UCLA-PPG-1323, 1991. 


\section{Appendix 3: Determination of Bed Thicknesses for Nested Shell Blanket}

This appendix describes the procedure used to determine the proper lithium oxide/beryllium mixed bed thicknesses used for Blanket Design 1.

The first step in determining the bed thicknesses is to find the volumetric heating as a function of position in the blanket in the mixed bed regions. To do this, the volumetric heating as a function of position is first found for the blanket, assuming the blanket is composed of a volumetrically uniform mixture of structure, breeder, and multiplier materials. For Blanket Design 1, the assumed volume fractions for this calculation are 0.11 MT $-9,0.12$ lithium oxide, and 0.36 beryllium. These volume fractions are the same as the ones used in the breeder/multiplier ratio optimization analysis discussed in Chapter 5 , and are justified there.

The operational heating of the volumetrically uniform blanket, calculated using the TWODANT code, is shown in Figure A3.1. The heating decreases exponentially along with the neutron flux for the volumetrically uniform case. Even if the blanket is not volumetrically uniform, the heating and neutron flux will tend to decrease exponentially within a particular, volumetrically uniform region. Hence, to determine a very good estimate of the heating in the mixed bed regions as a function of position in the blanket, all we need do is determine what the heating of the mixed bed region at the front of the blanket is, and assume that the heating of the mixed bed regions in the rest of the blanket drops off exponentially from that value. The appropriate exponential factor to use is the one which corresponds to the dropoff rate of the volumetrically uniform case shown in Figure A3.1. 
Figure A3.1. Volumetric Heating of Uniform MT-9/Li2O/Be Blanket

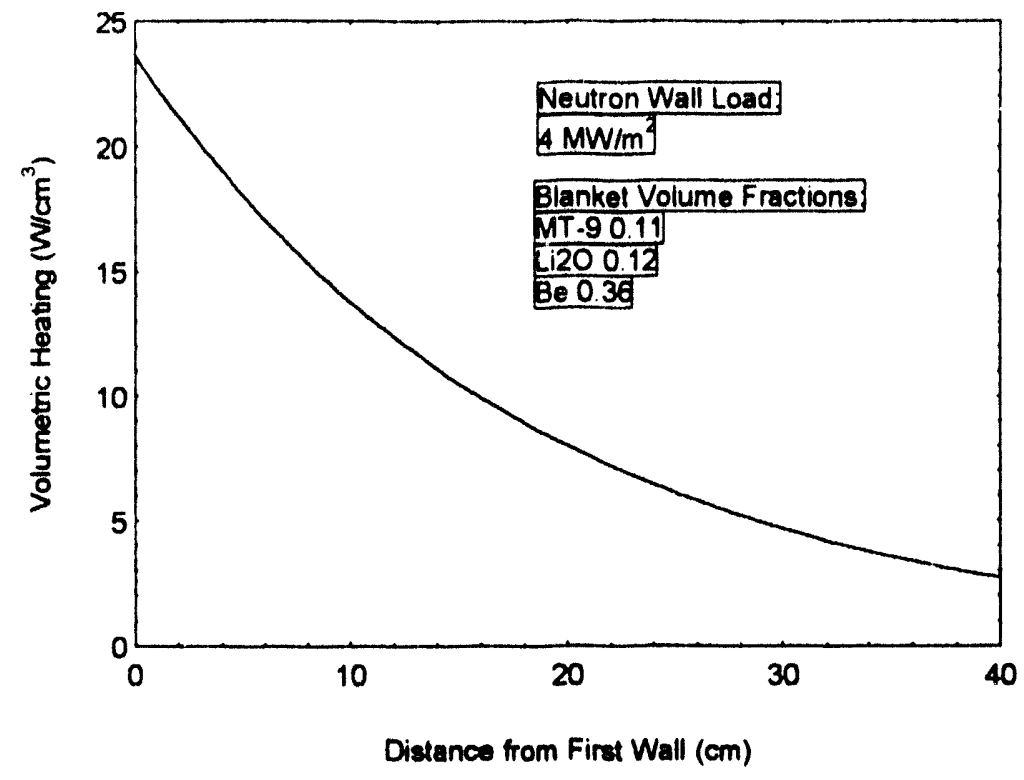

Figure A3.2 shows the resulting volumetric heating in the mixed bed regions alone.

Figure A3.2. Heating in Mixed Bed Regions

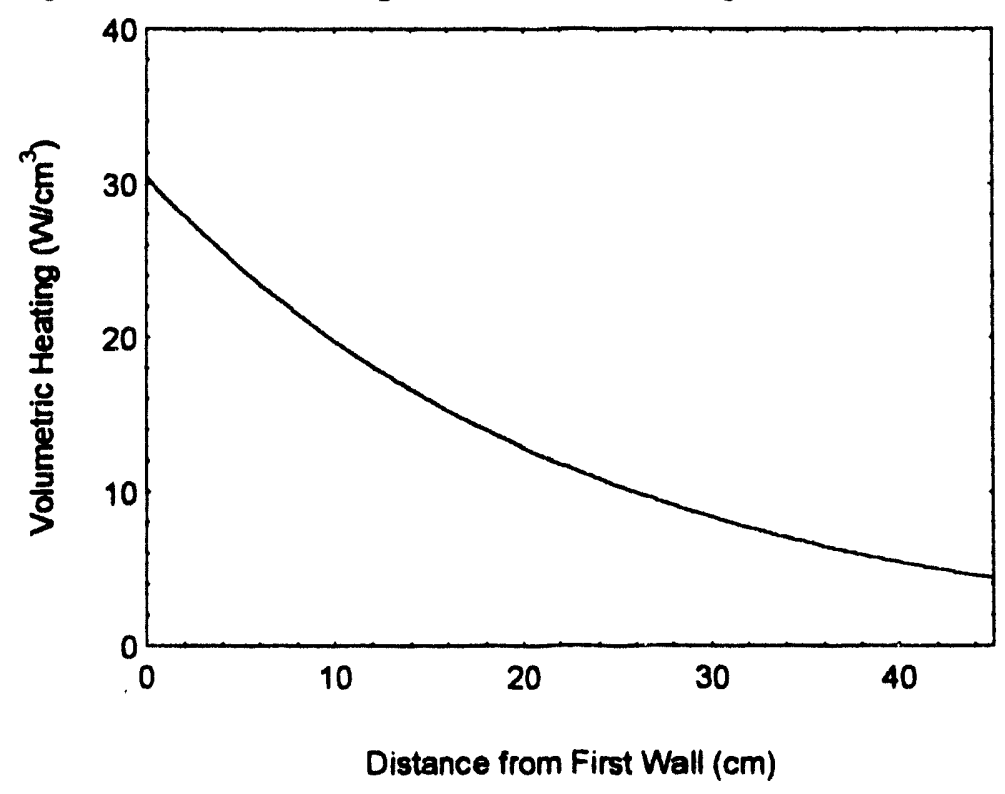


With the volumetric heating in the bed regions now determined, we can choose the appropriate thicknesses for the regions. It is desirable to have the mixed bed regions as thick as possible, for two reasons. Firstly, a thick region results in a higher heat flow to the helium coolant, and hence a higher temperature drop between the coolant and the breeder. This helps keep the breeder above the desired lower temperature limit in the region around the coolant inlet. Secondly, the thicker the mixed bed regions, the fewer structural shells are needed. Minimizing the number of structural shells minimizes the amount of metal in the blanket, thereby minimizing the amount of decay heat released and helping to mitigate the effects of a LOFA/LOCA accident.

However, the thickness of the bed regions is limited by the peak temperatures of the breeder and structural material in the vicinity of the coolant outlet. As the bed region's thickness is increased, both the temperature of the coolant channel adjacent to the bed and the peak temperature of the breeder near the center of the bed increase. We must ensure that these temperatures do not exceed the allowable maximum temperatures. 
Figure A3.3. Model for Bed

Thickness Calculations

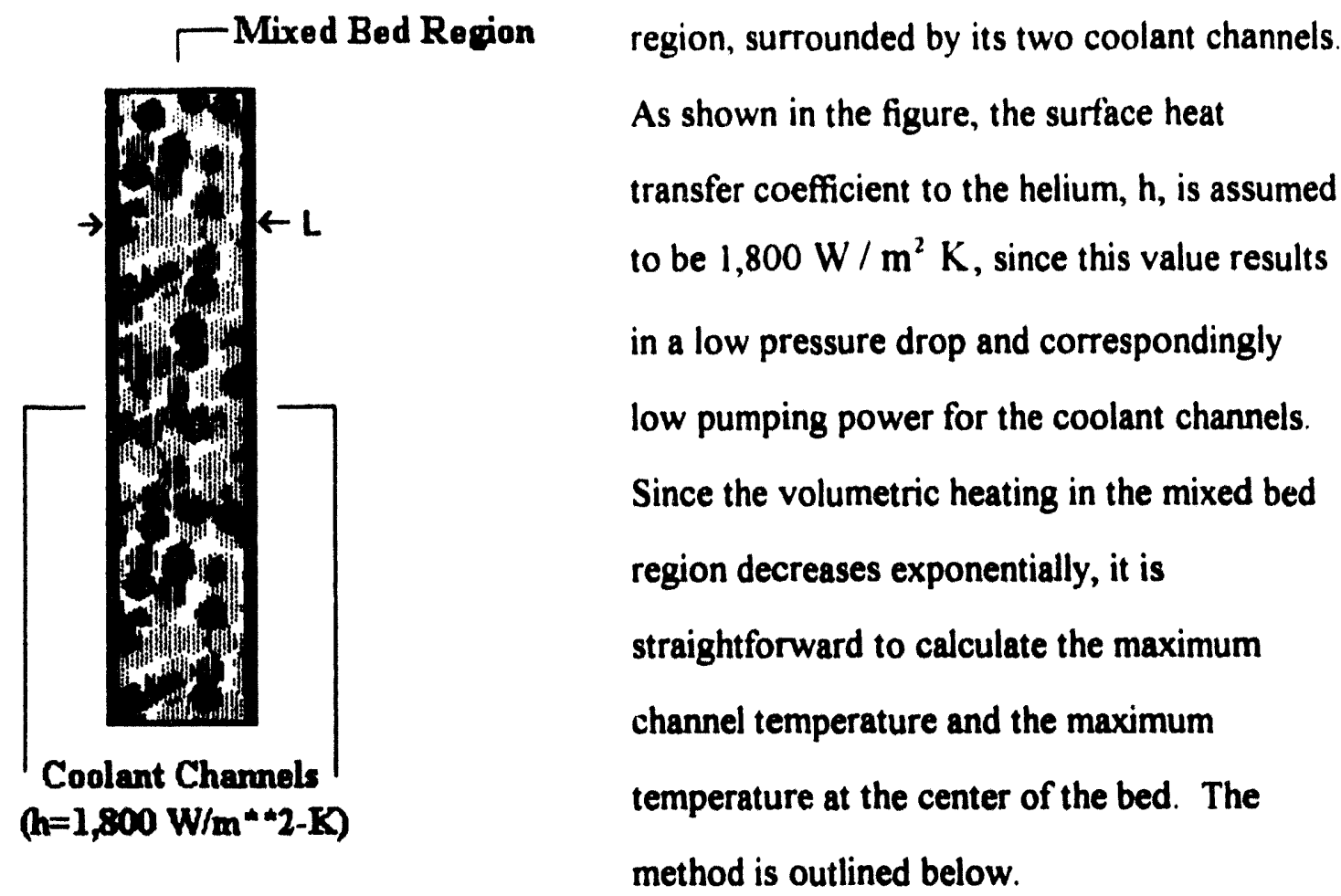

Figure A3. 3 shows a simple model of a bed
Figure A3.4. Model for Calculation of Maximum Pebble Bed Temperature

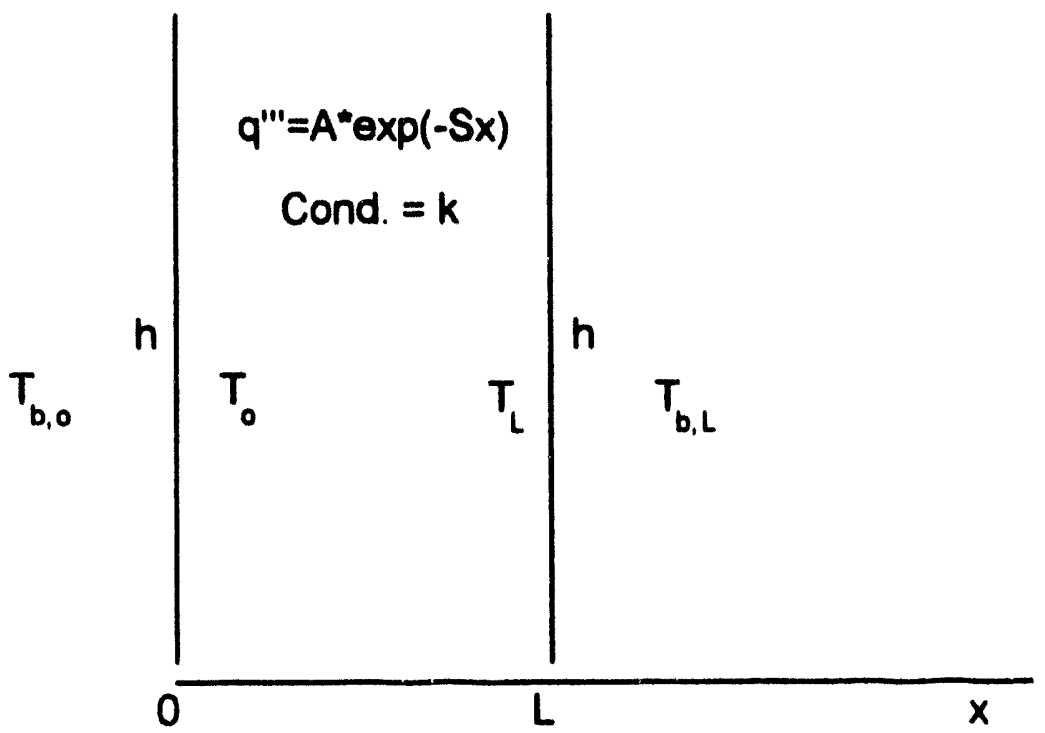

Figure A3.4 shows the model used to calculate the maximum temperature within the pebble bed. The model assumes that convective cooling of the region to the helium dominates over conductive cooling through the blanket

structure. The bed region is modeled as a one-dimensional slab of thickness $\mathrm{L}$ in the $\mathrm{x}-$ 
direction, with conductivity $\mathrm{k}$ and with volumetric heating given by the equation $\mathrm{q}^{\prime \prime \prime}=$ $A^{*} \exp (-\mathrm{Sx})$. This gives the exponentially-decaying volumetric heating discussed above The borders of the slab each have surface heat transfer coefficient $h$, with temperatures on either side of each border labeled as follows

$T_{b .0}$ and $T_{b . L}$ are the bulk helium temperatures corresponding to the slab borders at $x=$ 0 and $x=L$, respectively. $T_{0}$ and $T_{b}$ are the temperatures of the bed region at $x=0$ and $x=b$.

To calculate the temperature distribution within the slab, the one-dimensional steadystate heat conduction equation needs to be solved. For the present problem, this equation is as follows:

$$
k \frac{\partial^{2} T}{\partial^{2} x}=-q^{\prime \prime \prime}=-A \cdot \exp (-S x)
$$

The boundary conditions for this problem are as follows:

$$
q_{L}^{\prime \prime}=h\left(T_{L}-T_{b, L}\right)=-\left.k \frac{\partial T}{\partial x}\right|_{L} \text {, and }
$$

$$
q_{0}^{\prime \prime}=h\left(T_{0}-T_{b, 0}\right)=\left.k \frac{\partial T}{\partial x}\right|_{0}
$$

Integrating Equation A3.1 and using B.C.'s A3.2 and A3.3 yields the following relation for the temperature within the pebble bed region:

$$
\begin{aligned}
& T(x)=-\frac{A}{S^{2} k} \exp (-S x)+C_{1} x+C_{2} \text {, where } \\
& C_{1}=\frac{\frac{A}{S}\left[\exp (-S L)\left(\frac{1}{S k}-\frac{1}{h}\right)-\frac{1}{S k}-\frac{1}{h}\right]+T_{b, L}-T_{b, 0}}{L+\frac{2 k}{h}} \text {, and }
\end{aligned}
$$




$$
C_{2}=\frac{A}{S^{2} k}+T_{b, 0}+\frac{k}{h} C_{1}+\frac{A}{h S} .
$$

The maximum temperature of the region is where the following condition is satisfied

$$
\frac{\partial \mathrm{T}}{\partial \mathrm{x}}=0
$$

Using the above condition in Equation A3.4 yields the value of $x$ where the maximum temperature in the slab is, namely:

$$
x_{\text {hot }}=\frac{-\ln \left(\frac{-C_{1} S k}{A}\right)}{S} .
$$

This value of $x$ can be plugged into Equation $A 3.4$ to obtain the maximum temperature in the region.

The constants used in the above equations are as follows: $h=1,800 \mathrm{~W} / \mathrm{m}^{2} \cdot \mathrm{K}$, as discussed above; $k$ is as given in Section 5.2 .2 .1 (roughly $5.5 \mathrm{~W} / \mathrm{m} \cdot \mathrm{K}$ ); $\mathrm{S}$ is $4.3 \mathrm{~m}^{-1}$ (from Figure A3.2); and A is also given in Figure A3.2; the bulk coolant temperatures are specified as 450 degrees $C$, the helium coolant outlet temperature.

As a check on these calculations, a TWODANT run was performed with separated mixed bed and structural regions to confirm the volumetric heating of the bed regions, and the HEATING3 code was run to confirm that the operational temperatures are within the acceptable range. 


\section{Appendix 4: Calculation of Tritium Inventory in Blankets}

It is desirable to minimize the radioactive tritium inventory in the breeder and multiplier in a blanket for two reasons: (1) To minimize the amount of tritium that could potentially be released under accident conditions, and (2) To maximize the amount of tritium available to be processed and used as fuel for the reactor. The following section provides estimates for the tritium inventories of the Blanket Designs 1 and 2.

\section{Inventory in Lithium Oxide}

Estimation of the tritium inventory in the lithium oxide breeder requires a knowledge of the mechanisms of tritium transport in solid breeder materials. Determining the values of physical characteristics which are important to the tritium inventory, such as diffusion coefficients and grain sizes, is the subject of active research in the fusion community at the time of this writing. Enough data is available at this point, however, to make rough estimates of the expected tritium inventory in a fusion reactor blanket. The following discussion will develop tritium inventory estimates for the blanket designs which are developed in this work

In order for tritium generated in a grain of solid breeder material to become entrained in the purge flow stream and thereby be removed from the blanket, the following four processes must occur: ${ }^{1}$

1. Intragranular Diffusion, in which the tritium diffuses to the grain boundary;

2. Grain Boundary Diffusion, in which the tritium diffuses along grain boundaries to a pore;

3. Pore Diffusion, in which tritium diffuses through the porosity of the lithium oxide pebble to the surface of the pebble; and

'M. A. Abdou, et. al., "Thermal, Fluid Flow and Tritium Release Problems in Fusion Blankets," Fusion Technology, Vol. 18, September, 1990, p. 165. 
4. Purge Flow Convection, in which the tritium is entrained in the purge flow stream.

A fifth process, Surface Adsorption/Desorption, in which tritium is either sorbed or desorbed from surfaces, may be important with respect to tritium inventory of the breeder material. For lithium oxide, Precipitation of LiOT at temperatures below 300 degrees $\mathrm{C}$ can block pores and significantly retard the diffusion of tritium from the breeder. To minimize the precipitation of LiOT in lithium oxide, both Blanket Designs 1 and 2 are designed so that at least 90 percent of the lithium oxide material is above 300 degrees $\mathbf{C}$ during normal operation.

An analysis of the purge flow characteristics of typical solid breeder blanket geometries has been performed by Fujimura, et.al., showing that tritium convection into the purge flow stream happens much faster than diffusion from the lithium breeder pebbles. ${ }^{2}$ Hence purge flow convection (process 4 above) will not be limiting, and can be neglected with respect to the other processes. For temperatures above 300 degrees $\mathrm{C}$, the tritium release rate in lithium oxide is controlled by diffusion of $\mathrm{T}$ ions in the oxide, via processes 1 through 3 above. For lithium oxide particle sizes above about $1 \mathrm{~mm}$ in diameter, it has been shown that adsorption/desorption processes do not affect the tritium release rate significantly. ${ }^{3}$ If we assume the diffusion through the material's pores (process 3 ) is fast compared to diffusion through the grains and along the grain boundary, which is a reasonable assumption for a porous material, then diffusion through the grain and along the grain boundary (processes 1 and 2) govern the tritium release rate.

${ }^{2} \mathrm{~K}$. Fujimura, et. al., "Analysis of Helium Purge Flow in a Solid Breeder Blanket," Fusion Engineering. and Design, Vol. 8, 1989, p. 109.

${ }^{3}$ T. Terai, et.al., "In-Situ Tritium Release Experiments from Solid Breeding Materials (TTTEx) Released Chemical Form of Tritium and Its Diffusivities in Li2O," Fusion Engineering and Design, Vol. 7, 1989, p. 345. 
For this simplified, but realistic, case Fick's law can be used to derive a simple relationship for the tritium inventory in a particle, to wit: ${ }^{4}$

$$
I=\frac{S}{15}\left(\frac{a_{g}^{2}}{D_{g}}+\frac{a_{p}^{2}}{D_{1 n}}\right)
$$

where $\mathrm{I}$ is the tritium inventory in a particle, $\mathrm{S}$ is the tritium generation rate for a particle, $a_{g}$ and $a_{p}$ are the grain and particle radii, $D_{g}$ is the tritium diffusivity in a grain, and $D_{i n}$ is the effective tritium diffusivity through the grain boundaries in the particle.

Experiments have been performed to determine the in-situ tritium release from lithium oxide under fast neutron irradiation. ${ }^{5}$ Results from these experiments have determined the following relationships of $D_{B}$ and $D_{i}$ with respect to temperature:

$$
\begin{aligned}
& D_{g}\left(\mathrm{~m}^{2} / \mathrm{s}\right)=1.27 \times 10^{-9} \exp (-54.9(\mathrm{~kJ} / \mathrm{mol}) / R T) \\
& D_{\text {in }}\left(\mathrm{m}^{2} / \mathrm{s}\right)=1.61 \times 10^{-2} \exp (-95.1(\mathrm{~kJ} / \mathrm{mol}) / R T)
\end{aligned}
$$

where $\mathbf{R}$ is the gas constant. Although these two relationships were developed based on experiments in the temperature range 360-600 degrees C, we will assume that they hold down to the temperature where LiOT precipitation, rather than $T$ ion diffusion, begins to control the tritium release rate ( 300 degrees $C$ ). Figure $A 4.1$ shows plots of $D_{B}$ and $D_{\text {in }}$. as a function of temperature.

${ }^{4}$ See T. Terai, et. al. for derivation.

${ }^{5}$ T.Terai, et. al.. 
Figure A4.1. Lithium Oxide Diffusivities

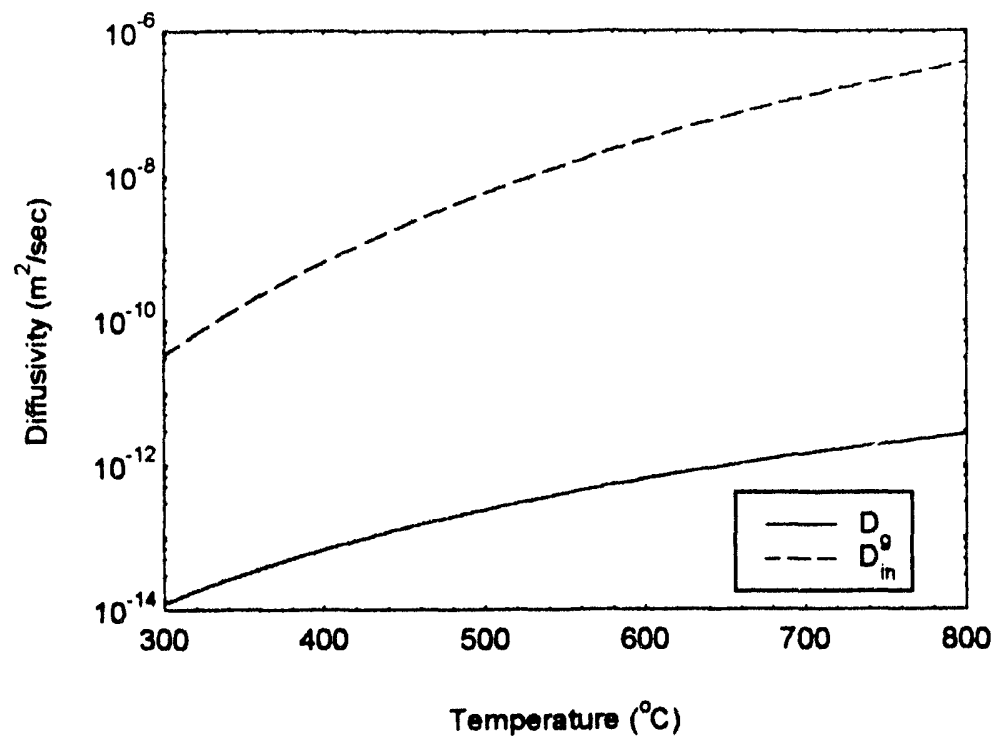

To calculate the tritium inventory in Blarket Designs 1 and 2, we use a lithium oxide pebble diameter which was chosen fo, the respective designs, namely $0.4 \mathrm{~mm}$ for Blanket Design 1 and $0.88 \mathrm{~mm}$ for Blanket Design 2. We assume a grain diameter of $10 \mu \mathrm{m}$ for the lithium oxide grains; lithium oxide with such a grain diameter has been fabricated. ${ }^{6}$ Although the majority (over 90 percent) of the lithium oxide in Blanket Designs 1 and 2 operates at temperatures significartly above 300 degrees $\mathrm{C}$, for simplicity and conservatism we will assume that this lithium oxide is all at exactly 300 degrees $C$. In addition, we will assume that for the lithium oxide which operates below 300 degrees $\mathrm{C}$ (less than 10 percent), the tritium inventory is increased by an order of magnitude over what the inventory would be at 300 degrees $\mathrm{C}$, due to LiOT precipitation. The table below shows the results of the tritium inventory estimates for Blanket Designs 1 and 2 .

${ }^{6} \mathrm{~K}$. Okuno and H. Kudo, "Tritium Diffusivity in Lithium-Based Ceramic Breeders Irradiated with Neutrons," Fusion Engineering and Design, Vol. 8, 1989, p. 355. 


\section{Tritium Inventories in Lithium Oxide Breeder}

\begin{tabular}{|l|l|l|}
\hline & Blanket Design 1 & Blanket Design 2 \\
\hline Lithium Oxide in Blanket & 40 tons & 40 tons \\
\hline Tritium in Breeder $(\mathbf{3 0 0} \mathrm{C})$ & $10 \mathrm{~g}$ & $20 \mathrm{~g}$ \\
\hline Tritium in Breeder $(<300 \mathrm{C})$ & $10 \mathrm{~g}$ & $20 \mathrm{~g}$ \\
\hline Total Tritium in Breeder & $20 \mathrm{~g}$ & $40 \mathrm{~g}$ \\
\hline
\end{tabular}

As shown in the table above, both blanket designs have tritium inventories below $50 \mathrm{~g}$, with Blanket Design 1 showing less inventory because of the smaller pebble size used in that design.

\section{Inventory in Beryllium}

Due to lack of irradiation data, little is known at the present time about the overall in-pile tritium retention behavior of beryllium. The lack of data is such that there are currently no good models for predicting tritium retention in beryllium. This is in contrast to the situation with breeder ceramics, which generally have fairly well-developed tritium retention models, such as the diffusion-driven model just used to estimate the inventory in lithium oxide.

It seems clear that a simple diffusion-oriented model will not suffice to describe the behavior of tritium in beryllium. Based on the limited available data, it appears that there is little or no release of tritium from beryllium below a certain temperature $\left(T_{0}\right)$ between 300-500 degrees $C$. Above a certain temperature $\left(T_{b}\right)$ between 600-900 degrees $C$ there is a burst release of virtually all of the tritium in the beryllium. For temperatures between 
$T_{o}$ and $T_{b}$ there is a gradual, quasi-linear increase in the fractional amount of tritium released as temperature is increased.

The burst-release temperature $T_{b}$ seems to be affected by the initial porosity as well as the irradiation-induced helium content of the samples. Increasing porosity or helium content both cause $T_{b}$ to decrease. ${ }^{7}$ This fact, coupled with data which shows that a majority of the trapped tritium is inside helium bubbles within the irradiated beryllium, ${ }^{8}$ indicates that the tritium may escape from the beryllium not by diffusion, but by passing through interconnected pores to the surface of the sample. If this is the case, then the tritium release behavior of beryllium would not be strongly dependent on parameters such as beryllium grain size and beryllium sample size and shape, which are necessary to calculate diffusion-controlled tritium inventories.

Figure A4.2. Tritium Retention Data for High-density

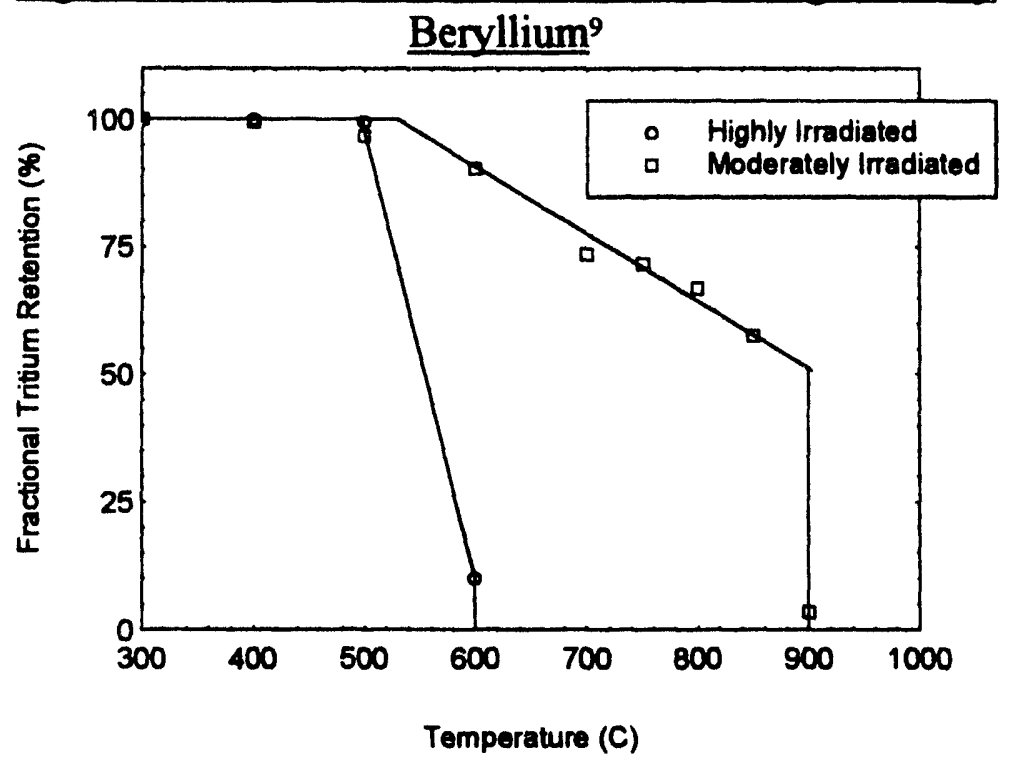

Figure A4.2 shows tritium retention data from ITER $\mathbf{R}$ and $D$ experiments on two high-density beryllium samples. Both samples had unirradiated porosities below 1 percent. One sample was highly irradiated (to a helium content of 26100 appm),

${ }^{7}$ M. Billone, Argonne National Laboratory Fusion Power Program, personal communication, June, 1993.

${ }^{8}$ G. Longhurst, Manager, E G and G Fusion Safety Program, personnel communication, April, 1993.

${ }^{9} \mathrm{M}$. Billone. 
the other sample was only moderately irradiated (to $872 \mathrm{appm}$ helium). After irradiation, the samples were annealed at increasing temperatures, and the fraction of retained tritium at the annealing temperature was determined.

As the figure shows, for temperatures above 600 degrees $C$, the highly irradiated beryllium retains virtually no tritium, indicating a burst release of all of the tritium at this temperature. Between 500 and 600 degrees $\mathrm{C}$ there is a significant decrease in the retained tritium of the highly irradiated sample, assumed to be linear. The moderately irradiated beryllium exhibits a gradual, linear decrease in tritium retention starting at 500 degrees C. At 900 degrees, however, there is a sudden burst release resulting in the loss of virtually all of the retained tritium. This burst release is similar to the release seen at 600 degrees for the highly irradiated sample.

One can conclude, based on this admittedly limited data, that for temperatures below about 600 degrees $\mathrm{C}$, virtually all of the tritium generated in highly dense beryllium is retained, no matter how highly irradiated the beryllium is. For temperatures above 600 degrees $C$, the fraction of retained tritium seems to depend on the fluence seen by the beryllium, as well as the temperature. It should be noted that no tritium retention experiments have yet been performed in-pile, and the tritium release behavior of beryllium while being irradiated may be different than the behavior seen during postirradiation anneals. Hence we use the present sparse data guardedly; the following tritium inventory estimates should be treated as preliminary estimates. 
Figure A4.3. Helium and Tritium Produced in Beryllium at EOL

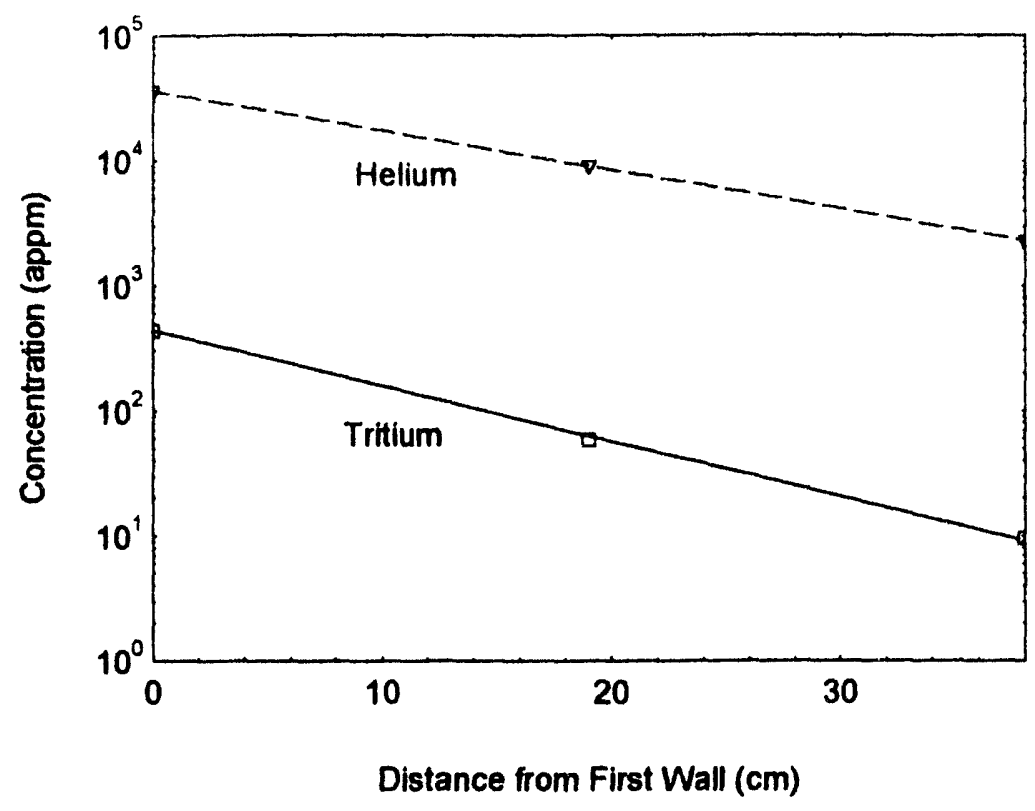

Figure A4.3 shows

the amount of helium and tritium generated in the beryllium of both Blanket Designs 1 and 2 at the end-oflife. The helium and tritium amounts are shown in the figure as concentrations (atomic parts-permillion), assuming

that all of the helium and tritium remains in the beryllium. The REAC3 and TWODANT codes were used to generate this data, along with ITER-relevant helium and tritium generation data. ${ }^{10}$ As can be seen in the figure, the majority of the tritium is generated in the front half of the blanket, where the helium concentration is above 10,000 appm. Hence, most of the tritium is generated in portions of the blanket which would be considered to be, in the context of Figure A4.2, "highly irradiated" by EOL.

It will be recalled that for Blanket Design 1, the beryllium temperature during normal operation ranges from 290 to 785 degrees C. For Blanket Design 2, the beryllium temperature ranges from 290 to 575 degrees C. For Blanket Design 2 it seems clear that since all of the beryllium is below 600 degrees $C$, most if not all of the tritium will be retained in the beryllium, regardless of the neutron fluence. For Blanket Design 1, the situation is less clear. For the beryllium in Blanket Design 1 which remains below 600

10 M. Billone, Argonne National Laboratory Fusion Power Program, personal communication, June, 1993. 
degrees $\mathrm{C}$, which amounts to roughly half the beryllium in the blanket, most of the tritium will be retained until end-of-life. But for the beryllium above 600 degrees $C$, the tritium will buildup until the fluence level reaches the point at which a burst release occurs, whereupon most of the retained tritium will be released. The fluence level at which this burst release occurs depends on the temperature of beryllium. For some portions of the beryllium at the back of the blanket, the fluence level may be low enough at end-of-life that no burst release occurs, even for beryllium which is above 600 degrees C.

Since the data is too sparse to develop a detailed model of the tritium-release behavior for the beryllium which is above 600 degrees in Blanket Design 1, we will make a simple, conservative assumption in order to estimate the end-of-life inventory for this beryllium. ${ }^{11}$ Roughly half of the beryllium in the blanket at end-of-life is irradiated to above 10,000 appm helium. Roughly half of this highly irradiated beryllium is above 600 degrees $\mathrm{C}$. The portion of highly-irradiated beryllium above 600 degrees $\mathrm{C}$ should contain virtually no tritium at end-of-life, according to Figure A4.2. The portion below 600 degrees $\mathrm{C}$ should contain virtually all of its tritium. Hence, one-fourth of the beryllium in Blanket Design 1 should have virtually no tritium, and one-fourth should have virtually all of its tritium. For the other "moderately-irradiated" half of the beryllium in Blanket Design 1, we will conservatively assume that all of the tritium is retained, no matter what the temperature of the beryllium is.

\footnotetext{
"II should be recognized that because the dependence of the retained tritium fraction on fluence for beryllium over 600 degrees $C$ is not fully understood yet, the end-of-life tritium inventory may not actually be the maximum tritium inventory which occurs over the life of the blanket for Blanket Design 1. As tritium inventory is building up in some beryllium sections, it is being released via burst-release in others. Only by modeling this behavior in detail (which is currenily impossible to do) will the absolute maximum tritium inventory be found. Of course, the upper bound for the tritium inventory assumes no tritium release at all, as was done for Blanket Design 2.
} 
The results of the above simple estimations of the end-of-life tritium inventory in the beryllium of the two blanket designs are shown below.

\section{Tritium Inventories in Beryllium at End-of-Life}

\begin{tabular}{|l|l|l|}
\hline & Blanket Design 1 & Blanket Design 2 \\
\hline Beryllium in Blanket & 140 tons & 140 tons \\
\hline Tritium in Beryllium & $3 \mathrm{~kg}$ & $4 \mathrm{~kg}$ \\
\hline
\end{tabular}




\section{Appendix 5: Thermal Stresses in Slabs}

This appendix derives relations useful in determining the thermal stresses in long, thin slabs. These relations are used in the determination of the thermal stresses to be expected in the beryllium slabs of Blanket Design 2.

Figure A5. 1 shows a schematic of a long, thin slab of length $L$, width $w$ and thickness $t$, where $\mathrm{L}>\mathrm{w}>\mathrm{t}$. The slab is assumed to have a temperature distribution which varies only in the width direction, $\mathrm{T}(\mathrm{y})$. The slab is also assumed to be unrestrained. The temperature distribution gives rise to stresses within the slab.

\section{Figure A5.1. Schematic of Slab with Temperature Distribution T(y)}
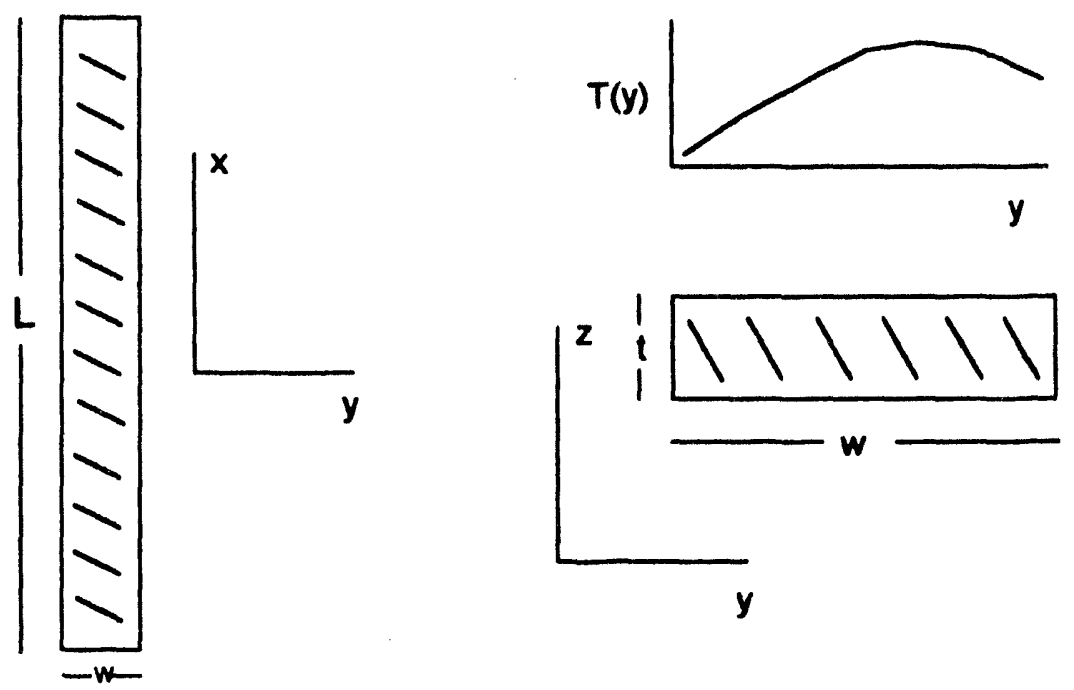

We use the so-called "strength-of-materials" approach to solve for the thermal stresses in this slab. ${ }^{1}$ This approach assumes that sections of the slab which are plane and perpendicular to the beam axis before loading remain so after loading (plane sections remain plane), and that lateral contraction may be neglected (that is, Poisson's Ratio can be taken as zero). This assumption, which is equivalent to neglecting the stresses in the

${ }^{1}$ The following derivation is derived from B. A. Boley and J. H. Weiner, Theory of Thermal Stresses, John Wiley and Sons, 1960, Chapter 10. 
$y$ and $z$ directions, results in only a very small error in the calculated thermal stresses for thin beams when the temperature does not vary in the long $x$ direction.

Assuming that plane sections remain plane results in a linear strain distribution in the $x$ direction of the form:

$$
\varepsilon_{x x}=\mathrm{a}+\mathrm{by},
$$

where $\mathrm{y}$ is the distance from the center of the rectangular beam in the $y$ direction, and a and $\mathrm{b}$ are constants (to be determined). Hooke's law for the stress in the $\mathrm{x}$ direction, neglecting Poisson's ratio, is given by:

$$
\sigma_{x x}(y)=E\left[\varepsilon_{x x}-\alpha T(y)\right]=E(a+b y-\alpha T(y)),
$$

where the thermal strain is given by the coefficient of thermal expansion $\alpha$ times the temperature $\mathrm{T}$. Note that in general $\mathrm{E}$ and $\alpha$ are dependant on temperature.

We now use the conditions of equilibrium to determine constants $a$ and $b$. If we assume we are far from the ends of an unrestrained beam, equilibrium requires that the force and moment on the slab cross section be equal to zero, or:

(A5.3) Force Equation $\quad \int_{A} \sigma_{x x} \mathrm{dA}=0$, and

$$
\text { (A5.4) Moment Equation } \quad \int_{A} y \sigma_{x x} d A=0
$$

where $\mathrm{A}$ is the cross sectional area. Substitution of Equation A5.2 into Equations A5.3 and A5.4 yields two equations which can be used to solve for $a$ and $b$, and hence for the $\mathrm{x}$-direction stress. Noting that

$$
\int \mathrm{y} d \mathrm{~A}=0
$$

and defining the moment of inertia about the $z$ axis as

$$
I_{z}=\int y^{2} d A,
$$


we obtain

(A5.7)

$$
\sigma_{\mathrm{xx}}(\mathrm{y})=-\alpha \mathrm{ET}(\mathrm{y})+\frac{\mathrm{P}_{7}}{\mathrm{~A}}+\frac{\mathrm{M}_{1:} \mathrm{y}}{\mathrm{I}_{z}} \text {, where }
$$

(A5.8)

$$
P_{T}=\int_{\Lambda} \alpha E T(y) d A, \text { and }
$$

(A5.9)

$$
\mathrm{M}_{\mathrm{T} z}=\int_{\lambda}-\alpha \mathrm{yET}(\mathrm{y}) d \mathrm{~A}
$$

Now, with a knowledge of the temperature distribution in the slab $T(y)$, we can determine the thermal stresses in the slab using the above equations. 


\section{Appendix 6: Cooling Tower/Auxiliary Heat Exchanger Sizing} for the Shield Water System

In the Chapter 6, the results of the cooling tower/Auxiliary Heat Exchanger sizing procedure for the single and two-loop loop shield water system (SWS) are discussed, and the optimum size for these two components is determined. In this section, the method by which these two components were sized is briefly discussed.' Following discussion of how the cooling tower/Auxiliary Heat Exchanger complex was sized for the single-loop SWS, the MATHCAD2 codes used for the sizing of components in the single- and two-loop SWS are provided.

\section{Auxiliary Heat Exchanger Sizing for Single-Loop SWS}

Table 6.2 and Table 6.4 in the Chapter 6 specify a number of SWS parameters which are fixed. Once the chimney height $(\mathrm{Ha})$ is specified in addition to these parameters, the heat exchanger and cooling tower can be sized. The first step in the sizing procedure is to estimate the mass velocities for both the air and water sides of the heat exchanger.

These mass velocities are defined as follows:

$$
G_{\text {air }}=\frac{W_{\text {ait }}}{\text { Aff }} ; \quad \text { Air Side Mass Velocity }
$$

$$
G_{\text {waer }}=\frac{W_{\text {tabe }}}{A_{\text {mbo }}} ; \quad \text { Water Side Mass Velocity, }
$$

where $\mathrm{W}_{\mathrm{air}}$ is the total air mass flow rate $(\mathrm{kg} / \mathrm{sec}), \mathrm{W}_{\text {tube }}$ is the water mass flow rate in a single heat exchanger tube, Aff is the air free-flow, or cross-sectional area, and $A_{\text {tube }}$ is the tube cross-sectional area.

\footnotetext{
'The method used to size the Auxiliary Heat Exchanger is adopted from W.M. Kays and A.L. London. Compact Heat Exchangers, McGraw-Hill. 1984, pp. 45-48.

${ }^{2}$ MATHCAD is a computer utility developed and copyrighted by MathSoft, Inc.
} 
Initial estimates for the mass velocities can be made using the following relation, which is derived from the equation for the velocity in the heat exchanger core: ${ }^{3}$

$$
\mathrm{G} \approx\left[\left(\frac{\mathrm{St} \operatorname{Pr}^{23}}{\mathrm{f}}\right)\left(\frac{\Delta \mathrm{P}}{\mathrm{NTU}}\right) \frac{\rho_{\mathrm{m}}}{\operatorname{Pr}^{23}}\right]^{1 / 2}
$$

If we note that the ratio $\mathrm{St} \operatorname{Pr}^{2 / 3} / \mathrm{f}$ is generally constant at about 0.3 for most surfaces and Reynolds numbers of interest, we can write:

$$
\mathrm{G} \approx\left[0.3\left(\frac{\Delta \mathrm{P}}{\mathrm{NTU}}\right) \frac{\rho_{\mathrm{m}}}{\mathrm{Pr}^{2^{\prime 3}}}\right]^{1 / 2}
$$

Since the inlet and outlet temperatures for both air and water are known, as are the relevant heights for natural circulation, the approximate natural circulation $\Delta \mathrm{P}$ 's for both the air and water sides can be determined. Likewise, since the heat load for the Auxiliary Heat Exchanger is known, the required NTU can be determined using the formulae for $\varepsilon$ and NTU in Chapter 6 . With these values, plus the average air or water density $\rho_{\mathrm{m}}$ known, the initial estimates for the G's can be determined using the above equation.

With these initial estimates for the G's, the following variables can be solved for in succession, for both air and water sides:

\section{UA, from Equation 6.4 from Chapter 6}

2. $\operatorname{Re}=\mathrm{G} \frac{\mathrm{D}}{\mu}$, where $\mathrm{D}$ is the characteristic length scale

3. St $\operatorname{Pr}^{2 / 3}$ and $\mathrm{f}$, from the figures and correlations in the Shield Water System chapter

4. $h$ (the heat transfer coefficient), from 3. above and $G$

5. U (the overall conductance), from the relations in the Shield Water System chapter

\footnotetext{
${ }^{3}$ See Kays and London, p. 47.
} 
6. A (the heat transfer area), from 1 . and 5 . above

7. $V$ (total heat exchanger volume), from 6 . above and $\beta$ from Table 6.2 of Chapter 6

8. Heat exchanger length, depth, and width, from 7. above, $\sigma$ from Table 6.2, the G's and W's.

Now that the heat exchanger dimensions have been completely determined, the pressure drops on both the water and air sides can be recalculated, and the estimates of the mass velocities adjusted as necessary. This process is then repeated, using the mass velocities as iteration variables, until convergence is reached. The Auxiliary Heat Exchanger volume and dimensions have then been determined.

\section{Cooling Tower Sizing for Single-Loop SWS}

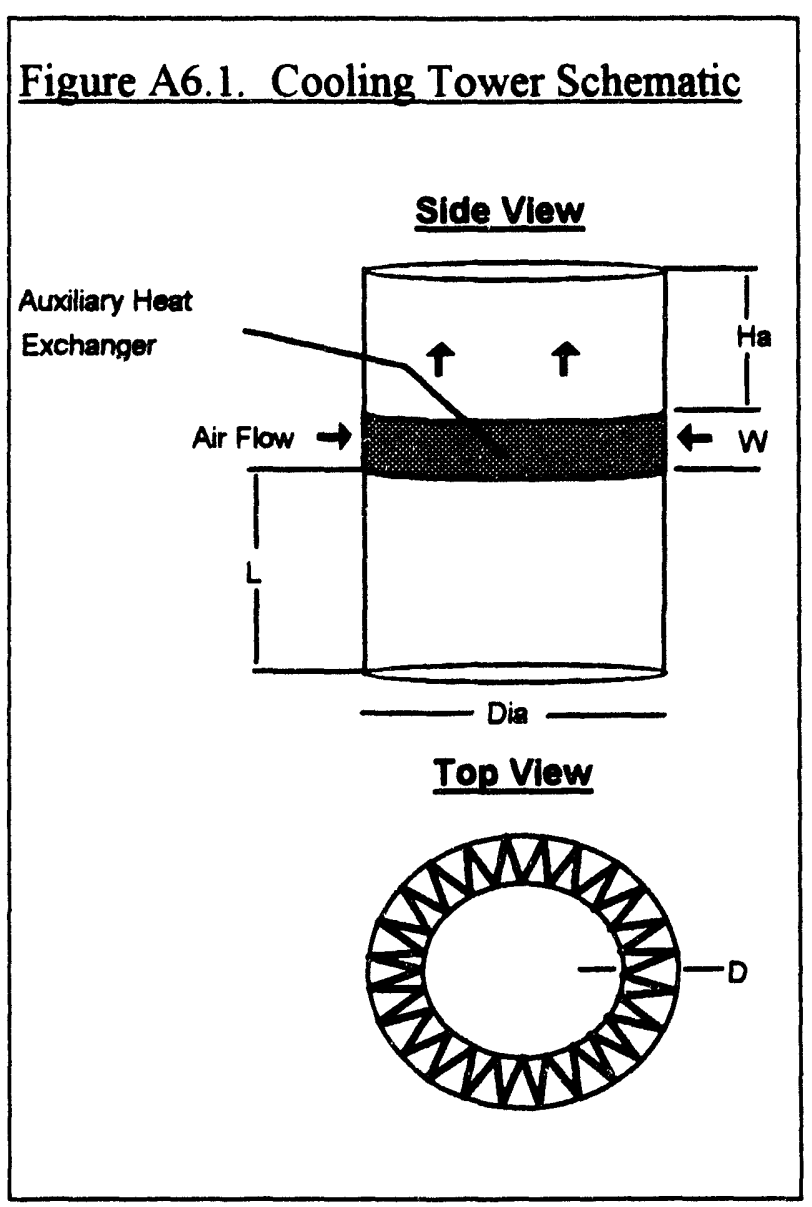

Figure A6.1 shows a simplified schematic of the cooling tower geometiy. The Auxiliary Heat Exchanger is elevated to a height $\mathrm{L}$ to provide for a natural circulation driving head for the primary water. The magnitude of $L$ is equal to the height of the shield centerline above ground (about 2.5 meters based on the tokamak/shield geometry) plus the specified height of the Auxiliary Heat Exchanger above the shield centerline $(\mathrm{Hw}=2$ meters, from Table 6.4 in Chapter 6). Hence L equals 4.5 meters. 
W is the width of the air inlet gap for the Auxiliary Heat Exchanger, and D is the depth of the heat exchanger arrangement in the cooling tower. Note that the width of the air inlet gap W is not generally equal to the width of the Auxiliary Heat Exchanger itself, as determined in the section above. Similarly, the depth of the heat exchanger arrangement $\mathrm{D}$ is not equal to the heat exchanger depth. As can be seen in the top view in Figure A6. 1 , the heat exchanger is arranged in a chevron arrangement around the diameter of the cooling tower. This arrangement maximizes the available air-side frontal area of the heat exchanger for a given cooling tower diameter and air gap width. For the present work, we will assume that the chevron arrangement is such that an (air-side frontal area)to-(air gap area) ratio of $4: 1$ results.

In Chapter 6, Ha was chosen as a variable to determine the optimum heat exchanger and cooling tower sizes. Once $\mathrm{Ha}$ is specified, $\mathrm{W}$ is determined by assuming that $\mathrm{W}=$ $\mathrm{Ha} / 10$. This assumption ensures that the area around the heat exchanger inlet is small relative to the total cooling tower area. Thus we need not be concerned with possible temperature anisotropies in this region affecting the overall air-side natural circulation driving head. Once $\mathrm{W}$ is determined, Dia (the cooling tower diameter) is determined based on the frontal area of the heat exchanger, which is known from the heat exchanger sizing procedure discussed above, and using the 4:1 (air-side frontal area)-to-(air gap area) ratio. Hence, we have:

$$
\text { Dia }=\frac{\text { HX Frontal Area }}{W \cdot \pi} \frac{1}{4}
$$

We have now determined all of the cooling tower dimensions except the thickness of the concrete tower shell. The shell thickness is assumed to be 0.33 meters, consistent with the shell thickness of existıng large cooling towers. 
Mathcad Code To Calculate Component Size and Cost for Single Loop Shield Water System

Max Power from Shield(incl. DH from shield and rad. from blanket)

$q=5 \cdot 10^{6}$

W

".."From waterhx.mod"*"

Air Cold Side Temp. Approx. Primary Side dp from Senvice $H X(@$ dmw=50kg/sec)

$$
\text { dpfservl }=45
$$

Tacold $=25 \quad \mathrm{C}$

Maximum Water side Temp

Twhot $=95$

Pick Tahot (say 40 is max)

Tahot $=40$

Cr must be less than or equal to 1.0

$\mathrm{Cr}=1$
Air chimney Height above HX(fixed) Height of $H X$ above CL He $=15$ of shield
$H w=2$

Assumed Concrete Cost

Pconcrete $=600 \cdot \frac{1}{(\mathrm{~cm} \cdot 100)^{3}}$ Dollars Pconcrete $=600 \cdot$ length $^{-3}$ Assumed ratio of $\mathrm{HX}$ cost/material cost

Reost $=5$

m."Shield Data (f. shidflow.med) ${ }^{\text {m.* }}$

Shield Tube Diameter(m)

Dashld $=0.022$

Number of Shield Tubes

Ntubes $=7435$

Pipe Data

Pipe Diameter(m) Pipe Length $(m)$

Dpipe $=1.1 \quad$ Lpipe $=\mathbf{2 0 0}$

Pipe Area

Apipe $=\pi \cdot\left(\frac{\text { Dpipe }}{2}\right)^{2} \quad$ Apipe $=0.95033$

Shiald Flow Area

Ashld $=$ Ntubes $\cdot \pi \cdot\left(\frac{\text { Dshld }}{2}\right)^{2}$

Ashld $=\mathbf{2 . 8 2 6 2 9}$

Pipe Surface Area

Vertical Shield Hoight

Length of shield tubes

VHshld $=4.9$

Lshld $=8.5$

SApipe $=\pi \cdot$ Dpipe $L$ pipe

SApipe $=691.15038$

Shiold Flow Area

Shiold Water Volume

Pipe Water Volume

SAshld $=$ Ntubes $\cdot$ Lshld $\cdot$ Dshld $\pi$

SAshild $=4.3679 \cdot 10^{3}$

Vshld $=$ Ntubes $\cdot$ Lshld $\cdot\left(\frac{\text { Dahld }}{2}\right)^{2} \cdot \pi$

Vpipe $=$ Lpipe-Apipe

Vpipe $=190.06636$

Vshld $=24.02344$

$\varepsilon=\frac{\text { Tabot }- \text { Tecold }}{\text { (Twhot }- \text { Treold })}$

$\varepsilon=0.21429$

Find NTU

$\mathrm{NTU}=\frac{\ln \left[\left(\ln (-\varepsilon-1)+\frac{1}{C r}\right) \cdot C r\right]}{C r}$

$\mathrm{NTT} \mathrm{I}=027597$ 


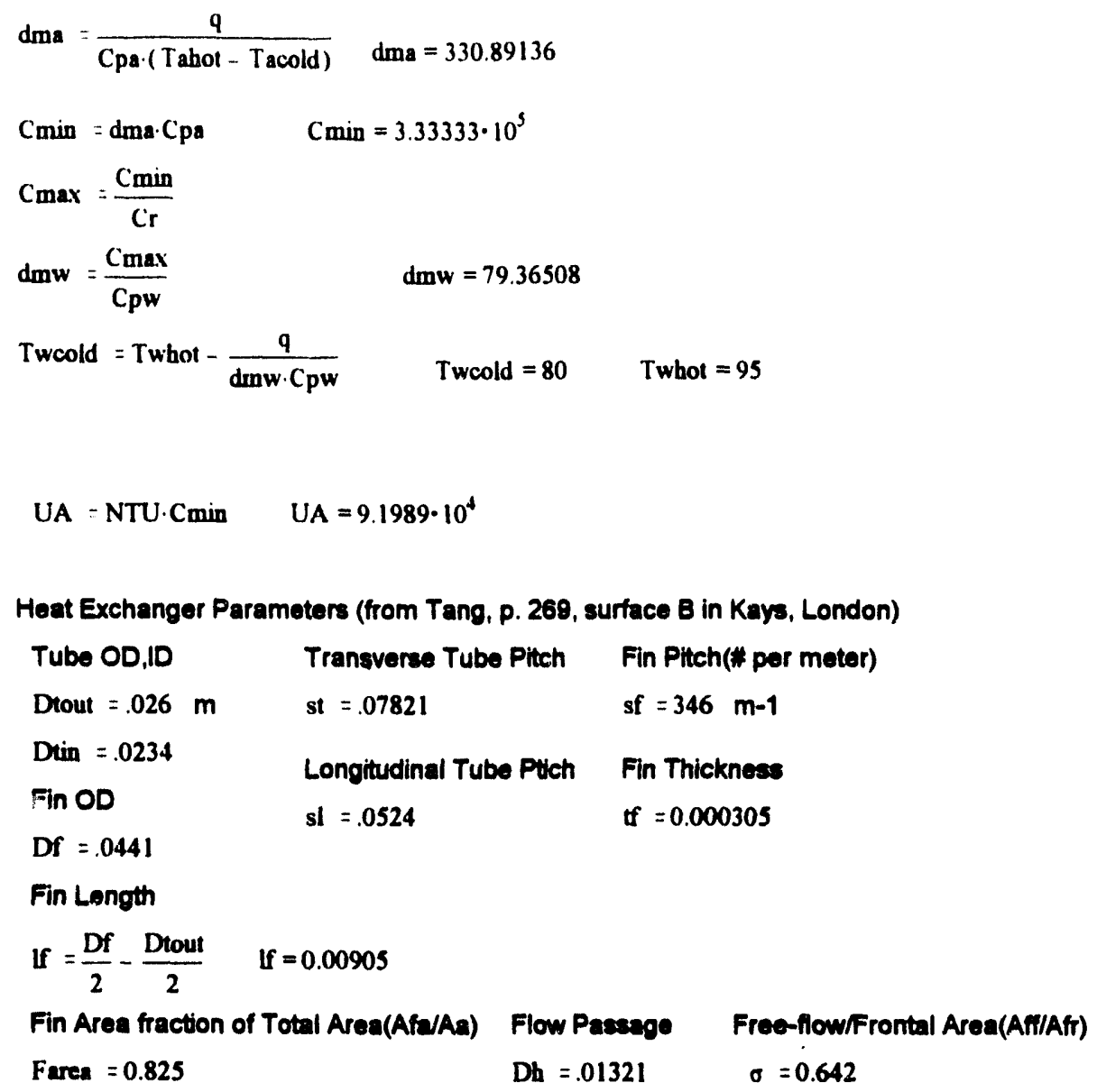

Fin Length

If $=\frac{\text { Df }}{2}-\frac{\text { Dtout }}{2} \quad$ if $=0.00905$

$\begin{array}{lll}\text { Fin Area fraction of Total Area(Afa/Aa) } & \text { Flow Pasaage } & \text { Free-flow/Frontal Area(Afr/Aft) } \\ \text { Farea }=0.825 & D h=.01321 & \sigma=0.642\end{array}$
$\mathrm{Dh}=.01321$

$\sigma=0.642$

Heat Transfer area Volume (AaMhx) (m2/m3)

$\beta=191 \mathrm{~m}-1$

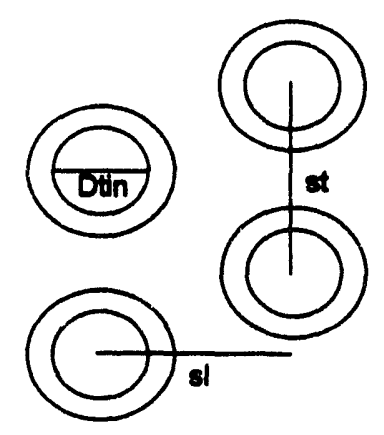

Ratio of water free flow area to $\mathrm{HX}$ area

$$
\begin{aligned}
& \sigma 2=\pi \cdot \frac{\left(\frac{\text { Dtin }}{2}\right)^{2}}{\text { sl.st }} \quad 02=0.10494 \\
& \text { AreaAHStube }=\pi \cdot\left(\frac{\text { Dtin }}{2}\right)^{2} \\
& \text { AreaAHStube }=4.30053 \cdot 10^{-4}
\end{aligned}
$$

\footnotetext{
Pressure head (air)

dpahead $=-g \cdot($ Ha $) \cdot($ pal Tahot $)-\operatorname{pa}($ Tacold $))$
} 


\section{Pressure head (water)}

dpwhead $=(g \cdot(\mathrm{Hw}) \cdot(\rho w(T w h o t)-\rho w(T w c o l d)))$

Initial Guess for Air Side Parameters(p. 47, Kays and London)

Approx HX Mass Velocitys(Initial Guess)

Gahx $=\left.3 \cdot \frac{\text { dpahead }}{\text { NTU }} \cdot \frac{p a(\text { Tahot })+p a(\text { Tacold })}{2 \cdot \text { Pra }^{.666}}\right|^{.5}$

Gahx $=3.64224$

$\mathrm{kg} / \mathrm{sec}-\mathrm{m} 2$

Prwav $=\frac{\text { Prw (Twhot })+\operatorname{Prw}(\text { Twcold })}{2}$

Gwbx $=\left.3 \cdot \frac{d p w h e a d}{\text { NTU }} \cdot \frac{p w(\text { Twhot })+p w(\text { Twcold })}{2 \cdot \text { Prwav }^{.66}}\right|^{.5}$

Gwhx $=348.06958$

koleec-m2

HX Reynold's Number

$\mu w a v e=\frac{\mu w(\text { Twhot })+\mu w(\text { Twcold })}{2}$

$\operatorname{Rewhx}(G w h x)=G w h x \cdot \frac{\text { Dtin }}{\mu w a v e} \operatorname{Rewhx}(G w h x)=2.48571 \cdot 10^{4}$

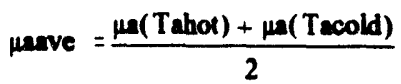

$\operatorname{Reahx}(\mathrm{Gahx})=\mathrm{Gahx} \cdot \frac{\mathrm{Dh}}{\mu a \mathrm{ve}} \quad \operatorname{Reahx}(\mathrm{Gahx})=7.14574 \cdot 10^{3}$

HX Ditus-Boolter

hwhx $(G w h x)=\frac{k w}{D \text { tin }} \cdot 0.023 \cdot \operatorname{Rewhx}(G w b x)^{8} \cdot \operatorname{Prwav}{ }^{3} \quad \operatorname{hwhx}(G w h x)=2.84905 \cdot 10^{3}$

HX Wator friction factor(McAdams, Blacius)

$f w h x(G w h x)=$ if $\left[\operatorname{Rewhx}(G w h x)<30000,0.316 \cdot \operatorname{Rewhx}(G w h x)^{(-.25)}, 0.184 \cdot \operatorname{Rewhx}\left(G_{w h x}\right)^{(-.2)}\right]$

fwhx $(G$ whx $)=0.02517$

From Tang

$\mathrm{jh}(\mathrm{Gahx})=0.2338 \cdot \operatorname{Reahx}(\mathrm{Gahx})^{-0.3962}$

jhy (Gahx $)=0.00695$

$\mathrm{fa}(\mathrm{G} \operatorname{ahx})=0.2836 \cdot \operatorname{Reahx}(\mathrm{Gehx})^{-0.22}$

$f(G$ ahx $)=0.03783$ 
St(Gahx) $=\frac{\text { jh(Gahx) }}{\text { Pra }^{666}}$ har(Gahx) $:$ St(Gahx) ( (jahx C Cpa $\quad$ ha (Gahx) $=32.32944 \quad$ W/m2-K

Fin Efficiency(Assume Aluminum Fins)

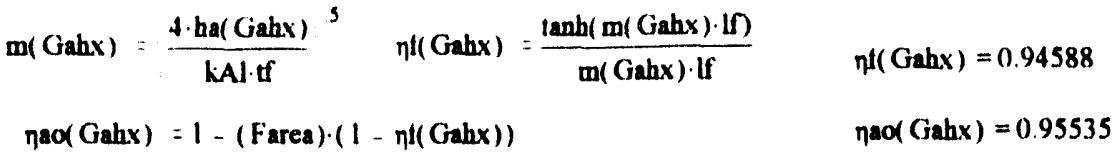

Calculate $U$ (based on Air Side Area)

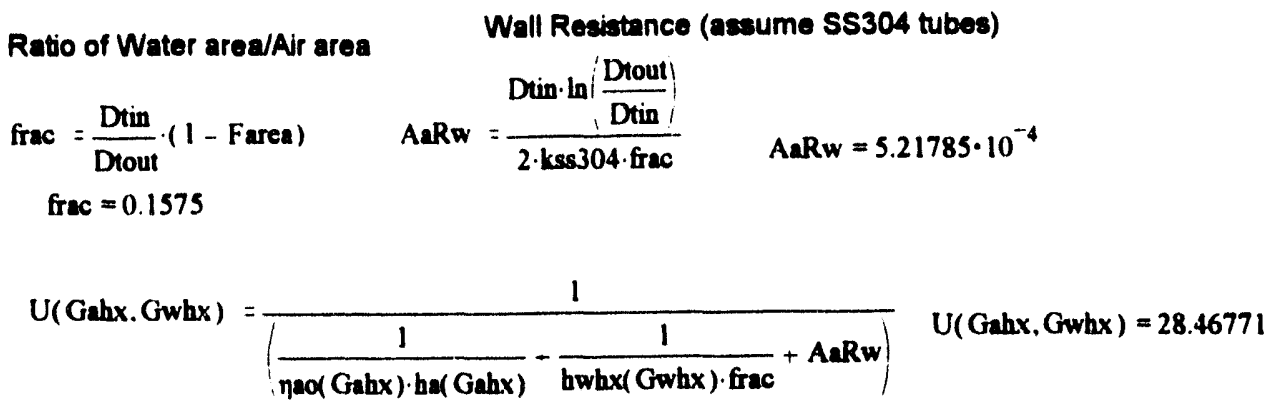

$A a(G a h x, G w h x)=\frac{U A}{U(G a h x \cdot G w h x)}$

$\operatorname{Aa}($ Gahx. Gwhx $)=3.23135 \cdot 10^{3}$

$\operatorname{Volhx}(G a h x . G w h x)=\frac{A a(G a h x, G w h x)}{\beta} \quad \operatorname{Volhx}(G a h x, G w h x)=16.91804$

Air Datn

Free Flow Area

Aff $(G a h x)=\frac{\text { dma }}{\text { Gahx }} \quad \operatorname{Aff}\left(G_{a h x}\right)=90.84819 \quad \operatorname{Afr}\left(G_{a h x}\right)=\frac{\text { Aff }(G a h x)}{\sigma} \quad \operatorname{Afr}\left(G_{a h x}\right)=141.50809$

$\operatorname{dann}\left(G_{a h x}\right)=\frac{A f(G \text { hax })}{58 \cdot 3.14} \quad \operatorname{dann}(G \operatorname{ahx})=0.777$

Weter Deth

$\operatorname{Affw}(G w h x)=\frac{d m w}{G w h x} \quad A f w(G w h x)=0.22801$

Heat Exchanger Depth, Width,Length

$\operatorname{Dhx}(G a h x, G w h x)=\frac{\text { Volhx (Gahx. Gwhx) }}{\operatorname{Afr}(G a h x)} \quad \operatorname{Dhx}(G a h x, G w h x)=0.11956$

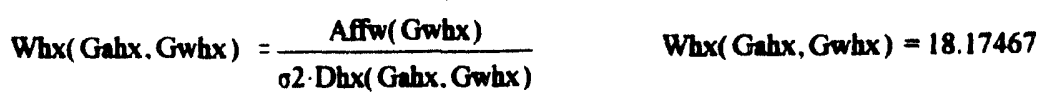

$\operatorname{Lhx}(G a h x, G w h x)=\frac{\operatorname{Volhx}(G a h x, G w h x)}{\operatorname{Dhx}(G a h x, G w h x) \cdot \text { Whx }(G a h x, G w h x)} \quad \operatorname{Lhx}(G a h x, G w h x)=7.78601$ 
HX Air-side friction dp(approx. value for rhomean used)

DP through $H X$ core

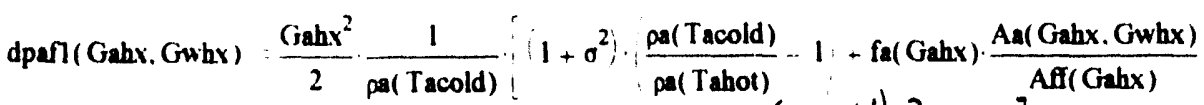

dpafl (Gahx, Gwhx) $=8.12814$

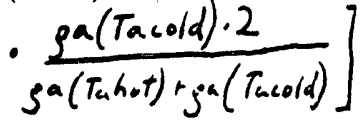

Flow Area of $\mathrm{HX}$

Aflowhx (Gahx. Gwhx) $=\frac{\text { Volhx (Gahx. Gwhx) }}{\operatorname{Dhx}(\text { Gahx. Gwhx) }} \quad$ Aflowhx (Gahx, Gwhx) $=141.50809$

Cooling tower diameter, assuming Whx is $1 / 10 \mathrm{Ha}$, cooling tower flow area $=1 / 4 \mathrm{HX}$ fow

area

$\mathrm{Wh \times 2}=\frac{\mathrm{Ha}}{10} \quad \mathrm{Wh} \times 2=1.5$

Lhx2(Gahx.Gwhx) $=\frac{\text { Allowhx(Gahx.Gwhx) }}{4 \cdot \text { Whx2 }}$

$\operatorname{Dia}\left(G \operatorname{ahx}\right.$. Gwhx) $=\frac{\operatorname{Lhx} 2 \text { (Gahx, Gwhx) }}{\pi} \operatorname{Din}\left(G_{a h x}, G w h x\right)=7.50724$

Cooling Tower Area

ATwr(Gahx, Gwhx) $=\pi \cdot\left(\frac{\operatorname{Dia}(G \operatorname{ahx}, G w h x)}{2}\right)^{2}$

DP through chimney outlet

$\operatorname{Gchim}\left(G a b x\right.$. Gwhx) $=\frac{\text { dma }}{\text { ATwr }(G \operatorname{abx}, \text { Gwhx) }}$

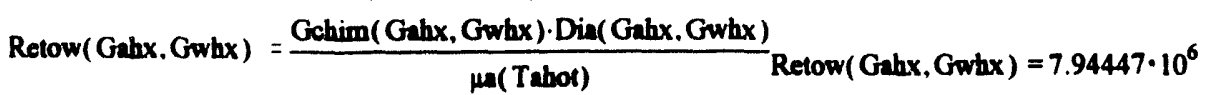

fatow (Gabx, Gwhx) = if $\operatorname{Retow}(G a h x, G w b x)<30000,0.316 \cdot \operatorname{Ret} o w(G a h x . G w h x)^{(-.25)}, 0.184$.

fatow $(G a h x . G$ whx $)=0.00767 \quad \cdot \operatorname{Refow}\left(G_{\text {aht }}, G_{w h x}\right)^{-.27}$

$\operatorname{dptow}(G a h x, G w h x)=\frac{H a \cdot f a t o w(G a h x, G w h x) \cdot G \operatorname{chim}(G a h x \cdot G w h x)^{2}}{2 \cdot \operatorname{Dia}(G a h x, G w h x) \cdot \operatorname{pa}(T a h o t)} \quad P_{2}=1 \cdot \operatorname{mass} \operatorname{length}^{-1} \cdot \operatorname{time}^{-2}$

$\operatorname{dptow}(G a b x, G w h x)=0.38019$

Total air friction op

$\operatorname{dpat}($ Gahx. Guhb ) = dplow (Gahx, Gwhx) $+\operatorname{dpafl}($ Gahx, Gwhx)

Friction Pressure Drops $\quad$ pwav $=\frac{\operatorname{pw}(\text { Twhot })+\operatorname{pw}(\text { Twcold })}{2}$ 
Shield:

Total shield pressure drop in loop (f. slhdtub2.mcd)

dpfishld $=14$

dpfshld $=14$

Piping

Gwpipe $=\frac{\text { dmw }}{\text { Apipe }} \quad$ Rewpipe $=$ Giwpipe $\frac{\text { Dpipe }}{\mu \text { wave }} \quad$ Rewpipe $=2.80359 \cdot 10^{5}$

fwpipe $=$ if Rewpipe $<30000 \cdot 0.316 \cdot$ Rewpipe $^{(25)} \cdot 0.184 \cdot$ Rewpipe $^{(-2)}$ fwpipe $=0.01497$

dpfpipe $=$ fwpipe $\frac{\text { Lpipe }}{\text { Dpipe }} \cdot \frac{G_{\text {wpipe }}^{2}}{2 \cdot p w a v} \quad$ dpfpipe $=9.81779$

Service $H X$

dpfserv $=\operatorname{dpfserv} I \cdot \frac{\text { dmw }{ }^{2}}{50 ;}, \quad$ dpfserv $=113.37868$

HX Water-side dp

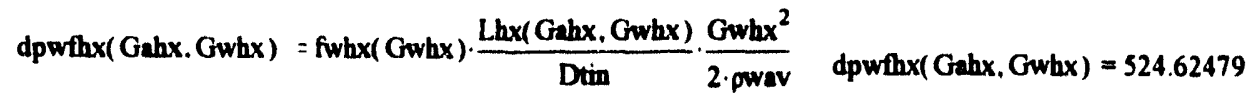

Total Water Friction Pressure Drop

dpwf $(G a h x$. Gwhx $)=d p w f h x(G a b x$. Gwhx $)+$ dpfpipe + dpfshld + dpfserv

Given

dpwf(Gahx, Gwhx) $=661.82127$

dpaf( Gahx, Gwhx $)=$ dpahead

dpwf(Gahx, Gwhx) $=$ dpwhead

$\left(\begin{array}{c}\text { Gaval } \\ \text { Gwval }\end{array}\right)=$ find (Gahx, Gwhx)

Gaval $=3.52884$

dpwthx (Gaval, Gwval $)=\$ 5.69421$

Gwval $=149.82986$

$\operatorname{dpwf}($ Gaval, Gwval $)=192.89069$

HX Final Dimenciong

$\operatorname{Dhx}($ Gaval, Gwval $)=0.12488 \quad \operatorname{Lhx}($ Geval, Gwval $)=3.61322$

Whx $($ Gaval. Gwval $)=40.42262 \quad$ Volhx $($ Gaval, Gwval $)=18.23885$

Concrete Volume(Assume $.33 \mathrm{~m}$ thick shell, inct height above center of shield)

Convol $=\left(\mathrm{Ha}+\frac{\mathrm{Whx} 2}{10}+\mathrm{Hw}+\frac{\mathrm{VH} \text { shld }}{2}\right) \cdot \operatorname{Dir}(\mathrm{Gaval}, \mathrm{Gwval}) \cdot \pi \cdot 33 \cdot(100 \cdot \mathrm{cm})^{3} \quad$ Coavol $=157.44819 \cdot \mathrm{length}{ }^{3}$ 
Price Data

Aluminum Tubes (Metal Goods, Marlborough, 11/11/93, for 500 th of 1 in dia tube)

$\mathrm{Pal}$

$\frac{1.61}{1 \cdot \mathrm{in} \cdot \pi \cdot 065 \cdot \mathrm{in} \cdot 1 \cdot \mathrm{f}}$

$\mathrm{Pal}=4.00941 \cdot 10^{4} \cdot$ length $^{-3} \quad$ Dollars

SS304 Tubes (Motal Goods, Marlboro, 11/11/93, for $500 \mathrm{n}$ of 1 in dia tube)

Pss304 $=\frac{1.71}{1 \cdot \text { in } \pi \cdot .065 \cdot \text { in } 1 \mathrm{ft}}$ Pss304 $=4.25844 \cdot 10^{4} \cdot$ length $^{-3}$

Aluminum Volume

Alvol $=$ Volhx $($ Gaval. Gwval $) \cdot \beta \cdot$ Farea $\cdot f \cdot(100 \cdot \mathrm{cm})^{3} \quad$ Alvol $=0.87657 \cdot$ length $^{3}$

Steel Volume

ss304vol $=$ Volhx (Geval. Gwval $) \cdot \beta \cdot(1-$ Farea $) \cdot \frac{\pi}{4} \cdot \frac{\text { Dtout }^{2}-\text { Dtin }^{2}}{\pi \cdot \text { Diout }^{2}} \cdot(100 \cdot \mathrm{cm})^{3}$

se304vol $=0.7529 \cdot$ length $^{3}$

HX Cost

HXXcost $=($ Alvol $\cdot \mathrm{Pal}+$ ss304vol $\cdot$ Pss304 $) \cdot$ Rcost

HXcost $=3.36034 \cdot 10^{5} \quad$ Dollars

Tower Cost

Towercost $=$ Convol $\cdot$ Pconcrete

Towercost $=9.44689 \cdot 10^{4}$ Dollars

Total Cost

Totcost $=$ Towercost $+H X \cos t \quad$ Totcost $=4.30503 \cdot 10^{5}$

\section{Constants}

$C_{p a}=4.18 \cdot 10^{3} \cdot 0.241 \quad C_{p a}=1.00738 \cdot 10^{3}$

J/ko-K (from Mark's)

Cpw $\equiv 4200 \mathrm{~J} / \mathrm{kg}-\mathrm{K}$ (from Todreess and $\mathrm{Kazimi}$ )

$\operatorname{par}(T) \equiv .0752 \cdot 16.0 \frac{293}{(T+273)} \mathrm{kg} / \mathrm{m} 3$ (both from Kays and London)

$\mu(T) \equiv(18.53+.0441 \cdot(T-300)) \cdot 10^{-6} \mathrm{Pa-s}$

Pra $\approx 0.7$

$g \equiv 9.8$

$k A J \equiv 200 \quad W / m-K \quad k s s 304 \equiv 15 \quad W / m-K$ 
Water density ( $T$ in $\mathrm{C}(0-100 \mathrm{C}))$

$\operatorname{pw}(T) \equiv 1000 \cdot 2.002461 \cdot 10^{2} \cdot T \cdot 5.882755 \cdot 10^{-3} \cdot T^{2}+1.534709 \cdot 10^{9} \cdot T^{3}$

Water PrandH Number(25-100 C)

$\operatorname{Prw}(T)=6.20034 \cdot \exp (\cdot 0.038745 \cdot(T-10))+0.0115698 \cdot T+2.85795$

Water Viscosity (20-110 C)

$\mu w(T) \equiv(397.674 \cdot \exp (0.0364205 \cdot(T-20))-1.91245 \cdot T+441.941) \cdot 10^{-6}$

Water conductivity (50-100 C)

$k w \equiv 0.7 \quad W / m-K$

\section{Densities}

pal $\equiv 0.1 \cdot \frac{\mathrm{lb}}{\mathrm{in}^{3}} \quad$ pal $=2.76799 \cdot 10^{3} \cdot$ mass $^{3} \cdot$ leagth $^{-3}$

pss304 $\equiv 8 \cdot \frac{g m}{\mathrm{~cm}^{3}} \quad$ pss304 $=8 \cdot 10^{3} \cdot$ mess $^{3} \cdot$ leagh $^{-3}$

Efrective HX Dimentions

Lhx2 = Lhx2(Gaval. Gwval)

$\sigma 2=0.10494 \quad H a=15$

$\mathrm{Wh} \times 2=1.5$

Dhx2 $=\frac{\text { Volhx (Gaval, Gwval) }}{\text { Lhx2. Whe2 }}$

$D b \times 2=0.4995$

$H a=15$

DP for all but Shiold (for shldilow.med)

dpfshld $=14$

dpwf(Gaval, Gwval) - dpfshld $=178.89069$

dpwf(Gaval. Gwval) $=192.89069$

Water-side Aux HX Surface Area

Pipe Surface Area

SAAHX = Aa(Gaval, Gwval) frec

SApipe $=691.15038$

SAAHX $=548.67027$

Shield Surface Area

SAshid $=4.3679 \cdot 10^{3}$

Service HX Survace Area(f. waterhx.med)

SASHX $=184$

Total Surface Area

SAtot = SAshld + SApipe + SAAHX + SASHX

SAtot $=5.79172 \cdot 10^{3}$

Service HX Primary Water Volume(f. waterhx.med)

Pipe Water Volume

PVSHX $=1.0$

$V_{\text {pipe }}=190.06636$ 
Aux HX Primary Water Volume

PVALDX 02 Volhv( Gaval. (iwval)

PVAHLX $=1.91393$

Total SWS Volume

Vtot = PVALX $-P V S H X-$ Vpipe - Vshld

$V 101=217.00372$
Shield Water Volume

Vshld $=24.02344$

Air Free Flow Area (for airhxte.med)

Aff $($ Gaval $)=93.76784$

Water Free Flow Aree

Affw $($ Gwval $)=0.5297$

Air Hest Transfor Area

Aa (Gaval. Gwval) $=3.48362 \cdot 10^{3}$

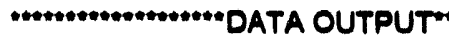

$\mathrm{Cr}=1 \quad \mathrm{Hw}=2 \quad \mathrm{Ha}_{\mathrm{a}}=15$

Twcold $=80$

Volhx (Gaval. Gwval $)=18.23885$

Dhx (Gaval. Gwval $)=0.12488$

$W h \times 2=1.5 \quad D h \times 2=0.4995$

$\operatorname{Dia}($ Gaval, Gwval) $=7.7485$

Convol $=157.44819 \cdot$ ength $^{3}$

Cooling Tower Height

$H$ tow $=H a+W h \times 2+H w+\frac{\text { VHshld }}{2}$

Tower Length-to-Diameter Ratio

$$
\frac{\text { Htow }}{\operatorname{Dia}(\text { Gaval. Gwval) }}=2.70375
$$

dptow( Gaval. Gwval) $=0.32663$

dpaf (Gaval, Gwval $)=8.334$

$$
\begin{aligned}
& \text { HXCost }=3.36034 \cdot 10^{5} \\
& \text { Towercost }=9.44689 \cdot 10^{4} \\
& \text { Totcost }=4.30503 \cdot 10^{5} \\
& \text { Affw }(\text { Gwval })=0.5297
\end{aligned}
$$$$
\text { Lhx (Gaval. Gwval })=3.61322
$$

$$
\operatorname{dmn}=330.89136
$$$$
\mathrm{dm} w=79.36508
$$$$
\text { SA }=\beta \cdot \text { Volhx (Gaval. Gwval })
$$$$
S A=3.48362 \cdot 10^{3}
$$$$
\frac{H D \cos t}{S A}=96.46113 \sin 2
$$

Number Tubes in AHX

$$
\text { Ntube }=\frac{\text { Affw (Gwval })}{\pi \cdot\left[\left(\frac{\text { Din }}{2}\right)^{2}\right]} \quad \text { Ntube }=1.23171 \cdot 10^{3}
$$


Mathcad Code To Calculate Component Size and Cost for Two-Loop Shield Water System

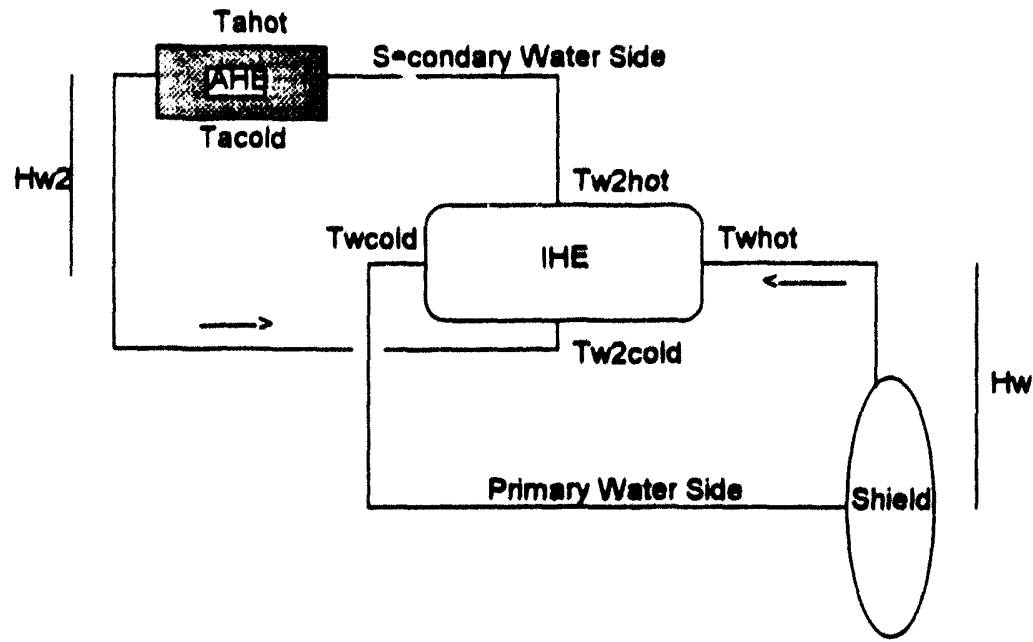

$\begin{array}{lll}\text { Equations: } & \text { Unknown Variables: } & \text { Aceumed variables: } \\ \text { dp primary side } & \text { Twcold } & \text { Tahot } \\ \text { dp secondary side } & \text { Tw2hot } & \text { Tacold } \\ \text { dp airhx } & \text { Tw2cold } & \text { Twhot } \\ \text { heat balance primary side } & \text { dma } & \\ \text { heat balance secondary side } & \text { dmw } & \\ \text { heat balance airhx } & d m w 2 & \end{array}$

Max Power from Shiold(incl. DH from shild and rad. from blanket)

$q=5 \cdot 10^{6} \cdot$ watt

Air Cold Side Tomp.

Troold $=25 \quad C$

Ar chimney Height above $\mathrm{HX}$ (fixed)

Maximum Primary Water side Temp

Twhot $=95$

$\mathrm{Ha}=1 \mathrm{~s} \cdot \mathrm{m}$

Hoight of IHX above CL

of shiold

$H w=5 \cdot m$

Height of AHE above IHX $H w 2=5 \cdot m$

Acaumed Concrote Cost (price for struct. col. for 5-etory bldg., if. "Moans Concrote Cost Datn, 1991"

Pick Tahot (aay 40 is max)

Tahot $=\mathbf{4 0}$

Pconcrete $=600 \cdot \frac{1}{(\mathrm{~cm} \cdot 100)^{3}} \quad$ Dollars Pconcrete $=600 \cdot$ length $^{-3}$ 
Cr must be less than or equal to 1.0

$\mathrm{Cr}=1$

"*Shield Data (f. shidflow.med)"**

Shiold Tube Diameter(m)

Dshld $=0.022 \cdot \mathrm{m}$

Number of Shield Tubes

Ntubes $=7435$

\section{Shield Flow Area}

Ashld $=$ Ntubes $\pi \cdot \frac{\text { Dshld: }}{2}$

Vertical Shield Height

VHishld $=4.9 \cdot \mathrm{m}$

Shiald Flow Area

SAshld $=$ Ntubes $\cdot$ Lshld $\cdot$ Dshld $\pi$

SAshld $=4.3679 \cdot 10^{3} \cdot$ length $^{2}$
Assumed ratio of $\mathrm{HX}$ cost/material cost

$R \cos t=5$

Secondary water Pipe Data

Pipe Diameter(m) Pipe Length $(m)$

Dpipes $=6 \cdot \mathrm{m} \quad$ Lpipes : $200 \cdot \mathrm{m}$

Pipe Ares

Apipes $=\pi \cdot \frac{\text { Dpipes }^{2}}{2}$

Apipes $=0.28274 \cdot$ length $^{2}$

Primary water pipe Data

Dpipep $=6 \cdot \mathrm{m} \quad$ Lpipep $=20 \mathrm{~m}$

Apipep $=\pi \cdot\left(\frac{\text { Dpipep }}{2}\right)^{2}$

Solve for secondary mase flow rate:

denw2 $=\frac{q}{C_{p w} \cdot C_{r} \cdot(\text { Tabot }- \text { Tecold })}$

Shield Water Volume

Vshld $=$ Nimbes. Lshidd $\cdot\left(\frac{\text { Dahld }}{2}\right)^{2} \cdot \pi$

Vshld $=24.02344 \cdot$ leagh $^{3}$

INTILAL GUESS for Tw2cold

Tw2cold $=60$

$T w 2 b o t(T w 2$ cold $)=T w 2$ cold $+\frac{q}{\operatorname{dmw} \cdot C_{p w}}$

$T w 2 \operatorname{bot}(T w 2 \operatorname{cold})=75$

Secondary side delta $T$

delT2 $=\frac{q}{\operatorname{dmw} 2 \cdot C_{p w}}$

delT2 $=15$

For aimplicity, "aseume" CR2 (ratio of capactly rates for IHE) in 1; thus the delta Te are equal for both primary and secondary sides:

$\mathrm{Cr} 2=1$

Hence, Primary side delta T.....

delT $=$ delT2

Twcold $=$ Twhot - delT 
and, primary and secondary side mass flow rates are equal:

$d m w=d m w 2$

$\mathrm{dmw}=79.36508 \cdot$ mass $^{*}$ time $^{-1}$

IHE diameter (assumed)

$D i=1 \cdot m$

IHE Efficiency(for $\mathrm{Cr}=1$ )

$\varepsilon 2($ Tw2cold $)=\frac{(\text { Tw2hol(Tw2cold) }- \text { Tw2cold })}{(\text { Twhot }- \text { Tw2cold })}$

$\varepsilon 2(T w 2$ cold $)=0.42857$

IHE NTU (Assuming CR=1)

$\operatorname{NTU}(T w 2$ cold $)=\frac{1}{C_{r} 2} \cdot \ln \left(C_{2} 2 \cdot \ln (1-22(T w 2 \operatorname{col} d))+1\right)$

$\operatorname{NTU}($ Tw2cold $)=0.82011$

IHE Cmin (asuming CR2=1)

$\mathrm{Cmin}_{\mathrm{m}}=\mathrm{dmw} 2 \cdot \mathrm{Cpw} \quad \mathrm{Cmin}=3.33333 \cdot 10^{5} \cdot$ mess length $^{2} \cdot$ time $^{-3}$

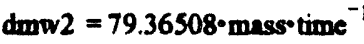

$C p w=4.2 \cdot 10^{3} \cdot$ length ${ }^{2} \cdot$ time $^{-2}$

IHE U*A

$U A(T w 2$ cold $)=N T U(T w 2$ cold $) \cdot C \mathrm{~min}$

$$
U A(T w 2 \text { cold })=2.73369 \cdot 10^{5} \cdot \text { mass } \text { length }{ }^{2} \cdot \text { time }^{-3}
$$

\section{HX Parmetors (for water-weter HX!- see 0.581. "Hest Exchongers")}

\section{Tube Drin}

Tube OD

Tube THickness Tube Plich

\section{Bafilo Spacing}

do $=1$.in

$1=\frac{d 0}{10}$

$p=1.25 \cdot \mathrm{in}$

$p=0.03175 \cdot$ length

$\operatorname{ls}(\mathbf{b x d})=\mathbf{b x l} \cdot \mathbf{l}$

do $\approx 0.0254 \cdot$ length

Tube ID

di : $=$ do -1

Pitch Paraliel and Normal to FLow (for triangular layout, dowe1 in, pos 1.25 in

$\mathrm{di}=0.02286 \cdot$ length

$$
\begin{aligned}
& \mathrm{pP}=1.082 \cdot \mathrm{in} \quad \mathrm{pn}=0.625 \cdot \mathrm{in} \\
& \mathrm{pP}=0.02748 \cdot \text { length } \quad \mathrm{pn}=0.01588 \cdot \text { length }
\end{aligned}
$$

Diametor of Outer Limit of Tubes (Dot), bame cut distance (IC) Inivial Guess for HX lenoth

$\operatorname{Dod}(\mathrm{Di})=\frac{7}{8} \cdot \mathrm{Di}$

$\mathbf{k}=\mathrm{Di}-\operatorname{Dot}(\mathrm{Di})$

\section{Shell Side Celculntions}

Crosentow Area

$\operatorname{Sm}(\mathrm{hxl})=\operatorname{ls}(\mathrm{hxd}) \cdot\left[\operatorname{Di}-\operatorname{Dov}(\mathrm{Di})+\frac{\operatorname{Dot}(\mathrm{Di})-\mathrm{do}}{p} \cdot(p-d o)\right] \quad \operatorname{Sm}(\mathrm{hxd})=0.88476 \cdot \operatorname{length}^{2}$ 
Shell Side Re number

$\mu$ aves $(T w 2$ cold $)=\mu w: \frac{\text { Tw2cold }- \text { Tw2hol(Tw2cold) }}{2}$

$\operatorname{Res}(\mathrm{bxl}$. Tw2cold $)=\frac{\text { do.dmw2 }}{\operatorname{\mu aves}(\mathrm{Tw} \cdot 2 \operatorname{cold}) \cdot \operatorname{Sm}(\mathrm{bxd})}$

$\operatorname{Res}(\mathrm{bxl}, \mathrm{Tw} 2 \mathrm{cold})=5.44025 \cdot 10^{3}$

Relation for ideal $j$ (assuming triangular layout, do=1 in, p=1.25 in, wor 100<Re<105

ji(bxl. Tw2cold $)=2.71228 \cdot \operatorname{Res}(\text { bxl. Tw2cold })^{(-0.892229)}+(0.0806972) \cdot \operatorname{Res}(\text { bxl. Tw2cold })^{(-0.25061)}$

ji $($ hxî. Tw2cold $)=0.01061$

Ideal $\mathrm{h}$ for shell side

hsideal(hxl. Tw2cold) $=j i(b x J . T w 2 \operatorname{cold}) \cdot C p w \cdot\left(\frac{d m w 2}{S m(b x l)}\right) \cdot\left(\frac{k w}{C_{p w} \cdot \mu \text { wes }(T w 2 \operatorname{cold})}\right)^{\frac{2}{3}}$

(conservatively neglecting

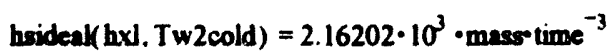
efrect of hot tube surface temperature)

Comversion to hareal, spprox. (p. 585, HEAT EXCHANGERS)

$$
\frac{\text { watt }}{m^{2}}=1 \cdot \text { mass } \cdot \text { time } e^{-3}
$$

hareal (hxl. Tw2cold) $=0.6 \cdot$ hsideak hxl. Tw2cold)

Iube(Primary) Side Calculation:

Averege Primary Side Prandil, viscodty

Prpave $=$ Prw $\frac{\text { Twbot }+ \text { Twoold }}{2}$

Mveep $=\mu w\left(\frac{\text { Twhot }+ \text { Twcold }}{2}\right)$

Number Tubee(curve iii)

$$
N t=\left(2.93-\frac{52 \cdot \operatorname{Dot}(\mathrm{Di})}{\mathrm{m}}\right)+\frac{907 \cdot \operatorname{Dot}(\mathrm{Di})^{2}}{\mathrm{~m}^{2}} \quad \mathrm{Nt}=651.85187
$$

Primary Side Flow Area

$$
\text { Afpri }=N \mathrm{~N} \cdot \pi \cdot\left(\frac{\mathrm{di}}{2}\right)^{2} \quad \text { Afpri }=0.26754 \cdot \text { length }{ }^{2}
$$

\section{Primary Side Re}

Gwp $=\frac{\text { denw }}{\text { Afpri }}$

Repri $=$ Gwp. do

Repri $=2.31309 \cdot 10^{4}$

Primary Sido h (Diture-Boeker) Conservatueno entrence efiocts

hp $=\frac{k w}{\mathrm{di}} \cdot 0.023 \cdot$ Repri $^{\cdot 8} \cdot$ Prpave $^{3}$

hp $=2.74817 \cdot 10^{3} \cdot$ mass time $e^{-3}$ 
Overall U (based on SHELL-SIDE AREA!)

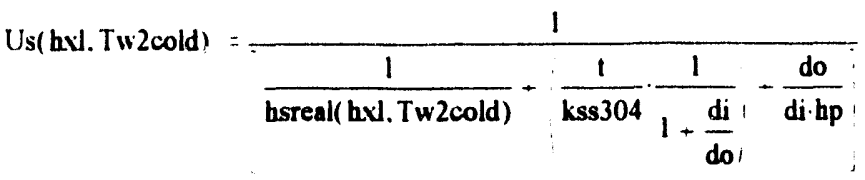

Us ( hvl. Tw2cold $)=790.94242 \cdot$ mass $^{\circ}$ time $e^{-3}$

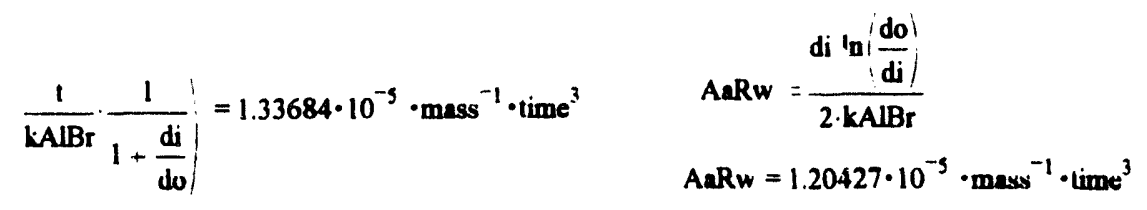

Shell-side Area

As( hxl. Tw2cold $)=\frac{U A(\text { Tw2cold })}{U s(\text { bxl. Tw2cold })} \quad A s($ hxl. Tw2cold $)=345.62471 \cdot$ length $^{2}$

\section{Primary Side Areo}

$A p(b x l, T w 2$ cold $)=A s(b x l, T w 2$ cold $) \cdot\left(\frac{d i}{d 0}\right)$

\section{Presirure Drops}

\section{Shell Side}

Number of Tube Rows Crosesd in Croseffow Section

$$
\mathrm{Nc}=\frac{\mathrm{Di} \cdot\left[\mathbf{I}-2 \cdot\left(\frac{\mathrm{lc}}{\mathrm{Di}}\right)\right]}{\mathrm{Pp}}
$$

Friction Factor (for triangular layout, doent in, p= $1.25 \mathrm{ln}, 100<R 0<105$ )

$f i=4872.38 \cdot \operatorname{Res}(\mathrm{hxd}, \mathrm{Tw} 2 \operatorname{cold})^{(-2.09811)}+1.32957 \cdot \operatorname{Res}(\mathrm{hxd} . \mathrm{Tw} 2 \operatorname{cold})^{(-0.216807)}$

$f i=0.20604$

Avg. SHell side Denaty

$\operatorname{prves}(T w 2$ cold $)=p w\left(\frac{T w 2 h o t(T w 2 c o l d)+T w 2 \text { cold }}{2}\right)$

Sec. side friction dp from IHE

$\operatorname{dpsiHE}(\mathrm{hxl}, \mathrm{Tw} 2 \mathrm{cold})=\frac{4 \cdot f \cdot d m w 2^{2} \cdot \mathrm{Nc}}{2 \cdot \operatorname{paver}(\mathrm{Tw} 2 \operatorname{cold}) \cdot \mathrm{Sm}(\mathrm{hxd})^{2}}$

$$
\operatorname{dpsIHE}(\mathrm{hxl}, \mathrm{Tw} 2 \text { cold })=92.40355 \cdot \text { mass } \text { length }^{-1} \cdot \text { time }^{-2}
$$


AHE calculations

$$
\varepsilon(\text { Tw2cold })=\frac{\text { Tahol - Tacold }}{\text { (Tw2hot( Tw2cold) - Tacold ) }}
$$

Find AHE NTU

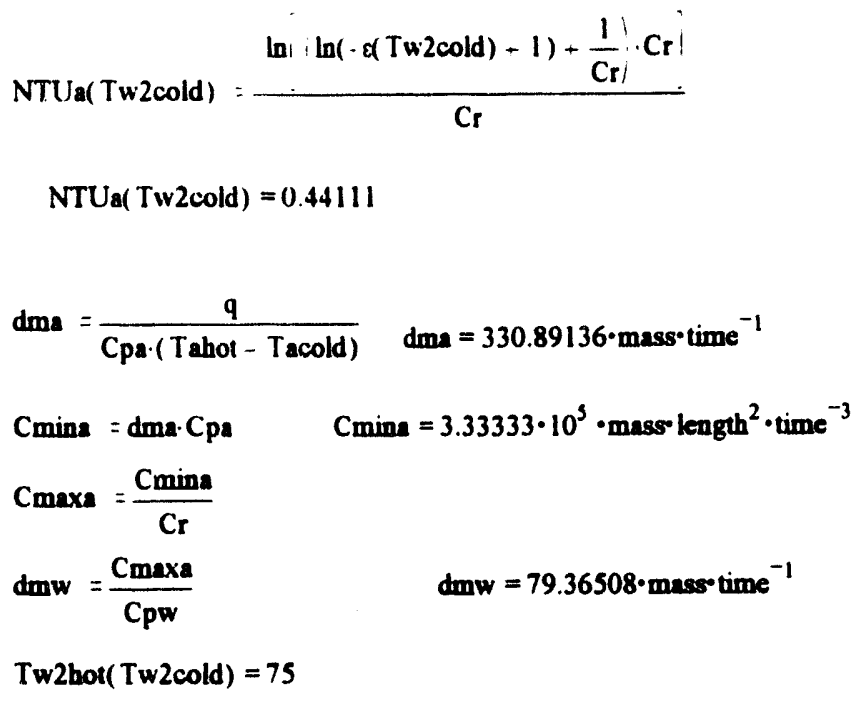

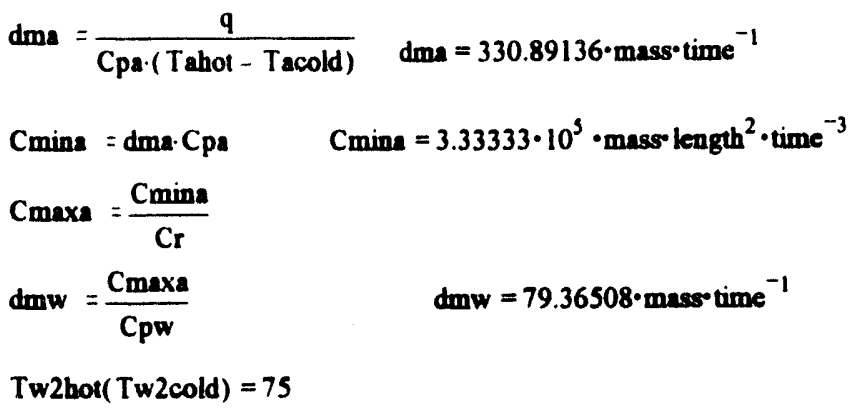


Heat Transfer areaNolume (AaNhx) (m2/m3)

$\beta=191 \cdot \frac{1}{m} \mathrm{~m}-1$

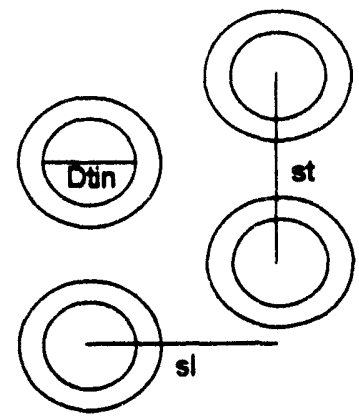

Ratio of water free flow area to $H X$ area

$\sigma 2=\pi \cdot \frac{\frac{\text { Dtin }^{2}}{2}}{\mathrm{sl} \cdot \mathrm{st}} \quad \sigma 2=0.10494$

AreaAHStube $=\pi \cdot \frac{\text { Din }}{2} !^{2}$

AreaAHStube $=4.30053 \cdot 10^{-4} \cdot$ length $^{2}$

\footnotetext{
Pressure head (air)

dpahead $=\cdot g \cdot(\mathrm{Ha}) \cdot(\operatorname{pa}($ Tahot $)-\operatorname{pa}($ Tacold $))$
}

Secondary side Pressure head (water)

dpwhead $(T w 2$ cold $)=(-g \cdot($ Hw2 $) \cdot(p w(T w 2 h o t(T w 2 \operatorname{cold}))-p w(T w 2 \operatorname{cold})))$

dpwhead $(T w 2$ cold $)=414.4598 \cdot$ mass $^{\text {length }}{ }^{-1} \cdot$ time $^{-2}$

Initial Guees for Air Side Parameters(p. 47, Kays and London)

Approx HX Mass Volocitys (Initial Guees)

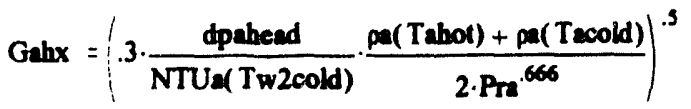

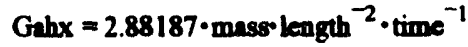

$\operatorname{Prwav}(T w 2$ cold $)=\frac{\operatorname{Prw}(T w 2 h o t(T w 2 c o l d))+\operatorname{Prw}(T w 2 \text { cold })}{2}$

$$
\begin{aligned}
& G w h x=\left(\frac{3 \cdot \frac{d p w h e a d}{(T w 2 c o l d)}}{N T U w(T w 2 c o l d)} \cdot \frac{\operatorname{pw}(T w 2 h o t(T w 2 c o l d))+p w(T w 2 c o l d)}{2 \cdot \operatorname{Prwav}(T w 2 c o l d)}\right)^{.666} \\
& \text { Gwhx }=374.03809 \cdot \text { mass length }^{-2} \cdot \text { time }^{-1}
\end{aligned}
$$

AHE Reynold's Number

$\mu w a v e(T w 2$ cold $)=\frac{\mu w(T w 2 h o t(T w 2 \operatorname{cold}))+\mu w(T w 2 \operatorname{cold})}{2}$ 


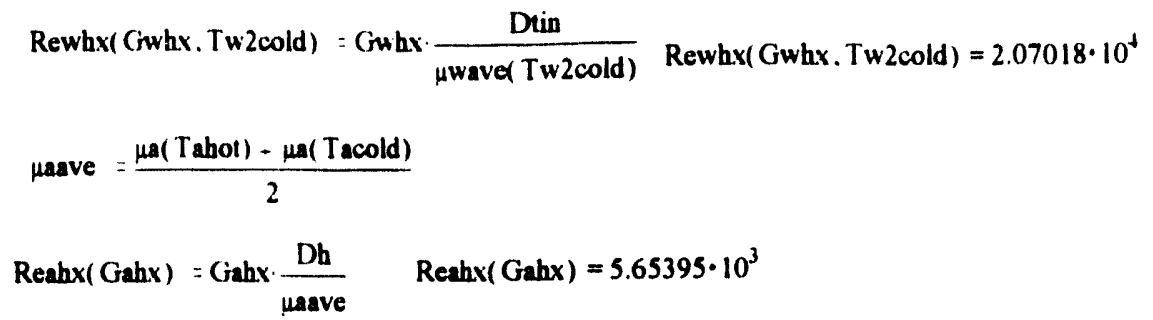

Calculate $U$ (beaced on Air Side Area)

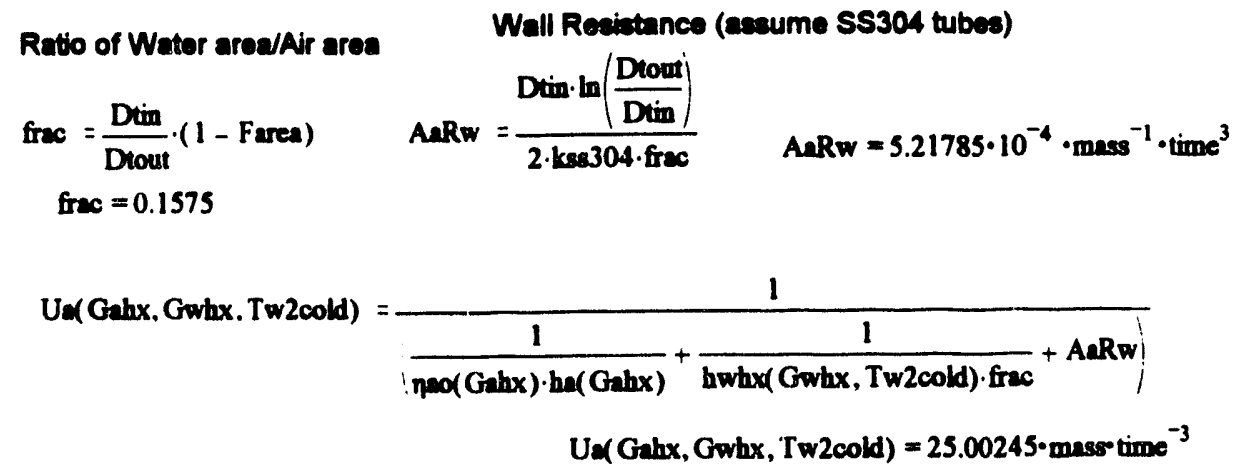




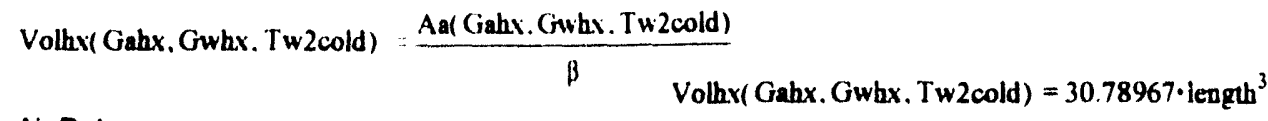

Air Data

Free Flow Area

Aff(Gahx) $=\frac{\text { dma }}{\text { Gahx }}$

Frontal Area

$\operatorname{Aff}($ Gahx $)=114.81839 \cdot$ length ${ }^{2}$

$\operatorname{dann}(G \operatorname{ahx})=\frac{\operatorname{Afr}(G \text { ahx })}{58 \cdot 3.14} \quad \operatorname{dann}(G$ ahx $)=0.98202 \cdot$ length $^{2}$

$\operatorname{Afr}(G a h x)=\frac{A f f(G a h x)}{\sigma}$

Afr $(G$ ahx $)=178.84485 \cdot$ length $^{2}$

Water Date

$\operatorname{Affw}($ Gwhx $)=\frac{\text { dmw }}{\text { Gwhx }} \quad \operatorname{Affw}($ Gwhx $)=0.21218 \cdot$ length $^{2}$

Heat Exchanger Depth, Width,Length

$\operatorname{Dhx}\left(\right.$ Gahx, Gwhx. Tw2cold) $=\frac{\text { Volhx (Gahx. Gwhx. Tw2cold) }}{\text { Afr(Gahx) }} \operatorname{Dhx}($ Gahx, Gwhx. Tw2cold $)=0.17216 \cdot$ length

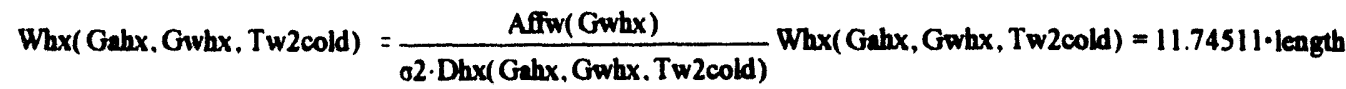

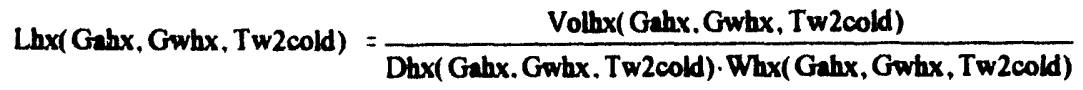

$\operatorname{Lhx}(\mathrm{Gahx}, \mathrm{Gwhx}, \mathrm{Tw} 2 \mathrm{cold})=15.22718 \cdot$ length

HX Air-aide friction dp(approx. value for homean used)

DP through $\mathrm{HX}$ core

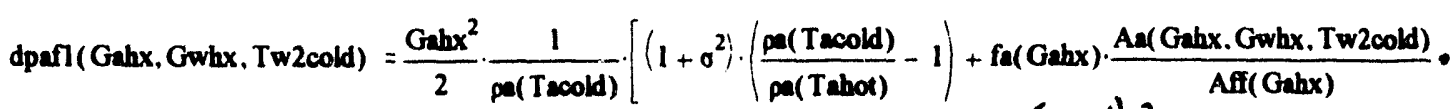
$\operatorname{dpaf1}($ Gahx, Gwhx, Tw2 cold $)=7.59822 \cdot$ mese length $^{-1} \cdot$ time $^{-2}$

Flow Ares of $\mathrm{HX}$

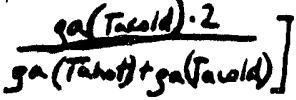

$f(G a h x)=0.0399$

Aflowhx (Gahx, Gwhx, Tw2cold $)=\frac{\text { Volhx (Gahx. Gwhx, Tw2cold })}{\operatorname{Dhx}(\text { Gahx, Gwhx, Tw2cold })}$

Aflowhx $($ Gahx, Gwhx. Tw2cold $)=178.84485 \cdot$ length $^{2}$

Cooling tower diameter, assuming Whx is $1 / 10 \mathrm{Ha}$, cooling tower flow areas $1 / 4 \mathrm{HX}$ flow

area

$\mathrm{Wh \times 2}=\frac{\mathrm{Ha}}{10}$

Whx2 $=1.5 \cdot$ length 


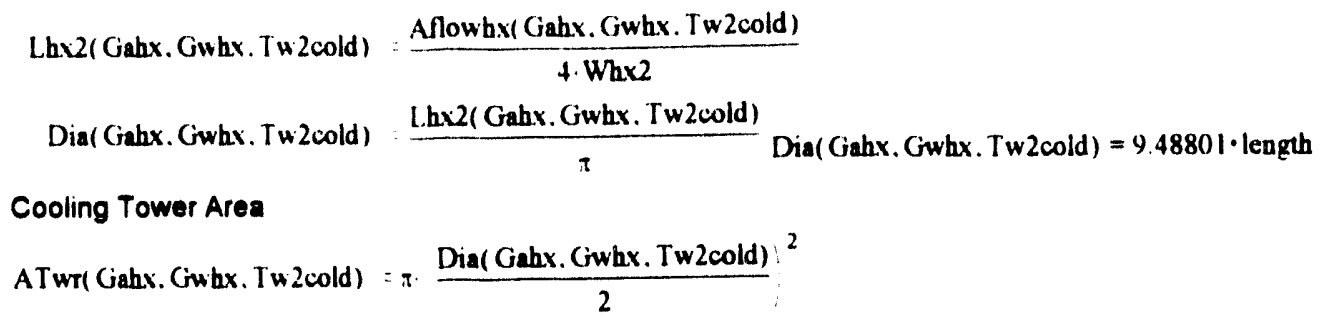




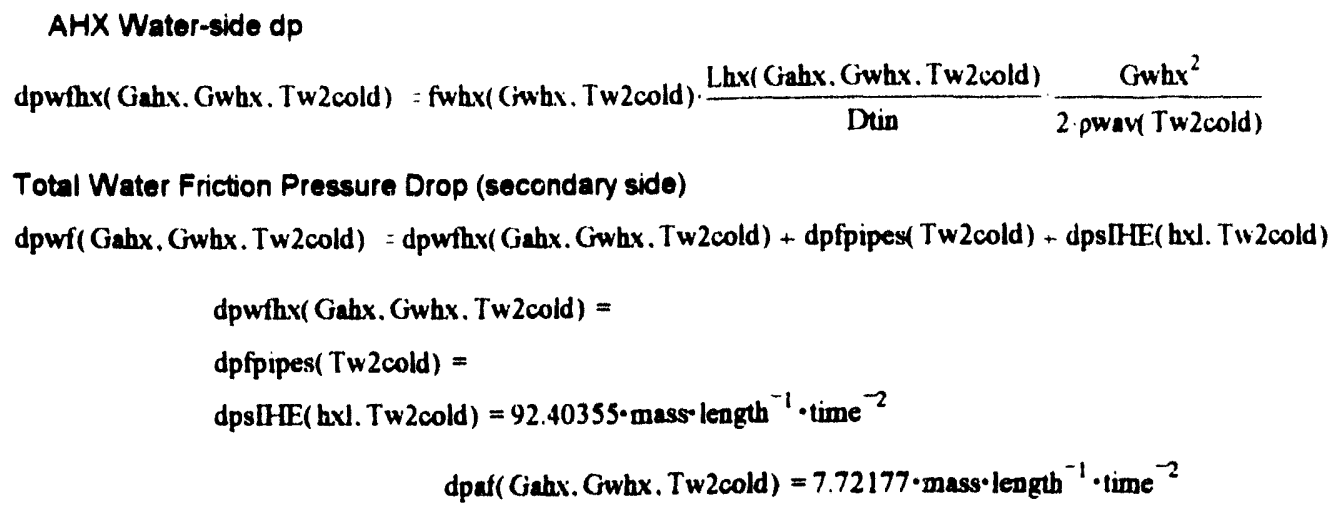

Primary side dp's

$\mu$ wpave $=\frac{\mu w(\text { Twhol })+\mu w(\text { Twcold })}{2}$

Shield

Gwshld $=\frac{\text { dmw }}{\text { Ashld }} \quad$ Gwshld $=28.08104 \cdot$ mass $^{\text {length }}{ }^{-2} \cdot$ time $^{-1}$

Rewsk:'1 = Gwshld $\frac{\text { Dshld }}{\mu \text { wpave }}$

Rewshld $=1.8854 \cdot 10^{3} \quad$ fwshld $=\operatorname{if}\left[\operatorname{Rewshld}<30000,0.316 \cdot \operatorname{Rewshld}^{(-25)}, 0.184 \cdot \operatorname{Rewshld}^{(-2)}\right]$ fwshld $=0.04796$

pwpeve $=\frac{p w(\text { Twhot })+p w(\text { Twcold })}{2}$

dpfshld $=$ fwshld $\frac{\text { Lshld }}{\text { Dshld }} \cdot \frac{\text { Gwshld }^{2}}{2 \cdot \text { pwpave }}$

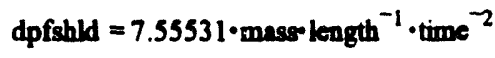

Primary piping dp

Gowpipep $=\frac{\text { dmw }}{\text { Apipep }} \quad$ Rewpipep $=$ Gwpipep $\frac{\text { Dpipep }}{\mu \text { wpreve }}$

Rewpipep $=5.13992 \cdot 10^{5}$

fwpipep $=\mathrm{if}\left[\operatorname{Rewpipep}<30000,0.316 \cdot \operatorname{Rewpipep}^{(-25)} \cdot 0.184 \cdot \operatorname{Rewpipep}^{(-2)}\right]$

fwpipep $=0.01326$ 


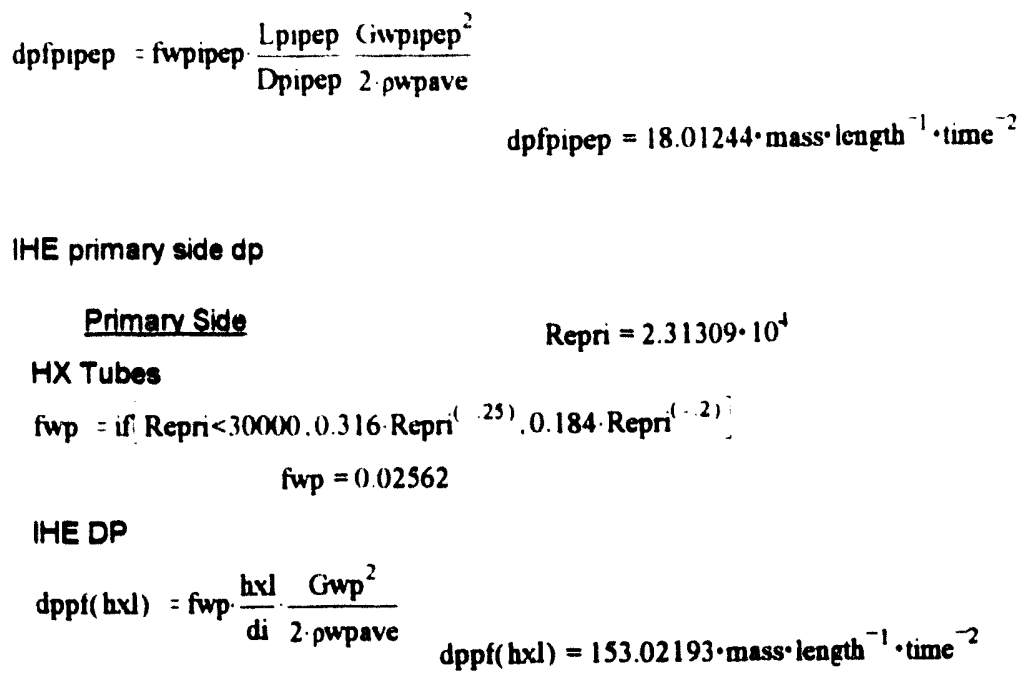

\section{Primany Side Total DP}

$d p p t(h x d)=d p p f(h x d)-d p f p i p e p+d p f s h l d$

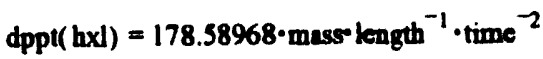

Primary side natural circulation DP

dpwphead $=(\cdot g \cdot(\mathrm{Hw}) \cdot(\operatorname{pw}($ Twhot $)-\operatorname{pw}($ Twcold $)))$

dpwphesd $=482.55394 \cdot$ mass $\cdot$ length $^{-1} \cdot$ time $^{-2}$

\section{Balance pressure drops on AHE}

Given

dpat(Gahx, Gwhx. Tw2cold)=dpahead

dpwf(Gahx, Gwhx. Tw2cold)=dpwhead (Tw2cold)

dppl( bxJ)=dpwphead

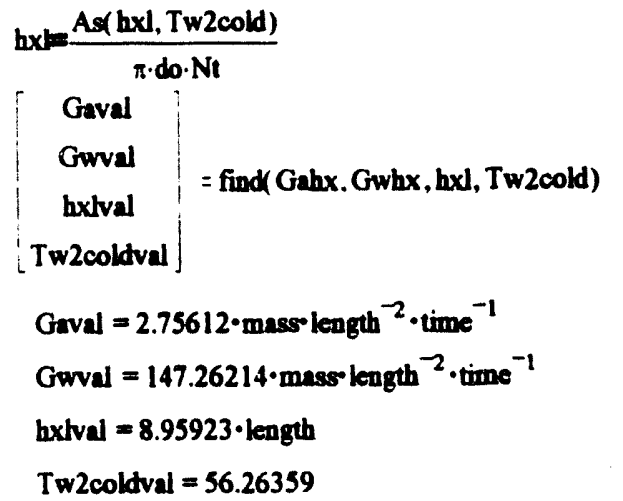

\section{HX Final Dimensions}

Dhx (Gaval, Gwval, Tw2coldval $)=0.20314 \cdot$ length

Lhx(Geval, Gwval, Tw2coldval $)=7.39669 \cdot$ length

Whx (Gaval, Gwval. Tw2coldval $)=25.2822 \cdot$ length Volhx (Gaval, Gwval, Tw2coldval $)=37.98809$ - length ${ }^{3}$ 
Concrete Volume(Assume $.33 \mathrm{~m}$ thick shell, incl height above center of shield)

Convol $=H a-\frac{W h x 2}{10}-H w 2 \cdot H w-\frac{V 1 \text { shld }}{2}$ Dial Gaval. Gwval. Tw2coldval) $\pi \cdot 33 \cdot 10(0) \cdot \mathrm{cm}$

$$
\text { Convol }=283.87283 \cdot \text { length }^{3}
$$

Price Data

Aluminum Tubes (Motal Goods, Marlborough, 11/11/93, for 500 if of 1 in dia tube)

$P_{\mathbf{a l}}=\frac{1.61}{1 \cdot \mathrm{in} \cdot \pi \cdot 065 \cdot \mathrm{in} \cdot 1 \cdot \mathrm{ft}}$

$$
\text { Pal }=4.00941 \cdot 10^{4} \cdot \text { length }^{-3} \text { Dollars }
$$

S\$304 Tubes (Metal Goods, Mariboro, 11/11/93, for $500 \mathrm{n}$ of 1 in dia tubo)

Pss304 $=\frac{1.71}{1 \cdot \text { in } \cdot \pi \cdot 065 \cdot \text { in } 1 \cdot \text { A }} \quad$ Pss304 $=4.25844 \cdot 10^{4} \cdot$ length ${ }^{-3}$ Dollars

\section{Aluminum Volume}

Alvol $=$ Volhx (Gaval. Gwval. Tw2coldval $) \cdot \beta \cdot$ Farea $\cdot$ if $\quad$ Afvol $=1.82572 \cdot$ length $^{3}$

Steel Volume

ss304vol $=$ Volhx (Gaval.Gwval. Tw2coldval $) \cdot \beta \cdot(1-$ Farea $) \cdot \frac{\pi}{4} \cdot \frac{\text { Dtout }^{2}-\text { Dinn }^{2}}{\pi \cdot \text { Dlout }^{2}}$

$s 8304 \mathrm{vol}=1.56814 \cdot$ length $^{3}$

\section{AHX Cost}

HXcost $=($ Atvol $\cdot$ Pal + ss304vol Pss304 $) \cdot$ Rcoa

$$
\text { HXcost }=6.99895 \cdot 10^{5} \quad \text { Dollars }
$$

Tower Cost

Towercost $=$ Convol $\cdot$ Pconcrete

Towercosa $=1.70324 \cdot 10^{5}$

Dollars

AlBr Cost (escume same as copper- copper quote from Admiral Metals in Woburn, for 1000 in of $3 / 4$ in copper tube)

PAlBr $=\frac{1.26}{0.75 \cdot \text { in } \cdot \pi \cdot 0.035 \cdot \text { in } \cdot 1 \cdot f t} \quad \mathrm{PABBr}=7.76978 \cdot 10^{4} \cdot$ length $^{-3}$

IHE Cost (se304 tubearse304 shell - acaume shell is $2 \mathrm{~cm}$ thick)

Tubevol $=\mathrm{Ni} \cdot \frac{\mathrm{do}^{2}-\mathrm{di}^{2}}{4} \cdot \pi \cdot \mathrm{bxtval} \quad$ Tubevol $=0.56225 \cdot \operatorname{length}{ }^{3}$ 
ss304vollHE hNval T. Di $2 \cdot \mathrm{cm} \quad$ ss304vollHE: $=0.56292 \cdot$ length ${ }^{3}$

IHEcost $=($ Tubevol Pss304 - ss304volIHE Pss304) Rcost

Total Cost

Totcost $=$ Towercost $-H C X \cos t+$ IHEcost $\quad$ Totcost $=1.10979 \cdot 10^{6}$

Towercost $=1.70324 \cdot 10^{5} \quad H X \cos t=6.99895 \cdot 10^{5} \quad$ LHEcost $=2.39575 \cdot 10^{5}$

Pss304 $=4.25844 \cdot 10^{4} \cdot$ length $^{-3} \quad \mathrm{PAlBr}=7.76978 \cdot 10^{4} \cdot$ length $^{-3} \quad \mathrm{Pl}=4.00941 \cdot 10^{4} \cdot$ length $^{-3}$

Constants $\quad J \equiv$ joule $\quad W \equiv$ wan $\quad N \equiv$ newton

$\mathrm{C}_{\mathrm{p} a} \approx 4.18 \cdot 10^{3} \cdot 0.241 \cdot \frac{\mathrm{J}}{\mathrm{lg} \cdot \mathrm{K}} \quad \mathrm{Cpa}=1.00738 \cdot 10^{3} \cdot$ leagh $^{2} \cdot$ time $^{-2}$

Cpw $\equiv 4200 \cdot \frac{1}{\mathrm{~kg} \cdot \mathrm{K}} \mathrm{J} / \mathrm{kg}-\mathrm{K}$ (from Todrease and Kerimi)

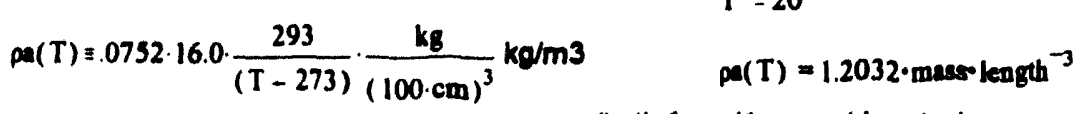

(both from Kaye and London)

$\mu(T) \equiv(18.53+.0441 \cdot(T-300)) \cdot 10^{-6} \cdot \mathrm{Pa} \cdot \sec \quad$ Pa- $\quad \mu(T)=6.182 \cdot 10^{-6} \cdot$ mass length ${ }^{-1} \cdot$ time $^{-1}$

Pra $\equiv 0.7$

$K A I \equiv 200 \cdot \frac{W}{100 \cdot \mathrm{cm} \cdot K} W / m-K \quad k e s 304=15 \cdot \frac{W}{100 \cdot \mathrm{cm} \cdot K}-W / m-K \quad K A I B r=100 \cdot \frac{\text { watt }}{\mathrm{m} \cdot K}$

Wator denaily (T in C (0-100 C))

$\operatorname{pw}(T) \equiv\left(1000+2.002461 \cdot 10^{-2} \cdot T-5.882755 \cdot 10^{-3} \cdot \mathrm{T}^{2}+1.534709 \cdot 10^{-5} \cdot \mathrm{T}^{3}\right) \cdot \frac{\mathrm{kg}}{(100 \cdot \mathrm{cm})^{3}}$

Water Prandu Number $(25-100 \mathrm{C}) \quad$ por $(\mathrm{T})=998.17017 \cdot$ mass length $^{-3}$

$\operatorname{Prw}(T) \equiv 6.20034 \cdot \exp (\cdot 0.038745 \cdot(T-10))+-0.0115698 \cdot T+2.83795$

Water Viscoeity $(20-110 \mathrm{C})$

$\mu \mathrm{W}(\mathrm{T}) \equiv(597.674 \cdot \exp (\cdot 0.0364205 \cdot(\mathrm{T}-20))-1.91245 \cdot \mathrm{T}+441.941) \cdot 10^{6} \cdot \mathrm{N} \cdot \frac{\mathrm{sec}}{(100 \mathrm{~cm})^{2}}$

Water conductivity (50-100 C)

$$
\mathrm{kw}=0.7 \cdot \frac{\mathrm{w}}{100 \cdot \mathrm{cm} \cdot \mathrm{K}} \quad \mathrm{W} / \mathrm{m}-\mathrm{K}
$$

Densities

$\mathrm{pal} \equiv 0.1 \cdot \frac{\mathrm{lb}}{\mathrm{in}^{3}} \quad \mathrm{pal}=2.76799 \cdot 10^{3} \cdot$ mass $\cdot$ leagth $^{-3}$

pss304 $\equiv 8 \cdot \frac{\mathrm{gm}}{\mathrm{cm}^{3}} \quad$ pese304 $=8 \cdot 10^{3} \cdot$ mase $^{3}$ length ${ }^{-3}$ 
Efrective $\mathrm{HX}$ Dimensions

Lhx2 : Lhx2(Gaval. Guval. Tw2coldval)

$\mathrm{Whx} 2=1.5 \cdot$ leagth

Dhx2 $=\frac{\text { Volhxi Gaval. Gwval. Tw2 coldval) }}{\text { Lhe2 Wha2 }}$

Dhx2 $=0.81256 \cdot$ leagth

$$
\mathrm{Ha}=15 \cdot \text { length }
$$

Air Free Flow Area (for airhxe.med)

Aff (Gaval) $=120.05689 \cdot$ length $^{2}$

Water Free Flow Area

Affw(Gwval) $=0.53894 \cdot$ length ${ }^{2}$

\section{Ar Heat Tranafor Area}

Aa (Gaval, Guval. Tw2coldval) $=7.25573 \cdot 10^{3} \cdot$ length $^{2}$

IHX volume

$$
\text { IHXXol }=\text { bxhval } \cdot \pi \cdot\left|\frac{D i}{\mid 2}\right|^{2} \quad \text { aHXVol }=7.03656 \cdot \operatorname{length}^{3}
$$

DATA OUTPUT

$\mathrm{Cr}_{\mathrm{r}}=1 \quad \mathrm{HW2}=5 \cdot$ length $\quad \mathrm{Hw}=5 \cdot$ leagth

$$
H_{\mathrm{a}}=15 \cdot \text { lougth } \quad \operatorname{Rewhx}(\text { Gwval, Tw20oldval })=7.72698 \cdot 10^{3}
$$

Twoold $=\mathbf{8 0}$

Volhx (Gaval. Gwval. Tw2coldval) $=37.98809 \cdot$ length $^{3}$ dime $=330.89136 \cdot \operatorname{mass} \cdot \operatorname{time}^{-1}$

$H X \cos t=6.99895 \cdot 10^{5}$

$\operatorname{Dhx}($ Gaval, Gwval. Tw2 coldval $)=0.20314 \cdot$ length $d m w=79.36508 \cdot$ masso time $e^{-1}$

Whe2 $=1.5 \cdot$ length

Towercost $=1.70324 \cdot 10^{3}$

$D h \times 2=0.81256 \cdot$ leagth

SA $=\beta \cdot$ Vollox (Gaval, Gwval, Tw2coldval)

Tolood $=1.10979 \cdot 10^{6}$

$\operatorname{Dir}($ Gaval, Gwval, Tw2 coldval) $=9.9209 \cdot$ leagth

Convol $=283.87283 \cdot$ length $^{3}$

Cooling Tower Heloht

Lhx (Gaval. Gwval, Tw2coldval) $=7.39669 \cdot$ length

Htow $=H_{2}+W_{h \times 2}+H_{w 2}+\frac{\text { VHshld }}{2}$

Hiow $=23.95 \cdot$ length

$$
\text { Hlow }-\mathrm{Ha}-\mathrm{Wh \times 2}=7.45 \text { - length }
$$

Tower Length-to-Diameter Ratio

Number Tubes in AHX

$\frac{\text { Hiow }}{\operatorname{Dia} \text { (Gaval, Gwval, Tw2coldval) }}=2.4141$

Ntube $=\frac{\text { Affw(Gwal) }}{\pi \cdot\left[\left(\frac{\text { Dinin }}{2}\right)^{2}\right]} \quad$ Ntube $=1.25319 \cdot 10^{3}$ 
Tw2coldval $=56.26359$

Twicold $=80$

Tacold $=25$

$T w 2$ coldval $=36.26359$
Tw2bol( Tw2coldval) $=71.26 .359$

$$
\begin{aligned}
& T \text { whot }=95 \\
& T \text { ahot }=40
\end{aligned}
$$

Tw2hol( Tw2coldval) $-T w 2$ coldval $=15$

Aff $($ Gaval $)=120.05689 \cdot$ length $^{2}$

Affw ( Gwval $)=0.53894 \cdot$ length $^{2}$

$$
\begin{aligned}
& H w=5 \cdot \text { length } \\
& H w 2=5 \cdot \text { length }
\end{aligned}
$$

Volhv( Gaval, Gwval. Tw2coldval) $=37.98809 \cdot$ length $^{3}$

Convol $=283.87283 \cdot$ length $^{3}$

IHXXol $=7.03656 \cdot$ length $^{3}$

Totcost $=1.10979 \cdot 10^{6}$

$\operatorname{Dia}($ Gaval. Gwval. Tw2coldval $)=9.9209 \cdot$ length

IHEcost $=2.39575 \cdot 10^{5}$

hxtval $=8.95923 \cdot$ length

ls $($ hxtval $)=8.95923 \cdot$ length

$H D \cos t=6.99895 \cdot 10^{5}$

Towercost $=1.70324 \cdot 10^{5}$ 


\section{Appendix 7: Shield Water System Pumping Power Calculations}

In the Shield Water System (SWS) chapter (Chapter 6), it was shown that the pumping power necessary to provide flow to the SWS is relatively small $(0.02 \mathrm{MW})$, despite the use of an inefficient jet pump to provide the required pressure head. This appendix will describe the method used to estimate the efficiency of the jet pump, and the electrical pumping power necessary for the SWS.

\section{Figure A7.1. Jet Pump Nozzle Configuration}

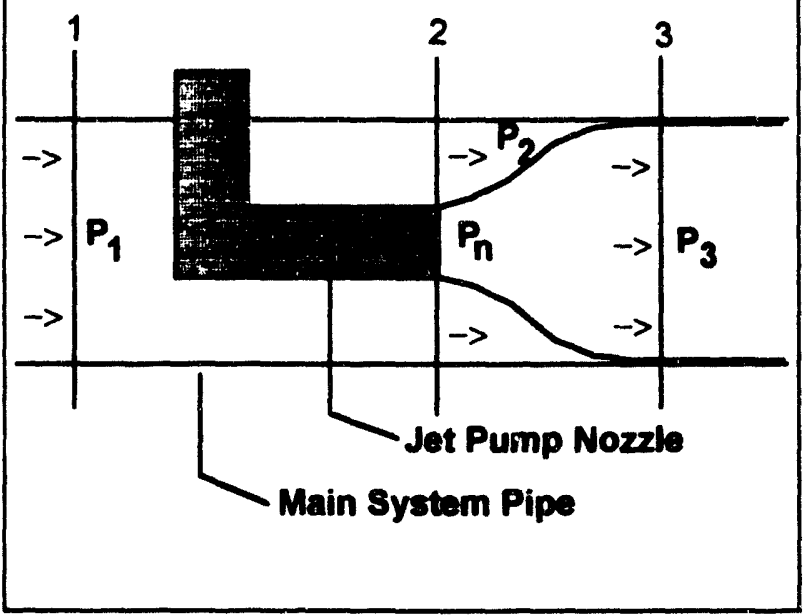

Figure A7.1 shows a simplified schematic of the jet pump nozzle inside the main SWS piping. The jet pumpdriven flow from the nozzle expands to fill the diameter of the main system pipe, as shown in the figure. The pressure upstream of the pump nozzle is $P_{1}$, and downstream of the nozzle the pressure is $P_{3}$. Hence the pressure head of the pump is $P_{3}-P_{1}$.

In the inviscid flow approximation, we can relate the pressure upstream of the nozzle $\left(P_{1}\right)$ to the pressure just before the nozzle outlet $\left(P_{2}\right)$ using the Bernoulli equation for a horizontal nozzle:1

$$
\mathrm{p}_{1}+\frac{\rho \mathrm{v}_{1}^{2}}{2}=\mathrm{p}_{2}+\frac{\rho \mathrm{v}_{2}^{2}}{2}
$$

The velocities at point 1 and 2 are related by conservation of mass:

\footnotetext{
'See, for example, N.E. Todreas and M.S. Kazimi, Nuclear Systems !, Thermal Hydraulic Fundamentals, Hemisphere, 1990,
} 


$$
v_{2}=v_{1} \frac{1}{1-\text { frac }}
$$

where "frac" is the fraction of flow area taken up by the nozzle diameter.

The pressures just upstream of the nozzle outlet $\left(P_{2}\right)$ and downstream of the nozzle $\left(P_{3}\right)$ can be related by conservation of momentum considerations. ${ }^{2}$ Namely, we have the following relation:

$$
\left(\int \rho \mathrm{v}^{2} \mathrm{dA}\right)_{2}+\mathrm{p}_{2} \mathrm{~A}=\left(\int \rho \mathrm{v}^{2} \mathrm{dA}\right)_{3}+\mathrm{p}_{3} \mathrm{~A}
$$

where $A$ is the cross-sectional area of the main system pipe in Figure 1. We can rewrite the above equation as follows:

$$
\rho\left[\mathrm{frac} \cdot \mathrm{v}_{\mathrm{n}}^{2}+(1-\mathrm{frac}) \cdot \mathrm{v}_{2}^{2}\right]+\mathrm{v}_{3}=\mathrm{p}_{3}-\mathrm{p}_{2},
$$

where $v_{n}$ is the velocity out of the nozzle. Note that $v_{3}=v_{2}=$ the flow velocity in the main system pipe.

Given the desired main system pipe flow velocity, the equations above can be used to determine the nozzle velocity required to obtain the required pump pressure head. The pumping power which we desire to calculate (that is, the electrical pumping power required for the system) is the power required to obtain the nozzle velocity $v_{a}$. The pressure head required to accelerate from rest to velocity $v_{n}$ is given by Bernoulli's equation with an initial velicity and pressure of zero, namely:

$$
\mathrm{p}_{\mathrm{a}}=\frac{\rho \mathrm{v}_{\mathrm{a}}^{2}}{2} \text {. }
$$

The required electrical pumping power, then, is given by multiplying the pressure $p_{n}$ by the mass flow rate through the nozzle, divided by the pump's electrical power-topumping power efficiency:

\footnotetext{
2ibid. p. 403.
} 


$$
\mathrm{PP}=\frac{\mathrm{p}_{\mathrm{a}} \cdot \mathrm{v}_{\mathrm{n}} \mathrm{A} \rho}{\varepsilon_{\mathrm{r}}},
$$

where $\varepsilon_{r}$ is the "raw" pump efficiency, which just accounts for the losses in the jet pump itself due to electrical to hydraulic power conversion losses. $\varepsilon_{r}$ is typically about 0.9 for a water pump. ${ }^{3}$

The effective pump efficiency is defined herein as the ideal pumping power required to sustain the flow through the main system piping divided by the actual required pumping power calculated above. The ideal pumping power for the main system pipe flow is simply the pressure drop across the jet pump $\left(\mathrm{P}_{3}-\mathrm{P}_{1}\right)$ multiplied by the main system mass flow rate. Hence, the effective pump efficiency is:

$$
\varepsilon_{\text {eff }}=\frac{\left(\mathrm{p}_{3}-\mathrm{p}_{1}\right) \cdot \mathrm{v}_{3} \mathrm{~A} \rho}{\mathrm{PP}}
$$

For a nozzle which takes up $1 / 3$ of the available main system piping flow area, we obtain an effective pump efficiency of 0.27 for the flow conditions of the Shield Water System.

${ }^{3}$ T. Baumeister. et.al., eds., Mark's Standard Handbook for Mechanical Engineers, Eighth Ed., McGraw-Hill, 1978. Chapter 14. 


\section{Appendix 8: Cost Data for Shield Water System Components}

This appendix describes the data used to generate cost estimates for the air-cooled Auxiliary Heat Exchanger/cooling tower complex and for the water-cooled Intermediate Heat Exchanger. These cost estimates are used only as guidance to help size these components, and to provide an order-of-magnitude estimate for the cost of the overall shield water system (SWS).

\section{Auxiliary Heat Exchanger and Intermediate Heat Exchanger Costing}

The Auxiliary Heat Exchanger (AHE) is composed primarily of stainless steel (CRES304) tubes with aluminum fins. In addition to the tubes and fins, there are structural members serving to support the heat exchanger tubes and fins in the required configuration. The Intermediate Heat Exchanger (IHE) is composed of a stainless steel shell (CRES304) containing aluminum brass tubes. Like the AHE, the IHE also has structural members to support the tubes, and to provide a foundation for the shell.

To estimate the cost of these heat exchangers, the required volumes of tubing are found for both exchangers. In addition, the fin volume for the AHE, and the shell volume for the IHE is found (assuming the IHE shell is $20 \mathrm{~mm}$ thick). The cost data in Table A8.1 is used to find the base material cost for the tubes, the AHE fins, and the IHE shell. Once the total cost for the base material for the AHE and IHE is found, the total base material cost is multiplied by a factor of 5 to account for the cost of the structural members, as well as the cost of heat exchanger design and fabrication. 
Table A8.1. Cost of Heat Exchanger Base Materials

\begin{tabular}{|c|c|}
\hline Material & Cost per cu. meter \\
\hline Stainless Steel (CRES304) & $\$ 43,000+$ \\
\hline Aluminum & $\$ 40,000 *$ \\
\hline Aluminum Brass & $\$ 78,000 f$ \\
\hline
\end{tabular}

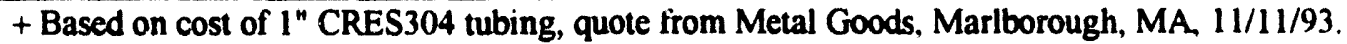

* Based on cost of 1" aluminum tubing, quote from Metal Goods, Marlborough, MA. 11/11/93.

$f$ Based on cost of 3/4" copper tubing: quote from Admiral Metals, Woburn, MA, 11/11/93.

\section{Cooling Tower Costing}

The cooling tower is a shell composed entirely of reinforced concrete. To estimate the cost of the tower, we assume that the shell thickness is 0.33 meters, which corresponds to the thickness for large ( $\sim 100$ meters tall) cooling towers used for the steam side of $1000 \mathrm{MW}$ power plants. ${ }^{1}$ This assumption is clearly conservative. Using the diameter and height of the tower, the volume of the reinforced concrete shell is determined. The cost of the concrete is determined using the as-fabricated cost for the reinforced concrete structural beams of a modest size (5-story) building, which is $\$ 600$ per cubic meter. $^{2}$

\footnotetext{
'Thickness of typical large cooling tower shell from Ron Chandren, Balke-Durr, private communication, $9 / 17 / 93$.

2From J.M. Goldman, Senior Ed, "Means Concrete Cost Data," 9th Annual Edition, R.S. Means and Co., 1991.
} 


\section{Appendix 9: Composition and Properties of HT/MT-9 Steels}

This appendix contains material property and composition information on the ferritic steel Sandvik HT-9, and the reduced-activation ferritic steel MT-9. The appendix also contains a discussion on the determination of the creep rupture curves for MT/HT-9 shown in Section 5.2.3.6.

Table A9.1 gives the composition, in weight percent, of HT-9 and MT-9.

\section{Table A9.1 Composition of MT-9 and HT-9}

\begin{tabular}{|c|c|c|}
\hline Element & HT-91 & MT-92 \\
\hline Fe & 84.5 & 85.5 \\
\hline Cr & 12 & 12 \\
\hline Mo & 1 & 0.02 \\
\hline V & 0.3 & 0.3 \\
\hline W & 0.5 & 1 \\
\hline C & 0.2 & 0.2 \\
\hline Si & 0.4 & 0.4 \\
\hline Mn & 0.6 & 0.6 \\
\hline $\mathbf{N i}$ & 0.5 & 0.0025 \\
\hline
\end{tabular}

${ }^{1}$ RL. Klueh, et.al., Ferritic/Martensitic Steels: Promises and Problems, in USDOE Fusion Reactor Materials Semiannual Progress Report for Period Ending March 31, 1992, USDOE Report \#DOE/ER/0313/12.

${ }^{2}$ S. Fetter, "The Radiological Hazards of Magnetic Fusion Reactors," Fusion Technology, Vol. 11, March 1987, p. 400. 
The basic material properties (thermal conductivity, thermal expansion, and volumetris heat capacity) of MT-9 steel used in the present work are assumed to be the same as for HT-9. To simplify the analysis performed using this data, the temperature dependence of these properties is assumed to be linear (the data is fit to a linear approximation.) This is consistent with what was done in a previous similar study, ${ }^{3}$ and results in minimal error. Table A9.2 shows the linear correlations used herein: They are based on data for the temperature range 500-1000 degrees $C$, which is the temperature range of most interest in the present work.

\section{Table A9.2 Material Properties of HT/MT-9}

\begin{tabular}{|c|c|}
\hline Property & Linear Correlation $(\mathrm{T}$ in $\mathrm{K})$ \\
\hline Thermal Conductivity $(\mathrm{W} / \mathrm{m} \cdot \mathrm{K})^{4}$ & $24.1+0.004 \cdot \mathrm{T}$ \\
\hline $\begin{array}{c}\text { Volumetric Heat Capacity }\left(\mathrm{J} / \mathrm{m}^{3} \cdot \mathrm{K}\right)^{5} \\
\text { Mean Thermal Expansion Coefficient } \\
\left(10^{-6} / \mathrm{K}\right)^{6}\end{array}$ & $1.989 \cdot 10^{6}+4920 \cdot \mathrm{T}$ \\
\hline
\end{tabular}

\footnotetext{
3J.E. Massidda and M.S. Kazimi, Thermal Design Considerations for Passive Safety of Fusion Reactors, MIT Plasma Fusion Center Report \#PFC/RR-87-18 dated October, 1987.

${ }^{4}$ Source, ibid.

${ }^{5}$ Source, ibid.

6Source, The Materials Handbook for Fusion Energy Systems, USDOE Report \#DOE/TIC-10122, McDonnell Douglas Astronautics Co., Initial Issue April 30, 1980.
} 


\section{Creep Rupture Curves}

The data used to generate the creep rupture curve for MT/HT-9 shown in Section 5.2.3.6 is based on 198 stress rupture data points obtained from 12 heats of Sandvik HT9. The temperature range for the data was 500-650 degrees $C .{ }^{7}$ To determine the appropriate creep rupture time curves from this data, the method described in ASME Code Case 1592 is used. This code case, which evolved into Code Case N-47 for Class 1 Components in Elevated Temperature Service, specifies the following method for determination of creep rupture curves: ${ }^{8}$ (1) obtain the data points indicating the rupture times for different samples at the same temperature and stress; (2) determine the mean $(\mu \mathrm{l})$ and the standard deviation $(\sigma \mathrm{l})$ of the logarithm of the rupture times for these points; (3) the logarithm of the minimum rupture time for the data set is given by: $\operatorname{minl}=$ $\mu l-1.65(\sigma l) ;(4)$ the creep rupture curves are them determined by the set of minl values for the various temperatures and stresses tested. This method ensures that the creep rupture curve thus defined is a lower boundary for approximately 95 percent of the data.

\section{Larson-Miller Parameter}

To estimate the creep rupture behavior for MT/HT-9 for temperatures above 650 degrees $\mathbf{C}$, for which there is no test data, use is made of the Larson-Miller parameter, which has been found to characterize the creep response of many metals. The LarsonMiller parameter is given by the following relation:

\footnotetext{
${ }^{7}$ Source, The Materials Handbook for Fusion Energy Systems, USDOE Report \#DOE/TIC-10122, McDonnell Douglas Astronautics Co., Initial Issue April 30, 1980.

${ }^{8}$ The method described is based on information in the booklet 1977 Design Criteria of Boilers and Pressure Vessels, papers presented at the 3rd International Conf. on Pressure Vessel Technology, Tokyo, Japan, April 19-22, 1977, The American Society of Mechanical Engineers, 1977.

${ }^{9}$ The discussion in this section is based on information in J.E. Massidda and M.S. Kazimi, Thermal Design Considerations for Passive Safety of Fusion Reactors, MIT Plasma Fusion Center Report \#PFC/RR-87-18 dated October, 1987, Chapter 3 and J.A. Collins, Failure of Materials in Mechanical Design, John Wiley and Sons, 1981.
} 


$$
\text { (A9.1) } \quad P=T\left(C+\log _{10} t_{r}\right) \text {, }
$$

where $t_{r}$ is the rupture time of the metal at temperature $T$ (in Rankine). It has been found that the Larson-Miller parameter $P$ is constant for a given metal and stress level, and that the constant $\mathrm{C}$ is usually about 20 for metals. Hence, if the rupture time is known at a particular temperature and stress, the rupture time at a different temperature can be estimated using the above equation. This equation is used to estimate the rupture stress curves for MT/HT-9 at temperatures above those for which test data is known. 


\section{Appendix 10: MATHCAD Code for Calculating Pebble Bed Conductivity'}

The following MATHCAD code incorprates the modified SZB model ${ }^{3} 45$ for calculating the pebble bed conductivity as a function of temperature for Blanket Design 1.

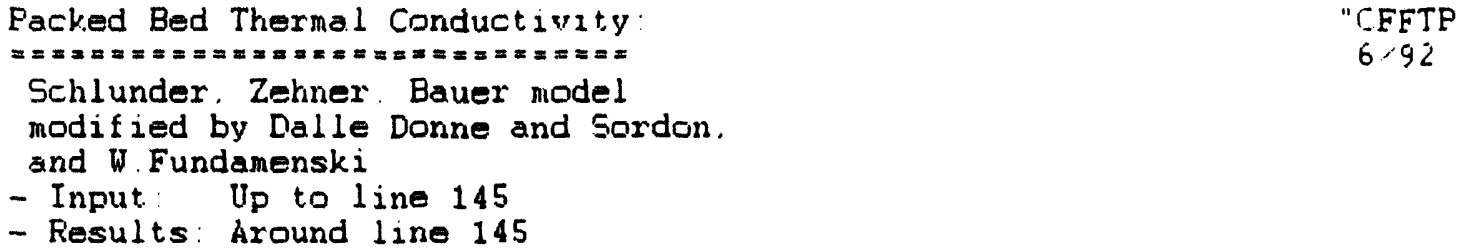

$n s:=2$

Number of pebble types

(This is $f$ ixed at 2 here. so model covers 1 or 2-size beds. Set second size volume fraction $v f 2$ to 0 if only one size pebble in bed.)

$j:=1 . . n s$

Diameters

Volume fraction of pebbles with respect to overall bed

$D p_{1}:=3.0 \cdot \mathrm{mm}$ This doesn't account for any porosity in pebbles: that is factored into the conductivity of the pebbles later on KMC

$D p_{2}:=0.4 \cdot \operatorname{mm} \quad \forall f_{2}:=0.2$

$v_{j}:=\frac{\nabla f_{j}}{v f_{1}+f_{2}} \quad \begin{aligned} & \text { Volume fraction of each pebble type } \\ & \text { relative to the total pebble volume }\end{aligned}$

${ }^{1}$ MathCAD is a computer utility developed by MathSoft, Inc.

${ }^{2}$ Provided to the author by P. Gierszewski of the Canadien Fusion Fuels Technology Project (CFFTP).

${ }^{3}$ W.R Fundamenski and P. Gierszewski, "Comparison of Correlations for Heat Transfer in Sphere-Pac Beds," Canadian Fusion Fuels Technology Project report G-9181, August 1991.

${ }^{4}$ E. Schlunder, "Particle Heat Transfer," Proc. 7th Intl. Heat Transfer Conf., Munich, Vol. 1, May 1982, p. 195.

${ }^{5}$ M. Dalle-Donne and G. Sordon, "Heat TRansfer in Pebble Beds for Fusion Blankets," Fusion Technology, Vol .17, 1990, p. 597. 


$$
D:=\left(\sum_{j} \frac{v_{j}}{D p_{j}}\right)^{-1}
$$

Effective dianeter for binary beds

$$
D=1.143 \mathrm{rm}
$$

$D D:=\frac{\sum_{j} \frac{v_{j}}{\left(D p_{j}\right)^{2}}}{\left(\sum \frac{v_{j}}{D p_{j}}\right)^{2}}$

Size distribution factor. from original SZB model, and as used by Dalle Donne/Sorden

$\mathrm{DD}=\mathbf{2 . 1 4 9 6 6}$

WF modification - use a bed of one size spheres of equivalent diameter. neglects SZB factor

$D D:=1$

$\Omega:=1-\left(\sum_{j} v_{j}\right)$

Bed Dorosity. fraction. . . . . . .

$$
\Omega=\mathbf{2 0} \%
$$

Contact area ratios between pebbles of type 1 and type 2 :

Following values are suggested for $(\rho k)^{\wedge} 2$ :

Low- $k$ ceramics - $0.5 \mathrm{E}-4$

High-k ceranics $(A 1203)-1.5 e-4$ steel - 5E-4

A1. Be - 10E-4 (???)

$$
\text { pk1 }:=\left(10 \cdot 10^{-4}\right)^{.3} \quad \quad p k 2:=\left(0.5 \cdot 10^{-4}\right)^{3}
$$

$p k:=\left[p k l^{3} \cdot\left(v_{1}\right)^{2}+p k 2^{2} \cdot\left(v_{2}\right)^{2}+p k 12^{2} \cdot 2 \cdot v_{1} \cdot v_{2}\right]^{5}$

$$
\mathrm{f}:=23 \cdot \frac{\Delta k^{2}}{1+22 \cdot p k^{1.3333}}
$$




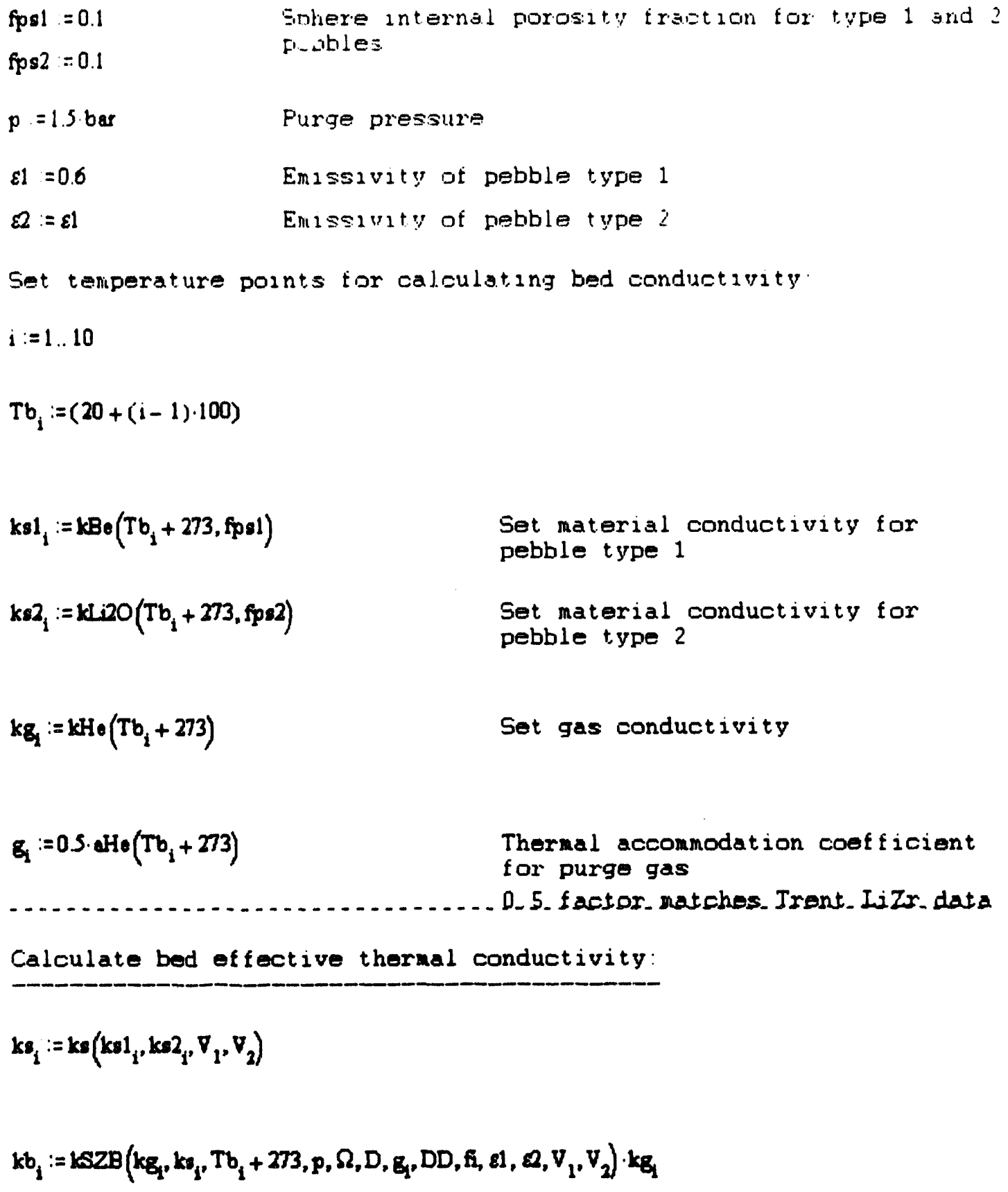



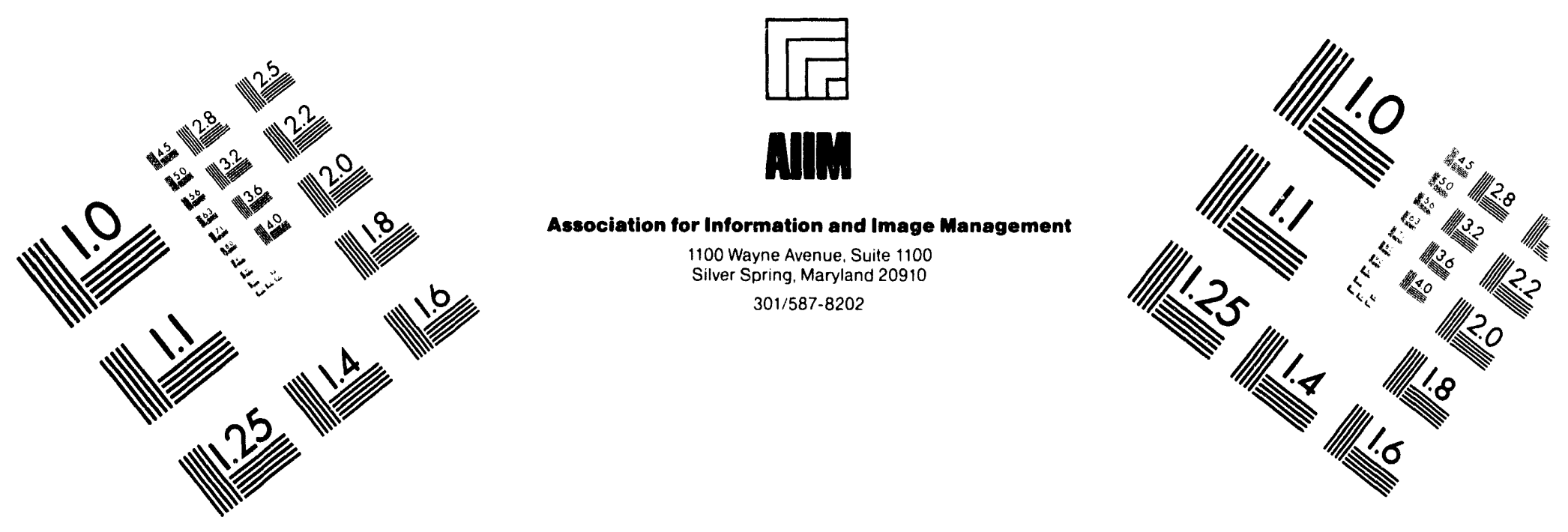

Centimeter

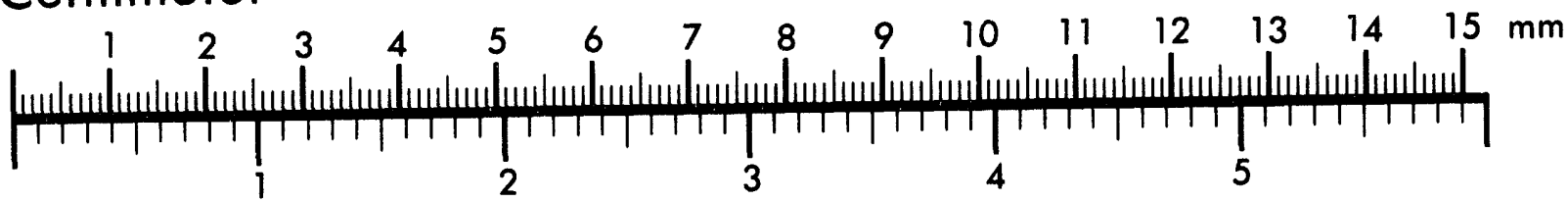
Inches
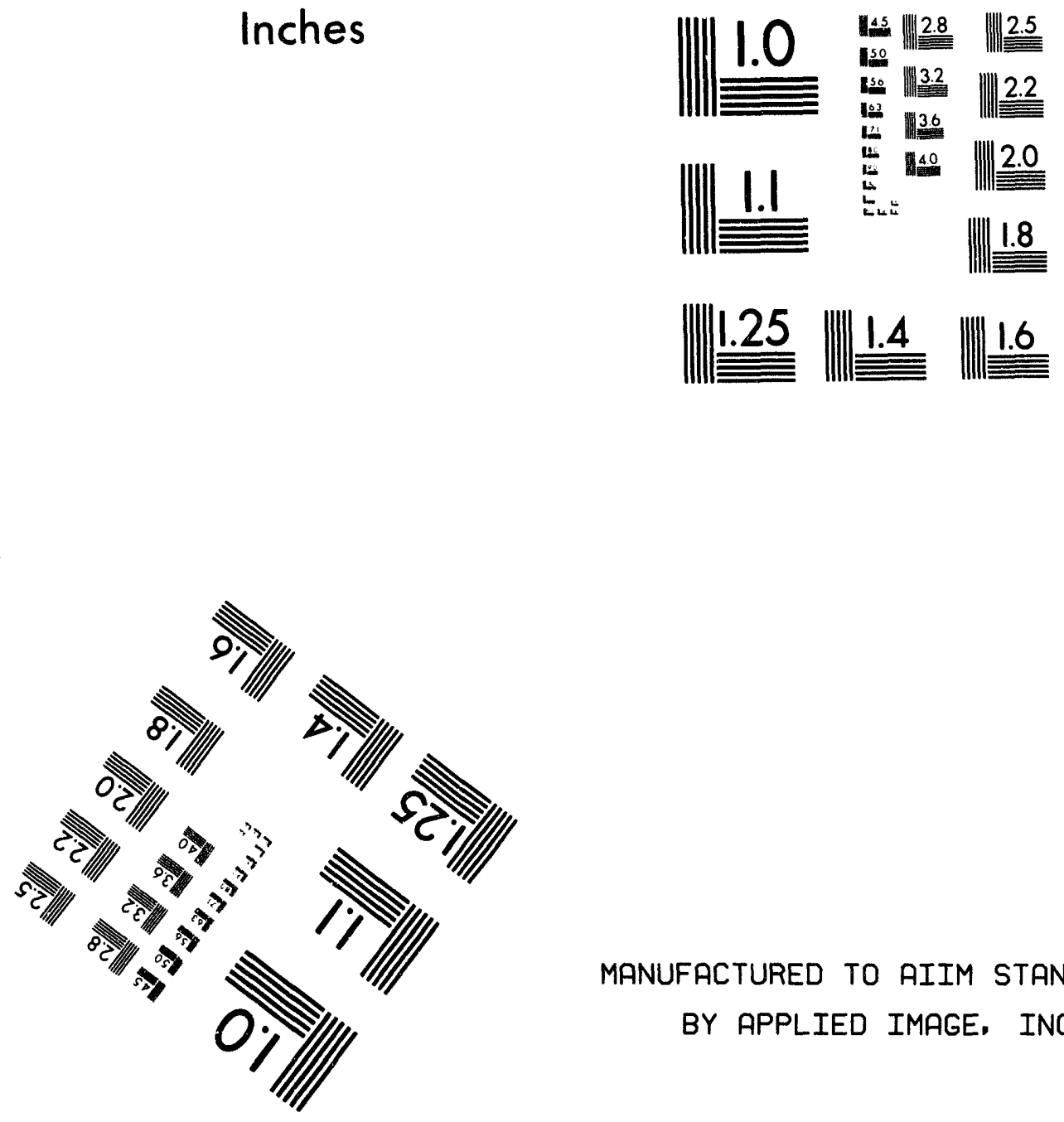

MANUFACTURED TO AIIM STANDARDS

BY APPLIED IMAGE. INC.

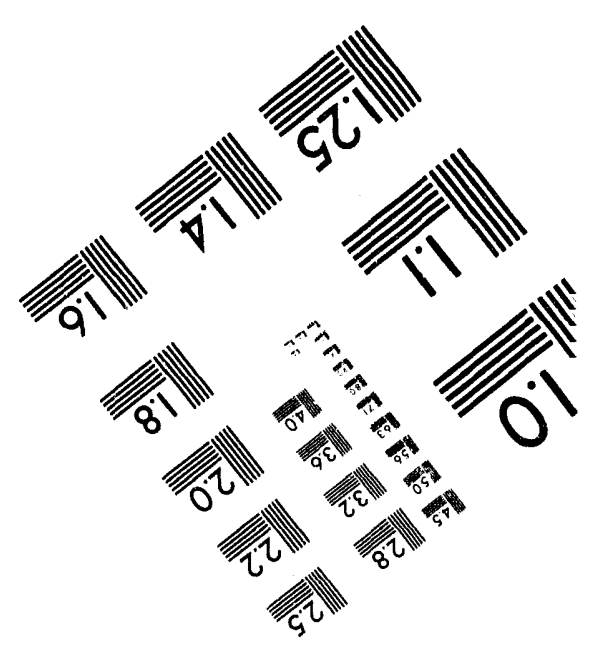



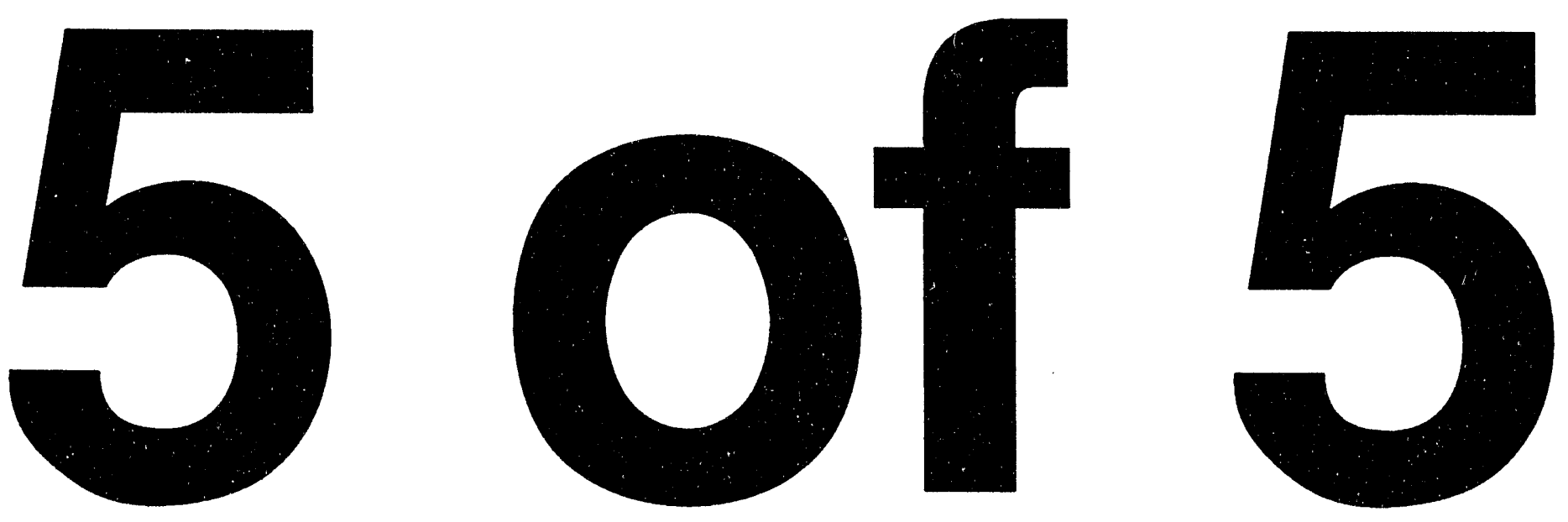


\begin{tabular}{|c|c|c|c|}
\hline \multirow{11}{*}{$\Omega=20 \%$} & $\mathrm{~Tb}_{i}$ & $k b_{i} \cdot m \cdot \frac{K}{W}$ & \\
\hline & 20 & 4.945 & 992 \\
\hline & 120 & 5.128 & 708 \\
\hline & 220 & 5.255 & 536 \\
\hline & 320 & 5.351 & 423 \\
\hline & 420 & 5.429 & 345 \\
\hline & 520 & 5.496 & \\
\hline & 620 & 5.552 & 246 \\
\hline & 720 & 5.59 & 212 \\
\hline & 820 & 5.597 & 181 \\
\hline & 920 & 5.552 & 151 \\
\hline
\end{tabular}

Unit dimensions:

ORIGIN $=1$

$\mathrm{kg}=1 \mathrm{M} \quad \mathrm{m}=1 \mathrm{~L} \quad \mathrm{~s}=1 \mathrm{~T} \quad \mathrm{~K}=1 \quad \mathrm{C}=1$

$\mathrm{cm}=0.01 \cdot \mathrm{m} \quad \operatorname{mm}=0.001 \cdot \mathrm{m} \quad u m=0.001 \cdot \mathrm{mm}$

$W=\mathrm{kg} \cdot \mathrm{m}^{2} \cdot \mathrm{s}^{-3} \quad \mathrm{~Pa}=\mathrm{kg} \cdot \mathrm{m}^{-1} \cdot \mathrm{s}^{-2} \quad \mathrm{MPa}=10^{6} \cdot \mathrm{Pa} \quad$ bar $=10^{5} \cdot \mathrm{Pa}$

Material property correlations:

$\mathrm{KLiZK}(\mathrm{TK}, f)=(1-f)^{\frac{2}{3}} \cdot\left(\frac{3.643}{1+0.001549 \cdot \mathrm{TK}}+7.579 \cdot 10^{-10} \cdot \mathrm{TK}^{3}\right) \cdot \frac{\mathrm{W}}{\mathrm{m} \cdot \mathrm{K}}$

ITER $90 \mathrm{fit}$ to $80 \% \mathrm{TD}$ data

$\mathrm{KLiZKTK,} f)=\left(\frac{1-f}{0.86}\right)^{\frac{5}{3}} \cdot\left[\frac{2.7699}{1+0.0008 \cdot(\mathrm{TK}-273)}+1.8034 \cdot 10^{-10} \cdot(\mathrm{TK}-273)^{3}-0.2284\right] \cdot \frac{\mathrm{W}}{\mathrm{m} \cdot \mathrm{K}} \cdot$

CFFTP/PG Fit to $86-87 \%$ TD data

KAIO $(T K, f)=\left(\frac{1-f}{0.90}\right)^{1.56} \cdot\left(44.85-0.07276 \cdot T K+3.543 \cdot 10^{-5} \cdot \mathrm{TK}^{2}\right) \cdot \frac{W}{\mathrm{~m} \cdot \mathrm{K}}$ 


$$
\begin{aligned}
& \begin{array}{l}
\mathrm{KAIO}(\mathrm{TK}, \mathrm{f}) \equiv\left(\frac{1-1}{0.90}\right) \quad\left(44.85-0.07276 \cdot \mathrm{TK}+3.543 \cdot 10^{-\lrcorner} \cdot \mathrm{TK}^{2}\right) \cdot \frac{\mathrm{v}}{\mathrm{m} \cdot \mathrm{K}} \\
\mathrm{KAKTK}, \mathrm{f}) \equiv(1-\mathrm{f})^{1.8} \cdot\left[2.05 \cdot \frac{\mathrm{TK}}{2.42+0.01117 \cdot(\mathrm{TK}-273)}+0.0503 \cdot \mathrm{TK}\right] \cdot \frac{\mathrm{W}}{\mathrm{m} \cdot \mathrm{K}}
\end{array} \\
& \mathrm{KLiSi}(\mathrm{TK}, \mathrm{f}) \equiv \frac{1-\mathrm{f}}{1+(1.95-0.0008 \cdot \mathrm{TK}) \cdot \mathrm{f}} \cdot\left(1.98+\frac{850}{\mathrm{TK}}\right) \cdot \frac{\mathrm{W}}{\mathrm{m} \cdot \mathrm{K}} \quad \begin{array}{l}
\text { Dalle-Donne } \\
\text { and Sorden }
\end{array} \\
& \mathrm{KL} \text { iSi }(\mathrm{TK}, \mathrm{f})=\left(1-\mathrm{f}^{\frac{s}{3}} \cdot 2.49 \cdot\left[\left(1+2.06 \cdot 10^{-3} \cdot \mathrm{TK}\right)^{-1}+1.85 \cdot 10^{-10} \cdot \mathrm{TK}^{3}\right] \cdot \frac{\mathrm{W}}{\mathrm{m} \cdot \mathrm{K}}\right.
\end{aligned}
$$

$\operatorname{KLiAKTK}, f)=\frac{1-f}{1+(2.08-0.00066 \cdot \mathrm{TK}) \cdot f} \cdot\left(\frac{20.6}{1+0.00642 \cdot \mathrm{TK}}\right) \cdot \frac{W}{\mathrm{~m} \cdot \mathrm{K}}$

$\mathrm{kLi20}(\mathrm{TK}, \mathrm{f})=(1-f)^{1.96} \frac{39.79}{1+0.007067 \cdot \mathrm{TK}} \cdot \frac{W}{\mathrm{~m} \cdot \mathrm{K}}$

$K L 20(T K, f)=\frac{1-f}{1+2.141 \cdot f-0.0007 \cdot T K \cdot f} \cdot \frac{100}{1.4+0.01828 \cdot T K} \cdot \frac{W}{m \cdot K}$

$\operatorname{LSS}(T K, f) \equiv(1-f)^{1.8} \cdot\left(2.985 \cdot \frac{T K}{0.111 \cdot T K-20}+1.674\right) \cdot \frac{W}{m \cdot K} \cdot \frac{1}{2}$

DD/Sorden. with PJG factor of 2 correction???

$\mathrm{KB} \cdot(\mathrm{TK}, \mathrm{f})=\frac{1-\mathrm{f}}{1+11 \cdot \mathrm{f}^{2}} \cdot 291 \cdot\left(1-1.65 \cdot 10^{-3} \cdot \mathrm{TK}+1.46410^{-6} \cdot \mathrm{TK}^{2}-5.125 \cdot 10^{-10} \cdot \mathrm{TK}^{3}\right) \cdot \frac{\mathrm{W}}{\mathrm{m} \cdot \mathrm{K}}$ 
$\operatorname{acoeff}(T K, \Delta \infty, \mathrm{TT})=\left[1+\left(\frac{1}{a \infty}-1\right) \cdot 10^{\frac{-\pi \mathrm{T}}{\mathrm{IK}}}\right]^{-1}$

$\operatorname{aH} e(T K) \equiv \operatorname{acoefff}(T K, 0.21,20)$

aHe (TK) = acooff $(T K, 0.21,20)$

$\operatorname{aAr}(T K)=\operatorname{acoeff(}(T K, 0.28,333)$

aN2 (TK) $=\operatorname{aAC}(T K)$

$\mathrm{aH} 2(\mathrm{TK})=$ acoeff $(T K, 0.26,20)$

$\mathrm{kH}(\mathrm{TK})=0.003366 \cdot \mathrm{TK}^{0.66 \theta} \cdot \frac{\mathrm{W}}{\mathrm{m} \cdot \mathrm{K}}$

$K A K(T K)=0.0002384 \cdot \mathrm{TK}^{0.74} \cdot \frac{W}{\mathrm{~m} K}$

$k H \chi(T K)=0.00265 \cdot \mathrm{TK}^{0.24} \cdot \frac{W}{\mathrm{~m} \cdot \mathrm{K}}$

$\mathrm{kN} 2(\mathrm{TK})=0.0325 \cdot\left(\frac{\mathrm{TK}}{400}\right)^{0.7624} \cdot \frac{\mathrm{W}}{\mathrm{m} \cdot \mathrm{K}}$

$\operatorname{kiir}(T K)=0.000270 \cdot T K^{0.700} \cdot \frac{W}{\mathrm{~m} \cdot \mathrm{K}}$
Mas lit values RT

H2 03 bright paint $N_{1}$

He 0016 is. 024 typ

N2. $\quad 0.5-0.9 \mathrm{~V}, 0.8 \mathrm{Ni}$

air $0.9 \mathrm{Fe} \mathrm{Al}$

hr: $0.85 \mathrm{~W} .0 .93 \mathrm{~N}$

$D D$ Sorden suggested form for $\mathrm{He}$ and Ar

DD/Sorden suggested form for He and Ar

Guess from abowe literature data

Fitted to dry air within $5 \%$,

-18 to $820 \mathrm{C}$ 
$\mathrm{ks}(\mathrm{ks} 1, \mathrm{ks} 2, \mathrm{~V} 1, \mathrm{~V} 2) \equiv \mathrm{ks} 1 \cdot \mathrm{V} 1^{2}+\mathrm{ks} 2 \mathrm{v2^{2 }}+2 \cdot \mathrm{V} 1 \cdot \mathrm{v} 2 \cdot 2 \cdot\left(\frac{1}{\mathrm{ks} 1}+\frac{1}{\mathrm{ks} 2}\right)^{-1}$

$\sigma(T, p)=0.1263 \cdot \frac{1}{p} \cdot \frac{T}{273.15} \cdot$ um bor

$$
o\left(273,10^{5} \cdot \mathrm{P}\right)=0.12623 \cdot \mathrm{um}
$$

$\operatorname{krkg}(T, D, \mathrm{~kg}, \varepsilon)=2.31 \cdot 10^{-7} \cdot \mathrm{D} \cdot \frac{\mathrm{T}^{3}}{\mathrm{~kg} \cdot\left(\frac{2}{\varepsilon}-1\right)} \cdot \mathrm{W} \cdot \mathrm{m}^{-2} \cdot \mathrm{K}^{-4}$

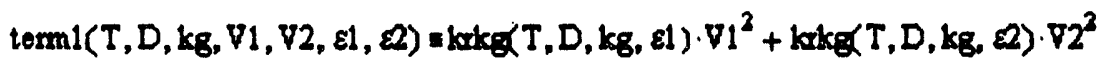

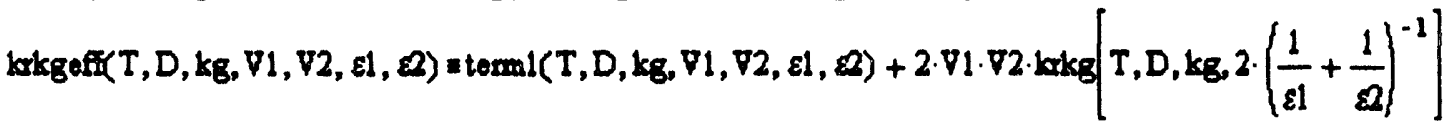
$B(\Omega, D D)=1.25 \cdot\left(\frac{1}{\Omega}-1\right)^{\frac{10}{9}} \cdot(1+3 \cdot \sqrt{D D-1})$

$A(k g k d$, kaks , B, kdks $)=(1+k$ kks $-B \cdot k d k s) \cdot k g k d-B \cdot(k g k d-1) \cdot(1+$ koks $)$

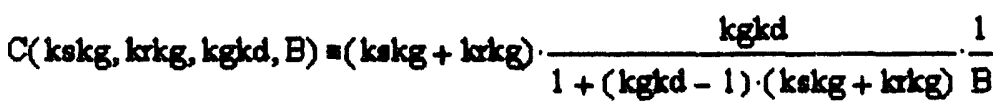

$F(A, B, k s k g, k \operatorname{kg}, \operatorname{kgkd}, C)=\frac{2}{A} \cdot\left[\frac{B}{A^{2}} \cdot(k \operatorname{kg} g+\operatorname{lokg}-1) \cdot \frac{\mathrm{kgkd}}{\mathrm{kskg}} \cdot \ln (C)-\frac{B-1}{A} \cdot \mathrm{kg} k \mathrm{kd} \ldots\right.$

$$
+0.5 \cdot\left(1+\frac{1}{B}\right) \cdot(\text { krkg } \cdot k g k d-B \cdot(1+(\text { kgkd }-1) \cdot \text { krkg }))
$$




$$
\begin{aligned}
& A v \equiv A\left(\operatorname{kgkd}(\sigma(T, p), g, D), \operatorname{krggeff}(T, D, k g, V 1, V 2, \varepsilon 1, \varepsilon) \cdot \frac{\mathrm{kg}}{\mathrm{ks}}, \mathrm{B}(\Omega, D D), \frac{\mathrm{kg}}{\mathrm{ks}} \frac{1}{\mathrm{kgkd}(\sigma(T, p), g, D)}\right) \\
& \mathrm{Cv} \equiv \approx\left(\frac{\mathrm{ks}}{\mathrm{kg}}, \operatorname{krkgeff}(T, D, \mathrm{~kg}, \mathrm{~V} 1, \mathrm{~V} 2, \varepsilon 1, \mathfrak{2}), \operatorname{kgkd}(\sigma(T, \mathrm{p}), \mathrm{g}, \mathrm{D}), \mathrm{B}(\Omega, D D)\right\} \\
& \left.\left.F v \equiv F\left(A v, B(\Omega, D D), \frac{k s}{k g}, \operatorname{krgeff(T,D,kg}, V 1, v 2, \varepsilon 1, \varepsilon\right), \operatorname{kgkd}(\sigma T, p), g, D\right), C v\right) \\
& \mathrm{kSZB}(\mathrm{kg}, \mathrm{ks}, \mathrm{T}, \mathrm{p}, \Omega, D, \mathrm{~g}, \mathrm{DD}, \mathrm{fi}, \varepsilon 1, \varepsilon, \mathrm{V} 1, \mathrm{~V} 2) \equiv(1-\sqrt{1-\Omega}) \cdot\left(\begin{array}{l}
\frac{\Omega}{\Omega-1+\operatorname{kgkd}(\alpha \mathrm{T}, \mathrm{p}), \mathrm{g}, \mathrm{D})} \ldots \\
+\Omega \cdot \operatorname{krkgeff}(T, D, \mathrm{~kg}, \mathrm{~V} 1, \mathrm{~V} 2, \varepsilon 1, \varepsilon)
\end{array}\right) \\
& +\sqrt{1-\Omega} \cdot f i \cdot \frac{k s}{k g}+(1-f i) \cdot F v
\end{aligned}
$$




\section{Appendix 11: Effect of Shield Water System Design on a Vanadium/Lithium/Lithium Blanket Design}

This appendix provides the results of a LOCA analysis of a vanadium alloy

structure/lithium coolant/lithium $(\mathrm{V} / \mathrm{Li} / \mathrm{Li})$ breeder blanket design. The analysis shows that having a Shield Water System (SWS) capable of natural circulation in the event of an accident can greatly reduce the potential for structural failure of the blanket in the event of a LOCA with air-ingress.

Vanadium alloyed with chromium and titanium (VCrTi) has been identified as a promising structural material for fusion reactor blankets because it has excellent lowactivation characteristics compared to other metal alloys. VCrTi also is compatible with liquid lithium coolant, as long as the proper alloying composition is chosen (note that due to concerns with impurities in helium coolant, vanadium alloy is not generally considered to be compatible with helium). ${ }^{12}$

A potentially severe safety problem with $\mathrm{VCrTi}$, however, is its behavior at high temperatures in an oxygenated atmosphere. Volatility experiments on $\mathrm{V} 15 \mathrm{Cr} 5 \mathrm{Ti}$, a vanadium alloy with 15 percent chromium and 5 percent titanium, indicate that exposure to an oxygenated atmosphere at temperatures above 650 degrees $C$ results in the formation of molten $\mathrm{V}_{2} \mathrm{O}_{3}$ oxide. With an oxygen partial pressure corresponding to air, formation of this molten oxide begins in less than 15 minutes. ${ }^{3}$

'D. L. Smith, et.al.. Blanket Comparison and Selection Study (BCSS) - Final Report, Argonne National Laboratory report \#ANL/FPP-84-1, September 1984.

${ }^{2}$ D. L. Smith, et.al., Reduced Activation Structural Materials Development for DEMO Fusion Reactor Applications, Argonne National Laboratory Report \#ANL/ER/CP-76020 dated September 1992.

${ }^{3}$ R. M. Neilson. Jr., Volatility of V15Cr5Ti Fusion Reactor Alloy, Idaho National Engineering Laboratory Report \#EGG-M-25985 dated December 1986. 
In the event a LOCA occurs in a blanket with vanadium structure, air from the reactor building could potentially enter into the blanket coolant system through the piping break. If this "LOCA with air ingress" occurs and the temperature of the vanadium structure exceeds 650 degrees $\mathrm{C}$ for any duration, formation of molten $\mathrm{V}, \mathrm{O}$, is likely to occur, possibly resulting in gross melting of the blanket structure. Two means exist to prevent this from occurring: (1) ensure that the reactor atmosphere has no oxygen (e.g. use a nitrogen atmosphere); and/or (2) ensure that the vanadium structure does not reach 650 degrees $\mathrm{C}$ during the LOCA.

Most reactor studies using $\mathrm{V} / \mathrm{Li} / \mathrm{Li}$ blankets assume that there will be a nitrogen atmosphere in the reactor building; this not only prevents the oxidation of vanadium but also reduces the risk of a lithium fire. However, reliance on a nitrogen atmosphere means that access to the reactor building will be hindered for the duration of the accident (unless oxygen breathing apparatus are used by personnel). Furthermore, during the course of the LOCA a common mode failure could result in partial or full loss of the nitrogen cover gas in the reactor building (the presence of oxygen at partial pressures much less than that found in air can cause rapid formation of molten vanadium oxide). Finally, if the LOCA could happen at a time when the nitrogen atmosphere was not in place (during maintenance or due to a sensor failure). For the reasons listed above, it is interesting to investigate whether it is possible to keep the temperature of a $\mathrm{V} / \mathrm{Li} / \mathrm{Li}$ blanket below 650 degrees $\mathrm{C}$ during a LOCA. If this can be done, there is no need for a nitrogen atmosphere to prevent gross melting of the vanadium structure.

The blanket design chosen for this analysis is the $\mathrm{V} / \mathrm{Li} / \mathrm{Li}$ blanket of the Blanket Comparison and Selection Study (BCSS). ${ }^{4}$ This design is shown in Figure A11.1.

${ }^{4}$ D. L. Smith, et.al., Blanket Comparison and Selection Study (BCSS) - Final Report, Argonne National Laboratory report \#ANL/FPP-84-1, September 1984. 


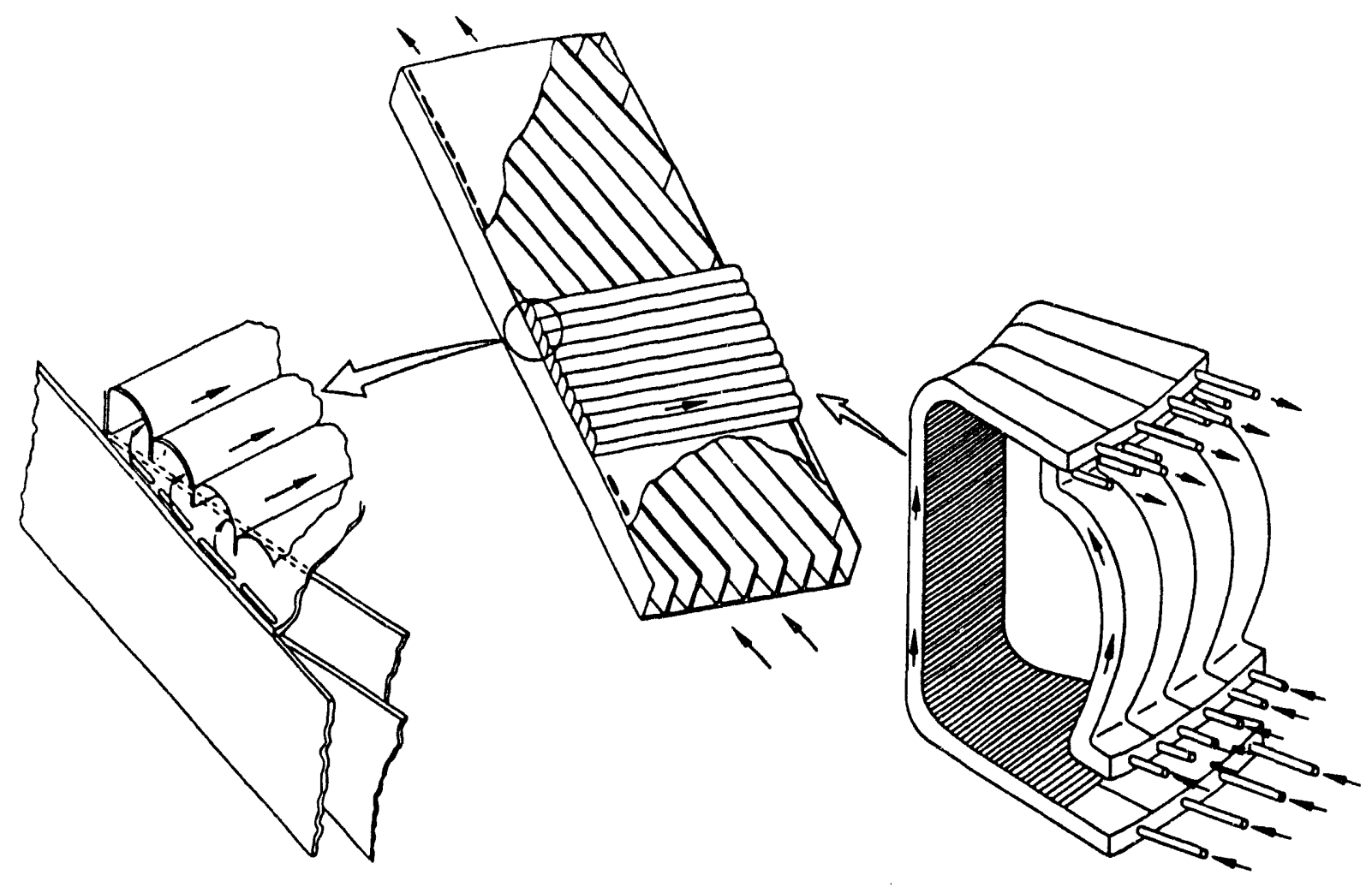

Figure A11.1 shows

the overall configuration of the blanket, and the liquid lithium flow paths. The righthand-side of the figure shows the inboard and outboard blanket segments as they would be arranged in a tokamak reactor. Moving to the left in the figure, the first wall region is shown, with toroidally-oriented coolant channels. Below the first wall region is the main tritium breeding region, where the lithium flows poloidally. Behind this region (not shown in the figure) is a second breeder region, followed by a lithium manifold region.

\footnotetext{
S.D.L. Smith, et. al., Blanket Comparison and Selection Study (BCSS) - Final Report, Argonne Nat1. Laboratory report ANL/FrP. 84-1, Sept. 1984
} 
The one-dimensional volumetric composition of the inboard $\mathrm{V} / \mathrm{Li} / \mathrm{Li}$ blanket is shown in Figure A11.2. The VCrTi alloy used in the BCSS design is V15Cr5Ti. The composition

Figure A11.2. One-Dimensional Model of Inboard BCSS V/Li/Li Blanket

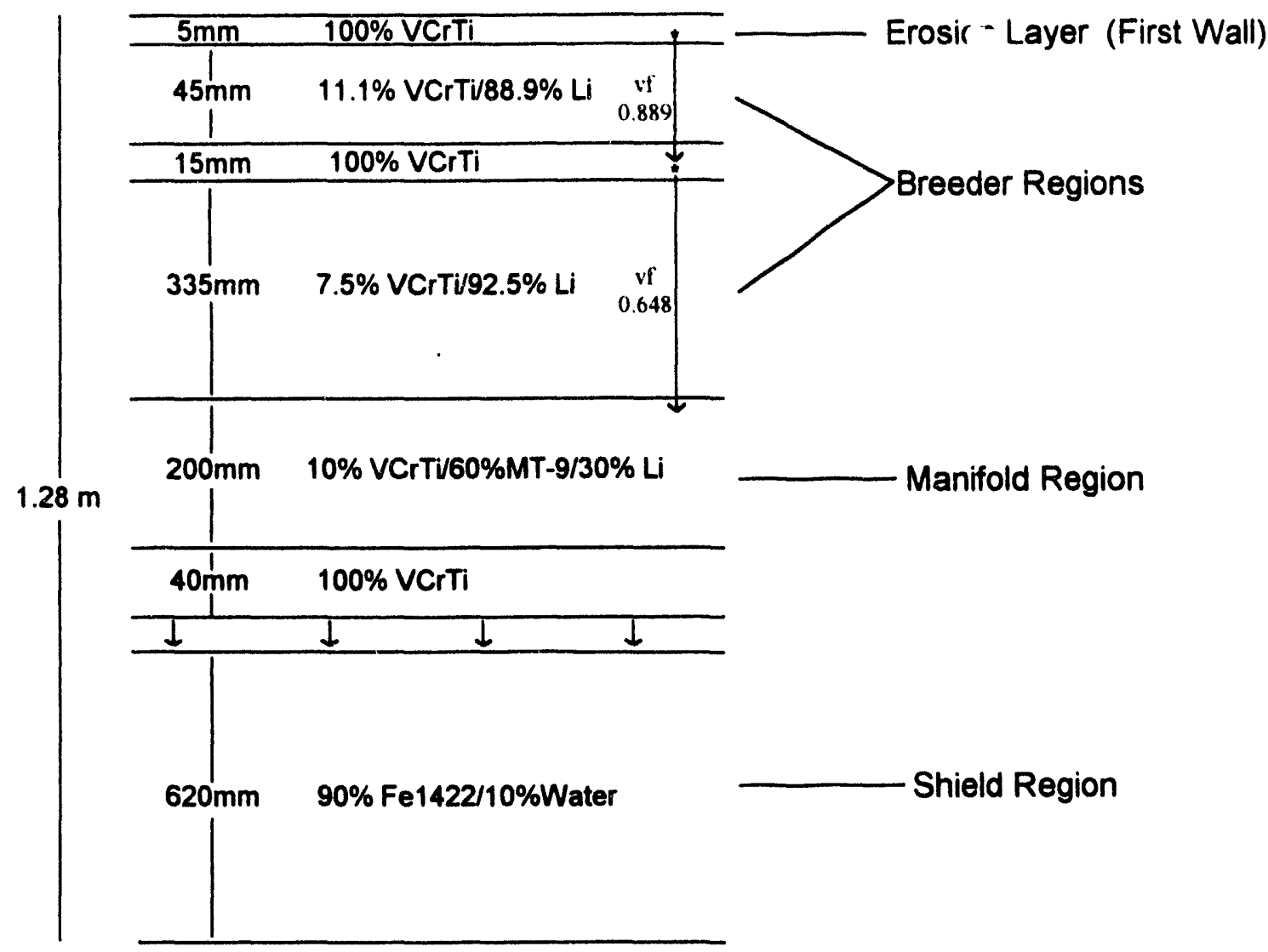

is taken from BCSS. This figure shows the first wall followed by the two breeder regions (separated by a wall of 100 percent $\mathrm{VCrTi}$ ) followed by the marifold region. The manifold region contains MT-9 ferritic steel as well as VCrTi. Behind the manifold region is a $40 \mathrm{~mm}$ thick VCrTi wall followed by a vacuum gap and then the shield. The shield is composed of water and Fe1422 ferritic steel, which is composed of 14 percent manganese, 2 percent nickel and 2 percent chromium, the remainder being iron. Fel 422 has a lower strength than MT-9, but is also significantly less expensive, and can be used in the low-pressure shield region. The arrows in Figure Al1.2 indicate radiant heat flow 
paths which exist following a LOCA in which the lithium coolant is fully drained out of the blanket. Because of the blanket geometry, radiant heat transfer can occur between the first wall and the wall separating the breeder regions, and between the wall separating the breeder regions and the manifold region. The view factors ( $v f$ ) for radiant heat transfer are indicated in the figure. ${ }^{6}$ Of course, a radiant heat flow path with a view factor of 1.0 always exists between the back of the blanket and the front of the shield.

The method for the LOCA analysis of the $\mathrm{V} / \mathrm{Li} / \mathrm{Li}$ blanket proceeds in the same manner as did the analysis for Blanket Designs 1 and 2 in Chapter 5. Hence, only the substantial data relevant to the present analysis will be presented here. The afterheat of the V15Cr5Ti structure as a function of time after shutdown is shown in Figure A11.3 for various distances behind the first wall of the BCSS V/Li/Li blanket. The lithium coolant exhibits negligible afterheat compared to the VCrTi structure, and, in any event, is assumed to drain away during the LOCA.

\footnotetext{
${ }^{6}$ Note that a similar analysis for this blanket design was performed by J.E. Massidda and M.S. Kazimi in Thermal Design Considerations for Passive Safety of Fusion Reactors, MIT Plasma Fusion Center Report \#PFC/RR-87-18 dated October 1987. Massidda and Kazimi included a radiant heat flow path from the wall between the breeder regions to the rearmost wall of the blanket. This view path is possible given the $\mathrm{V} / \mathrm{Li} / \mathrm{Li}$ blanket geometry, but has a view factor of only 0.278 , and does not change the results of the analysis significantly. For the present work, this radiant heat flow path is conservatively neglected.
} 
Figure A11.3. Afterheat in V15Cr5Ti for the BCSS V/Li/Li Blanket

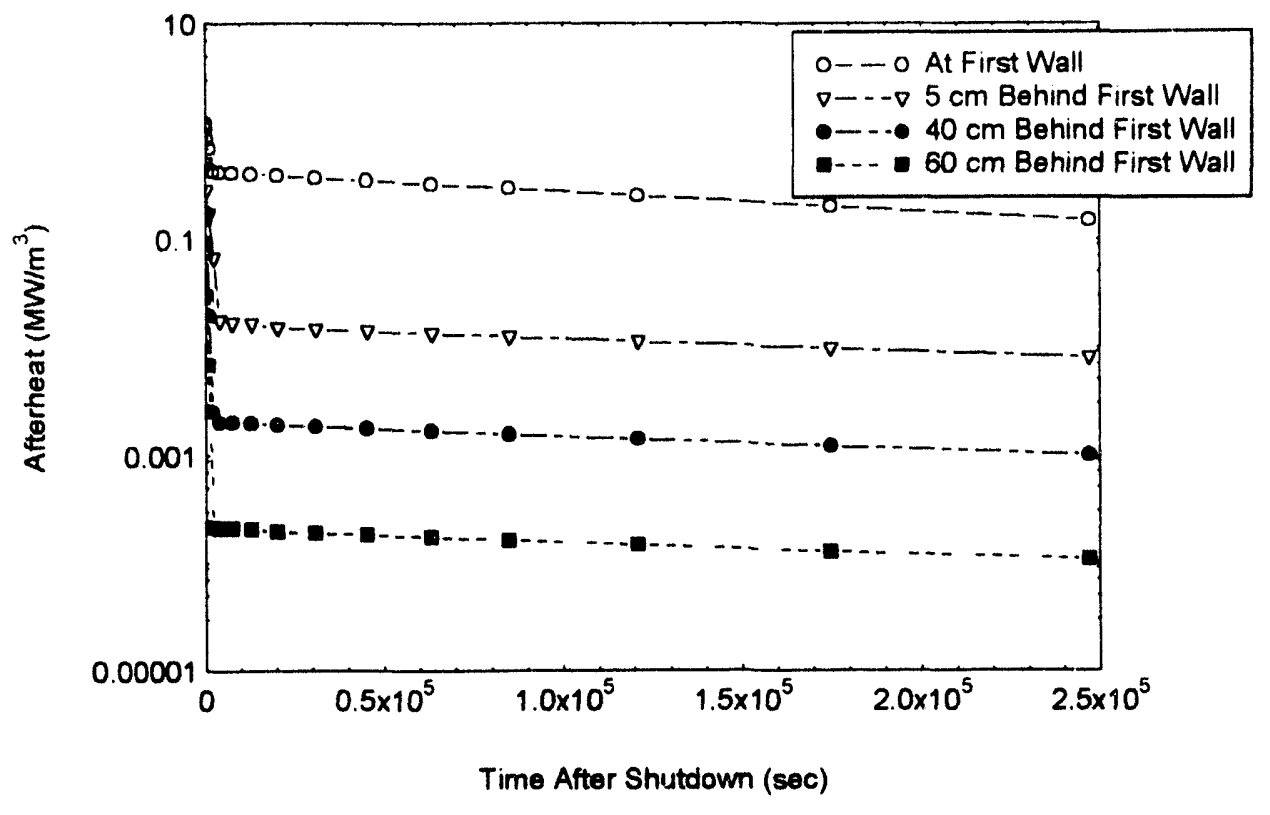

The afterheat of the MT-9 in the manifold region of the BCSS V/Li/Li blanket is shown in Figure Al1.4 for the front and back regions of the manifold.

Figure A11.4. Afterheat of MT-9 in Manifold of the BCSS V/Li/Li Blanket

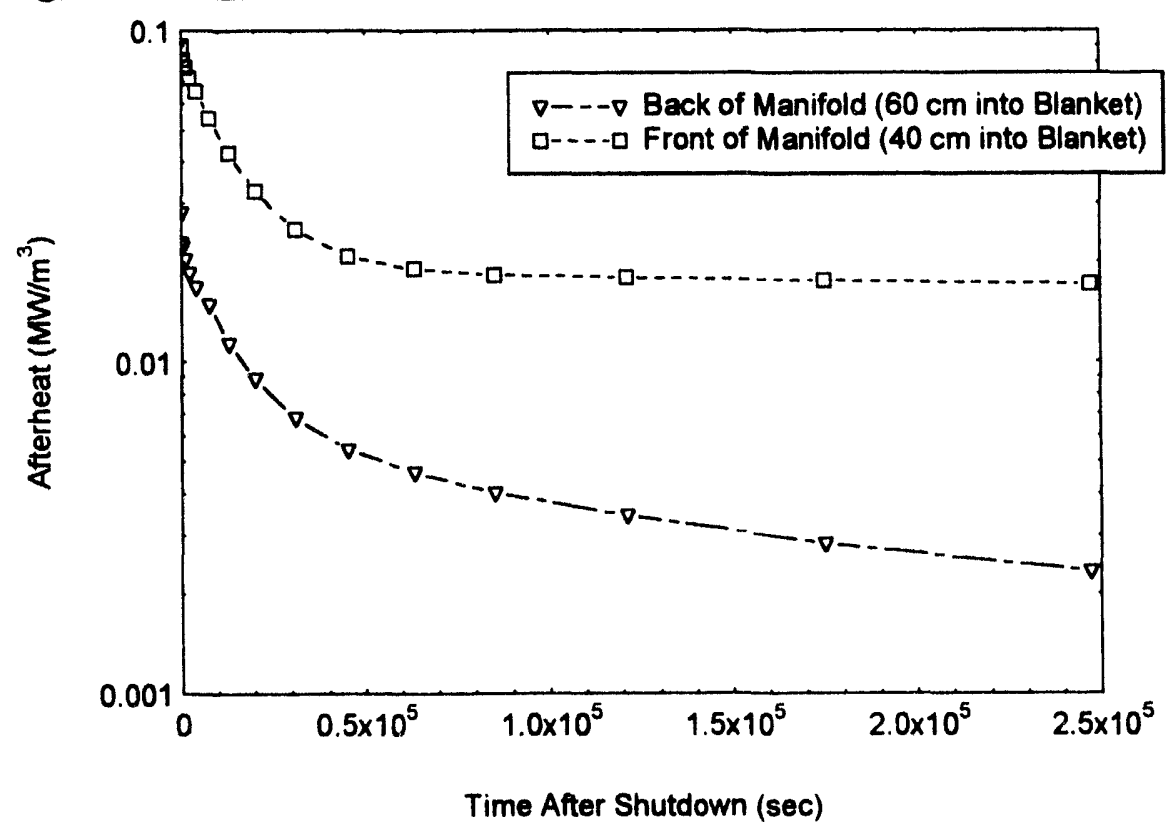


The afterheat of the Fe142.2 in the shield region is shown in Figure A11.5. The afterheat generated by the water in the shield is negligible compared to the structural afterheat.

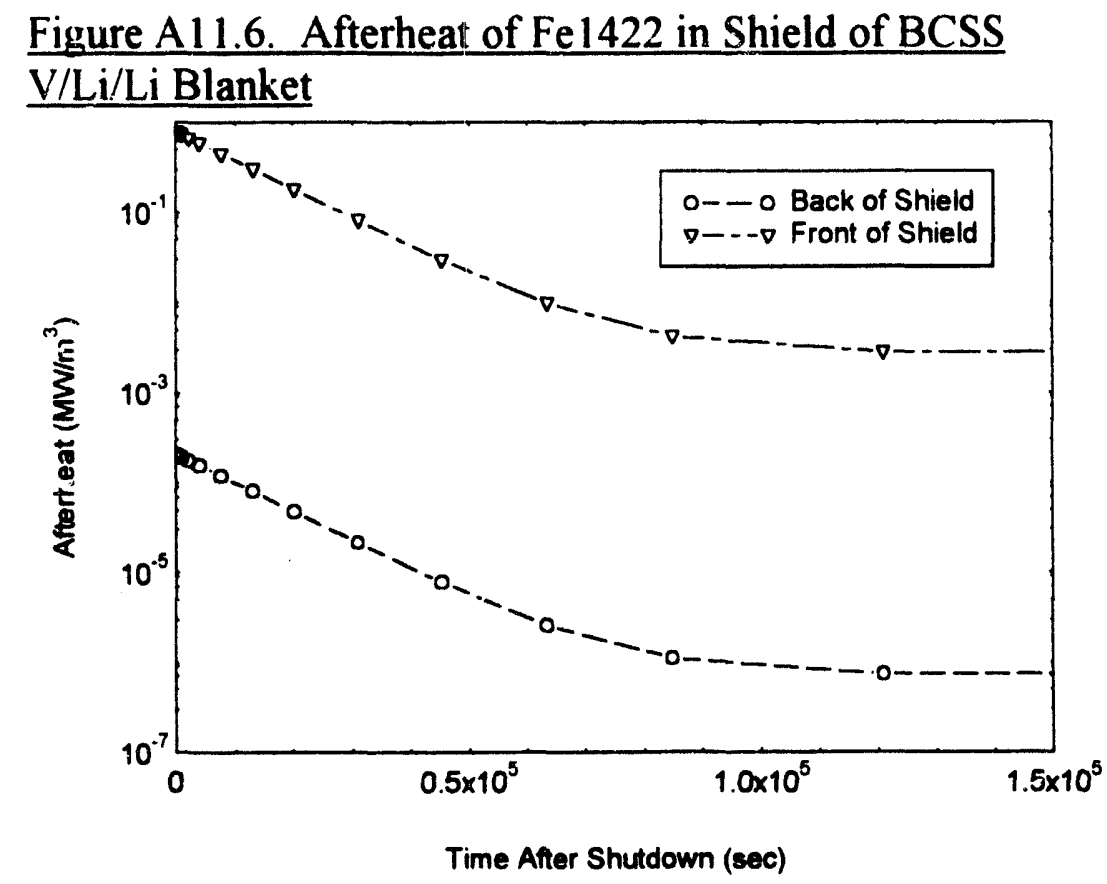

Figure A11.7. Afterheat of MT-9 in Blanket Design 2 Shield

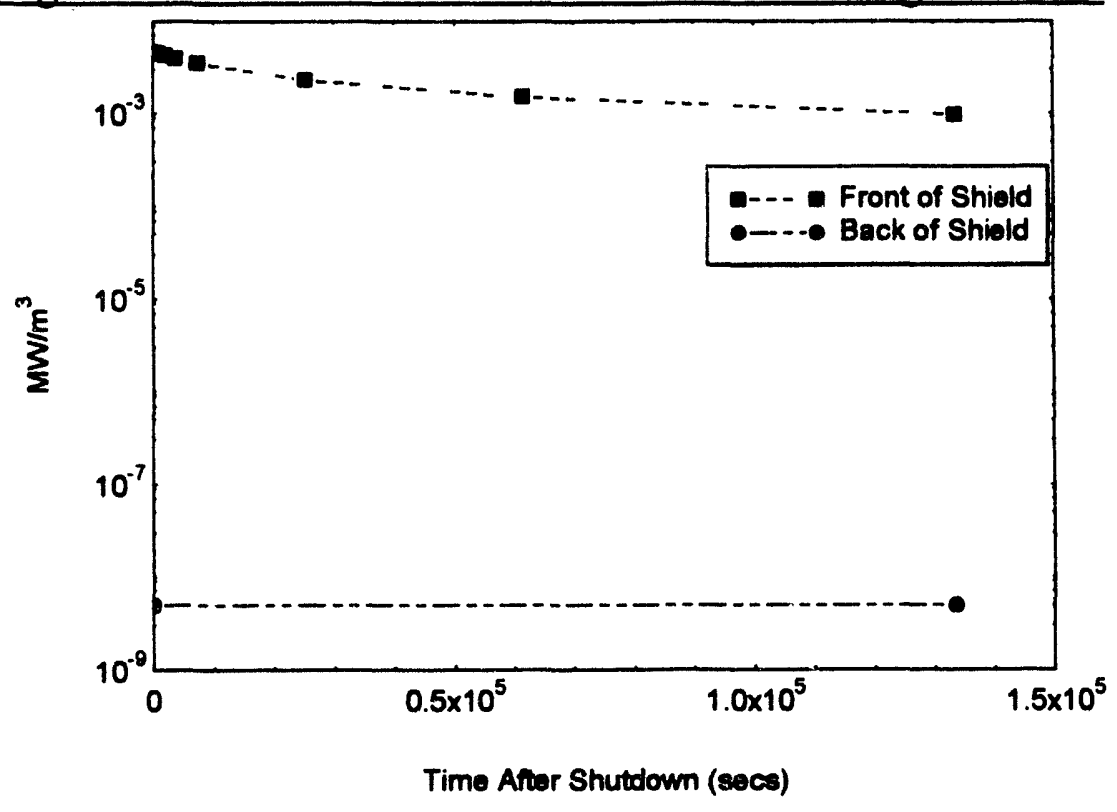

It is interesting to compare the afterheat generated by the Fe1422 in the $\mathrm{V} / \mathrm{Li} / \mathrm{Li}$ blanket shield to the afterheat generated by the MT -9 in the shield of Blanket Design 2. The afterheat of the Blanket Design 2 shield was discussed in Section 6.1.2, and the results are reproduced again here in Figure A11.7. The afterheat in the shield of the $\mathrm{V} / \mathrm{Li} / \mathrm{Li}$ blanket is much higher than the afterheat in the shield of Blanket Design 2.

There are two main 
reasons for the large difference in afterheating in the two shields. Firstly, the MT-9 of the Blanket Design 2 shield has lower activation characteristics than the Fe1422 used in the $\mathrm{V} / \mathrm{Li} / \mathrm{Li}$ shield. Secondly, and most significantly, the V Li Li blanket is significamlly Ihinner than Blanket Design 2. This results in a higher, harder neutron flux impacting on the front of the $\mathrm{V} / \mathrm{Li} / \mathrm{Li}$ shield than impacts the shield for Blanket Design 2. This higher, harder neutron flux results in significantly more activation in the shield structure. As will be shown, the higher afterheat in the shield will have an important impact on the performance of the $\mathrm{V} / \mathrm{Li} / \mathrm{Li}$ blanket during a LOCA.

Important inputs to the LOCA analysis are the emissivities of the surfaces which can radiate heat in Figure A11.2. For the blanket-to-shield radiant heat flow path, and emissivity of 0.7 is assumed for both surfaces, as was done for Blanket Designs 1 and 2 and justified in Section 5.2.3.3. For the in-blanket surfaces, the emissivity is assumed to be 0.5 . This emissivity is typical for oxidized metal surfaces, ${ }^{7}$ and in the absence of data for $\mathrm{VCrTi}$, is used herein. ${ }^{8}$

\footnotetext{
${ }^{7}$ T. Baumeister, et.al., Mark's Standard Handbook for Mechanical Engineers, Eighth Ed, Mc-Graw Hill, 1978.

${ }^{8}$ Oxidation of the VCrTi surfaces will occur in the presence of oxygen, even at temperatures below 650 degrees C. However, below 650 degrees the oxidation is not as rapid, and does not result in formation of an oxide with a low melting point. Since we are concerned in the present analysis with a LOCA with air ingress, it is reasonable to assume an emissivity associated with an oxidized metal.
} 
The normal operating temperature as a function of distance from the first wall for the BCSS V/Li/Li blanket/shield is given in Figure A11.8. The distribution is linear within

Figure A11.8. Operating Temperature Distribution in V/Li/Li Blanket and Shield 9

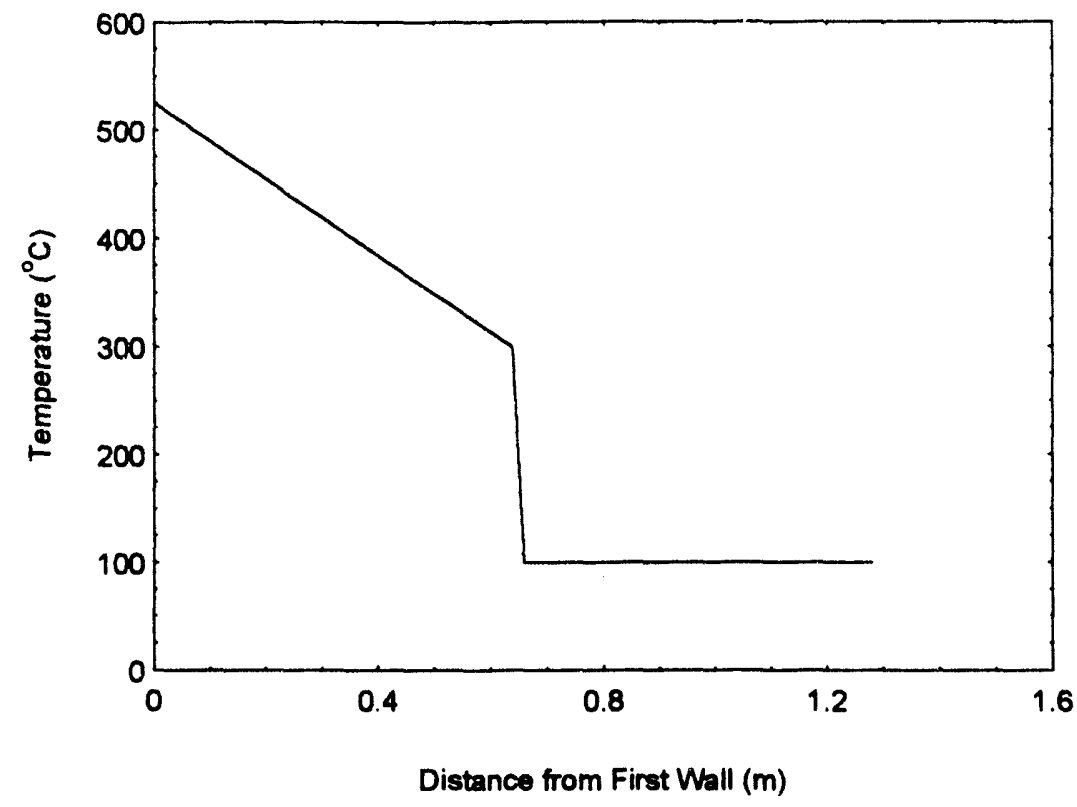

the blanket region, ranging from a first wall temperature of 525 degrees $\mathrm{C}$ to a temperature of 300 degrees $\mathrm{C}$ at the back of the blanket. The shield region is assumed to be 100 degrees $\mathrm{C}$ during normal operation.

Two LOCA cases are now presented. The first case assumes a LOCA with the plasma extinguishing at the onset of the LOCA, and with no cooling of the shield region after the onset of the accident. This could occur if there was a common-mode failure which caused both a LOCA in the lithium loop and a failure of the shield water system pump(s). This is the worst-case undercooling accident with respect to the oxidation concern, since both the blanket and the shield heat up, resulting in the highest temperatures in the VCrTi structure. The inboard blanket/shield configuration is analyzed here since the thermal mass of the inboard shield is lower than that of the outboard shield. This results in more rapid heating of the shield region, and hence more rapid heating of the blanket structure as well. As was done for the analysis of Blanket Design 2 at the beginning of

${ }^{9}$ From D. L. Smith, et.al., Blanket Comparison and Selection Study (BCSS) - Final Report, Argonne National Laboratory report \#ANL/FPP-84-1, September 1984. 
Chapter 6 , the blanket/shield configuration is assumed to be thermally isolated from the surroundings. Figure Al1.8 shows the results of this analysis. This figure shows the Figure A11.8. LOCA in Inboard V/Li/Li Blanket/Shield with temperature of the No Shield Water Circulation

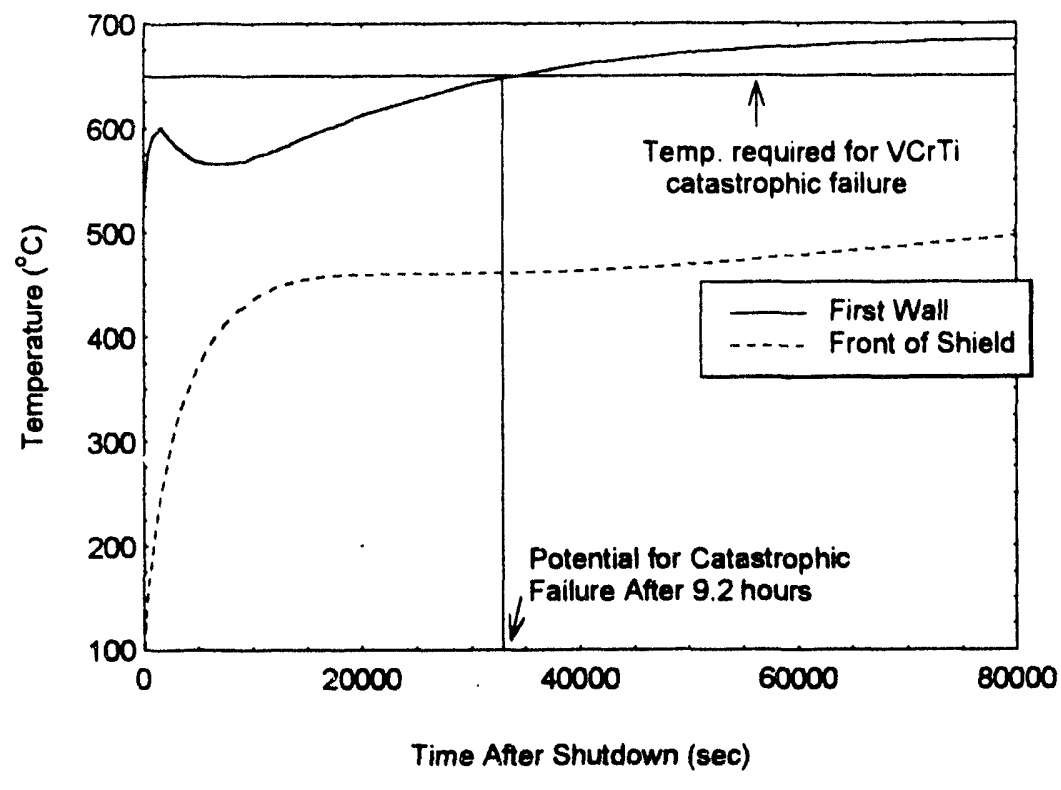

inboard $\mathrm{V} / \mathrm{Li} / \mathrm{Li}$

blanket first wall

and the front of

the shield. The

first wall

temperature rises

from an initial

temperature of 525

degrees $C$ to 600

degrees, then

drops temporarily

as the VCrTi afterheating near the first wall drops early in the transient (see Figure

A11.3). The first wall temperature then rises at a more moderate rate reaching 650 degrees after 9.2 hours. At this point, air ingress coupled with the LOCA could cause gross structural failure of the first wall region. The front of the $\mathrm{V} / \mathrm{Li} / \mathrm{Li}$ blanket shield rapidly heats up to 450 degrees $C$, then continues to rise in temperature more slowly. This heat up of the shield prevents heat from rapidly radiating from the blanket to the shield, exacerbating the heatup of the first wall.

The second LOCA analysis to be run assumes that the Shield Water System (SWS) has natural circulation capability. Hence, the front of the shield stays at 100 degrees $C$ throughout the transient. Except for this new boundary condition, the analysis is the same as the first LOCA analysis just presented. The results of the natural circulating SWS analysis are shown in Figure A11.10. 
Figure A11.10. LOCA in Inboard V/Li/Li Blanket/Shield The figure shows that with Natural Circulation in SWS

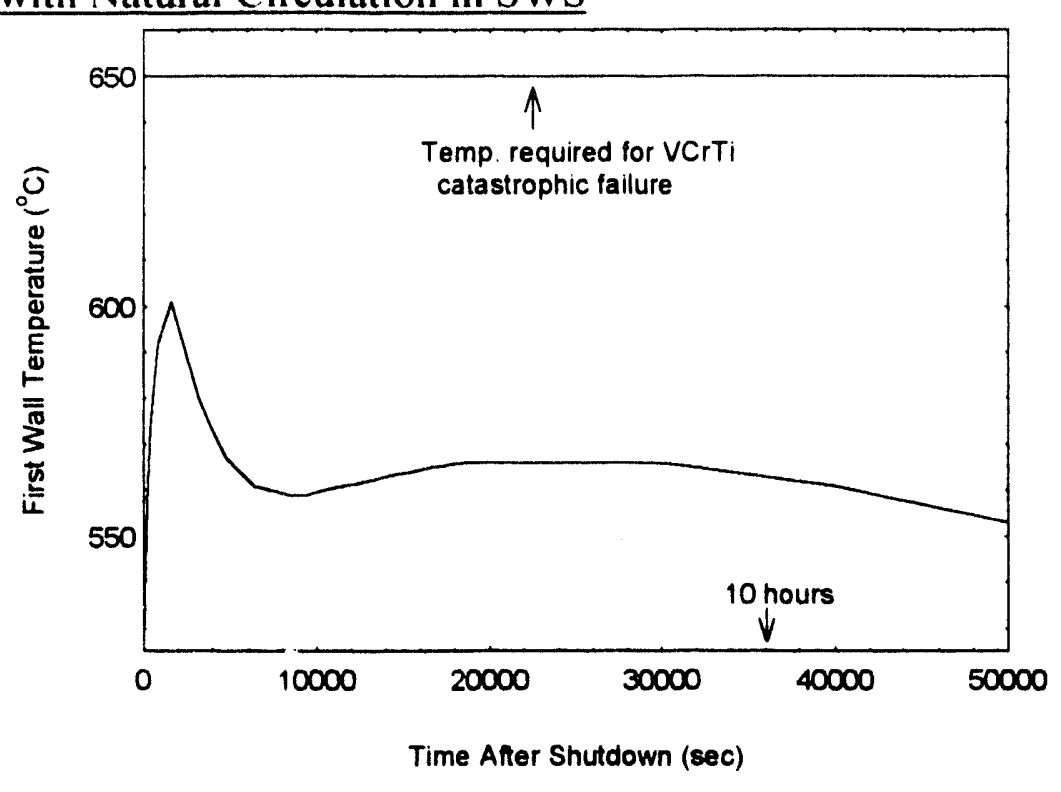

by keeping the front of the shield at 100 degrees $\mathbf{C}$ via natural circulation of the SWS, the $\mathrm{V} / \mathrm{Li} / \mathrm{Li}$ first wall is kept well below 650 degrees $\mathrm{C}$ for the entire transient, thus eliminating the risk of gross structural failure in the event of air ingress.

In Chapter 6 it was shown that for Blanket Design 2 the effect of a SWS capable of natural circulation ensured long-term (10 days after accident onset) passive safety of the blanket in the event of a No-Flow LOFA. This appendix has shown that for a different blanket design with a different worst case accident, having a SWS capable of natural circulation can greatly improve the short-term ( 9.2 hours after accident onset) passive safety of the blanket. Although a cost estimate for a natural circulation-capable SWS is not performed herein for the $\mathrm{V} / \mathrm{Li} / \mathrm{Li}$ blanket shield, based on the results of the analysis shown in Chapter 6 the cost would be expected to be minimal compared to the cost of the overall blanket/shield complex. 

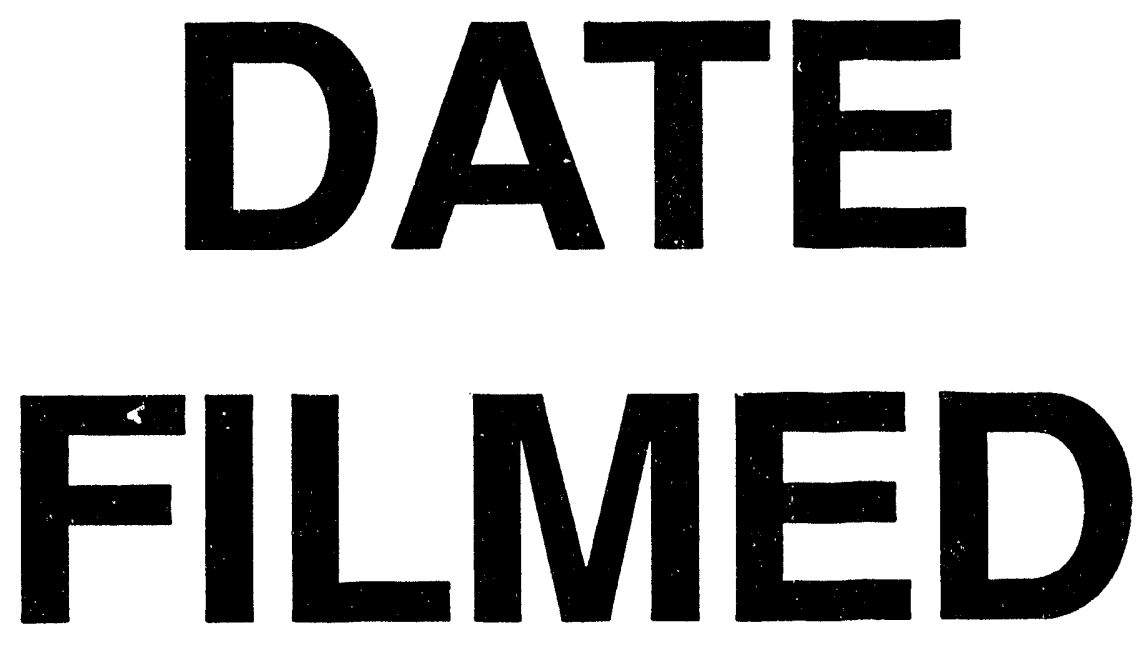

$10 / / 9 / 94$
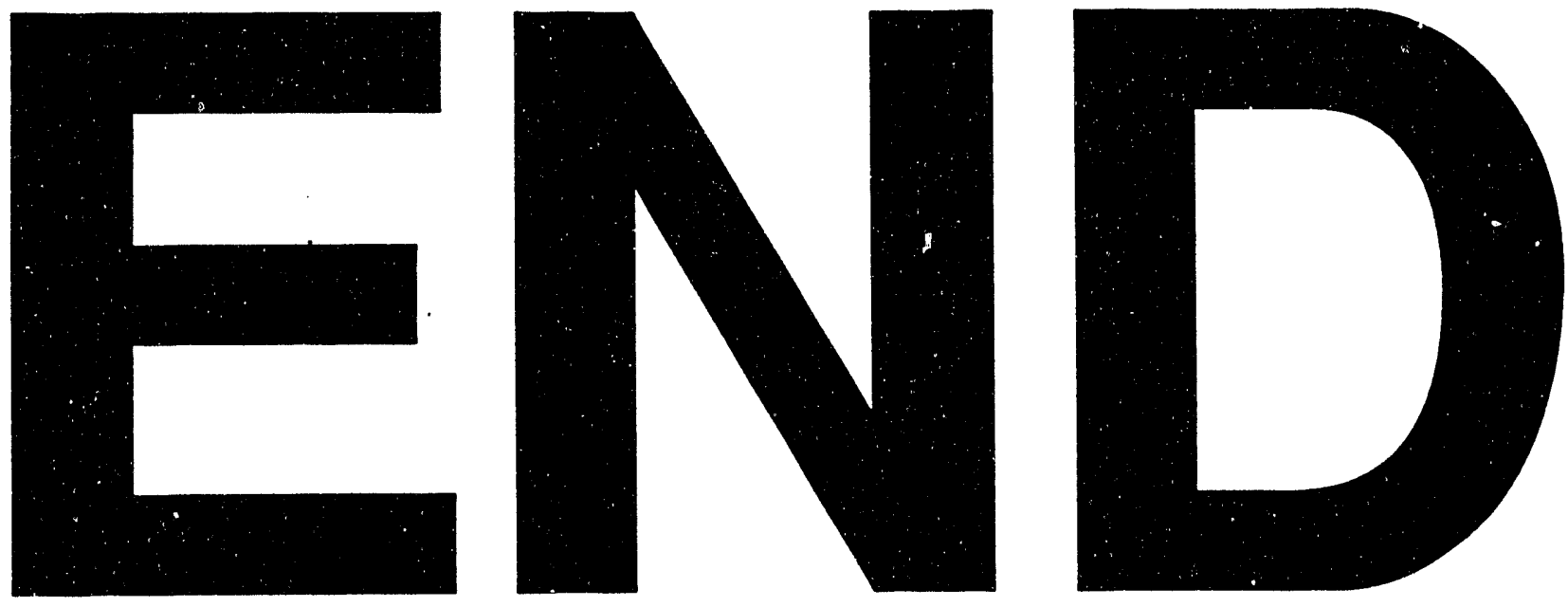
